\title{
THATCH: A Computer Code for Modelling Thermal Networks of High-Temperature Gas-Cooled Nuclear Reactors
}

Prepared by

P. G. Kroeger, R. J. Kennett. J. Colman, T. Ginsberg

Brookhaven National Laboratory

Prepared for

U.S. Nuclear Regulatory Commission 


\section{AVAILABILITY NOTICE}

Availability of Reference Materials Cited in NRC Publications

Most documents cited in NRC publications will be avallable from one of the following sources:

1. The NRC Public Document Room, 2120 L Street. NW., Lower Level, Washington. DC 20555

2. The Superintendent of Documents, U.S. Government Printing Office, P.O. Box 37082, Washington, DC 20013-7082

3. The National Technical Information Service, Springfield, VA 22161

Although the listing that follows represents the majority of documents clted in NRC publicaticns, it is not intended to be exhaustive.

Referenced documents available for inspection and copying for a fee from the NRC Public Document ?:oom include NRC correspondence and internal NRC memoranda; NRC bulletins, circulars, infiormation notices, Inspection and investigation notices; licensee event reports; vendor reports and correspondence; Commission papers; and applicant and licensee documents and correspondence.

The following documents in the NUREG series are available for purchase from the GPO Sales Program: formal NRC staff and contractor reports. NRC-sponsored conference proceedings, international agreement reports, grant publications, and NRC booklets and brochures. Also available are regulatory guides, NRC regulations in the Code of Federal Regulations, and Nuclear Regulatory Commission Issijances.

Documents avalable from the National Technical Information Service include NUREG-series reports and technical reports prepared by other Federal agencies arid reports prepared by the Atomic Energy Commission. iorerunner agency to the Nuclear Regulatory Commission.

Documents available from public and special technical libraries include all open literature items, such as books. Journal articles, and transactions. Federal Register notices. Federal and State legislation, and congrescional reports can usually be obtained fromi these libraries

Documents such as theses, dissertations. foreign reports and translations, and mon-NRC conference proceedings are available for purchase from the organization sponsoring the publication cited.

Single copies of NRC draft reports are available free, to the extent of supply, upon written request to the Office of Administration. Distribution and Mail Services Section. U.S. Nuclear Regulatory Commission. Washington, DC 20555.

Copies of industry codes and standards used in a substantive manner in the NRC regulatory process are maintained at the NRC Library, 7920 Norfolk Avenue. Bethesda, Maryland. for use by the public. Codes and standards are usually copyrighted and may be purchased from the originating organization or. If they are American National Standa!ds, from the American National Standards Institute, 1430 Broadway. New York. NY $100: 8$.

\section{DISCLAIMEA NOTICE}

This report was prepared as an account of work sponsored by an agency of the United States Government. Neither the United States Government nor any agency thereof, or any of their employees, makes any warranty. expressed or implied, or assumes any legal liability of responsibility for any third party's use, or the results of such use, of any information, apparatus, product or process disclosed in this report, or represents that its use by such third party would not infringe privately owned rights. 
NUREG/CR--5620

TI92 003261

\section{THATCH: A Computer Code for Modelling Thermal Networks of High-Temperature Gas-Cooled Nuclear Reactors}

Manuscript Completed: September 1990

Date Published: October 1991

Prepared by

P. G. Kroeger, R. J. Kennett, J. Colman, T. Ginsberg

Brookhaven National Laboratory

Upton, NY 11973

Prepared for

Division of Regulatory Applications

Office of Nuclear Regulatory Research

U.S. Nuclear Regulatory Commission

Washington, DC 20555

NRC FIN A3827

Under DOE Contract No. DE-AC02-76CH00016

\section{MASTER}




\begin{abstract}
This report documents the THATCH code, which can be used to model general thermal and flow networks of solids and coolant channels in two-dimensional r-z geometries.

The main application of THATCH is to model reactor thermo-hydraulic transients in HighTemperature Gas-Cooled Reactors (HTGRs). The available modules simulate pressurized or depressurized core heatup transients, heat transfer to general exterior sinks or to specific passive Reactor Cavity Cooling Systems, which can be air or water-cooled. Graphite oxidation during air or water ingress can be modelled, including the effects of added combustion products to the gas flow and the additional chemical energy release. A point kinetics model is available for analyzing reactivity excursions; for instance due to water ingress, and also for hypothetical no-scram scenarios. For most HTGR transients, which generally range cver hours, a user-selected nodalization of the core in r-z geometry is used. However, a separate model of heat transfer in the symmetry element of each fuel element is also available for very rapid transients. This model can be applied coupled to the traditional coarser $\mathbf{r}-\mathbf{z}$ nodalization.
\end{abstract}

This report describes the mathematical models used in the code and the method of solution. It describes the code and its various sub-elements. Details of the input data and file usage, with file formats, is given for the code, as well as for several preprocessing and postprocessing options. The THATCH model of the currently applicable $350 \mathrm{MW}_{\text {th }}$ reactor is described. Input data for four sample cases are given with output available in fiche form. Installation requirements and code limitations, as well as the most common error indications are listed. 
ABSTRACT $\ldots \ldots \ldots \ldots \ldots \ldots \ldots \ldots \ldots \ldots \ldots \ldots \ldots \ldots \ldots \ldots$ ii

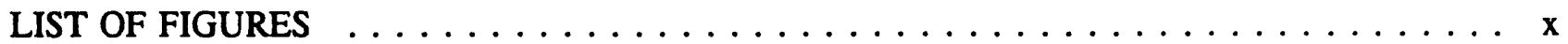

LIST OF TABLES $\ldots \ldots \ldots \ldots \ldots \ldots \ldots \ldots \ldots \ldots \ldots \ldots \ldots \ldots \ldots \ldots \ldots$ xii

ACKNOWLEDGEMENTS $\ldots \ldots \ldots \ldots \ldots \ldots \ldots \ldots \ldots \ldots \ldots \ldots \ldots$ ivx

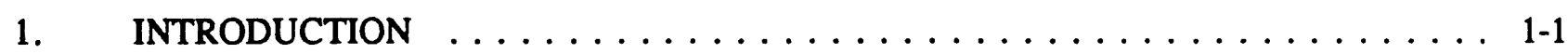

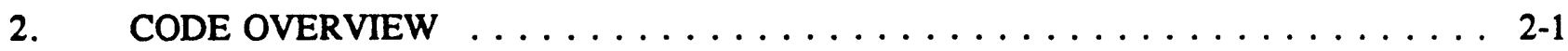

2.1 Description of the THATCH Main Code $\ldots \ldots \ldots \ldots \ldots \ldots$

2.2 Sub-Module Descriptions $\ldots \ldots \ldots \ldots \ldots \ldots \ldots \ldots \ldots \ldots \ldots \ldots$

2.2.1 PASCOL Module for Air-Cooled RCCS . . . . . . . . 2-1

2.2.2 WATCOL Module for Water-Cooled RCCS . . . . . . . . . . 2-2

2.2.3 FLOXI Module for Reactor Gas Flow, Heat

Transfer, and Chemical Reactions . . . . . . . . . . . 2-2

2.2.4 PKIN Module for Point Kinetics Option . . . . . . . . . 2-3

2.2.5 SYMELAN Module for Rapid Thermal Core

Transients Using Symmetry Element

Nodalization ...................... 2-3

3. CODE MODELS AND METHOD OF SOLUTION $\ldots \ldots \ldots \ldots \ldots \ldots \ldots \ldots$

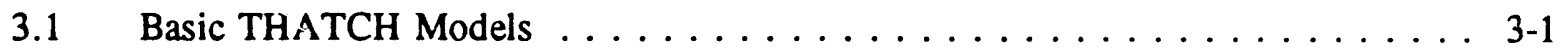

3.1.1 THATCH Conduction/Radiation

Heat Transfer in a Solid Network . . . . . . . . . . . . . 3-1

3.1.2 THATCH Plena Two-Dimensional

Radiation Heat Transfer . . . . . . . . . . . . 3-8

3.1.3 Method of Solution for THATCH Models $\ldots \ldots \ldots \ldots \ldots$. . . . . .

3.2 PASCOL Models for Air-Cooled RCCS $\ldots \ldots \ldots \ldots \ldots \ldots \ldots$ 3-11

$3 ? .1$ Heat Transfer Across Reactor Cavity . . . . . . . . . . 3-12

3.2.2 Heat Transfer from the RCCs

Panels to the Coolant and Coolant

Energy Equation . . . . . . . . . . . . . . . . . . . 3-14 


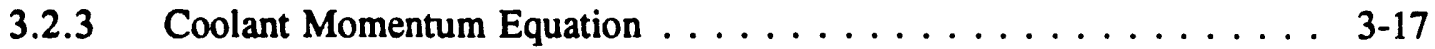

3.2.4 Method of Solution for PASCOL Module $\ldots \ldots \ldots \ldots \ldots \ldots$ 3-19

3.3 WATCOL Module for Water-Cooled RCCS $\ldots \ldots \ldots \ldots \ldots \ldots \ldots$ 3-20

3.3.1 Assumptions $\ldots \ldots \ldots \ldots \ldots \ldots \ldots \ldots \ldots \ldots \ldots \ldots \ldots$

3.3.2 Reactor Vessel $\ldots \ldots \ldots \ldots \ldots \ldots \ldots \ldots \ldots \ldots \ldots \ldots$ 3-22

3.3.3 Water Heat Transport Loop $\ldots \ldots \ldots \ldots \ldots \ldots \ldots \ldots \ldots$ 3-22

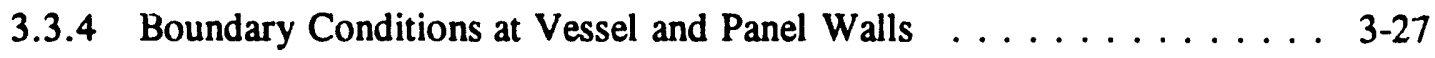

3.3.5 Air-Water Heat Exchanger $\ldots \ldots \ldots \ldots \ldots \ldots \ldots \ldots . \ldots \ldots$

3.3.6 Constitutive Relations $\ldots \ldots \ldots \ldots \ldots \ldots \ldots \ldots \ldots \ldots$ 3-31

3.3.7 Method of Solution for WATCOL Module $\ldots \ldots \ldots \ldots \ldots \ldots$ 3-32

3.4 FLOXI Module for Reactor Gas Flow, Heat Transfer and Chemical Reactions $\ldots \ldots \ldots \ldots \ldots \ldots \ldots \ldots \ldots \ldots \ldots \ldots$

3.4.1 Gas Flow in the Reactor Vessel $\ldots \ldots \ldots \ldots \ldots \ldots \ldots$ 3-33

3.4.2 Reactor Coolant to Solid Heat Transfer . . . . . . . . . . 3-36

3.4.3 Chemical Reactions During Gas Ingress Scenarios . . . . . . . 3-37

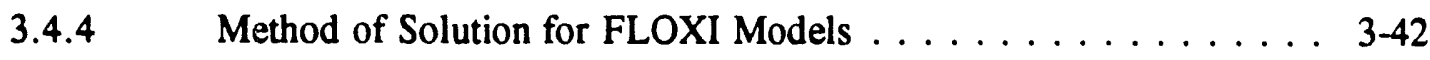

3.5 PKIN Module for Point Kinetics Model $\ldots \ldots \ldots \ldots \ldots \ldots \ldots \ldots \ldots$ 3-43

3.5.1 Point Kinetics Model $\ldots \ldots \ldots \ldots \ldots \ldots \ldots \ldots$ 3-43

3.5.2 Method of Solution for Point Kinetics Model $\ldots \ldots \ldots \ldots$ 3-43

3.6 SYMELAN Module for Rapid Thermal Core Transients Using

Symmetry Element Nodalization . . . . . . . . . . . . . . . . 3-47

3.6.1 Symelan Active Core Heat Transfer Model $\ldots \ldots \ldots \ldots$. $\ldots$.48

3.6.2 Method of Solution $\ldots \ldots \ldots \ldots \ldots \ldots \ldots \ldots \ldots$. $\ldots \ldots 6$ 
4. PROGRAM STRUCTURE $\ldots \ldots \ldots \ldots \ldots \ldots \ldots \ldots \ldots$ 4-1

4.1 THATCH Subroutines $\ldots \ldots \ldots \ldots \ldots \ldots \ldots \ldots \ldots \ldots$ 4-1

4.1.1 THATCH Main Program $\ldots \ldots \ldots \ldots \ldots \ldots \ldots \ldots$ 4-1

4.1.2 THATCH Input Subroutines $\ldots \ldots \ldots \ldots \ldots \ldots \ldots \ldots$ 4-1

4.1.3 THATCH Subroutines for Internal Conductance

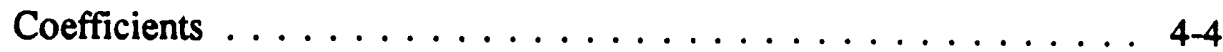

4.1.4 THATCH Subroutines for Time Integration of Solid Temperatures . . . . . . . . . . . . . . . . . 4-7

4.1.5 THATCH Solid and Gap Property Routines . . . . . . . . 4-12

4.1.6 THATCH Output Routines $\ldots \ldots \ldots \ldots \ldots \ldots \ldots \ldots$ 4-13

4.1.7 THATCH Utility Subroutines $\ldots \ldots \ldots \ldots \ldots \ldots \ldots \ldots$. . . . . . . .

4.1.8 THATCH Interface Subroutines $\ldots \ldots \ldots \ldots \ldots \ldots \ldots$ 4-14

4.2 BIG Subroutines $\ldots \ldots \ldots \ldots \ldots \ldots \ldots \ldots \ldots \ldots \ldots \ldots \ldots \ldots$ 4-14

4.2.1 PASCOL Subroutines $\ldots \ldots \ldots \ldots \ldots \ldots \ldots \ldots$ 4-14

4.2.2 WATCOL Subroutines $\ldots \ldots \ldots \ldots \ldots \ldots \ldots \ldots$. . . . . . . . .

4.2.3 FLOXI Subroutines $\ldots \ldots \ldots \ldots \ldots \ldots \ldots \ldots \ldots$ 4-32

4.2.4 PKIN Subroutines $\ldots \ldots \ldots \ldots \ldots \ldots \ldots \ldots \ldots . \ldots .4 .43$

4.2.5 SYMELAN Subroutines $\ldots \ldots \ldots \ldots \ldots \ldots \ldots \ldots \ldots$. . . . . . . .

4.2.6 GENLIB Subroutines $\ldots \ldots \ldots \ldots \ldots \ldots \ldots \ldots \ldots$. $\ldots \ldots 1$

4.2.7 THTCHLIB Subroutines $\ldots \ldots \ldots \ldots \ldots \ldots \ldots \ldots$ 4-66

4.2.8 GASPRLIB Subroutines $\ldots \ldots \ldots \ldots \ldots \ldots \ldots \ldots$ 4-70

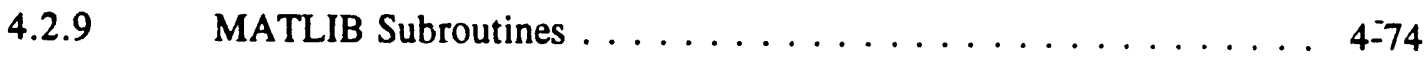

4.2.10 THPROCS Subroutines . . . . . . . . . . . . . . . 4-77

4.2.11 THRADLIB Subroutines $\ldots \ldots \ldots \ldots \ldots \ldots \ldots \ldots$ 4-84 
TABLE OF CONTENTS (CONT'D)

4.3 Code Maintenance via UPDATE/ HISTORIAN $\ldots \ldots \ldots \ldots \ldots \ldots$

4.4 THATCH and BIG Commons $\ldots \ldots \ldots \ldots \ldots \ldots$

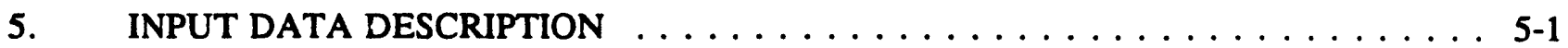

5.1 Input to THATCH Runs, Including THATCH Submodules $\ldots \ldots \ldots$. . . .

5.1 .1 THATCH Input Processor $\ldots \ldots \ldots \ldots \ldots \ldots \ldots \ldots \ldots \ldots \ldots$ 5-1

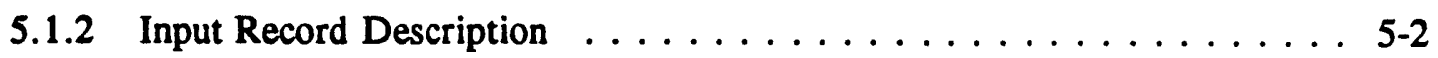

5.2 Input to THRADLIB Preprocessor for Radiation

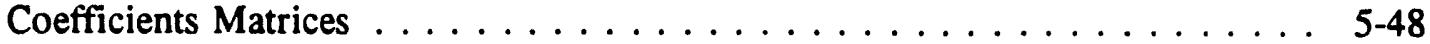

5.3 Input to THPROCS Post Processing and Plot File Writing . . . . . . . . 5-50

6. FILE USAGE OF THATCH AND IT'S SUB MODULES $\ldots \ldots \ldots \ldots \ldots$

7. PREPROCESSING REQUIREMENTS AND POST PROCESSING OPTIONS $\ldots \ldots \ldots$. . $7-1$

7.1 Preprocessing Requirements $\ldots \ldots \ldots \ldots \ldots \ldots \ldots \ldots \ldots \ldots \ldots$

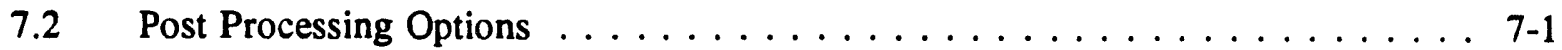

8. THATCH MODEL OF THE COMMERCIAL MHTGR $\ldots \ldots \ldots \ldots \ldots$

8.1 Standard Nodalization and Block Description of the THATCH Model of the Commercial MHTGR $\ldots \ldots \ldots \ldots \ldots \ldots \ldots$. . . . . . . .

8.2 PROPMAT Subroutine for Solid and Gap Material Properties . . . . . . . . . . 8-1

8.3 Irradiation Damage and Annealing Model for Thermal Conductivity

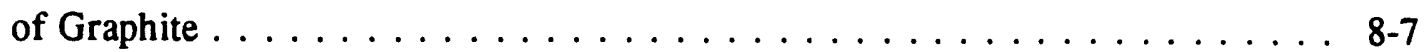

8.4 Modelling of Solid Heating Rate in THATCH $\ldots \ldots \ldots \ldots \ldots$

8.5 Upper Plenum Natural Convection Model . . . . . . . . . . . . . . . . 8 8-9

9. SAMPLE CASES AND INSTALLATION REQUIREMENTS $\ldots \ldots \ldots \ldots$. . . . . . 9-1 
TABLE OF CONTENTS (CONT'D)

10. THATCH CODE CAPABILITIES AND LIMITATIONS $\ldots \ldots \ldots \ldots \ldots$

10.1 Code Capabilities $\ldots \ldots \ldots \ldots \ldots \ldots \ldots \ldots \ldots \ldots \ldots \ldots$

10.2 Code Limitations $\ldots \ldots \ldots \ldots \ldots \ldots \ldots \ldots \ldots \ldots$

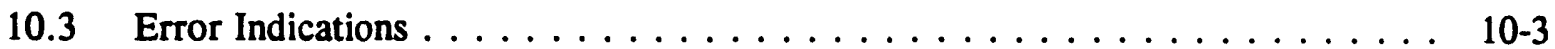

10.4 Code Service $\ldots \ldots \ldots \ldots \ldots \ldots \ldots \ldots \ldots \ldots \ldots \ldots \ldots$

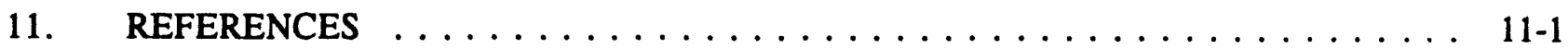

Appendix A - Radiation Exchange between Gray Surfaces in a Closed Cavity with a Transparent Gas . . . . . . . . . . . . . . A-1

Appendix B - Radiation View Factors for Prescribed Geometries . . . . . . . . . . . . B-1

B.1 Cylindrical Cavities $\ldots \ldots \ldots \ldots \ldots \ldots \ldots \ldots \ldots \ldots \ldots$ B-1

B.2 Annular Cylindrical Cavities $\ldots \ldots \ldots \ldots \ldots \ldots \ldots \ldots$

Appendix C - Internal Evaluation of Heat Transfer in Finned RCCS

Upflow Panels ....................... C-1

Appendix D - Properties of Gasses and Gas Mixtures . . . . . . . . . . . . D-1

D.1 Thermal Gas Properties $\ldots \ldots \ldots \ldots \ldots \ldots \ldots$ D-1

D.2 Gas Viscosities ..................... D-3

D.3 Gas Thermal Conductivities $\ldots \ldots \ldots \ldots \ldots \ldots \ldots \ldots$

Appendix E - Tabular Summary of THATCH Subroutines and Commons $\ldots \ldots \ldots$. . . E-1

r.ppendix F - Input Decks for Sample Cases of Section $9 \ldots \ldots \ldots \ldots \ldots$ 


\section{LIST OF FIGURES}

Figure No,

Title

Page

1-1

MHTGR in Underground Silo $\ldots \ldots \ldots \ldots \ldots \ldots$ 1-2

1-2

Schematic of MHTGR Primary Loop . . . . . . . . . . . . . . 1-2

1-3

Passive Decay Heat Removal System . . . . . . . . . . . . 1-3

Nodalization Convention of the THATCH Code

3-2 Schematic of a Solid Network Broken Down

Into "Blocks" for Modelling by the THATCH Code . . . . . . . . . 3-3

3-3 Schematic of Node Interface and Block

Boundary Designations . . . . . . . . . . . . . . . . . . 3-4

3-4 Radial Gap Between Solid Structures in the

THATCH Code ...................... 3-6

3-5 Schematic of Heat Flows in Nodes Adjacent

to Plena . . . . . . . . . . . . . . . . . . . . 3-10

3-6 Schematic of Internally Finned RCCS Up-

Flow Channel of the RCCS . . . . . . . . . . . . . . . 3-15

3-7 Schematic of the Flow Loop of the Water-

Cooled RCCS ......................... 3-21

.3-8 Typical Axial Node Through Vessel and Cooling Panel . . . . . . . . 3-23

3-9 Schematic of Natural Draft Water/Air Heat Exchanger . . . . . . . 3-30

3-10 Standard Fuel Element of the MHTGR

(Bechtel, 1986) . . . . . . . . . . . . . . . . . . 3-49

3-11 Symmetry Element Contained in MHTGR Fuel Element . . . . . . 3-50

4-1 Flow Chart of Time Step Integration Loop

in TIMSOL . . . . . . . . . . . . . . . . . . . 4-8

4-2 Flow Chart of TEMPSOL Solid Temperature Field

Solution Procedure . . . . . . . . . . . . . . . . . . . . . 4-9

4-3 Y-Vector Indices Versus Axial Node Indices in

PASCOL for Case with Thermal Insulation Between

Flow Passages. 


\section{FIGURES (CONT'D)}

Figure No.

Title

$\underline{\text { Page }}$

4-4 Y-Vector Indices Versus Axial Node Indices in

PASCOL for Case without Thermal Insulation Between

Flow Passages . . . . . . . . . . . . . . . . . . . . . . 4 4-22

4-5 Schematic of PASCOL Solution of Air-Cooled RCCS

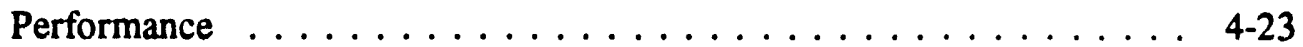

4-6 Flow Chart of FLOXIEX Main Loop for Coupled Flow,

Heat Transfer and Chemical Reaction Computations . . . . . . . . . . 4 4-35

4-7 Flow Chart of Point Kinetics Time Integration

in PKINEX . . . . . . . . . . . . . . . . . . . . . . 4-47

4-8 Flow Chart of TIMLOP Time Loop in SYMELAN $\ldots \ldots \ldots \ldots$. . . . 4-51

8-1 Schematic of THATCH Block Nodalization of MHTGR $\ldots \ldots \ldots$. .

A-1 Schematic of Incident, Absorbed, Emitted and

Reflected Energy and a Gray Surface Element . . . . . . . . . . . A-2

B-1 Schematic of Geometry for Disk and Annulus

Radiation View Factors $\ldots \ldots \ldots \ldots \ldots \ldots$ B-2

B-2 Schematic of Annular Cylindrical Cavity $\ldots \ldots \ldots \ldots \ldots \ldots$

C-1 Schematic of Nodalized Internal Fin Structure

of Air-Cooled RCCS . . . . . . . . . . . . . . . . . . . C-2

C-2 Schematic of Node Indices for RCCS Upflow Channel $\ldots \ldots \ldots \ldots$

C-3 Schematic for View Factors Between Surfaces

Parallel in the Z-Direction . . . . . . . . . . . . . . . . C-5 


\section{LIST OF TABLES}

Table No.

Title

Page

3-1 Reaction Rate Constants of THATCH/FLOXI Model for

Air and Water Ingress . . . . . . . . . . . . . . . . 3-40

3-2 Reactor Physics Data Which Can Be Varied by Use

of Namelist VARYINP . . . . . . . . . . . . . . . . . 3-51

3-3 Currently Available Options for Symmetry Element

Nodalization ...................... 3-52

4-1 Function of Sub-Libraries $\ldots \ldots \ldots \ldots \ldots \ldots \ldots$

4-2 THATCH and BIG Effective Main Programs $\ldots \ldots \ldots \ldots \ldots$

4-3 Header Records on THATCH Output File . . . . . . . . . 4-15

4-4 Time Records on THATCH Output File $\ldots \ldots \ldots \ldots \ldots$. . . . . .

4-5 THATCH-PASCOL Interface Routines $\ldots \ldots \ldots \ldots \ldots$ 4-18

4-6 THATCH-FLOXI Interface Routines $\ldots \ldots \ldots \ldots \ldots$. . . . . .

4-7 Variable Vector of WATCOL Module $\ldots \ldots \ldots \ldots$. . . . . . 4-29

4-8 WATCOL Function Type Subroutine for Liquid Water Properties

As Well As Friction and Heat Transfer Correlations for Flow

in Tubes and Across Tube Bank ............... . . . . . . .

4-9 FLOXI Output Data Record for THATCH Output File . . . . . . . 4-37

4-10 Y-Vector of Point Kinetics Module $\ldots \ldots \ldots \ldots \ldots$. . . . . . . .

4-11 Interface Subroutines for SYMELAN/THATCH

Floxi Interface . . . . . . . . . . . . . . . 4-56

4-12 SYMELAN Plot File Variable Vector on Time Records . . . . . . 4-59

4-13 SYMELAN Output File of Temperature Arrays $\ldots \ldots \ldots$. . . . . 4-60

4-14 Control of Integration in SOLTRAN via DIVPAG or
DGEAR Based on Character Control Variable CALLTYP $\ldots \ldots \ldots$. . $4-65$

4-15 Records on THPROCS Plot Files $\ldots \ldots \ldots \ldots \ldots$ 


\section{LIST OF TABLES (CCNT"D)}

4-19 View Factor Subroutines for Surface Elements

in Ânnular Cylindrical Cavities . . . . . . . . . . . . . . . . . . 4 4-87

5-1 Type of Boundary Resistances $\ldots \ldots \ldots \ldots \ldots \ldots \ldots$

5-2 Input Data in NAMELIST INPUT for THRADLIB $\ldots \ldots \ldots \ldots$

$6-1 \quad$ Input Files for THATCH Runs $\ldots \ldots \ldots \ldots \ldots \ldots \ldots$

$6-2 \quad$ Output Files for THATCH Rurs $\ldots \ldots \ldots \ldots \ldots$

7-1 Input Files for THPROCS THATCH Post Processing Runs $\ldots \ldots$. . .

7-2 Output Files for THPROCS THATCH Post Processing Runs $\ldots \ldots$. . $7-3$

8-1 Node Block Description of the Commercial MHTGR $\ldots \ldots \ldots$. . .

8-2 Solid Node Properties of the THATCH Commercial MHTGR Model ...................... . 8-4

8-3 Gap Effective Thermal Conductivities of the THATCH Cornmercial MHTGR Model . . . . . . . . . . . . 8 8-6

D-1 Thermodynamic Properties of Air $\ldots \ldots \ldots \ldots$

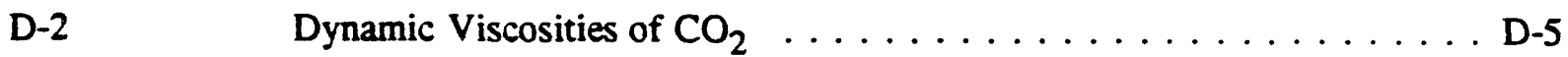

D-3 Dynamic Viscosities of $\mathrm{H}_{2} \mathrm{O} \ldots \ldots \ldots \ldots \ldots$

D-4 Dynamic Viscosity of $\mathrm{H}_{2} \ldots \ldots \ldots \ldots \ldots \ldots$ 


\section{ACKNOWLEDGEMENTS}

This document was created in a relatively short time and under a very demanding schedule. All the more credit is due to Ms. Kathleen Ratto, Mrs. Linda Hanlon, and Ms. Mary Mc Grath for working very hard, overcoming additional obstacles with the authors' handwriting and the mysteries of Word Perfect 5.1. The authors sincerely thank them for all their help and endurance.

Over the ten years since the inception of THATCH, many people have contributed to its development and supported it. The development of the code was funded by the U.S. Nuclear Regulatory Commission. During the development of the original code, Mr. Ronald Foulds was our contract monitor. In the last five years, under the guidance of Dr. Peter Williams, many code extensions were implemented, and the code was extensively applied to the MHTGR. Mr. Cesar Sastre provided early guidance, and in particular, some of the early radiation view factor routines were adopted from him. Dr. Hsiang-Shou Cheng helped to implement the original point kinetics model. Dr. James G. Guppy and Dr. Gregory J. Vail Tuyle have provided guidance and assistance in the last six years.

The authors thank all who have helped to create in THATCH an efficient and useful MHTGR computer code. 


\section{INTRODUCTION}

The "Thermal Analysis for Temperatures in the Core of an $\underline{H}$ TGR" computer code (THATCH) was originally developed under NRC funding for thermal analysis of core heatup accidents in large HighTemperature Gas-Cooled Reactors (HTGRs). In its original form, THATCH solved conduction and radiation in two-dimensional cylindrical network of solids and plena. The first applications were for the $2240 \mathrm{MW}(\mathrm{t})$ reactor in 1984.

As the modular HTGR concept developed, the code was modified and extended; several submodules were added for air-cooled Reactor Cavity Cooling Systems (RCCS), for point kinetics, for forced and natural circulation gas flows, and for chemical reactions of the core graphite with air or water at elevated temperatures.

In its recent version, the code has been applied extensively to model various transients in the commercial modular HTGR (MHTGR) [Kroeger, 1989], under funding by the Nuclear Regulatory Commission. A detailed description of the MHTGR can be found in a concept description report [Bechtel National, 1986]. The most essential features of the reactor are briefly given here, for those readers not familiar with the concept.

The reactor vessel and the steam generator are located in two adjacent cavities of an underground silo as shown in Figure 1-1. A schematic of the reactor with the steam generator is shown in Figure 1-2. Power is generated in a ceramic graphite moderated core using inert helium as the primary coolant fo: energy transfer to the steam generator. The active core fuel elements are arranged in an annular cylinder, surrounded in the center and on all sides by graphite reflector elements. Helium flow is normally provided by the main circulator. If the main circulator or any other steam generator component is not available for service, the reactor would be scrammed, and a Shutdown Cooling System (SCS), located at the bottom of the reactor vessel, would generally be used for decay heat removal.

A third heat removal system, one of the inherent safety features of the MHTGR, is a completely passive air cooling system, the Reactor Cavity Cooling System (RCCS), shown schematically in Figure 1-3. If neither of the above forced flow cooling systems is available, the reactor is scrammed, and then the heat rejection is predominantly by conduction and radiation from the active core via the side reflectors and the reactor vessel, across the reactor cavity to the RCCS cooling panels. Inside the cooling panel an airflow is created by natural circulation assisted by an outlet stack. The reactor vessel is not thermally insulated and during normal power operation, a parasitic heat loss to the RCCS of about $0.8 \mathrm{MW}(0.25 \%$ of $350 \mathrm{MW}_{\text {th }}$ design power) is allowed. Thus, the RCCS is a completely passive system, without valves, damper or other active components. It is always in operation, self-activated, whenever there is a heat flow from the reactor vessel.

This report documents the code in its current form, including a new sub-module for a watercooled RCCS as used in the New Production Reactor (NPR-MHTGR). Section 2 presents an overview of the code and its sub-modules, outlining the types of problems it can be applied to. The physical models, equations and solution methods are described in Section 3, with some of the details relegated to the appendices.

Section 4 documents the code structure and interface between THATCH and its submodules. Detailed descriptions of input data are given in Section 5, followed by a summary of the input and output file usage in Section 6. Section 7 outlines the preprocessing requirements and the postprocessing options. 


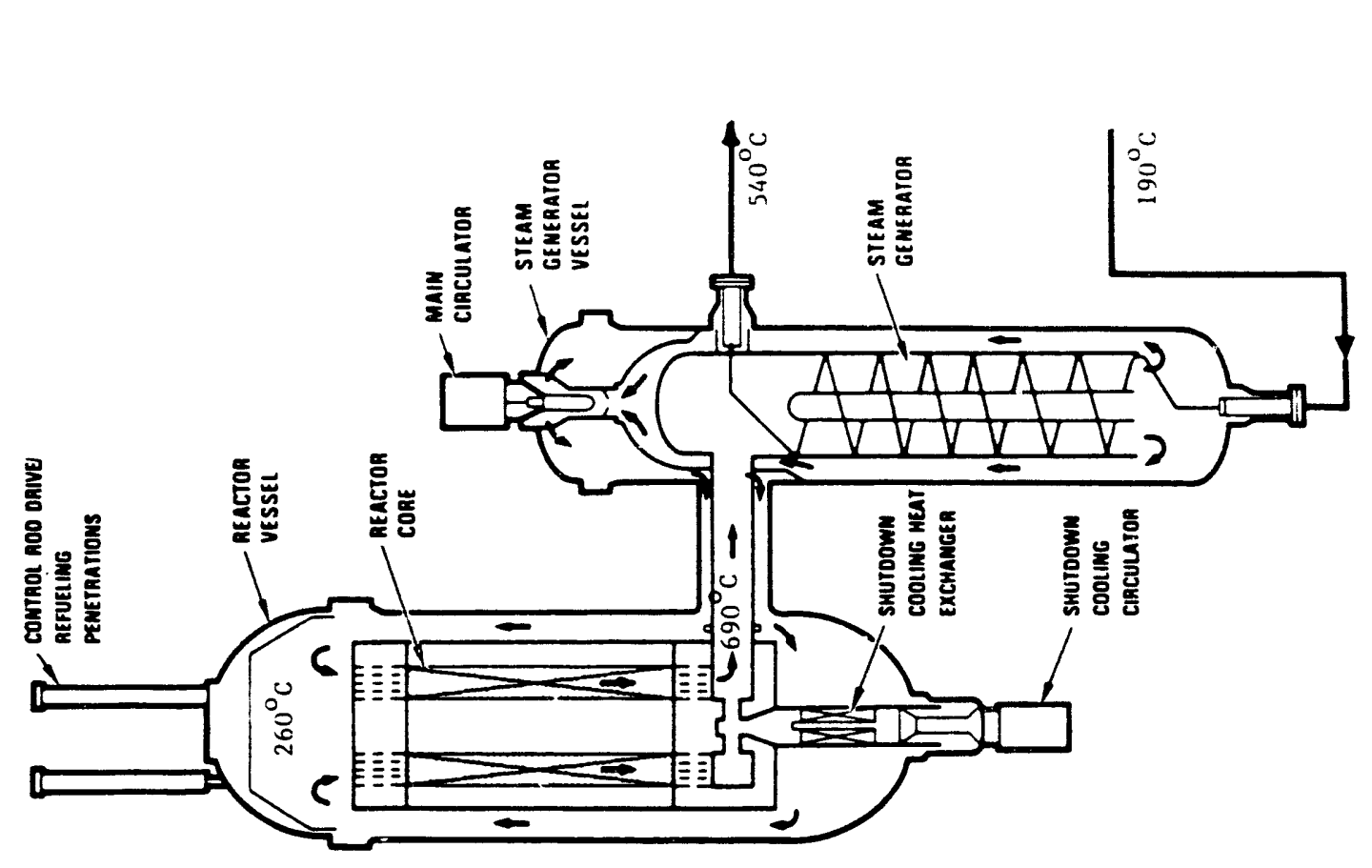

웅

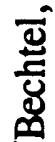

용

㿣

定

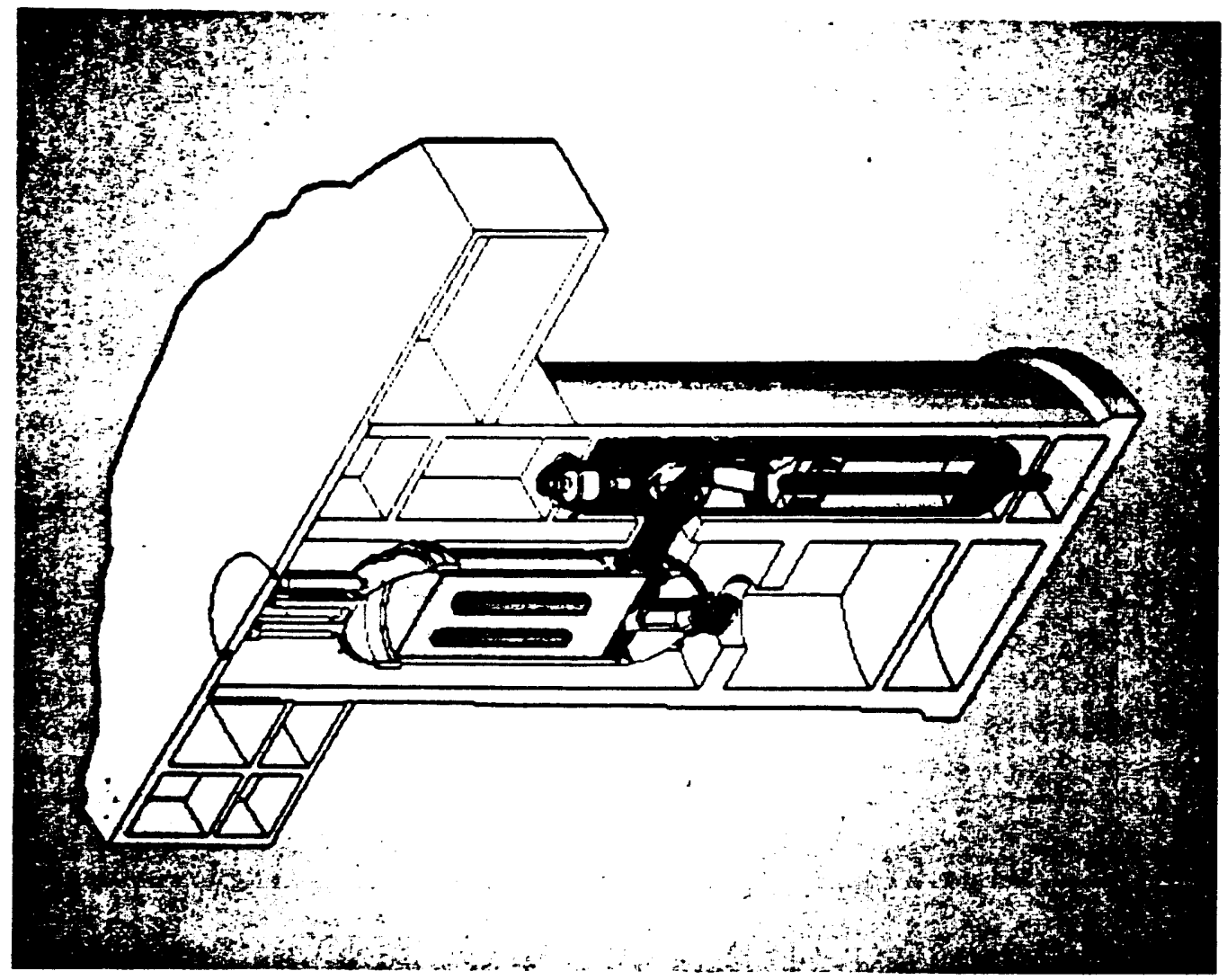

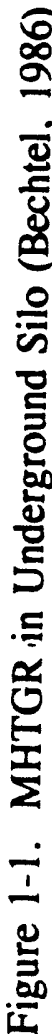




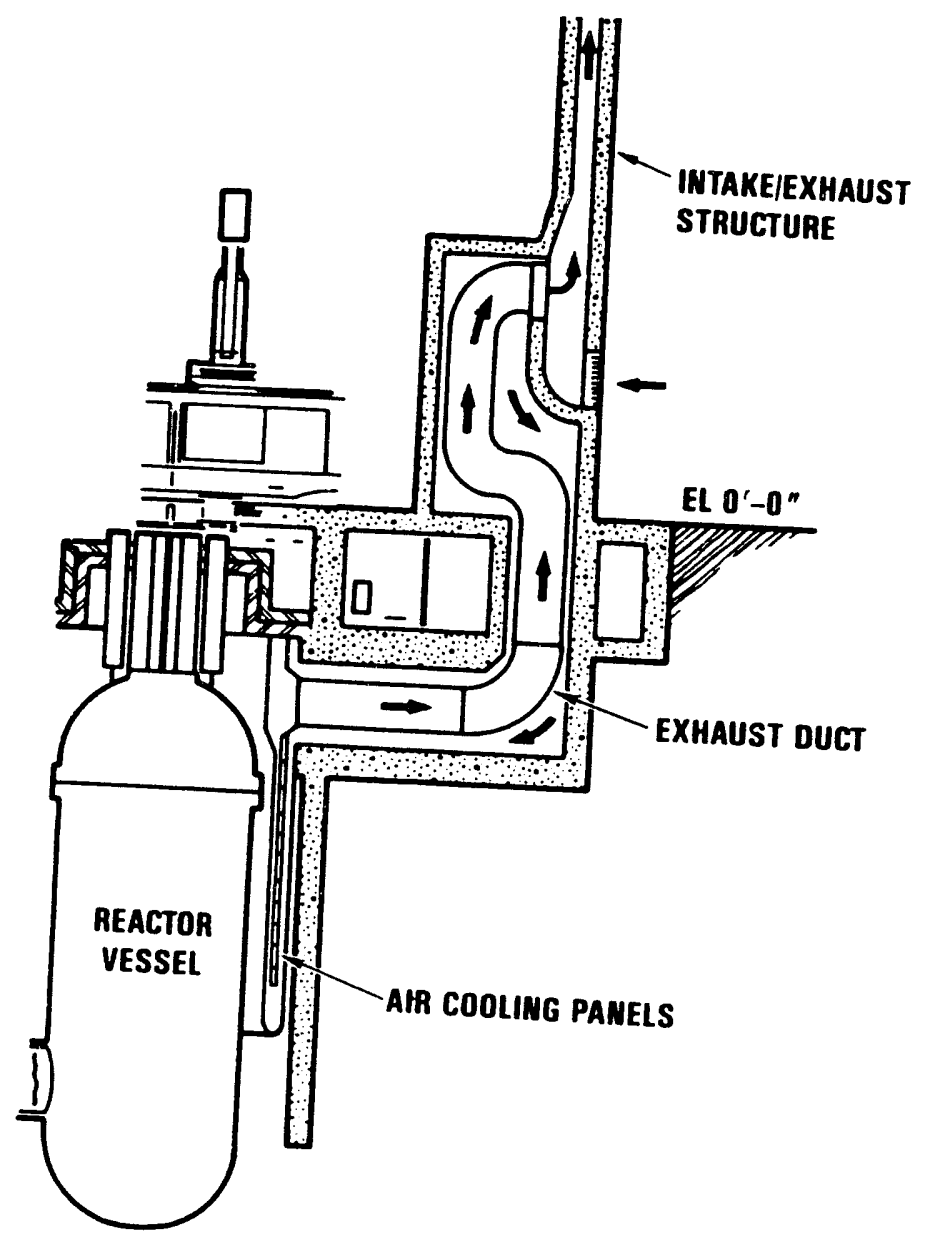

Figure 1-3. Passive Decay Heat Removal System

(Bechtel, 1986) 
Section 8 describes the THATCH modal used for the commercial 350-MW MHTGR, including nodalization and material property models and upper plenum natural convection models. Section 9 outlines the sample cases to be used during code installation and contains the required notes on code installation. Currently, the full code is operational on the IBM 3090 at BNL and on the CRAY at INEL.

Section 10 lists the code capabilities as well as the known, significant code limitations and error indications. 


\section{CODE OVERVIEW}

The original THATCH code was developed to model conduction and radiation transients during core heatup transients in large MHTGRs. This main code is fairly general and can be applied to any network of solid noc'es, which can be suitably described by a two-dimensional cylindrical coordinate $(\mathrm{r}-\mathrm{z})$ nodalization.

Recently, during the application of the code to MHTGR transients, several submodules were added, which apply specifically to nuclear reactors or even only to HTGRs. In this section, an overview of the function of the main code and the various sub-modules is given.

\subsection{Description of the THATCH Main Code}

The basic THATCH Main Code models the transient thermal conduction and radiation heat transfer in a two-dimensional nodal network presented in cylindrical $(r-z)$ coordinates. The user selects the nodalization. The solid network is generally subdivided into "blocks" of equal materials or equal function.

The THATCH model includes internal heat generation, for instance from neutronics or decay heat, from chemical reactions (graphite oxidation), or from convective heating/cooling by a gas stream. Internal thermal resistances in gaps within the solid network or in plena with multi-dimensional radiation can be included. In some cases, internal layers of materials which are of relatively small thermal capacitance, such as an insulation blanket, can be treated optionally as quasi-steady solids, generally referred to here as "layers". The exterior boundary condiitons can be selected by the user with a great

amount of freedom. Material properties are generally supplied by a property library, but can also be called from any specified user - library if so desired.

Typical code applications have been for long-term core heatup accidents, initially in large HTGRs, and more recently in the MHTGR. Originally, the code was developed for depressurized scenarios, where convective heat transfer is negligible. Full convection modelling capability now exists and is discussed below with the FLOXI module.

\subsection{Sub-Module Descriptions}

Several sub-modules were added to the code during application to the commercial MHTGR. These modules can all be executed as free-standing codes or integrated with THATCH.

\subsubsection{PASCOL Module for Air-Cooled RCCS}

As a passive Reactor Cavity Cooling System (RCCS) was added to the MHTGR, the PASCOL module was developed, using air as the cooling medium.

The PASCOL module models the heat iransfer across the reactor cavity to the air-cooled RCCS panels. Heat transfer is by radiation and natural convection across the reactor cavity, then by conduction and radiation in the finned RCCS panels, and ultimately by convection to the air rising inside the panels. The model includes the solution of quasi-steady energy and momentum equations for the coolant in the RCCS panels, in the ducting and in the stack. As any conceivable RCCS transients are very slow, quasi- 
steady evaluations are completely satisfactory.

This module essentially provides the exterior boundary condition for the reactor vessel transient analysis of THATCH. The module has also been used to evaluate the passive air cooling systems of the advanced LMR concepts SAFR and PRISM.

\subsubsection{WATCOL Module for Water-Cooled RCCS}

The option for a water-heat transport loop to transfer energy from the reactor cavity to an airwater heat exchanger was incorporated into the MHTGR design. Therefore, the WATCOL Module was jeveloped and added to the THATCH code, which models transport of energy from the reactor vessel to the water coolant, and subsequent transport of energy by convection to the air flow in the air-water heat exchanger.

The model assumes that RCCS cooling panels receive energy from the reactor vessel via radiation and natural convection from the cavity gas surroundings. The energy is transferred by conduction and convection to the water coolant located within the array of "D-tubes", which are welded to the back of the panels. The water flows by natural circulation within a closed loop, which transports the energy to an air-water heat exchanger, where the water is cooled by convective flow of air. The cooled water then flows back to the RCCS panels. The air-water heat exchanger is contained within a "natural draft" stack structure. Natural convection air flow past the heat exchanger tubes removes the energy from the water transport system.

The WATCOL module provides the boundary condition for the reactor vessel transient analysis of THATCH. The coupled, quasi-steady mass, momentum and energy equations are solved for the closed water loop and the once through air flow, driven by natural convection, in the air-water heat exchanger stack.

The present version of the water-cooled RCCS model is preliminary. The input data of the heat exchanger model should be updated as soon as a design is firmed up. The accuracy of the model should be established by comparison with either experimental data or with a set of benchmark calculations performed using an industry standard, if available.

\subsubsection{FLOXI Module for Reactor Gas Flow, Heat Transfer, and Chemical Reactions}

The FLOXI sub-module models gas flow, heat transfer, and chemical reactions in the reactor. This module can analyze natural or forced convection in the core. The convective heat transfer is generally only significant in the pressurized mode. However, during ingress transients, the gas to solid heat transfer in the core barrel region can be essential because it affects the overall buoyancy driving force. The flow and heat transfer computations allow for change in composition and mass addition due to chemical reactions and handle mixtures of all relevant gases. A simplified model for upper plenum heat transfer is included, but the treatment given here cannot substitute for multi-dimensional upper plenum turbulent heat transfer models, which should be used separately as free-standing models, and can ultimately provide inputs to improve the current simplified one-dimensional model.

The graphite oxidation process is a function of the temperature regime. At low temperatures, the carbon/oxygen reaction kinetics control the reaction rate. At intermediate temperatures, the in-pore diffusion is controlling, while at high temperatures the coolant to surface mass transfer by diffusion, here 
in a laminar flow field, is controlling.

To consider all three regimes, a model including external mass transfer, in-pore diffusion, and chemical reaction kinetics is required, as in the Oxide-3 code [Peroomian et al., 1974]. For the current applications, we decided to use a simpler approach, combining the in-pore diffusion and chemical reaction process into a single Langmuir-Hinshelwood type semi-empirical equation as done, for instance by Moormann and Petersen [1982]. In particular, since some of the data on in-pore diffusion are not very certain, this approach is well justified at this time. As better data becomes available, a refinement of the model may become desirable.

\subsubsection{PKIN Modul for Point Kinetics Option}

The PKIN Module, the point kinetics sub-module of THATCH, analyzes no-scram scenarios and reactivity insertions coupled with thermohydraulic transients.

The model uses six delayed neutron groups and considers change of reactivity feedbacks due to:

$$
\begin{aligned}
& \text { changes in fuel temperature (Doppler) } \\
& \text { changes in moderator temperature } \\
& \text { changes in reflector temperature } \\
& \text { changes in xenon concentration } \\
& \text { changes in samarium concentration } \\
& \text { changes in water concentration. }
\end{aligned}
$$

The PKIN module has been used to evaluate no-scram scenarios during core heatup transients and water ingress transients. For rapid transients, the general THATCH nodalization is too coarse to model the resulting changes in temperature and in particular, the Doppler feedback. For such applications, the PKIN module can be used with the SYMELAN module, either in a free-standing mode of SYMELAN with PKIN, or also coupled with THATCH.

\subsubsection{SYMELAN Module for Rapid Thermal Core Transients Using Symmetry Element Nodalization}

While the THATCH reactor vessel model describes thermal and flow transients in steady state, scrammed, or other relatively slow transients, it cannot model rapid core transients where fuel and moderator temperatures may vary significantly.

For this purpose, the SYMELAN code is available as a separate code, but it can also be run integrated in THATCH. It nodalizes the symmetry element contained within the fuel elements where typically 6 fuel sticks surround each coolant hole (see Figures 3.7 and 3.8 below). This small symmetry element is generally nodalized into 6 fuel nodes and 12 moderator nodes.

The SYMELAN module has the capability to model very rapid transients, such as reactivity insertions due to severe water ingress, and also to accurately evaluate steady-state temperature distributions.

In general, the SYMELAN module is executed in the free-standing mode, with prescribed inlet flows and temperatures, and assumes no heat conduction across the active core boundaries. Both conditions are justified for many rapid scenarios; however, where desired, for example, when the effects of decreases in gas flow due to core heatup in fast transients are to be modelled, it can be run jointly as part of THATCH. 


\section{CODE MODEL AND METHOD OF SOLUTION}

This section describes the models used in the various code modules, gives the equations, and describes the method of solution. Where appropriate, details have been relegated to Appendices.

\subsection{Basic THATCH Models}

\subsubsection{THATCH Conduction/Radiation Heat Transfer in a Solid Network}

For THATCH modelling of conduction/radiation heat transfer in a solid network, the body under consideration must be broken down into a two-dimensional r-z geometry. The user selects the number of radial or axial nodes, as well as the node spacing. Figure 3-1 shows the nodalization convention applied in the THATCH code. Index " $\mathrm{i}^{\text {" }}$ applies to radial nodes, increasing with the outward pointing $\mathrm{r}$-coordinate. Index " $\mathrm{j}$ " applies to axial nodes, increasing with the downward pointing $\mathrm{z}$-coordinate. Nodes are separated by "interfaces", and the outward (or downward) interface to node $(i, j)$ are interfaces $\mathrm{i}$ (or $\mathrm{j}$ ). After selecting a suitable nodalization, the user describes the system to be modelled in terms of "blocks" of equal functions or material properties, and assigns a proper "material index" to each block. An arbitrary example of such a network is shown in Figure 3-2. Note that Block 5 is a plenum (Material Index > 100; for details see Section 5). The "gap" separating Blocks 2 and 4 from Block 6 is in terms of the nodalization, a part of Blocks 2 and 4; i.e., Blocks 2,4,5, and 7 have the same outer radius and node/interface index. Gaps are always considered to be on the outside (downside) of a block; (for details see Section 5). Outer boundaries have to be specified for the corresponding boundaries of Blocks 7,6,2, and 3. A further convention is to label sides of blocks or nodes as North, East, South, or West, or use the "side numbers" (for instance, in array indices) as shown in Figure 3-3.

Heat transfer within the solid structures of the system is described by the transient heat conduction equation which can be written as follows:

$$
\rho c \frac{\partial \theta}{\partial t}=\frac{1}{r} \frac{\partial}{\partial r} k r \frac{\partial \theta}{\partial r}+\frac{\partial}{\partial z} k \frac{\partial \theta}{\partial z}+q^{\prime \prime \prime}
$$

where the material properties $\rho c$ and $\mathbf{k}$ are functions of temperature.

In finite difference form this equation can be written as follows:

$$
\begin{aligned}
v_{i, j}(\rho c)_{i, j} \frac{\theta_{i, j}-\theta_{i, j}^{\circ}}{\Delta t} & \\
& =k_{r+} A_{r+} \frac{\theta_{i+1, j}-\theta_{i, j}}{\Delta r_{+}}-k_{r-} A_{r-} \frac{\theta_{i, j}-\theta_{i-1, j}}{\Delta r_{-}} \\
& +k_{z+} A_{z+} \frac{\theta_{i, j+1}-\theta_{i, j}}{\Delta z_{+}}-k_{z-}-A_{z-} \frac{\theta_{i, j}-\theta_{i, j-1}}{\Delta z_{-}} \\
& +V_{i, j} q^{\prime \prime \prime}
\end{aligned}
$$



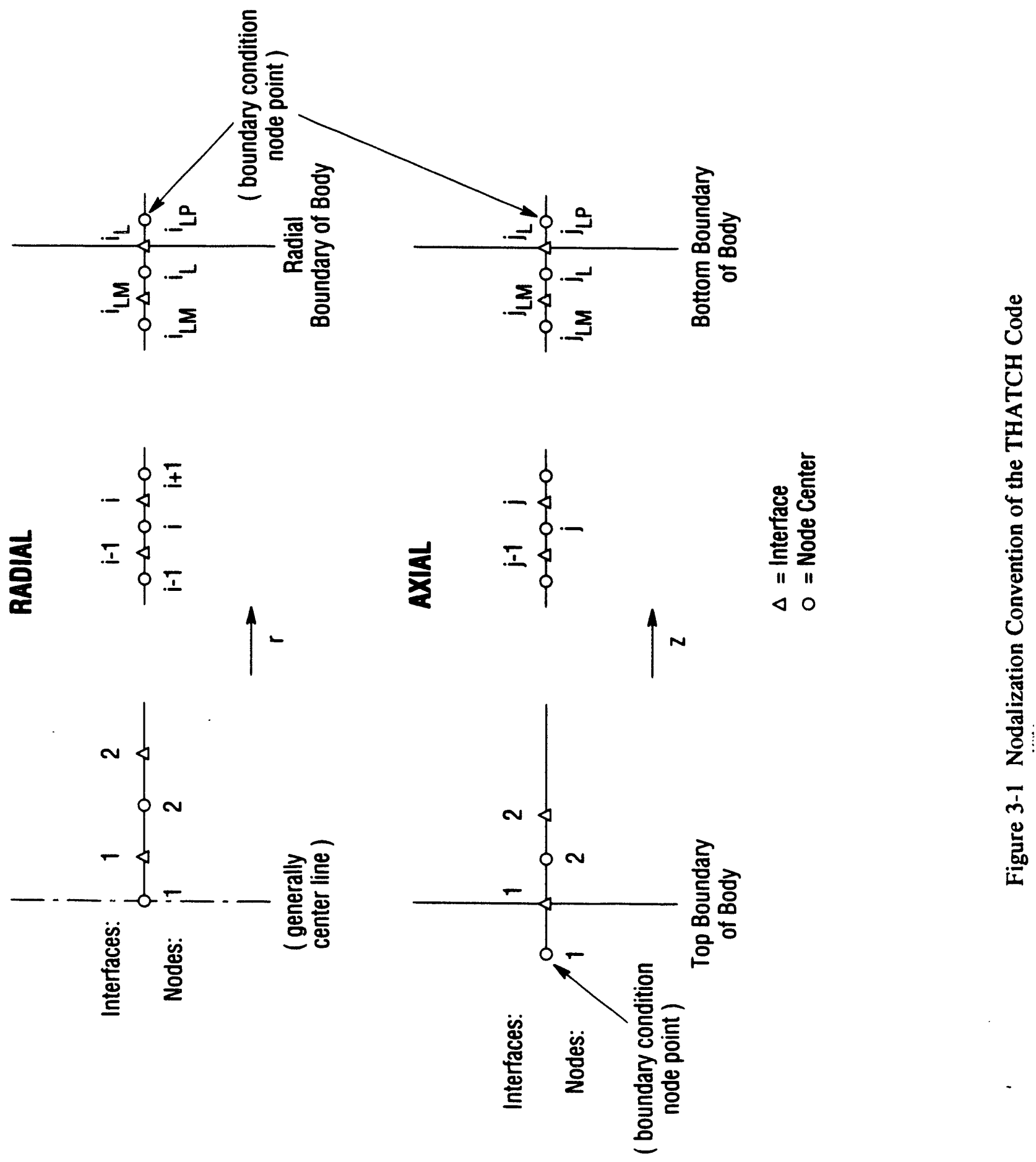


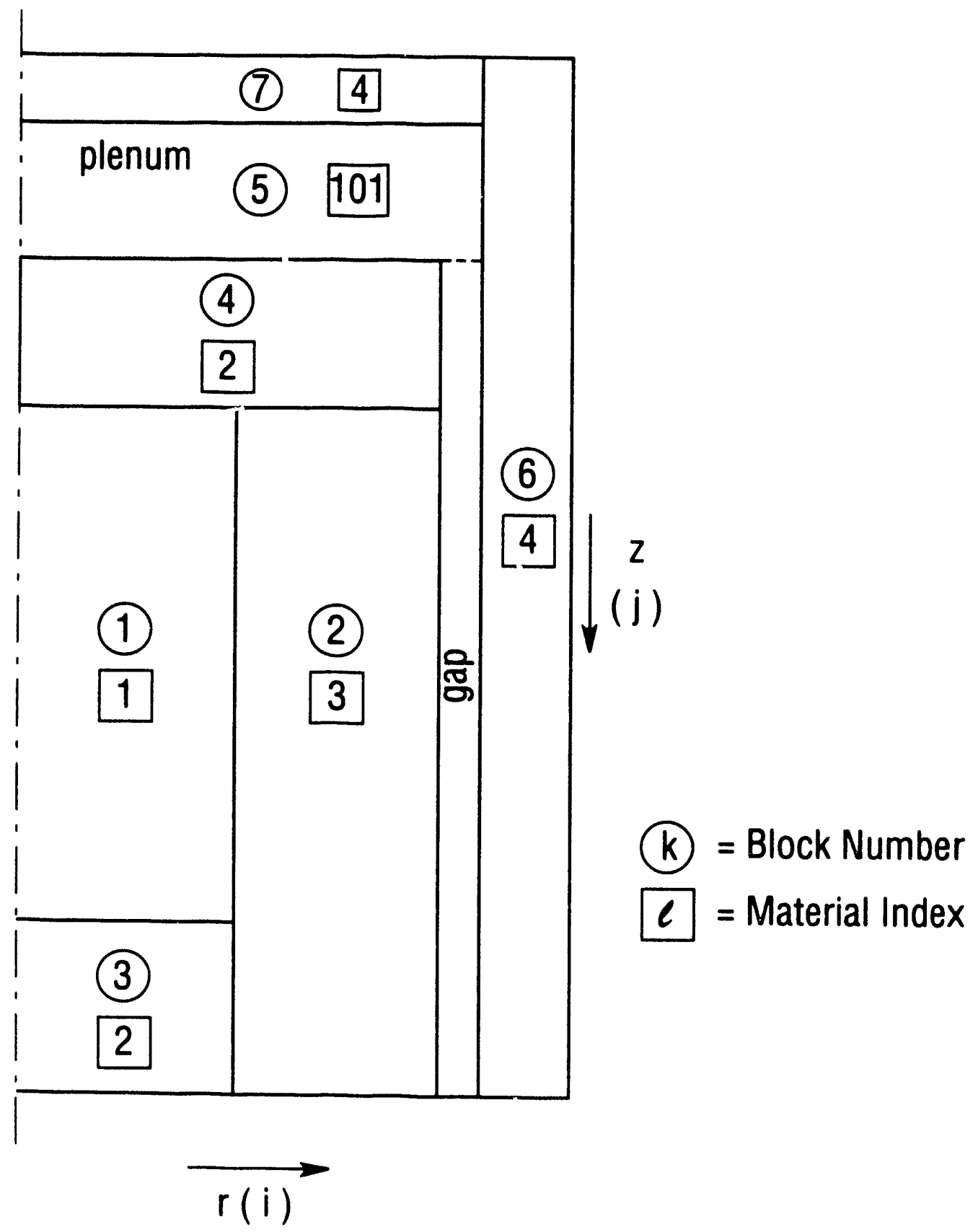

Figure 3-2 Schematic of a Solid Network Broken Down Into "Blocks" for Modelling by the THATCH Code 

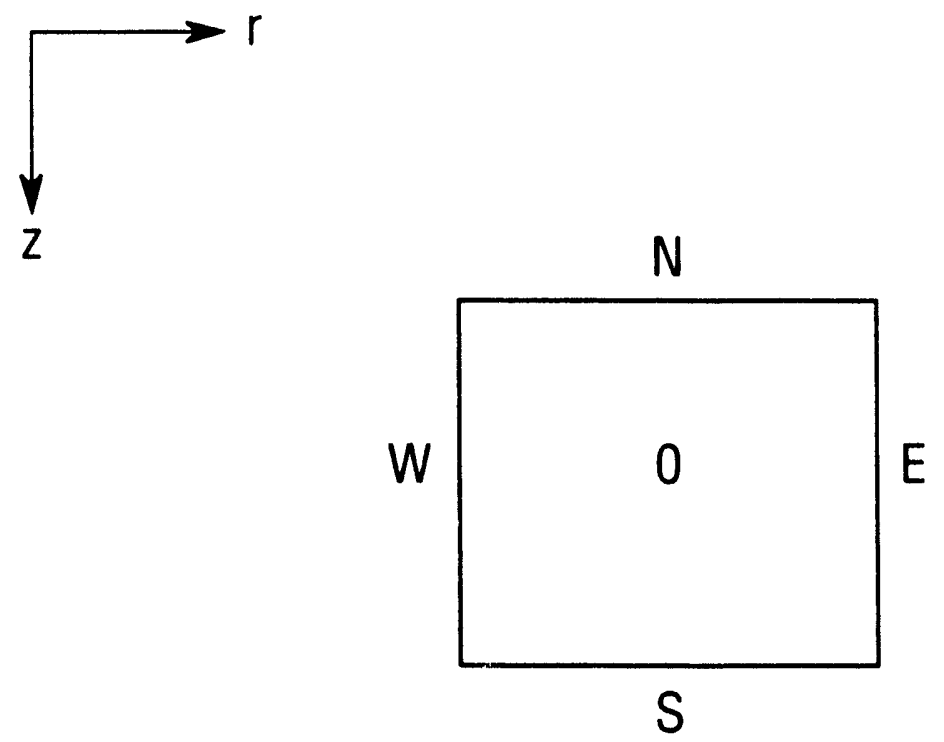

and / or

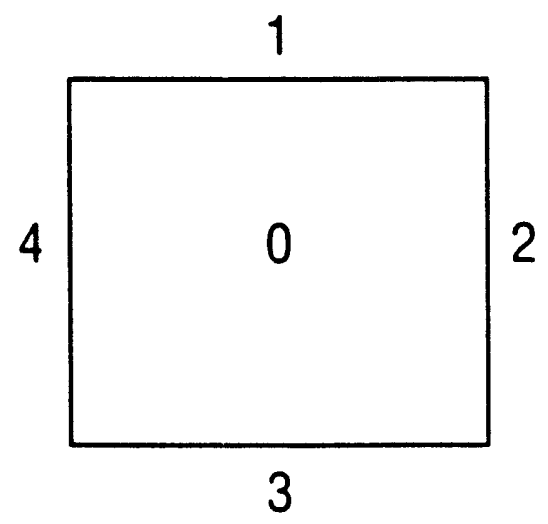

Figure 3-3 Schematic of Node Interface and Block Boundary Designations 
where $\theta^{\circ}$ denotes the temperature of the preceding time step, the subscripts $r_{+}, r_{-}, z_{+}, z_{-}$denote values of the corresponding node interface, and $\Delta r_{+}$etc. denote the corresponding distances between node centers.

This equation can be rearranged as follows:

$$
\begin{aligned}
\theta_{i, j}-\theta^{\circ}{ }_{i, j} & =C_{N i, j}\left(\theta_{i, j-1}-\theta_{i, j}\right) \\
& +C_{E i, j}\left(\theta_{i+1, j}-\theta_{i, j}\right) \\
& +C_{S i, j}\left(\theta_{i, j+1}-\theta_{i, j}\right) \\
& +C_{W i, j}\left(\theta_{i-1, j}-\theta_{i, j}\right)+C_{Q i, j}
\end{aligned}
$$

where,

$$
C_{Y_{i, j}}=\frac{\Delta t}{V_{i, j}(\rho c)_{i, j}} A_{Y} \alpha_{Y}
$$

where $\alpha_{Y}=k_{Y} / \Delta x_{Y}$ is the conductance between neighboring nodes, $\Delta x$ represent $\Delta r$ or $\Delta z$, and $Y$ represents N,E,S or W;

$$
C_{Q}=\frac{\Delta t}{(\rho c)_{i, j}} q_{i, j}^{\prime \prime \prime}
$$

Note that the $C_{Y}$ are dimensionless and $C_{Q}$ has the dimension of temperature.

Equation 3.1-3 can be used to solve the temperature field in all solid structures if the Ccoefficients of Equations 3.1-4 and 5 are known. This can be done, in principal, by evaluating these coefficients, using areas and node intervals from the selected nodalization, and thermal properties $\rho c$ and $\mathbf{k}$ from the preceding time step. The internal heating rates $\mathrm{q}^{\prime \prime \prime}$ of the original THATCH code were from decay heat only, which is available as a function of space and time, as specified with the input (see Section 5).

Modifications of the C- coefficients are required at exterior boundaries, at interior gaps or other heat transfer resistances, and at solid block to plena boundaries.

Internal thermal resistances between adjacent nodes can be modelled in the code by prescribing conductive and/or radiative resistances. To permit modelling of some solids of relatively small thermal capacitances in a quasi-steady mode, the concept of a "layer" of material is introduced. Any "gap" in the THATCH network can contain, in addition to the physical gap, an additional "layer" of a solid material. This solid layer can be located on the " + side" or the "-side" of the gap, always progressing with increasing $\mathrm{r}$ or $\mathrm{z}$ coordinate. The total gap conductance model is outlined here for a radial gap, with a layer on the "+ side". Such a gap is shown schematically in Figure 3-4. Note that a "radial gap" is defined as a vertical gap at constant radial coordinates, while an "axial gap" is horizontal at constant axial coordinates. 


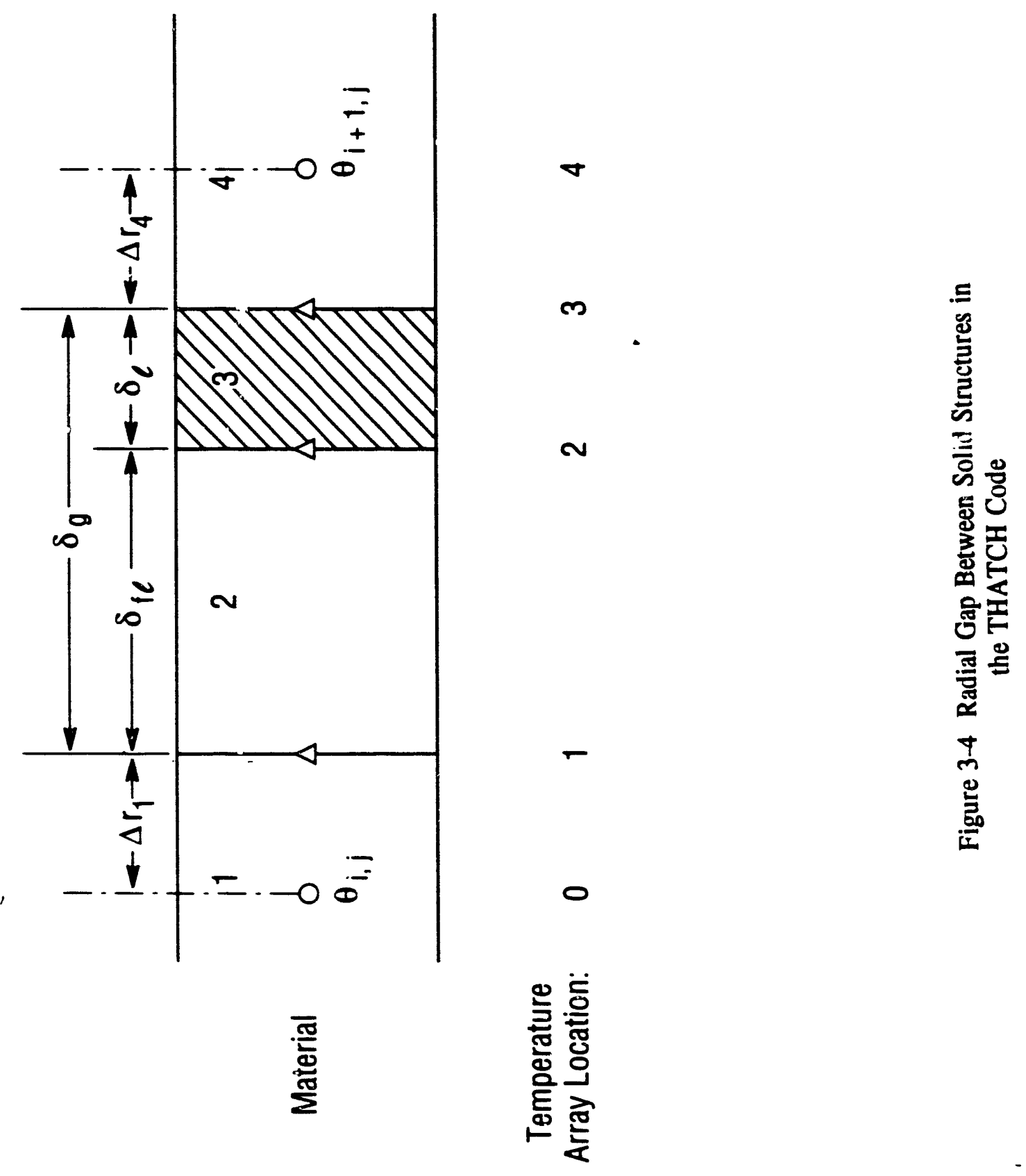



follows:

The total heat transfer resistance between the shown node center points can be obtained as

$$
\sum R=\frac{\Delta r_{1}}{k_{1}\left(\theta_{1 \mathrm{av}}\right)}+R_{2}+\frac{\delta_{1}}{k_{3}\left(\theta_{3} \mathrm{av}\right)}+\frac{\Delta r_{4}}{k_{4}\left(\theta_{4} a v\right)}
$$

where $\theta_{1}$ av is the average of $\theta_{0}$ and $\theta_{1}$,

$\theta_{2}$ av is the average of $\theta_{1}$ and $\theta_{2}$,

$\theta_{3}$ av is the average of $\theta_{2}$ and $\theta_{3}$, and

$\theta_{4}$ av is the average of $\theta_{3}$ and $\theta_{4}$.

Heat transfer across the fluid gap can be either

1. zero resistance, in which case

$$
\mathbf{R}_{2}=0 \text {. }
$$

2. Conduction only, in which case

$$
\mathbf{R}_{2}=\frac{\delta_{\mathrm{fl}}}{\mathrm{k}_{2}\left(\theta_{2 \mathrm{av}}\right)}
$$

3. Conduction plus $1-\mathrm{d}$ radiation, where

$$
\mathrm{h}_{\mathrm{rad}}=\epsilon_{1,3} \sigma\left(\theta_{1}^{2}+\theta_{2}^{2}\right)\left(\theta_{1}+\theta_{2}\right)
$$

and

$$
R_{2}=\frac{1}{\frac{k_{2}\left(\theta_{2}\right. \text { av }}{\delta_{\mathrm{fl}}}+h_{\mathrm{rad}}}
$$

$\epsilon_{1,3}$ the effective emissivity between materials 1 , and 3 is from a user input.

4. Radiation only, where

$$
\mathbf{R}_{2}=\frac{1}{\mathbf{h}_{\mathrm{rad}}}
$$

with $h_{\mathrm{rad}}$ from Equation 3.1-9.

The conductance terms of Equation (3.1-4), $\alpha_{Y}$, are replaced at internal boundaries by the inverse of the $\Sigma R$ of Equation (3.1-6), namely with 


$$
\alpha_{Y}=\frac{1}{\sum R}
$$

Computation of the gap conductance is iterative at each time step with the nodal temperatures known, and the internal temperatures being recomputed till convergence is achieved.

A fifth option is an isolated gap, in which case $\alpha_{\mathbf{Y}}$ is set to zero.

For external boundaries, one can similarly specify an insulated face, or a prescribed heat transfer crefficient with $1-d$ radiation. The conductance $\alpha_{Y}$ in the $C$ - coefficients of Equation $3.1-4$ is then rnodified as follows:

For the insulated rase,

$$
\alpha_{Y}=0
$$

For the case of $1-d$ radiation and a prescribed convective heat transfer coefficient, $h$, one obtains

$$
\frac{1}{\alpha_{Y}}=\frac{\Delta x_{n}}{2 k_{s}\left(\theta_{\text {avg }}\right)}+\frac{1}{h+h_{\mathrm{rad}}}
$$

with

$$
h_{\mathrm{rad}}=\epsilon_{\mathrm{n}, \mathrm{s}} \sigma\left(\theta_{\mathrm{ns}}^{2}+\theta_{\mathrm{s}}^{2}\right)\left(\theta_{\mathrm{ns}}+\theta_{\mathrm{s}}\right)
$$

where $\Delta x_{n}$ is either $\Delta r_{n}$ or $\Delta z_{n}$ of the last node and $k_{s}$ is the conductivity of the last node material at the average temperature between the center of the node and the outside surface of the node. $\epsilon_{\mathrm{n}, \mathrm{s}}$ is the prescribed emissivity between node and sink material, $\theta_{\mathrm{ns}}$ is the node surface temperature, and $\theta_{\mathrm{s}}$ the sink temperature. Evaluation of the conductance is again iterative, with known node and sink temperature.

\subsubsection{THATCH Plena Two-Dimensional Radiation Heat Transfer}

The two-dimensional radiation model in THATCH assumes a transparent gas in an open plenum neglecting the presence of control rods and/or participating gases. Convection between the gas and the solid surfaces is neglected here, but will be included with the FLOXI sub-module in Section 3.4.

The radiation exchange between gray surfaces of a cavity is modelled following Sparrow and Cess (1966). For temperature-independent emissivities, the radiation exchange coefficient matrix $\Lambda$ can be computed once for a given geometry and given nodal emissivities. Thereafter, the radiation exchange coefficient matrix can be used to compute the total heat flow from node $\ell$ to all plena nodes $\ell=1$, L as follows:

$A_{\ell}$ is the surface area of node $\ell$ facing the plenum, and $\theta_{\text {sm }}$ is the nodal surface temperature of node $m$. Details on the computation of the radiation exchange coefficient matrix are given in Appendix 


$$
\mathrm{Q}_{1}=\mathrm{A}_{1} \sum_{\mathrm{m}=1}^{\mathrm{L}} \Lambda_{\mathrm{lm}} \sigma \theta_{\mathrm{sm}}^{4}
$$

A. If one wanted to use temperature-dependent emissivities, one would have to modify the code, and recompute the $\Lambda$ array at each time step.

The 2-dimensional plena radiation heat flow computations must be coupled with the conduction heat transfer computations of the surrounding solids.

For solid node $(i, j)$ bounded by a plenum on the north side, as shown schematically in Figure 3-5, the condition

can be expressed as follows:

$$
Q_{\text {cond }}=Q_{\text {rad }}
$$

$$
\begin{gathered}
\mathrm{Q}_{1}=\frac{2 \mathrm{~A}_{1}}{\Delta \mathrm{z}_{\mathrm{n}}} \mathrm{k}\left(\theta_{\mathrm{i}, \mathrm{j} \text { avg }}\right)\left(\theta_{\mathrm{i}, \mathrm{j}}-\theta_{\mathrm{sl}}\right) \\
=\mathrm{A}_{1} \sum_{\mathrm{m}=1}^{\mathrm{L}} \Lambda_{\mathrm{lm}} \sigma \theta_{\mathrm{sm}}^{4}
\end{gathered}
$$

Note that each node adjacent to a plenum can be identified either by the general set of solid node indices $(i, j)$ or by the "plenum node" indices $(\ell)$.

Equation 3.1-17 represents a set of non-linear simultaneous equations, which is solved at each time step for the set of surface temperatures $\theta_{\mathrm{s} \ell}$.

For known surface temperatures, the heat flows at the node to the surface of the plenum can then be computed from Equation (3.1-17). For nodes adjacent to plena, the solid node finite difference equations must be modified as follows: In Equation (3.1-3) the C-coefficients corresponding to the plena surfaces are set to zero, and the corresponding terms

$$
C_{Y i, j}=\frac{\Delta t}{V_{i, j}(\rho c)_{i, j}} Q_{l}
$$

are added to the corresponding $\mathrm{C}_{\mathrm{Q}} \mathrm{i,j}$ coefficients.

\subsubsection{Method of Solution for THATCH Models}

Solution of the THATCH equations describing the heat conduction in solid structures with interior and/or exterior interface resistances and coupled with 2 -d radiation in plena, requires the advancement of Equation (3.1-3) at each time step, after modification of the appropriate C-coefficients to account for interface resistances. Coupled with this process, Equation (3.1-17) for the plena heat flows must-be solved as a system of simultaneous non-linear algebraic equations.

For the solution of the solid network finite difference equations, the Alternate Direction Implicit (ADI) method was selected (Isaacson and Keller, 1966 or Carnahan, Luther and Wilkes, 1969). This 


\section{Plenum}

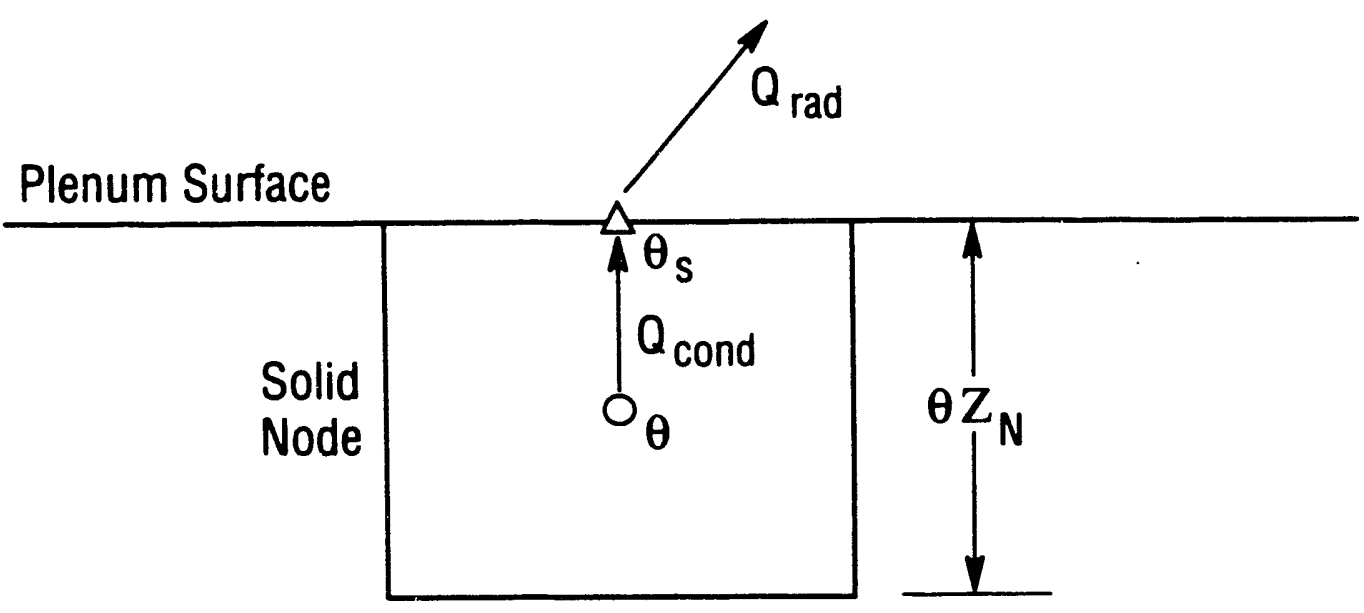

Figure 3-5 Schematic of Heat Flow's in Nodes Adjacent to Plena 
method computes the solution at $t+\Delta t$ in two steps, first processing from $t$ to $t+1 / 2 \Delta t$, solving by rows and treating all row terms as implicit, and then progressing from $t+1 / 2 \Delta t$ to $t+\Delta t$, this time sweeping by columns, with in-column terms being treated as implicit. The advantage of this method is that it has the stability of fully implicit methods and requires only the solution of tridiagonal simultaneous equations, which can be done with very efficient solution algorithms.

Equations (3.1-3) can be written for the sweep by row as follows:

$$
\begin{aligned}
-C_{W i, j} \theta_{i-1, j}^{m} & +\left(1+C_{W i, j}+C_{E i, j}\right) \theta_{i, j}^{m}-C_{E i, j} \theta_{i+1, j}^{m} \\
=\theta_{i, j}^{\circ} & +C_{N i, j}\left(\theta_{i, j-1}^{\circ}-\theta_{i, j}^{\circ}\right) \\
& +C_{S i, j}\left(\theta_{i, j+1}^{\circ}-\theta_{i, j}^{\circ}\right)+C_{Q i, j}
\end{aligned}
$$

where superscripts $0, m$, and $n$ designate old $(t=t)$, mid point $(t=t+1 / 2 \Delta t)$, or new $(t=t+\Delta t)$.

For the second sweep by columns, we use

$$
\begin{aligned}
-C_{N i, j-1} \theta_{i, j-1}^{n}+(1 & \left.+C_{N i, j}+C_{S i, j}\right) \theta_{i, j}^{n}-C_{S i, j} \theta_{i, j+1}^{n} \\
=\theta_{i, j}^{m} & +C_{W i, j}\left(\theta_{i-1, j}^{m}-\theta_{i, j}^{m}\right) \\
& +C E i, j\left(\theta_{i+1, j}^{m}-\theta_{i, j}^{m}\right)+C_{Q i, j}
\end{aligned}
$$

Note that the $\Delta t$ of Equations (3.1-4,5, and 18) must be the incremental step, i.e., $1 / 2 \Delta t$.

The simultaneous algebraic equations for the plena are from Equation $(3.1-17)$

$$
\sum_{m=1}^{L}\left(\delta_{l m} \alpha_{1} \theta_{s l}+\Lambda_{l m} \sigma \theta_{s m}^{4}\right)=\alpha_{1} \theta_{i, j} \quad 1=1, L
$$

where the conductance is

$$
\alpha_{1}=\frac{2 k\left(\theta_{i, j} \text { avg }\right)}{\Delta z_{n}}
$$

This set of equations is solved by NEWTON, the non-linear, simultaneous equation solver, providing an analytical Jacobean matrix and residual vector computed from Equation 3.1-20.

\subsection{PASCOL Models for Air-Cooled RCCS}

The PASCOL module for the passive air-cooled RCCS was originally developed before some of the current RCCS design detail became available. This module includes several of the original options, but they are currently not of interest. These options have not been used recently, but are still part of the 
code. This report covers the currently used options. Specifically, the following input options are affected:

1. There is therrnal insulation between the downflow channels and the finned riser channels, (INSUL = true); with INSUL = false regenerative heating of the incoming air could be modelled.

2. The stack is connected to the inner RCCS channels, facing the reactor vessel (ISTACON =1). In principle, a RCCS with downflow in the inner channels and the stack connected to the outer upflow channels (ISTACON=2) has some operating advantages, but it is not self starting and, therefore, not completely passive.

3. Arithmetic average fluid temperatures are used in the solution of the fluid energy equation (EXPO = false); EXPO = true would provide the exponential formulation of a rise in gas temperature in a heated channel, corresponding to a logarithmic average temperature difference model.

4. User supplied fin effectiveness data will be used (FINK = false). With FINK =true, the simultaneous radiation and conduction in the finned RCCS panels can be internally computed. Such internal evaluations of the fin performance were used during parametric performance evaluations (Kroeger, 1989, App. B). We found that once the fin effectiveness has been determined for a specific configuration, prescribed data were much more efficient to use for general THATCH runs. Details of the internal fin evaluation are given in Appendix $\mathrm{C}$.

\subsubsection{Heat Transfer Across Reactor Cavity}

In current MHTGR concepts, the reactor vessel is housed inside the reactor cavity, which is lined with RCCS panels. For the commercial MHTGR, these panels are cooled by a natural convection air flow inside the channels enclosed by the panels. The gap between the reactor vessel and the RCCS front panel is of the order of $1 \mathrm{~m}$ wide, and the cavity is about $22 \mathrm{~m}$ high. At the prevailing temperatures of vessel temperatures between $200^{\circ} \mathrm{C}$ and $500^{\circ} \mathrm{C}$ (during core heatup accidents) and RCCS panel temperatures of about $80^{\circ} \mathrm{C}$ to $160^{\circ} \mathrm{C}$, the heat transfer across the reactor cavity is predominantly by radiation aided by turbulent natural convection (with Rayleigh numbers of about $10^{9}$ to $10^{19}$, which contributes about 10 to $15 \%$ of the total heat transfer.

In principle, the radiation heat transfer across the cavity is best modelled as two-dimensional radiation through a transparent gas. The radiation exchange coefficient matrix is precomputed for prescribed constant emissivities, following the procedure in Appendix A and using the view factors in Appendix B; Section B-2. The radiation heat flow from any node $\ell$ is then, as already stated in Equation (3.1-16):

$$
\mathrm{Q}_{1}=\mathrm{A}_{1} \sum_{\mathrm{m}=1}^{\mathrm{L}} \Lambda_{\mathrm{lm}} \sigma \theta_{\mathrm{sm}}^{4} \quad \text { for } \mathrm{l}=1, \mathrm{~L}
$$


This procedure was used in some of the THATCH evaluations of core heatup scenarios without a functioning RCCS. However, peak vessel temperatures obtained with the above two-dimensional radiation model were only slightly lower than those obtained with the much simpler one-dimensional radiation model. The uncertainty in the vessel and panel emissivities, where textbook values of 0.8 or 0.85 are generally being applied, is sufficiently large, that use of the above two-dimensional radiation model was not justified for reactor cavity heat transfer with functioning RCCS.

In the computations for the one-dimensional reactor cavity radiation heat transfer, the radiative flow across the cavity is computed for each vertical node as follows:

$$
Q_{\text {rad } j}=A_{V} \epsilon_{1,2} \sigma\left(\theta^{4}{ }_{V_{s j}}-\theta^{4}{ }_{F P j}\right)
$$

where $\theta_{\mathrm{V}_{\mathrm{aj}}}$ and $\theta_{\mathrm{FPj}}$ are temperatures of the vessel surface, and the RCCS front panel at node $\mathrm{j}$. The radiation exchange coefficient $\epsilon_{1,2}$ is for concentric cylinders

$$
\epsilon_{1,2}=\frac{1}{\frac{1}{\epsilon_{\mathrm{V}}}+\frac{\mathrm{R}_{\mathrm{V}}}{\mathrm{R}_{\mathrm{FP}}}\left(\frac{1}{\epsilon_{\mathrm{FP}}}-1\right)}
$$

where $\mathbf{R}_{\mathbf{V}}$ and $\mathbf{R}_{\mathrm{FP}}$ are reactor cavity side radii of the reactor vessel surface and the RCCS front panel, respectively. Note that with this formulation for $\epsilon_{1,2}$ the vessel surface must be used for heat flow computations.

For turbulent natural convection, the following correlation is used (Holman, 1990).

$$
\begin{aligned}
& N u_{D}=.197\left(R a_{d}\right)^{1 / 4}\left(\frac{H}{D}\right)^{-1 / 9} \quad R a<149,200 \\
& N u_{D}=.073\left(R a_{d}\right)^{1 / 3}\left(\frac{H}{D}\right)^{-1 / 9} \quad R a<149,200
\end{aligned}
$$

Where $\mathrm{D}$ is the cavity width and $\mathrm{H}$ its height ${ }^{1}$.

The cross-over Raleigh number is actually given by Holman as 200,000 . However, we arbitrarily use the value of 149,183 to avoid a discontinuity in the Nusselt number. Since RCCS Raleigh numbers are five orders higher, this practice would only affect results if the routine were used for other applications, or if the cavity dimensions changed drastically.

Convective turbulent heat transfer in such cavities would be expected to form several circulating cells in the cavity. Computation of a heat transfer coefficient at each node level, as is currently done, is not quite correct. However, the above correlation is an overall correlation for isothermal walls, and convection is only a minor contributor to the total heat transfer. Therefore, the convective heat transfer coefficient is evaluated at each node and added to the radiative coefficient. For coupling between the

1 Note that the PASCOL module uses as width and height of the cavity the distance from reactor vessel outside radius to RCCS panel inside radius, and the sum of all nodal heights in the RCCS panels. 
THATCH nodal network and the PASCOL RCCS model, the THATCH node temperatures of the outermost reactor vessel node $\theta_{i_{L}}$ are provided, and the total heat transfer resistance across the reactor cavity is evaluated, consisting of vessel conduction plus radiation augmented by convection across the cavity. The conductive resistance of the relatively thin RCCs panels is neglected. Thus, the following relationship.is used for total heat transfer across the reactor cavity at each node level:

$$
Q_{\text {cav } j}=A_{j}\left(\theta_{i_{L} j}-\theta_{F P j}\right)
$$

where,

$$
\frac{1}{\mathrm{AU}_{\mathrm{j}}}=\frac{1}{\mathrm{~A}_{\mathrm{V}} \mathrm{h}_{\mathrm{rad}}+\mathrm{A}_{\mathrm{avg}} \mathrm{h}_{\mathrm{conv}}}+\frac{\Delta \mathrm{r}_{\mathrm{n}, \mathrm{j}}}{\mathrm{A}_{\mathrm{V}} 2 \mathrm{k}\left(\theta_{\mathrm{av}}\right)}
$$

i.e., the convective heat flow is based on an averaged heat flow area, while cavity radiation and vessel conduction are based on the vessel heat transfer area. The RCCS panel temperature is $\theta_{\mathrm{FPj}}$. The radiation heat transfer coefficient here is derived from Equation (3.2-2).

\subsubsection{Heat Transfer from the RCCS Panels to the Coolant and Coolant Energy Equation}

The riser panels of the RCCS are finned internally, as shown schematically, in Figure 3-6. Heat radiated from the reactor vessel will be transferred by conduction and radiation to the fins and the back panel, and then by forced convection to the rising coolant. The code has the option to do these evaluations at each node and at each time step. Parametric evaluations showed that for the current RCCS design about $85 \%$ of the heat flow is by conduction and $15 \%$ by radiation. Details of these conduction/radiation computations are given in Appendix C.

For most code applications, a simpler and satisfactory approach is to specify a fin effectiveness coefficient. Several slightly differing definitions are in the literature. The definition to be used here is the following:

$$
\Phi=\frac{\text { Total Convective Heat Flow to Coolant }}{\text { Convective Heat Flow to Coolant from Unfinned Front Panel }}
$$

For the current MHTGR design (Bechtel, 1986), the value most representative for normal operation and for core heatup transients was established in parametric evaluations as $\Phi=4.4$, which is specified as input parameter for the finned section of the RCCS.

The convective heat flow to the coolant can then be expressed for node $\mathrm{j}$ as follows:

$$
Q_{c j}=A_{F P j} \Phi h_{c o o l j}\left(\theta_{F P j}-\theta_{f l j}\right)
$$

The convective heat transfer correlations used for $h_{\text {cool }}$ are optionally (user-specified option) evaluated from the following:

$$
\mathrm{Nu}_{\mathrm{D}}=0.0243 \quad \mathrm{Re}^{0.8} \operatorname{Pr}^{0.4}
$$




\section{DOWN-FLOW CHANNEL}

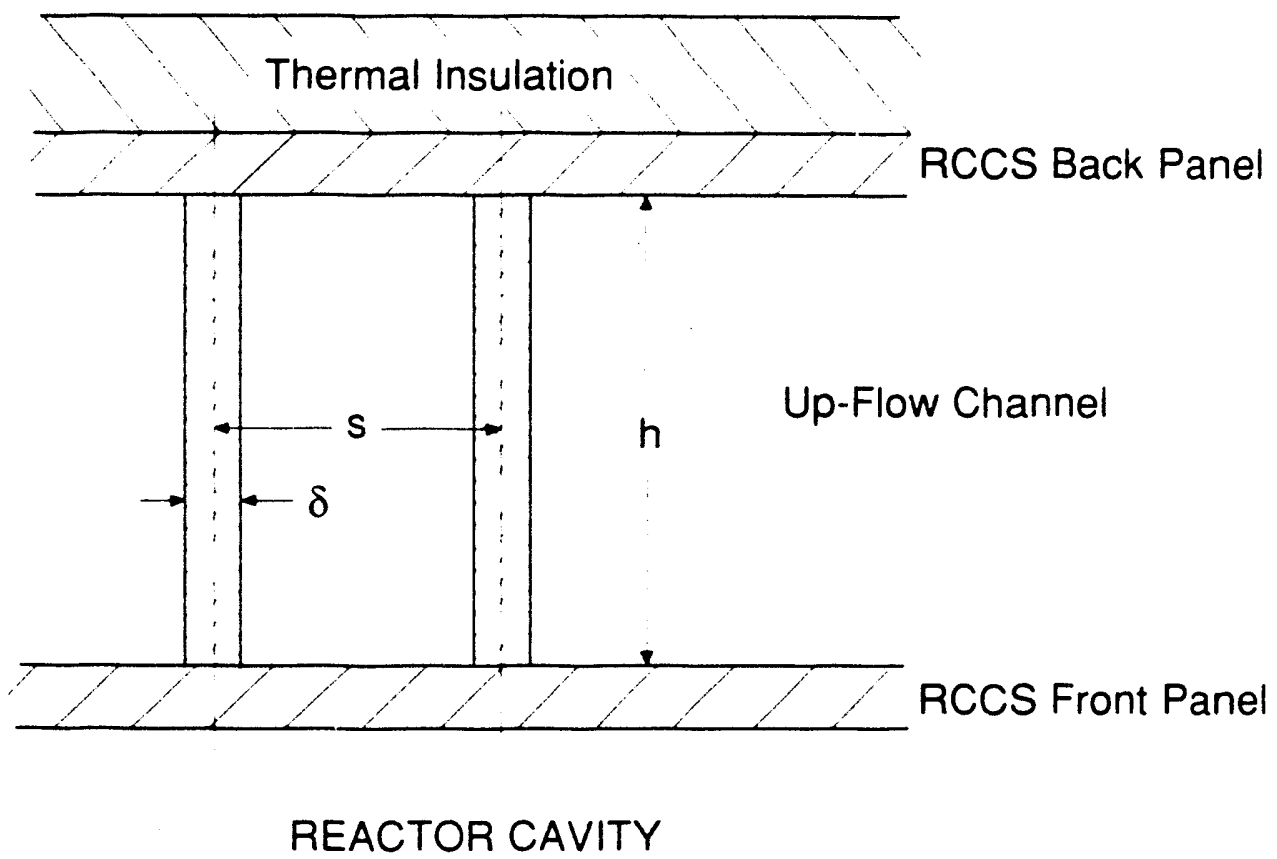

Figure 3-6 Schematic of an Internally Finned Up-Flow Channel of the RCCS 
or from

$$
N u_{D}=0.021 \quad \operatorname{Re}^{0.8} \operatorname{Pr}^{0.6}
$$

At normal operating conditions of the RCCS, some Reynolds numbers are as low as 8,000 . Laminar flow has not been encountered, and is currently not provided with this correlation. (The above correlations were chosen early in the development of THATCH code. To extend the subroutine HHCONVF to include lower Reynolds number domains, it might be preferable to replace these correlations with the more general correlations used by FLOXI and SYMELAM in subroutine HETCOF).

In unfinned sections of the RCCS (currently the top region), heat is transferred from the front panel to the back panel by radiation and then from both panels by convection to the coolant.

In this case, the radiation transfer from the front panel to the back panel is as follows:

$$
\mathrm{Q}_{\mathrm{rad} \text { int } \mathrm{j}}=\epsilon_{1,2} \sigma\left(\theta_{\mathrm{FPj}}^{4}-\theta_{\mathrm{BPj}}^{4}\right)
$$

The convective heat flow to the coolant from the front panel is the following:

$$
Q_{c 1 j}=A_{F P j} h_{\text {cool } j}\left(\theta_{F P j}-\theta_{f l j}\right)
$$

and from the back panel

$$
Q_{c 2 j}=A_{B P j} h_{c o o l ~ j}\left(\theta_{B P j}-\theta_{f l j}\right)
$$

For the finned section, $Q_{\text {cav } j}$ of Equation (3.2-5) must equal $Q_{c j}$ of Equation (3.2-7), while for the unfinned section we have the following:

$$
Q_{\text {cavj }}=Q_{\text {rad int } j}+Q_{c 1 j}
$$

and

$$
Q_{\text {rad int } j}=Q_{c 2 j}
$$

The quasi-steady coolant energy equation can be written for any node $\mathrm{j}$ as follows:

$$
\mathrm{dQ}=\mathrm{A}^{\prime} \mathrm{dz} h\left[\theta_{\mathrm{w}}-\theta_{\mathrm{f}}(\mathrm{z})\right]=W c_{\mathrm{p}} \mathrm{d} \theta_{\mathrm{fl}}
$$

where $\mathrm{d} z$ is positive in flow direction, $A^{\prime}$ is the heat transfer surface per unit length, $\theta_{\mathrm{w}}$ is the wall temperature, considered constant in axial direction within one node, and $\theta_{\mathrm{fl}}$ is the node fluid temperature. By integrating the equation within the node, we obtain the exponential formulation. This formulation was provided in PASCOL as an option, but did not provide any advantage, because the vertical nodalization was rather fine and the temperature changes were small. Therefore, this option has not been used recently. We applied the simpler option of using arithmetic average temperature differences. 
Recasting Equation (3.2-14) as a difference equation we obtain:

$$
Q_{j}=A_{j} h\left(\theta_{w j}-\theta_{f l a v g}\right)=W c_{p}\left(\theta_{f l e x j}-\theta_{f l \text { in } j}\right)
$$

With Equations (3.2-7), we can write for finned sections the following:

$$
Q_{c j}=W c_{p}\left(\theta_{f l e x j}-\theta_{f i \text { in } j}\right)
$$

where

$$
\theta_{\mathrm{fl} \mathrm{j}}=.5\left(\theta_{\mathrm{fl} \mathrm{ex} \mathrm{j}}-\theta_{\mathrm{fl} \text { in } \mathrm{j}}\right)
$$

and

$$
\theta_{\mathrm{fl} \text { in } \mathrm{j}}=\theta_{\mathrm{fl} \mathrm{ex} \mathrm{j-1}}
$$

with $\mathrm{j}$ increasing in flow direction.

For an unfinned region, Equation (3.2-16) must be expressed as follows:

$$
\left(Q_{c 1 j}+Q_{c 2 j}\right)=W c_{p}\left(\theta_{f 1 e x j}-\theta_{f 1 \text { in } j}\right)
$$

\subsubsection{Coolant Momentum Equation}

The quasi-steady coolant momentum equation in heated or unheated flow passages of the RCCS can be written as a balance of friction buoyancy and momentum forces.

$$
\Delta \mathrm{p}_{\text {frict }}+\Delta \mathrm{p}_{\text {mom }}+\int \rho \mathrm{gdz}=0
$$

where the $z$ coordinate is positive pointing downward.

For quasi-steady flow we have the following:

$$
\rho \mathrm{VA}=\mathrm{W}=\text { const. }
$$

Within each node of the upflow channel, the friction term can be expressed as follows:

$$
\Delta \mathrm{p}_{\mathrm{fr} j}=4 \mathrm{f} \frac{\Delta \mathrm{z}_{\mathrm{j}}}{\mathrm{d}_{\mathrm{h}}} \rho \frac{\mathrm{v}^{2}}{2}
$$


where

$$
\begin{array}{ll}
f=f_{L}=\frac{16}{\operatorname{Re}} & \operatorname{Re}<2300 \\
f=f_{T}=\frac{0.04}{\operatorname{Re} 0.16} & \operatorname{Re}>8000
\end{array}
$$
8000.

with linear interpolation between $f_{\mathrm{L}}(2300)$ and $f_{\mathrm{T}}(8000)$ for values of Re between 2300 and

Using Equation 3.2-21 and the gas equation of state for air at essentially atmospheric pressure:

$$
\rho=\frac{p_{0}}{R \theta_{\mathrm{f}}}
$$

Equation 3.2-22 can be expressed as the following:

$$
\Delta p_{f r j}=\left(\frac{R}{2 p_{0} A^{2}}\right)\left(4 f_{j} \theta_{f l j}\right)\left(\frac{\Delta z_{j}}{d_{h}}\right)|W| W
$$

where the sign change in case of reverse mass flow has been incorporated into the equation.

For the total upflow channel, we obtain:

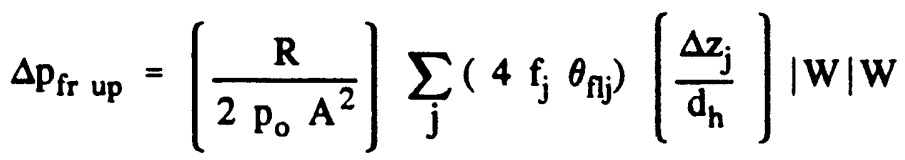

Similarly, the buoyancy term for the upflow channel is:

$$
B_{u p}=-\frac{g p_{o}}{R} \sum_{j} \frac{\Delta z_{j}}{\theta_{f l j}}
$$

The momentum pressure drop over the heated section can be written as follows:

$$
\Delta \mathrm{p}_{\mathrm{m} \text { up }}=\left(\frac{\mathrm{W}}{\mathrm{A}}\right)^{2}\left(\frac{1}{\rho_{\mathrm{ex}}}-\frac{1}{\rho_{\mathrm{in}}}\right)
$$

or

$$
\Delta \mathrm{p}_{\mathrm{m} \text { up }}=\frac{\mathrm{R}}{\mathrm{p}_{\mathrm{o}}}\left(\frac{\mathrm{W}}{\mathrm{A}}\right)^{2}\left(\theta_{\mathrm{fl} \text { in }}-\theta_{\mathrm{fl} \text { ex }}\right)
$$


Friction losses in the stack, unheated ducting and in the downflow channel, as well as the stack buoyancy effects must be added to these terms.

For the stack of height $h$ and diameter $d$ :

$$
\Delta \mathrm{p}_{\mathrm{fr} \text { st }}=\frac{\mathrm{R}}{2 \mathrm{p}_{\mathrm{o}} A_{\mathrm{st}}^{2}} 4 \mathrm{f} \frac{\mathrm{h}}{\mathrm{d}} \theta_{\mathrm{flex}}|\mathrm{W}| \mathrm{W}
$$

and

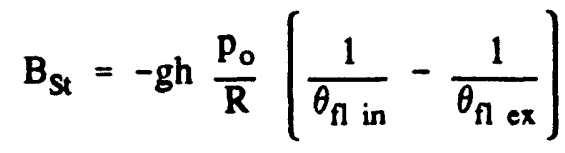

where, as in Equations (3.28) and (3.29) subscript "ex" refers to fluid leaving the heated section, while subscript "in" designates fluid entering the heated section, which is identical to ambient.

The loss coefficients for the inlet ducting, and the downflow channel and the bottom bend, preceding the heated section can be written as follows:

$$
\Delta \mathrm{p}_{\mathrm{fr} \text { in }}=\frac{\mathrm{R}}{2 \mathrm{p}_{\mathrm{o}} \mathrm{A}^{2}}\left(4 \mathrm{f} \frac{\mathrm{h}}{\mathrm{d}}+\mathrm{K}_{\mathrm{in}}+\mathrm{K}_{\mathrm{bot}}\right) \theta_{\mathrm{fl} \text { in }}|\mathrm{W}| \mathrm{W}
$$

while for the exit ducting from the heated section to the stack

$$
\Delta p_{\text {fr ex }}+\frac{R}{2 \text { po } A^{2}} K_{e x} \theta_{\text {fl ex }}|W| W
$$

The loss coefficients $K_{\mathrm{in}}, \mathrm{K}_{\mathrm{bot}}$, and $\mathrm{K}_{\mathrm{ex}}$ are user inputs.

Combining the corresponding expressions, the total momentum balance is the following:

$$
\Delta \mathrm{p}_{\mathrm{fr} \text { in }}+\Delta \mathrm{p}_{\mathrm{fr} \text { up }}+\Delta \mathrm{p}_{\mathrm{m} \text { up }}+\Delta \mathrm{p}_{\mathrm{fr} \text { ex }}+\Delta \mathrm{p}_{\mathrm{fr} \mathrm{St}}+\mathrm{B}_{\mathrm{up}}+\mathrm{B}_{\mathrm{St}}=0
$$

which can be solved for the mass flow in the RCCS.

\subsubsection{Method of Solution for PASCOL Module}

The equations for the RCCS, as given in the previous sections, constitute a system of simultaneous non-linear algebraic equations.

In particular, at each time step the vessel temperatures are given from the THATCH evaluations, and the air inlet temperature is given. For each node in the RCCS upflow channel, the fluid temperature leaving the node must be solved for, together with temperatures for vessel surface and the RCCS front panel for finned nodes, while in un-finned nodes, the temperatures for vessel surface, the RCCS front panel plus the back panel must be obtained. Finally, the mass flow must be computed. 
Solving all of these in one system of non-linear equations proved to be cumbersome. Due to the loose coupling between some of the equations (small coefficients in their Jacobean matrix), the following solution method was found to execute very rapidly.

Consider $j_{L}$ RCCS nodes. The fluid energy equations (3.2-16) for finned nodes and (3.2-19) for unfinned nodes are solved together with Equation (3.2-34) for the mass flow as a system of $N=j_{L}+1$ equations, to be solved by a Newton-Raphson Solver.

In the Jacobean matrix only the terms

$$
\begin{array}{cc}
\mathbf{J}_{\mathbf{j}, \mathrm{j}-1} ; \mathrm{J}_{\mathbf{j}, \mathbf{j}} ; \mathbf{J}_{\mathbf{j}, \mathbf{N}} & \mathrm{j}=1, \mathbf{j}_{\mathrm{L}} \\
\mathbf{J}_{\mathbf{N}, \mathbf{N}} & \mathrm{j}=\mathbf{N}
\end{array}
$$

were considered, which allowed for a very simple matrix inversion.

Simultaneously, at each iteration and at each node, Equations (3.2-5) and (3.2-7) must be solved for finned sections of the RCCS as two simultaneous non-linear equations in temperatures for the vessel surface and the front panel of the RCCS. For unfinned sections, Equations (3.2-5) and (3.2-9) to (3.2-13) can be considered as three equations in temperatures for the vessel surface plus front and back panels of the RCCS. The solution of these two or three additional equations is affected by a Newton-Raphson procedure internal to the above Newton-Raphson solution for fluid temperatures and mass flows, with analytical inversion of the resulting internal 2 or 3 equation systems.

\subsection{WATCOL Module for Water-Cooled RCCS}

\subsubsection{Assumptions}

Figure 3-7 is a schematic of the water-cooled RCCS. The THATCH code model will characterize the following:

(i) the rate of heat transfer from the heat source, the reactor vessel, to the "D-tubes",

(ii) the mass, energy and momentum processes within the water loop shown as A-B-C-D-A in Figure 3-7,

(iii) the mass, energy and momentum transfer processes within the air-water heat exchanger.

The models for the various elements are presented in the sections which follow. The THATCH energy equation for the vessel is a transient conduction equation. For all other processes within the reactor cavity, it is assumed that quasi-steady flow and heat transfer conditions apply. One-dimensional mass, energy and momentum balance equations will be used to characterize the phenomena within the water heat removal loop.

While the cavity cooling panels are distributed irregularly around the periphery of the reactor vessel, it will be assumed here that the panels are distributed uniformly around the vessel. The total surface area exposed to thermal radiation from the vessel is conserved. This assumption simplifies the radiation treatment considerably, as discussed in more detail in Section 3.4. The assumption also permits axisymmetric treatment of the reactor vessel. 


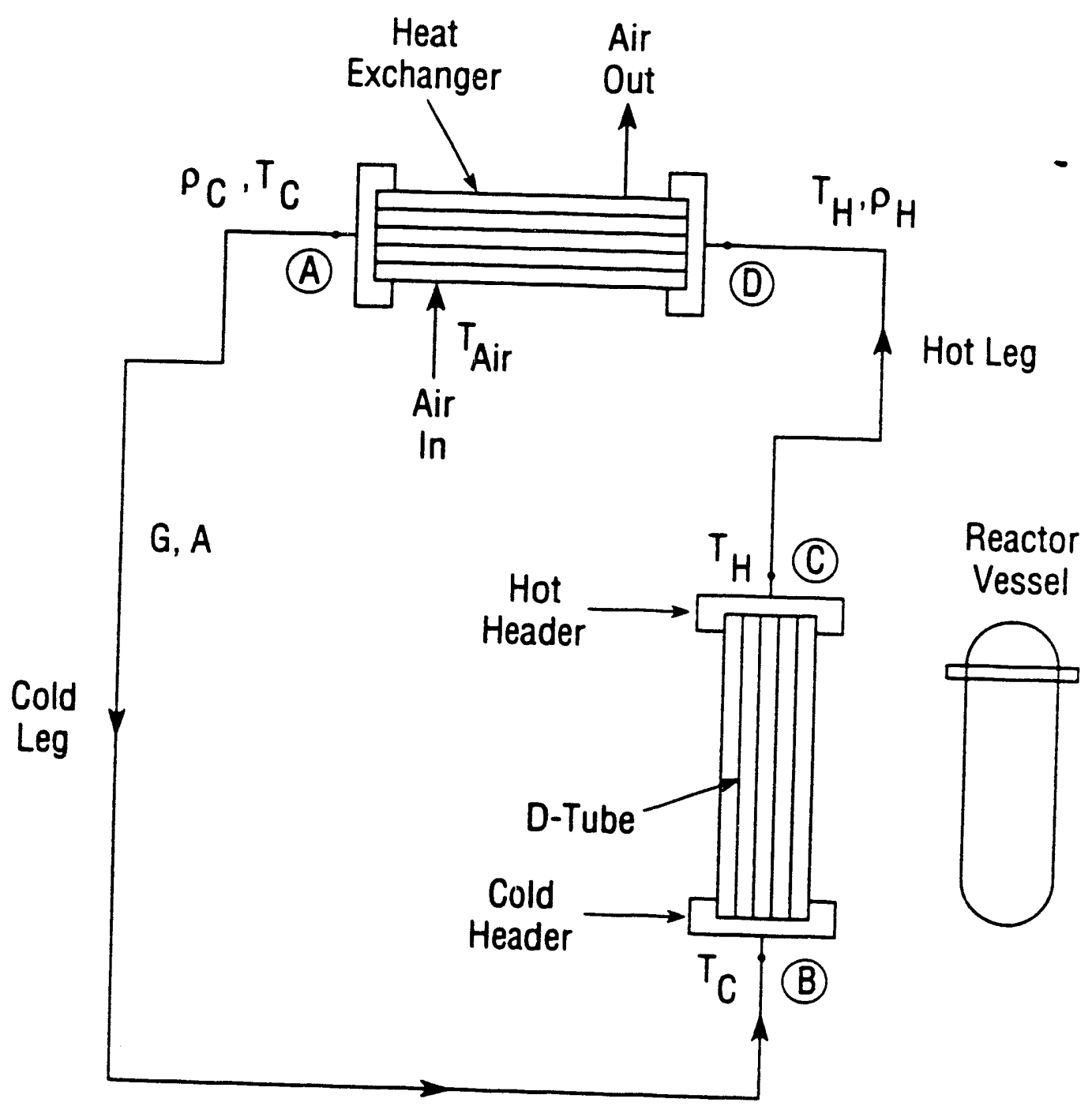

Figure 3-7 Schematic of the Flow Loop of the Water-Cooled RCCS 


\subsubsection{Reactor Vessel}

Decay heat generated within the reactor vessel is transferred to the vessel surface, from where energy is transferred to the cavity cooling panels by thermal radiation and to the cavity gases by natural convection along the vessel surface. The temperature distribution around the vessel is assumed axisymmetric.

The length of the reactor vessel is divided into $n_{1}$. nodes, one of which is shown in Figure 3-8. It is assumed that radiative heat transfer between the vessel and the cavity cooling panels is onedimensional, i.e., radial. The heat transfer model is represented schematically, showing the transport paths from the vessel wall to the cavity gases and to the cavity cooling panels. The coupling between the THATCH model of the reactor vessel and the water-cooled RCCS module is via the outermost node of the reactor vessel. The temperature of the reactor vessel is computed within THATCH, based on WATCOL supplied overall heat transfer coefficients and temperatures of the RCCS panel. WATCOL uses the vessel temperature as input, to evaluate temperatures for vessel and panel surface as well as reactor cavity and RCCS heat transfer. The total heat transfer across the reactor cavity is the following:

$$
Q_{c a v} j=A U_{j}\left(\theta_{f, j}-\theta_{s p, j}\right)
$$

where $\theta_{\mathrm{f}, \mathrm{j}}$ is the last vessel node temperature, $\theta_{\mathrm{sp}, \mathrm{j}}$ is the surface temperature of the RCCS panel and $U_{j}$ is the overall cavity heat transfer coefficient, based on heat transfer area $A$. The heat flux from the vessel internals to the outside surface of the vessel is represented by:

$$
q_{v s, j}=\frac{k_{f, j}}{\Delta r_{j}}\left(\theta_{f, j}-\theta_{v s, j}\right)
$$

where $k_{f, j}$ is the effective conductivity of the vessel internals at the node $j$ and $\Delta_{\mathrm{rj}}$ is the half-width of the node $\mathrm{j}$, and $\theta_{\mathrm{vsj}}$ is the outside surface temperature of the vessel wall. The overall heat transfer coefficient $U_{j}$ is obtained from the following:

$$
\frac{1}{A U_{j}}=\frac{\Delta r_{J}}{A_{v} k_{f, j}}+\frac{1}{\langle A\rangle_{j} h_{v g, j}+A_{r, j} h_{r v, j}}
$$

where $A_{v}$ is the vessel surface area, $h_{r v, j}$ is the radiative heat transfer coefficient across the reactor cavity, as defined in Section 3.3.4, and $h_{\mathrm{vg}, \mathrm{j}}$ is the convective heat transfer coefficient, defined in Section 3.3.6, with $A_{r, j}$ and $\langle A\rangle_{j}$ being the respective heat transfer surface areas.

The boundary condition at the vessel surface is discussed in further detail in Section 3.3.4.

\subsubsection{Water Heat Transport Loop}

As a result of natural circulation within the loop, cold water flows down the downcomer pipe along Section A-B in Figure 3-7. The pipe diameter is $d_{c}$, and the flow rate is $W$. At the cold water header, the flow splits into $n_{t} D$-tube riser channels, each with flow rate $W_{t}$. The heated water is collected in the hot water header and is transported along path C-D, with flow rate $W$ in a pipe of diameter $d_{h}$ to the heat exchanger. It is assumed that a header within the heat exchanger splits the water flow into $n_{x}$ horizontal tubes, each with flow rate $W_{x}$, and the water cools along path $D-A$. Sections $A-B$ 


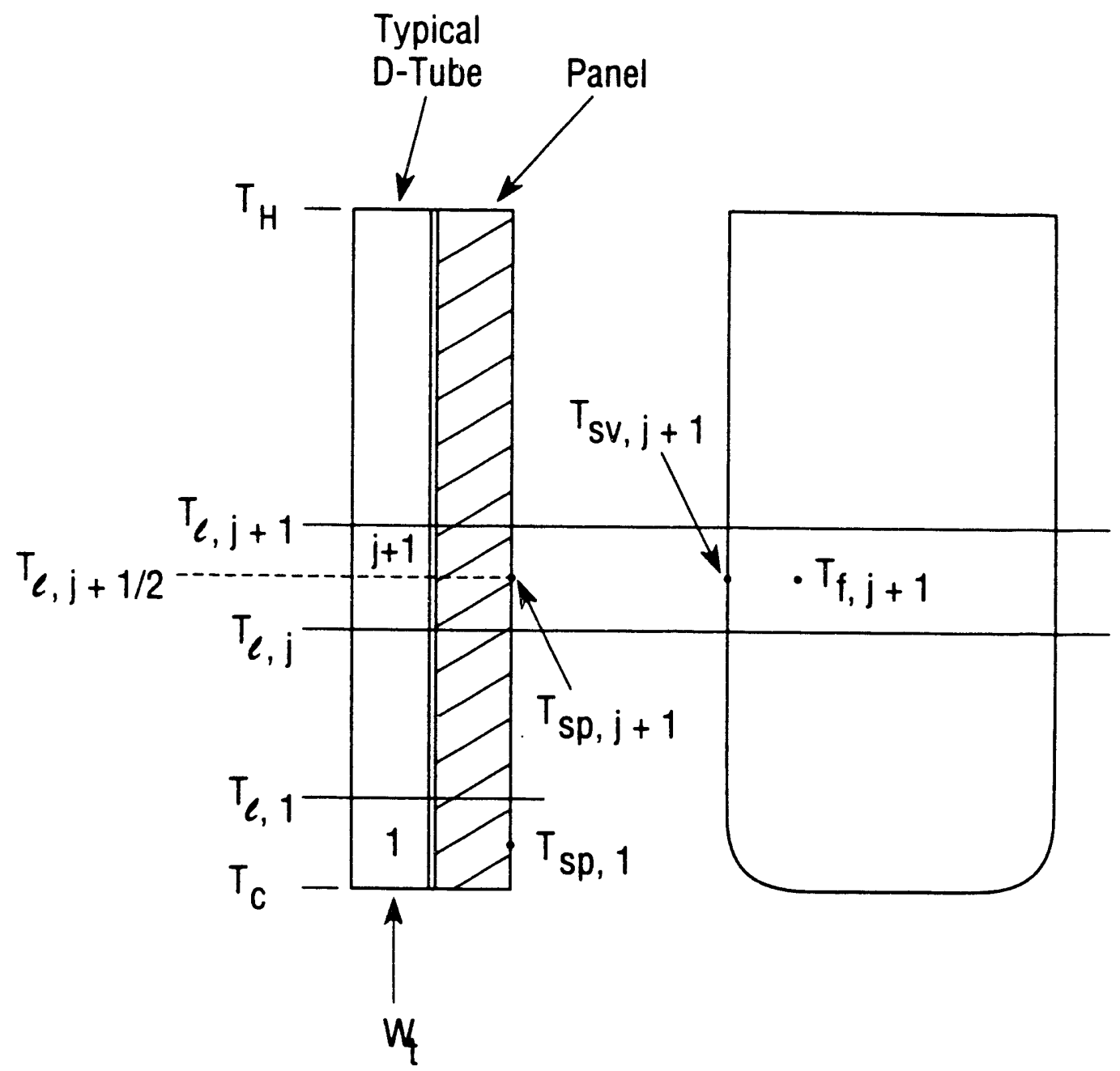

Figure 3-8 Typical Axial Node Through Vessel and Cooling Panel 
and C-D in Figure 3-7 are assumed thermally insulated. Momentum, energy and mass balance equations are derived for the four sections of the water transport path.

Single-phase water is assumed to flow through the loop. The steady-state, one-dimensional mass, momentum and energy equations for the water within any section of the loop are:

Mass Balance

$$
\frac{d W}{d x}=0, \frac{d W_{t}}{d x}=0, \frac{d W_{x}}{d x}=0
$$

where $W$ represents the total water mass flow rate, $W_{t}$ is the flow rate through any single $D$-tube, $W_{x}$ is the flow rate through a heat exchanger tube, and $\mathbf{x}$ is the coordinate in flow direction.

\section{Momentum Balance Around Loop}

The general momentum balance equation for single phase water through a constant diameter pipe is as follows:

$$
\frac{d p}{d x}=( \pm) \rho g-\tau_{w} \frac{p_{w}}{A}-G \frac{d u}{d x}
$$

where $G=W / A$. The negative sign in the gravity term applies to the down flow and the positive sign to the up flow, portion of the loop. The gravity term is zero in horizontal sections of the loop. The shear stress at the wall is given by the following:

$$
\tau_{\mathrm{w}}=\frac{1}{2} \mathrm{f} \rho \mathrm{u}^{2}
$$

Equation 3.3-5 is integrated around the water loop giving a zero total pressure drop, i.e.,

$$
\Delta \mathrm{p}=\sum(\Delta \mathrm{p})_{\mathrm{j}}=0
$$

where

$$
\left(\Delta \mathrm{p}_{\mathrm{j}}\right)=\left(\Delta \mathrm{p}_{\text {gravity }}\right)_{\mathrm{j}}+\left(\Delta \mathrm{p}_{\text {friction }}\right)_{\mathrm{j}}+\left(\Delta \mathrm{p}_{\text {acceleration }}\right)_{\mathrm{j}}+\left(\Delta \mathrm{p}_{\text {losses }}\right)_{j}
$$

where the losses are due to pipe fittings, elbows, etc.

The acceleration pressure drop is due to heating of the water and passage of the fluid through the D-tube and heat exchanger tube banks, which act as manifolds. The acceleration pressure drop across the D-tube bank, from a point just upstream to a point just downstream is given by:

$$
\left(\Delta \mathrm{p}_{\mathrm{BC}}\right)_{\mathrm{acc}}=-\frac{\mathrm{G}^{2}}{2}\left(\frac{1}{\rho_{\mathrm{C}}}-\frac{1}{\rho_{\mathrm{H}}} \frac{\mathrm{A}^{2}}{\mathrm{~A}_{\mathrm{h}}^{2}}\right)-\frac{\mathrm{G}^{2}}{2} \frac{\mathrm{A}^{2}}{\mathrm{~A}_{\mathrm{t}}^{2}}\left(\frac{1}{\rho_{\mathrm{C}}}-\frac{1}{\rho_{\mathrm{H}}}\right)
$$


Where $\rho$ is t.a water density and subscripts $C$ and $H$ denote cold and hot. $A_{h}$ and $A$ are the flow areas in the hot leg and in the cold leg, respectively. $A_{t}$ and $A_{x}$ are the total flow areas of the bank of D-tubes and heat exchanger tubes, respectively.

Similarly, the acceleration pressure drop across the heat exchanger is given by:

$$
\left(\Delta \mathrm{P}_{\mathrm{DA}}\right)_{\mathrm{acc}}=\frac{\mathrm{G}^{2}}{2}\left(\frac{1}{\rho_{\mathrm{C}}}-\frac{1}{\rho_{\mathrm{H}}} \frac{\mathrm{A}^{2}}{\mathrm{~A}_{\mathrm{h}}^{2}}\right)+\frac{\mathrm{G}^{2}}{2} \frac{\mathrm{A}^{2}}{\mathrm{~A}_{\mathrm{x}}^{2}}\left(\frac{1}{\rho_{\mathrm{C}}}-\frac{1}{\rho_{\mathrm{H}}}\right)
$$

Therefore, the total acceleration pressure drop is the following:

$$
(\Delta p)_{a c c}=-G^{2} \frac{1}{2}\left(\frac{A^{2}}{A_{x}^{2}}-\frac{A^{2}}{A_{t}^{2}}\right)\left(\frac{1}{\rho_{H}}-\frac{1}{\rho_{C}}\right)
$$

The pressure drop due to losses in pipe fittings is given, for fitting $k$, by:

$$
(\Delta \mathrm{p})_{\text {loss }}=-\frac{\mathrm{K}_{\mathrm{fk}}}{2 \rho_{\mathrm{k}}} \mathrm{G}_{\mathrm{k}}^{2}
$$

where $K_{f k}$ defines the fitting loss coefficient.

Application of Equations 3.3-5 - 3.3-12 to the loop shown in Figure 3-7 yields the result

$$
\begin{gathered}
-G^{2} \sum_{i}\left(2 \frac{f_{i}}{\rho_{i}} \frac{l_{i}}{D_{i}} \frac{A^{2}}{A_{i}^{2}}\right)-G^{2} \sum_{m} \frac{1}{2 \rho_{m}} K_{f m} \frac{A^{2}}{A_{m}^{2}}-G^{2} \frac{1}{2}\left(\frac{A^{2}}{A_{x}^{2}}-\frac{A^{2}}{A_{t}^{2}}\right)\left(\frac{1}{\rho_{H}}-\frac{1}{\rho_{C}}\right) \\
+\sum_{j} \rho_{C j} g l_{j}-\sum_{k} \rho_{H k} g l_{k}=0
\end{gathered}
$$

where $\mathrm{j}$ represents all piping elements, and $\left(\mathrm{l}_{\mathrm{j}} / \mathrm{D}_{\mathrm{j}}\right)$ includes allowance for fittings in the segment. The sum over $j$ includes only vertical piping elements in the cold leg and the sum over $k$ are over vertical elements in the hot leg. The sum over $m$ represents all fittings in the loop. Equation 3.3-13 can be solved for $\mathrm{G}$, giving the following:

$$
G^{2}=\frac{\sum \rho_{C_{j}} g l_{j}-\sum \rho_{H k} g l_{k}}{\sum\left(2 \frac{f_{j}}{\rho_{j}} \frac{l_{j}}{D_{j}} \frac{A^{2}}{A_{j}^{2}}\right)+\sum \frac{1}{2 \rho_{m}} K_{f m} \frac{A^{2}}{A_{m}^{2}}+\frac{1}{2}\left(\frac{A^{2}}{A_{x}^{2}}-\frac{A^{2}}{A_{t}^{2}}\right)\left(\frac{1}{\rho_{H}}-\frac{1}{\rho_{C}}\right)}
$$

where the numerator represents the buoyancy term, which drives the flow around the loop, and the denominator the friction term and other losses.

\section{Energy Balance for Water in D-Tubes}

It is assumed that the D-tubes receive energy from the from the front face of the Cavity Cooling Panels and are insulated in the direction facing the cavity walls. The energy equation for the water in 
one of the D-tubes is as follows:

$$
W_{t} \frac{d h_{1}}{d x}=U_{p l}\left(\theta_{s p}-\theta_{1}\right) P_{h p}
$$

and is based upon the nomenclature shown in Figure 3-8. $P_{h p}$ is defined in Equation 3.3-19. Equation 3.3-15 is integrated across an axial node $j$ shown in Figure 3-8, yielding the result for the axial variation of water temperature,

$$
\theta_{1, j+1}-\theta_{1, j}=<\frac{U_{p l} P_{h p} \Delta x_{j}}{W_{t} c_{p}}>\left(\theta_{a p, j+1}-\theta_{1, j+1}\right)
$$

where $\mathrm{U}_{\mathrm{pl}}$ is the conductance which includes conduction in the steel panel, conduction in the D-tube wall and the convective conductance of the water. $\theta_{\mathrm{sp}}$ is the surface temperature of the panel. Equation 3.316 can be solved for $\theta_{1, j}$, giving

$$
\theta_{1, j+1}=\frac{\theta_{1, j}+C_{j} \theta_{s p, j+1}}{1+C_{j}}
$$
given by:

The quantity $C_{j}$ represents the factor in brackets in Equation 3.3-16. The conductance $U_{p l}$ is

$$
\frac{1}{U_{\mathrm{Pl}}}=\frac{\delta_{\mathrm{P}}}{\mathrm{k}_{\mathrm{p}}}+\left(\frac{\delta_{\mathrm{t}}}{\mathrm{k}_{\mathrm{t}}}+\frac{1}{\mathrm{~h}_{1}}\right) \frac{\mathrm{P}_{\mathrm{hp}}}{\mathrm{P}_{\mathrm{ht}}}
$$

The quantity $P_{h p}$ represents the perimeter of the panel surface which transfers energy to a single D-tube and depends on the number of D-tubes in a single loop and on the number of loops (1 or 2) assumed to be operational, and is given by:

$$
P_{h p}=\frac{P_{p}}{n_{t} n_{\text {loop }}}
$$

where $n_{\text {loop }}$ is the number of loops operational, and $n_{t}$ is the number of $D$-tubes in a single loop. $P_{p}$ is the total panel perimeter, and $P_{h t}$ is the perimeter of a single $D$-tube.

\section{Energy Balance for Water in Heat Exchanger}

It is assumed, as shown schematically in Figure 3-7, that the water line feeding the heat exchanger is manifolded and that a bank of horizontal tubes is cooled by air flowing across the tubes.

The energy equation for the water in one of the tubes is given by:

$$
W_{x} c_{p w} \frac{d \theta_{1}}{d x}=U_{x} P_{w x}\left(\theta_{1}-\theta_{\text {air }}\right)
$$

where $P_{w x}$ is the perimeter of a bare heat exchanger tube. 
Assuming that the air temperature and conductance are constant along the horizontal tubes, the water temperature at the exit of the exchanger is as follows:

$$
\theta_{C}=\bar{\theta}_{\text {air }}+\left(\theta_{\mathrm{H}}-\bar{\theta}_{\text {air }}\right) e^{-U_{x} A_{x} /\left(w_{x} c_{p w}\right)}
$$

where $\theta_{\mathbf{H}}$ is the hot leg temperature at the inlet to the heat exchanger, $A_{\mathbf{x}}$ is the surface area of a bare heat exchanger tube and, $c_{p w}$ is the average specific heat of the water in the heat exchanger.

The heat exchanger tubes may be finned, and the overall heat exchanger conductance is given by:

$$
\frac{1}{A_{x} U_{x}}=\frac{1}{h_{a i r} A_{t}}+\frac{1}{h_{b x} A_{1}}+\frac{\delta_{x}}{A_{x} k_{x}}
$$

The heat transfer correlations, $h_{\text {air }}$ for either bare or finned tubes are discussed in Section 3.6. The heat transfer coefficient on the water-side of the heat exchanger is $h_{1 x}$, and $\delta_{x}$ is the tube wall thickness.

Equation 3.3-21 applies to an "average" tube within the heat exchanger and the air temperature; $\theta_{\text {air }}$ represents the air temperature adjacent to this "average" heat exchanger tube. The

heat transfer model represented by Equation 3.3-21 is preliminary at this point. When further design information is available, a more detailed model may be incorporated.

\subsubsection{Boundary Conditions at Vessel and Panel Walls}

\section{Thermal Radiation Model}

It is assumed that the surface of each axial segment of the reactor vessel exchanges radiation with the surface of the opposite cooling panel node. The radiation is modeled as one-dimensional radiation transport between infinite concentric cylinders. The radiative heat flux from the vessel, $q_{\mathrm{rv}, \mathrm{j}}$, is given by:

$$
q_{r v j}=\frac{\sigma\left(\theta_{s v, j}^{4}-\theta_{s p, j}^{4}\right)}{\left.\left[\frac{1}{\varepsilon_{v}}+\frac{A_{v j}}{A_{p, j}} \frac{1}{\varepsilon_{p}}-1\right)\right]}
$$

and the heat flux to the cooling panel, $\mathrm{q}_{\mathrm{rp} j}$, is as follows:

$$
q_{r p, j}=\frac{A_{v}}{A_{p}} q_{r, j}
$$

where $A_{v}$ and $A_{p}$ are the vessel and panel surface areas. 


\section{Boundary Condition at the Vessel Wall}

The heat balance equation at the vessel wall stipulates that the rate of conduction to the vessel wall from within the vessel is balanced by the sum of the rate of convection from the vessel wall to the cavity gas by natural convection and the radiative transfer from the vessel wall to the panel,

$q_{\text {conduction to vessel wall }}=q_{\text {convection from vessel wall to panel }}+q_{\text {radiation from vessel wall to panel }}$

The balance equation is written as the following:

$$
\left.\frac{k_{f, j}\left(\theta_{f, j}-\theta_{s v, j}\right)}{\Delta r_{j}} A_{v, j}=h_{v g, j}\left(\theta_{s v, j}-\theta_{s p, j}\right)<A\right\rangle_{j}+h_{r v, j}\left(\theta_{s v, j}-\theta_{s p, j}\right) A_{v, j}
$$

where $\theta_{\mathrm{spj}}$ is the panel surface temperature, and $\mathrm{h}_{\mathrm{rv}, \mathrm{j}}$, the radiation heat transfer coefficient, is defined, using Equation 3.3-23, by:

$$
h_{r v, j}=\frac{\sigma\left(\theta_{s v, j}^{2}+\theta_{s p, j}^{2}\right)\left(\theta_{s v, j}+\theta_{s p, j}\right)}{\frac{1}{\varepsilon_{v}}+\frac{A_{v j}}{A_{p j}}\left(\frac{1}{\varepsilon_{p}}-1\right)}
$$

$\mathrm{h}_{\mathrm{vg}, \mathrm{j}}$ represents the convective heat transfer coefficient in the cavity, and $\left.<\mathrm{A}\right\rangle$ represents an average area for cavity convective heat transfer.

Equation 3.3-25 can be solved for the vessel surface temperature, $\theta_{\mathrm{sv}, \mathrm{j}}$, at axial node $\mathrm{i}$,

$$
\theta_{s v, j}=\frac{\frac{k_{f, j}}{\Delta r_{j}} \theta_{f, j}+h_{r g, j} \theta_{s p, j}+h_{r g, j} \theta_{s p, j}}{\frac{k_{f, j}}{\Delta r_{j}}+h_{r g, j}<A>_{j} / A_{v, j}+h_{r v, j}}
$$

\section{Boundary Condition at the Surface of the Cooling Panel}

The heat balance at the cooling panel surface specifies that the rate of heat transfer from the gas to the panel wall by natural convection and the radiative transfer rate from the vessel to the panel surface, is balanced by the rate of transfer from the panel wall to the water in the D-tubes.

$q_{\text {convection from vessel to panel wall }}+q_{\text {radiation from vessel to panel wall }}=q_{\text {conductance from panel wall to water }}$ The balance equation is written as follows:

$$
\left.h_{p g, j}\left(\theta_{s v j}-\theta_{s p, j}\right)<A\right\rangle_{p, j}+h_{r v, j}\left(\theta_{s v, j}-\theta_{s p, j}\right) A_{p, j}=U_{p l, j}\left(\theta_{s p, j}-\theta_{1, j}\right) A_{p, j}
$$

where the panel conductance $\mathrm{U}_{\mathrm{plj}}$ is given by Equation 3.3-18. 
Equation 3.3-28 can be solved for the panel surface temperature at axial node $\mathrm{j}$,

$$
\theta_{\mathrm{spj}}=\frac{\langle A\rangle_{j} / A_{p, j} h_{p g, j} \theta_{g a s}+h_{r p, j} \theta_{s p j}+U_{p l, j} \theta_{1, j}}{\langle A\rangle_{j} / A_{p, j} h_{p g, j}+h_{r p j}+U_{p l, j}}
$$

\subsubsection{Air-Water Heat Exchanger}

A detailed design for the heat exchanger is not available at this time. A simplified model is used which contains the essential features of a buoyancy-driven, air-water heat exchanger. Figure 3-9 schematically shows a triangular array of heat exchanger tubes, each of length $l_{x}$, stacked to a height $H_{x}$. The overall height of the stack is $\mathrm{H}_{\mathrm{xtot}}$. The heat exchanger tubes may be finned. Air enters the heat exchanger at temperature $\theta_{\text {air }}$ and exits at $\theta_{\text {airx }}$. Heat, transferred to the air from the water flow through the heat exchanger tubes, heats the air and reduces its density relative to the air surrounding the air stack. The resultant buoyant force drives the air flow.

\section{Momentum Balance Across the Stack}

The momentum balance for the air flow in the heat exchanger stack is obtained by equating the pressure drop in the stack to the gravitational head of the air column in the atmosphere. The resulting equation is solved for the mass flux of air in the stack, in analogy to Equation 3.3-14 for the liquid flux in the water loop. The mass flux is given by:

$$
\mathrm{G}_{\text {air }}^{2}=\frac{\rho_{\text {air }} \mathrm{gH}_{\mathrm{s}}-\left[\bar{\rho}_{\text {air }} \mathrm{g} \mathrm{H}_{\mathrm{x}}+\rho_{\mathrm{air}, \mathrm{x}} \mathrm{g}\left(\mathrm{H}_{\mathrm{s}}-\mathrm{H}_{\mathrm{x}}\right)\right]}{\frac{2 \mathrm{f}_{\mathrm{t}}}{\rho_{\text {air }}}\left(\frac{\mathrm{A}_{\mathrm{x}}}{\mathrm{A}_{\min }}\right)^{2} \mathrm{n}_{\mathrm{rows}}+\sum_{\mathrm{i}=1,2} 2\left(\frac{\mathrm{f}}{\bar{\rho}} \frac{1}{D_{\mathrm{h}}}\right]_{\mathrm{i}}+\left(\frac{1}{\rho_{\text {air }, \mathrm{x}}}-\frac{1}{\rho_{\text {air }}}\right)}
$$

where the numerator represents the buoyancy term which drives the flow, and the denominator represents the flow resistances due to friction along the walls of the stack and the flow losses across the bank of the heat exchanger tubes. The quantity $f_{t}$ is the friction factor for flow across the bank, which can be finned

if desired. The summation in the denominator represents friction losses along the walls of the stack adjacent to the bank and friction losses in the bare stack above the bank.

\section{Energy Balance Across the Heat Exchanger}

A global energy balance across the heat exchanger equates the energy removed by the air flow to the energy lost by the water. The energy balance leads to the following equation for the exit temperature of the air:

$$
\theta_{\mathrm{air}, \mathrm{x}}=\theta_{\mathrm{air}}+\frac{\mathrm{W} \overline{\mathrm{c}}_{\mathrm{p}, \mathrm{w}}}{\mathrm{W}_{\mathrm{air}} \overline{\mathrm{c}}_{\mathrm{p}, \mathrm{a}}}\left(\theta_{\mathrm{lH}}-\theta_{\mathrm{lC}}\right)
$$

where $\mathrm{W}_{\mathrm{air}}$ is the air mass flow rate through the stack, and $\mathrm{W}$ is the liquid loop flow rate. 


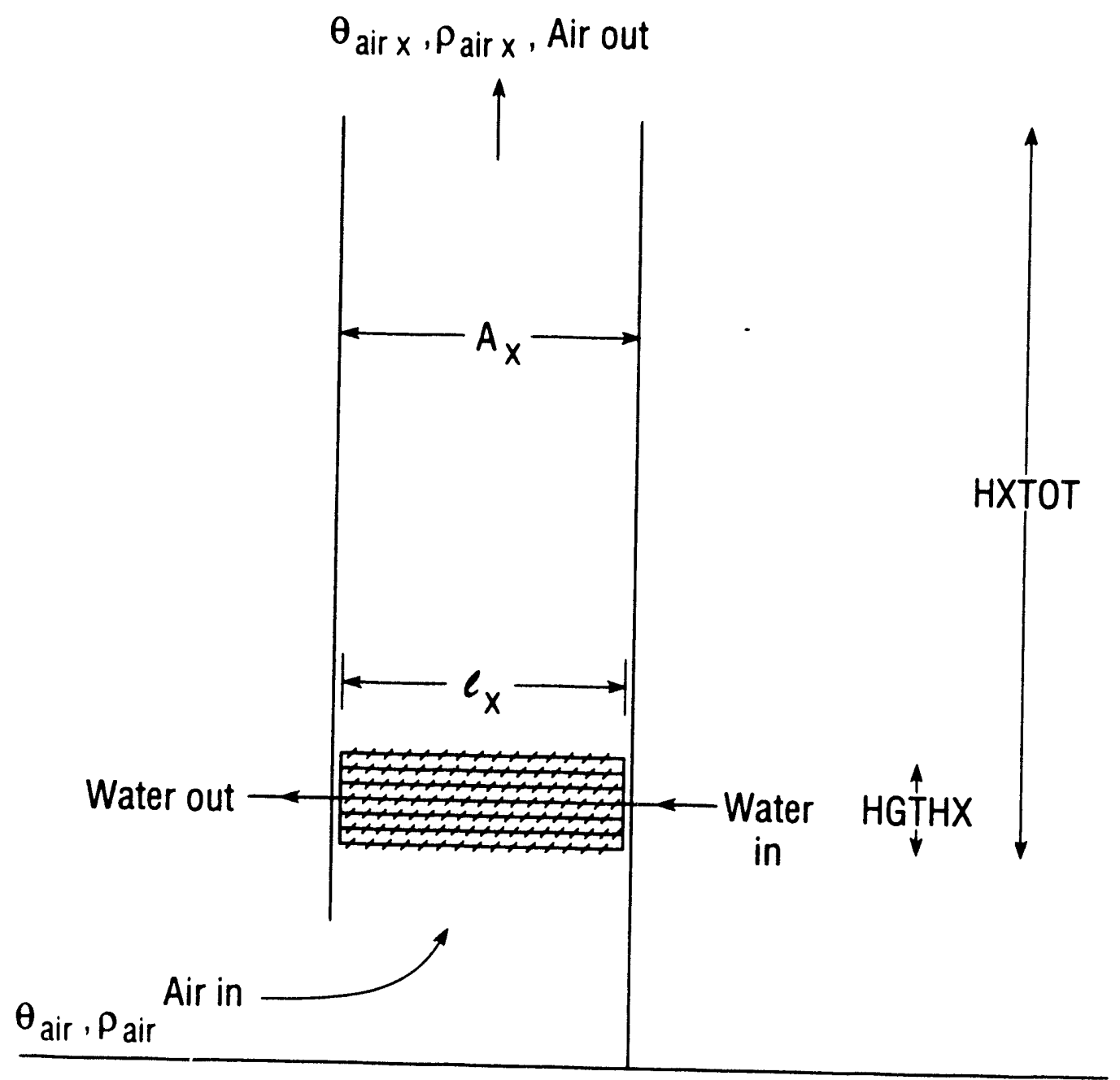

Figure 3-9 Schematic of Natural Draft Water/ Air Heat Exchanger 


\subsubsection{Constitutive Relations}

\section{Friction Factor in D-Tubes}

A smooth-tube friction factor is used for the D-tubes and is given by the following:

$$
\begin{aligned}
f & =16 / \operatorname{Re} \text { for } \operatorname{Re}<2300 \\
& =0.04 / \operatorname{Re}^{0.16} \text { for } \operatorname{Re}>8000
\end{aligned}
$$

wh.ere $\mathrm{Re}$ is the Reynolds number based on the mass flux of liquid through a tube. A linear interpolation is used between the above Reynolds' number limits.

\section{Friction Factor on the Air-Side of the Heat Exchanger}

The flow losses within the air stack are due to flow past the bank of the tube and friction along the walls of the stack. For friction along the wall, the smooth-tube friction factor correlation, given above, is used. For the pressure drop across the bank of the tube, a friction factor for either an array of bare tubes, or an array of finned tubes is used. For either case, the pressure drop is given by:

$$
\Delta P=\frac{2 f_{t}}{\rho}\left[\frac{A_{s}}{A_{\min }}\right]^{2} n_{\text {Rows }} G_{\text {Air }}^{2}
$$

For bare tubes in a triangular array, the friction factor is given by [Zukauskas, 1983(a)]:

$$
f_{t}=0.355\left(\frac{1}{\eta-1}\right)^{0.33} \operatorname{Re}^{-0.15}
$$

where $\eta$ is the pitch-to-diameter ratio of the tube bundle.

For finned tubes with ring-shaped fins, the friction factor is [Zukauskas, 1983(b)]:

$$
f_{t}=1.35\left(\frac{d^{*}}{d_{e}}\right)^{0.3} \operatorname{Re}_{d}^{-0.25}
$$

where $d^{*}$ and $d_{e}$ are defined as follows:

$$
d^{*}=d \frac{A_{\text {tube }}}{A_{\text {total }}}+\frac{A_{\text {fin }}}{A_{\text {total }}} \sqrt{0.785\left(D^{2}-d^{2}\right)}
$$

and

$$
\mathrm{de}=\frac{2\left[s\left(s_{1}-d\right)-2 \delta \mathrm{h}\right]}{2 \mathrm{~h}+\mathrm{s}}
$$

and where $d$ is the bare tube diameter; $D$ is the fin diameter; $A_{\text {tube }}$ is the bare tube surface area per unit tube length; $A_{f i n}$ is the fin surface area per unit tube length; and $A_{\text {total }}$ is the total surface area per unit 
tube length. The quantity $s$ is the fin spacing; $s_{1}$ is the distance between tube centers; $h$ is the fin height; and $\delta$ is the fin thickness. The Reynolds number $R_{d^{*}}$ is based upon $d^{*}$ given above, and upon the maximum fluid velocity between tubes.

\section{Natural Convection Heat Transfer of Gas in the Reactor Cavity}

Equation $3.2-4$ is used for the natural convection heat transfer in the reactor cavity.

\section{Forced Convection Heat Transfer of Water in D-Tubes}

The Dittus-Boelter correlation is used to characterize the convective heat transfer conductance within the D-Tubes and on the water side of the heat exchanger.

$$
\mathrm{Nu}=0.023 \mathrm{Re}^{0.8} \operatorname{Pr}^{0.04}
$$

\section{Forced Convection Heat Transfer for Air in the Heat Exchanger Stack}

For forced convective heat transfer past an equilateral triangular array of bare tubes (no fins) in crossflow, the following correlation [McAdams, 1954] was used:

$$
\mathrm{Nu}=0.33 \mathrm{Re}^{0.6} \mathrm{Pr}^{0.333}
$$

For flow past an equilateral triangular array of finned tubes in a crossflow, the heat transfer correlation [Gnielinski, 1983] used is:

$$
\mathrm{N}=0.2183\left(\mathrm{~s}^{*}\right)^{0.18}\left(\mathrm{~h}^{*}\right)^{-0.14} \mathrm{Re}^{0.65} \mathrm{Pr}^{0.33}
$$

where $s^{*}$ is the ratio of fin spacing to tube diameter, and $h^{*}$ is the ratio of fin height to tube diameter.

\subsubsection{Method of Solution for the WATCOL Module}

The system of simultaneous non-linear algebraic equations outlined in the preceding sections is solved, very similar to the PASCOL module equations, by a system of nested Newton Raphson methods. As in the air-cooled RCCS, the coolant temperatures and flow rates for water and air are functions of the vessel temperature profile and the ambient air temperature only.

For each node in the heated upflow channel, the fluid energy balance (Equation 3.3-17) is used to compute the coolant exit temperature as function of the coolant inlet temperature. These are coupled to the temperatures of the panel surface and the vessel surface via Equations 3.3-27 and 29. In addition, the water loop flow rate must be obtained from the momentum balance of Equation 3.3-14, while the cold leg liquid temperature is computed from the heat exchanger energy balance of Equation 3.3-21. The airside mass flow follows from the momentum balance of Equation 3.3-30, with the air exit temperature being obtained from the energy balance of Equation 3.3-31. For a model using $N$ nodes in the upflow channel, this results in a system of $3 \mathrm{~N}+4$ simultaneous equations. However, by solving at each node, Equations 3.3-27 and 3.3-29 in an internal Newton Raphson procedure for panel and vessel surface temperatures, we can reduce the size of the set of simultaneous equations to $N+4$ equations, which are solved by an outer Newton Raphson procedure. Because the coolant exit temperature of any node $n$ 
depends only on the coolant inlet temperature, the upper left square of $\mathrm{N}$ entries in the total matrix is bidiagonal, with only diagonal and left-of-diagonal terms. This matrix structure permits the implementation of a special simultaneous equation solver, which reduces the system of $\mathrm{N}+4$ equations to a system of only four simultaneous non-linear equations, in the last four variables, the cold leg liquid temperature, the liquid side mass flow, the air side mass flow and the air-side exit temperature. The liquid temperatures in the heated channel are then readily obtained by back substitution.

\subsection{FLOXI Module for Reactor Gas Flow, Heat Transfer, and Chemical Reactions}

The FLOXI module can be called at each time step in a THATCH run, to solve the quasi-steady momentum and energy equations for gas flow and heat transfer in the reactor vessel and for the graphite oxidation chemical reactions in case of air or water ingress. The solid temperatures in the reactor vessel are inputs to the FLOXI computations, which solve for gas flow rates, gas temperatures, heat transfer and mass transfer rates, changes in gas composition and mass addition due to chemical reactions.

The gas flow rates, the fluid temperatures, and the change in gas composition due to chemical reaction are coupled to each other. The FLOXI module will solve for these independently and then iterate, if so desired, among the three sets of computations, as described in Section 4.2.3.

\subsubsection{Gas Flow in the Reactor Vessel}

The gas flow in the reactor vessel is modelled by solving the quasi-steady momentum equation for a network of parallel channels. The number of channels is user-prescribed.

In free-standing FLOXI runs, the number is a completely free user selection. Within THATCH runs, for practical purposes, the number of parallel channels must be coordinated with the THATCH nodalization. This number is generally equal to the number of radial nodes in the active core plus one return channel, representing the up-flow channel of the core barrel and flow through the steam generator. Upper and lower plenum are the junction points for this network, and external effects such as a circulator head and the flow losses in the steam generator can be added in the return flow channel.

For each parallel channel the quasi-steady momentum equation can be expressed as follows:

$$
\Delta \mathrm{p}_{\mathrm{o}}=\mathrm{p}_{\text {UPL }}-\mathrm{p}_{\text {LOPL }}=\Delta \mathrm{p}_{\mathrm{fr} \mathrm{i}}+\Delta \mathrm{p}_{\ell \mathrm{i}}+\Delta \mathrm{p}_{\mathrm{m} i}+\Delta \mathrm{p}_{\text {buoy } \mathrm{i}} \quad \mathrm{i}=1, \mathrm{i}_{\mathrm{CHP}}
$$

where $i_{C H}$ is the number of in-core flow channels, and $i_{C H P}=i_{C H}+1$ represents the return flow channel.

Each of the above equations can be expressed as a function of mass flow in the channel, $W_{i}$. A final mass balance for the upper or lower plenum

$$
\sum w_{i}=0
$$

results in a system of $i_{C H P}+1$ simultaneous non-linear algebraic equations for the mass flows $W_{i}$ and the plena pressure difference $\Delta \mathrm{p}_{0}$. 
The friction pressure drop term of Equation (3.4-1) can be derived from the following:

$$
\mathrm{dp}_{\mathrm{fr}}=4 \mathrm{f} \frac{\mathrm{dz}}{\mathrm{d}_{\mathrm{h}}} \rho \frac{\mathrm{v}^{2}}{2}
$$

In finite difference form, summed over the channel, and in terms of the mass flow

$$
\mathbf{W}=\mathbf{A} \rho \mathbf{v}
$$

This can be written for any channel $i$

$$
\Delta p_{f r i}=\sum_{j=1}^{j_{N O D}} \frac{1}{2 A_{i}^{2}} 4 f_{i, j} \frac{\Delta z_{j}}{d_{h_{i, j}}} \frac{1}{\rho_{i, j}}\left|W_{i, j}\right| W_{i, j}
$$

where mass flows are defined positive for downflow, and $j_{\text {NOD }}$ is the number of axial nodes in each channel.

While the crosssection for flow remains essentially constant in the reflector regions and in the core, the hydraulic diameter varies significantly. Therefore, the flow area was assumed to be constant, and then can be taken out of the sum.

In case of chemical reactions, gas composition and mass flow vary within the channel. Defining

$$
\lambda_{i, j}=\frac{w_{i, j}}{w_{o i}}
$$

where $W_{o}$ is the inlet flow into channel $i$, Equation (3.4-5) can be expressed as follows:

$$
\Delta p_{f r i}=\frac{1}{2 A_{i}^{2}}\left[\sum 4 f_{i, j} \frac{\Delta z_{j}}{d_{h i, j}} \frac{\lambda^{2}, j}{\rho_{i, j}}\right]\left|w_{o i}\right| w_{o i}
$$

In cases with chemical reaction, the viscosity, Reynolds number, and friction factor are computed using the mixture viscosity relations of Appendix D, and evaluating $R e$ and at each node using Equation (3.2-23).

The entrance and exit loss terms are of the following general form:

$$
\Delta \mathrm{p}_{\ell}=k \frac{\rho \mathrm{v}^{2}}{2}=\left(\frac{\mathrm{k}}{2 \mathrm{~A}^{2}}\right) \frac{1}{\rho} \mathrm{W}|\mathrm{W}|
$$

With one "top" and "one" bottom loss coefficient, and considering changes in mass flow due to chemical reactions, the loss pressure drop for the core channels $\left(i=1, i_{C H}\right)$ is the following: 


$$
\Delta p_{\ell \mathrm{i}}=\left[\left[\frac{\mathrm{k}_{\text {top i }}}{2 \mathrm{~A}_{\text {top } \mathrm{i}}^{2}}\right]\left(\frac{\lambda_{\text {top } \mathrm{i}}}{\rho_{\text {top } \mathrm{i}}}\right)^{2}+\left(\frac{\mathrm{k}_{\text {bot i }}}{2 \mathrm{~A}_{\text {bot } \mathrm{i}}^{2}}\right)\left(\frac{\lambda_{\text {bot } \mathrm{i}}}{\rho_{\text {bot i }}}\right)^{2}\right]\left|\mathrm{w}_{\text {oi }}\right| \mathrm{w}_{\text {oi }}
$$

where top and bot designate flow channel inlet at the upper plenum and outlet at the lower plenum, respectively. The loss coefficients $k_{\text {top }}$, and $k_{b o t}$ are user prescribed inputs. In the return flow channel, $\mathfrak{i}_{\text {CHP }}$ steam generator flow losses can be included by specifying a corresponding, larger bottom loss for that channel. Furthermore, a circulator head can be specified for that channel with the input in form of an absolute pressure rise, i.e., Equation (3.4-9) is for channel $i_{C H P}$

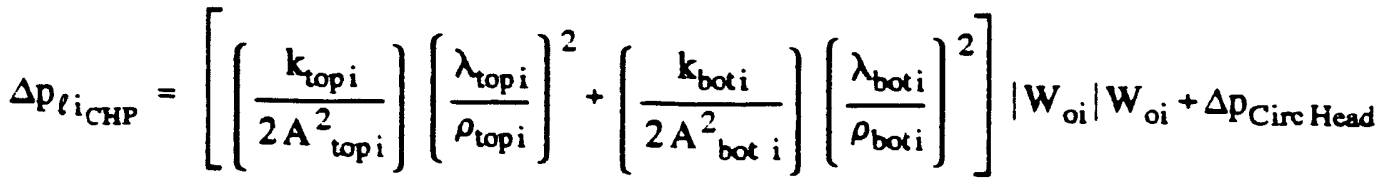

where $\Delta \mathrm{p}$ Cire Head is a user-prescribed input.

The momentum pressure drop term is derived from the following:

$$
d p_{m}=\rho v \frac{\partial v}{\partial z} d z
$$

which can be expressed in finite difference form, summed over the channel length as follows:

$$
\Delta \mathrm{p}_{\mathrm{mi}}=\frac{\mathrm{W}_{\mathrm{oi}}}{\mathrm{A}_{\mathrm{i}}^{2}} \sum_{\mathrm{j}=1}^{\mathrm{j}_{\mathrm{NOD}}} \lambda_{\mathrm{avg} \mathrm{j}}\left[\left(\frac{\lambda}{\rho}\right)_{\mathrm{j}}-\left(\frac{\lambda}{\rho}\right)_{\mathrm{j}-1}\right]
$$

the $\lambda$ and $\rho$ values in the bracket present node inlet and exit values and the nodal average value of $\lambda$ is

$$
\lambda_{\text {avg } j}=\frac{1}{2}\left(\lambda_{j-1}+\lambda_{j}\right)
$$

For the case of no chemical reaction, Equation (3.4-11) simplifies to

$$
\Delta \mathrm{p}_{\mathrm{m} \mathrm{i}}=\frac{\mathrm{W}_{\text {oi }}}{\mathrm{A}_{\mathrm{i}}^{2}}\left(\frac{1}{\rho_{\text {bot }}}-\frac{1}{\rho_{\text {top }}}\right)
$$

The hydrostatic head follows from

$$
\mathrm{dp}_{\text {buoy }}=\mathrm{g} \rho \mathrm{dz}
$$


expressed in finite difference form and summed over the channel as follows:

$$
\Delta p_{\text {buoy } i}=g \sum_{j=1}^{j_{N O D}} \rho_{i, j} \Delta z_{j}
$$

\subsubsection{Reactor Coolant to Solid Heat Transfer}

In the parallel channel network of the active core and the return flow channel, convective heat transfer between coolant and solid structures can be modelled.

Within a node $j$ in channel $i$, the solid-surface temperature of the node $\theta_{s}$ is considered to be constant at each time step.

Then

$$
\mathrm{dQ}=-W c_{\mathrm{p}} \mathrm{d} \theta=\mathrm{h} \mathrm{A}^{\prime} \mathrm{dz}\left(\theta(\mathrm{z})-\theta_{\mathrm{s}}\right)
$$

where $A^{\prime}$ is the heat transfer surface area per unit length, and $\theta(z)$ is the fluid temperature varying in flow direction. For convenience, the node subscripts $i$ and $j$ will be omitted here. Integrated along the node, we obtain

$$
\theta(z)-\theta_{8}=\left(\theta_{\text {in }}-\theta_{3}\right) \mathrm{e}^{-\psi^{\prime} z}
$$

where

$$
\psi^{\prime}=\frac{\mathrm{hA}}{\mathrm{W} c_{\mathrm{p}}}
$$

or for the node exit

$$
\theta_{\text {ex }}-\theta_{s}=\left(\theta_{\text {in }}-\theta_{s}\right) e^{-\psi}
$$

with

$$
\Psi=\frac{h A}{W c_{p}}=\frac{h A^{\prime} \Delta z}{W c_{p}}
$$

The integrated average fluid temperature for the node is

$$
\theta_{\mathrm{av}}=\theta_{\mathrm{s}}+\left(\theta_{\mathrm{in}}-\theta_{\mathrm{s}}\right) \frac{1-\mathrm{e}^{-\Psi}}{\Psi}
$$


and the heat exchanged is

$$
Q=h A\left(\theta_{a v}-\theta_{s}\right)=W c_{p}\left(\theta_{\text {in }}-\theta_{\text {ex }}\right)
$$

The changes in mass flow and gas composition, due to chemical reactions, are considered in evaluating the channel heat transfer, including the variations in $c_{p}$ and $R e$.

The heat transfer coefficients are those for convective heat transfer in turbulent or laminar flow in circular tubes [Kaminsky, 1990].

For the laminar flow regime $(\operatorname{Re} \leq 2300)$

$$
\begin{aligned}
& \mathrm{Nu}=4 \\
& \mathrm{St}=\frac{\mathrm{Nu}}{\operatorname{RePr}}
\end{aligned}
$$

For the turbulent flow regime $(\operatorname{Re} \geq 8000)$

$$
\text { St }=0.022 \operatorname{Re}^{-0.2} \operatorname{Pr}^{-0.6}
$$

and

$$
\mathbf{h}=\mathbf{G} \mathrm{cp} \mathrm{St}
$$

where $G$ is the mass flux.

For the transition regime, the Stanton number is computed by linear interpolation between St $(2300)$ and St (8000).

\subsubsection{Chemical Reactions During Gas Ingress Scenarios}

At high temperatures, graphite will oxidize in the presence of oxygen or water, but for the high purity and high density graphite grades used in the gas-cooled reactor industry in the United States, the chemical reactivity is relatively small.

For the case of air ingress, the following chemical reactions have to be considered:

$$
\begin{aligned}
2 \mathrm{C}+\mathrm{O}_{2} & \rightarrow 2 \mathrm{CO} \\
\mathrm{C}+\mathrm{O}_{2} & \rightarrow \mathrm{CO}_{2}
\end{aligned}
$$

and

$$
\mathrm{C}+\mathrm{CO}_{2} \rightarrow 2 \mathrm{CO}
$$


Equations 3.4-25 and 3.4-26 are often combined as follows:

$$
2 \mathrm{C}+\mathrm{n}_{1} \mathrm{O}_{2} \rightarrow \mathrm{n}_{2} \mathrm{CO}+\mathrm{n}_{3} \mathrm{CO}_{2}
$$

In the temperature range of gas-cooled reactor cores, the equilibrium is strongly shifted towards $\mathrm{CO}$, so that the reaction of Equation 3.4-25 dominates [Moormann et al, 1980]. While some $\mathrm{CO}_{2}$ could be formed initially in the cooler, bottom sections of the core, such $\mathrm{CO}_{2}$ would react in the hotter regions of the core with graphite to $\mathrm{CO}$ following the Boudouard reaction (Equation 3.4-27).

The energy release per $\mathrm{kmol}$ of oxygen from graphite oxidation to $\mathrm{CO}$ (Equation 3.4-25) is 242 $\mathrm{MJ} / \mathrm{kmol} \mathrm{O}$, while oxidation to $\mathrm{CO}_{2}$ (Equ. 3.4-26) results in a release of $393 \mathrm{MJ} / \mathrm{kmol} \mathrm{O}$. However, this energy release generally amounts to less than $10 \%$ of the decay heat, and because oxidation is essentially complete in cooler core regions, its contribution to raise peak fuel temperatures is negligible. The main concern is the graphite corrosion which leads to some release of fission product due to platedout and embedded fission products, and also could reduce the structural strength of the graphite. As the reaction to $\mathrm{CO}$ (Equation 3.4-25) dominates and is also conservative with respect to the amount of graphite consumed per kmol of oxygen, while the slightly higher energy release of reaction to $\mathrm{CO}_{2}$ (Equation 3.4-26) is not of much concern, the current model considers, as a conservative approach, that all oxidation is to $\mathrm{CO}$, i.e., using equation $3.4-25$.

The graphite oxidation is a function of the temperature regime. At low temperatures, the carbon/oxygen reaction kinetics control the reaction rate. At intermediate temperatures (about $700^{\circ} \mathrm{C}$ to $900^{\circ} \mathrm{C}$ ), the in-pore diffusion is controlling, while at higher temperatures, the coolant to surface mass transfer by diffusion, here in a laminar flow field, is controlling [Rossberg and Wicke, 1956; Moormann et al 1980].

To consider all three regimes, a model including external mass transfer, in-pore diffusion, and chemical reaction kinetics is required, as for instance, in the Oxide-3 code [Peroomian et al., 1974]. For the current applications, we decided to use a simpler approach, combining the in-pore diffusion and chemical reaction process into a single Langmuir-Hinshelwood type semi-empirical equation, as done, for instance, by Moormann and Peterson [1982]. This equation is the following:

$$
\Gamma_{\mathrm{O}_{2}}^{\prime \prime}=-\mathrm{C} \frac{\mathrm{k}_{1} \mathrm{e}^{-\mathrm{c}_{1} / \theta} \mathrm{P}_{\mathrm{O}_{2} \mathrm{w}}}{1+\mathrm{k}_{2} \mathrm{e}^{\mathrm{c}^{\prime}{ }^{\prime \theta}} \sqrt{\mathrm{PO}_{\mathrm{O}_{2} \mathrm{w}}}} \cdot \sqrt{\frac{\mathrm{D}}{\mathrm{D}_{\mathrm{o}}}}\left[\frac{\mathrm{kmol} \mathrm{O_{2 }}}{\mathrm{m}^{2} \mathrm{~s}}\right]
$$

where $\Gamma_{\mathrm{O}_{2}}^{\prime \prime}$ is the oxygen reaction rate per unit surface area, $\mathrm{p}_{\mathrm{O}_{2}}$ is the partial pressure of oxygen, and $D$ is the binary diffusion coefficient of oxygen in the gas mixture. $\theta$ is the gas temperature and $k_{1}, k_{2}$, $c_{1}, c_{2}$ are empirical constants. $D_{0}$ is the binary diffusion coefficient of oxygen in helium at a reference temperature of $800^{\circ} \mathrm{C}$ and a pressure of $1.5 \mathrm{bar}$. $\mathrm{C}$ is a conversion constant to convert from the units of $k_{1}$ (generally given in $\mathrm{mg}$. carbon $/ \mathrm{cm}^{2} \mathrm{~s}$ ) to $\mathrm{kmol} \mathrm{O}_{2} / \mathrm{m}^{2} \mathrm{~s}$. The minus sign indicates that oxygen is being consumed in the reaction. 
The corresponding generation and depletion rates for $\mathrm{CO}$ and $\mathrm{C}$, respectively, follow stoichiometrically from Equation 3.4-25:

$$
\Gamma_{\mathrm{C}}^{\prime \prime}=2 \Gamma_{\mathrm{O}_{2}}^{\prime \prime}
$$

and

$$
\Gamma_{\mathrm{CO}}^{\prime \prime}=-\Gamma_{\mathrm{C}}^{\prime \prime}
$$

Equation 3.4-29 is solved simultaneously with the coolant to surface mass transfer relationship

$$
\Gamma_{\mathrm{O}_{2}^{\prime \prime}}=-\frac{\beta}{\hat{\mathrm{R}} \theta}\left(\mathrm{P}_{\mathrm{O}_{2} \text { str }}-\mathrm{P}_{\mathrm{O}_{2} \mathrm{w}}\right)\left[\frac{\mathrm{kmol} \mathrm{O}_{2}}{\mathrm{~m}^{2} \mathrm{~s}}\right]
$$

where the mass transfer coefficient $\beta$ is computed from the Sherwood number correlation

$$
\mathrm{Sh}=\frac{\beta \mathrm{d}}{\mathrm{D}}=\mathrm{f}\left(\operatorname{Re}, \frac{\nu}{\mathrm{D}}\right)
$$

and for purely laminar flow

$$
\mathrm{Sh}=\frac{\beta \mathrm{d}}{\mathrm{D}}=4.3
$$

$\hat{R}$ is the universal gas constant; $\mathrm{D}$ the coolant hole diameter; $\operatorname{Re}$ the Reynolds number; and $\nu$ the kinematic gas viscosity. The subscripts $w$ and str refer to wall and bulk stream values, respectively.

There is corsiderable uncertainty in the available in-pore diffusion data, and during most air ingress scenarios, the mass transfer in the coolant stream is controlling. Essentially, all incoming oxygen will react. Therefore, the total amount of carbon that reacts is determined by the gas inflow rate, which, in turn, is limited by the high core friction pressure drop. Thus, this model is completely sufficient for evaluating air ingress scenarios. Its shortcoming is that for the in-pore diffusion regime it cannot predict the lateral distribution of the oxidation process, i.e., the depth of the reaction zone in radial direction. In the chemical reaction controlled regime, the burn-off is essentially uniform throughout the graphite, while for the coolant to surface mass transfer controlled regime, oxidation occurs predominantly at the surface.

The binary diffusion coefficients for various non-polar gas mixtures were computed based on the Chapman-Enskog kinetic theory [Bird et al., 1960]. For air ingress scenarios, assuming conservatively pure air to enter the reactor vessel, the diffusion coefficient for oxygen in nitrogen was approximated as follows: 


$$
\mathrm{D}_{\mathrm{O}_{2}-\mathrm{N}_{2}}=1.7 \times 10^{-4}\left(\frac{\theta}{\theta_{o}}\right)^{1.5} \quad\left[\frac{m^{2}}{s}\right]
$$

where the reference temperature is $1000 \mathrm{~K}$.

For the diffusion coefficient of oxygen in helium we used the following approximation:

$$
\mathrm{D}_{\mathrm{O}_{2}-\mathrm{He}}=6.5 \times 10^{-4}\left(\frac{\theta}{\theta_{0}}\right)^{1.5} \quad\left[\frac{m^{2}}{\mathrm{~s}}\right]
$$

For the current model, the reaction rate constants of Katcher and Moormann [1986] for A3-3 graphite, a typical core graphite, were used, because these reaction rate constants were readily available in the required format. We shall substitute corresponding data representative of H451 core graphite as they become available. The reaction rate constants currently used are given in Table 3-1.

Table 3-1

Reaction Rate Constants of THATCH/FLOXI

Model for Air and Water Ingress

(Equation 3.4-29)

\begin{tabular}{||c|c|c|c|c|c||}
\hline Ingress & $\mathrm{k}_{1}{ }^{*}$ & $\mathrm{c}_{1}$ & $\mathrm{k}_{2}$ & $\mathrm{c}_{2}$ & \multicolumn{1}{|c|}{ Reference } \\
\hline Air & 2.34 & 12,850 & .00399 & 1569 & Katcher, 1986 \\
\hline Water & 0.072 & 17,000 & $1.202 \times 10^{-5}$ & 7000 & Moormann, 1985 \\
\hline
\end{tabular}

* The units of $k_{1}$ are [mg carbon $/ \mathrm{cm}^{2} \mathrm{~s}$ ]

Because there are considerable uncertainties in the reaction rate constants, as well as in the diffusion coefficients used, these have been varied parametrically. The reaction rates were raised up by one order and down by two orders. The result for several very conservative air ingress transients during core-heatup transients showed that the effect of all these variations on the amount of graphite oxidized was negligible, because essentially all oxygen entering the core will react and the amount of carbon oxidized is limited by the gas flow through the core, which remains small due to its long and narrow coolant passages [Kroeger, 1989].

Using the above rate equations, the quasi-steady mass conservation equations are solved for each gas species involved in the chemical reaction in each parallel flow channel. For species $\ell$, we have the following:

$$
\frac{\partial}{\partial z} c_{\ell} v=\Gamma_{\ell}^{\prime \prime \prime}
$$

where $c_{\ell}$ is the molar concentration of species $\ell ; v$ is the local flow velocity; and $\Gamma_{\ell}^{\prime \prime \prime}$ is the volumetric generation rate of species $\ell$. 
In finite difference form for axial node $j$, this can be expressed as follows:

$$
c_{j, \ell} v_{j}=c_{j-1, \ell} v_{j-1}+\Gamma_{j, \ell}^{\prime \prime \prime} \Delta z
$$

Multiplying by the flow area and noting that the molar flow rate of species $\ell$ in $\mathrm{kmol} / \mathrm{s}$ is

$$
C_{\ell}=c_{\ell} v A_{\text {flow }}=\frac{W}{M_{m}} \nu_{\ell}
$$

we obtain

$$
C_{j, \ell}=C_{j-1, \ell}+A_{\text {surf }}^{\prime} \Delta z \Gamma_{j, \ell}^{\prime \prime}
$$

where $C_{j, \ell}$ is the local molar flow rate of species $\ell$ leaving node $j$, and $\Gamma_{j, \ell}^{\prime \prime}$ is the reaction rate as given in equations 3.4-29 to $31 . \mathrm{M}_{m}$ is the mixture mole weight and $\nu_{\ell}$ the volume fraction of species $\ell$. It has to be noted that the integration of these equations must be done in flow direction. Therefore, here the node index $\mathrm{j}$ counts in flow direction and does not necessarily correspond to the node index $\mathrm{j}$ fixed by THATCH. reaction":

For the case of water ingress, the chemical reaction to be considered is the so-called "water gas

$$
\mathrm{C}+\mathrm{H}_{2} \mathrm{O}=\mathrm{CO}+\mathrm{H}_{2}
$$

This reaction is endothermal with the absorption of $118.8 \mathrm{MJ} / \mathrm{kmol} \mathrm{H}_{2} \mathrm{O}$. The model for water ingress follows the same equations as the air ingress model, except that the constants of reaction rates differ.

$$
\Gamma_{\mathrm{H}_{2} \mathrm{O}}^{\prime \prime}=-\mathrm{C} \frac{\mathrm{k}_{1} \mathrm{e}^{-\mathrm{c}_{1} / \theta} \mathrm{p}_{\mathrm{H}_{2} \mathrm{Ow}}}{1+\mathrm{k}_{2} \mathrm{e}^{\mathrm{c}{ }^{\prime \theta}} \sqrt{\mathrm{p}_{\mathrm{H}_{2} \mathrm{Ow}}}}\left[\frac{\mathrm{kmol} \mathrm{\textrm {H } _ { 2 } \mathrm { O }}}{\mathrm{m}^{2} \mathrm{~s}}\right]
$$

where $\Gamma^{\prime \prime} \mathrm{H}_{2} \mathrm{O}$ is the water reaction per unit surface area; $\mathrm{p}_{\mathrm{H}_{2} \mathrm{O}}$ is the water partial pressure; and $\mathrm{C}$ is a conversion constant from the units of $k_{1}$ (generally $\mathrm{mg}$ carbon $/ \mathrm{cm}^{2} \mathrm{~s}$ ) to $\mathrm{kmol} \mathrm{H}_{2} \mathrm{o} / \mathrm{m}^{2} \mathrm{~s}$. The reaction constants for water ingress are given in Table 3-1. 
From equation 3.4-41 it follows that

$$
\begin{gathered}
\Gamma_{\mathrm{C}}^{\prime \prime}=\Gamma_{\mathrm{H}_{2} \mathrm{O}}^{\prime \prime} \\
\Gamma_{\mathrm{CO}}^{\prime \prime}=-\Gamma_{\mathrm{H}_{2} \mathrm{O}}^{\prime \prime}
\end{gathered}
$$

and

$$
\Gamma_{\mathrm{H}_{2}}^{\prime \prime}=-\Gamma_{\mathrm{H}_{2} \mathrm{O}}^{\prime \prime}
$$

The diffusion coefficient of $\mathrm{H}_{2} \mathrm{O}$ in $\mathrm{He}$ [Bird et al., 1960, pg. 505] is approximated by:

$$
\mathrm{D}_{\mathrm{H}_{2} \mathrm{O}-\mathrm{He}}=3.396 \times 10^{-10}[\theta(\mathrm{K})]^{2.334}
$$

\subsubsection{Method of Solution for FLOXI Models} follows:

The FLOXI system of equations consists of three distinct but coupled sets of equations, as

1. the flow model,

2. the heat transfer model, and

3. the chemical reaction model.

These are solved separately and, if so specified as user option, an external iteration scheme assures that all coupling has been accounted for in the final solution.

In the external iteration scheme, the heat transfer and chemical reaction computations must progress in flow direction and, therefore, are done separately for up and down flow channels. The computations include plena models, which assume complete mixing of the incoming flows and uniform temperature and composition to the outflowing channels. A schematic of this loop is shown in Figure 4-6, below.

\section{Solution of Parallel Channel Flow Equations}

Equation (3.4.-1) applied for each channel and Equation (3.4-2) form a system of $\dot{i}_{\mathrm{CHP}}+1$ simultaneous, non-linear algebraic equations for the inflow into each channel $W_{\text {oi, }}$ and the overall pressure difference between the plena $\Delta \mathrm{p}_{\mathrm{o}}$.

The first $\mathrm{i}_{\mathrm{CHP}}$ equations are obtained from Equation 3.4-1, and of the independent variables only the flow rate of its channel and the overall pressure difference enter. In the last equation (Equation 3.42), all mass flows enter, but the overall pressure difference does not. With this structure of the equations, a very efficient Newton-Raphson iteration scheme can readily be implemented, using the sparse structure of the Jacobeam maxtrix to solve for the residual vector. The Newton-Raphson procedure is 
included in the FLOXI flow-computation routine, FLXFLOW, using sub-routine SOLSPEC for the inversion of the Jacobean matrix.

\section{Solution of Coolant to Solid Heat Transfer Equations}

Based on a known flow distribution, gas composition and solid temperature distribution, the fluid temperatures and heat flows can be readily computed by indexing through each of the parallel flow channels in flow direction. With heat transfer coefficients from Equation 3.4-23 and 24, and $\psi$ coefficient from Equation 3.4-20, we obtain the fluid average temperature and fluid exit temperature of each node from Equations 3.4-21 and 3.4-19, respectively. The fluid-to-solid heat exchange follows from Equation 3.4-22.

\section{Solution of Chemical Reaction and Mass Transfer Equations}

Equating Equations (3.4-29) and (3.4-32), we obtain at each axial node of each parallel flow channel in the core, a non-linear equation for the stream and surface values of the reactant partial pressure $\left(\mathrm{O}_{2}\right.$ for air ingress and $\mathrm{H}_{2} \mathrm{O}$ for water ingress).

Applying the mass balance of the reactant Equation (3.4-38) for the node exit, using $\Gamma$ from Equation (3.4-32) and expressing the left-hand side as follows:

$$
C_{j, \ell}=\nu_{j, \ell} \sum_{\ell=1}^{L} C_{j, \ell}
$$

noting

$$
\mathrm{p}_{\mathrm{j}, \ell}=\nu_{\mathrm{j}, \ell} p_{o}
$$

we obtain a second simultaneous equation for the reactant partial pressures. $\nu_{j, \ell}$ is the volume fraction of species $\ell$ at interface $j$.

These two equation are solved in each parallel flow channel and at each axial node, again indexing in flow direction. A Newton-Raphson procedure is used with inversion of the linear equations by Cramer's Rule. Once the reactant partial pressures are known, the source terms for the various species that change can be computed and molar flows leaving a node follow from equation (3.4-40), completing the mass balance equations.

\subsection{PKIN Module for Point Kinetics Option}

\subsubsection{Point Kinetics Model}

The point kinetics model of THATCH is based on a previous model by Cheng [1976] and also follows Knief [1981]. This model is based on one equation for the prompt neutron power plus -six delayed neutron groups: 


$$
\begin{gathered}
\frac{d \hat{P}}{d t}=\frac{\rho-\beta}{\ell^{*}} \hat{P}+\sum \lambda \hat{C} \\
\frac{d C_{i}}{d t}=\frac{\beta_{i}}{\ell^{*}} \hat{P}-\lambda_{i} C_{i} \quad i=1,6
\end{gathered}
$$

where $\hat{P}=P / P_{0}=N / N_{o}$, with $P$ the reactor power; $P_{0}$ the steady-state full power level; $N$ the neutron flux; $N_{o}$ is the neutron flux at steady-state full power; and $\hat{C}_{i}=C_{i} / N_{o}$.

$$
\begin{array}{lll}
\mathrm{P} & = & \text { reactor power } \\
\mathrm{C}_{\mathrm{i}} & = & \text { delayed precursor concentrations of group } \mathrm{i} \\
\lambda_{\mathrm{i}} & = & \text { decay constant of group } \mathrm{i}\left(\mathrm{s}^{-1}\right) \\
\beta_{\mathrm{i}} & = & \text { delayed neutron fraction of group } \mathrm{i} \\
\beta & = & \text { total delayed neutron fraction } \\
\rho & = & \text { total reactivity } \\
\ell^{*} & = & \text { prompt neutron generation time }(\mathrm{s})
\end{array}
$$

At initial steady-state, $\hat{\mathrm{P}}=1$ and $\rho=0$, with

$$
\hat{C}_{i}=\frac{\beta_{i}}{\ell^{*} \lambda_{i}}
$$

The Xenon-135 concentration is obtained from the two following equations for iodine and xenon [Knief, 1981]:

$$
\begin{gathered}
\frac{d I}{d t}=\gamma^{\text {Te }} \sum_{f} \Phi-\lambda^{I} I \\
\frac{d X}{d t}=\gamma^{X} \sum_{f} \Phi+\lambda^{I} I-X \sigma_{a}^{X} \Phi-\lambda^{X} X
\end{gathered}
$$

where

I I-135 concentration $\left[\mathrm{m}^{-3}\right]$

$\mathrm{X} \quad \mathrm{Xe}-135$ concentration $\left[\mathrm{m}^{-3}\right]$

$\gamma^{\mathrm{Te}} \quad$ fission yield of tellurium or iodine (due to short half-life of Te-135)

$\gamma^{\mathrm{X}} \quad$ fission yield of Xenon-135

$\sum_{\mathrm{f}} \phi$ the fission rate [fissions $/ \mathrm{m}^{3} \mathrm{~s}$ ]

$\lambda^{I}, \lambda^{X} \quad$ iodine and xenon decay constants $\left[\mathrm{s}^{-1}\right]$

$\sigma_{\mathrm{a}}^{\mathrm{X}}$ microscopic Xenon-135 absorption cross section $\left[\mathrm{m}^{2}\right]$

$\Phi \quad$ the neutron flux $\left[\mathrm{m}^{-2} \mathrm{~s}^{-1}\right]$ 
Scaling the above equations with the full-power, steady-state concentrations yields the following:

$$
\frac{\mathrm{d} \hat{l}}{\mathrm{dt}}=\dot{\lambda}^{\mathrm{J}}(\hat{\mathbf{P}}-\hat{\mathrm{I}})
$$

and

$$
\frac{d \hat{X}}{d t}=\frac{\lambda^{X}+G}{\gamma^{X}+\gamma^{T e}}\left(\gamma^{X} \hat{P}+\gamma^{T e} \hat{I}\right)-\left(G \hat{P}+\lambda^{X}\right) \hat{X}
$$

where

$$
\begin{aligned}
& \hat{X}=X / X o, \hat{I}=I / I o \\
& \hat{P}=P / P o=\sum_{f} \Phi /\left(\sum_{f} \Phi\right)_{o}
\end{aligned}
$$

and

$$
G=\left(\frac{\sigma_{\mathrm{a}}^{\mathrm{X}}}{\Sigma_{\mathrm{f}}}\right)\left(\Sigma_{\mathrm{f}} \Phi\right)_{\mathrm{o}}=\left(\frac{\sigma_{\mathrm{a}}^{\mathrm{X}}}{\Sigma_{\mathrm{f}}}\right)\left(\frac{\mathrm{P}_{\mathrm{o}}}{\mathrm{E}}\right)
$$

where $X_{o}$ and $I_{o}$ are the steady-state full power xenon and iodine concentrations; $\Sigma_{f}$ is the macroscopic fission cross-section; $P_{0}$ the steady-state, full-power power density; and $E$ is the energy per fission.

For some of the very slow core heatup transients, Samarium-149 poisoning must be considered in the model. The corresponding ODEs for Promethium and Samarium [Knief, 1981] are:

$$
\begin{aligned}
\frac{\mathrm{dPm}}{\mathrm{dt}} & =\gamma^{\mathrm{Nd}} \Sigma_{\mathrm{f}} \Phi-\lambda^{\mathrm{Pm}} \mathrm{Pm} \\
\frac{\mathrm{dS}}{\mathrm{dt}} & =\lambda^{\mathrm{Pm}} \mathrm{Pm}-\mathrm{S} \sigma_{\mathrm{a}}^{\mathrm{S}} \Phi
\end{aligned}
$$

where

Pm Concentration of Promethium $-149\left[\mathrm{~m}^{-3}\right]$

$S$ Concentration of Samarium- $149\left[\mathrm{~m}^{-3}\right]$

$\gamma^{\mathrm{Nd}} \quad$ fission yield of neodymium or promethium (due to short half-life of $\mathrm{Nd}-149$ )

$\lambda^{\mathrm{Pm}} \quad$ decay constant of promethium $\left[\mathrm{s}^{-1}\right]$

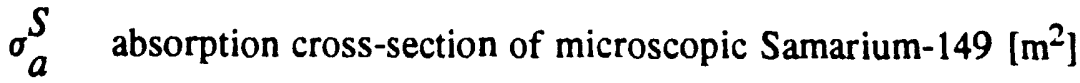


Scaling these equations with the full power, steady-state concentration yields the following:

$$
\begin{aligned}
& \frac{d \hat{P}_{m}}{d t}=\lambda^{P m}(\hat{P}-\hat{P} m) \\
& \frac{d \hat{S}}{d t}=G_{1}(\hat{P} m-\hat{P} \hat{S})
\end{aligned}
$$

with

$$
G_{s}=\left(\frac{\sigma_{a}^{s}}{\Sigma_{f}}\right)\left[\frac{P_{o}}{E}\right)
$$

The reactivity feedbacks included in the THATCH point kinetics model are the following:

1. Change in fuel temperature

2. Change in moderator temperature

3. Change in reflector temperature

4. Change in xenon concentration

5. Change in samarium concentration

6. Change in water concentration

These can be expressed as follows:

$$
\begin{aligned}
d \rho & =C_{D} d \theta_{\text {fuel }}+C_{m} d \theta_{\text {moderator }} \\
& +C_{R} d \theta_{\text {reflector }} \\
& +C_{x} d \hat{X}+C_{S} d \hat{S}+C_{W} d W
\end{aligned}
$$

where $\mathrm{W}$ refers to the water concentration.

The xenon feedback coefficient is

$$
C_{x}=-\frac{\sigma_{a}^{X}}{\Sigma_{t}} x_{0}
$$

where $\mathrm{X}_{\mathrm{o}}$ is the steady-state, full power xenon concentration

$$
X_{o}=\left(\frac{\gamma^{X}+\gamma^{T e}}{\lambda^{X}+G}\right)\left(\frac{P_{o}}{E}\right)
$$


and $\sum_{t}$ is the total macroscopic cross section.

Correspondingly, the Samarium feedback coefficient is

$$
C_{3}=-\frac{\sigma_{a}^{S}}{\Sigma_{1}} S_{0}
$$

with

$$
S_{0}=\frac{\gamma^{N d} \Sigma_{f}}{\sigma_{\mathrm{a}}^{\mathrm{s}}}
$$

The temperature coefficients, water worth data, macroscopic cross section data and delayed neutron group fractions and decay constants are based on PSID data. Other constants used are

$$
\begin{aligned}
& \gamma^{\mathrm{Te}}=0.056 ; \quad \gamma^{\mathrm{x}}=0.003 \quad ; \quad \gamma^{\mathrm{Nd}}=.01406 \\
& \mathrm{E}=200 \mathrm{Mev} / \text { fission }=3.2042 \times 10^{-11} \mathrm{~J} / \text { fission } \\
& \mathrm{P}_{\mathrm{o}}=5.96 \mathrm{MW} / \mathrm{m}^{3} \\
& \lambda^{I}=2.9 \times 10^{-5} 1 / \mathrm{s} ; \gamma^{\mathrm{X}}=2.1 \times 10^{-5} 1 / \mathrm{s} ; \lambda^{\mathrm{Pm}}=3.63 \times 10^{-6} 1 / \mathrm{s} \\
& \sigma_{\mathrm{a}}^{\mathrm{X}}=2.65 \times 10^{6} \mathrm{~b} ; \sigma_{a}^{S}=25.03 \times 10^{3} \mathrm{~b}
\end{aligned}
$$

\subsubsection{Method of Solution for Point Kinetics Model}

The Point Kinetics Model consists of eleven ODEs, one for the reactor power, six for delayed neutron concentrations, two for the iodine and xenon concentrations, and two for the promethium and samarium concentrations. For convenience, the integrated scaled power is added as a twelfth equation, i.e., for integrated power

$$
\pi=\int \hat{\mathrm{P}} \mathrm{dt}
$$

we have as ODE

$$
\frac{d \pi}{d t}=\hat{P}
$$

This set of twelve ODEs is solved by a standard stiff ODE solver. The IMSL routine DIVPAG is used for this purpose and is available in the IMSL library, Version 10.0. The ODE solver DGEAR which is available in the earlier IMSL library, Version 9.2, can also be used. As the equations are generally stiff, we found that the Gear method (MTHGEAR $=2$ in PKIN input) with iteration method 
MTTER = 1 gave best results. Because this option requires a user-supplied Jacobian matrix, a subroutine computing the matrix (JACK) is provided in addition to the derivative routine (DERIV).

Much of the reactor physics data used were obtained from the PSID and are "hard-wired" in PKINL.B Block Data Routine BLDPINK. To change these data, we can optionally override these entries by using special input data records. The variables included in this option are summarized in Table 3.-2.

The point kinetics and solid node temperature computations are coupled. Fuel and graphite temperatures are functions of the heating rate or power. The power depends on the reactivity feedbacks, which, in turn, are a function of fuel and graphite temperatures. In particular, with strong reactivity incursions this coupling can be essential. The point kinetics and solid node temperature computations, therefore, can be executed iteratively, if so specified by the user (COMPMOD = 'Iterate' in Input Record 5001D). In this case, the core temperatures and the point kinetics vectors are iteratively advanced from time $t_{1}$ to time $t_{2}$. As the point kinetics integration proceeds at its time steps, controlled by its integration routine, we assumed that the core reactivity feedbacks vary lineariy between $\rho\left[\theta\left(t_{1}\right)\right]$ and $\rho\left[\theta\left(t_{2}\right)\right]$. Convergence is accepted when the core average temperatures used for core reactivity feedback evaluations vary less than some $\epsilon$ between subsequent iterations (general $10^{-3} \mathrm{~K}$ ).

The water reactivity feedback uses PSID data on water reactivity worth versus mass of water in the core. The code computes the maximum theoretical concentration of water vapor in the core at the set point pressure of the relief valve and at the average temperature of the core coolant, assuming an ideal gas equation of state for the vapor. Depending on the input specified, beginning of ingress time and ramp time, the code then computes the current concentration of water vapor as a fraction of the maximum theoretical vapor concentration, where this fraction is specified with the input data.

\subsection{SYMELAN Module for Rapid Thermal Core Transients, Using Symmetry Element Nodalization}

\subsubsection{Symelan Active Core Heat Transfer Model}

The SYMELAN module can evaluate rapid core thermal transients where detailed modelling of heat conduction in the fuel stick and in the moderator web is essential.

The active core is again divided into a number of parallel flow channels selected by the user. Each channel represents some number of fuel element columns. Because the SYMELAN free-standing model neglects horizontal conduction between parallel flow channels, any flow channel can represent just one fuel element column, a part of a fuel element column or a group of fuel element columns, as desired. The core is also divided into a number of axial zones selected by the user, generally with two zones per fuel element. similar to the general THATCH nodalization.

In each fuel element, a coolant hole is generally surrounded by six fuel holes, as shown in Figure 3-10. The heat conduction between the center line of the fuel pin and the coolant hole can be characterized by that in the symmetry element of Figure 3-11, where the sides of the triangle, due to symmetry, can be modelled as adiabatic surfaces. Each axial node region, j, of each parallel flow channel, i, contairis many symmetry elements (approximately 1200 per fuel element). The mudel assumes uniform heating rate and coolant inflow in node region $i, j$. Therefore, the temperature distribution in one symmetry element applies for all elements in node region $i, j$. 

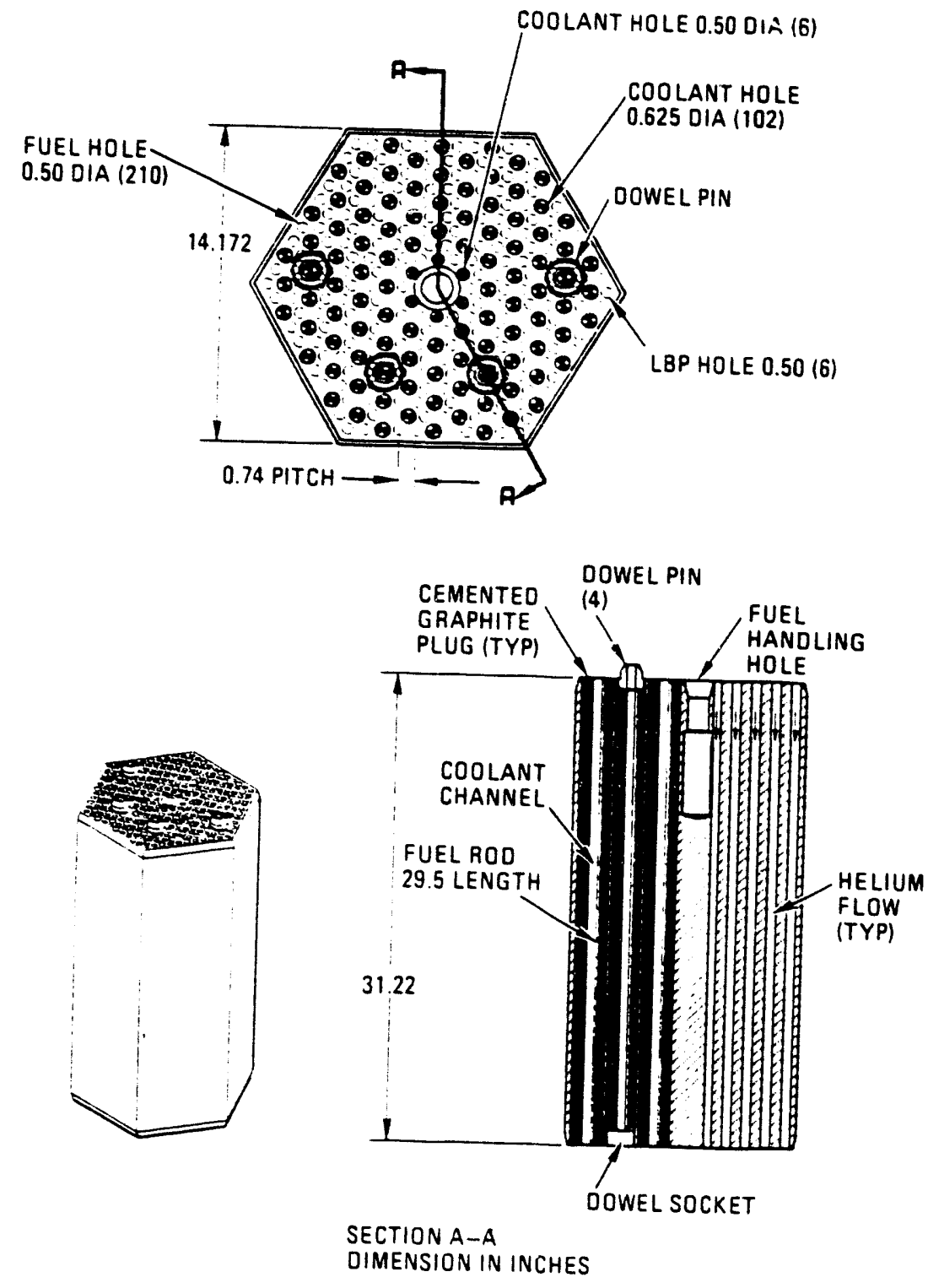

Figure 3-10 Standard Fuel Element of the MHTGR (Bechtel, 1986) 


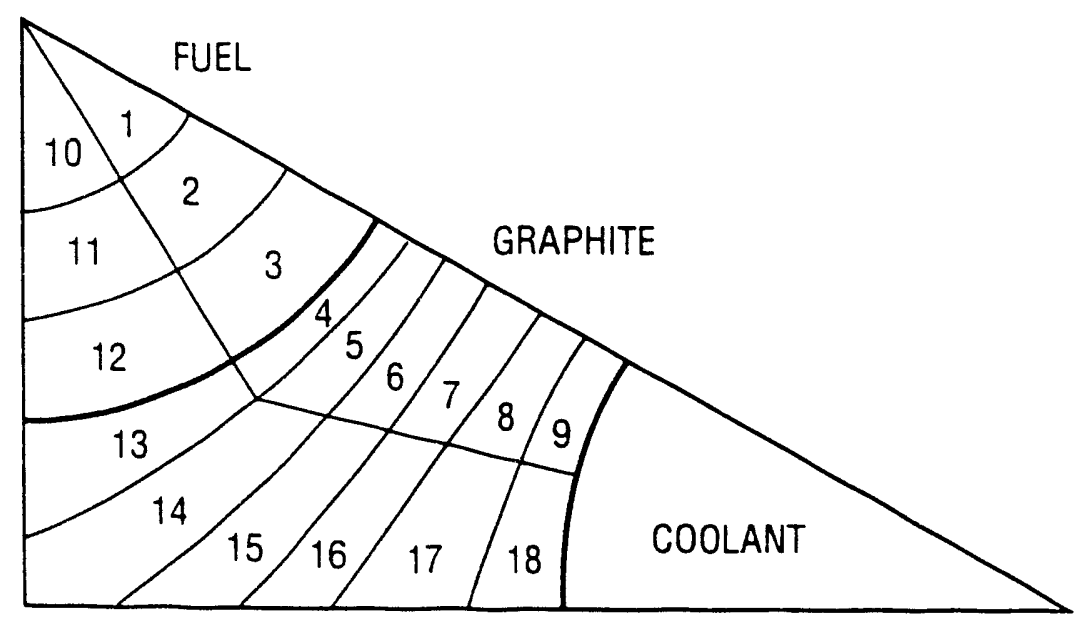

Figure 3-11 Symmetry Element Contained in MHTGR Fuel Element 
Table 3.-2 Reactor Physics Data Which Can Be Varied by Use of Input Records 5004 to 5010D of Section 5.1

\begin{tabular}{|c|c|c|c|c|c|}
\hline Variable & Symbol & Variable Name & Positions & Units & $\begin{array}{l}\text { Input } \\
\text { Record } \\
\text { No. } \\
\end{array}$ \\
\hline Delayed Neutron Fractions & $\beta_{\mathrm{i}}$ & BETA & 6 & & $5004 \mathrm{D}$ \\
\hline $\begin{array}{l}\text { Decay Constants for Delayed Neutron } \\
\text { Groups }\end{array}$ & $\lambda_{\mathrm{i}}$ & DECAY & 6 & $\mathbf{s}^{-1}$ & $5005 \mathrm{D}$ \\
\hline Prompt Neutron Generation Time & $\ell^{*}$ & GENT & & $s$ & $5006 \mathrm{D}$ \\
\hline Decay Constants for Iodine & $\lambda^{I}$ & XLAM (1) & 1 & $\mathrm{~s}^{-1}$ & $5007 \mathrm{D}$ \\
\hline Decay Constants for Xenon & $\lambda^{x}$ & XLAM (2) & 1 & $s^{-1}$ & 5007D \\
\hline Fission Yield of Tellurium & $\gamma^{\mathrm{Te}}$ & GAM (1) & 1 & & 5008D \\
\hline Fission Yield of Xenon & $\gamma^{\mathrm{X}}$ & GAM (2) & 1 & & $5008 \mathrm{D}$ \\
\hline Power Density & $P_{0}$ & POWD & 1 & $\mathrm{~W} / \mathrm{m}^{3}$ & 5009D \\
\hline $\begin{array}{l}\text { Xenon Microscopic Absorption Cross } \\
\text { Section }\end{array}$ & $\sigma_{\mathrm{a}}^{\mathrm{X}}$ & SIGA & 1 & $\mathrm{~m}^{2}$ & 5009D \\
\hline Macroscopic Fission Cross Section & $\Sigma_{\mathrm{f}}$ & SIGF & 1 & $m^{-1}$ & 5009D \\
\hline Total Macroscopic Cross Section & $\Sigma_{\mathrm{t}}$ & SIGT & 1 & $\mathrm{~m}^{-1}$ & $5009 \mathrm{D}$ \\
\hline Fission Yield of Neodymium & $\gamma^{N d}$ & GAMND & 1 & & $5010 \mathrm{D}$ \\
\hline Decay Constant of Promethium & $\lambda^{\mathrm{Pm}}$ & XLAMPM & 1 & $\mathbf{s}^{-1}$ & $5010 \mathrm{D}$ \\
\hline $\begin{array}{l}\text { Samarium Microscopic Absorption } \\
\text { Cross-Section }\end{array}$ & $\sigma_{\mathrm{a}}^{\mathrm{S}}$ & SIGASAM & 1 & $\mathrm{~m}^{2}$ & 5010D \\
\hline
\end{tabular}


The nodalization within the fuel stick of the symmetry element is regular, i.e., node boundaries are circles of constant radius and straight lines. In the graphite web, irregular boundaries were drawn, following estimated isotherms and flux lines. Three nodalizations were used, as summarized in Table 3-3.

Nodalization 1 is shown in Figure 3-10. The chosen graphite dimensions for these nodalizations are tabulated in Block Data SYMDAT in the SYMELAN library. The data file contains node volumes $\left[\mathrm{mm}^{3} / \mathrm{mm}\right]$, heat flow areas at the node boundaries $\left[\mathrm{mm}^{2} / \mathrm{mm}\right]$, and node center distances in "radial" direction [mm] (i.e., from fuel hole to coolant hole) and in transverse direction (between node rows) for the case of Nodalization 1 with two node rows. The data on radial node distance are the distances between node centers, except for the first and last entries which are from the fuel/graphite interface to the first node center, and from the last node center to the coolant hole surface. Some numerical experimentation with these nodalizations was performed, and we found that moderate variations in changes in dimensions between graphite nodes had no significant effect on the results. Further, ever, in the most rapid transients the conduction between rows remained very small relative to the radial conduction from fuel to coolant. Therefore, we prefer Nodalization 3 and used it in most studies. Also, we found twenty axial core nodes to be sufficient for all cases. For the number of parallel flow channels, either three or six channels were generally used. Six channels must be used in runs coupled to THATCH, to correspond with the general THATCH nodalization.

The transient heat conduction equation within the nodal network of the symmetry element can be written for any node as follows:

$$
\rho c V^{\prime} \frac{\partial \theta}{\partial t}=-\sum A^{\prime} q_{\text {out }}^{\prime \prime}+V^{\prime} q_{g}^{\prime \prime \prime}
$$

Table 3-3

Currently Available Options for

Symmetry Element Nodalization

\begin{tabular}{|c|c|c|c|}
\hline $\begin{array}{c}\text { Nodalization } \\
\text { No. }\end{array}$ & $\begin{array}{c}\text { Number of } \\
\text { Node Rows }\end{array}$ & $\begin{array}{c}\text { Number of } \\
\text { Fuel Node Columns }\end{array}$ & $\begin{array}{c}\text { Number of } \\
\text { Graphite Node } \\
\text { Columns }\end{array}$ \\
\hline \hline 1 & 2 & 3 & 6 \\
2 & 1 & 3 & 6 \\
3 & 1 & 6 & 12 \\
\hline
\end{tabular}

* Entries are values typically used; user is free to vary actual value by input data specification 
With row index $m$ and column index $n$, this can be written in finite difference form, for implicit solution as follows:

$$
\begin{array}{r}
{\left[\left(\rho c V^{\prime}\right)_{n, m}+\left[\sum_{N} \frac{A^{\prime}}{\Delta y} k\right] \Delta t\right] \theta_{n, m}-\sum_{N} \frac{A^{\prime}}{\Delta y} k \Delta \theta_{N}} \\
=\left(\rho c V^{\prime}\right)_{n, m} \theta_{n, m}^{\circ}+V_{n, m}^{\prime} q_{g}^{\prime \prime \prime} \Delta t
\end{array}
$$

where $V^{\prime}$ and $A^{\prime}$ are node volumes and heat transfer areas per unit length.

In this equation, the $\sum_{N}$ represents summing over all neighboring nodes, and $\theta^{\circ}$ denotes the temperature at the preceding time step.

This equation applies within each node of the symmetry element and for each axial node region $j$, and each parallel flow channel $i$, i.e., all $n, m$ arrays actually have four indices. For brevity the $i, j$ indices will only be used where necessary.

The internal heating rate in the fuel is considered constant across the fuel stick and within the node region $i, j$. However, it varies based on prescribed axial and radial power profiles between axial node regions $j$ and parallel flow channels $i$.

Expressing the conduction coefficients again as $C_{N}, C_{E}, C_{S}$, and $C_{W}$, we have for any node the following:

$$
\mathrm{C}_{\mathrm{Y}}=\frac{\mathrm{A}^{\prime}}{\delta \mathrm{y}} \mathrm{k} \Delta \mathrm{t}
$$

where $A^{\prime}$ is the heat flow area at the node boundary per unit length; $\delta y$ the node center to node center distance; and $\mathrm{k}$ is evaluated at the average temperature between nodes. Optionally, an axial conduction term between node regions of the axial can be included as an explicit term (however, we found this term to be small at all times). 
Then

$$
\begin{aligned}
& {\left[\left(\rho c V^{\prime}\right)_{n, m}+C_{N_{n, m}}+C_{E_{n, m}}+C_{S_{n, m}}+C_{W_{n, m}}\right] \theta_{n, m}} \\
& -C_{N_{n, m}} \theta_{n, m-1}+C_{E_{n, m}} \theta_{n+1, m}+C_{S_{n, m}} \theta_{n, m+1}+C_{W_{n, m}} \theta_{n-1, m} \\
& =\left(\rho c V^{\prime}\right)_{n, m} \theta_{n, m}^{\circ}+V_{n, m}^{\prime} q_{g}^{\prime \prime \prime} \Delta t \\
& \quad+\frac{1}{\Delta z}\left[\frac{A}{\Delta z_{a x}}\right] \Delta t\left[k_{-}\left(\theta_{n, m}^{\circ}-\theta_{n, m}^{\circ}\right)\right. \\
& \left.\quad-k_{+}\left(\theta_{n, m}^{\circ}-\theta_{n, m, j}+1\right)\right]
\end{aligned}
$$

where the additional $\Delta Z$ in the axial conduction term is for conversion of this term to unit length, corresponding to the other terms.

$\left[\frac{\mathrm{A}}{\Delta \mathrm{z}}\right]$ are the axial node to node heat transfer surfaces divided by node distance, $\mathbf{k}_{-}$and $\mathbf{k}_{+}$are the conductivities evaluated at average temperatures between nodes.

For single row nodalization, the north and south terms disappear and the system of equations is tridiagonal. Even for two-row nodalization, only one off-tridiagonal would remain, and it most likely could have been treated explicitly. However, the code currently treats it implicitly and does not use the tridiagonal system in case of two-row nodalizations.

Boundary conditions must be applied at the fuel centerline, at the fuel graphite interface, and at the coolant hole surface.

At the fuel centerline

$$
\frac{\partial \theta}{\partial \mathrm{r}}=0
$$

applies and the $C_{W}$ terms of Equation (3.6-4) disappear.

At the fuel graphite interface, radiation, and conduction across a gap of user-prescribed thickness is assumed. The corresponding $C_{\mathbf{Y}}$ coefficients at the fuel graphite interface are the following:

$$
C_{Y}=A^{\prime} U \Delta t
$$


where

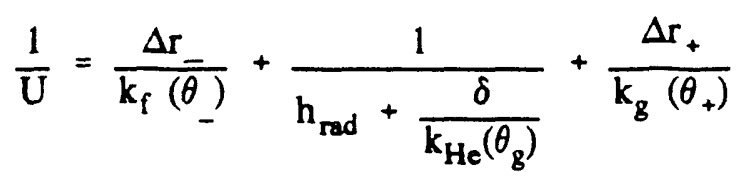

where $\Delta r_{-}$and $\Delta r_{+}$are the node center to interface distances; $k_{f}\left(\theta_{-}\right)$and $k_{g}\left(\theta_{+}\right)$are the fuel and graphite conductivities evaluated at the respective node temperatures; $\delta$ is the gap thickness; $\mathbf{k}_{\mathrm{He}}\left(\theta_{\mathrm{g}}\right)$ is the thermal conductivity of helium at the gap temperature; and $h_{\text {rad }}$ is the radiation heat transfer coefficient at a user-provided fuel to graphite effective emissivity (see Equation 3.1-15).

At the graphite and coolant interface, convection from solid to coolant is modelled as follows: within each axial node region, temperatures of the symmetry element remain constant in axial direction, and between the last node, its surface and the fluid average temperature, we have the following:

$$
\mathrm{Q}=\mathrm{A}^{\prime} \frac{\mathrm{k}}{\Delta \mathrm{r}}\left(\theta_{\mathrm{n}}-\theta_{\mathrm{s}}\right)=\mathrm{A}^{\prime} \mathrm{h}\left(\theta_{\mathrm{s}}-\theta_{\mathrm{flav}}\right)
$$

or

$$
Q=A^{\prime} U\left(\theta_{n}-\theta_{f l a v}\right)
$$

where

$$
\frac{1}{\mathrm{U}}=\frac{\Delta r}{\mathrm{k}}+\frac{1}{\mathrm{~h}}
$$

$\mathrm{h}$ is the convective heat transfer coefficient, and $\mathrm{Q}$ is the total heat flow out of the last symmetric element node to the coolant. Note that in the SYMELAN formulation all heat transfer rates, surfaces, and volumes are expressed in terms of unit length, i.e., the dimension of the above equation is $[\mathrm{W} / \mathrm{m}]$, and the surface area $A^{\prime}$ is in $\left[\mathrm{m}^{2} / \mathrm{m}\right]$.

For a node of length $\Delta z$, representing one parallel flow channel, we have as in Section 3.4-2

$$
\left(\theta_{n}-\theta_{n \text { av }}\right)=\left(\theta_{n}-\theta_{f 1 \text { in }}\right) \frac{1-e^{-\Psi}}{\Psi}
$$

where

$$
\Psi=\frac{A_{N} U}{W c_{p}}
$$

and 


$$
A_{N}=A^{\prime} \Delta z n m
$$

where $\Delta z$ is axial node length; $n$ is the number of symmetry elements per flow channel; and $m$ is the number of node rows per symmetry element.

Substituting Equations (3.6-10) to (3.6-12) into Equation (3.6-8), we obtain:

$$
\mathrm{Q}=\frac{\left(1-\mathrm{e}^{-} \Psi\right) W c_{p}}{\Delta z \mathrm{~m}}\left(\theta_{\mathrm{n}}-\theta_{\mathrm{n} \text { in }}\right)
$$

and, correspondingly, for the last node conductance coefficient

$$
C_{E}=\frac{\left(1-e^{-\Psi}\right) W c_{P}}{\Delta z n m} \Delta t
$$

For each axial node we also have, as in Section 3.4-2, for the fluid exit temperature from each axial nude region

$$
\theta_{\text {flex }}-\theta_{n}=\left(\theta_{\text {n in }}-\theta_{n}\right) e^{-\Psi}
$$

\subsubsection{Method of Solution}

From the equations given above, for any flow channel and any axial node region with given fluid inlet temperature, the temperature field of the symmetry element can be solved, using the set of Equations 3.6-4 with conductance coefficients from Equations (3.6-3,5,6, and 14). Thereafter, Equation (3.6-15) is solved for the coolant inlet temperature to the next axial node.

Since axial stepping in flow direction is required, the symmetry element equations of all parallel flow channels $i$ for one axial node region $j$ are solved together. Then the fluid exit temperatures of the axial node region are evaluated for each flow channel, and the next axial node region is evaluated for all channels. For the generally used nodalizations of one single node row, a tridiagonal algorithm can be applied and one set of $i_{C H} \times n_{N}$ equations is solved, where $i_{C H}$ is the number of flow channels, and $n_{N}$ the number of symmetry element nodes. For nodalizations with two node rows, $i_{C H}$ sets of $n_{N}$ simultaneous equations are solved. (If such nodalizations were to be used extensively, one should consider to treat the $C_{N}$ and $C_{S}$ terms of Equation 3.6-4 explicitly and use the tridiagonal solution algorithm). 


\section{PROGRAM STRUCTURE}

The THATCH code and its sub-modules are subdivided into two components containing specific sets of subroutines. The first component, called THATCH, contains the general THATCH routines. The second is currently assembled in a component called BIG. Because both components are maintained via Update or Historian, there are no COMMON decks shared by the two components.

BIG includes many general service routines and also the sub-module libraries. In many cases, it is convenient to subdivide BIG into several individual libraries, particularly if we only work with one or two sub-modules. Table E-1 in Appendix E lists all THATCH subroutines and entries, including their location in THATCH or BIG and to which library the routine belongs. The libraries and their general purpose are summarized in Table 4-1.

The THATCH and BIG components include several "subroutines" which actually present main programs (Table 4-2). These can be executed as free-standing programs that are called by exterior 3-line dummy programs. For example, the "subroutine" THATCH in the THATCH component must be called by a user-supplied 3-line program as follows:

\section{PROGRAM THATCHM \\ CALL THATCH \\ END}

The major functions of the THATCH and BIG subroutines are described in this section, in order by libraries.

\section{$4.1 \quad$ THATCH Subroutines}

In the heading of most THATCH subroutines, a significant amount of information about the routine is provided in the form of comments. These comments were mainly written during the original development of the code, and have not been updated recently. In many cases, they will be very useful to new users.

\subsubsection{THATCH Main Program}

Subroutine THATCH. This subroutine is actually the main program. After reading the control variables for sub-modules (records 201D and 202D), the program sets the control variable CALMODE, which is 'THATCH' for THATCH runs without SYMELAN, and 'COMBIN' for combined THATCH/SYMELAN runs. The program calls the various THATCH input routines, as well as the submodule input routines, and then calls TIMSOL, the actual time step integration routine.

\subsubsection{THATCH Input Subroutines}

The names of the THATCH input routines generally begin with IN _ _. Entries to these subroutines are named PRE _ _ for initialization before input data processing; $\mathrm{INP}_{\text {. . . }}$ for the main input section; and PST . . . for data processing after completion of input data processing. 
Table 4-1

Function of Sub-Libraries of BIG

\begin{tabular}{||l|l||}
\hline Sub-Library & Function \\
\hline FLOXLIB & FLOXI Subroutines \\
PKINLIB & PKIN Subroutines \\
SYMELLIB & SYMELAN Subroutines \\
PASCOLIB & PASCOL Subroutines \\
WATCOLIB & WATCOL Subroutines \\
GENLIB & $\begin{array}{l}\text { General Purpose Subroutines (Interpolation, } \\
\text { Friction and Heat Transfer Correlations, etc.) }\end{array}$ \\
GASPRLIB & Gas Property Routines \\
MATLIB & Solid Material Properties \\
THTCHLIB & THATCH-specific general routines, including NEWTON \\
& AE solver and MHTGR Data Bank Routines \\
THPROCS & THATCH Post Processing Routines (pre-plotting) \\
THRADLIB & Radiation Viewfactor Routines \\
\hline
\end{tabular}

Table 4-2

THATCH and BIG Effective

Main Programs

\begin{tabular}{||l|l||}
\hline \hline Subroutine & Function \\
\hline THATCH & Execute THATCH \\
THRAD & Execute Radiation View Factor Computations \\
THPROCS & Execute THATCH Post-Processing \\
FLOXIMS & Free Standing FLOXI Execution \\
SYMELAN & Free Standing SYMELAN Execution \\
\hline
\end{tabular}


Subroutine INGEOM, with entries PREGEOM, INPGEOM, and PSTGEOM, reads, processes, and prints node geometry data. After reading radial and axial interface arrays, this subroutine computes node volumes, surface and flow areas, and other geometry coefficients for later conductance computations. The latter computations are done in entry PSTGEOM, which is called after gap data have been read in INBND, so that solid volumes, areas and node distances properly consider the gap dimensions. In entry INPGEOM, the logical variable UNIFIN from input record 301D is passed as call argument.

Subroutine INBLOCK, with entries PREBLCK and INPBLKS, reads, processes, and prints the subdivision of the structure into blocks, including the specification of boundary locations, identifying for each block face the boundary numbers of the boundaries covering it. A schematic print-out of the block breakdown is included with the output.

Subroutine INBNDS with entries PREBND and INPBND reads, processes, and prints the boundary descriptions, including types and dimensions of boundaries, characteristics of heat transter and layer information. Maps of the radial and axial boundaries within the nodal network are produced with the output.

Subroutine INHTGEN, with entries PREHTGN, INPHTGN, and PSTHTGN, reads and processes data describing the in-core heat generation, including initial power, radial and axial power profile specification, and the after heat option to be applied. This subroutine produces the two-dimensional power distribution arrays for decay heat and fission power, the latter only if the point kinetics option is used. In entry INPHTGN, the logical variable UNIFIN from input record 301D is passed as a call argument.

Subroutine INPLENA, with entries PREPLNA, INPPLNA, and PSTPLNA, initializes the plena arrays. Entry INPPLNA has the following call sequence:

$$
\text { LP100,I1,I2,J1,J2,GEOM }
$$

where LP100 is the plenum number plus 100 , as specified on input record $603 \mathrm{D}$; I1,I2,J1,J2 are the range of node numbers for this plenum, and GEOM is the plenum geometry descriptor, as specified on input record 651D. In entry INPPLNA, the array IJPLENA is loaded, identifying for each plenum surface node, $\mathrm{K}=1, \mathrm{KMX}$, progressing clockwise around the plenum the $\mathrm{I}$ and $\mathrm{J}$ indices of the corresponding solid nodes. In entry PSTPLNA, heat transfer surface areas, node center to surface distances, and other parameters for later conductance coefficient computations are computed.

Subroutine INTIME, with entries PRETIME and INPT, provides for input of time step information. This subroutine also reads and processes the specifications for temperature print-out formats and for the selection of block surface temperatures to be printed.

Subroutine INTHETA, with entries PRETHETA and INPTH, reads initial temperature files and loads the corresponding internal and boundary temperature arrays from prescribed binary files and/or from the input file. To accommodate the free-field user specification of temperature by blocks or lines (see Section 5), it calls on THETAIN to process the input records. 
Subroutine THETAIN receives the character strings specified for initial node temperatures in the input file, converts them to the proper temperatı!re values, and loads the corresponding locations in the temperature array.

Subroutine PREINIT does initialization before input data processing by calling the various PRE . . . entries and sets some of the system constants for COMMON/CONST/.

Subroutine PSTINIT does final initialization after completing the general THATCH input processing. This subroutine calls the various post-initialization entries PST _ _ _ and also reads the binary files for the two-dimensional radiation coefficient matrices. For restart runs, it locates and loads the initial temperatures and other required data from the restart file.

Subroutine FREEIN is used to unpack the boundary number specifications provided for block boundaries in input records 604D to 607D.

\subsubsection{THATCH Subroutines for Internal Conductance Coefficients}

Subroutine CONDUC computes the conductances for all interfaces between internal nodes and also beiween nodes and boundaries during time integration at each time step. At the beginning of each call, the damage parameter array for graphite irradiation damage is updated by calling ANNELEX, before the thermal conductivities are called from PROPMAT via CONDF calls. Where boundary resistances are required, BNDCONI and BNDCONX are called.

Subroutine BNDCONI computes the effective conductance between internal node centers wherever finite boundary resistances have been specified. During the iterative solution the node surface temperatures are also obtained. The conductance computations follow Equations 3.1-6 to 15 and the geometry of Figure 3-4. The call sequence to BNDCONI is as follows:

COND,I,J,TINT,IJR,THM,THP,THINT,THSURFM,THSURFP,MAT1, MAT2,MAT3,MAT4,DX1,DX2,DX3,DX4,KTYPE,NCOND,LAYFL,NITMAX, EMMI,DAM1,DAM2,DAM3,DAM4

where

$\begin{array}{ll}\text { COND } & \text { effective conductance (to be returned) }\left[\mathrm{W} / \mathrm{m}^{2} \mathrm{~K}\right] \\ \text { I,J } & \begin{array}{l}\text { radial and axial location of interface } \\ \text { current time [s] }\end{array} \\ \text { TINT } & \begin{array}{l}\text { boundary number } \\ \text { IJB }\end{array} \\ \text { nHM } & \text { node temperature on minus-side }[\mathrm{K}] \\ \text { THP } & \text { solid node/solid layer surface temperature }[\mathrm{K}] \\ \text { THINT } & \text { solid/gas surface temperature on minus-side [K] } \\ \text { THSURFM } & \text { solid/gas surface temperature on plus-side [K] } \\ \text { THSURFP } & \text { material property index for use in subroutine PROPMAT. For layers not } \\ \text { MAT1 TO MAT4 } & \text { present, the corresponding entry must be set to zero by the calling } \\ & \text { routine, and its resistance is then set to zero here. } \\ \text { DX1 TO DX4 } & \text { conductance lengths [m] } \\ \text { KTYPE } & \text { interior boundary type (see Table 5-1) } \\ \text { NCOND } & \text { conductance direction }(1=\text { axial; } 2=\text { radial) }\end{array}$


LAYFL

NITMAX

EMMI

DAM1 TO DAM4 index identifying fluid layer location. $L A Y F L=2$, fluid layer precedes solid layer (as in Figure 3-4); LAYFL $=3$, the sequence of the two layers is reversed.

maximum number of iterations to be used in BNDCONI iterative computations. Convergence is achieved when at iteration $\mathbf{n}$

$$
\frac{\sum R-\sum R_{n-1}}{\sum R_{n}}<\epsilon
$$

where $\epsilon-10^{-6}$

effective emissivity between the two solid surfaces of the fluid gap

damage parameter for irradiation damage in thermal conductivity calls to PROPMAT

Subroutine BNDCONX computes the effective conductance between boundary nodes and the exterior boundary points. During the iterative solution, the node surface temperatures are also obtained. The call sequence to BNDCONX is as follows:

COND,I,J, TINT, IBB, THM, THP, THS, THSURFM,THSURFP, NCOND,KTYPE, NITMAX,MATM,MATP,EMMI,HCONV,DXACM,DXACP,DAM

where

COND
I,J
TINT
IJB
THM
THP
THS
THSURFM
THSURFP
NCOND
KTYPE
NITMAX

effective conductance (to be returned) $\left[\mathrm{W} / \mathrm{m}^{2} \mathrm{~K}\right]$

radial and axial location of interface

current time [s]

boundary number

node temperature on minus-side (used if boundary is on plus-side) [K] node temperature on plus-side (used if boundary is on minus-side) [K] sink (boundary) temperature [K]

node surface temperature on minus-side (used if boundary is on plusside) $[\mathrm{K}]$

node surface temperature on plus-side (used if boundary is on minusside) $[\mathrm{K}]$

conductance direction $(1=$ axial $; 2=$ radial $)$

interior boundary type (see Table 5-1)

maximum number of iterations to be used in BNDCONX iterative computations (convergence is achieved when at iteration $n$

$$
\frac{\sum R_{n}-\sum R_{n-1}}{\sum R_{n}}<\epsilon
$$

where $\epsilon=10^{-3}$ )

MATM to MATP

material property nodes for use in subroutine PROPMAT.

EMMI

effective emissivity between the two solid surfaces of the boundary.

HCONV

DXACM,DXACP

convective heat transfer coefficient $\left[\mathrm{W} / \mathrm{m}^{2} \mathrm{~K}\right]$

conductance lengths $[\mathrm{m}]$ 
Subroutine COMCON computes the effective conductance across two layers of differing materials. During the iterative solution, the surface temperature at the interface of the two layers is also obtained. The call sequence to COMCON is as follows:

\section{CC,THO,TH1,TH2,DX1,DX2,MAT1,MAT2,NDIR,NITMAX,DAM1,DAM2}

where

CC
THO
TH1
TH2
DX1,DX2
MAT1,MAT2
NDIR
NITMAX

DAM1,DAM2 effective conductance (to be returned) $\left[\mathrm{W} / \mathrm{m}^{2} \mathrm{~K}\right]$

node temperature on minus-side [K]

surface temperature between nodes $[\mathrm{K}]$

node temperature on plus-side $[\mathrm{K}]$

conductan:e lengths $[\mathrm{m}]$

material property nodes for use in subroutine PROPMAT

conductance direction $(1=$ axial; $2=$ radial $)$

maximum number of iterations to be used in COMCON iterative computations (convergence is achieved when at iteration $\mathrm{n}$

$$
\frac{\sum R_{n}-\sum R_{n-1}}{\sum R_{n}}<\epsilon
$$

where $\epsilon-10^{-6}$ )

damage parameter for irradiation damage in thermal conductivity calls to PROPMAT

Subroutine CONDAPL computes the effective conductance of nodes adjacent to plena between node centers and the node/plenum interface.

Subroutine ANNEL, with entries ANNELIN and ANNELEX, computes the damage parameter array for the thermal conductivity of irradiated graphite based on initial irradiation damage data and a prescribed annealing temperature range for graphite. In entry ANNELIN, called by THATCH, the input records of Group 10 (Section 5-1) are read and loaded. In entry ANNELEX, called by CONDUC, the damage parameter array is recomputed, if annealing was specified based on the model of Section 8.3.

Subroutine FAILURE, with entries PREFAIL, INFAILP, FAILS, and ALLPLFL, permits the "failure" of certain layers and nodes. In previous HTGR designs, in pr: icular the thermal barrier cover plates, were expected to fail as certain failure temperature levels are reached. This subroutine, with its input data from Group 9, permits the simulation of such nodes or layers to disappear or to be "redeposited", for instance from the ceiling of a plenum to the same radial position on the floor.

This code feature has not been used for the MHTGR, and surrently might not be interfaced properly with other sections of the code. At this time there is no need for this feature; however, it should be tested carefully before being used again. 
Subroutine HEATGEN is called to update the heat generation data at each time step. It primarily produced the QTRIP array for each node, corresponding to the volumetric heating rate $q^{\prime \prime \prime}$. which is used in computing the node solid temperatures, including the effects of afterheat, neutronic heating (PKINL = true), as well as heat from chemical reactions and convective heat transfer from the gas stream (the latter two if FLOXI=true). For further details of the model for $q^{\prime \prime \prime}$, see Section 8.4.

\subsubsection{THATCH Subroutines for Time Integration of Solid Temperatures}

Subroutine TIMSOL, called from THATCH, contains main time-step, loop. It calls subroutine TEMPSOL for time integration of the transient temperatures in the solid structures. Furthermore, it activates the various available modules, depending on the sub-module options of the run, to provide quasisteady evaluations of gas tlows, chemical reactions, convective heat transfer (FLOXIEX), RCCS performance (AIRCLEX), and plena heat transfer (RADTN), as well as transient point kinetics evaluations (PKINEX), and core transient temperatures from SYMELAN (TEMPSYM). The transient evaluations of core solid temperatures and point kinetics can be done iteratively if so specified (RHOINT = true, set in PKIN module if input parameter COMPMOD ='Iterate').

A tlow chart of the TIMSOL integration loop is presented in Figure 4-1.

Subroutine TEMPSOL evaluates the transient temperature field in the solid nodes of the THATCH network. The ADI method, used to solve the simultaneous equations, requires the solution of tridiagonal sets of equations, first progressing by rows, and then progressing by columns. For vectorized solution of these equations, it is efficient to solve one set of equations, for all rows at once, plus another set for all columns at once.

Figure 4-2 is a flow chart for the main section of TEMPSOL, solving for the solid temperature array.

During the first pass through TEMPSOL, several index arrays are set, to be used later to load the conductunce and beat generation coefficients (CN,CE,CS,CW,CQ) into the tridiagonal system matrix arrays (ATR,BTR,CTR,FTR).

These arrays are as follows:

$\operatorname{KSETT}(1, J)$

which is set for each node equal to its material index KMAT(I,J), except if that node is an active core node and this is a run with SYMELL = true, in which case it is set to 200. In runs using SYMELAN, these temperatures are not to be solved here, but in THYSYM, and therefore, these nodes will be bypassed in the TEMPSOL solution.

ILOC index in tridiagonal system arrays during row-wise solution

JLOC

IINDXR (ILOC) and

JINDXR (ILOC)

index in tridiagonal system arrays during column-wise solution

II A (J) and

I2A (J)

contain node indices $\mathrm{I}$ and $\mathrm{J}$, corresponding to ILOC position. in tridiagonal arrays

contain ILOC, corresponding to tirst and last node of row J (i.e. for nodes $1, \mathrm{~J}$ and IL.J) 
TIme Siep Integration Loop in TIMSOL

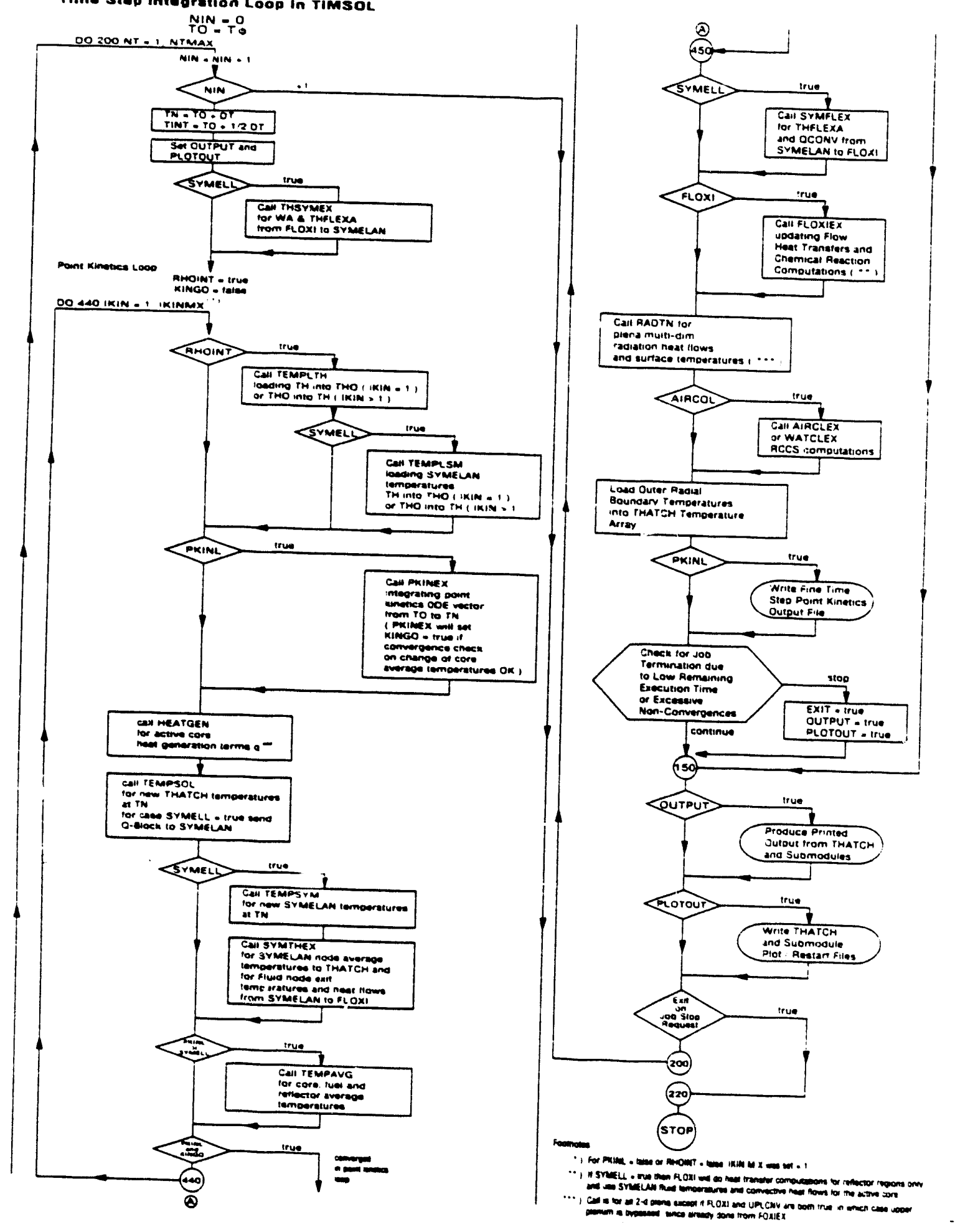

Figure 4-1 Flow Chart of Time Step Integration Loop in This OL 
Transient Solution for Solld Node Tomperatures in TEMPSOL

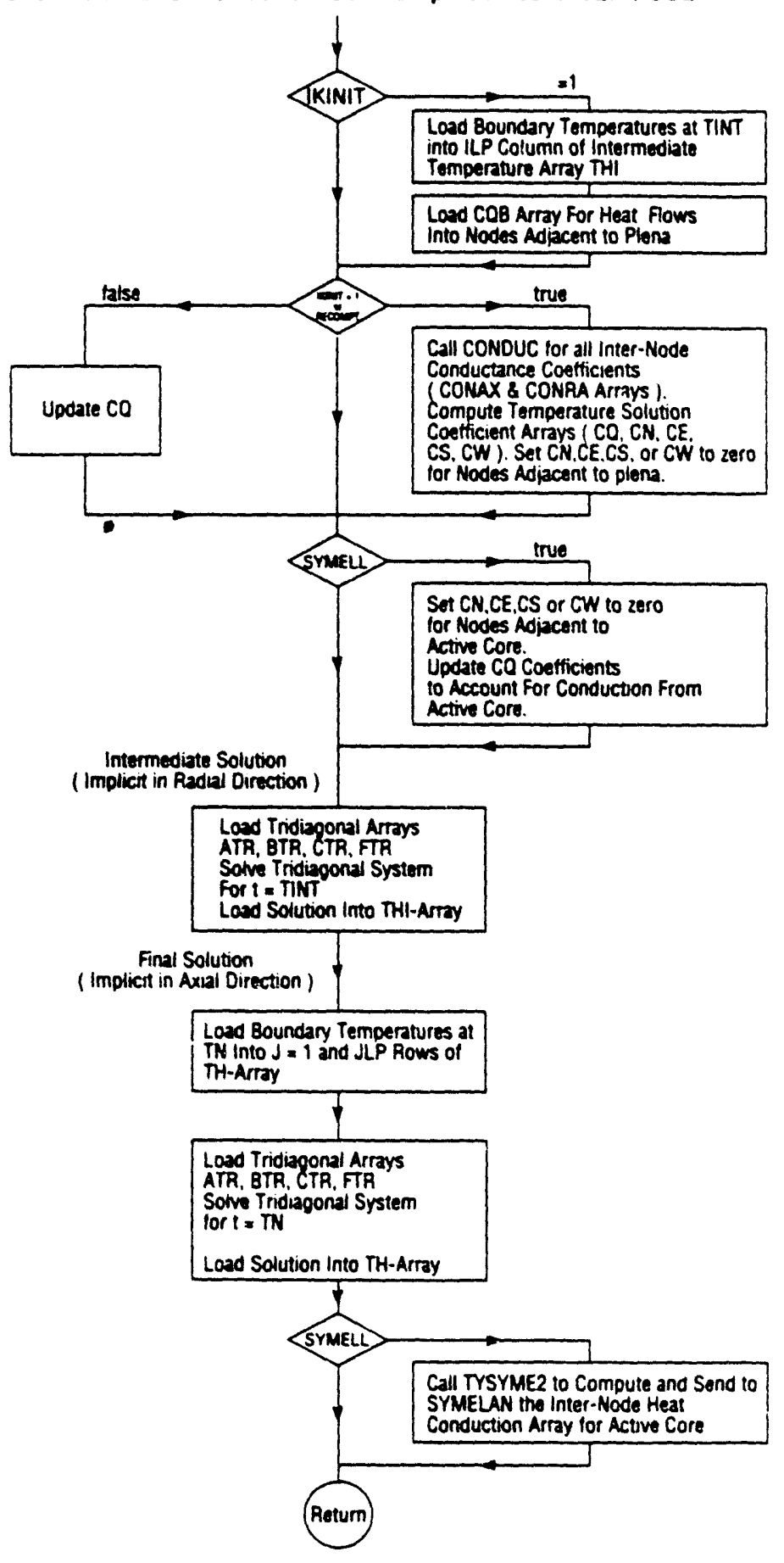

Figure 4-2 Flow Chart of TEMPSOL Solid Temperature Field Solution Procedure 
IINDXC (JLOC) and JINDXC (JLOC)

$\mathrm{J} 1 \mathrm{~A}(\mathrm{I})$ and

J2A (I) contain node indices $\mathrm{I}$ and $\mathrm{J}$, corresponding to JLOC position in tridiagonal arrays

contain JLOC, corresponding to first and last node of column I (i.e. for nodes $\mathrm{I}, 2$ and $\mathrm{I}, \mathrm{JL}$ )

ISUMEQ and JSUMEQ are the total number of nodes to be solved for in TEMPSOL (The two must be equal to each other).

Function THETABC provides boundary temperatures for range $\mathrm{J}=1$ (side $\mathrm{L}=1$ ), column JLP (side $L=3$ ) and column ILP (side $L=2$ ), where the side numbers follow the convection of Figure 3.3. The call sequence is as follows:

\section{$\mathrm{T}, \mathrm{I}, \mathrm{J}$}

where $T$ is time $[\vec{s}]$, and $I$ and $J$ are the indices of a boundary node for which the temperature is to be returned. These temperatures are currently loaded from the THSINKA array, which is user-specified on input records $1502 \mathrm{D}$ to $1504 \mathrm{D}$.

The reason not to load these input values into the main temperature array, but to reload them node by node at each time step via calls to THETABC, is to allow for time varying boundary temperatures. By modifying the extremely simple routine THETABC, the user can readily simulate time varying boundary temperatures.

Subroutine THTMPAV, with entry THTMPMX, computes average and maximum temperatures for the active core and for the reflector regions of the temperature array TH of the THATCH model. The call sequence for THTMPAV is as follows:

\section{T THAVCOR THAVREF}

where
$T$
time [s]
THAVCOR
average core temperature $[\mathrm{K}]$
THAVREF
average reflector temperature $[\mathrm{K}]$

If CALMODE = 'THATCH', i.e. THATCH alone, without SYMELAN, both temperatures will be returned. In CALMODE = 'COMBIN', i.e. THATCH with SYMELAN run, only the average reflector temperature will be computed. (Averages of active core temperature are then provided by SYMELAN subroutine SYTMPAV via call to TEMPAVG.

Subroutine TEMPLTH is called in TIMSOL within the point kinetics loop. If IKINIT $=1$ (first iteration), it stores the solid temperature array $\mathrm{TH}$ into temporary storage THO. In later iterations (IKINIT > 1), it provides the original temperature again as the starting point, by loading THO into TH. 
Subroutine RADTN evaluates plena surface temperatures and heat flows for two-dimensional radiation, coupled with conduction from adjacent nodes. Three calculation methods are provided for the iterative solution of plenum surface temperatures:

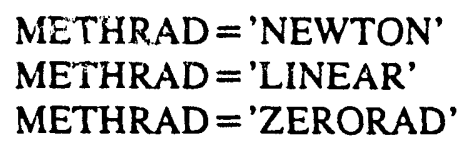

uses the Newton-Raphson Solver NEWTON from THTCHLIB. uses iteration with under relaxation

assumes that the conduction from node centers to node surfaces is the predominant heat transfer resistance and that all node surface temperatures are essentially equal.

'LINEAR' and 'ZERORAD' have not been used recently and 'NEWTON' is the preferred method.'ZERORAD' is a valid method at temperatures of several thousand $\mathrm{K}$, as they occur in some hypothetical accidents in large HTGRs.

After return of plenum surface temperatures, the plenum heat flows are computed and the plenum surface temperatures are stored in the appropriate node surface temperature arrays. If the upper plenum convection model is used, the appropriate calls to subroutine UPLCNV are made.

The call sequence to subroutine RADTN is as follows:

\section{IDEBUGL MODCALL}

where

IDEBUGL

MODCALL ='ALL" ='LP1' $=$ 'RMP' is a debug print control ( $>0$ triggers progressively more output as IDEBUGL is raised)

a character control variable

this call is for all plena in the system

this call is for upper plenum only

this call is for all but upper plenum

'LP1' and 'RMP' are used in runs with upper plenum convection, where separate calls for upper plenum only or the "remainder of plena" are required.

Subroutine RESJAC is called by NEWTON when NEWTON is called by RADTN. This subroutine computes for NEWTON the residual vector RES and the Jacobian matrix XJAC, following Equations 3.1-20 and 3.1-21. If UPLCONV=true, the model modifications of Section 8.5 are included. UPLCONV = true is transmitted by RADTN to RESJAC via logical variable CONVEC in Common PLENAS.

The call sequence for RESJAC is as follows:

$$
\text { Y,NEQ,XJAC,RES,BIGR,JBIGR,IDXJAC,IDEBUG }
$$

$Y$

NEQ

XJAC

RES variable vector (input)

number of equations (input)

Jacobean matrix (output)

residual vector (output) 


$\begin{array}{ll}\text { BIGR } & \text { largest absolute residual (output) } \\ \text { JBIGR } & \text { location of BIGR in RES (output) } \\ \text { IDXJAC } & \begin{array}{l}\text { dimension of Jacobean array } \\ \text { debug control variable }\end{array}\end{array}$

Subroutine UPLCONV evaluates the upper plenum convection model of Section 8.5. Its call sequence is as follows:

\section{NU,THS,AAR,IDPLNOD,IDPLENA,LRP,LP,IJPLMM,IWRITE}

where

$\begin{array}{ll}\text { NU } & \text { a control variable, described below } \\ \text { THS } & \text { plenum surface temperature array }[\mathrm{K}] \\ \text { AAR } & \text { plenum surface area array }\left[\mathrm{m}^{2}\right] \\ \text { IDPLNOD } & \text { first dimension of the above arrays } \\ \text { IDPLENA } & \text { second dimension of the above arrays } \\ \text { number of nodes in plenum } & \text { plenum number (must be } 1 \text { at this time) } \\ \text { LP } & \text { array of plenum node corner indices } \\ \text { IJPLMM } & \text { IJPLMM(1,LP)=I1 } \\ & \text { IJPLMM }(2, \mathrm{LP})=\mathrm{I} 2 \\ & \text { IJPLMM }(3, \mathrm{LP})=\mathrm{J} 1 \\ & \text { IJPLMM }(4, \mathrm{LP})=\mathrm{J} 2 \\ \text { IWRITE } & \text { output print control. }\end{array}$

On the first call to UPLCONV various surface area parameters and helium properties are set.

General control of execution is through control variable NU as follows:
$\mathrm{NU}=0$
Compute convective heat flow for each node, based on given temperatures and heat transfer coefficients.
$N U=1$
$N U=2$
$N U=3$
Compute Grashof and Nusselt numbers and convective heat transfer coefficient 'sing Equation (8.5-5).
Same as NU $=1$, but use Equation $(8.5-6)$.
The convective heat transfer coefficient is part of the simultaneous $t_{i}$ "'lations. Only Grashof and Nusselt numbers and some derivative coefficients for the Jacobean matrix are computed here.

\subsubsection{THATCH Solid and Gap Property Routines}

The material properties for solids and composite structures are computed in THATCH in subroutine PROPMAT, which must generally be user-supplied for a specific model.

The subroutine must compute effective thermal conductivities $(k)$ and thermal capacitances (pc) for all solid materia! numbers that were specified in the material index designations of input record 603D. It also must compute effective thermal conductivities for all gap material indices specified in input records $901 \mathrm{D}$ or $902 \mathrm{D}$. The calls for thermal conductivities are as follows: 
where
MATL
material index (1-99 for solids, 101-199 for
DAM
THK
$\mathbf{N}$ effective gap conductivities) irradiation damage parameters (see below) temperature [K] directional index $(1=$ axial, 2 = radial $)$

The conductivities must be returned in units of $\mathrm{W} / \mathrm{mK}$. The calls for thermal capacitances are as follows:

$$
\text { ROCF(MATL,THK,N) }
$$

where
MATL
material index (1-99)
THK
temperature $[\mathrm{K}]$
$\mathrm{N}$
not used

The thermal capacitances must be returned in units of $\mathrm{J} / \mathrm{m}^{3} \mathrm{~K}$.

Through common /THGASPR/ the following gas properties are available for property computations in PROPMAT:

$\begin{array}{ll}\text { RGASHEL } & \text { helium gas constant }[\mathrm{J} / \mathrm{kgK}] \\ \text { RGASAIR } & \text { air gas constant }[\mathrm{J} / \mathrm{kgK}] \\ \text { XMOLHEL } & \text { helium mole mass }[\mathrm{kg} / \mathrm{kmol}] \\ \text { XMOLAIR } & \text { air mole mass }[\mathrm{kg} / \mathrm{kmol}] \\ \text { POUPL } & \text { upper plenum pressure }\left[\mathrm{N} / \mathrm{m}^{2}\right]\end{array}$

The routine currently embodied in the main section of THATCH models the commercial MHTGR as described in the PSID, and is described in more detail in Section 8.2.

\subsubsection{THATCH Output Routines}

Subroutine PRINTER, with entries BLKPRNT, PLENPRT, and SURFPRT, provides for printed output, as specified via input data records 1203D with 1301D, 1401D, and 1402D. BLKPRNT provides for printing of solid node temperatures; PLENPRT for plenum surface temperatures and summary heat flows. SURFPRT prints solid block surface temperatures.

Subroutine HTFLUX computes heat flows over block boundaries, providing these data also to the THATCH output file and prints a map of system heat flows, if the call parameter IPR is set larger than 0 .

Subroutine PLOTTER, with entries BASEPLT and TIMEPLT, writes the THATCH output file (File 14) for post-processing or restarts (as File 15). 
In entry BASEPLT, initial run information and header data are written, while every call to TIMEPLT produces one set of records at that time step. A summary of the records written to File 14 is available as printed output file from File 7.

The header records provided by calls to BASEPLT are given in Table 4-3. The records written at specified time steps by calls to TIMEPLT are given in Table 4-4.

Block Data THBLDAT contains a series of constants used in various sections of the code, primarily for formatting of printed output.

\subsubsection{THATCH Utility Subroutines}

Subroutine ERROR is used to analyze some of the earlier error messages; however, most error messages are issued directly from the routines sensing the error.

Subroutine PAGER, with entries LINEON, LINETST, and NEWPAGE provides page control during printing of output.

Subroutine NLOCF is currently not used.

\subsubsection{THATCH Interface Subroutines}

The interface subroutines between THATCH and its sub-modules transfer data between THATCH and the sub-modules. These subroutine: are generally included in the main THATCH component and contain THATCH Commons. However, they are called by the sub-modules, with the respective submodule variables being transferred as call arguments. A few exceptions to this design were required in the interface to SYMELAN, as described in Section 4.2.5.

Subroutines FTHIN, FTHEX, and TTHEX provide the required interfacing between THATCH and PASCOL. Their function is schematically described in Table 4-5.

Subroutines THFLXIN. THFLXEX, and FLXTHEX provide the required interfacing between THATCH and FLOXI. Their function is schematically described in Table 4-6.

Subroutine THSYMIN provides for some of the interfacing between THATCH and SYMELAN. It is called from SYMELAN routine SYMELIN during initialization and provides the initial power POW0 and the logical variable PKINL from THATCH to SYMELAN. The remaining interface routines between THATCH and SYMELAN will be described with the SYMEL.AN library in Section 4.2.5.

\subsection{BIG Subroutine}

\subsubsection{PASCOL Subroutines}

The PASCOL module models the heat removal by the air cooled RCCS. It computes the coolant temperatures and mass flows in the RCCs, based on input-prescribed ambient air conditions and on vessel temperatures that are provided by THATCH. 
Table 4-3

Header Records on THATCH Output File

\begin{tabular}{|c|c|c|}
\hline $\begin{array}{l}\text { Record } \\
\text { Number }\end{array}$ & $\begin{array}{c}\text { Writing } \\
\text { Controlled } \\
\text { by }\end{array}$ & Variables \\
\hline 1 & & $\begin{array}{l}\text { JDATE, JTIME, TITLE } \\
\text { (Date, Time, Run Title) }\end{array}$ \\
\hline 2 & & $\begin{array}{l}\text { IPL,JLP,NBLOCK,NPLENA,NFAIL } \\
\begin{array}{l}\text { ILP = Number of node columns (including } \\
\quad \text { boundary) }\end{array} \\
\begin{aligned} \text { JLP }= & \text { Number of node rows (including } \\
& \text { boundaries }\end{aligned} \\
\text { NBLOCK = Number of blocks } \\
\text { NPLENA = Number of plena } \\
\text { NFAIL = Number of nodes that can fail }\end{array}$ \\
\hline 3 & & $\begin{array}{l}(\mathrm{RIFA}(\mathrm{I}), \mathrm{I}=1, \mathrm{ILP}) \\
\text { Radial node interface array }\end{array}$ \\
\hline 4 & & $\begin{array}{l}(\mathrm{ZIFA}(\mathrm{J}), \mathrm{J}=1, \mathrm{JLP}) \\
\text { Axial node interface array }\end{array}$ \\
\hline 5 & & $\begin{array}{l}\text { (JLO(K),JHI(K),ILO(K), IHI(K), } \mathrm{Ns}(\mathrm{K}), \mathrm{K}=1, \mathrm{NBLOCK}) \\
\text { JLO low axial interface number } \\
\text { JHI high axial interface number } \\
\text { ILO low radial interface number } \\
\text { IHI high radial interface number } \\
\text { NS number of block node surface temperatures } \\
\end{array}$ \\
\hline 6 & & $\begin{array}{l}\text { ( } L R(L P), L P=1, N P L E N A) \\
\text { LR number of surface nodes in plenum LP }\end{array}$ \\
\hline 7 & FLOXI & $\begin{array}{ll}\text { FLOXI Input Data: } \\
\text { TYPINGR,PRTCTRL,IREACT, ICHF, ICHFP,JNODF,LGAS } \\
\text { TYPINGR } & \text { ingress type, see input record 3103D } \\
\text { PRTCTRL } & \begin{array}{l}\text { print control, see input record 3212D } \\
\text { chemical reaction control, see input }\end{array} \\
\text { IREACT } & \begin{array}{l}\text { record 3102D } \\
\text { number of parallel channels in core }\end{array} \\
\text { ICHF } & \text { number of parallel channels including } \\
\text { ICHFP } & \text { return flow channel } \\
& \text { number of axial nodes } \\
\text { JNOD } & \text { number of gases, see input record 3104D } \\
\text { LGAS } & \end{array}$ \\
\hline
\end{tabular}


Table 4-4

Time Records on THATCH Output File

\begin{tabular}{|c|c|c|c|}
\hline $\begin{array}{l}\text { Record } \\
\text { Number }\end{array}$ & $\begin{array}{l}\text { Number of } \\
\text { Records } \\
\text { Written }\end{array}$ & $\begin{array}{l}\text { Writing } \\
\text { Controlled } \\
\text { by }\end{array}$ & Variables \\
\hline 1 & 1 & & Time [hr] \\
\hline 2 & 1 & & $\begin{array}{l}((\mathrm{TH}(\mathrm{I}, \mathrm{J}), \mathrm{I}=1, \mathrm{ILP}), \mathrm{J}=1, \mathrm{JLP}) \\
\text { Node temperatures }[\mathrm{K}]\end{array}$ \\
\hline 3 & NPLENA & & $\begin{array}{l}\text { (THETARS }(\mathrm{L}, \mathrm{LP}), \mathrm{L}=1, \mathrm{LR}(\mathrm{LP})) \text {, } \\
\text { Plenum surface temperatures }[\mathrm{K}]\end{array}$ \\
\hline 4 & 1 & & 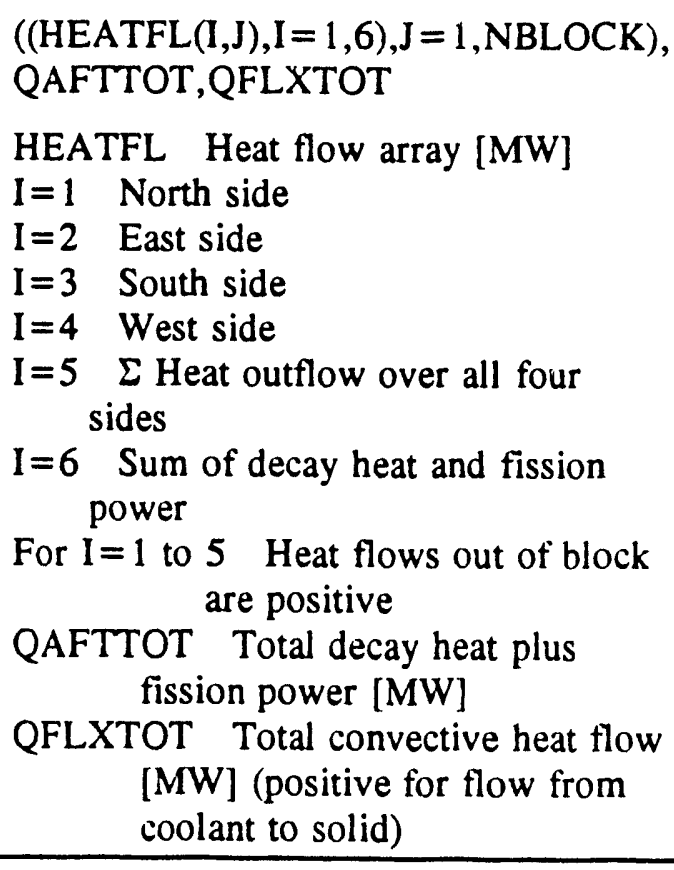 \\
\hline 5 & NBLOCK & & $\begin{array}{l}\text { NSF, (THSPLOT }(N), N=1, N S F) \\
\text { Block surface temperature }[K] \\
\text { NSF must equal corresponding entry } \\
\text { in NS array (Record } 5 \text { of Table 4-3) }\end{array}$ \\
\hline 6 & 1 & FLOXI & $\begin{array}{l}\text { Floxi output data record, see } \\
\text { Table 4-9 for details }\end{array}$ \\
\hline
\end{tabular}


Table 4-4 (Cont'd)

\begin{tabular}{|c|c|c|c|}
\hline 7 & 1 & AIRCOL & 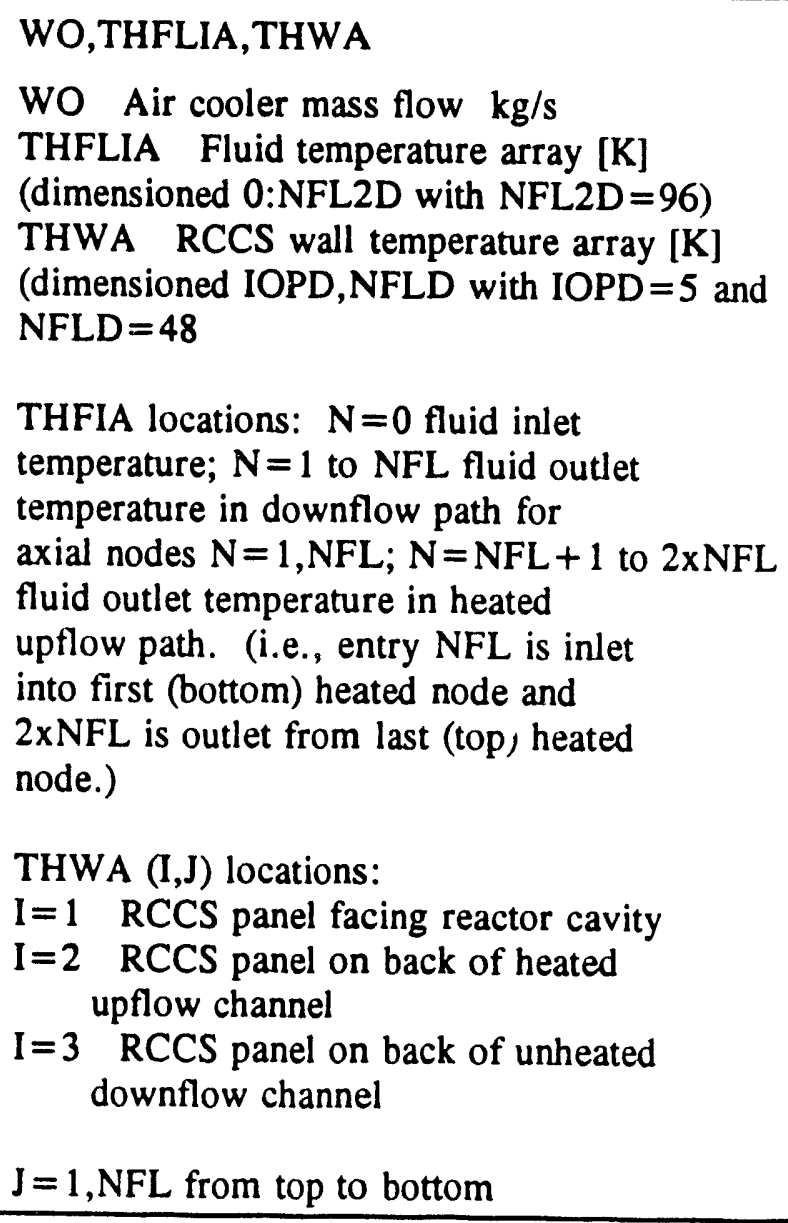 \\
\hline 8 & 1 & PKIN & $\begin{array}{l}\text { Y Vector of PKIN-Module } \\
\text { dimensioned IDYPKIN }=26 \\
\text { For details, see Table } 4-6\end{array}$ \\
\hline 9 & 1 & & $\begin{array}{l}((\mathrm{DAMPAR}(\mathrm{I}, \mathrm{J}), \mathrm{I}=1, \mathrm{ILP}), \mathrm{J}=1, \mathrm{JLP}) \\
\text { Annealing damage parameter array }\end{array}$ \\
\hline 10 & 1 & NFAIL $>0$ & $\begin{array}{l}\text { (LFAIL(K),K=1,NFAIL) } \\
\text { Integer index indicating whether and } \\
\text { which failure has occurred }\end{array}$ \\
\hline
\end{tabular}




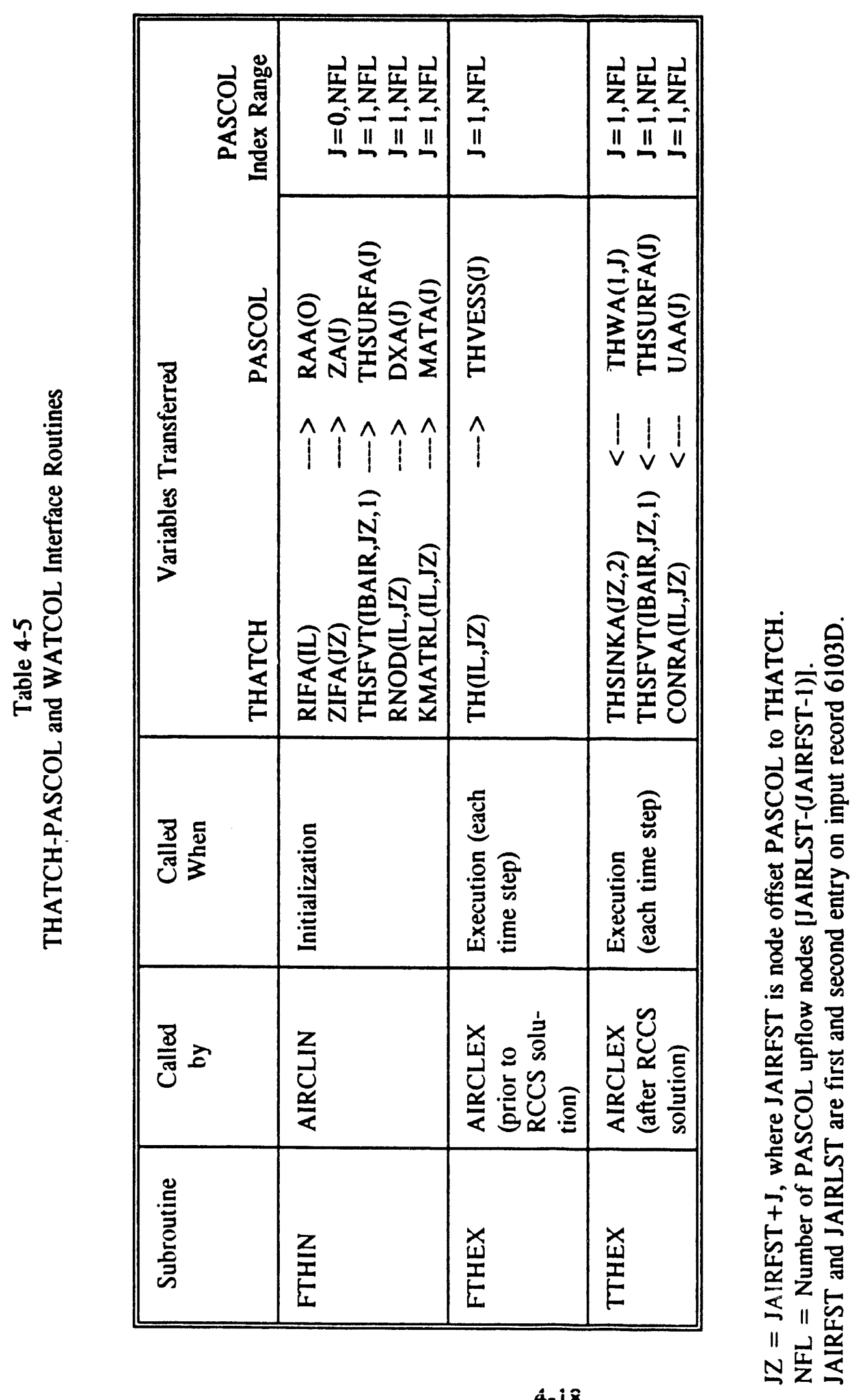


Table 4-6

THATCH-FLOXI Interface Routines

\begin{tabular}{|c|c|c|c|c|}
\hline Subroutine & $\begin{array}{l}\text { Called } \\
\text { by }\end{array}$ & $\begin{array}{l}\text { Called } \\
\text { When }\end{array}$ & \begin{tabular}{lr}
\multicolumn{2}{c}{ Variables } \\
Transferred \\
THATCH & FLOXI \\
\end{tabular} & $\begin{array}{c}\text { FLOXI } \\
\text { Index Range } \\
\end{array}$ \\
\hline THFLXIN & FLOXIIN & Initialization & $\begin{array}{cc}\text { ZIFA(J) } & \rightarrow \text { ZIFA(J) } \\
& \text { processed in } \\
& \text { FLOXIIN into } \\
& \text { DZA(J) } \\
\end{array}$ & $\mathrm{J}=1, \mathrm{JNODF}$ \\
\hline THFLXEX & $\begin{array}{l}\text { FLOXIEX } \\
\text { (Load Mode } \\
\text { 'Full' } \\
\text { before FLOXI } \\
\text { computations; } \\
\text { Load Mode } \\
\text { 'Partial'after } \\
\text { RADTN call) }\end{array}$ & $\begin{array}{l}\text { Execution (each } \\
\text { time step) }\end{array}$ & 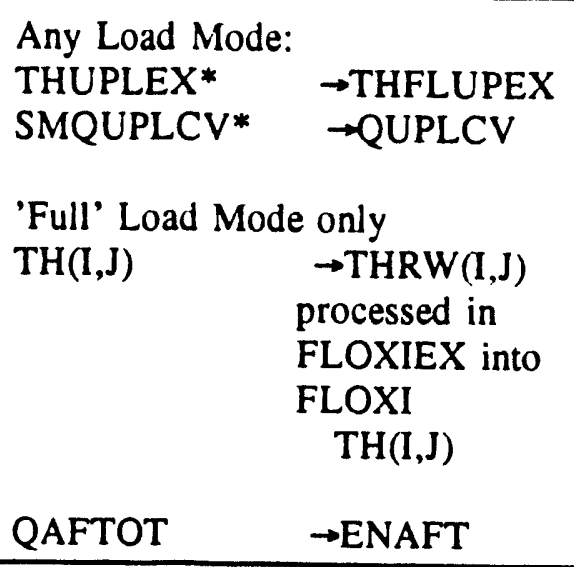 & $\begin{array}{l}\mathrm{I}=1, \mathrm{ICHFP} \\
\mathrm{J}=1, \mathrm{JNODF}\end{array}$ \\
\hline FLXTHEX & $\begin{array}{l}\text { FLOXIEX } \\
\text { (Load Mode } \\
\text { 'Full' } \\
\text { after FLOXI } \\
\text { computa- } \\
\text { tions; } \\
\text { Load Mode } \\
\text { 'Partial' } \\
\text { prior to } \\
\text { RADTN call) }\end{array}$ & $\begin{array}{l}\text { Execution } \\
\text { (each time } \\
\text { step) }\end{array}$ & \begin{tabular}{ll} 
Any Load Mode: & \\
THUPLIN* & -THFLUPIN \\
POUPL* & -PO \\
WINUPL* & -SUMWCUP2 \\
\multicolumn{1}{c}{ or } & \\
& SUMWCUP2 \\
& + WCORBAR \\
& \\
'Full' Load Mode oniy \\
QFLOX $(\mathrm{I}, \mathrm{J})$ & - QRW $(\mathrm{I}, \mathrm{J})$
\end{tabular} & $\begin{array}{l}\mathrm{I}=1, \mathrm{ICHFP} \\
\mathrm{J}=1, \mathrm{JNODF}\end{array}$ \\
\hline
\end{tabular}

*Variables in Common UPLCNV. 
Subroutine AIR COOL, with entries AIRCLIN, AIRCLEX, AIRCLOT, AIRCLWR and AIRCLRD, constitutes the effective main program of this module. The call sequence is as follows:

\section{MODE, TSEC, IDEBUG}

$\begin{array}{ll}\text { MODE } & \text { character variable identifying run type (= 'THATCH' in THATCH runs) } \\ \text { TSEC } & \text { time [s] } \\ \text { IDEBUG } & \text { debug print control }\end{array}$

Entry AIRCLIN obtains the run input data, initializes various arrays and prints the input data and options specified. It computes various design data of the system such as flow areas and heat transfer areas. If fin performance parameters are to be computed internally (FINK=true), it calls for the computation of the relevant radiation view factors.

Entry AIRCLEX is generally called once at each time step to evaluate, based on the reactor vessel temperature array and the air inlet temperature to the RCCS, all RCCS solid and coolant temperatures as well as the coolant mass flow rate, following the model of Section 3.2. The reactor vessel temperature array is obtained by a call to the THATCH/PASCOL interface routine FTHEX (see Table 4-5).

The system of simultaneous non-linear equations to be solved in AIRCLEX consists of quasisteady fluid energy equations, one per node, plus a single quasi-steady momentum equation. This system of equations is solved by a call to NEWTON (see THTCHLIB, Section 4.2-7). The number of fluid energy equations and their arrangement in the $\mathrm{Y}$-vector depends on the run options selected.

The interface points JAIRFST and JAIRLST, specified by the user, correspond to

$$
\text { NFL }=\text { JAIRLST }- \text { JAIRFST }
$$

nodes in vertical direction. In early versions of the RCCS, there was no thermal insulation between the down-flowing and up-flowing air paths, and regenerative heating of the incoming, downward flowing air from the upflow side was possible. The code permits this option, even though it is not used in current designs. If this option is selected, then two-fluid energy equations must be solved at each axial node, one for the downflow channel and one for the upflow channel. Thus, the number of fluid energy equations becomes

$$
\text { NFL2 }=2 \text { NFL }
$$

The $y$-vector variable is the fluid outlet temperature of each node, in flow direction. For thermal insulation between the downflow and upflow paths, integration begins at the bottom of the upflow path and ends at the last interface, NFL, as shown schematically in Figure 4-3. Without thermal insulation, integration begins at the top of the downflow path and ends at the last interface of the upflow path, NFL2, as shown schematically in Figure 4-4. The last equation in either case is the momentum equation, with the mass flow being the last variable in the Y-vector.

The residuals and Jacobian matrix are provided to NEWTON from subroutine JACAIR0 as shown in Figure 4-5. As the fluid temperatures are coupled to the solid temperatures at each node, the solid temperatures are computed at each node level in an internal Newton-Raphson procedure, which is embedded in subroutine WATSOLO. 


\section{Y-Vector Entry of Fluid Exit Temperature}

\section{Node Index}

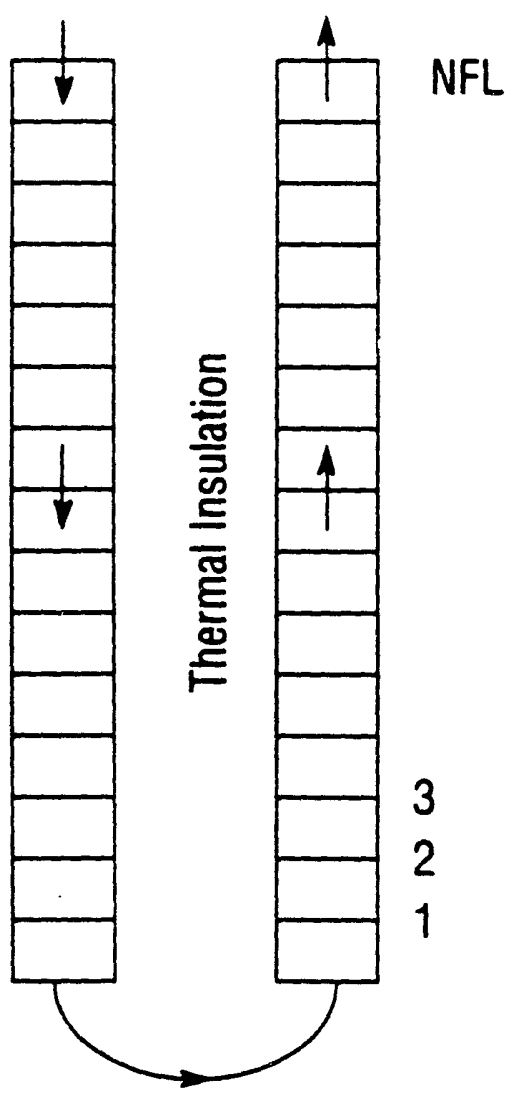

Figure 4-3 Y-Vector Indices versus Axial Node Indices in PASCOL for Case with Thermal Insulation Between Flow Passages 


\section{Y-Vector Entry of Fluid Exit Temperature}

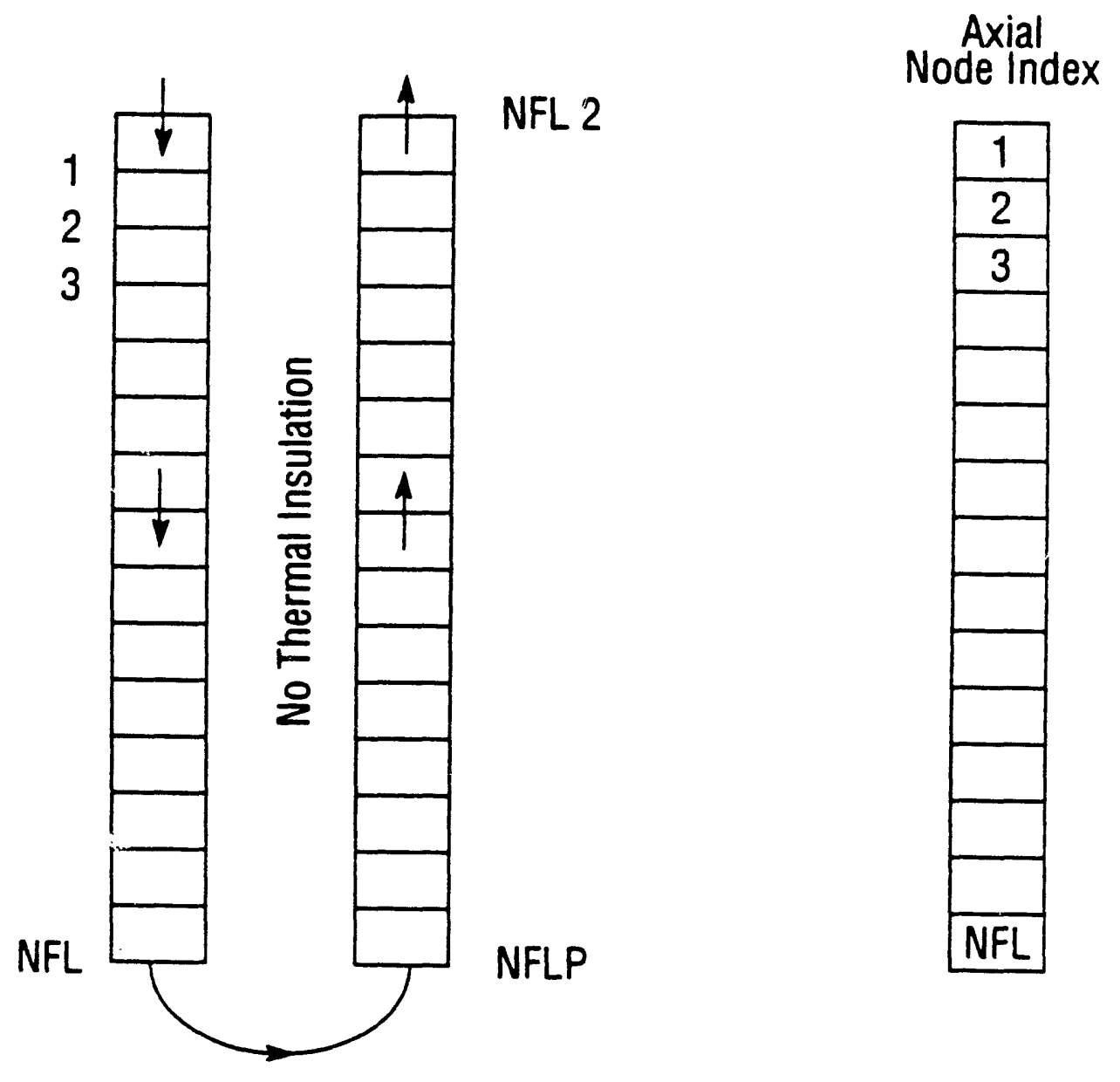

Figure 4-4 Y-Vector Indices versus Axial Node Indices in PASCOL for Case without Thermal Insulation Between Flow Passages 


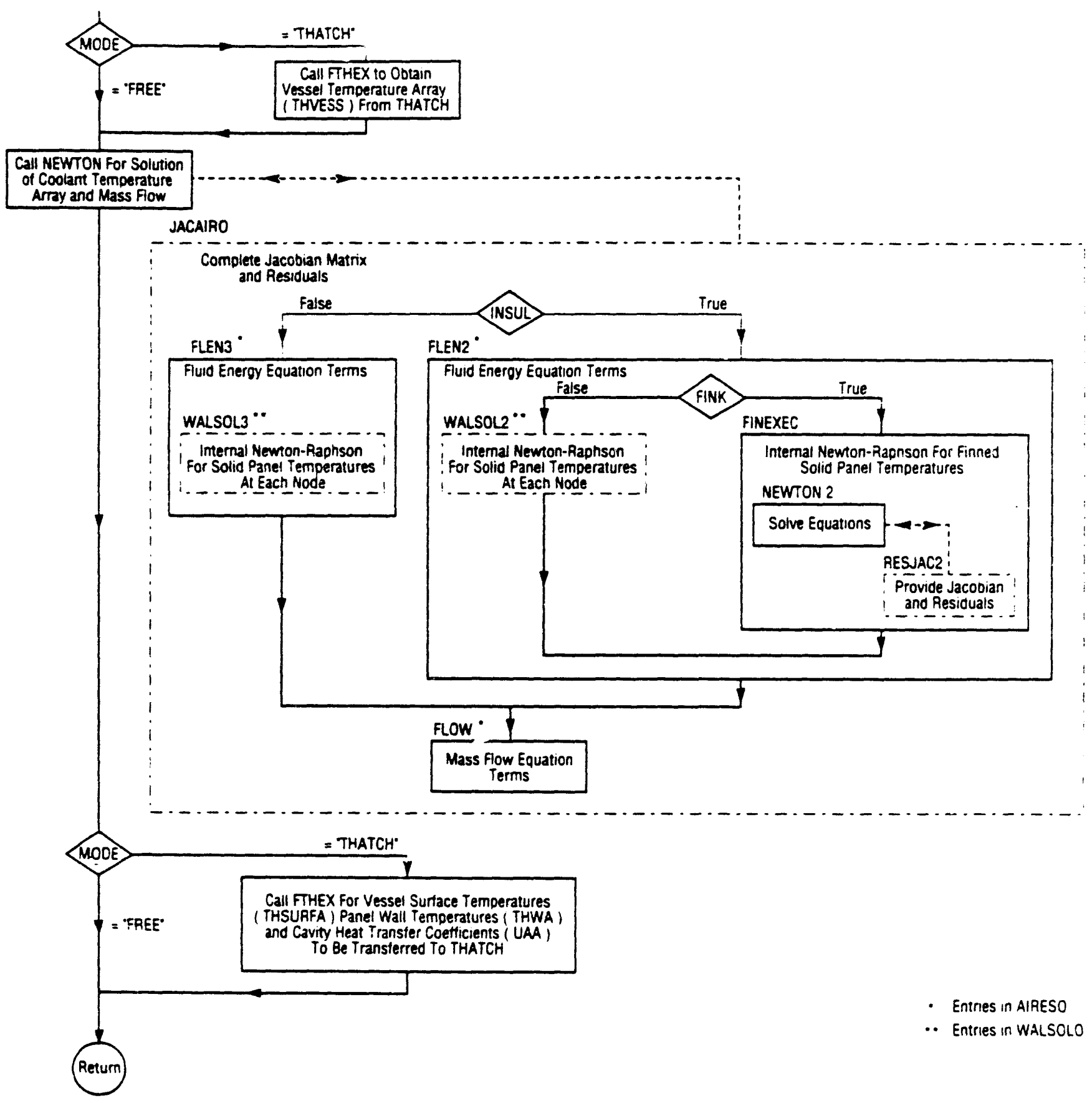

Figure 4-5 Schematic of PASCOL Solution of Air-Cooled RCCS Performance 
Entry AIRCLOT provides for printed output at the current time step.

Entries AIRCLWR and AIRCLRD will write or read one record to or from the THATCH output/restart file as per Table 4-4.

Subroutine JACAIRO is specified in the call to NEWTON from AIRCLEX as the subroutine to provide the Jacobian matrix and the residual vector. Its call sequence is specified by NEWTON (Section 4.2-7) as follows:

$$
\text { Y, NEQ, XJAC, RHS, BIGR, JBIGR, IDIMJAC, IDEBUG }
$$

where

$\begin{array}{lll}\text { Y } & \text { independent variable vector } & \text { (input) } \\ \text { NEQ } & \text { number of equations } & \text { (input) } \\ \text { XJAC } & \text { Jacobian matrix } & \text { (output) } \\ \text { RHS } & \text { residual vector } & \text { (output) } \\ \text { BIGR } & \text { absolute value of largest residual } & \text { (output) } \\ \text { JBIGR } & \text { location of largest residual in RHS array } & \text { (output) } \\ \text { IDIMJAC } & \text { leading dimension of XJAC array } & \text { (input) } \\ \text { IDEBUG } & \text { debug print control variable } & \text { (input) }\end{array}$

As shown in Figure 4-5, JACAIR0 calls on entries FLEN2 or FLEN3 in AIRES0 to supply the residuals and Jacobian terms for the fluid energy equation, and an entry FLOW in AIRESO for the residual and Jacobian coefficients of the quasi-steady momentum equation.

Subroutine AIRESO computes the residuals and the non-zero Jacobian matrix terms for Subroutine JACAIR0, via its three entries FLEN2, FLEN3 and FLOW. The call sequence for all entries is as follows:

\section{J, JJ, Y, NEQ, RES1, RES2, IDEBUG}

where

J

JJ

Y

NEQ

RES1, RES2

IDEBUG is the Y-vector entry for which the residual and Jacobian terms are to be evaluated. (Note that, in generai, J does not correspond to axial node or interface numbers as shown in Figure 4.3 and 4.4.)

is a control index, which is generally set $=0$ and will cause reloading of surface temperatures and overall heat transfer coefficients. $\mathrm{JJ} \neq 0$ will bypass the reloading and is only used in debug calls.

Y-vector supplied by NEWTON

number of equations

residuals to be returned via JACAIRO to NEWTON (two residuals in case of FLEN3 (INSUL = false), and one for FLEN2 or FLOW calls). debug print control variable. 
Subroutine WALSOLO, with entries WALSOL2 and WALSOL3, provides for computation of the solid panel temperatures and cavity heat transfer, which are coupled to the fluid temperatures and flow rate. Its call sequence consists of the debug control variable IDEBUG only.

For the case of INSUL = true, i.e., with thermal insulation between the downflow and upflow paths, the WALSOL2 entry is used, and the set of three simultaneous equations for temperatures of front and back panels of the RCCS up-flow channel and vessel surface temperatures is solved for any vertical node $\mathrm{J}$, using the current values for the coolant flow rate and coolant temperatures as well vessel node temperature, as described in Section 3.2.4. The solution is obtained from a Newton Raphson procedure, embedded in WALSOL2.

For the case INSUL = false, i.e., without thermal insulation between the downflow and upflow paths, entry WALSOL3 is used, and a set of four simultaneous equations for front center and back panel temperatures, plus vessel surface temperature is solved. In this case, the three panel temperatures are obtained from a Newton-Raphson procedure, embedded in the WALSOL3 section of the code and the vessel surface temperature follows from an iteration loop internal to the Newton-Raphson loop.

Subroutine STACK supplies the stack friction and buoyancy terms for the fiuid momentum equation, when it is called from entry FLOW of Subroutine AIRESO. Its call sequence is as follows:

$$
\text { W0, TH1, TH2, STFRIC, STBUOY, IDEBUG }
$$

where

$\begin{array}{lll}\text { W0 } & \text { air flow rate } & {[\mathrm{kg} / \mathrm{s}]} \\ \text { TH1 } & \text { air inlet temperature of the RCCS } & {[\mathrm{K}]} \\ \text { TH2 } & \text { air outlet temperature of the RCCS } & {[\mathrm{K}]} \\ \text { STFRIC } & \text { stack friction pressure drop term } & {\left[\mathrm{P} /(\mathrm{kg} / \mathrm{s})^{2}\right]^{2}} \\ \text { STBUOY } & \text { stack buoyancy pressure drop term } & {[\mathrm{P}]} \\ \text { IDEBUG } & \text { debug print control } & \end{array}$

Subroutine YTHETA loads the fluid temperature array THFLIA from the corresponding Y-vector entries. Its call sequence is as follows:

$$
\text { Y, THFLIA, NFL, NFL2, N1, N2, ISTACON, INSUL }
$$

where

Y

THFLIA

NFL2

N1

N2
Y-vector

fluid exit temperature of node [K]. Note that array THFLIA always contains the air inlet temperature in location 0 , and beyond that counts as shown for the $\mathrm{Y}$-vector in Figure 4-4; i.e., for the general case of thermal insulation, it will have constant temperatures equal to the air inlet temperature in locations 0 to NFL, and the $\mathrm{Y}$-vector entries $1^{-}$to NFL in locations NFL +1 to NFL2. number of downflow and upflow fluid nodes $(N L F 2=2 N F L)$ beginning of THFLIA locations to be loaded end of THFLIA locations to be loaded 
ISTACON

INSUL stack connection control variable

control variable for thermal insulation between downflow and upflow paths

Subroutine SOLVAIR solves the set of simultaneous linear equations required for the NewtonRaphson procedure called by entry AIRCLEX. It uses the specific sparse structure of this matrix as outlined in Section 3.2.4. Its call sequence is as follows:

$$
\text { XJAC, IDJAC, RHS, NEQ, IDEBUG }
$$

where

$\begin{array}{ll}\text { XJAC } & \text { Jacobian matrix } \\ \text { IDJAC } & \text { leading dimension of Jacobian matrix } \\ \text { RHS } & \text { on input, right-hand side vector; on return, the solution vector } \\ \text { NEQ } & \text { number of equations } \\ \text { IDEBUG } & \text { debug control variable }\end{array}$

Subroutine EVALAIR is called by entry AIRCLOT to print output of the air-cooled RCCS performance at designated time intervals. Its call sequence is as follows:

\section{TSEC,Y,NEQ,IDEBUG}

where

$\begin{array}{ll}\text { TSEC } & \text { time [s] } \\ \mathrm{Y} & \mathrm{Y} \text {-vector } \\ \text { NEQ } & \text { nurnber of equations } \\ \text { IDEBUG } & \text { debug print control }\end{array}$

In addition to printing output, EVALAIR also checks whether the Reynolds numbers may have been out of the bounds of the correlations used, and ascertains the accuracy of the energy balance.

Subroutine FINNER, with entries FININ1, FININ2, and FINEXEC, is used for input and execution during optional use of internal fin performance evaluation (FINK=true).

Under entry FININ1, the required input data for fin spacing and nodalization from input records 6201D and 6202D are loaded and used to initialize internal arrays for surface areas. By calling RADCOEF, the internal radiation coefficient matrices are produced.

Under entry FININ2, an array of reactor vessel conductances is initialized.

Entry FINEXEC is called from Entry FLEN2 of Subroutine AIERESO to compute upflow channel temperatures of the RCCS as well as reactor cavity solid temperatures, following the model- of Appendix $C$, as shown in Figure 4-5.

Subroutine NEWTON2 provides a Newton-Raphson solution to the set of simultaneous equations for the panel surface temperatures of the RCCS. Its call sequence is as follows: 


\section{Y, NEQ, EPSR, EPSY, NITMX, FRLX, IDEBUG, BIGR, ITERSNR, IER, RHS, DY, XJAC, IDIM}

where

$\mathbf{Y}$

NEQ

EPSR

EPSY

NITMX

FRLX

IDEBUG

BIGR

ITERSNR

IER

RHS

DY

XJAC

IDIM solutions vector (output)

(on call, $Y$ must contain an initial estimate of $Y$ )

number of equations (input)

convergence criterion with respect to residual vector (input)

convergence criterion with respect to change in Y-vector (input)

maximum number of iterations allowed (input)

underrelaxation coefficient to be applied to change in Y-vector

debug print control (input)

largest residual (computed in FCTJAC, output)

number of iterations used (output)

error indicator (output $=0$ converged, $=3$ not converged)

right-hand side vector (computed for NEWTON2 in FCTJAC)

change in Y-vector (output)

Jacobean matrix (computed for NEWTON2 in FCTJAC)

leading dimension of XJAC (input)

It must be noted that NEWTON2 is called from AIRESO via JACAIR0, which provides residuals and Jacobian coefficients to NEWTON; i.e., this Newton-Raphson procedure for the solid node temperatures in the RCCS upflow channel is called here internal to the Newton-Raphson procedure for overall RCCS coolant temperatures and flow rates. While the procedure of NEWTON2 is identical to that of NEWTON, subroutine NEWTON could not be used here because a subroutine is not allowed to call itself.

Subroutine RESJAC2 provides the equation residuals and the Jacobian coefficients for NEWTON2. Its call sequence is as follows:

$$
\text { Y, NEQ, PHI, XJ, IDIM, IDEBUG }
$$

where

$\begin{array}{ll}\text { Y } & \text { variable vector } \\ \text { NEQ } & \text { number of equations } \\ \text { PHI } & \text { residual vector } \\ \text { XJ } & \text { Jacobian matrix } \\ \text { IDIM } & \text { leading dimension of Jacobian matrix } \\ \text { IDEBUG } & \text { debug control variable }\end{array}$

The residuals and Jacobian coefficients are computed from Equations C-1 to C-5.

Function UCAVF evaluates the overall heat transfer coefficient across the reactor cavity, from the last temperature of the vessel node to the temperature of the RCCS front panel, following Equations $(3.2-5)$ and $(3 \cdot 2-6)$. Its call sequence is as follows: 


\section{THRCCS, IDEBUG}

where
THRCCS
temperature of the RCCS front panel [K]
IDEBUG
debug print control

Subroutine RADCOEF is called by FINNER (Entry FININ1) to obtain the prescribed internal fin nodalization for the radiation view factors. From these, the radiation coefficient matrix is then obtained, following the procedure in Appendix A. Subroutine RADCOEF uses the functions VIEWPAR and VIEWREC to evaluate the view factors.

Function NZFCT is a utility function, called by RADCOEF to identify the side node index for a given cell node, following the nodalization schematic of Figure C-2. Its call sequence is as follows:

\section{I, NN}

where
I
cell node index
NN
total number of side nodes

The returned value of NZFCT is the side index $\mathrm{N}$.

The functions VIEWPAR, and VIEWREC provide the view factors between nodes in the upflow channel of the RCCS, as required for the model of Appendix C.

Function VIEWPAR provides for view factors between parallel nodes, i.e., an opposite panels or fins of Figure C-1. Its call sequence is as follows:

$$
\mathrm{X} 11, \mathrm{X} 12, \mathrm{X} 21, \mathrm{X} 22, \mathrm{DY}
$$

where

$\mathrm{X} 11$ and $\mathrm{X} 12$

$\mathrm{X} 21, \mathrm{X} 22$

DY coordinates of the beginning and the end of node 1 in its plane corresponding coordinates for node 2 normal distance between the parallel planes

The returned value of VIEWPAR is the view factor $F_{1,2}$ i.e., from node 1 to node 2.

Function VIEWREC provides for view factors between nodes on adjacent sides of the rectangular cavity of Figure C-1, i.e., for nodes at right angles to each other. Its call is sequence is as follows:

$$
\mathrm{X} 11, \mathrm{Y} 11, \mathrm{X} 12, \mathrm{Y} 12, \mathrm{X} 21, \mathrm{Y} 21, \mathrm{X} 22, \mathrm{Y} 22
$$

These are the end-point coordinates for nodes 1 and 2 in accordance with Figure C-3. The returned value of VIEWREC is the view factor $F_{1,2}$ from node 1 to node 2 . 


\subsubsection{WATCOL Subroutines}

The WATCOL module computes the liquid and air side coolant temperatures and mass flows, based on THATCH reactor vessel temperatures and an ambient air temperature prescribed with the input data. The arrangement of the required variables is given in Table 4-7.

Table 4-7

Variable Vector of WATCOL Module

\begin{tabular}{||c|c|c|l|c||}
\hline $\begin{array}{c}\text { Variable } \\
\text { Location }\end{array}$ & $\begin{array}{c}\text { Index } \\
\text { Name }\end{array}$ & $\begin{array}{c}\text { Variable } \\
\text { Name }\end{array}$ & \multicolumn{1}{|c|}{$\begin{array}{c}\text { Variable } \\
\text { Description }\end{array}$} & Units \\
\hline $1-\mathrm{N}$ & 1-NAXV & TL & $\begin{array}{l}\text { Liquid Temperature } \\
\text { Leaving Node } \mathrm{n}\end{array}$ & $\mathrm{K}$ \\
\hline $\mathrm{N}+1$ & NAXVP & TC & $\begin{array}{l}\text { Cold Leg } \\
\text { Temperature }\end{array}$ & $\mathrm{K}$ \\
\hline $\mathrm{N}+2$ & NAXVP2 & WTOT & Liquid Mass Flow & $\mathrm{kg} / \mathrm{s}$ \\
\hline $\mathrm{N}+3$ & NAXVP3 & WAIR & Air Mass Flow & $\mathrm{kg} / \mathrm{s}$ \\
\hline $\mathrm{N}+4$ & NAXVP4 & TAIRX & $\begin{array}{l}\text { Air Exit } \\
\text { Temperature }\end{array}$ & $\mathrm{K}$ \\
\hline \hline
\end{tabular}

* for $\mathrm{N}$ axial nodes

Subroutine WATCLIN is called by THATCH to read the WATCOL input data file, and for various initialization. Its call sequence is as follows:

MODE, TSEC, IIN

where
MODE, Character Variable, = 'THATCH' for THATCH runs, and
$=$ 'FREE' for free-standing runs
TSEC
Problem time [s]
IIN Input data file identifier (currently not used)

In addition to reading and printing the input data, WATCLIN computes the required node and variable indices and various geornetrical constants such as flow and surface areas. It also calls subroutine CAVIN, to estimate the initial system variables.

It obtains the vessel axial nodalization, vessel radius, and material index data from THATCH using the same interface routine as PASCOL (see Table 4-5).

Subroutines WATCLEX is called TIMSOL at each time step to provide the RCCS solution based on given vessel temperatures. Its call sequence is as follows: 
where the arguments are the same as identified above with WATCLIN.

The subroutine obtains the last radial vessel node temperatures from THATCH via interface routine FTHEX (Table 4-5).

WATCLEX calls NEWTON for a solution to its set of equations, specifying WATRESD as the routine to provide the Jacobean matrix and the residuals, and SPECWAT as the routine to solve the set of equations.

WATCLEX returns to THATCH via interface routine TTHEX (Table 4-5) the temperatures of the RCCS panel surface, the temperatures of the vessel surface, and the overall heat transfer coefficient from vessel node to panel surface temperature.

Subroutine WATCLOT with entries WATCLRD and WATCLWR, prints output. Its call sequence is as follows:

\section{MODE, TSEC}

where the arguments are the same as identified above with WATCLIN.

Entry WATCLWR writes the WATCOL variable vector YWAT and the array of the panel surface temperature THWA, onto the THATCH output file (File 14). Entry WATCLRD reads the same records from the restart file (File 15) during restart runs.

Subroutine WATRESD is called by NEWTON to provide for a given variable vector $Y$ the Jacobean matrix and the residuals. Its call sequence is as follows:

Y, NEQ, XJAC, F, BIGR, JBIGR, IDJAC, IDEBUG

$\begin{array}{ll}\text { Y } & \text { variable vector (input) } \\ \text { NEQ } & \text { number of equations (input) } \\ \text { XJAC } & \text { Jacobean matrix (input) } \\ \text { F } & \text { residual vector (output) } \\ \text { BIGR } & \text { largest absolute residual (output) } \\ \text { JBIGR } & \text { location of BIGR in F (output) } \\ \text { IDJAC } & \text { dimension of Jacobean array } \\ \text { IDEBUG } & \text { debug control variable }\end{array}$

Subroutine WATRESD calls subroutine WATRR for the actual computation of the residuals and of the Jacobean terms. The Jacobean coefficients provided by WATRR are approximations, which is generally the most efficient procedure for solving simultaneous non-linear equations (The Jacobean coefficients do not affect the accuracy of a solution, but only the speed at which convergence is attained). To check on the accuracy of these terms, subroutine WATRESD permits as a debug option the computation of a full numerical Jacobean matrix. 
Subroutine WATRR does the actual computation of residuals and Jacobean matrix coefficients. Its call sequence is as follows:

$$
\mathrm{Y}, \mathrm{F}, \mathrm{XJAC}, \text { IDJAC, NEQ, IDEBUG }
$$

where all entries are identical to the variables described above under subroutine WATRESD. Based on a given $Y$ vector, the residuals and the most significant Jacobian terms are computed. The structure of the $Y$ vector is given in Table 4-7.

Subroutine SPECWAT, called by Newton, solves the system of simultaneous equations, using the special form of the Jacobian matrix, as described in Section 3.3.7. Its call sequence is as follows:

$$
\text { XJAC, IDJAC, RHS, NEQ, IDEBUG }
$$

where

$\begin{array}{ll}\text { XJAC } & \text { Jacobean matrix (input) } \\ \text { IDJAC } & \text { dimension of XJAC array } \\ \text { RHS } & \text { on input, right-hand side vector; on output, solution vector } \\ \text { NEQ } & \text { number of equations } \\ \text { IDEBUG } & \text { debug control variable }\end{array}$

Subroutine CAVIN establishes an initial estimate of the Y-vector during initialization, using several input parameters prescribed by the user. Once a satisfactory initial estimate has been established, further changes in those parameters should not be required. Its call sequence is as follows:

$$
\text { Y,NEQ }
$$

where

$$
\begin{array}{ll}
\mathrm{Y} & \mathrm{Y}-\text { vector } \\
\text { NEQ } & \text { number of equations }
\end{array}
$$

Subroutine DMAT loads a two-dimensional indicator array for a specified number of axial nodes, indicating terms of the Jacobean matrix that are significant and should be used in the solution. This routine is currently not used, but it can be activated with model changes for debugging. Its call sequence is as follows:

$$
\text { ND, IDIMND, NEQ }
$$

where
ND
IDIMND
two-dimensional indicator array
NEQ
dimension of ND
number of equations

On return, the ND array contains 0 for insignificant terms and 1 for significant terms. 
The WATCOL module inciudes several function type subroutines for water properties, as well as friction and heat transfer across tube banks (Table 4-8).

\subsubsection{FLOXI Subroutines}

Subroutine FLOXIMS is the actual main program for free-standing FLOXI runs. For these runs, a solid temperature history file must be available. This information is produced from THATCH output files by the THPRCCS post processor.

FLOXIMS is not used in THATCH runs. For free standing runs it requires input records 3105D and $3106 \mathrm{D}$ to be read.

Subroutine FLOXIIN is cailed by THATCH or by FLOXIMS and provides for FLOXI initialization, loading the input records of Groups 31 and 32 (Section 5), and calling CORINFL, the unified input option routine, if so specified. Its call sequence is as follows:

\section{MODE, UNIFIN, TSEC, IDEBUG, IIN}

where

MODE
UNIFIN
TSEC
IDEBUG
IIN

Character variable for THATCH runs = 'THATCH' for free-standing runs $=$ 'FREE' Logical variable specifying that the unified input option is in effect. problem time [s] debug print control variable input data file identifier (currently not used)

Subroutine FLOXIEX executes the main FLOXI computations for gas flows, convective heat transfer, and chemical reactions, as specified by the input data. If user-specified, the coupling between heat flow, chemical reactions and gas-flows is considered in the FLOXIEX 350-Loop. Details of this loop are presented in Figure 4-6. Subsequent to the execution loop, several sums of heat flows and energy balance checks are computed and are included in the output file record.

Its call sequence is as follows:

\section{MODE, TSEC, NONCONV, UPLCNV, IDEBUG}

where
MODE
Character variabre
for THATCH runs = 'THATCH'
for free standing runs = 'FREE'
TSEC
NONCONV
problem time [s]
Non-convergence indicator. Increased by one every time that non- convergence occurs in main FLOXI loop (350-Loop). 


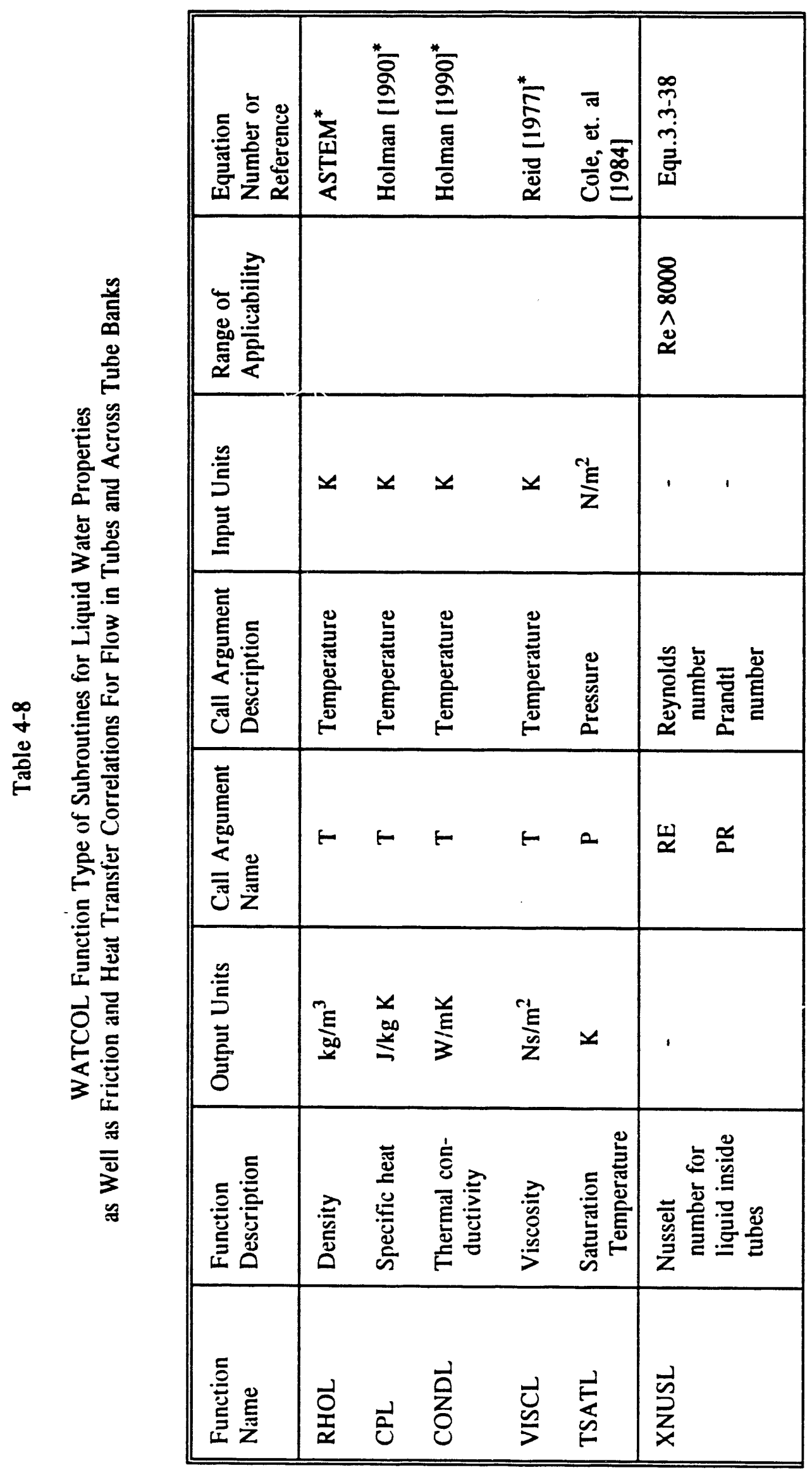




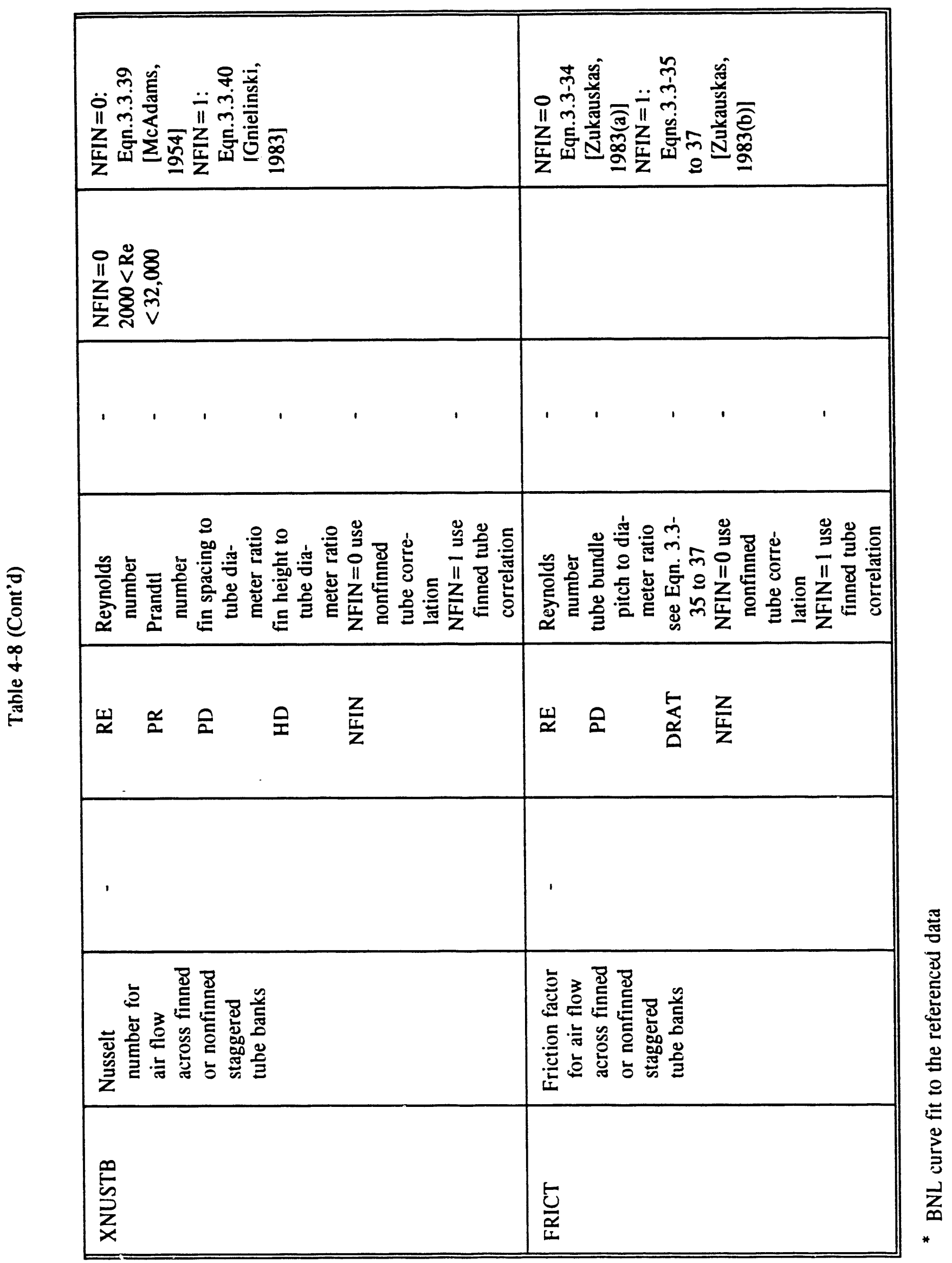


FLOXIEX Iteration Procedure

Between Flow, Heat Transter and

Chemical Reaction Computations

FRLXOP $=$ FRLXOPO

FRLX = FRLXO

NCTFLRV $=0$

DO 360 MNM $=1$, NITRMX $\cdot \cdot$

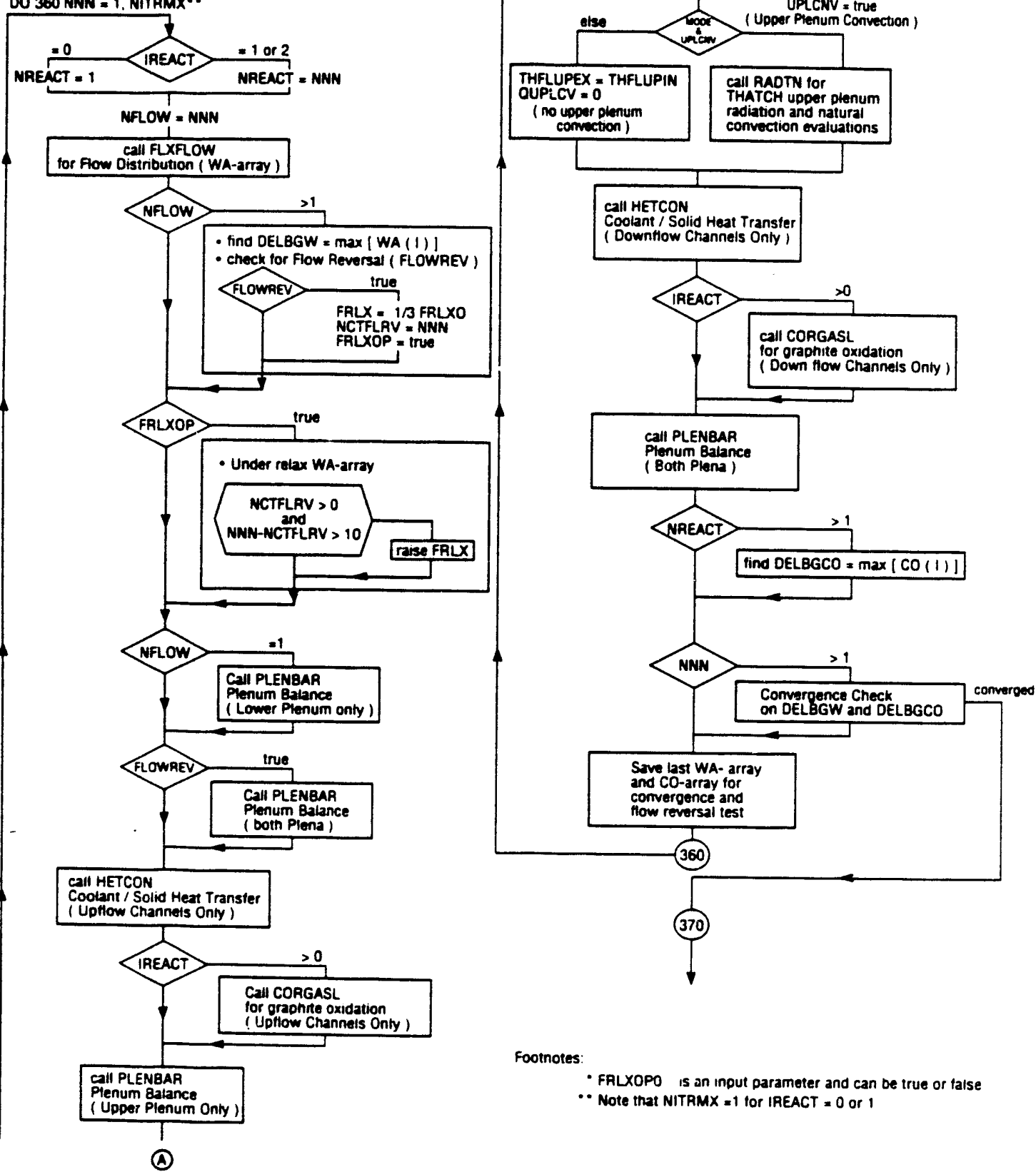

Figure 4-6 Flow Chart of FLOXIEX Main Loop for Coupled Flow, Heat Transfer and Chemical Reaction Computations 
UPLCNV Logical variable, if true upper plenum convective heat flow is to be computed by calling THATCH routine RADTN

IDEBUG debug print control variable

Subroutine FLOXIOT, with entries FLOXIRD and FLOXIWR, provides for printed output as well as for writing output file records and plot file records.

The following is the call sequence for FLOXIOT (printed output) :

MODE, TSEC, IDEBUG

where

$\begin{array}{ll}\text { MODE } & \begin{array}{l}\text { Character variable } \\ \text { for THATCH runs = 'THATCH' } \\ \text { for free-standing runs = 'FREE' } \\ \text { problem time [s] } \\ \text { TSEC }\end{array} \\ \text { IDEBUG print control variable }\end{array}$

For entries FLOXIRD and FLOXIWR, the call sequence is as follows:

MODE, TSEC, IDEBUG, ITP_-

where ITP _ is either ITPRD or ITPWR.

MODE a character variable controlling the type of record to be read or written

TSEC

IDEBUG

(see below)

problem time [s]

ITP. -

debug print control variable

identifier for the file to be read from or written to.

FLOXIOT calls the major FLOXI routines with IDEBUG $=1$, which causes the normal printed output to be produced.

There are three different output records that can be written and read by entries FLOXIWR or FLOXIRD, depending on the MODE variable. For Mode= 'THATCHO', a control variable record is written or read, generally for the THATCH output file. The contents of this record are described in Table 4-3 (Record 7).

For Mode= 'THATCH' a large output record of time-dependent variables is written or read, generally as part of a THATCH output file. This is Record 6 of Table 4-4; Details of its contents are in Table 4-9.

For Mode= 'PLOT', a plot record will be written by the post processor THPROCS at each time step. In accordance with all time-dependent plot output records, this record begins with a counting integer, followed by the time [s], and the dependent variable vector. This dependent variable vector is identical to the first 59 records of the THATCH output file as described in Table 4-9. 
Table 4-9

FLOXI Output Data Record

For

THATCH Output File

\begin{tabular}{|c|c|c|c|}
\hline $\begin{array}{l}\text { Location in } \\
\text { Output Record* }\end{array}$ & $\begin{array}{l}\text { Variable } \\
\text { Name }\end{array}$ & Units & Description \\
\hline 1 & WTOLOPL & $\mathrm{kg} / \mathrm{s}$ & Mass flow to lower plenum \\
\hline 2 & WCORBAR & $\mathrm{kg} / \mathrm{s}$ & Core barrel mass flow \\
\hline 3 & WINFLOW & $\mathrm{kg} / \mathrm{s}$ & Inflow to reactor vessel \\
\hline 4 & SUMWCUP1 & $\mathrm{kg} / \mathrm{s}$ & $\begin{array}{l}\text { Sum of core channel upflows at } \\
\text { bottom reflector inlet }\end{array}$ \\
\hline 5 & SUMWCUP2 & $\mathrm{kg} / \mathrm{s}$ & $\begin{array}{l}\text { Sum of core channel upflows at } \\
\text { top reflector outlet }\end{array}$ \\
\hline 6 & SUMWCDN1 & $\mathrm{kg} / \mathrm{s}$ & $\begin{array}{l}\text { Sum of core channel downflows at } \\
\text { top reflector inlet }\end{array}$ \\
\hline 7 & SUMWCDN2 & $\mathrm{kg} / \mathrm{s}$ & $\begin{array}{l}\text { Sum of core channel downflows at } \\
\text { bottom reflector outlet }\end{array}$ \\
\hline $8-14$ & WA & $\mathrm{kg} / \mathrm{s}$ & Channel mass flows $(I=1, I C H F P)$ \\
\hline 15 & TSUMCAR & $\mathrm{kg} / \mathrm{s}$ & Rate of graphite oxidation \\
\hline 16 & XMAXCTH & $\mathrm{kg} / \mathrm{s}$ & $\begin{array}{l}\text { Maximum theoretical oxidation if } \\
\text { all of incoming oxidizer }\left(\mathrm{O}_{2} \text { or }\right. \\
\left.\mathrm{H}_{2} \mathrm{O}\right) \text { reacts }\end{array}$ \\
\hline 17 & $\mathrm{PO}$ & $\mathrm{N} / \mathrm{m}^{2}$ & System pressure \\
\hline 18 & DELPCR & $\mathrm{N} / \mathrm{m}^{2}$ & Core pressure drop \\
\hline 19 & DPFRAV & $\mathrm{N} / \mathrm{m}^{2}$ & $\begin{array}{l}\text { Average core friction pressure } \\
\text { drop (absolute value) }\end{array}$ \\
\hline 20 & ENREACTO & $\mathrm{J} / \mathrm{kg}$ Carb & $\begin{array}{l}\text { Specific energy release of } \\
\text { oxidation }\end{array}$ \\
\hline 21 & ENAFT & W & After heat plus fission power \\
\hline $22-28$ & THFLINA & $\mathbf{K}$ & $\begin{array}{l}\text { Coolant inlet temperature for } \\
\text { each channel }\end{array}$ \\
\hline $29-35$ & THFLEXA & $\mathrm{K}$ & $\begin{array}{l}\text { Coolant exit temperature for each } \\
\text { channel }\end{array}$ \\
\hline 36 & THFLUPIN & $\mathrm{K}$ & $\begin{array}{l}\text { Upper plenum coolant inlet } \\
\text { temperature }\end{array}$ \\
\hline
\end{tabular}


Table 4-9 (Cont'd)

\begin{tabular}{|c|c|c|c|}
\hline $\begin{array}{l}\text { Location in } \\
\text { Output Record* }\end{array}$ & $\begin{array}{l}\text { Variable } \\
\text { Name }\end{array}$ & Units & Description \\
\hline 37 & THFI_UPEX & $\mathbf{K}$ & $\begin{array}{l}\text { Upper plerum coolant exit } \\
\text { temperature }\end{array}$ \\
\hline 38 & THFLLOPL & $\mathbf{K}$ & $\begin{array}{l}\text { Lower plenum coolant exit } \\
\text { temperature }\end{array}$ \\
\hline $39-45$ & SUMQPOS & W & $\begin{array}{l}\text { Sum of positive heat flows } \\
\text { within each channel** }\end{array}$ \\
\hline 46 & SMSMQP & $\mathbf{W}$ & Sum of all positive heat flows** \\
\hline $47-53$ & SUMQNEG & W & $\begin{array}{l}\text { Sum of negative heat flows within } \\
\text { each channel** }\end{array}$ \\
\hline 54 & SMSMQN & W & Sum of all negative heat flows** \\
\hline 55 & SUMQCON & W & $\begin{array}{l}\text { Sum of convective heat flow over } \\
\text { all flow channels }\end{array}$ \\
\hline 56 & SUMQRCT & W & $\begin{array}{l}\text { Sum of chemical reaction heat } \\
\text { release over all core nodes }\end{array}$ \\
\hline 57 & QUPLCV & W & $\begin{array}{l}\text { Upper plenum convective heat flow } \\
\text { to solid }\end{array}$ \\
\hline 58 & SMSMQCNV & W & $\begin{array}{l}\text { Sum of convective heat flows over } \\
\text { flow channels and upper plenum }\end{array}$ \\
\hline 59 & ENCOLNT & W & $\begin{array}{l}\text { Net energy removed from reactor } \\
\text { by coolant loop }\end{array}$ \\
\hline $60-67$ & NUAUPL & & $\begin{array}{l}\text { Gas volume fraction array for } \\
\text { upper plenum }\end{array}$ \\
\hline $68-75$ & NUALOPL & & $\begin{array}{l}\text { Gas volume fraction array for } \\
\text { lower plenum }\end{array}$ \\
\hline
\end{tabular}


Table 4-9 (Cont'd)

\begin{tabular}{|c|l|c|l|}
\hline $\begin{array}{l}\text { Location in } \\
\text { Output Record* }\end{array}$ & $\begin{array}{l}\text { Variable } \\
\text { Name }\end{array}$ & Units & \multicolumn{1}{|c|}{ Description } \\
\hline \hline $76-215$ & THFL & K & $\begin{array}{l}\text { Coolant temperature array } \\
(7 \times 20 \text { entries })\end{array}$ \\
\hline $216-355$ & QCONV & W & $\begin{array}{l}\text { Convective heat flow array } \\
(7 \times 20 \text { entries })\end{array}$ \\
\hline $356-495$ & QREACT & W & $\begin{array}{l}\text { Chemical reaction heat array } \\
(7 \times 20 \text { entries })\end{array}$ \\
\hline
\end{tabular}

*Locations in output record are for 6 in-core flow channels plus one return flow channel and 20 axial nodes. The THATCH and FLOXI write statements will work for any consistent nodalization, but the variable locations in the output record will vary for other nodalizations.

**Note that FLOXI convective heat tlows are defined as positive for flow from coolant to solid.

***During post-processing (in mode 'PLOT') only variables 1 to 59 are read and written. 
Subroutine FLOXISM permits a summary print out of a previously analyzed transient. It is generally called in free standing runs from FLOXIMS or in post processing runs from the THPROCS module. Its call sequence is as follows:

\section{MODE, IDEBUG, ITPOUT}

where
MODE
a character variable controlling the type of record to be read or written (see below)
IDEBUG debug print control variable
ITPOUT
File from which transient data are to be read ('PLOT' format required)

FLOXISM will read a file written by FLOXIWR in mode 'PLOT' and print a summary output.

Subroutine FLXFLOW is the main routine solving for the gas flow distribution in the prescribed parallel channels using its own Newton-Raphson method. If call variable IDEBUG is set $=1$, the normal printec output is produced after execution (see Section 3.4.1 and 3.4.4).

Subroutine AVAL provides various arrays of friction and buoyancy terms to FLXFLOW which do not change during FLXFLOW iterations.

Subroutine FLOWAPR can be called by FLXFLOW for an initial approximate flow solution if so desired. (APPROX, Input Record 3102D)

Subroutine SOLSPEC solves the simultaneous system of equation in FLXFLOW, using the sparse and special structure of the Jacobean matrix (see Section 3.4.4).

Subroutine CORINIT sets initial volume fractions, mole mass, temperature, and density arrays during its first call from FLOXIEX. At each time step it sets before the main FLOXI loop, initial values for fluid temperatures and densities.

Subroutine CORGASL solves for the mass balance during chemical oxiation computations, advancing through the active core flow channel in flow direction.

The call variable CALMDFX can be set by FLOXIEX or FLOXIOT to 'UP', 'DOWN' or 'ALL' to evaluate up-flow channels only, down-flow channels only, or all channels.

Subroutine REACT, called from CORGASL, evaluates the actual chemical reaction rate for each node based on local component partial pressures, temperatures, etc. Its call sequence is as follows:

TIME,IDEBUGL,CAVM,SUMCAVM,CAVP,SUMCAVP,GAM,GAMCARB,RGCT,RGC,NUA,II,JJ SUMGMKG,XMCARB,PO,THFLL,THSL,PREACTSTR,PREACTW,LGAS,LDM,TYPINGR

where
TIME
time [s]
IDEBUGL
local debug print control
CAVM
molar inflow rate array for gas species (input) $\left[\frac{\mathrm{kmol}}{\mathrm{s}}\right]$ 


\begin{tabular}{|c|c|}
\hline SUMCA $: M$ & molar inflow rate of gas mixture (input) $\left[\frac{\mathrm{kmol}}{\mathrm{s}}\right]$ \\
\hline CAVP & molar outflow rate array for gas species (output) $\left[\frac{\mathrm{kmol}}{\mathrm{c}}\right]$ \\
\hline SUMCAVP & molar outflow rate of gas mixture (output) $\left[\frac{\mathrm{kmol}}{{ }^{s}}\right]^{s}$ \\
\hline GAM & gas generation rate array for gas species (output) $[\underline{\mathrm{kmol}}$ \\
\hline GAMCARB & molar oxidation rate for carbon $\left[\frac{\mathrm{kmol}}{\mathrm{s}}\right]$ \\
\hline RGCT & mass oxidation rate for carbon (output) $\left[\frac{\mathrm{kg}}{\mathrm{g}}\right]$ \\
\hline RGC & mass oxidation rate of carbon per unit surface area \\
\hline $\begin{array}{l}\text { NUA } \\
\text { II,JJ }\end{array}$ & $\begin{array}{l}\text { gas volume fraction array at node inlet (input) } \\
\text { node indices }\end{array}$ \\
\hline SUMGMKG & mass increase of gas mixture (output) $\left[\frac{\mathrm{kg}}{\mathrm{g}}\right]$ \\
\hline XMCARB & mole mass of carbon $\left[\frac{\mathrm{kg}}{\mathrm{kmol}}\right]$ \\
\hline PO & system pressure $\left[\mathrm{N} / \mathrm{m}^{2}\right]$ \\
\hline $\begin{array}{l}\text { THFLL } \\
\text { THSL }\end{array}$ & $\begin{array}{l}\text { fluid temperature }[\mathrm{K}] \\
\text { solid temperature }[\mathrm{K}]\end{array}$ \\
\hline PREACTSTR & reactant partial pressure in stream (output) \\
\hline PREACTW & reactant partial pressure at wall (output) $\left[\frac{N}{\mathrm{~m}^{2}}\right]$ \\
\hline $\begin{array}{l}\text { LGAS } \\
\text { LDM } \\
\text { TYPINGR }\end{array}$ & $\begin{array}{l}\text { number of gases } \\
\text { dimension of arrays in call sequence } \\
\text { type of ingress }\end{array}$ \\
\hline
\end{tabular}

Subroutine RUFF2OX provides the Newton-Raphson solution for the two simultaneous equations for the chemical reaction as given in Section 3.4.4. The call variable XX presents the variable vector consisting of dimensionless free stream and surface reactant partial pressure.

Subroutine HETCON solves the convective heat flows in the coolant channels, advancing within each channel in flow direction. Fluid average and fluid exit temperatures are also computed for each node, following the model of Section 3.4.2.

The call variable CALMDFX can be set by FLOXIEX or FLOXIOT to 'UP', 'DOWN' or 'ALL' to evaluate up-flow channels only, down-flow channels only, or all channels.

Subroutine PLENBAR sets up local plena inflow data and calls the plenum subroutine PLENSUM, which sums over inflows and averages outflow temperatures and gas compositions.

The call variable CALMDFX can be set by FLOXIEX or FLOXIOT to 'UPPER', 'LOWER' or 'ALL' to evaluate upper plenum only, lower plenum only, or both plena. When CALMDFX ='ALL' is set, the nodal gas densities as well as gas densities of entry and exit into the plena are also evaluated. 
Subroutine PLENSUM evaluates plenum inflow data to compute averages for temperatures of plenum outlet gas, gas compositions, and mole mass.

It's call sequence is as follows:

MOLAR,POSIN,BALANCED,WA,TH,CA,CAT,MOLM,NUAIN,NUAPL,THPL,THINFL, NUAINFL,ISET,ICHF,ICHFP,ICHPD,LGAS,LGASDD,LVLPRT,IRLA,PLENAM,PRINTU

where

MOLAR

POSIN

BALANCED

WA

TH

CA

CAT

MOLM

NUAIN

NUAPL

THPL

THINFL

NUAINFL

ISET

ICHF and ICHFP

ICHPD

LGAS

LGASDD

LVLPRT is a logical variable, if true, then component molar inflows are given in CA-array, or else they must be computed here.

is a logical variable, if true, then inflows in WA-array are positive, and outflows negative, otherwise, the reverse.

is a logical variable, if true, then the WA-array is expected to be balanced, i.e., $\Sigma W A=0$ (general mode for upper plenum). Otherwise, the core channel flows through the various core flow channels, $I=1, I C H F$, are summed and the return flow channel, ICHFP, is set to the negative of the summed core flows (general mode for lower plenum). mass flow array of gas mixture at all plenum connections to flow channels $[\mathrm{kg} / \mathrm{s}]$. (For sign convention, see variable POSIN above. Input except for WA(ICHFP), if BALANCED = false.)

fluid temperature at plenum connection to flow channels [K] (input for inflows, output for ICHFP, if BALANCED = false and WA(ICHFP) > O).

molar flow array for gas species at plenum connection to flow channels $\left[\frac{k m o l}{s}\right]$ (input if MOLAR = true; otherwise output)

total molar inflow rate into plenum of gas species

$$
\left[\frac{\mathrm{kmol}}{\mathrm{s}}\right] \text { (output) }
$$

mole mass of plenum gas mixture $\left[\frac{k g}{k m o l}\right]$ (output)

volume fraction array for gas species at plenum connection to flow channels (input for inflows, output for ICHFP, if BALANCED = false and WA(ICHFP) > 0).

volume fraction of gas species in plenum (output)

gas temperature of plenum gas mixture [K] (output)

gas inlet temperature to reactor loop [K] (input, input data record 3103D)

gas composition at inlet to reactor loop (input, input data record 3104D)

inflow/outflow control index array (output). ISET is $s e t=1$ for inflow and $=0$ for outflows.

flow channel indices

dimension of all arrays in call sequence that use ICHF index

index for number of gas species

dimension of all arrays in call sequence that use LGAS index

output print control 
IRLA

PLENAM

PRINTU array for index storage location to compute of mixture properties from spline functior.s (see HHMSPL and HHISPL calls) character variable for plenum name, used in printed output. character control for printed output. PRINTU = 'HR' flows in $[\mathrm{kg} / \mathrm{hr}]$, else in $[\mathrm{kg} / \mathrm{s}]$.

Subroutine RENCH checks the largest Reynolds number in the parallel channel flow network, which is generally printed in the printed output file. This check is primarily for ingress scenarios, where laminar flow in the coolant channels has always been observed, and is assumed to exist as part of the model.

Subroutine FLUINUP simulates varying coolant inlet temperatires as the gas exit temperatures vary, delayed by a time-lag prescribed by the user (input record 3103D). For most cases, this feature will not be desired, and is bypassed by setting the time-lag $>10^{6}$.

Block Data FLOXDAT contains sume constants which are used by more than one subroutine.

\subsubsection{PKIN Subroutines}

The point kinetics module is generally called from THATCH or from SYMELAN. It can also be called from a free-standing main program, PKINRUN, which requires the availability of a THATCH output file of core temperatures. This mode has not been used lately, and PKINRUN would require some updates. It is currently not part of the PKIN module, but could be updated and added within days, if desired.

Subroutine PKINQ with entries PKININ, PKINEX, PKINOUT, PKINRD, and PKINWR is the major subroutine of this module.

In entry PKININ, the module is initialized, the input data records are processed, and the initial $\mathrm{Y}$-vector is loaded by call to subroutine CALIC. The first twelve locations of the Y-vector are the integration variables, following the model of Section 3.5. For convenience, 14 more variables are added to this vector before it is written to output files, and when it is read for restart or for post processing. The total Y-vector is given in Table 4-10.

The call sequence for PKININ is as follows:

CALMODE,T1,POWKIN,RHOINT,IKINMX,IIN,THCORAV,THREFAV,THFUELAV,THFLUDAV

where

\begin{tabular}{|c|c|c|}
\hline \multirow{4}{*}{ ALMODE } & \multicolumn{2}{|c|}{ character variable controlling run mode } \\
\hline & $=$ 'ТHATCH' & THATCH run with point kinetics \\
\hline & $=$ 'SYMELAN' & SYMELAN run with point kinetics \\
\hline & $=$ 'COMBIN' & $\begin{array}{l}\text { THATCH run coupled with SYMELA } \\
\text { and using point kinetics }\end{array}$ \\
\hline & & \\
\hline & \multicolumn{2}{|c|}{ dimensionless fission power (output) } \\
\hline
\end{tabular}


Table 4-10

Y-Vector of the Point Kinetics Module

\begin{tabular}{|c|c|c|c|}
\hline Location & $\begin{array}{l}\text { Variable } \\
\text { Name }\end{array}$ & Units & Description \\
\hline 1 & & & $\begin{array}{l}\text { Non-dimensional fission power } \\
\mathrm{P} / \mathrm{P}_{\mathrm{o}}=\mathrm{N} / \mathrm{N}_{\mathrm{o}}\end{array}$ \\
\hline $2-7$ & & & $\begin{array}{l}\text { Non-dimensional delayed neutron } \\
\text { fractions } \mathrm{Ci} / \mathrm{No}\end{array}$ \\
\hline $\begin{array}{c}8 \\
9 \\
10 \\
11\end{array}$ & & & $\begin{array}{l}\text { Non-dimensional iodine concentration } \\
\mathrm{I} / \mathrm{I}_{\mathrm{o}} \\
\text { Non-dimensional xenon concentration } \\
\mathrm{X} / \mathrm{X}_{\mathrm{o}} \\
\text { Non-dimensional promethium concentra- } \\
\text { tion } \mathrm{Pm} / \mathrm{Pm}_{\mathrm{o}} \\
\text { Non-dimensional samariium concentration } \\
\mathrm{S} / \mathrm{S}_{\mathrm{o}}\end{array}$ \\
\hline 12 & & $\mathbf{S}$ & $\begin{array}{l}\text { Integral of scaled fission power } \\
\text { (see Equ 3.5-19) }\end{array}$ \\
\hline $\begin{array}{l}13 \\
14 \\
\end{array}$ & $\begin{array}{l}\text { THCORAV } \\
\text { THREFAV } \\
\end{array}$ & $\begin{array}{l}\mathbf{K} \\
\mathbf{K} \\
\end{array}$ & $\begin{array}{l}\text { Core average temperature } \\
\text { Reflector average temperature }\end{array}$ \\
\hline $\begin{array}{l}15 \\
16 \\
17 \\
18\end{array}$ & $\begin{array}{l}\text { SUMRHOC } \\
\text { RHOREF } \\
\text { RHOXEN } \\
\text { SUMRHO } \\
\end{array}$ & & $\begin{array}{l}\text { Sum of core reactivity feedbacks } \\
\text { Reflector reactivity feedback } \\
\text { Xenon reactivity feedback } \\
\text { Sum of all reactivity feedbacks }\end{array}$ \\
\hline 19 & THCORMX & $\mathbf{K}$ & Maximum core temperature \\
\hline $\begin{array}{l}20 \\
21 \\
\end{array}$ & & $\begin{array}{l}\mathrm{W} \\
\mathrm{W}\end{array}$ & $\begin{array}{l}\text { Decay heat } \\
\text { Fission power }\end{array}$ \\
\hline $\begin{array}{l}22 \\
23 \\
24\end{array}$ & $\begin{array}{l}\text { RHOSAM } \\
\text { RHOWAT } \\
\text { RHODOP }\end{array}$ & & $\begin{array}{l}\text { Samarium reactivity feedback } \\
\text { Water reactivity feedback } \\
\text { Doppler reactivity feedback }\end{array}$ \\
\hline $\begin{array}{l}25 \\
26 \\
\end{array}$ & & & $\begin{array}{l}\text { Not used } \\
\text { Not used }\end{array}$ \\
\hline
\end{tabular}


RHOINT logical variable controlling execution mode, set based on input variable COMPMOD (see below)

IKINMX maximum number of iterations in point kinetics loop (from input record 5001D) (output)

IIN input data file identifier (currently not used)

THCORAV average core temperature [K] (input)

THREFAV average reflector temperature [K] (input)

THFUELAV average fuel temperature [K] (input)

THFLUDAV average core coolant temperature [K] (inp't)

The input variable COMPMOD controls the coupling between point kinetics and thermal/flow computations, as well as the internal update of reactivity feedbacks during time step integration.

The modes currently provided and their effect on the computations are as follows:

COMPMOD = 'Iterate'

This is the mode generally used in most applications. Logical variable RHOINT is set to true; the point kinetics ard core temperature computations are solved iteratively in the point kinetics loop of TIMSOL in THATCH, as shown in Figure 4-1 (or TIMLOP in SYMELAN for free-standing SYMELAN runs, Figure 4-8). In this case, all reactivity feedbacks are computed internally during time integration.

COMPMOD = 'CONSTCOR'

In this case, there is no coupling between point kinetics and core temperature computations. RHOINT is set $=$ false, and the input value of IKINMX is overridden by IKINMX $=1$. However, the feedbacks for xenon, samarium and water are computed internally, controlled by RHOXINT = true.

COMPMOD $=$ 'CONSTALL'

None of the feedbacks are updated internally during time integration, and the last time step values are used for all of them (RHOXINT = false).

PKININ also calls subroutine RHOOFTH for initialization of the temperature coefficients for core reactivity feedbacks. as follows:

In entry PKINEX, the time integration from time $\mathrm{T} 1$ to time $\mathrm{T} 2$ is executed. Its call sequence is T1,T2,POWKIN,IKINIT,KINGO,THCORAV,THREFAV,THFUELAV,THFLUDAV

where

$\mathrm{T} 1$ and $\mathrm{T} 2$

POWKIN

IKINIT beginning and end time for current time integration step [s] (input) average non-dimensional fission power between $T 1$ and $T 2$ (output) point kinetics loop iteration counter 
KINGO

THCORAV

THREFAV

THFUELAV

THFLUDAV logical variable controlling point kinetics loop convergence. Was set $=$ false before the point kinetics loop, and will be set $=$ true here, if convergence check in PKINEX shows convergence, for terminating point kinetics loop.

average core temperature [K] (input)

average reflector temperature [K] (input)

average fuel temperature $[\mathrm{K}]$ (input)

average core coolant temperature $[\mathrm{K}]$ (input)

The PKINEX routine sets the required reactivity feedback data depending on the mode of execution, and calls the SOLTRAN routine, which, in turn, calls the IMSL ODE integration routines DIVPAG or DGEAR for integration from T1 to T2. A flow chart of the PKINEX section of PKINO is shown in Figure 4-7.

Entries PKINRD ard PKINWR will read one record containing a Y-vector (Table 4-8) from File 15, or write one record with a Y-vectc: to File 14. These records are used in THATCH for reading and writing THATCH output files. (Variables $Y_{19}$ to $Y_{21}$ are only set in PKINOUT, and therefore, will only be available for PKINWR, if the call frequency is equal or coarser than that for PKINOUT, and if PKINOUT calls, within a time step, precede calls to PKINWR. This is true for THATCH subroutine TIMSOL.) Entry PKINIOUT will write a record of time and Y-vector to output File 33 for later use in THPROCS.

Subroutine CALIC is called during initialization to compute some of the initial values in the Yvector and other constants. It prints output of some of the initial data.

Subroutine PKSOL and Function SEXPAM are not used.

Subroutine UERTXXX is used to bypass the corresponding IMSL error subroutine called by DGEAR.

Block Data BLDPINK contains scme of the input data for the point kinetics model, which can be overridden by the use of input records 5004 to 5010D. (The exception is variable XJPF $=3.2042 \times 10^{-}$ $11 \mathrm{~J} /$ fission.)

Function RHOCORF evaluates core reactivity feedbacks, drawing on the design temperature coefficients, which are given in subroutine RHOOFTH. The following is the call sequence for RHOCORF:

\section{MODE,CORAVG,THCORAV,THFUELAV,THREFAV}

where

MODE

CORAVG is the character variable RHOMODE of input data record 5001D. Currently, it must be set to "INTEGR' (see RHOOFTH below).

is a logical variable controlling the computation mode of the Doppler feedback. It is also user-specified on input record $5001 \mathrm{D}$. 


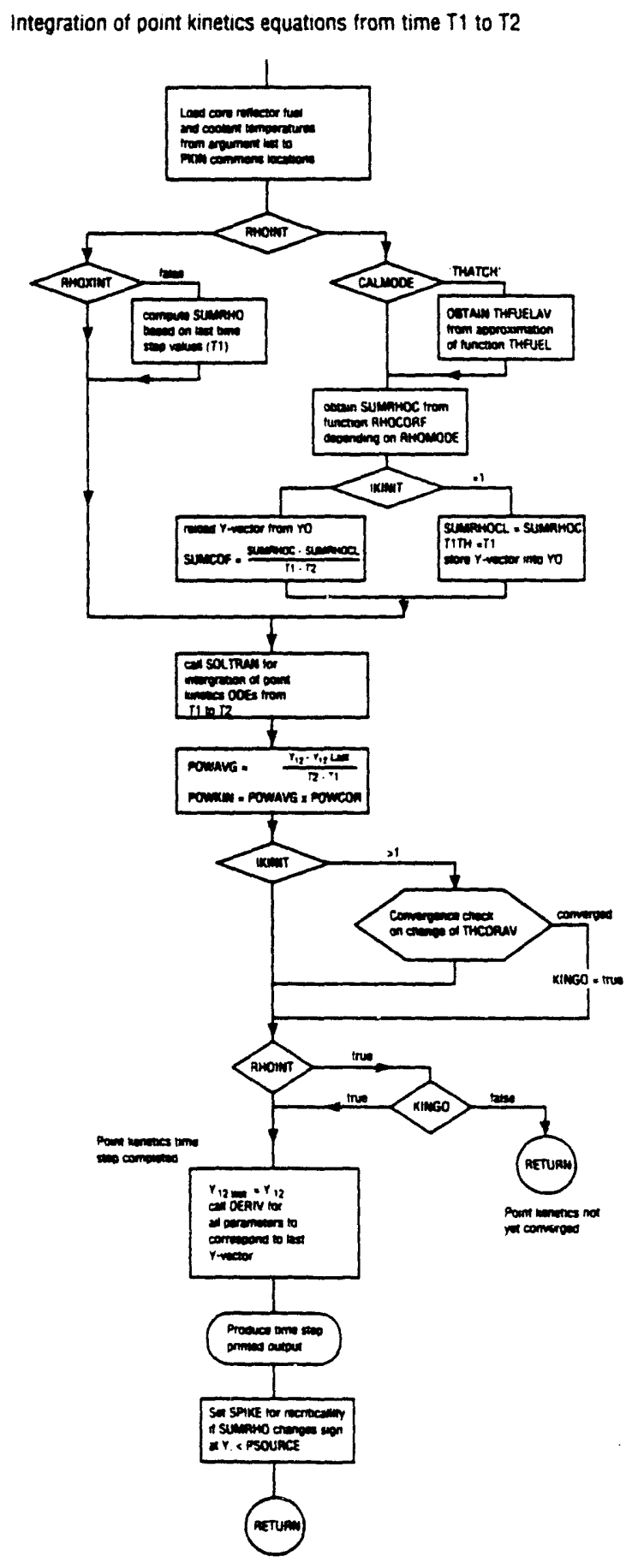

Figure 4-7 Flow Chart of Point Kinetics Time Integration in PKINEX 


\section{THCORAV}

THFUELAV

THREFAV

are the average temperatures for the core, fuel, and reflector $[\mathrm{K}]$

This function obtains the component feedbacks from subroutine RHOOFTH and returns the sum of core feedback coefficients as follows:

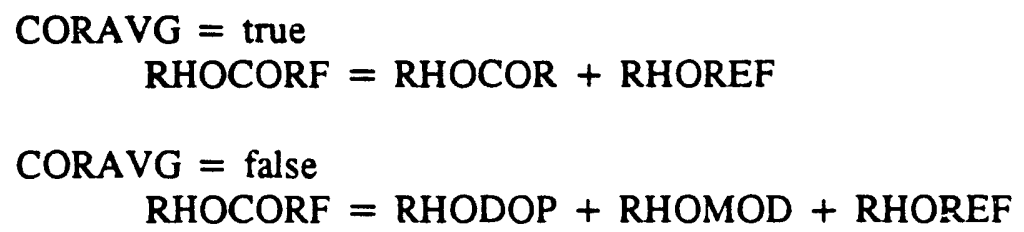

Subroutine RHOOF [H contains the PSID temperature coefficients for the core for EOC-EQ conditions. For the integrated mode, which is generally used, the local coefficients are used to compute integrated coefficients, based on a cold reference temperature of $200^{\circ} \mathrm{C}$

$$
\tilde{c}(\theta)=\frac{1}{\theta-\theta_{\text {ref }}} \int_{\theta_{\text {ref }}}^{\theta} c(\theta) d \theta
$$

and in terms of these, at any temperature $\theta$, the change in reactivity with respect to steady state is

$$
\Delta \rho=\rho-\rho_{S t S t}=\bar{c}(\theta)\left[\theta-\theta_{r e f}\right]-\tilde{c}_{S t S t}\left[\theta_{S t S t}-\theta_{r e f}\right]
$$

On any call to RHOOFTH, the average core temperatures must be available in Common RHO3. For initialization, these must be the initial steady-state temperatures, not restart temperatures. During initialization, called from PKININ, the integrated coefficients and the steady-state terms of Equation (4.2-2) are computed for Doppler, moderator, total core, and reflector coefficients.

During the transient, called from the function RHOCORF, the $\Delta \rho$ of Equation $4.2-2$ is referred to as RHODOP,RHOMOD, RHOCOR, or RHOREF in subroutine RHOOFTH and in function RHOCORF.

The actual ODE-integration is carried out by the IMSL ODE-solvers, DIVPAG or DGEAR. These require the availability of a subroutine to compute the derivative vector and, if MITER $=1$ is specified with integration control input (Input Record 5003D), also a routine for the Jacobean matrix.

Subroutines DERIV and JACK compute for a given time and Y-vector, the corresponding derivative vector and the Jacobean matrix using the equations of Section 3.5.1.

Function SUMRHOF is called during time integration by subroutines DERIV and JACK to obtain the required reactivity feedbacks. 
In the case of iterative solution of point kinetics and solid core temperatures, RHOINTL = true, the core reactivity changes were assumed to vary linearly between times $\mathrm{T} 1$ and $\mathrm{T} 2$ (see Section 3.5.2). These are then computed, based on last iteration values, for the time interval $T 1$ to $T 2$. The xenon and samarium feedbacks are computed based on their concentrations (in the Y-vector), and the water reactivity feedback is obtained from Function RHOWATF as a function of time. During non-iterative computations, last time step values for core reactivities are used, and the other terms are updated in SUMRHOF as described above, unless COMPMOD was specified as 'CONSTALL', in which case all reactivities remain constant during iniegration, at last time step values.

Function RHOWATF computes the water reactivity based on PSID data. In the given correlation for the reactivity change, RHOWL, variable XMWAT is the mass of water in the active core [kg]. XMWATTH is the maximum mass of water that the core could contain at safety relief valve pressure and given average coolant temperature, assuming $100 \%$ water concentration and considering the superheated steam to be an ideal gas. The "hard-wired" constant $C=1.677226 \mathrm{E} 5$ represents active core gas volume times pressure divided by the gas constant for water. $\left(\mathrm{V}=10.76 \mathrm{~m}^{3} ; \mathrm{p}=72 \times 10^{5} \mathrm{~N} / \mathrm{m}^{2}\right)$.

The actual mass of water in the core, XMWAT, is obtained by the ramp data of Input Record 5002D, which include an initial time of no ingress and a "ramp time" over which the water mass rises linearly to the maximum theoretical value.

The input parameter RHOWPAR (Input Record 5002D) is used for parametric variation of water reactivity effects, and multiplies the values obtained from the above reactivity correlation.

Function THFUEL is used in THATCH runs if separate consideration of Doppler and moderator coefficients is desired (CORAVG $=$ false). Because the relatively large THATCH nodes cannot provide separate values for fuel and moderator temperatures, a simple approximation for this effect is applied here, setting the average fuel temperature above the average core temperature to an input value DTHO (Input Record 5001D), which represents the full-power, steady-state difference between average fuel temperature and average core temperature. During a transient, DTHO is then multiplied by the dimensionless core power $\mathrm{P} / \mathrm{P}_{\mathrm{o}}$ at time $\mathrm{t}$ to obtain the current fuel to average core temperature difference. (SYMELAN computes its own average fuel temperatures and does not call this function.)

苚.

\subsubsection{Symelan Subroutines}

The SYMELAN submodule can be executed as a free standing module from dummy subroutine SYMELAN, or by calling several other of its subroutines from THATCH.

In free standing transient runs (SYRUNMD = 'Transient'), SYMELAN will call TIMLOP, the main time-loop routine of this module. (SYRUNMD = 'TEMPOUT'), another free standing mode that can be used, will only access a previous SYMELAN temperature output file and print the desired sections of the temperature history.

Subroutine SYMELAN serves as an effective main program to call the free-standing SYMELAN code by setting CALMODE =SYMEL. After call to the input routine SYMELIN, SYMELAN will branch, depending on input variable SYRUNMD, either to the transient routine TIMLOP or to the routine TEMPRIN to process a previous SYMELAN temperature output file. Currently, the free-standing mode uses its own input data and bypasses the unified input option of THATCH. However, there should be no problem in accessing this option, and we plan to implement this feature in the near future. Following 
a free-standing transient run, SYMELAN calls FILEFIN to finalize a SYMELAN plot output file with output data from SYMELAN and PKIN for immediate plotting or summary output printing. The file format will be given below.

Subroutine TIMLOP contains the time loop of SYMELAN, and it corresponds to TIMSOL of THATCH, but is much simpler. The time loop is shown schematically in Figure 4-8.

Subroutine SYMELIN is the input routine, reading input data, initializing various code sections and reading the specified initial temperature files. It also calls SYMELDT for loading the symmetry element description based on the chosen nodalization. For combined THATCH/SYMELAN runs, the interface routine is THSYMIN (see THATCH subroutines). In the SYMELAN free-standing mode, the point kinetics are initialized by determining average temperatures from subroutine TEMPAG and calling PKININ.

Subroutine SYMTPIN will read and load initial temperature files from a prescribed input file NIN searching for a time record at time TREAD.

Subroutine SYMELDT initializes the geometry of symmetry element. Node volumes, heat transfer surfaces and center distances of nodes are computed based on the selected nodalization via data specified by users for:

$\begin{array}{ll}\text { NOFUCOL } & \text { number of fuel column nodes } \\ \text { NOGRCOL } & \text { number of graphite column nodes } \\ \text { MOROW } & \text { number of node rows nodes }\end{array}$

(See Input Record 4006D)

The basic geometry data specified in Block Data SYMDAT are used. Internal units in this routine are in [mm], and all areas and volumes are expressed per unit length. For use in the remainder of SYMELAN, all data are then converted to units of [m], again expressed per unit length.

Subroutine TEMPSYM obtains the solution of the temperature field of the symmetry element for each axial node region in each parallel flow channel. Its call sequence is as follows:

$$
\text { T, DT, CALMODE, POWKIN, IDEBUG }
$$

where

$\begin{array}{ll}\text { T } & \text { new time }[s] \\ \text { DT } & \text { time step [s] } \\ \text { CALMODE } & \text { character control variable } \\ & \text { 'SYMEL' for free standing SYMELAN runs } \\ & \text { 'COMBIN' for THATCH/SYMELAN runs } \\ \text { POWKIN } & \text { dimensionless fission power }\left(P / P_{o}\right) \\ \text { IDEBUG } & \text { debug print control (normally }=0) .\end{array}$

The average volumetric heating rate at time $T$ is either obtained from POWKIN, the point kinetics output, or from POWPROF, a utility routine that is user-supplied (see page 4-81). 


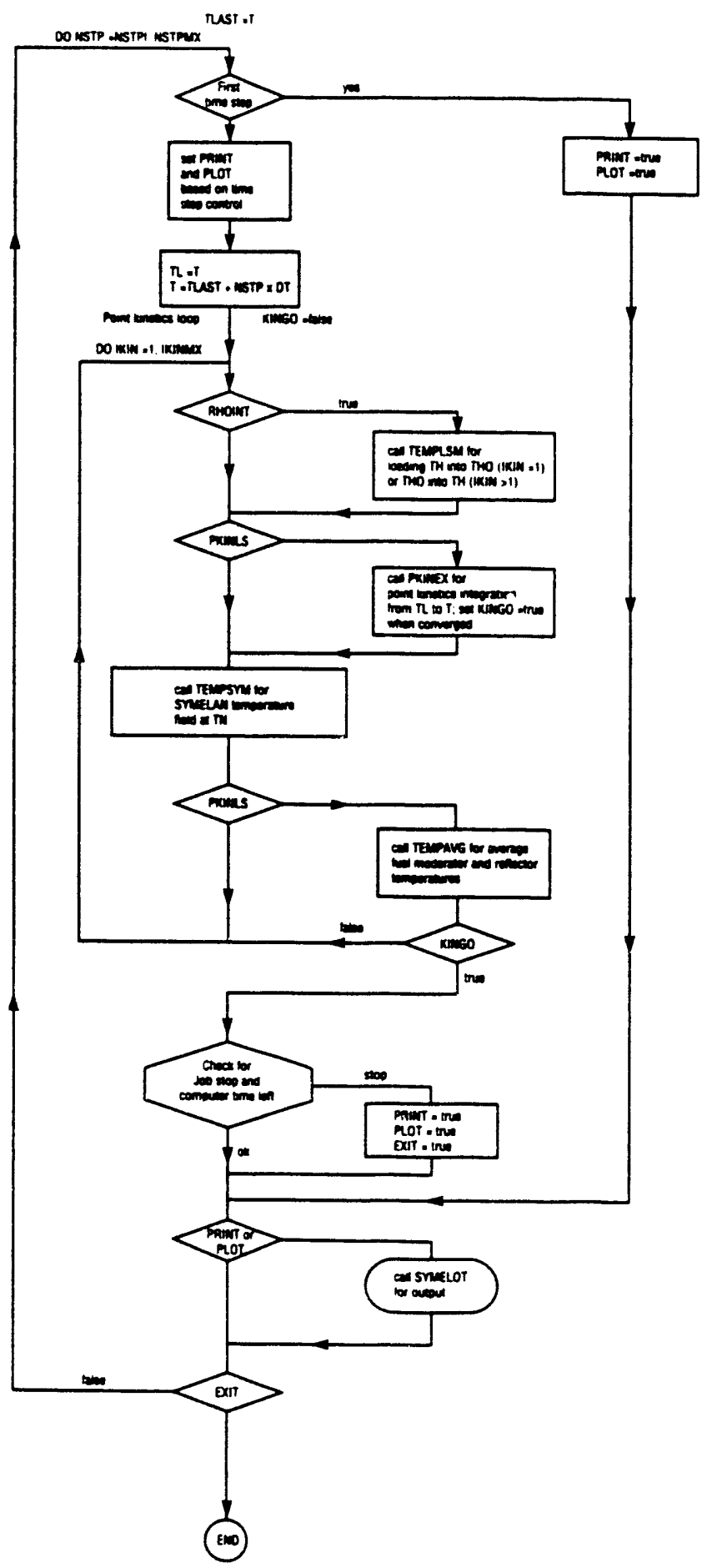

Figure 4-8 Flow Chart of TIMLOP Time Loop in SYMELAN 
After a call to subroutine PROPLOD for property and local transfer data, the coefficients for the solution of the simultaneous sets of equation sets are obtained from calls to subroutines COEFFLD or COEFFL2, depending on the number of node rows in the nodalization.

The temperature field is obtained by solving the sets of simultaneous equations, solving simultaneously for all symmetry element nodes of each parallel flow channel for one axial node region. Fluid exit temperatures, convective heat flows, and node surface temperatures are then computed for the axial node region of each parallel flow channel. Thereafter, the solution is repeated for the next axial node region, stepping down through the core in flow direction.

Subroutines COEFFLD and COEFFL2 load the simultaneous equation coefficients for single-node row nodalizations or for two-node row nodalizations, respectively, using the geometry data provided by subroutine SYMELDT, and the property and heat transfer arrays from subroutine PROPLOD.

Subroutine PROPLOD loads property arrays and other data on flow and heat transfer at the beginning of each time before coefficients are loaded in COEFFLD or COEFFL2.

In the computation of Reynolds numbers and heat transfer coefficients, pure helium is assumed as the coolant. The coefficient of convective heat transfer is obtained from subroutine HETCOF of GENLIB which models Equations (3.4-23 and 24). The fuel to graphite interface resistance is obtained from function HFUGRF, and the solid properties are obtained from function PROPT via calls to CONDFSM and ROCFSM (see below).

If, axial conduction is requested per user option, $\mathrm{AXCON}$, in the free-standing mode, the explicit term for the right-hand side of Equation (3.6.4) will be computed here and loaded into the QUIBLCON array, for use in the coefficient load routines. In combined THATCH/SYMELAN runs, this array will be loaded from data on axial conduction computed by THATC' $\boldsymbol{H}$.

Function PROPF with entries CONDFSM and ROCFSM, provides thermal conductivities and thermal capacitance, for fuel and graphite to SYMELAN. The call sequence for PROPF is as follows:

$$
\text { TH, MAT }
$$

where

$\begin{array}{ll}\text { TH } & \text { temperature }[\mathrm{K}] \\ \text { MAT } & \text { material index } \\ & 1=\text { fuel } \\ & 2=\text { graphite }\end{array}$

The returned values of CONDFSM and ROCFSM are in SI units $[\mathrm{W} / \mathrm{mK}]$ and $\left[\mathrm{J} / \mathrm{m}^{3} \mathrm{~K}\right]$.

For fuel, the data from GA-A12985 are used, as presented in MATLIB. For graphite, the data for irradiated $\mathrm{H}-451$ are used, with radial thermal conductivity.

The radial conductivity was also used to evaluate the axial conduction term in free-standing runs of SYMELLAN. Strictly speaking, this evaluation is not correct. As Figure A-3 of Kroeger [1984] shows, the axial conductivity is slightly higher than the radial conductivity. This evaluation should be 
corrected in future revisions of the code; however, the effect of this correction is expected to be very minor because axial conduction is very small during the rapid transients for which SYMELAN is used.

Function HFUGRF models the gap heat transfer resistance of the fuel to graphite interface including conduction and radiation, as described in Section 4.6. Its call sequence is as follows:

THNFU, THNGR, NOITS, RES1, RES3, IDEBUG

where

$\begin{array}{ll}\text { THNFU } & \text { temperature of last fuel node }[\mathrm{K}] \\ \text { THNGR } & \text { temperature of first graphite node }[\mathrm{K}] \\ \text { NOITS } & \text { maximum number of iterations } \\ \text { RES1 } & \text { thermal resistance of } 1 / 2 \text { fuel node }\left[\mathrm{m}^{2} \mathrm{k} / \mathrm{W}\right] \\ \text { RES3 } & \text { thermal resistance of } 1 / 2 \text { graphite node }\left[\mathrm{m}^{2} \mathrm{k} / \mathrm{W}\right] \\ \text { IDEBUG } & \text { debug print control }\end{array}$

If NOIrS is specified $=1$, then the node temperatures will be used to evaluate the gap resistance. If NOITS is larger than 1, then iteration will occur and the gas resistance is computed based on the internal surface temperatures. The returned value of HFUGRF is in units $\left[\mathrm{W} / \mathrm{m}^{2} \mathrm{~K}\right]$.

Subroutine TEMPLSM stores and reloads the node temperature array of SYMELAN as required in the point kinetics loop. Prior to the first iteration, the temperature array THA is stored in a THOO array. On each subsequent iteration, THA is reloaded from THOO.

The following routines are available to compute average and maximum temperatures as well as "hot fractions" of the core, where "hot fractions" are the core volume fraction exceeding a specific temperature:

Subroutines AVERAGE and AVERAG2, with entries THMAX, TEMPMX2, HOTFRAC and HOTFRC2, compute average and maximum temperatures as well as hot fractions. AVERAGE, THMAX, and HOTFRAC are called for one-node row nodalizations, while AVERAG2, TEMPMX2, and HOTFRC2 are used for two-node row nodalizations. The following arrays are computed by these routines:

Node region averages for $\mathrm{I}=1$, ICHS and $\mathrm{J}=1$, JNODS:

average temperature of graphite

THAVGRN $(\mathrm{I}, \mathrm{J})$

average temperature of fuel THAVFUN (I,J)

Average values for each parallel flow channel $I=1$, ICHS with overall average stored in position ICHSP:

average temperature of graphite

average temperature of fuel

average temperature of coolant
THAVGR(I)

THAVFU(I)

THAVFL(I)

Maximum values for each parallel flow channel $I=1$, ICHS with overall maximum stored in position ICHSP: 
maximum temperature of graphite maximum temperature of fuel
THMXGR(I)

THMXFU(I)

Volume fractions exceeding specific values of THHOT array (four input values) in each parallel flow channel:

fraction of graphite exceeding temperature fraction of fuel exceeding temperature
THOT(K)

THOT(K)
FRACGR(I,K) FRACFU(I,K)

Array locations ICHSP contain the overall volume fractions exceeding the specified temperatures.

Subroutine TEMPAVG, with entry TEMPMAX is used by THATCH and SYMELAN to obtain average temperatures of the core and/or reflector for the evaluations on the feedback of point kinetics reactivity, as well as maximum core temperatures. TEMPAVG does not compute these, but controls the call to the appropriate routines, depending on the type of run, as controlled by variable CALMODE. The call sequence for TEMPAVG is as follows:

T, THCORAV, THREFAF, THFUELAV, THFLUDAV, CALMODE

and for entry TEMPMAX

\section{T, THCORM}

where

$\begin{array}{lll}\text { T } & \text { time }[\mathrm{s}] & \text { (input) } \\ \text { THCORAV } & \text { Average temperature of core }[\mathrm{K}] & \text { (output) } \\ \text { THREFAV } & \text { Average temperature of reflector }[\mathrm{K}] & \text { (output) } \\ \text { THFUELAV } & \text { Average temperature of fuel }[\mathrm{K}] & \text { (output) } \\ \text { THFLUDAV } & \text { Average temperature of coolant [K] } & \text { (output) } \\ \text { THCORMX } & \text { Maximum temperature of core }[\mathrm{K}] & \text { (output) } \\ \text { CALMODE } & \text { Character control variable } & \text { (input) } \\ & =\text { 'THATCH' THATCH run without SYMELAN } \\ & =\text { 'COMBIN' combined THATCH/SYMELAN run } \\ & =\text { 'SYMELAN' free standing SYMELAN run }\end{array}$

For free-standing SYMELAN runs, routine SYTMPAV is called for average fuel and coolant temperatures of the core, and SYTMPMX for maximum temperatures of the core. The reflector temperatures are not computed in SYMELAN, and a constant input value from Input Record 4013D is returned here.

For THATCH runs, the THATCH routine THTMPAV is called for average temperatures of the core and reflector. Average temperatures of the coolant and fuel are then set equal to the average temperature of the core. The maximum temperature of the core is obtained from entry THTMPMX in subroutine THTMPAV of THATCH. 
For combined runs, the average temperatures of the core, fuel, and fluid are obtained from SYMELAN temperatures via SYTMPAV, and the maximum temperature of the core via SYTMPMX. The average temperature of the reflector is obtained from routine THTMPAV of THATCH.

Subroutine SYTMPAV with entry SYTMPMX calls the above routine AVERAGE or AVERAG2, and loads from its output arrays, the average temperatures of the core, fuel, and coolant, or the maximum temperatures of the core. Note that the average temperature of graphite, not an average temperature of fuel and graphite is returned as the average temperature of the core.

Interfacing between SYMELAN, THATCH, and FLOXI is handled in the following subroutines:

THSYMIN (see THATCH, Section 4.1)

THSYMEX

SYMTHEX

THSYME2

SYMFLEX (Entry in SYMTHEX)

Their function is illustrated in Table 4-11.

Subroutine SYMTHEX is the first interface routine called from THATCH. It also computes the coordinate off-sets between the active core nodes of SYMELAN, THATCH, and FLOXI and stores some of these in SYMELAN/FLOXI combined common SYMFLX. The call sequence for subroutine SYMTHEX is as follows:

T, THS, IDIM, JDIM, IAFTLO, IAFTHI, JAFTLO, JAFTHI, IDEBUG

$\mathrm{T}$

THS

IDIM, JDIM

IAFTLO, IAFTHI,

JAFTLO, JAFTHI

IDEBUG time [s]

solid node temperature array of THATCH [K]

dimension of THS array in THATCH

range of active core nodes in THATCH

debug print control

Subroutine THSYME2 computes and transfers from THATCH to SYMELAN the core heat flow array. The call sequence to subroutine THSYME2 is as follows:

T, THS,IDMTH,JDMTH,CONAX,CONRA,IDIM,JDIM,IAFTLO,IAFTHI,JAFTLO,JAFTHI, IDEBUG

where $\mathrm{T}$

\section{THS}

IDHTH, JDHTH

CONAX

CONRA

IDIM, JDIM time [s]

solid node temperature array of THATCH [K]

dimensions of THS array in THATCH

axial conductance array [W/K]

radial conductance array $[\mathrm{W} / \mathrm{K}]$

dimension of CONAX and CONRA arrays in THATCH 


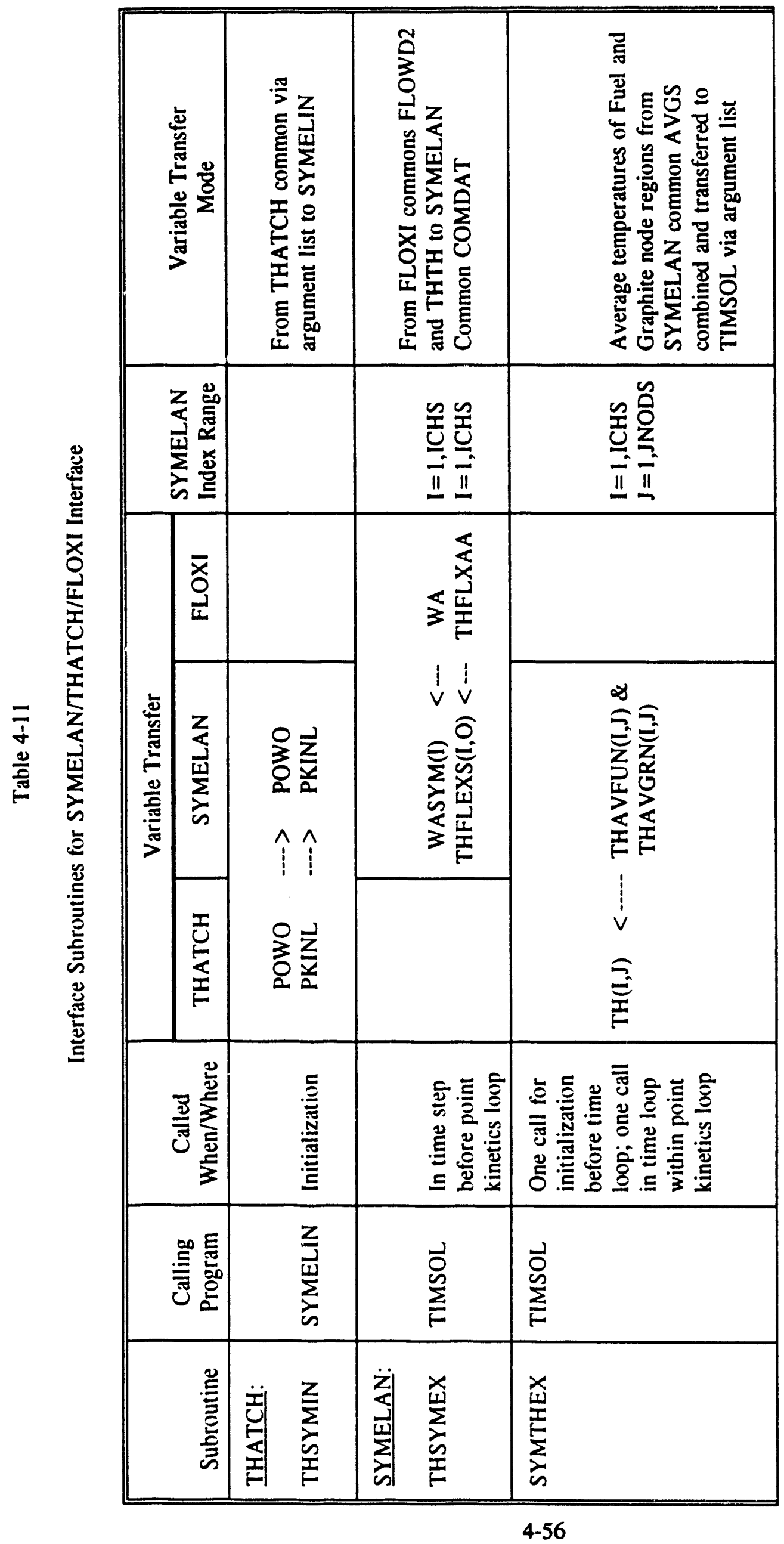




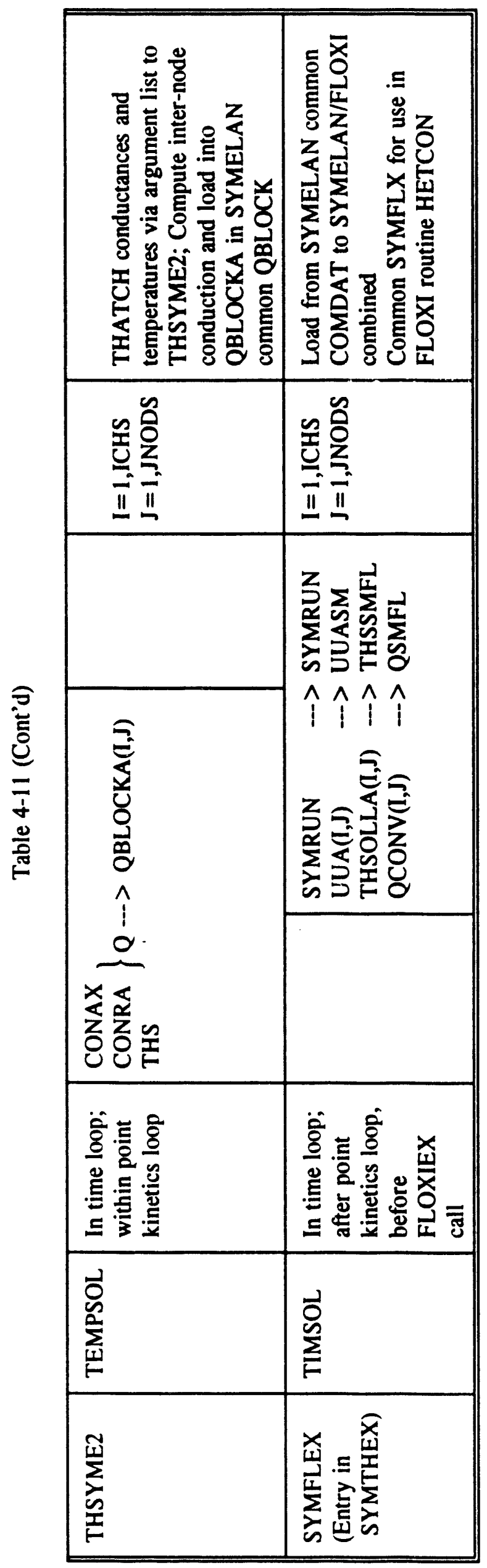


IAFTLO, IAFTHI,

JAFTLO, JAFTHI range of active core nodes in THATCH

IDEBUG debug print control

Subroutine SYMELOT provides printed output, as well as an output file in the plot file format, and an output file of complete temperature arrays. Its call sequence is as follows:

\section{T,PRINT,PLOT,WRITMP,IDEBUG}

where
T
PRINT, PLOT, WRITMP
time [s]
logical control variables to produce printed output, a plot file time record and/or a set of temperature
IDEBUG array records.
debug print control.

A list of the plot file time records for SYMELAN is given in Table 4-12, and Table 4-13 lists the temperature arrays on the temperature output file.

The total plot file for SYMELAN follows the format used in the THPROCS, the post processing module of THATCH. The file contains one title record (CHARACTER * 240) and record IREC,NOREC, where NOREC is the number of time records to follow. Each time record has the following format:

$$
\text { JNO, Time (s), (Y(I), I=1, IREC). }
$$

(See also subroutine FILEFIN below).

Subroutine TEMPRIN is called from the main program, SYMELAN, if run mode 'TEMPOUT' was specified in a free-standing SYMELAN run. In this case, TEMPRIN reads a previous SYMELAN temperature file and produces selected printed output at time points specified with the input data from SYMELIN.

Subroutine FILEFIN finalizes a plot file at the end of a SYMELAN run and adds the number of time records to record number 2 of the file (see SYMELOT above). If the run was with point kinetics, FILEFIN adds the point kinetics vector (26 locations) of Table 4-10 in locations $I=101$ to 126 to the SYMELAN vector of Table 4-12.

Subroutine TIMVAR permits the user to vary mass flow and fluid inlet temperatures as a function of time in free-standing SYMELAN runs. The call sequence for TIMVAR is as follows:

where

$$
\text { T,TLAG }
$$

$$
\begin{array}{ll}
T & \text { time }[s] \\
\text { TLAG } & \text { time-lag. }
\end{array}
$$

If TLAG is set $>10^{6}$, TIMVAR is bypassed. This time-lag is currently set to $10^{7}$ to bypass TIMVAR. 
Table 4-12

SYMELAN Plot File

Variable Vector on Time Records

\begin{tabular}{||c|c|c|l||}
\hline Variable Name & Units & $\begin{array}{c}\text { Number of } \\
\text { Entries* }\end{array}$ & \multicolumn{1}{|c||}{ Description } \\
\hline THAVFUC & ${ }^{\circ} \mathrm{C}$ & ICHSP & Average temperature of fuel \\
\hline THAVGRC & ${ }^{\circ} \mathrm{C}$ & ICHSP & Average temperature of graphite \\
\hline THAVFLC & ${ }^{\circ} \mathrm{C}$ & ICHSP & Average temperature of coolant \\
\hline THMXFUC & ${ }^{\circ} \mathrm{C}$ & ICHSP & Maximum temperature of fuel \\
\hline THMXGRC & ${ }^{\circ} \mathrm{C}$ & ICHSP & Maximum temperature of graphite \\
\hline QTOFL & $\mathrm{W}$ & ICHSP & Heat to Coolant \\
\hline FRACFU & & 4 & $\begin{array}{l}\text { Core fraction of fuel exceeding } \\
\text { specified temperatures }\end{array}$ \\
\hline FRACGR & & 4 & $\begin{array}{l}\text { Core fraction of graphite exceeding } \\
\text { specified temperatures }\end{array}$ \\
\hline THFLINC & ${ }^{\circ} \mathrm{C}$ & ICHS & Inlet temperature of coolant \\
\hline THFLEXC & ${ }^{\circ} \mathrm{C}$ & ICHS & Exit temperature of coolant \\
\hline THFLXAVC & ${ }^{\circ} \mathrm{C}$ & 1 & Average exit temperature of coolant \\
\hline POWL & & 1 & Dimensionless fission power P/P \\
\hline
\end{tabular}

* ICHS = Number of parallel flow channels

ICHSP $=$ Number of parallel flow channels plus one 
Table 4-13

SYMELAN Output File

of

Temperature Arrays

\section{Heading Records}

Record Number

1

Title (Character x240)

2

$\begin{array}{ll}\text { ICHS } & \text { Number of pit allel flow channels } \\ \text { JNODS } & \text { Number of axial nodes } \\ \text { NOFUCOL } & \text { Number of fuel column nodes } \\ \text { NOFUCOLP } & \text { nofucol }+1 \\ \text { NONODES } & \text { Number of symmetry element nodes } \\ \text { DEGK } & \text { Kelvirícentrigrade offset }=273.15 \mathrm{~K}\end{array}$

Time Records Repeated at Each Write Step

1

Time [s]

2

$(((T H A(N, J, I), i=1, I C H S), J=1, J N O D S), N=1$, NONODES $) \quad$ Solid Node Temperatures [K]

((THFLAP(I,J),I=1,ICHS), J = 1,JNODS) Average Temperature of the Node Region between Coolant and Surface [K]

4

((THSURFA(I,J),I=1,ICHS), $\mathrm{J}=1, \mathrm{JNODS})$ Surface Temperatures of the Coolant Holes of the Node Regions [K]

$((T H F L A(I, J), I=1, I C H S), \mathrm{J}=1, \mathrm{JNODS})$ Average Coolant Temperature of the Node Region [K]

((THFLEXS(I,J),I = 1,ICHS), J =0,JNODS) Coolant Exit Temperature of the Node Region [K] 
Subroutine POWPROF is called in TEMPSYM if this run is without point kinetics. The user can specify in POWPROF a time-varying, core-averaged volumetric heating rate $\left[\mathrm{W} / \mathrm{m}^{3}\right]$ as a fraction of QTRIPO, the initial, core average heating rate. Currently, as required for steady-state sirnulations, this subroutine keeps power constant.

Subroutine ENCHECK was used for checks of debug energy conversion. It has not been used lately, and may require updates if it were to be used again.

Functions FMAX2 and FMAX3 find the maximum entry in a two-or-three-dimensional array. The arrays are loaded into a one-dimensional array and ESVVLIB routine IDMAX is used to locate the maximum value. These routines are not used.

Block Data SYMDAT contains primarily the data on symmetry element geometry for the three internally provided nodalizations, plus a few other general constants.

\subsubsection{GENLIB Subroutines}

The GENLIB subroutines are general subroutines and functions for file handling, interpolation friction, and heat transfer correlations, after heat functions and other subroutines.

The following subroutines are not used within THATCH and its sub-modules and therefore, are not described here at this time:

$\begin{array}{lll}\text { ERFCF } & \text { PRINMAT } & \text { SOLV2 } \\ \text { FRACT } & \text { RATDRV } & \text { SQRTF } \\ \text { MATVEC } & \text { RATF } & \text { SQUAPR } \\ \text { MODFCT } & \text { RELOD } & \text { SUMNT } \\ \text { ORDER } & \text { REPACK } & \text { TRIPR }\end{array}$

The after-heat computations are done by subroutine AFTCOFS(IIN) and functions AFTANS(T),AFTER(T) and AFTPSID(T). IIN identifies the input file from which data are read (currently not used) and $\mathrm{T}$ is the time [s]. AFTCOFS serves for initialization and reads coefficients, as required (Input Records 1108 to 1111D). The logical variable AFTMODE controls which correlation to use.

AFTFR computes the dimensionless after-heat $\mathrm{P} / \mathrm{PO}$ as a function of time by linear interpolation in the log-log table of P/PO vs time, if AFTMODE is 'NOML', 'LTR', 'PSID1', or 'READ'. For AFTMODE = 'ANS' or 'PSID2', AFTFR calls functions AFTANS or AFTPSID, respectively.

The following subroutines represent friction factor and heat transfer correlations: 
Function FRIC(RE,IOPT) gives the friction pressure drop for smooth pipes as a function of Reynolds rumber, following Equation 3.2-23. Under IOPT $=1$, linear interpolation will occur between $\operatorname{Re}=2300$ and $\operatorname{Re}=8000$, i.e. the friction factor increases between the lower and upper limit of the transition region. With IOPT $=2$, the friction factor remains constant between $R e=1685$ and 8000 of $\mathrm{f}(8000)$. Option 2 is used in THATCH.

Function HETCOF (RE,PR,G,CP,NU,ST) is used for convective heat transfer coefficients of gases or gas mixtures in tubes or channels.

$\begin{array}{ll}\text { RE } & \text { Reynolds number (input) } \\ \text { PR } & \text { Prandtl number (input) } \\ \text { IJ } & \text { mass flux }\left[\mathrm{k} / \mathrm{m}^{2} \mathrm{~s}\right] \text { (inut) } \\ \text { IP } & \text { specific heat }[\mathrm{J} / \mathrm{kgK}] \text { (input) } \\ \text { NU } & \text { Nusselt number (output) } \\ \text { ST } & \text { Stanton number (output) } \\ \text { HETCOF } & \text { heat transfer coefficient }\left[\mathrm{W} / \mathrm{m}^{2} \mathrm{~K}\right] \text { (output) }\end{array}$

The correlation of Equation 3.4-23 is used, with linear interpolation in the transition region.

Function HHRADBL (TH1,TH2) computes the equivalent heat transfer coefficient between two black surfaces of temperatures $\mathrm{TH} 1$ and $\mathrm{TH} 2$ in units $\left[\mathrm{W} / \mathrm{m}^{2} \mathrm{~K}\right]$.

Function HHCAVTY computes a heat transfer coefficient of turbulent natural convection for a closed cavity with a hot and a cold vertical surface (used for reactor cavity). Its call sequence is as follows:

TH1,TH2,XL,XD,PT,LGAS

where
TH1, TH2
hot and cold surface temperatures [K] (input)
$\mathrm{XL}, \mathrm{XD}$ cavity height and width [m] (input)
PT gas pressure $\left[\mathrm{N} / \mathrm{m}^{2}\right]$ (input)
LGAS gas index (only LGAS $=1$ helium or LGAS $=2$ air are currently allowed) (input) HHCAVTY heat transfer coefficient $\left[\mathrm{W} / \mathrm{m}^{2} \mathrm{~K}\right]$ (output)

Function HHCONVF is an earlier correlation for convective heat transfer to air. It is still used in the air cooled RCCS model of PASCOLIB, and should be replaced by function HETCOF (above).

The following subroutines or functions are used for interpolation and function evaluations:

Subroutine TBLLK (X,XA,I1,IL,INDEX,IERR) will find in an array of monotonously increasing values $\mathrm{XA}$, the index of the last XA value below $\mathrm{X}$, and return it as INDEX. The index range of XA is Il to IL, and IERR is an error indicator.

$$
\begin{aligned}
\text { IERR } & =0 & & \text { INDEX was found; } X \text { was within range of table. } \\
& =-1 & & X \text { below range; INDEX set }=I 1 \\
& =1 & & X \text { above range; INDEX set }=\text { IL-1 }
\end{aligned}
$$


This routine works by dividing the index range into half at each step which is generally faster than a sequential search from II to IL.

Subroutine TBLLKSEC (X,XA,IL,INDEX,IERR) has the same purpose as TBLLK for an index range of XA from 1 to IL; however, the search is sequential here, which is sometimes preferable.

Subroutine TBLLKF (X,XA,I1,IL,INDEX,NAME) is a substitute call to TBLLK; the first five arguments of the call sequence are identical. TBLLKF calls TBLLK and handles the printing of the error message IERR $\neq 0$ on return. NAME is a character variable of up to 30 characters, and is used to identify the call to TBLLKF.

Function Spline2 (X,XA,IIP,C,IDMC,IRL,IERR,IDER) evaluates a cubic spline fit as defined by the dependent coefficient array $C$ of the spline function and independent variable vector $X A$. $X$ is the value at which the function is to be evaluated. IIP is the number of entries in XA; IDMC is the leading dimension of C; IRL is on output, the range in XA where X was found (INDEX of TBLLK). On return, an initial check is made, whether or not $X$ is still in the range of IRL, to bypass the table search if possible. IERR is the same error indicator as in TBLLK. IDER $=0$ results in the function evaluation, while for $\operatorname{IDER}=1$, the first derivative will be computed.

Subroutine REDCOF2 (XA,C,IDIM,II,IIP,NTAPE) reads a set of spline coefficients for a cubic spline fit from input file NTAPE.

The three file records on file NTAPE are the following:

$$
\begin{array}{ll}
\text { II } & \begin{array}{l}
\text { number of intervals in spline fit } \\
\text { independent variable at node points } 1 \text { to IIP, where IIP }=\text { II }+1 \\
(\mathrm{XA}(\mathrm{I}), \mathrm{I}=1, \mathrm{IIP})
\end{array} \\
\begin{array}{ll}
(\mathrm{C}(\mathrm{I}, \mathrm{M}), \mathrm{M}=1,4), & \text { spline coefficients. } \\
\text { I }=1, \mathrm{II}) & \text { the leading dimension of the C-array }
\end{array} \\
\text { IDIM }
\end{array}
$$

Subroutines FRITE, FCOND, and COMBINE handle plot files. The plot file format is described in Section 4.2.5 with the SYMELAN output file.

Subroutine FRITE (NSEG,NTPIN,NTPOUT,Y,IRECMX,FILTYPE) reads a plot file from NTPIN, reading only the entry IREC from the second record. FRITE then counts the number of time records, and writes a new file onto NTPOUT, including both IREC and NOREC on record number two. IRECMX is the dimension of $\mathrm{Y}$ in the calling program and must be larger than IREC. Currently, NSEG $=1$ and FILTYPE $=$ 'COLWISE'. range.

Subroutine FCOND reduces time records on plot files and eliminates points outside of a specified

The call sequence is as follows:

NSEG,NTPIN1,NTPOUT1,NOTAPES,NPTS,XMIN,XMAX,Y,IRECMX, NTPSCR1, NTPSCR2,MINREC 


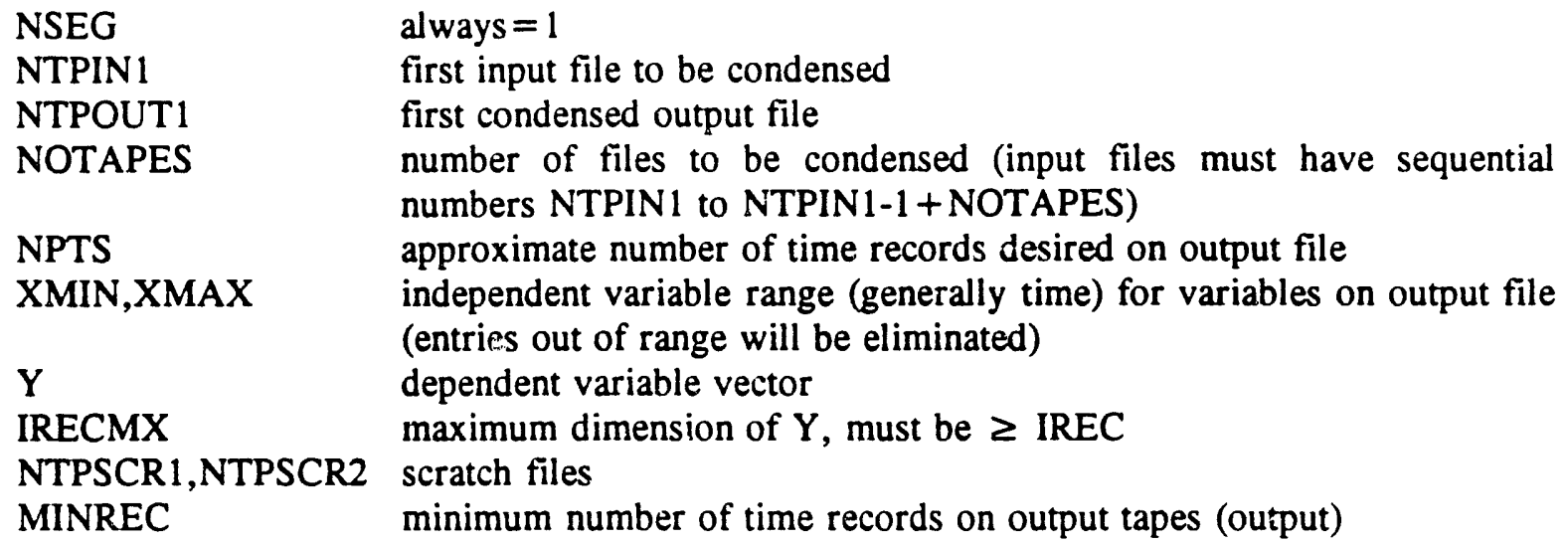

Subroutine COMBINE (NTIN1,NOTAPES,NTOUT,SCR,IRECMX,FILTYPE) will combine sequential plot file NTIN1 to NTIN1-1 + NOTAPES onto output file NTOUT. SCR is a scratch vector of size IRECMX $\geq$ IREC. FILTYPE is always 'COLWISE'.

Subroutine CONDENS, NORECF,POSITN, and RERITE are service functions for the three preceding routines and are never called by themselves. Details can be provided by the authors on request.

Subroutine RUFF (FCT,DFCT,SCALE,X,IER,EPS1,EPS2,NMX,LVLPRT) solves a single transcendental equation of the form $F(X)=0$, as prescribed in FCT and DFCT, using the NewtonRaphson method.

FCT(X) and DFCT(X) are external functions defining the function and its derivative in te-ms of the independent variable X. FCT and DFCT must be provided by the user.

Convergence is obtained when

$$
\frac{|F C T(X)|}{S C A L E}<E P S 1
$$

or

$$
|F C T(X)|<E P S 2
$$

NMX is the maximum number of iterations and LVLPRT provides debug print control.

Function EXPF $(X)$ computes $\operatorname{EXP}(X)$, but avoids overflow and underflow messages by limiting $\mathrm{X}$ to a machine-dependent range.

$(|X|<174$. on $1 B M 3090)$ 
Subroutine TRIDG (A,B,C,F,X,N1,N2) solves a tridiagonal system of simultaneous linear equations where
A left of diagonal array
B diagonal array
C right of diagonal array
$\mathrm{F}$ right hand side vector
$\mathrm{X}$ solution vector
N1 location of first coefficient in arrays
N2 location of last coefficient in arrays (i.e., N2-(N1-1) equations are being solved).

Subroutine TRIDG2 (A,B,C,F,N1,N2) is identical to TRIDG, except that the original right hand side vector and the solution vector show the same storage location as the F-array.

Subroutine SOLTRAN is a generalized routine for integration of a set of ODEs. It calls either DGEAR or DIVPAG and handles its input requirements and error analysis.

Its call sequence is as follows:

\section{TIN,TOUT,Y,YDOT,NEQ,CALLTYP,RWKSP,IIWK,IDEBUG}

Time integration is for NEQ equations from TIN to TOUT. Y is the variable vector and YDOT is its derivative.

Control variables INTMTHD, MTHGEAR, and MITER (user-specified, Record 5003D) are transferred to SOLTRAN via Commons.

Character variable CALLTYP is set by the calling program to control the integration process based on the different requirements of DIVPAG and DGEAR (see IMSL documentation). Table 4-14 describes the implementation of the selected ODE solver in SOLTRAN.

Table 4-14

Control of Integration in SOLTRAN via DIVPAG or DGEAR Based on Character Control Variable CALLTYP

\begin{tabular}{|l|l|l|}
\hline CALLTYP & \multicolumn{1}{|c|}{ DIVPAG } & \multicolumn{1}{c|}{ DGEAR } \\
\hline \hline 'FIRST' & $\begin{array}{l}\text { IDO=1 } \\
\text { Load PARAM array }\end{array}$ & $\begin{array}{l}\text { INDX }=1 \\
\text { HSTEP }=\text { HSTEPO }\end{array}$ \\
\hline 'RESET' & HSTEP=HSTEPO & $\begin{array}{l}\text { INDX }=-1 \\
\text { HSTEP }=\text { HSTEPO }\end{array}$ \\
\hline 'RESTART' & $\begin{array}{l}\text { IDO }=3 \\
\text { CALL DIVPAG( ) } \\
\text { IDO }=1\end{array}$ & $\begin{array}{l}\text { INDX }=1 \\
\text { HSTEP }=\text { HSTEPO }\end{array}$ \\
\hline 'LAST' & $\begin{array}{l}\text { IDO }=3 \\
\text { CALL DIVPAG ( ) }\end{array}$ \\
\hline
\end{tabular}


Block Data ODEDAT contains some of the control data and default values for the ODE integration.

Subroutine MAPRINT (A,NN,IOPD,ICOLPR0,MODE,NAME) is used for two-dimensional matrix printing. Matrix $A$ of NNxNN entries and of dimension IOPDxIOPD is printed, ICOLPRO columns at a time. If Mode $=0$ only the matrix is printed. If Mode $=1$, the row sum is added as the last column. The routine is primarily used by THRADLIB. Character variable NAME identifies the matrix.

Subroutine READIN (IIN,NAME) reads an input file from File 5, strips it of its comments (lines beginning with $\mathrm{Cbb}$ ), and copies it to file IIN. Character variable NAME (up to 80 characters) identifies the file being read.

Subroutine STRACE is a system-dependent routine that indicates an error, and traces back the error location (triggered on IBM3090 by arbitrarily dividing by zero).

Function Second $(T)$ provides elapsed execution time for code execution time control $(T$ is a dummy variable).

Function MINATOR (DUMMY) provides execution time remaining for this run, to permit orderly run termination.

\subsubsection{THTCHLIB Library Subroutines}

The THTCHLIB Library contains several THATCH utility subroutines which might also be used by other codes or in free-standing FLOXI or SYMELAN runs. Furthermore, it contains the subroutines used for the unified input option, computing current commercial MHTGR geometry data, based on the design data of Block Data COREDTS. None of the subroutines in THTCHLIB contain any of the THATCH Commons.

Subroutines CORFEL, COREDTS, CORINDT, and CORINPS process the input data required under the unified input option.

Subroutine CORFEL (NREAD, LVLPRT) is called by THATCH to initialize the unified input subroutines, if this option is requested (UNIFIN = true on Input Record 301D). NREAD identifies the input file (no longer used), and LVLPRT controls the amount of printed output.

Based on the core design data of the PSID, as stored in Block Data COREDTS, the data on fuel element geometry such as flow areas, heat transfer surfaces, fuel, moderator and coolant volumes are computed and printed. Active core-wide data of the same variables are produced and printed.

The radial core nodalization is treated by considering the following five zones:

1 the central reflector zone

2 to 4 three active core power zones (corresponding to the three radial power zones of the PSID)

5 one side reflector zone, comprising replaceable and permanent side reflectors.

Based on the input data specified by the user (Records 302D to 303D), the radial nodalization of each zone is set up for either equal cross section area or equal radial width of the nodes within a zone. 
If equal area were specified (SPACING='AREA'), then another option is available in the allocation of flow and heat transfer areas in the first of the three radial core power zones, provided that two radial nodes were specified for this zone. This region contains the fuel elements with Reserve Shutdown Control channels, which have less fuel and also less coolant holes. Optionally, this effect can be "smeared" over the whole inner power zone or just into the inner one of the two node rings.

Axially, the active core consists of ten fuel elements, while top and bottom reflector regions have data for four sections each. At the top, the first section represents plenum elements, and the other three represent the actual graphite top reflectors. At the bottom, the four sections have different flow and heat transfer areas for each section corresponding to the bottom reflector design. The unified input option permits the user to specify subdivisions into finer nodes. At this time, only the axial node spacing of the active core is supplied by the unified input option, with each fuel element being divided into a number of nodes of equal length specified by the user. For the reflector regions, the axial node spacing is currently not supplied by this option, but the flow and heat transfer surface areas are supplied (in FLOXI).

The unified input option includes the following radial and axial power profiles which the user can call for via Input Records 305D to 308D:

$\begin{array}{ll}\text { Radial 3-Zone } & \text { Fission Power Profile } \\ \text { Axial 3-Zone } & \text { Fission Power Profile } \\ \text { Axial 10-Zone } & \text { Fission Power Profile } \\ \text { Axial 10-Zone } & \text { Decay Heat Profile }\end{array}$

Aside from these profiles, which were specified in the PSID, the user can ask for 1-zone models, which imply uniform power distributions.

Block Data COREDITS contains the core design data as given in the PSID. The user has the option to override any of these entries, for instance for parametric studies.

Subroutine CORINDT, with entries CORINDM, CORINFL, and CORINSY, is called from THATCH, FLOXI, and/or SYMELAN to load specified core data computed in CORFEL to the appropriate THATCH or Submodule Commons.

Entry CORINDM (LABIN, XX, ID1, ID2) will load, depending on LABIN being = 'RIFA' or $=$ 'ZIFA', the radial node interface array for central reflector, active core and side reflectors, or the axial node interface array for the active core. The corresponding data are loaded into the $\mathrm{XX}$ array which is dimensioned XX(ID1:ID2).

Entry CORINFL (IC1, IC2, JC2, JC2, ICHF, JNODF, AFLOW, AFLOCOR, ASURFP, DHF, REFCORF, IDIM, JDIM) is called by FLOXI and will return to FLOXI the following:

IC1

IC2

JC1

JC2
THATCH radial node corresponding to the first parallel flow channel THATCH radial node corresponding to the last parallel flow channel THATCH axial node corresponding to the first axial flow channel node (upper plenum element)

THATCH axial node corresponding to the last axial flow channel node (last bottom reflector node) 
$\mathrm{ICHF}$

JNODF

AFLOW

AFLOCOR

ASURFP

DHF

REFCORF
Number of parallel flow channels in the core

Number of axial nodes in FLOXI

Flow cross section area $\left[\mathrm{m}^{2}\right],(I=1, \mathrm{ICHF}+1)$

Total active core flow area $\left[\mathrm{m}^{2}\right]$

Specific heat transfer surface area $\left[\mathrm{m}^{2} / \mathrm{m}\right],(I=1, \mathrm{ICHF}+1)$

Hydraulic diameter array $[\mathrm{m}],(\mathrm{I}=1, \mathrm{ICHF}+1 ; \mathrm{J}=1, \mathrm{JNOD})$

Correction array for heat transfer surface of the active core (see input record 3207D) $(\mathrm{J}=1, \mathrm{JNOD})$

IDIM and JDIM are the dimensions of the above arrays in FLOXI.

Entry CORINSY (ICHS, JNODS, AFLOW, AFLOCOR, ASURFP, RADFRACA, XX, IDIM, JDIM, ID1, ID2) is called by SYMELAN and will return to SYMELAN the following:

ICHF

JNODF

AFLOW

AFLOCOR

ASURFP

RADFRACA

$\mathrm{XX}$
Number of parallel flow channels

Number of axial nodes in FLOXI

Flow cross section area $\left[\mathrm{m}^{2}\right],(\mathrm{I}=1, \mathrm{ICHF}+1 \mathrm{I}=1, \mathrm{ICHS})$

Total active core flow area $\left[\mathrm{m}^{2}\right]$

Specific heat transfer surface area $\left[\mathrm{m}^{2} / \mathrm{m}\right],(I=1, \mathrm{ICHS})$

Fraction of core flow area (AFLOW(I)/AFLOCOR), (I=1,ICHS)

DZA array of axial node length in active core $[\mathrm{m}],(\mathrm{J}=1, \mathrm{JNOD})$

The XX array is dimensional X(ID1..J2). IDIM and JDIM are the dimensions of the other arrays in SYMELAN.

Subroutine CORINPW, with entry CORINPS, loads radial and axial power profiles to THATCH or SYMELAN, respectively.

The call sequence for CORINPW is as follows:

PHIR1, PMIR2, PHIX1, PMIX2, ID1, ID2

The call sequence to CORINP5 is the same, except that PMIR1 AND PHIX1 are omitted.

PHIR1 Radial power profile for after-heat I=1, NFLO

PHIR2 Radial power profile for fission power, I=1, NFLO

PHIX1 Axial power profile for after-heat $J=1, N O D Z$

PHIX2 Axial power profile for fission power $\mathrm{J}=1, \mathrm{NODZ}$

where NFLO is the number of radial active core nodes, and NODZ is the number of axial active core nodes. ID1 and ID2 are the dimensions of the radial and axial power profile arrays in the calling programs.

Subroutine NEWTON provides a Newton-Raphson solution for a set of non-linear, simultaneious algebraic equations, based on a function specified by a user, which supplies the Jacobean matrix and the residual vector. Its call sequence is as follows: 
Y, NEQ, EPSR, EPSY, NITMX, XJAC, IDJAC, RHS, DY, DYMXFR, IDEBUG, BIGR, ITERSNR

IER, FCTJAC, WRK, IDWRK, IIWRK, IDIIWRK, LINMETM, SPECSOL

where

$\mathbf{Y}$

NEQ

EPSR

EPSY

NITMX

XJAC

IDJAC

RHS

DY

DYMXFR

IDEBUG

BIGR

ITERSNR

IER

FCTJAC

WRK

IDWRK

IIWRK

IDIIWRK

LINMETH

Solutions vector (output)

(on call, $\mathrm{Y}$ must contain initial estimate of $\mathrm{Y}$ )

Number of equations (input)

Convergence criterion with respect to residual vector (input)

Convergence criterion with respect to change in $Y$-vector (input)

Maximum number of iterations allowed (input)

Jacobean matrix (computed for NEWTON in FCTJAC)

Dimension of XJAC (input)

Right-hand side vector (computed for NEWTON in FCTJAC)

Change in Y-vector (output)

Maximum fractional change permitted in $\mathrm{Y}$-vector at one iteration (input)

Debug print control (input)

Largest residual (computed in FCTJAC, output)

Number of iterations used (output)

Error indicator (output; $=0$ converged; $=3$ not converged)

Name of external subroutine supplying Jacobean matrix and residual vector (input). The call sequence of function FCTJAC is as follows:

$Y, N E Q, X J A C, R H S, B I G R, J B I G R$, IDJAC, IDEBUG

with all entries having the same meaning as in the call sequence for NEWTON.

Work array (needed with LINMETM $=3$ or 4 only; to be set in accordance with requirements of SLATEC or NAG library)

Dimension of WRK-array

Integer work array (size must be $\geq N E Q$ )

Dimension of IIWRK-array

Control index to select method for solving of linear system of equations.

Currently,

$=1$ ESVVLIB library routines DGEF and DGES

$=2$ not used

$=3$ NAG library routine, FO4ARF

$=4$ SLATEC library routine, DGEFS

$\geq 5$ special routine for particular structure o Jacobean, see SPECSOL below.

SPECSOL

name of subroutine for solving system of linear equations, using the of special sparse structure of Jacobean (currently used in PASCOL) The call sequence of the subroutine SPECSOL is as follows:

XJAC, IDJAC, RHS, NEQ, IDEBUG, with all entries as defined in the call sequence for NEWTON.

Subroutine GASLOAD (RGASHEL, RGASAIR, XMOLHEL, XMOLAIR) initializes the gas property routines of GASPRLIB and makes the following call variables available to the calling program: 


\section{RGASHEL Gas constant of helium [J/kgK] \\ RGASAIR Gas constant of air [J/kgK] \\ XMOLHEL Molar mass of helium $[\mathrm{kg} / \mathrm{kmol}]$ \\ XMOLAIR Molar mass of air [kg/kmol]}

Subroutine TIMER, with entries TIMEIN, TIMEOUT, and TIMEPR provides the execution time in specified program sections. A call to entry TIMEIN (JSECT) begins to count the execution time for program section JSECT until terminated by calling entry TIMEOUT (JSECT). A call to entry TIMEPR (NPR1, NPR2) prints the total time accumulated for program sections, NPR1 to NPR2. Currently, the routine is set up for Sections 1 to 16 for THATCH runs and Sections 21 to 24 for SYMELAN runs.

Function POLF (A, II, X) evaluates an II-order polynomial with $\mathrm{X}$ as the independent variable and coefficient array $A(0: I I)$.

Subroutine TIMEX (ITIME) loads character variable ITIME with time in format HH.MM.SS., and subroutine DATE (IDATE) loads character variable IDATE with the date in format MM/DD/YY.

\subsubsection{GASPRLIB Subroutines}

The mixture property routines for gases are contained in the GASPRLIB section of BIGLIB.

For the thermal properties, the tabulated internal energy data of Owczarek [1964] are contained in the BLOCK DATA section GASDATA together with mol weights, universal gas constant, and the energy of formation (required for combustion computations).

The gas indices used in THATCH are as follows:

$\begin{array}{ll}\ell=\quad 1 & \text { helium } \\ 2 & \text { air } \\ 3 & \mathrm{CO} \\ 4 & \mathrm{CO}_{2} \\ 5 & \mathrm{H}_{2} \mathrm{O} \\ 6 & \mathrm{H}_{2} \\ 7 & \mathrm{O}_{2} \\ 8 & \mathrm{~N}_{2}\end{array}$

The data are for all gases but helium and air. For helium the ideal gas correlations of Appendix D are used. During an initialization call to GASENER the gas properties of air are computed from those for $\mathrm{O}_{2}$ and $\mathrm{N}_{2}$. It should be noted that the water properties here apply for an ideal gas, i.e., for a gas of sufficient superheat and sufficiently low partial pressure.

The subroutine GASENER initializes the gas property library and returns for a specified temperature the internal energies of specified gases, obtained by linear interpolation of the data. The call sequence for GASENER is as follows:

TH

gas temperature $[\mathrm{K}]$ 


$$
\begin{array}{ll}
\text { ENA } & \text { internal energy array }\left[\frac{J}{k m o l}\right] \\
\mathrm{J} 1, \mathrm{~J} 2 & \text { indices to indicate for which gases internal } \\
\text { energies are to be obtained (from } \mathrm{J} 1 \text { to } \mathrm{J} 2, \\
\text { i.e. } 1,8 \text { means all, and } 5,5 \text { means } \mathrm{H}_{2} \mathrm{O} \text { only, } \\
\text { etc.) }
\end{array}
$$

In any code run which uses the GASPRLIB thermal properties an initialization call to GASENER must be included. In this call variable $\mathrm{J} 1$ must be set to zero. The other variables are then not used during this call.

The mixture or single gas properties are obtained from the following subroutines:

Single gas with properties from linear interpolation:

For Function CV1 with entries CP1, ENINT1, and ENTHAL1, the call sequence is as follows:

$$
\text { TH, IG }
$$

where
TH
IG
Gas Temperature [K]
Gas Species
The returned properties are in $\left[\frac{J}{k m o l}\right]$ or $\left[\frac{J}{k m o l K}\right]$.

Gas mixtures with properties from linear interpolation:

For Function CVMIX with entries CPMIX, ENINTRL, and ENTHAL, the call sequence is as follows:

$$
\text { TH, NUAL, IG1, IG2 }
$$

where

TH

NUAL

IG1,IG2

\section{Gas Temperature [K]}

Array of volume fractions defining gas mixture Range (from IG1 to IG2) for which mixture properties are desired.

The returned properties are in molar units, as given above.

Single gas with properties from cubic spline: follows:

For Function CV1SPL with entries CP1SPL, EE1SPL, and HH1SPL, the call sequence is as

TH, IG, IRL 
where

TH Gas Temperature [K]

IG Gas Species

IRL Range index in table data at last call (spline computation routine SPLINE2 will first test whether range remains the same, thus potentially bypassing range search.

Gas mixture with properties from cubic spline:

For Function CVMIXSP with entries CPMIXSP, EEMSPL, and HHMSPL, the call sequence is as follows

$$
\text { TH, NUA, IG1, IG2, IPL }
$$

where

TH Gas Temperature [K]

NUA Array of volume fractions defining gas mixture

IG1,IG2 Range (from IG1 to IG2) for which mixture properties are desired

IPL $\quad$ Range index in table data at last call (spline computation routine

SPLINE2 will first test whether range remains the same, thus potentially bypassing range search.

Function THFROMH will compute the gas temperature for a given gas mixture and enthalpy.

Its call sequence is as follows:

HH, XNUA, IDX, IG1, IG2, TH

where

$\mathrm{HH}$

enthalpy $\left[\frac{J}{k m o l}\right]$

XNUA volume fraction array

IDX dimension of XNUA array

IG1,IG2 range of gases in the mixture

(from IG1 to IG2)

TH initial estimate of temperature [K]

This subroutine uses the general purpose solver RUFF (see GENLIB) which calls the required functions HFCT and DHFCT.

Viscosit: Subroutines as follows:

Function VISGSPK, provides viscosities for a single gas or a gas mixture. Its call sequence is

TH, NUAL, IGAS, TYPINGR 
where

TH Gas temperature [K]

NUAL Gas volume fraction array

IGAS Number of gas species in current

$\begin{array}{ll} & \text { problem (input variable) } \\ \text { TYPINGR } & \text { Character variable specifying }\end{array}$

type of ingress (input variable)

The returned viscosity is in $\left[\frac{N s}{m^{2}}\right]$.

For IGAS = 1, the helium viscosity is returned. Otherwise, for TYPINGR = 'air', three gases are used, or for TYPINGR = 'water', six gases are loaded (see Appendix D). Function VISGAS is then called to obtain the mixture viscosities.

Function VISGAS computes the component viscosities of up to six gases and then calls Function VISMIX to solve for the mixture viscosities. This function is generally called by Function VISGSPK.

Function VISMIX will compute mixture viscosities from given component viscosities, volume fractions, and mole masses, using Equation D-13. This function is generally called by VISGAS.

$\begin{array}{ll}\text { Functions } & \frac{\text { VISHEL }}{\text { VISCO }} \\ \frac{\text { VISCO2 }}{\text { VISAIR }} \\ \frac{\text { VISH2 }}{\text { VISH2O }}\end{array}$

Compute the component viscosities of the respective gases.

Their call sequence is as follows:

\section{TH}

where

TH Gas temperature [K]

The returned compound viscosities are in $\left[\frac{N s}{m^{2}}\right]$

Thermal Conductivity Subroutines

Functions CONHEL

CONAIR compute the component thermal conductivities 
Their call sequence is as follows:

TH

where

TH Gas temperature [K]

The returned thermal conductivities are in $\left[\frac{W}{m k}\right]$.

Function CONDGM provides a thermal conductivity for a mixture of helium and air, weighted by volume fractions. Its call sequence is as follows:

\section{TH,NUAL,LGAS}

where

$\begin{array}{ll}\text { TH } & \text { Gas temperature [K] } \\ \text { NUAL } & \text { Gas volume fraction array } \\ \text { LGAS } & \text { Number of gases }\end{array}$

The returned conductivity is in $\left[\frac{W}{m k}\right]$.

\section{Subroutine PROPAIR}

With this subroutine, we obtain within one call viscosity, thermal conductivity and Prandtl number of air.

\subsubsection{MATLIB Subroutines}

MATLIB subroutines contain solid material property routines for various materials used in the thermal analysis of MHTGRs.

The properties of primary interest are thermal conductivities $k$ and thermal capacitances $\rho c$. The main function of the library PROPTY contains all material properties as entries.

All inputs and outputs are in SI units, i.e.,

Temnaratures (input) [K]

Thermal conductivities [W/mK]

Specific heat $[\mathrm{J} / \mathrm{kgK}]$

Density $\left[\mathrm{kg} / \mathrm{m}^{3}\right]$

Thermal capacitance $(\rho c)\left[\mathrm{J} / \mathrm{m}^{3} \mathrm{~K}\right]$

All property calls, unless otherwise noted, are functions with a single-call argument, the temperature.

The following functions for graphite thermal conductivities are available: 


$\begin{array}{ll}\text { CONRDGR } & \text { H327 radial } \\ \text { CONAXGR } & \text { H327 axial } \\ \text { CONRDG2 } & \text { H451 radial (based on LTR-3 data) } \\ \text { CONAXG2 } & \text { H451 axial based on LTR-3 data) } \\ \text { CON4RU } & \text { H451 radial unirradiated* } \\ \text { CON4RD } & \text { H451 radial irradiated* } \\ \text { CON4AU } & \text { H451 axial unirradiated* } \\ \text { CON4AD } & \text { H451 axial irradiated* } \\ \text { CONFERU } & \text { effective radial conductivity of unirradiated H451 fuel element* } \\ \text { CONFERD } & \text { effective radial conductivity of irradiated H451 fuel element* } \\ \text { CONR20F } & \text { radial conductivity of stack pole } 2020 \text { graphite* } \\ \text { CONA20F } & \text { axial conductivity of stack pole } 2020 \text { graphite* }\end{array}$

The following functions for graphite specific heat are available:

CPGREX older exponential fit for graphite specific heat

CPGRAF polynomial fit for graphite specific heat (preferred correlation)

The following function for thermal conductivity of boronated graphite pellets (upper plenum elements) is available:

\section{CONBORG Boronated graphite pellets*}

The following data for steel are available:

General carbon steel:

$\begin{array}{ll}\text { CONCS } & \text { thermal conductivity } \\ \text { ROCCS } & \text { thermal capacitance }\end{array}$

Medium carbon steel (reactor vessel):

$\begin{array}{ll}\text { CONMCS } & \text { thermal conductivity } \\ \text { ROCMCS } & \text { thermal capacitance }\end{array}$

Alloy 800 (core barrel, upper plenum shroud):

$\begin{array}{ll}\text { CON800 } & \text { thermal conductivity } \\ \text { ROC800 } & \text { thermal capacitance }\end{array}$

General Alloyed Steel (currently not used):

$\begin{array}{ll}\text { CONALST } & \text { thermal conductivity } \\ \text { ROCALST } & \text { thermal capacitance }\end{array}$

*GA-propietary information; not available at all installations 
Hastelloy-X Alloy:

$\begin{array}{ll}\text { CONHA } & \text { thermal conductivity } \\ \text { ROCHA } & \text { thermal capacitance }\end{array}$

The following data for insulating materials are available:

Kao wool fiber mats:

CONKA thermal conductivity

ROCKA thermal capacitance

Alumina (core floor):

CONALU thermal conductivity

ROCALU thermal capacitance

The following functions for concrete are available:

$\begin{array}{ll}\text { CONCC } & \text { thermal conductivity } \\ \text { ROCCC } & \text { thermal capacitance }\end{array}$

The following data for fuel are available (GA-A12985):

$\begin{array}{ll}\text { CONFUEL } & \text { thermal conductivity } \\ \text { CPFUEL } & \text { specific heat }\end{array}$

Other entries are as follows:

CONRAD effective thermal conductivity for radiation in axial direction in coolant holes (contribution

is small at all temperature levels of MHTGRs.

CONPEB effective thermal conductivity of a pebble bed. (not used)

Subroutine COND45 is only executed on first-call to any of the H451 graphite conductivity routines to initialize the corresponding data arrays.

Function CON451A, with entries CON451R and CONFER, combines the corresponding unirradiated and irradiated conductivities by linear combination, depending on the damage parameter DAM.

The call sequence is as follows:

TH,DAM

where

TH

temperature [1] 
Subroutine TBLLKER is no longer used and will be deleted with the next revision (use TBLLKF, see Section 4.2.6).

\subsubsection{THPROCS Subroutines}

The THPROCS submodule post-processes THATCH output files, extracts and summarizes results in various tables, searches for peak fuel and vessel temperatures, computes gas inventories, and produces condensed output files for plotting.

Its effective main program is Subroutine THPROCS, which reads the input data (see Section 5.3) to control post-processing runs. Up to nine THATCH output files can be handled in one run. The files can be either sequential in time or parallel (i.e., for the same time period.) If PROCESS $=$ true on the first input data record, then routine PROCRAW is called for post-processing of the output file(s), and writing of a plot file, in the format of Table 4-15.

Depending on the time points found and on the NPTS specified on the second input record, the plot file created by PROCRAW may be condensed by a call to FCOND. If COMB = true was specified on the first input record, the files are considered to be sequential, and the plot files produced by PROCRAW will be combined into a single plot file. If Plot =true was specified, plots can be produced via call to FILEPLT, which calls on DISSPLA to produce plots (This option is currently only available at BNL).

Subroutine PROCRAW is the main routine for post-processing. It is called from a loop in THPROCS, with one call for each output file to be processed. Its call sequence is as follows:

IIN,IOUT,IFLOW,IKIN,TSTART, TEND, TSTEP,ILEVOUT,NTPSCR1,NTPSCR2, NTPSCR3, AIRCOL,PTKINS,FLOXI,WRK,IDWRK,IRECMX,TTMPWRT

where

$\begin{array}{ll}\text { IIN } & \text { File number for THATCH output file to be processed } \\ \text { IOUT } & \text { File number for plot file to be produced } \\ \text { IFLOW } & \text { File number for FLOXI temperature file to be produced } \\ \text { IKIN } & \text { File number for output file of fine step point kinetics data to be processed } \\ \text { TSTART,TEND } & \text { Start and end time for processing of files to be processed [hr] } \\ \text { TSTEP } & \text { Desired time increment between steps [hr] } \\ \text { ILEVOUT } & \text { Printed output control (larger entries produce more data tables) } \\ \text { NTPSCR1,NTPSCR2 } & \\ \text { NTPSCR3 } & \text { Identifiers for scratch files } \\ \text { AIRCOL } & \text { Logical variable from second input record (PASCOL was used) } \\ \text { PTKINS } & \text { Logical variable from second input record (PKIN was used) } \\ \text { FLOXI } & \text { Logical variable from second input record (FLOXI was used) } \\ \text { WRK } & \text { Work space } \\ \text { IDWRK } & \text { Dimension of work space } \\ \text { IRECMX } & \text { Maximum length of Y-vector on time records of plot files }\end{array}$


Table 4-15

Records on THPROCS Plot Files

Record No.

1

2

3 TO ( 2 + NOREC) JNO, TIME, (Y(I),I=1,IREC)

where the contents of the $\mathrm{Y}$-vector are as follows:

\begin{tabular}{|c|c|c|c|}
\hline Location & Variable & Units & Description \\
\hline $\begin{array}{c}1-16 \\
17-32 \\
33-36\end{array}$ & $\begin{array}{l}\text { THAVA } \\
\text { THMAX } \\
\text { QENV }\end{array}$ & $\begin{array}{l}{ }^{\circ} \mathrm{C} \\
{ }^{\circ} \mathrm{C} \\
\mathrm{MW}\end{array}$ & $\begin{array}{l}\text { Block average temperatures for Blocks } 1-16 \\
\text { Block maximum tempertures for Blocks } 1-16 \\
\text { Conductive heat flow across four envelopes } \\
\begin{array}{l}1=\text { active core } \\
2=\text { total core } \\
3=\text { vessel } \\
4=\text { to } \text { RCCS }\end{array}\end{array}$ \\
\hline $\begin{array}{l}37 \\
38 \\
39\end{array}$ & $\begin{array}{l}\text { QTOSOLD } \\
\text { QQAFTTOT } \\
\text { QFLXTOT }\end{array}$ & $\begin{array}{l}\text { MW } \\
\text { MW } \\
\text { MW }\end{array}$ & $\begin{array}{l}\text { Total heat flow to solid } \\
\text { Decay heat plus fission power } \\
\text { Total convection and chemical reaction heat } \\
\text { to solid }\end{array}$ \\
\hline $\begin{array}{c}40-51 \\
52 \\
53 \\
\\
54 \\
55 \\
\\
56\end{array}$ & $\begin{array}{l}\text { GASA } \\
\text { GASVESL } \\
\text { GASCAV } \\
\text { GASCOR } \\
\text { GASUPL } \\
\text { GASBOT }\end{array}$ & $\begin{array}{l}\mathrm{kmol} \\
\mathrm{kmol} \\
\mathrm{kmol} \\
\\
\mathrm{kmol} \\
\mathrm{kmol} \\
\\
\mathrm{kmol}\end{array}$ & $\begin{array}{l}\text { Gas inventory array (see Table 4-18) } \\
\text { Gas inventory, reactor vessel (sum of } 1 \text { to } 9 \text { ) } \\
\text { Gas inventory, reactor cavity (sum of } 10 \text { to } \\
\text { 12) } \\
\text { Gas inventory, core (sum of } 1 \text { to } 3 \text { ) } \\
\text { Gas inventory, upper head region (sum of } 4 \\
\& 6 \text { ) } \\
\text { Gas inventory, bottom region (sum of } 8 \& \\
9 \text { ) }\end{array}$ \\
\hline $57-60$ & FRCHT & - & $\begin{array}{l}\text { Volume fraction of active core exceeding } \\
1000,1200,1400 \& 1600^{\circ} \mathrm{C}\end{array}$ \\
\hline $\begin{array}{l}61 \\
62 \\
63 \\
64\end{array}$ & $\begin{array}{l}\text { WAIR } \\
\text { THAIREX } \\
\text { THGVEAV } \\
\text { THGCAAV }\end{array}$ & $\begin{array}{l}\mathrm{kg} / \mathrm{s} \\
{ }^{\circ} \mathrm{C} \\
{ }^{\circ} \mathrm{C} \\
{ }^{\circ} \mathrm{C}\end{array}$ & $\begin{array}{l}\text { RCCS air flow } \\
\text { RCCS air exit temperature } \\
\text { Average gas temperature in reactor vessel } \\
\text { Average gas temperature in reactor cavity }\end{array}$ \\
\hline
\end{tabular}


Table 4-15 (Cont'd)

\begin{tabular}{|c|c|c|c|}
\hline Location & Variable & Units & Description \\
\hline $\begin{array}{l}65 \\
66 \\
67\end{array}$ & $\begin{array}{l}Y_{1} \\
Y_{9} \\
Y_{11}\end{array}$ & & $\begin{array}{l}\text { Point kinetics vector variable } \mathrm{P} / \mathrm{P}_{\mathrm{o}} \\
\text { Point kinetics vector variable } \mathrm{X} / \mathrm{X}_{\mathrm{o}} \\
\text { Point kinetics vector variable } \mathrm{Sm} / \mathrm{Sm}_{\mathrm{o}}\end{array}$ \\
\hline $\begin{array}{l}68 \\
69 \\
70 \\
71 \\
\end{array}$ & $\begin{array}{l}Y_{15} \\
Y_{16} \\
Y_{17} \\
Y_{18} \\
\end{array}$ & & $\begin{array}{l}\text { Point kinetics vector variable } \Sigma \rho_{\text {core }} \\
\text { Point kinetics vector variable } \rho_{\text {ref } \ell} \\
\text { Point kinetics vector variable } \rho_{\text {xen }} \\
\text { Point kinetics vector variable } \Sigma \rho \\
\end{array}$ \\
\hline \multicolumn{4}{|c|}{ The following five entries are only valid for THATCH runs without functioning RCCS: } \\
\hline $\begin{array}{l}72 \\
73 \\
74 \\
75 \\
76\end{array}$ & $\begin{array}{l}\text { THAVA (KCONTOP) } \\
\text { THMAX (KCONTOP) } \\
\text { THAVA (KCONSID) } \\
\text { THMAX (KCONSID) } \\
\text { TH(IVSSUP,JVSSUP) }\end{array}$ & $\begin{array}{l}{ }^{\circ} \mathrm{C} \\
{ }^{\circ} \mathrm{C} \\
{ }^{\circ} \mathrm{C} \\
{ }^{\circ} \mathrm{C} \\
{ }^{\circ} \mathrm{C}\end{array}$ & $\begin{array}{l}\text { Average temperature of concrete top slab } \\
\text { Maximum temperature of concrete top slab } \\
\text { Average temperature of concrete side wall } \\
\text { Maximum temperature of concrete side wall } \\
\text { Concrete temperature of vessel supports }\end{array}$ \\
\hline $\begin{array}{l}77 \\
78 \\
79\end{array}$ & $\begin{array}{l}\mathrm{Y}_{23} \\
\mathrm{Y}_{15}+\mathrm{Y}_{16} \\
\text { THPKFUEL }\end{array}$ & ${ }^{\circ} \mathrm{C}$ & $\begin{array}{l}\text { Point kinetics vector variable } \rho_{\text {wat }} \\
\text { Point kinetics vector variable } \rho_{\text {core }}+\rho_{\text {refe }} \\
\text { Peak fuel temperature (not a SYMELAN } \\
\text { value but approximation only) }\end{array}$ \\
\hline $\begin{array}{l}80 \\
81 \\
82\end{array}$ & $\begin{array}{l}\text { WWAT } \\
\text { THWATC } \\
\text { THWATH }\end{array}$ & $\begin{array}{l}\mathrm{kg} / \mathrm{s} \\
{ }^{\circ} \mathrm{C} \\
{ }^{\circ} \mathrm{C}\end{array}$ & $\begin{array}{l}\text { RCCS water flow } \\
\text { RCCS water cold leg temperature } \\
\text { RCCS water hot leg temperature }\end{array}$ \\
\hline
\end{tabular}


Subroutine PROCRAW calls for internal gas volume computations and reading of the files to be processed. It affects computation of average temperatures, peak temperatures and gas inventories, and writes the files shown in Table 4-16 (see also Table 4-17).

Subroutine READTOP (IIN, FLOXI, IDEBUG) reads the header records of the the THATCH output file as described in Table 4-3. If FLOXI = true, the corresponding FLOXI record of the header section is also read.

Subroutine READTIM reads the time records from the output file of THATCH, as described in Table 4-4. The call sequence is as follows:

\section{TIME,IIN,FLOXI,AIRCOL,PTKINS,SURFPRT,IDEBUG}

where

$\begin{array}{ll}\text { Time } & \text { time [hr] } \\ \text { IIN } & \text { file number of THATCH output file to be processed } \\ \text { AIRCOL } & \text { logical variable from second input record (PASCOL was used) } \\ \text { PTKINS } & \text { logical variable from second input record (PKIN was used) } \\ \text { FLOXI } & \text { logical variable from second input record (FLOXI was used) } \\ \text { SURFPR } & \text { logical debug control of surface temperature printing } \\ \text { IDEBUG } & \text { debug print control }\end{array}$

Subroutine VOLCOMP (IDEBUG) computes the gas volumes for various sections of the reactor vessel and the reactor cavity. These data are then used to compute based on system pressure and section temperature the gas inventory in the reactor vessel as a function of time.

Table 4-18 lists the sections and the computed volumes, based on PSID design data. Some of the computations use "hard-wired" design data included in Block Data CORVDAT.

Block Data CORVDAT contains core design data used in the above gas volume computations (VOLCOMP).

Subroutine GASINVT (TIME, AIRCOL, IDEBUG) computes the gas inventory in the reactor vessel and reactor cavity volumes of Table 4-18 based on system pressure and component temperatures. For most components with rather stagnant gas masses, the average temperature of gas is wellapproximated by the average of the component solid surface temperatures, which is used to compute the gas inventory.

Subroutine THAVMAX (IDEBUG) computes for each solid block the volume weighted average temperature and determines the maximum temperature of the nodes in the block. 
Table 4-16

Output Files Produced in Post-Processing of

THATCH Output Files

\begin{tabular}{|c|c|c|c|}
\hline File & File No. & $\begin{array}{l}\text { File Record } \\
\text { Format }\end{array}$ & Remarks \\
\hline THATCH plot file & $21 \mathrm{ff}$ & Table 4-15 & \\
\hline FLOXI plot file & $21 \mathrm{ff}$ & $\begin{array}{l}\text { Plot file format; for } \\
\text { Y-vector see Table } \\
4-9\end{array}$ & $\begin{array}{l}\text { if FLOXI = true; records } \\
\text { are added to THATCH } \\
\text { plot file as records } 101 \\
\text { to } 159\end{array}$ \\
\hline $\begin{array}{l}\text { Temperature file for free- } \\
\text { standing FLOXI runs }\end{array}$ & $41 \mathrm{ff}$ & Table 4-17 & \\
\hline $\begin{array}{l}\text { Fine-step point kinetics plot } \\
\text { file }\end{array}$ & $71 \mathrm{ff}$ & $\begin{array}{l}\text { Plot file format; six } \\
\text { Y-vector entries from } \\
\text { point kinetics vector: } \\
Y_{1}, Y_{9}, Y_{11}, Y_{12} \text {, } \\
Y_{20}, Y_{20}+Y_{21}\end{array}$ & \\
\hline
\end{tabular}

Table 4-17

Records on THPROCS Temperature Output File for Free Standing FLOXI Runs

\begin{tabular}{||c|c|}
\hline \multicolumn{1}{|c|}{ Record Number } & Content \\
\hline Top Only: & \\
\hline 1 & Title (Character *240) \\
\hline 2 & IMAXP, JMAXP \\
\hline 3 & (RIFA(I),I=1,IMAX \\
\hline 4 & (ZIFA(J),J =1,JMAX) \\
\hline One Set of Record per Time Step: & Time \\
\hline 5 & IMAXP records: $($ TH $(I, J)$ J $=1$, JMAXP) \\
\hline 6 &
\end{tabular}


Table 4-18

Gas Volumes in Reactor Vessel and Reactor Cavity

\begin{tabular}{|c|l|c||}
\hline $\begin{array}{c}\text { Index } \\
\text { Reactor Vessel: }\end{array}$ & \multicolumn{1}{|c|}{ Volume } & $\begin{array}{c}\text { V } \\
{\left[\mathrm{m}^{3}\right]}\end{array}$ \\
\hline 1 & Active Core & 10.98 \\
\hline 2 & Top reflectors and plenum elements & 2.20 \\
\hline 3 & Bottom reflectors & 2.49 \\
\hline 4 & Upper plenum & 51.70 \\
\hline 5 & Lower plenum & 23.78 \\
\hline 6 & Upper plenum shroud to vessel gap & 22.10 \\
\hline 7 & Core barrel to vessel gap & 68.52 \\
\hline 8 & Core support structure & 32.90 \\
\hline 9 & Bottom plenum \& SCS & 59.50 \\
\hline & & 274.18 \\
\hline 12 & SUM VESSEL & 285.00 \\
\hline 11 & Bottom & 313.20 \\
\hline & & SUM CAVITY \\
\hline
\end{tabular}


Subroutine PEAKERO (TIME, IDEBUG), with entry PEAKER, searches for active core and reactor vessel whether the block maximum temperature, varying in time, has reached a temporal maximum, referred to here as "peak" temperature. If so, a flag is printed.

Furthermore, the conductive heat flow across four enevelopes (entries 33 to 36 in Table 4-15) is compared against total heat flow to solid in the core, and when the heat flow across the envelope exceeds the heat flow to the solid, a "cross-over point" message is printed. is as follows:

Subroutine OUPPUT will produce further printed output tables, as requested. Its call sequence

\section{ILEVOUT,ITPINT,ITPFLX,FLOXI,AIRCOL,PTKINS,WRK,IDWRK,IDEBUG}

where

ILEVOUT
ITPINT
ITPFLX
FLOXI
AIRCOL
PTKINS
WRK
IDWRK
IDEBUG

printed output control (larger entries produce more data tables)

file number of file containing data to be printed

file number of FLOXI plot output file

logical variable from second input record (FLOXI was used)

logical variable from second input record (PASCOL was used)

logical variable from second input record (PKIN was used)

work space

dimension of WRK

debug print control

Subroutine PTKOUT reads the fine time step point kinetics output file produced by THATCH, prints output from it, and writes a brief plot file, as noted in Table 4-16. Its call sequence is as follows:

\section{TSTART,TEND,IKIN,TITLE,WRK,IDWRK,NTPSCR3,IRECMX,IDEBUG}

where

$\begin{array}{ll}\text { TSTAKT,TEND } & \text { start and end time for processing of files to be processed [hr] } \\ \text { IKIN } & \text { file number for fine step point kinetics output file to be processed } \\ \text { TITLE } & \text { run title (Character } * 240) \\ \text { WRK } & \text { work space } \\ \text { IDWRK } & \text { dimension of WRK } \\ \text { NTPSCR3 } & \text { identifiers for scratch files } \\ \text { IRECMX } & \text { maximum length of Y-vector on time records of plot files } \\ \text { IDEBUG } & \text { debug period control }\end{array}$

Subroutine THFLPLT (NTPTH,NTPFL,NTPOUT,WRK,IDWRK,IDEBUG) combines the THATCH plot file NTPTH and the FLOXI plot file NTPFL in File NTPOUT, adding the FLOXI records in positions 101 to 159 . The routine also does several data conversions (temperatures from units $\mathrm{K}$ - to ${ }^{\circ} \mathrm{C}$, mass flows from $\mathrm{kg} / \mathrm{s}$ to $\mathrm{kg} / \mathrm{hr}$, so that it is specific to the current FLOXI plot file format (see Section 4.2.3) and would require revisions if that section changes.

Block Data PLOTDAT contains values of $\pi$ and DEGK $=273.15$. 
At this time, the THPROCS post-processor does contain some "hard-wired" entries of data, characterizing the current model of the commercial MHTGR. Such entries will be removed with the next code revision. For that purpose, and as caution to the user, they are listed here.

\begin{tabular}{lll} 
ROUTINE & LOCATION & \multicolumn{1}{c}{ DATA } \\
PROCRAW & ISN26-31 & $\begin{array}{l}\text { PASCOL Data JAIRFST, JAIRLST } \\
\text { NFL, NFL2 } \\
\text { PROCRAW }\end{array}$ \\
ISN3 & $\begin{array}{l}\text { Parameter statements setting block } \\
\text { numbers (KCORE=2, etc.) }\end{array}$ \\
CORVDAT & all & $\begin{array}{l}\text { Block data for core volume } \\
\text { computations }\end{array}$ \\
THFLPLOT & $\begin{array}{l}\text { Plot variable unit conversions are hard } \\
\text { wired and would cause problems if } \\
\text { nodalization changed. Also, they } \\
\text { assume mass flows are in kg/s, which } \\
\text { is not necessarily true in FLOXI; see } \\
\text { FLOXI input variable PRTCTRL on } \\
\text { Input Record 3212D. }\end{array}$
\end{tabular}

\subsubsection{THRADLIB Subroutines}

The THRADLIB subroutines constitute a separate module which is executed by calling subroutine THRAD, the effective main program of this module, which serves as pre-processor to obtain the necessary radiation coefficient matrices for two-dimensional radiation in plena and cavities.

Subroutine THRAD initializes the pre-processor and controls the calls to the various routines for view factors and radiation coefficient routines. Input to this routine is still via Namelist reading of Namelist INPUT, as described in Section 5.2 (The THATCH input data processor should replace this in the near future). Depending on input variable GEOM, either view factors and radiation coefficients for a cylindrical cavity or for an annular cylindrical cavity will be computed.

For view factor computations, either subroutine VIEWFAC or subroutine ANVIEW are called to obtain the view factors for cylindrical cavities or annular cylindrical cavities, respectively. Thereafter, the algebra of Appendix $A$ is executed to obtain the LAMBDA-matrix of radiation coefficients to be used in THATCH. After some printouts with consistency checks, the LAMBDA-matrix is written to File 8 for later use as an input file to THATCH.

Subroutine VIEWFAC (F,X,NIS,IDIM,NRA,RA,NZA,ZA,AA,EMMIS) computes the view factors for a cylindrical cavity as described in Section B-1.

F

$\mathrm{X}$ view factor array dimensioned IDIMxIDIM (output)

geometry array dimensioned IDMx6 (output),

where each surface is designated by coordinates $R 1, R 2, H 1, H 2$, where $R$ is radius, and $\mathrm{H}$ is height (or $\mathrm{z}$-coordinate). 


$\begin{array}{ll}\text { NIS } & \mathbf{X}(\mathrm{N}, 6)=\text { surface area } \\ \text { NRA } & \text { is the number of surface elements in the cavity (input) } \\ \text { RA } & \text { number of radial surface elements on top and bottom disks (input) } \\ \text { NZA } & \text { radial coctdinates (input) } \\ \text { ZA } & \text { number of surface elements (rings) on cylinder side walls (input) } \\ \text { AA } & \text { axial coordinates (input) } \\ \text { EMMIS } & \text { surface area array of surface elements (output) }\end{array}$

After computing the above geometric coordinates of each surface element, VIEWFAC determines an index, IQ, for the interaction between any two surfaces.
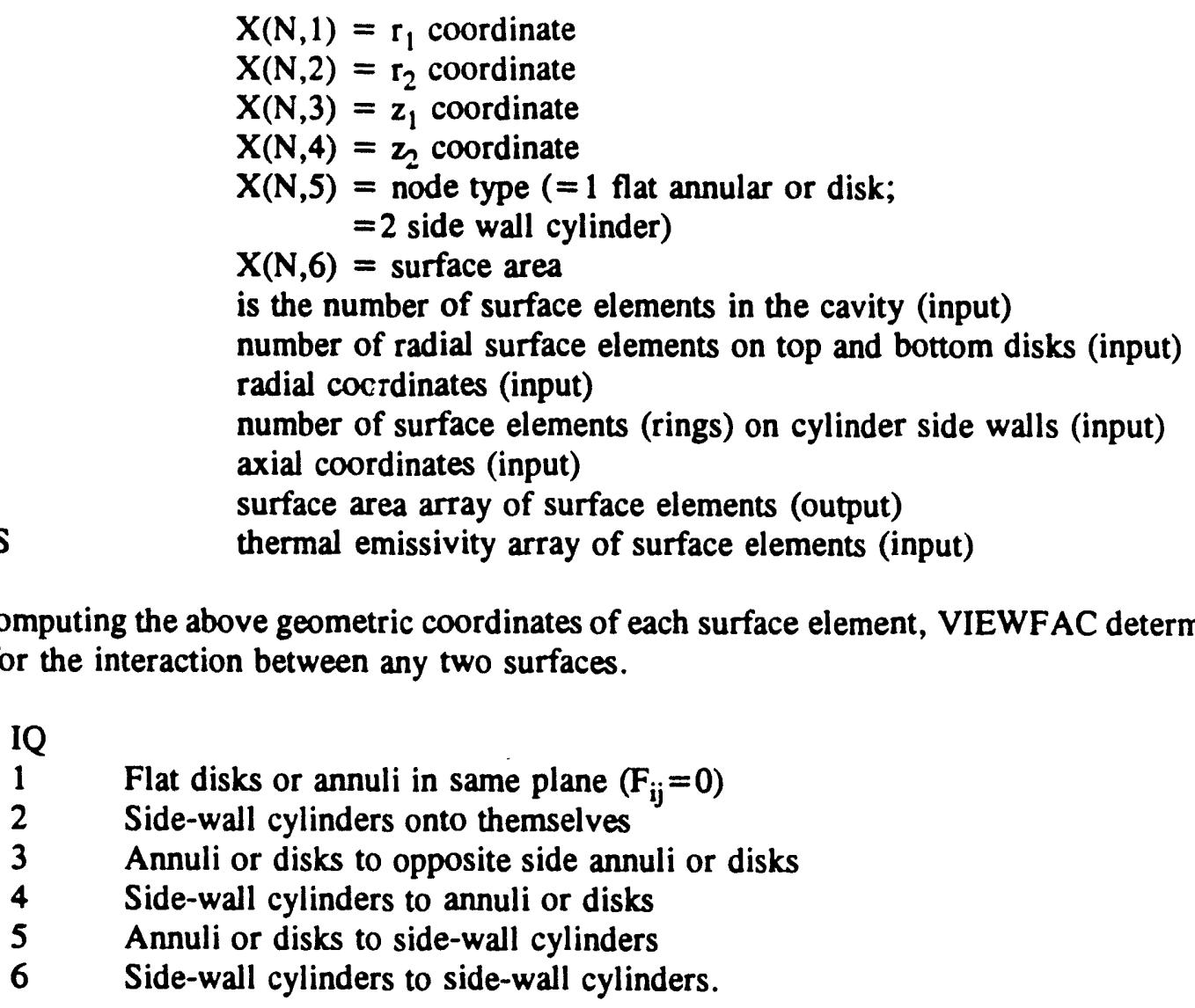

For each of these, the required algebra is then executed calling on routine FDISK12, which represents Equation (B-5). Upon completion of all view factor computations they are checked against Equation (B-2) for consistency.

Subroutine ANVIEW (ZAO,JOMX,ZAI,JIMX,RA,NRA,AA,FF,IRAD,IDIMFF,IPRINT) controls the computing of view factors for annular cylindrical cavities.

$\mathrm{ZAO}$ and ZAI

JOMX and JIMX

RA and NRA axial coordinates on the outer and inner cylinder

the number of node intervals in ZAO and ZAI (ZAO and ZAI arrays range from 0:JOMX or JIMX)

the radial coordinates (same for top and bottom annuli), with NRA being the number of entries (Currently, ANVIEW requires NRA $=1$, i.e. single top and bottom annuli. This restriction could be removed easily.) surface area of nodes (output) view factor array dimensioned IDIMFFxIDIMFF (output) number of nodes in cavity (input) leading dimension of FF array output print control

Function KTYPE (ZO1,ZO2,ZI1,ZI2) facilitates the view factor computation for arbitrary cylindrical rings on the outer and inner surfaces of the cavity, where $\mathrm{ZO} 1, \mathrm{ZO} 2, \mathrm{ZI} 1$, and $\mathrm{ZI2}$ are the coordinates of the two rings. The following are the returned types: 


$\begin{aligned} \text { KTYPE } & =0 & & \text { rings exactly opposite } \\ & =1 & & \text { no overlap } \\ & =11 & & \text { inner ring is larger and envelopes outer ring } \\ & =12 & & \text { outer ring is larger and envelopes inner ring } \\ & =13 & & \text { partial overlap }\end{aligned}$

The various functions for specific view factors, required to complete the view factor array of the annular cylindrical cavity, are summarized in Table 4-19.

These routines follow the formulas of Appendix B and include the corresponding asymptotic approximations where some parameters get small or large.

Functions ASINF $(X)$. ACOSF $(X)$ and ATANF $(X)$ compute the corresponding trigonometric functions, but branch for $\mathrm{x}<10^{-4}$ to the corresponding asymptotic approximations.

\subsection{Code Maintenance via UPDATE/HISTORIAN}

The THATCH code is stored in an UPDATE compatible program library format. Currently, it is installed on both the INEL CRAY and the BNL IBM 3090. The submodule codes (SYMELL, PASCOL, FLOXI and PKIN) and the auxiliary codes (MATLIB, GENLIB, GASPRLIB, THRADLIB and THPROCS) are stored in a similar manner in a second library.

The codes at all installations should be maintained by a single administrator (the only user with $\mathrm{read} / \mathrm{write}$ authorization, see Section 10 ). All other authorized users should be granted read only access. For example, at INEL users belonging to the NPR group may read from either library, but may not alter them. This type of program management is essential maintaining the integrity of any code.

All system-dependent coding has been conditionally included in the program libraries at both sites to maintain line for line compatibility between all installed versions of the code automatically generated at each installation.

\subsection{THATCH AND BIG COMMONS}

The two code sections are maintained in separate component files, with no Commons being used jointly. Within each component, all Commons are in COMDECKS, following the format requirements for Update/Historian.

In many of the large THATCH Commons, additional dummy COMDECKS are available to provide nomenclature to these Common decks. These are customarily listed in the THATCH main program and are generally not repeated later in the code.

Throughout THATCH and BIG, all Commons must always have identical variable names and identical lengths, to provide traceability and reduce possibilities for inadvertent errors.

Table E-3 lists of all Commons used in THATCH and the routines using it. The same list for component BIG is given in Table E-4. 


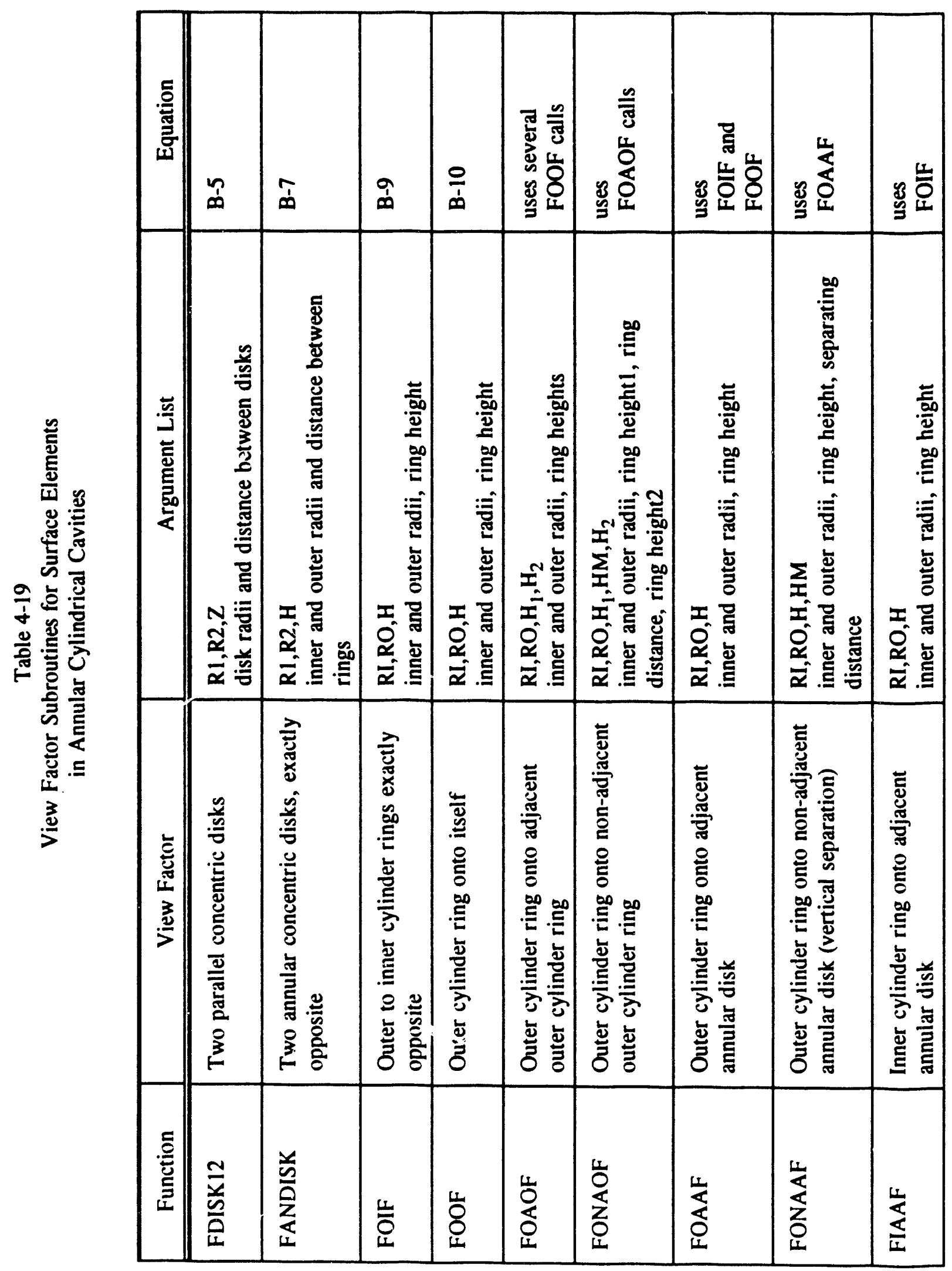




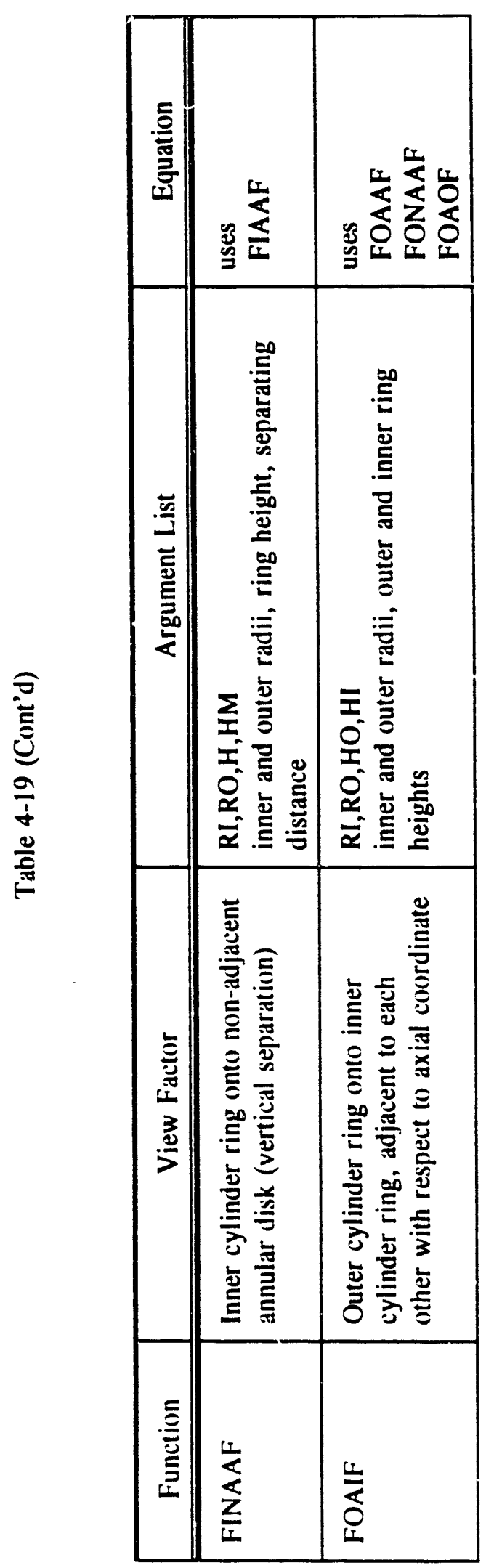




\section{INPUT DATA DESCRIPTION FOR THATCH AND ITS SUBMODULES}

\subsection{Input to THATCH Runs, Including THATCH Submodules}

\subsubsection{THATCH Input Processor}

The THATCH input data is processed by a version of the GENRD [Cox, 1972] input processor, which was originally developed at Los Alamos National Laboratory. GENRD interprets free-format alphanumeric data from 80 byte card-image records. The input is free-format in the sense that field location, field length, and type of conversion are determined solely by the user, not by an external format.

In addition to data nrocessed by GENRD, the THATCH input processor will accept as comment, records which begin with the character string 'Cbb' (bytes 1 through 3 ). These records are subsequently ignored by the input processor. As a result, they may be injected at any point within the data file. Comment may also be entered on data records processed by GENRD. One method is to use the construct

$$
<\text { data }>\$ \text {...comment...\$< data }>
$$

anywhere on a data record. This method, while perfectly acceptable, is somewhat less efficient than the alternate construct

$$
<\text { data }>\text { /...comment... }
$$

where there is no data following the comment on the record.

\section{THATCH INPUT FILE STRUCTURE}

The THATCH input file structure is based on a serial model, where all repeating records are of the same type (DATA RECORD). The overall structure of the THATCH input file is:

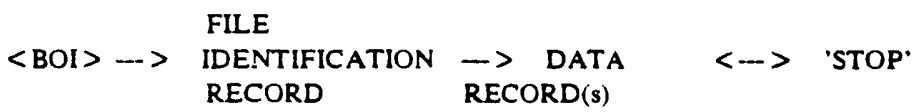

where

and

$$
\text { FILE IDENTIFICATION RECORD: FILE VERSION NUMBER } \rightarrow>\cdot \mathrm{V} \cdot \rightarrow \quad b \rightarrow>\text { 'THATCH' }
$$

DATA RECORD: RECORD IDENTIFIER $->\quad D^{\prime} \rightarrow>\quad b \quad->$ DATA-LIST

The file identification record is the first record processed. On this record, the file version number is the only entry defined by the user. It may be assigned any positive integer value; it is a simple means of identifying data sets and has no significance to the functioning of the code. A typical THATCH file identification record would be:

\section{THATCH}

It should be noted that GENRD is case-sensitive. All of its control information must be in its upper case. In particular, lower case 'V's and 'D's will not be processed properly.

Ali of the THATCH data processed by GENRD is read from data type records. For data to be properly defined, each data record is assigned a unique record identifier, which implicitly defines the sequence of data entries which constitute the record's data-list. The data-lists for each of the THATCH data records is presented later in this section.

Each data record is processed completely before the next record is interpreted. A data record 
is defined to have a maximum length equal to one or more card-image records ( 80 characters). It is terminated either by a slash $(/)$, or by the beginning of another data record, or a STOP record (a record containir the string "STOP"). Information following a slash is not processed and may be used as a comrr From each record, GENRD parses information into discrete numeric and/or character packets. Jimma and/or one or more blanks separates numeric information, which may be either a single-valued decimal constant, or a specification calling for the repetition of numeric constants. Character information is delimited by single quote marks and may contain any alphanumeric character, except the single quote mark. Character constants may not span a card-image record. If a terminal quote mark is omitted, the string is terminated at the record boundary (this method of terminating a character string may cause blank characters to be added to the end of the intended string). Logical constants, while similar to character constants, form a distinct third type of data. Logical constants may assume one of two values (TRUE and FALSE) and are delimited by periods. If an error is detected, a message describing the error is printed, processing of the current field is halted, and the next field on the record is read and processed.

GENRD provides a simple shorthand for the repetition of one or more numeric constants. The repeat $(R)$ specification provides this function for any sequence of real and/or integer values. For example,

is equivalent to

$$
1.2,3 \mathrm{R}
$$

and

$$
1.2,1.2,1.2
$$

is equivalent to

$$
(0.1,2.3,456.78,9.0), 4 \mathrm{R}
$$

$$
0.1,2.3,456.78,9.0,0.1,2.3,456.78,9.0,0.1,2.3,456.78,9.0,0.1,2.3,456.789 .0
$$

\subsubsection{Input Record Description}

The input records for the THATCH code are described in the following pages, in tabular format, including variable names and variable types as well as units, where applicable. In Group 3, of the THATCH Main Module Input Data a unified input option can be specified. If this option is chosen, various inputs for THATCH and its submodules are provided from a set of data bank subroutines, with data for the commercial MHTGR as described in the PSID. This option also assures that identical data are used by THATCH and its submodules. If this option is used, some variable entries from the normal input steam will be disregarded. In the list which follows, those variables are marked with "*". 
$\stackrel{2}{\Xi}$

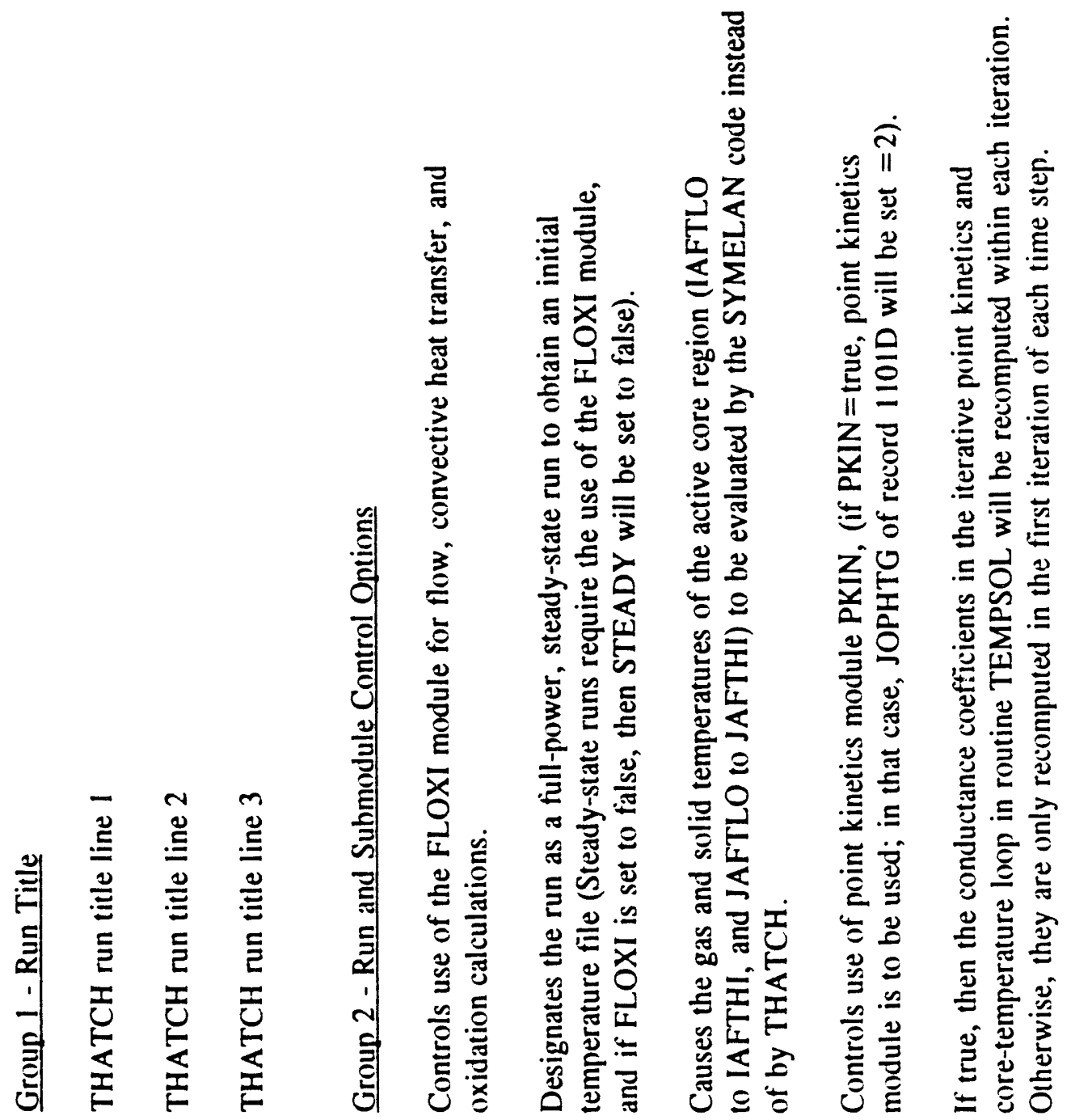

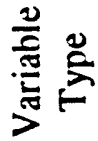

产焉

齐

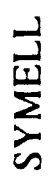

$\sum_{\frac{1}{2}} \sum_{\frac{1}{\alpha}}^{\frac{5}{0}}$

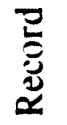

을 옹 융

$\stackrel{0}{?}$

ิㅗㅇ 
产

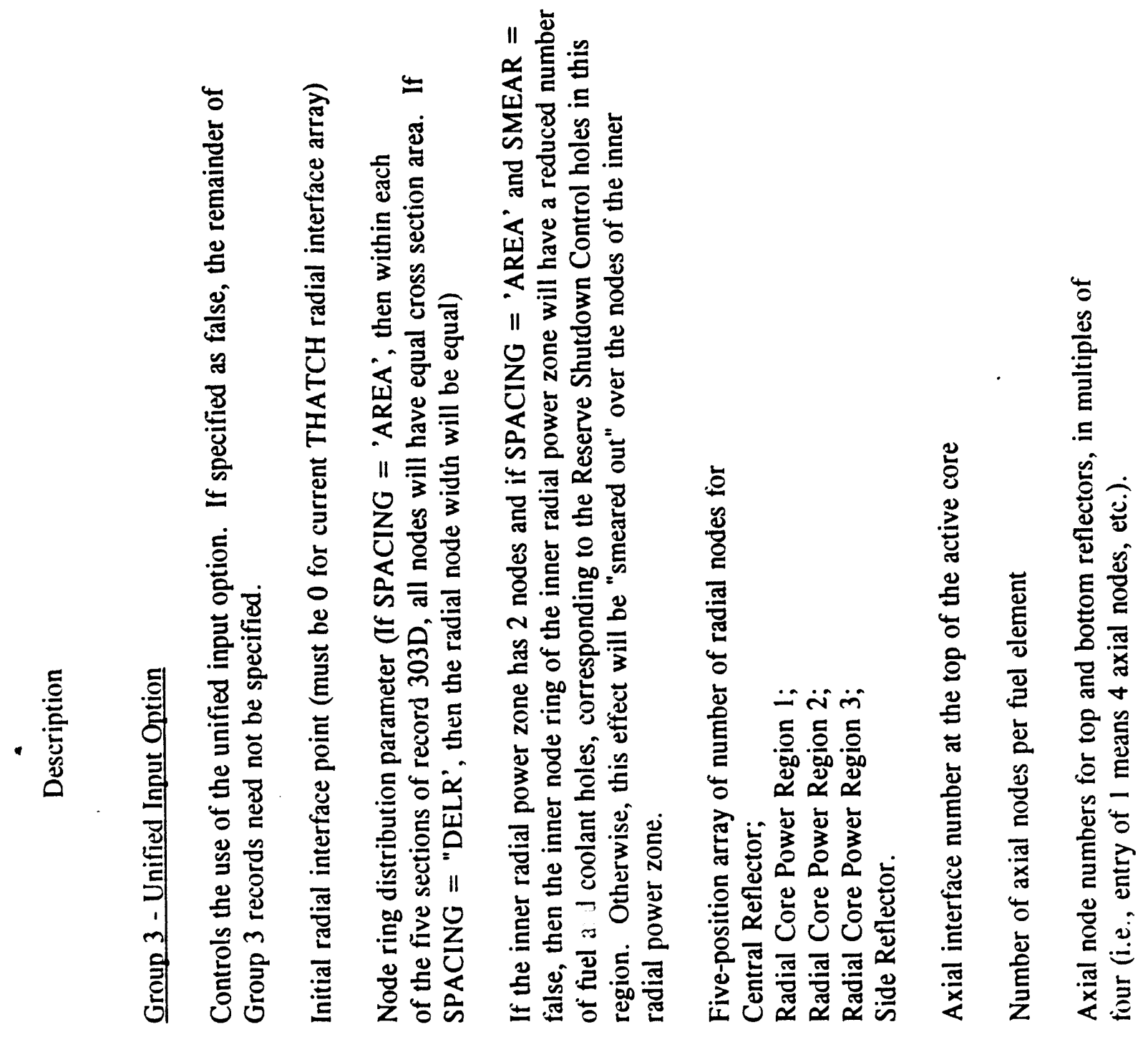

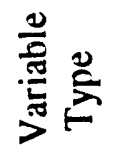

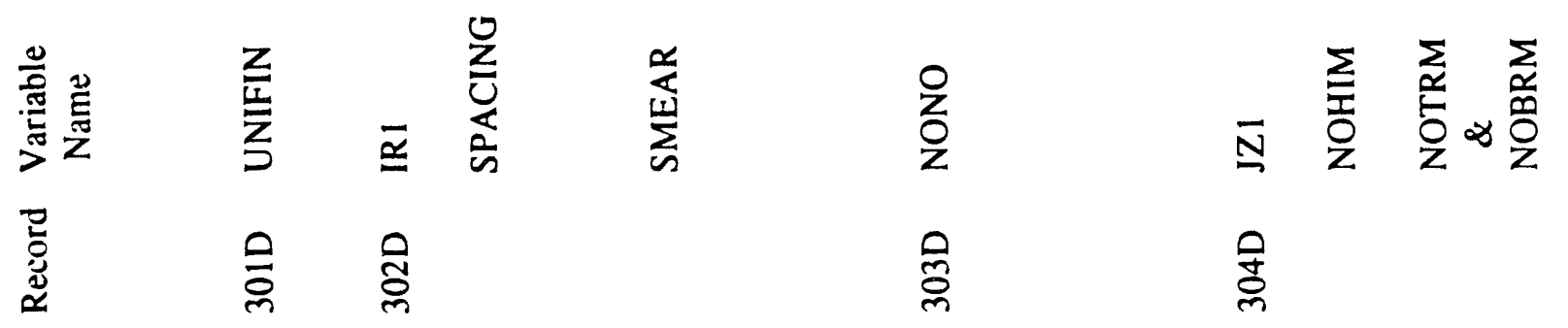


旁

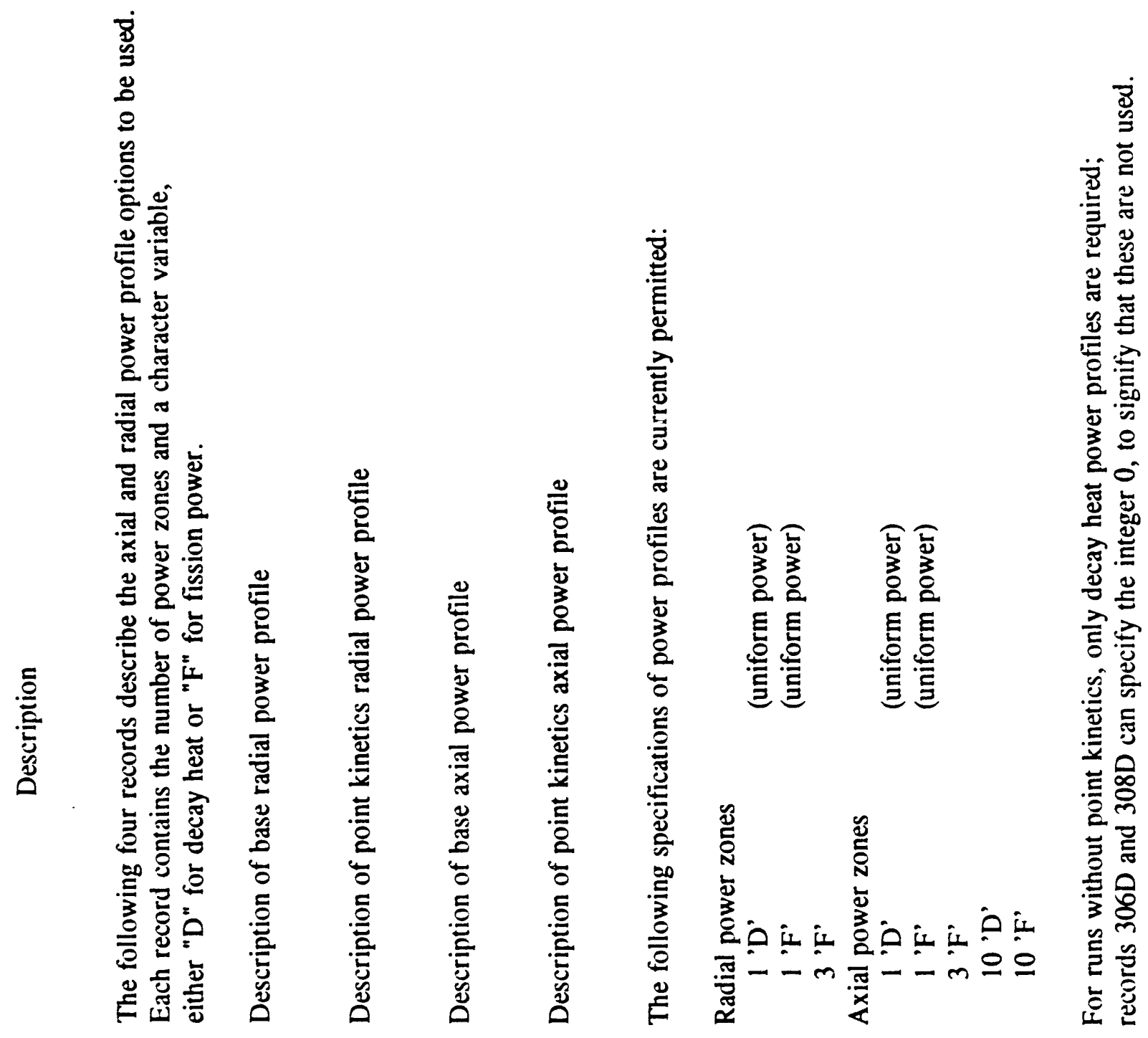

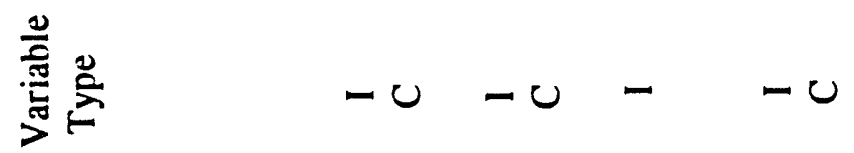

高量

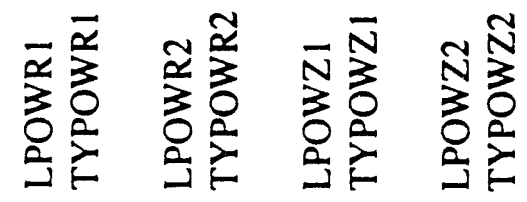

竞

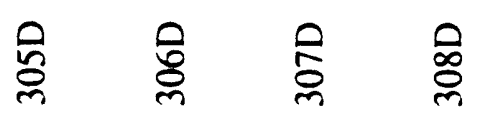


爱

暠

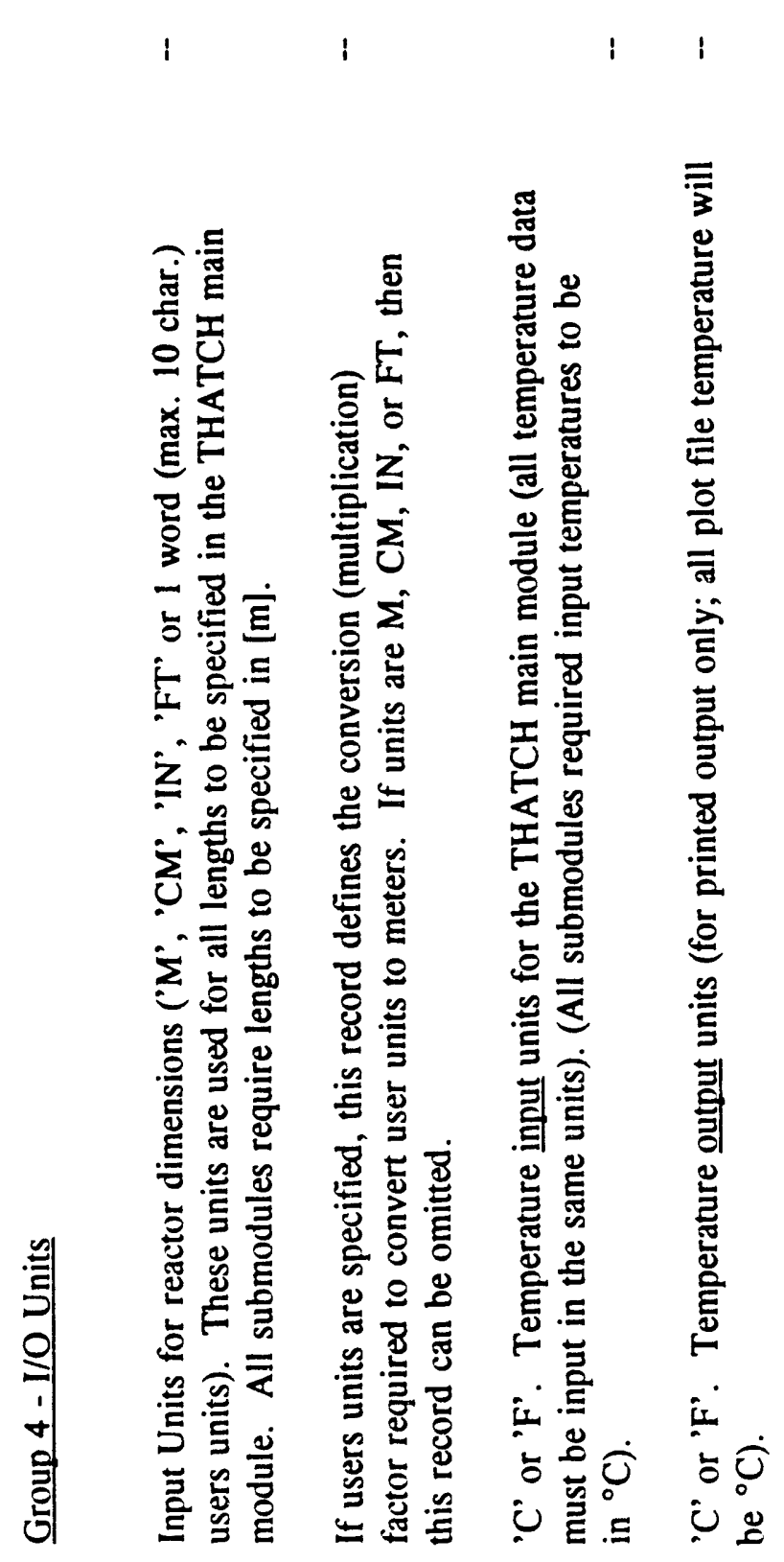

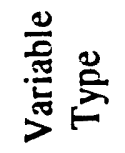

高量

齿

产

後

产

竞

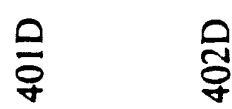

高

率 


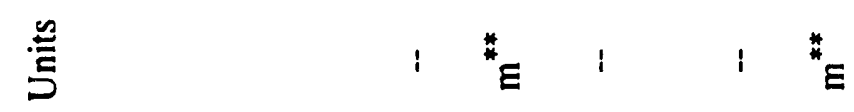
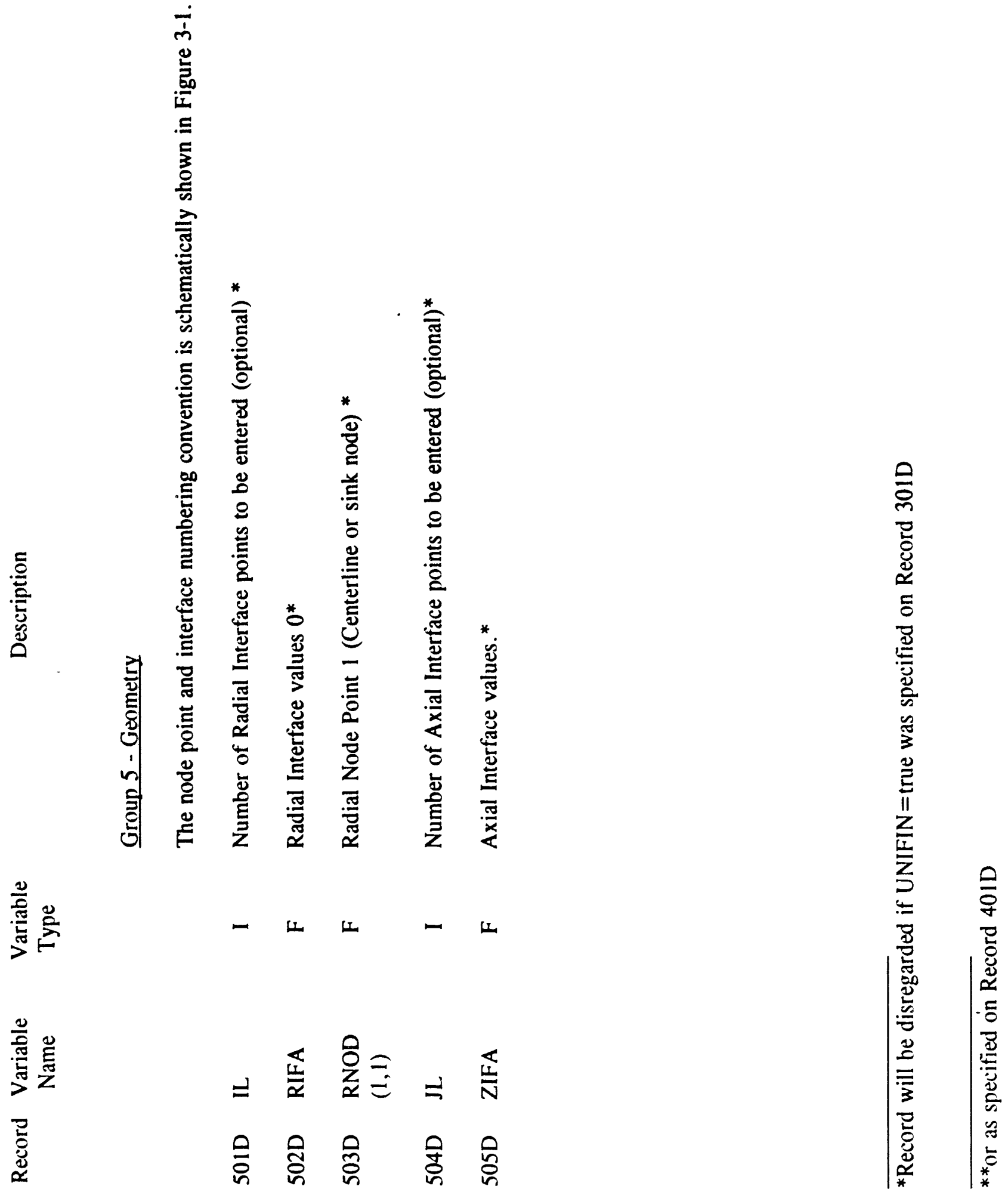
䜌

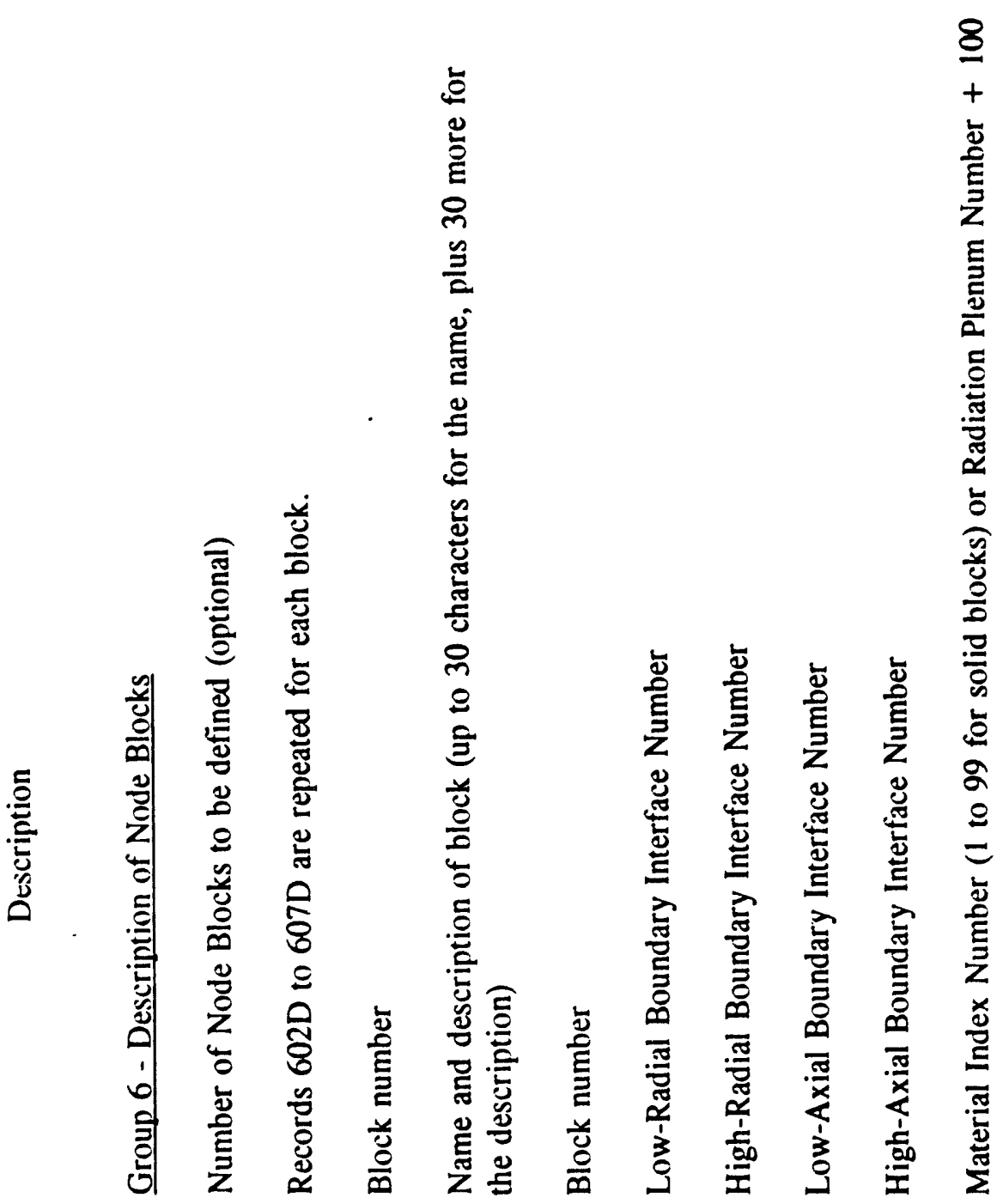

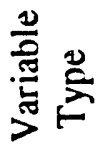

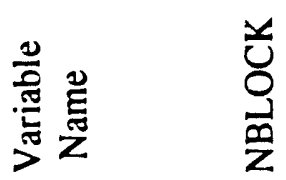

苍

믐

\%

ริ

爱 
善
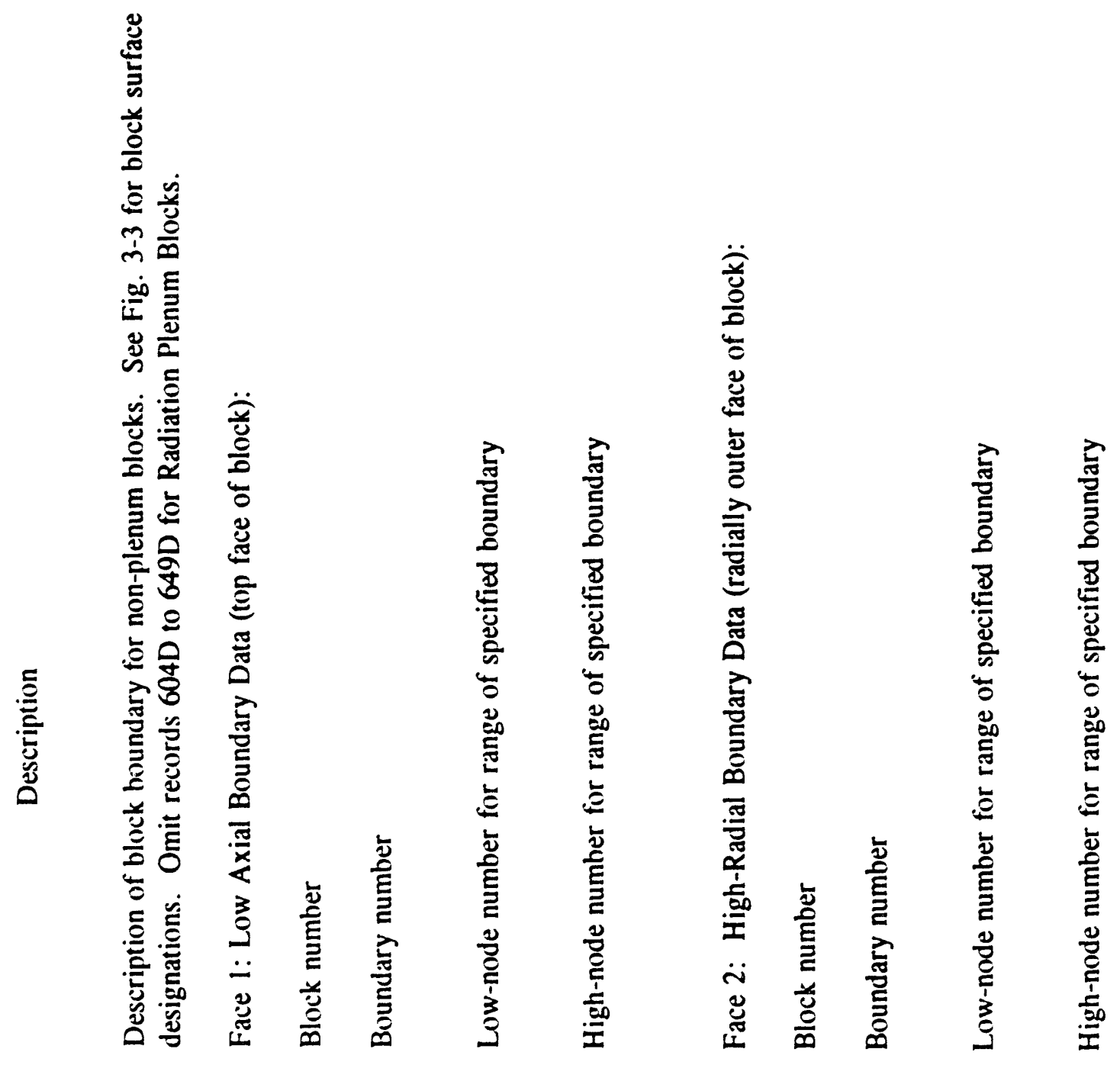

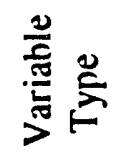

高量

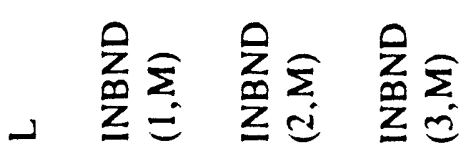

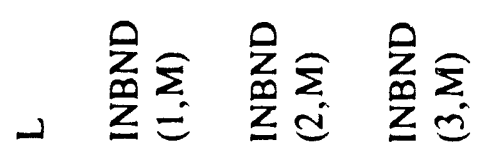

竞

웅

安 
产

(

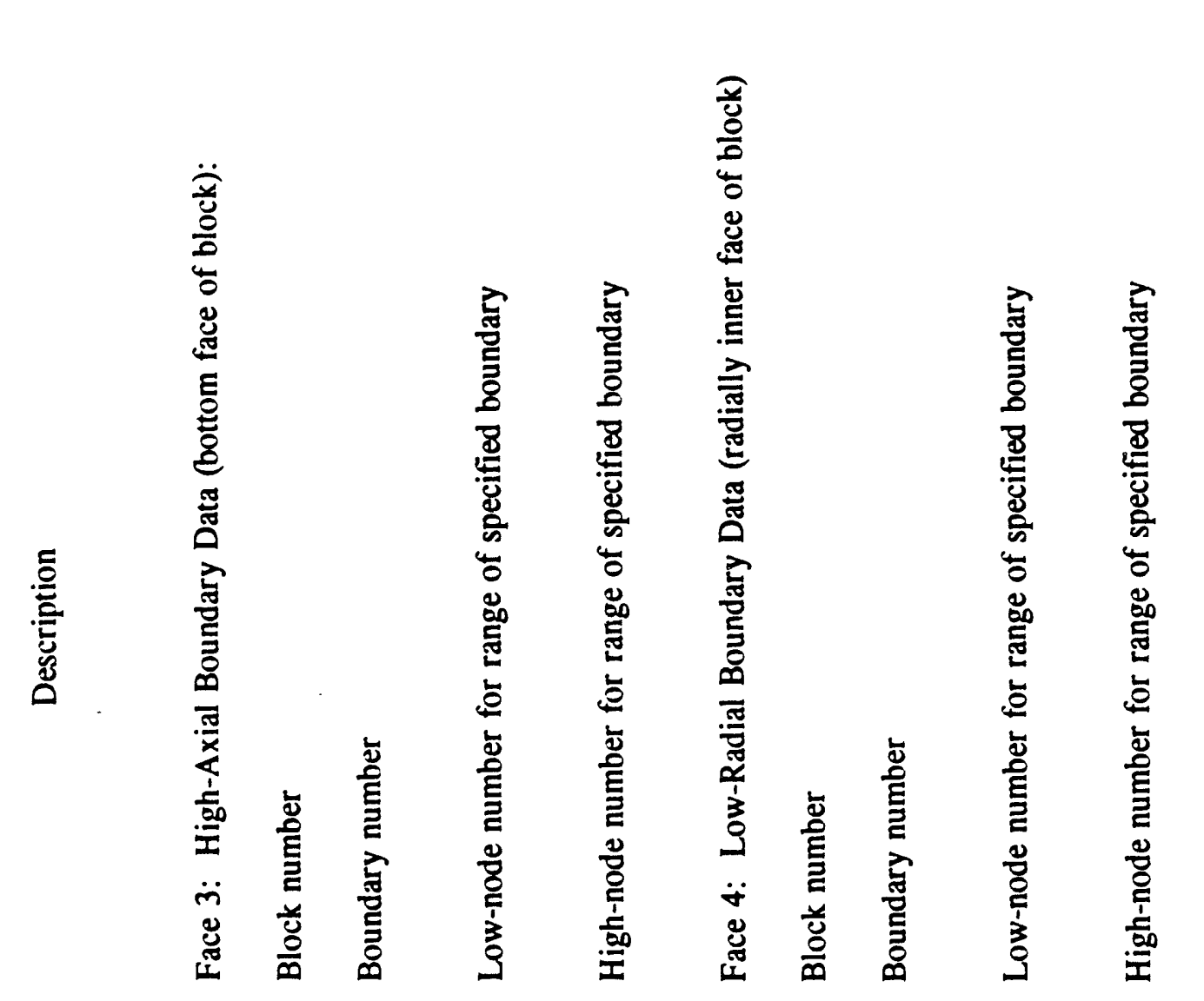

咅嚾

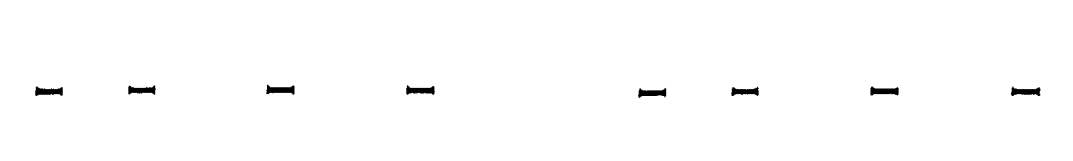

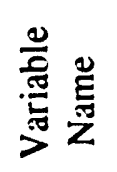

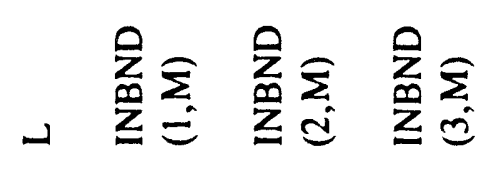

$$
\begin{aligned}
& \text { \& }
\end{aligned}
$$

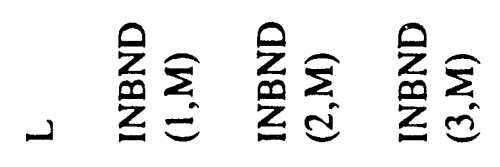

\%
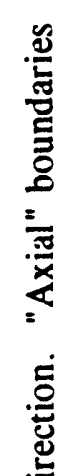

꽁

홍

.

은

종

훈

is.

禀

넣

氖

넌

ปั

๙

는

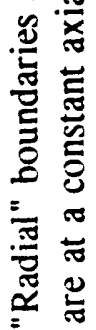

릉

¿ัญ 


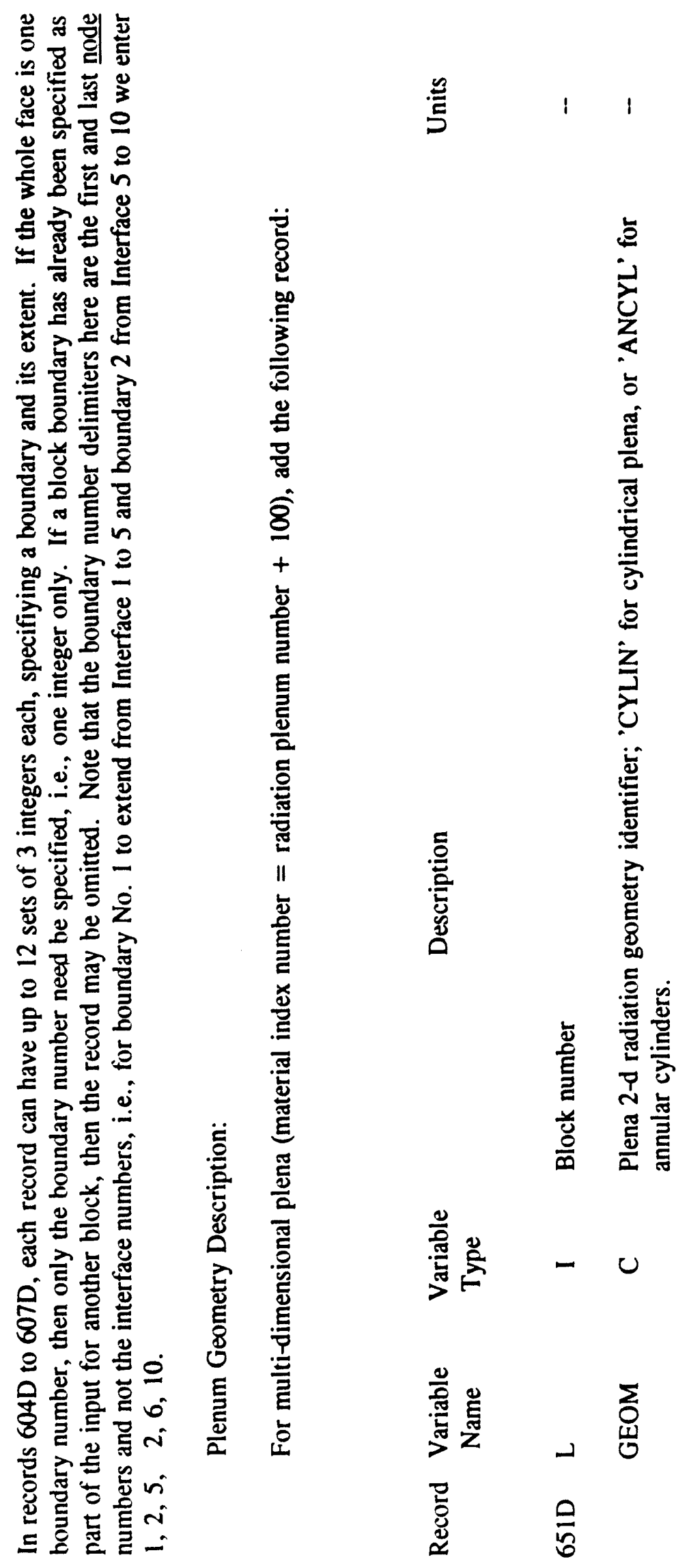


$\stackrel{n}{5}$

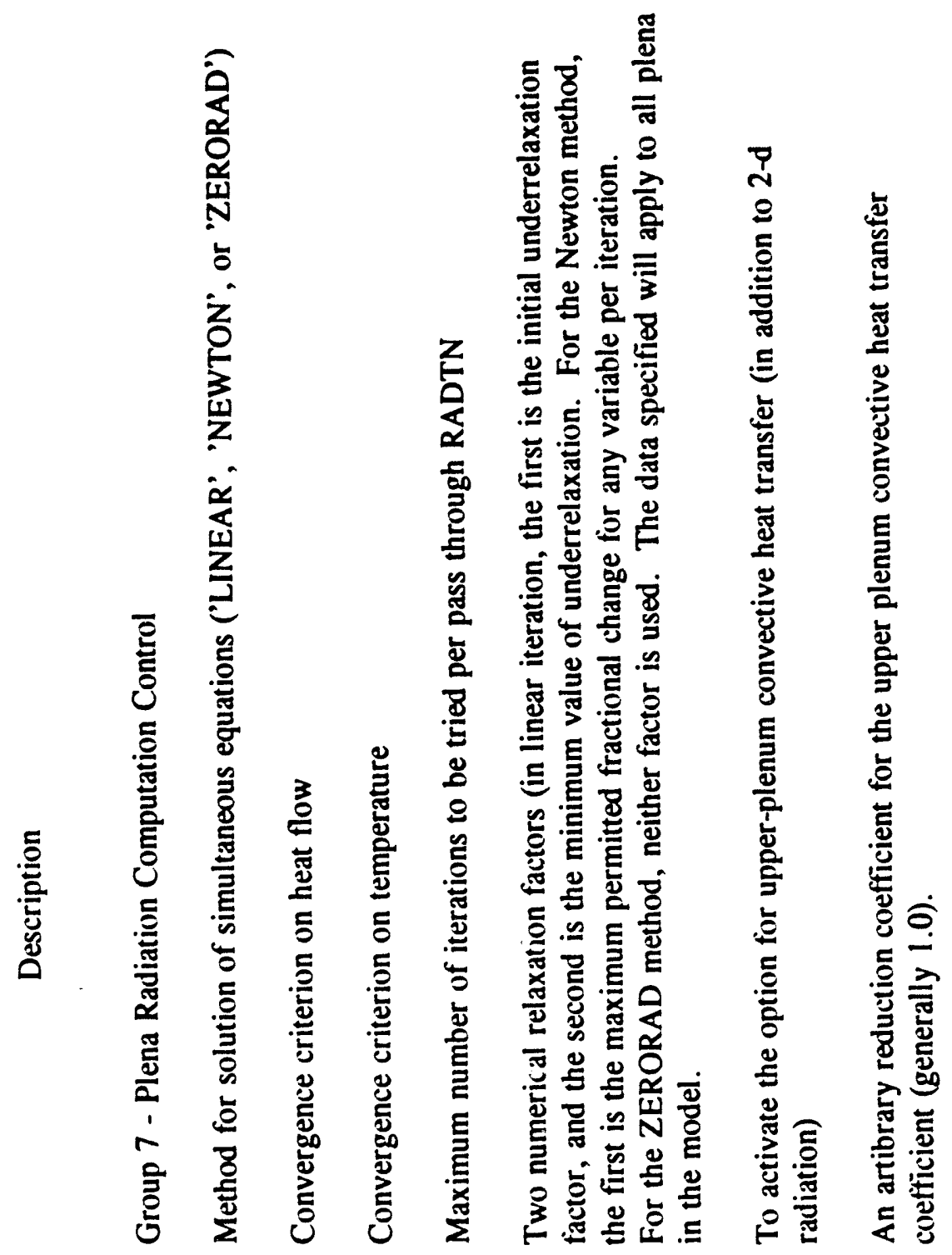

苞导

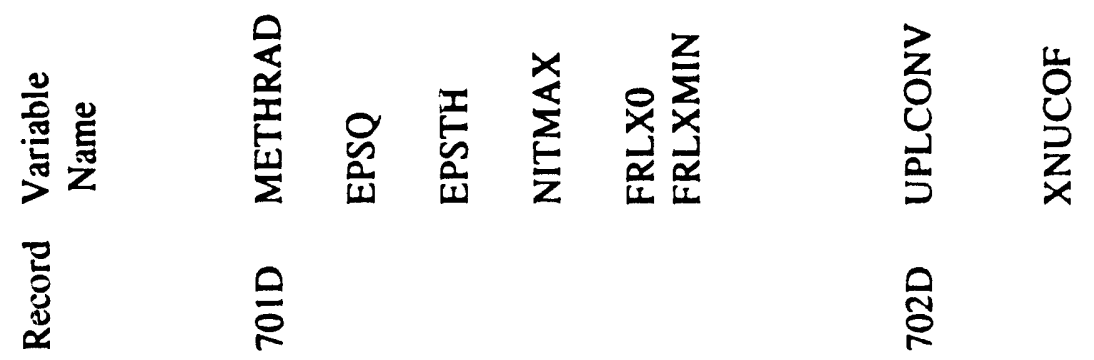


$\stackrel{5}{5}$

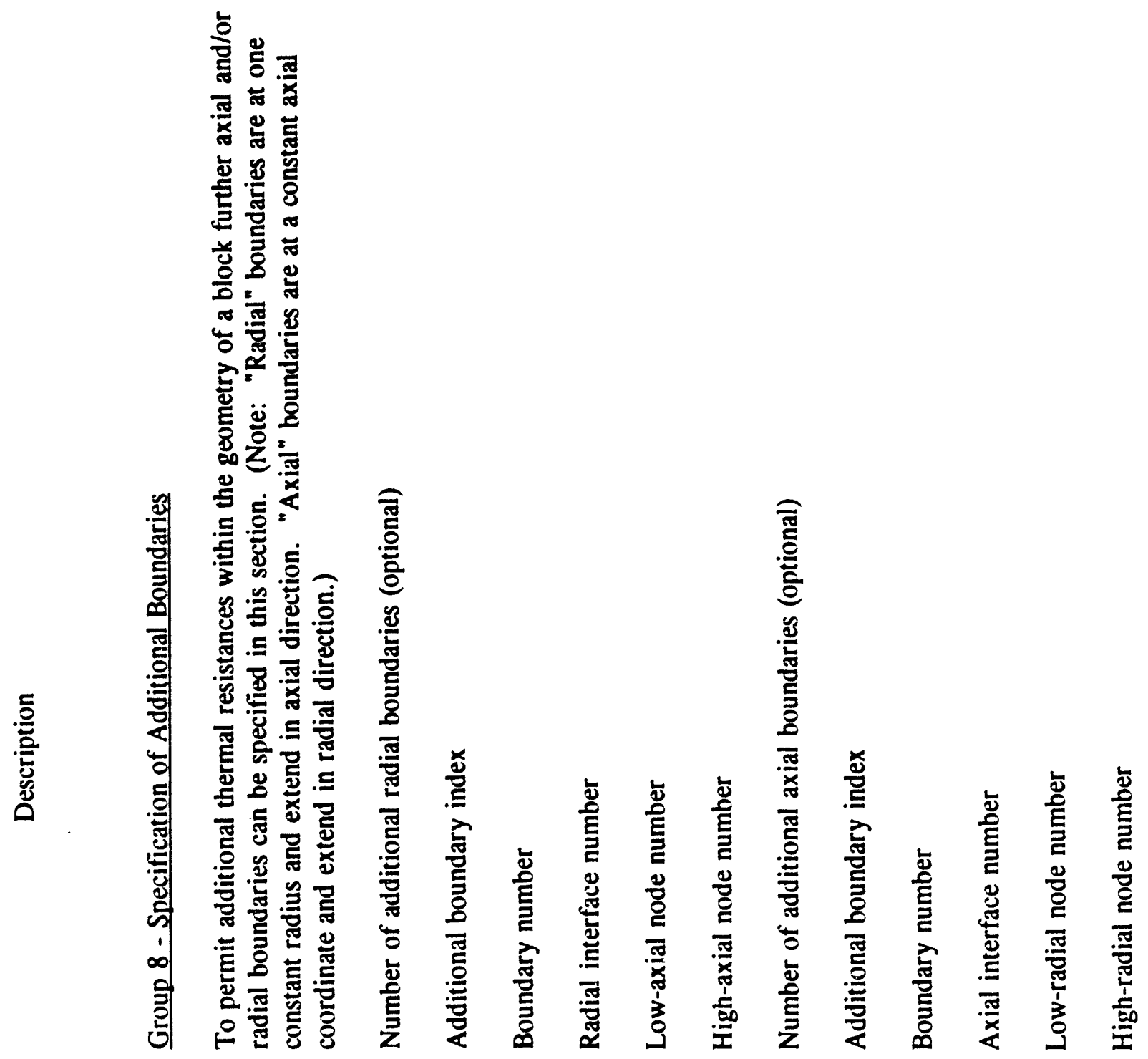

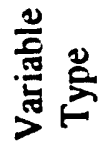

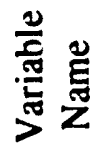

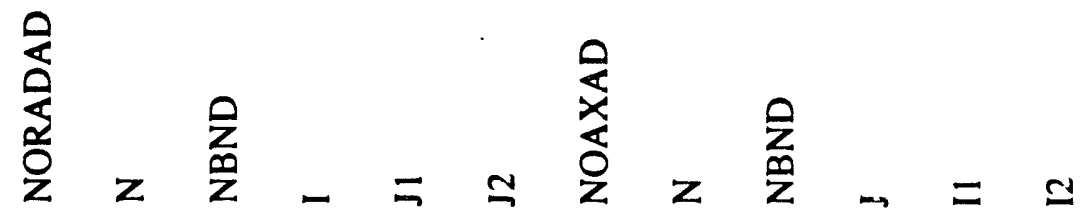

릉

$\frac{0}{\infty} \stackrel{0}{8}$

ஜํㅇ ஓ 
$\stackrel{3}{.2}$

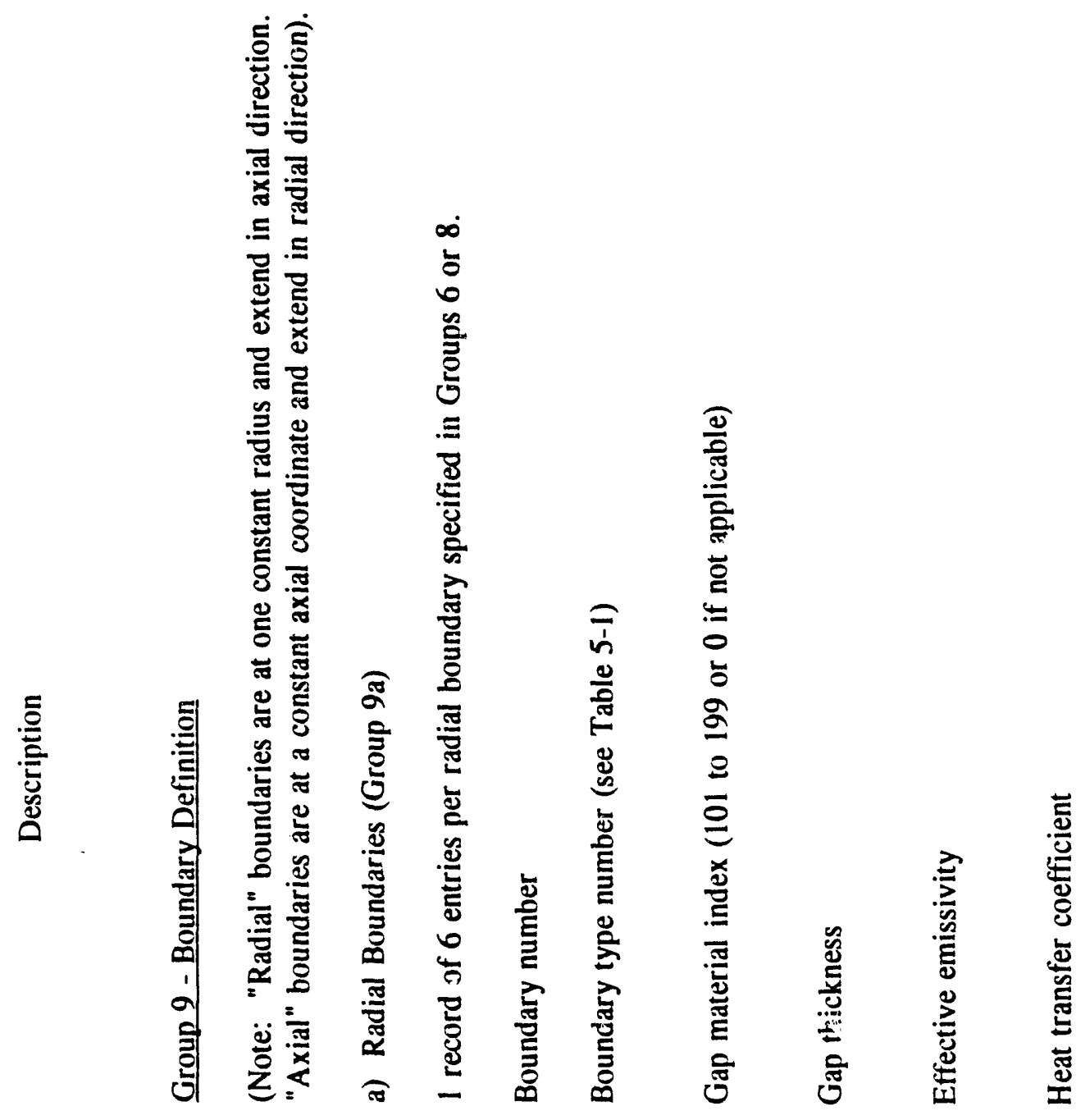

竞

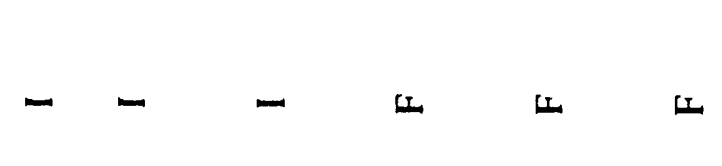

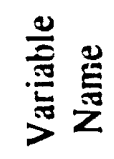

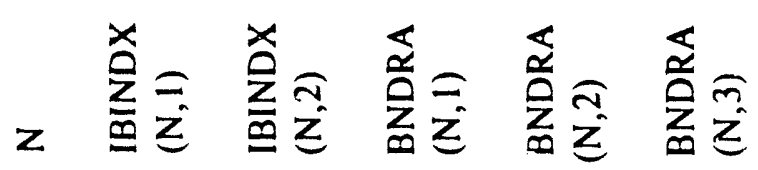

를

$\overline{8}$ 


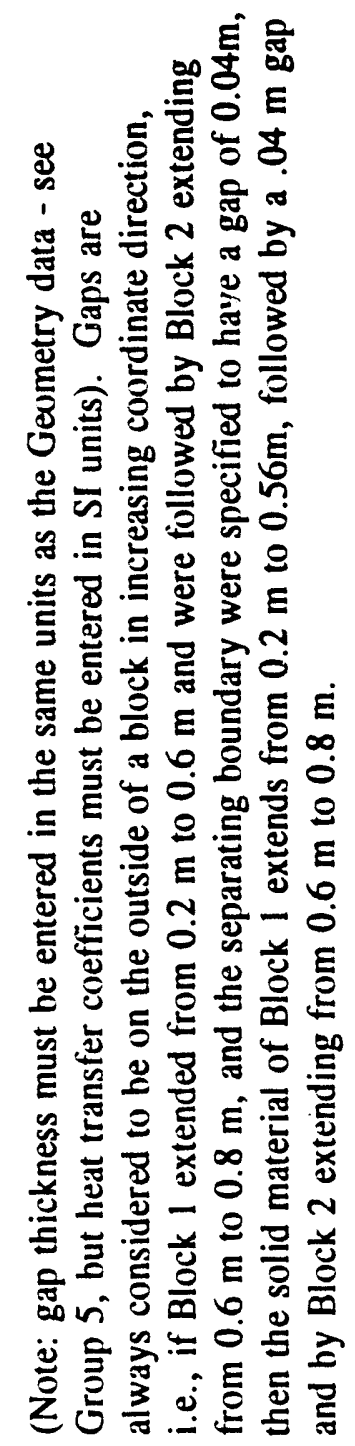


$\stackrel{\mathscr{2}}{5}$
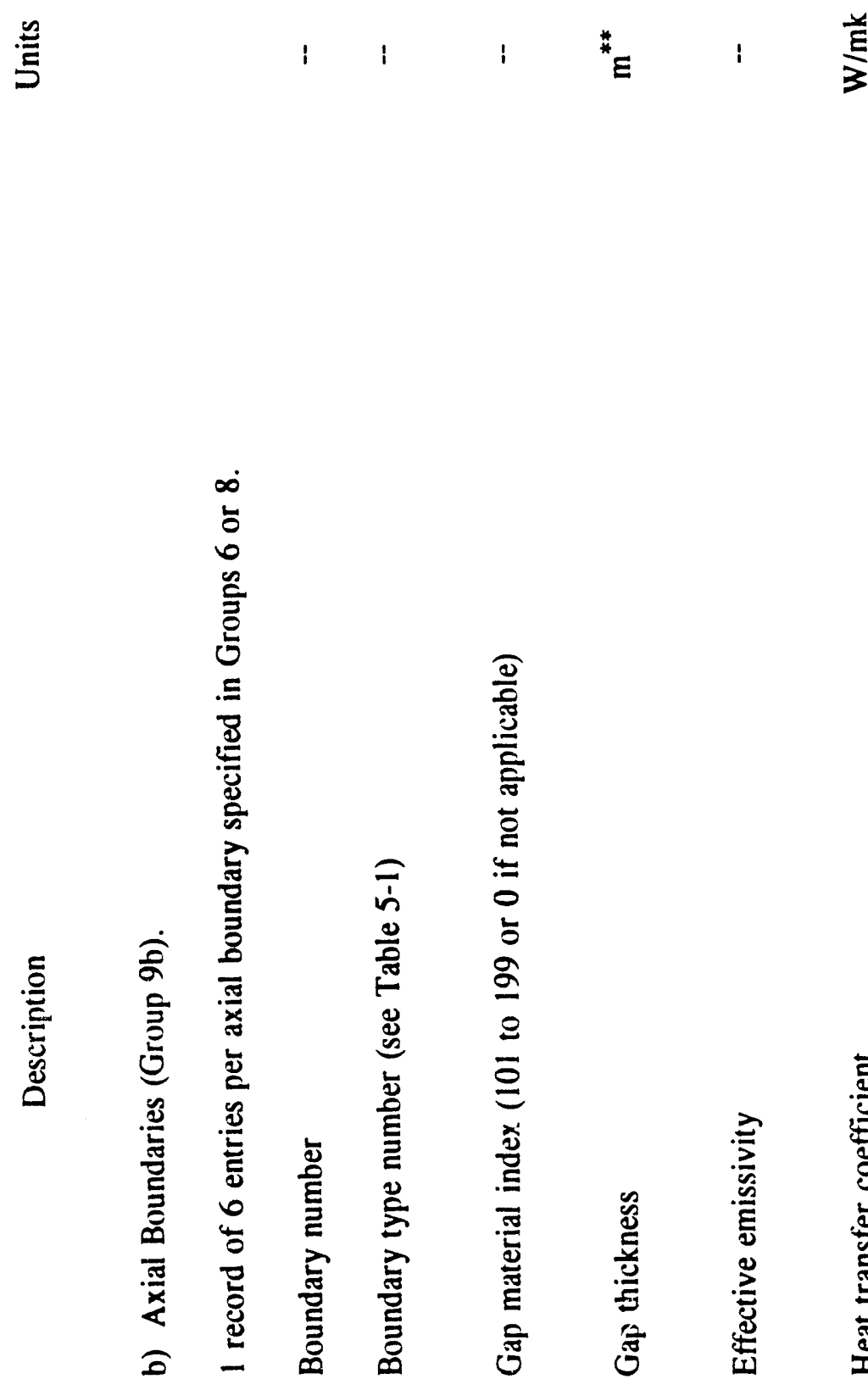

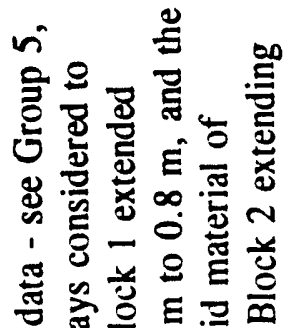

곯

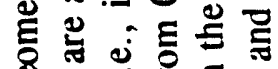
仓

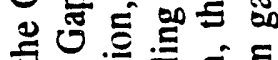

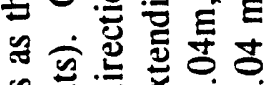
气 :

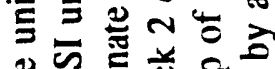

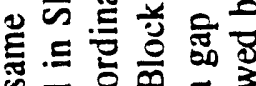

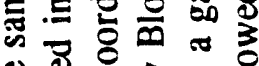
包造远范

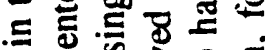

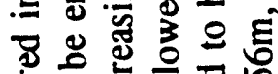

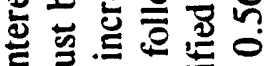
के

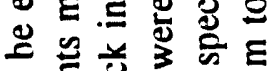

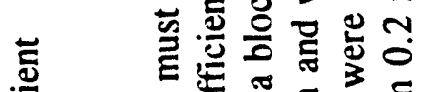

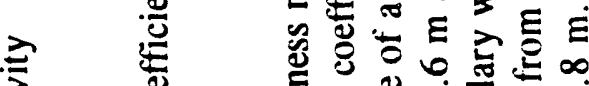
尊虫

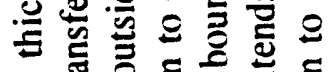
ㄷํำ

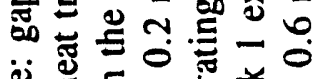

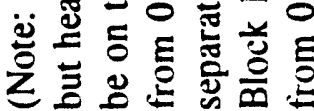

产旁突

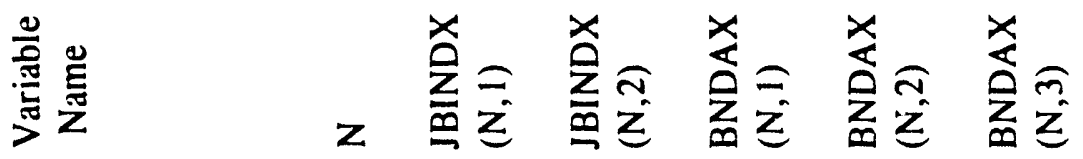

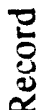

茯 


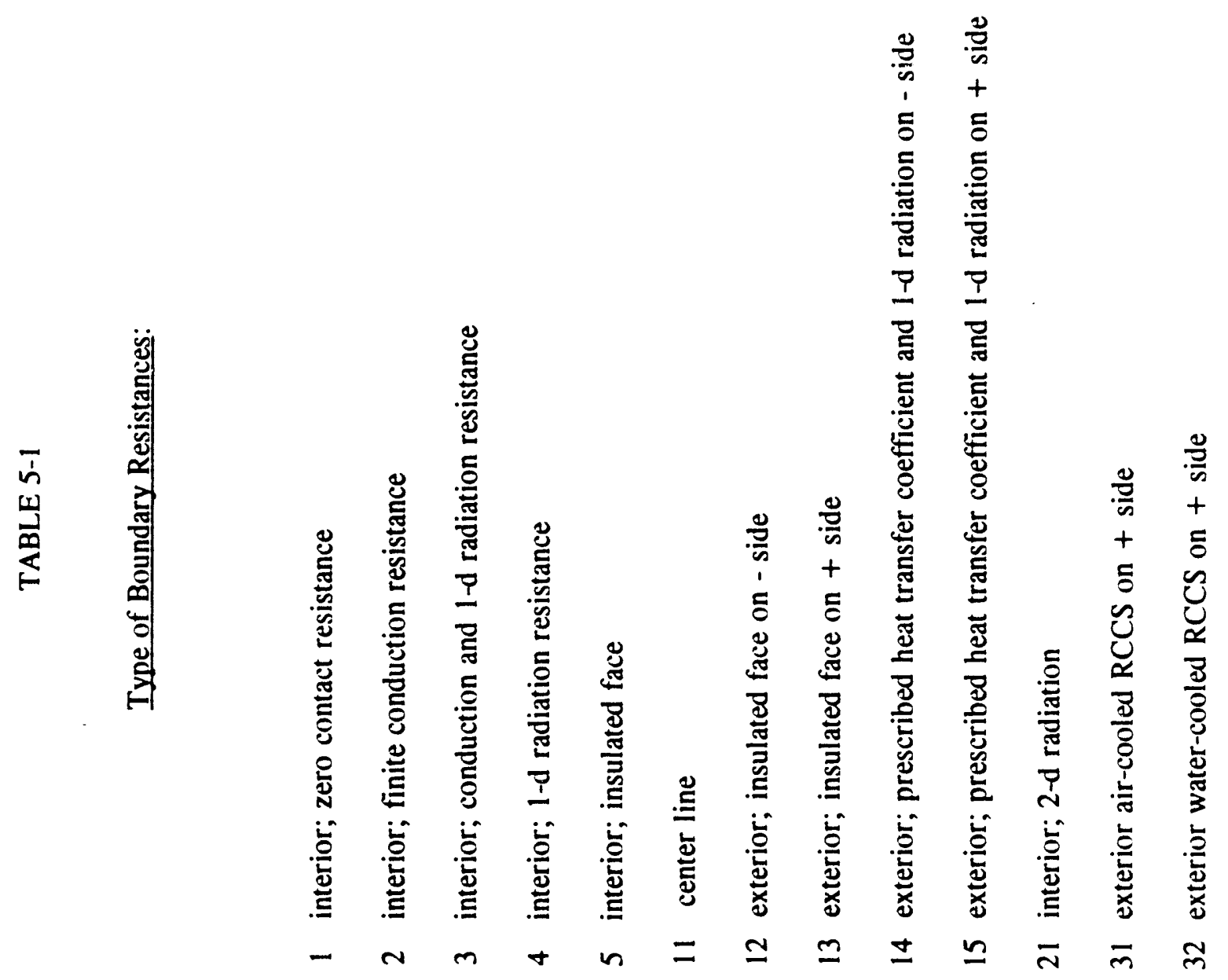


$\stackrel{\mathscr{Z}}{\Xi}$

ป气.

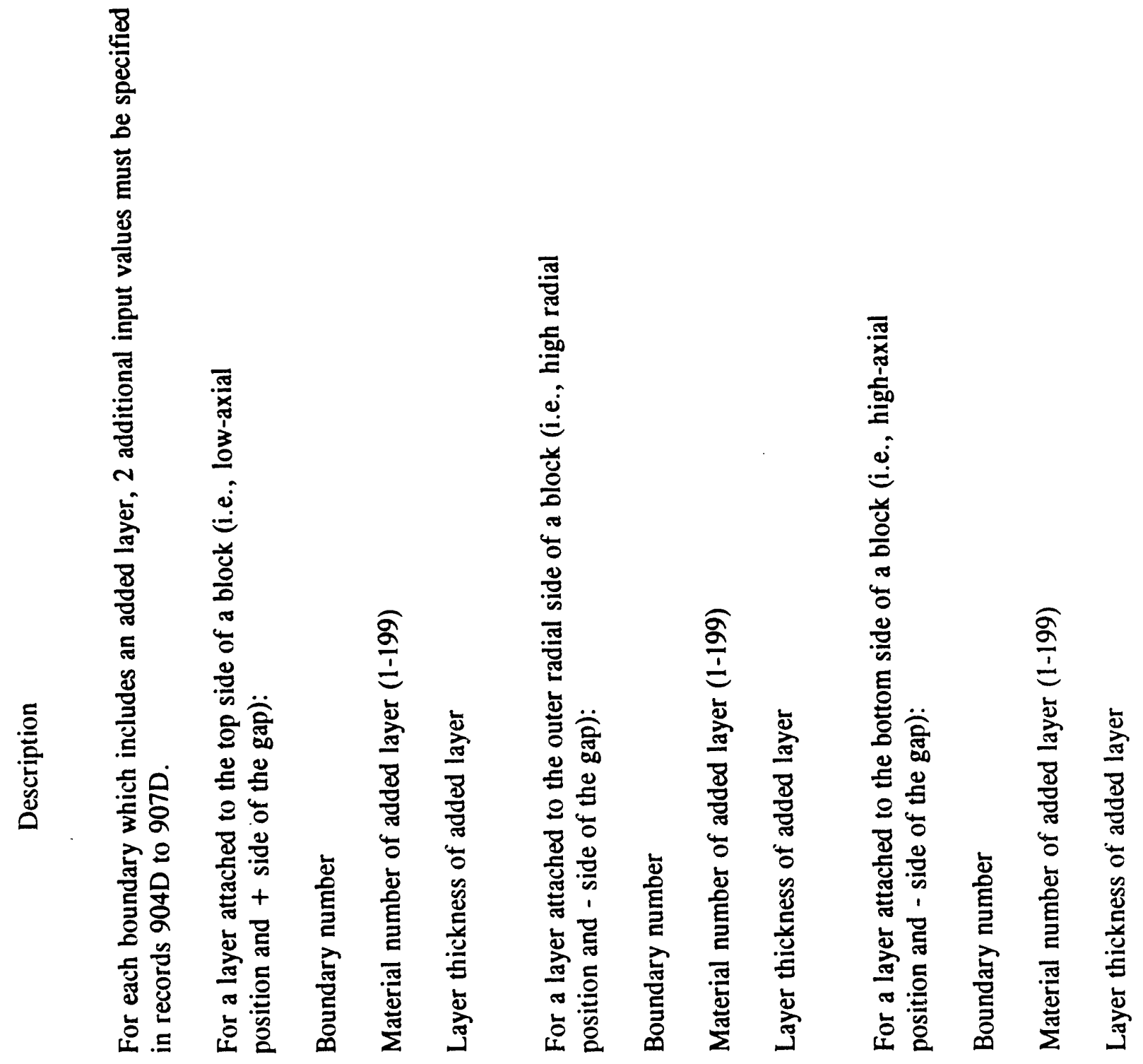

包

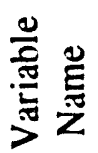

$z \sum_{\Sigma}^{5} \frac{\alpha}{2}$

$z \sum_{j}^{\xi} \frac{\alpha}{\alpha}$

己્ّ

용

อ 
竞 $\quad 1 \quad$ *
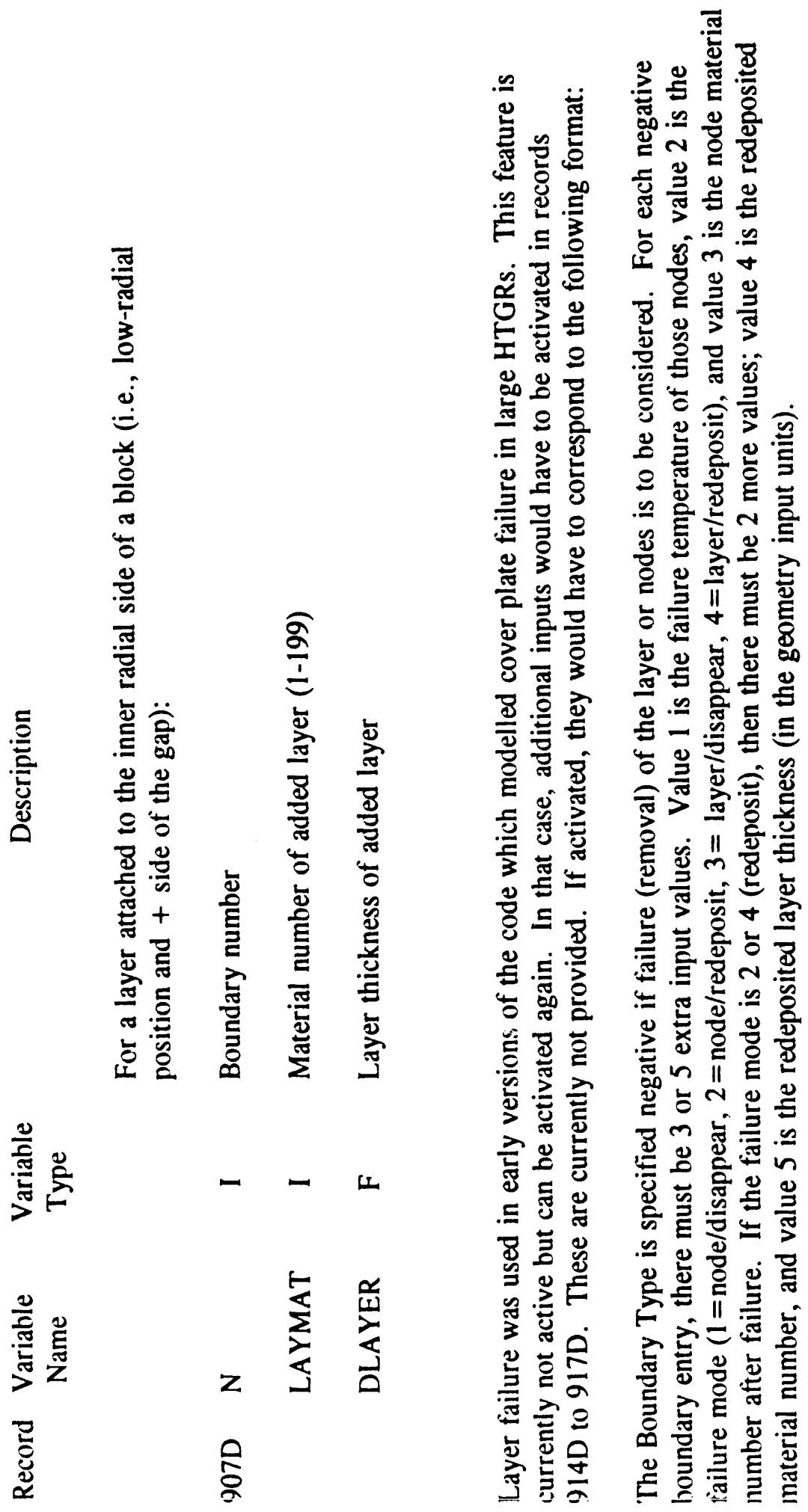
产

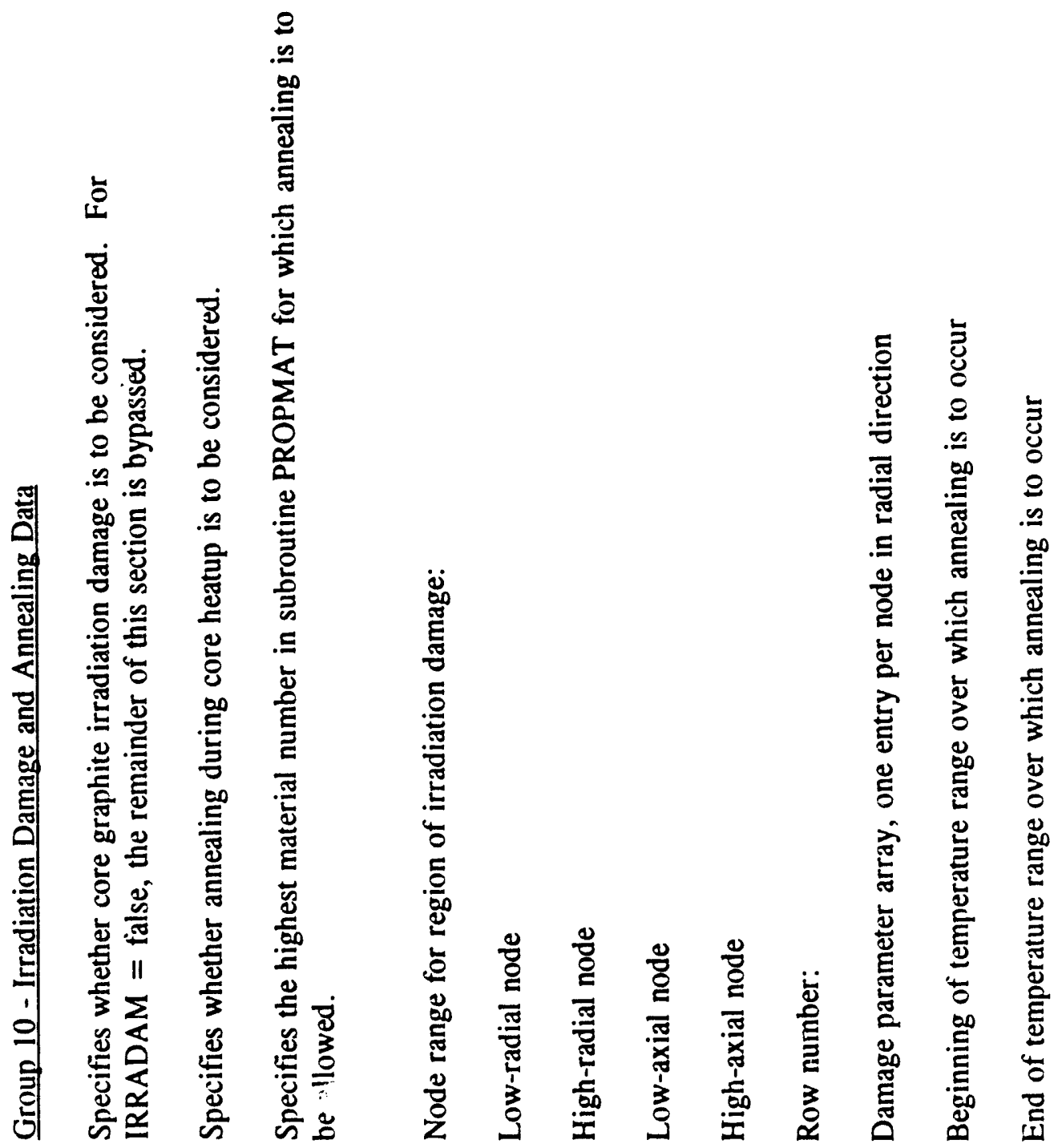

紊

旁

衰喜喜

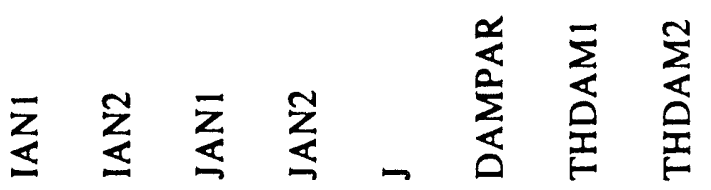

高

言

毫

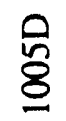

星 
善

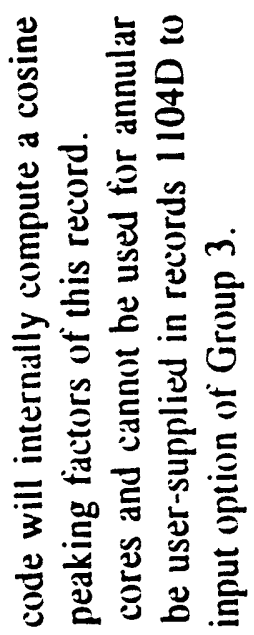

중

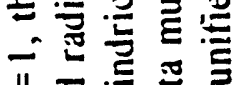

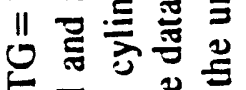

중

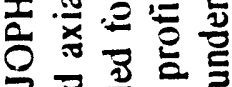

=

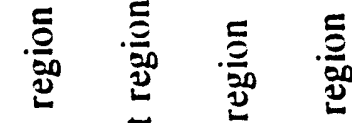

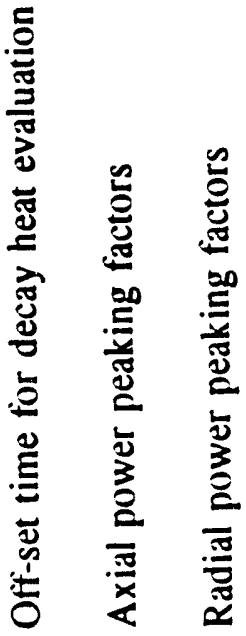

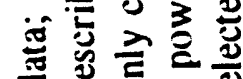

क्व

늘 제에

政

늘

蛋

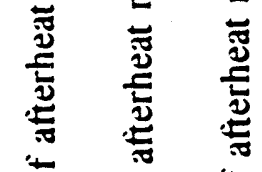

글

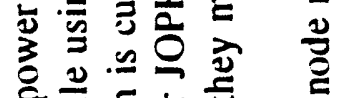

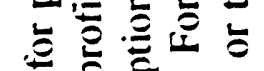

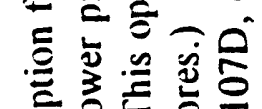

을

के

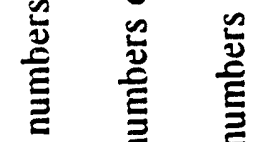

苞

总

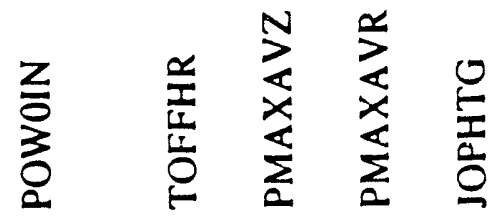

焉豆

竞

言

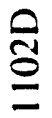


高

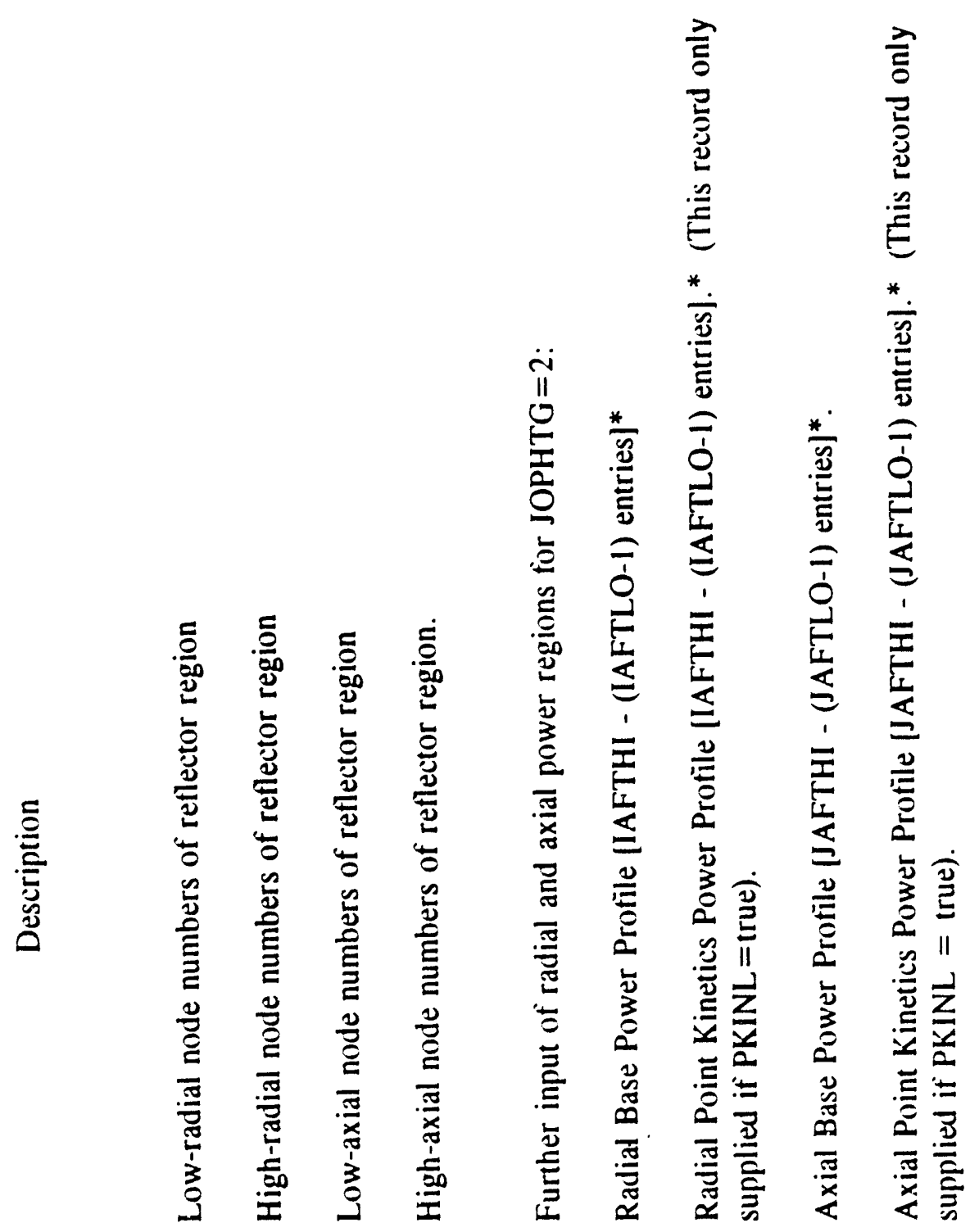

善 몰

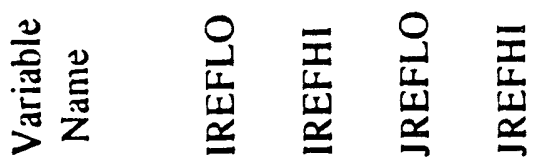

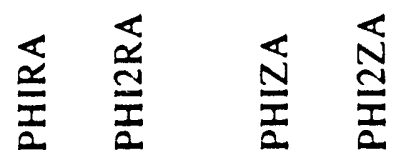

吝 总

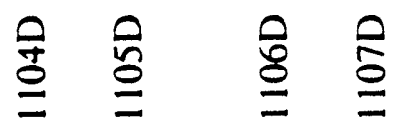


器!

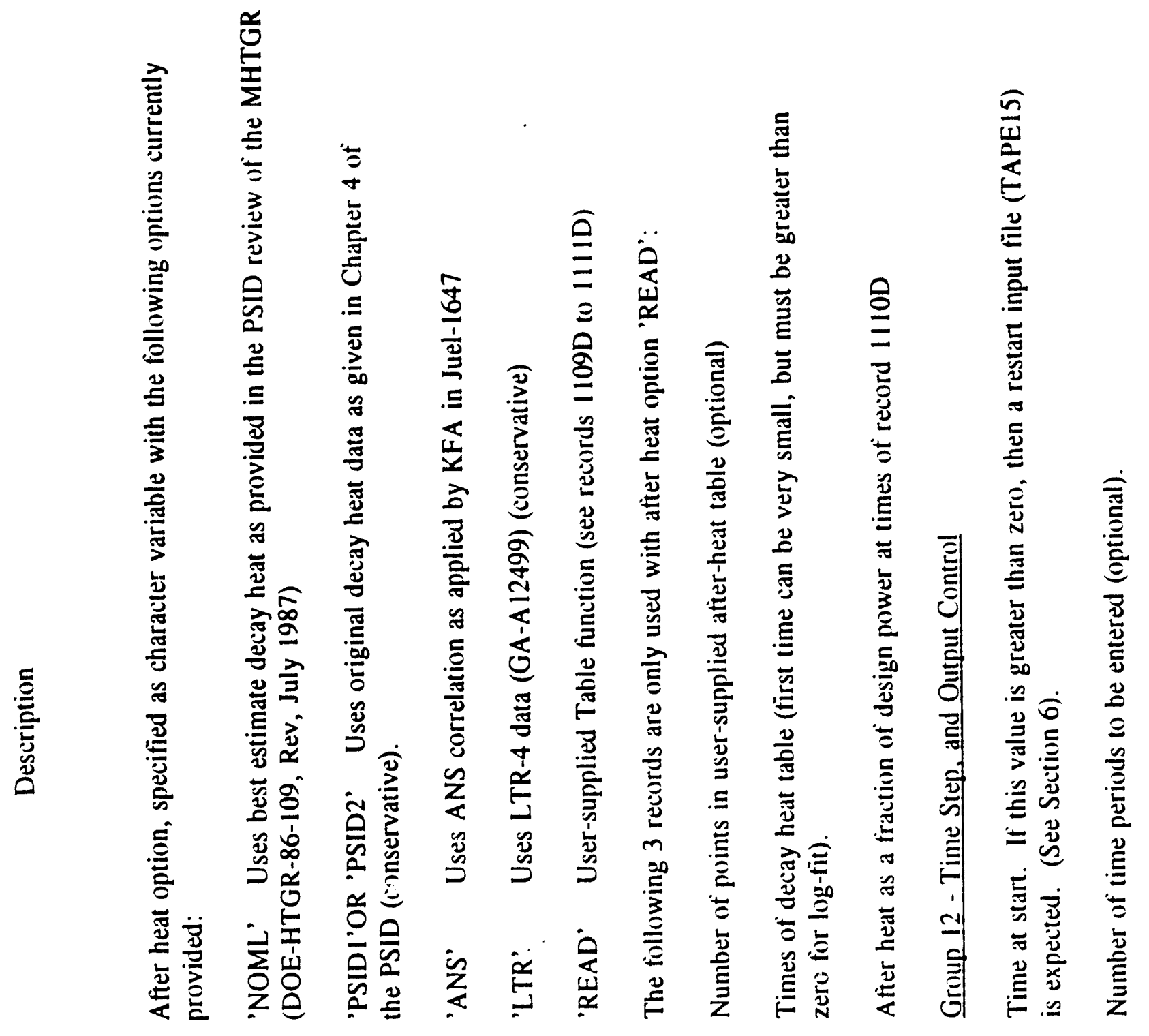

善誉。

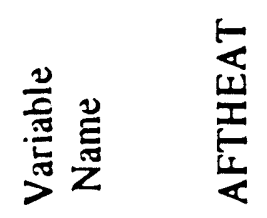

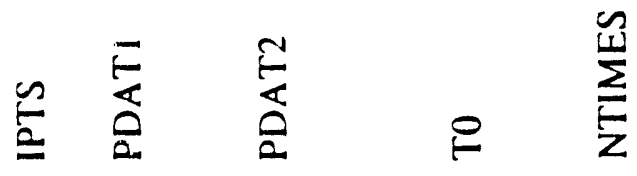

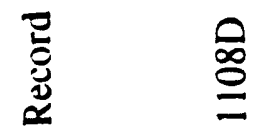

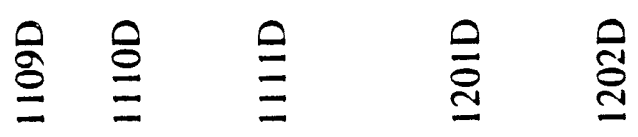



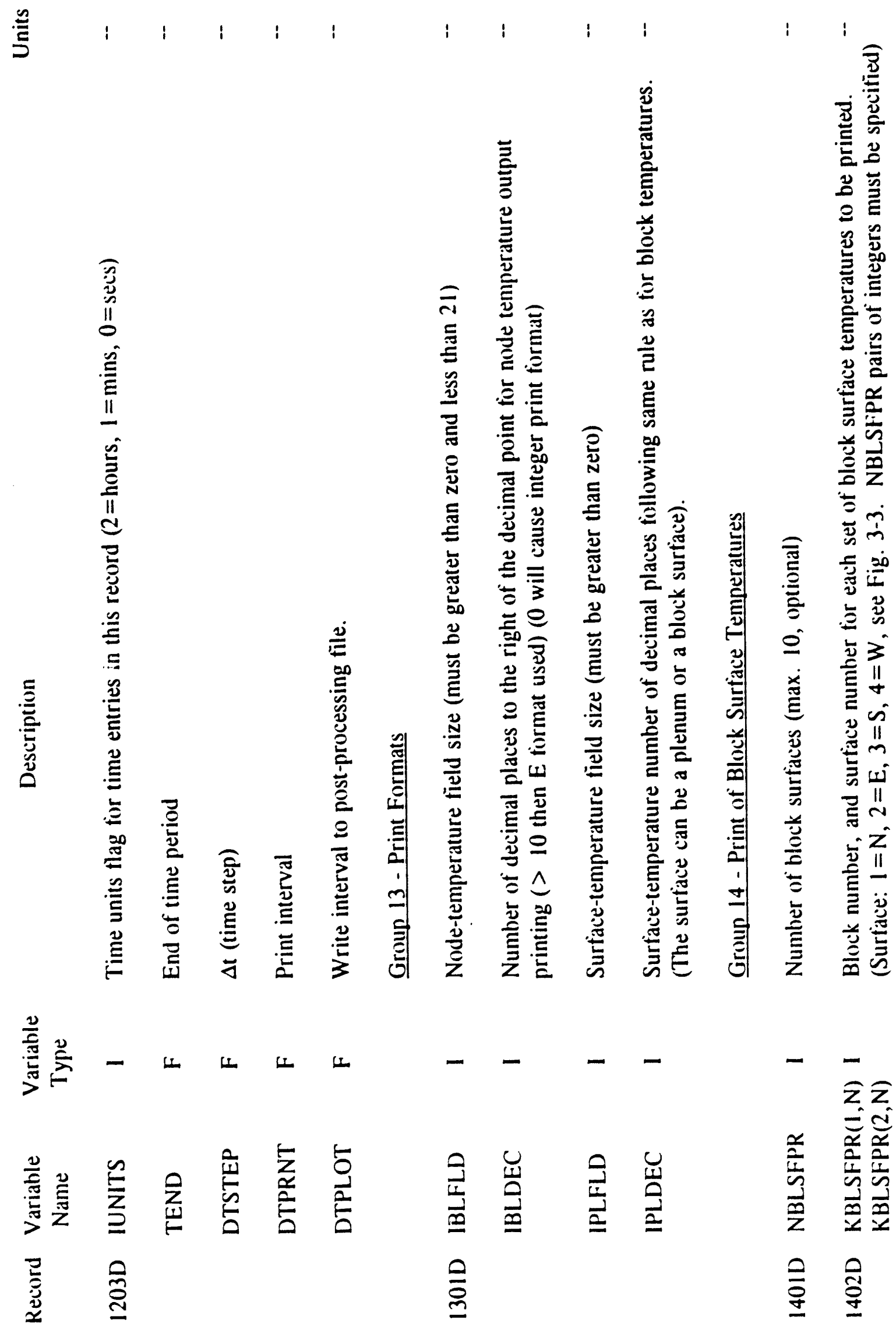
$\stackrel{\mathscr{E}}{\stackrel{\Xi}{\Xi}}$

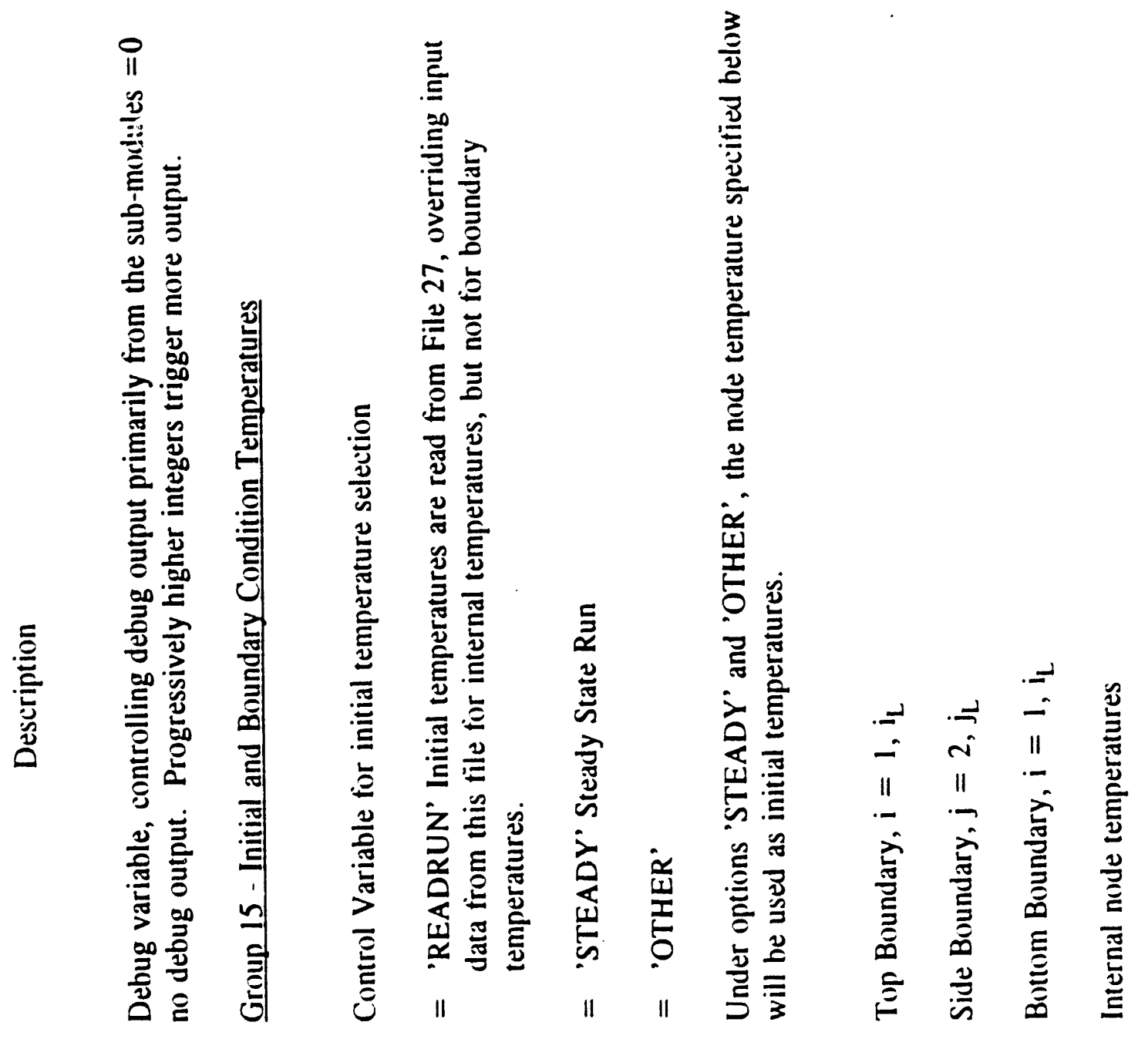

$\div \div$
产

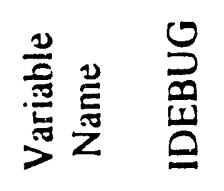

幽

喜兽

言
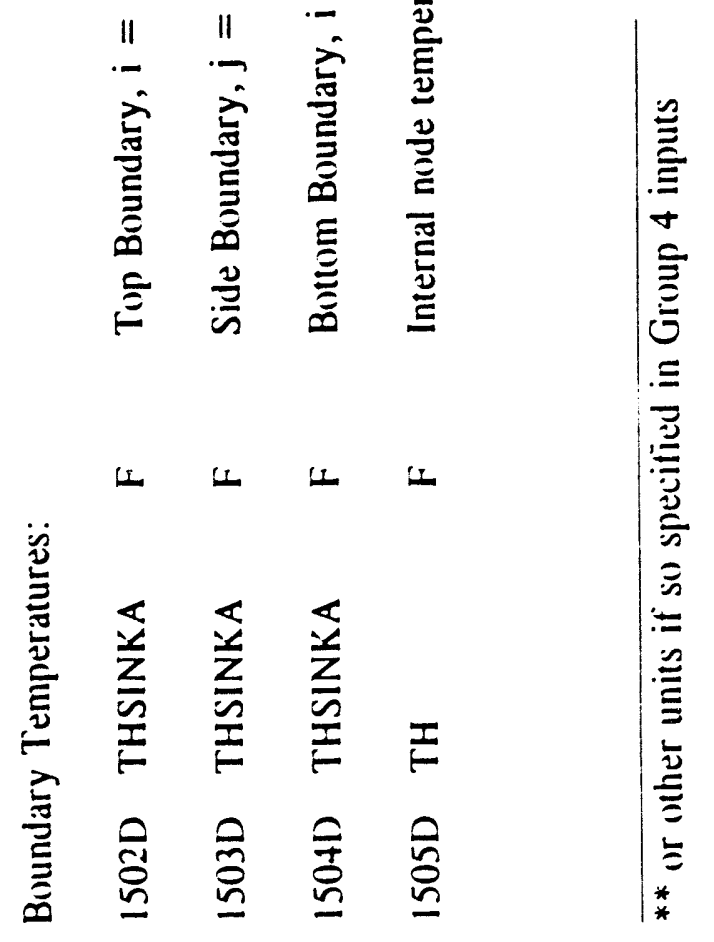
旁

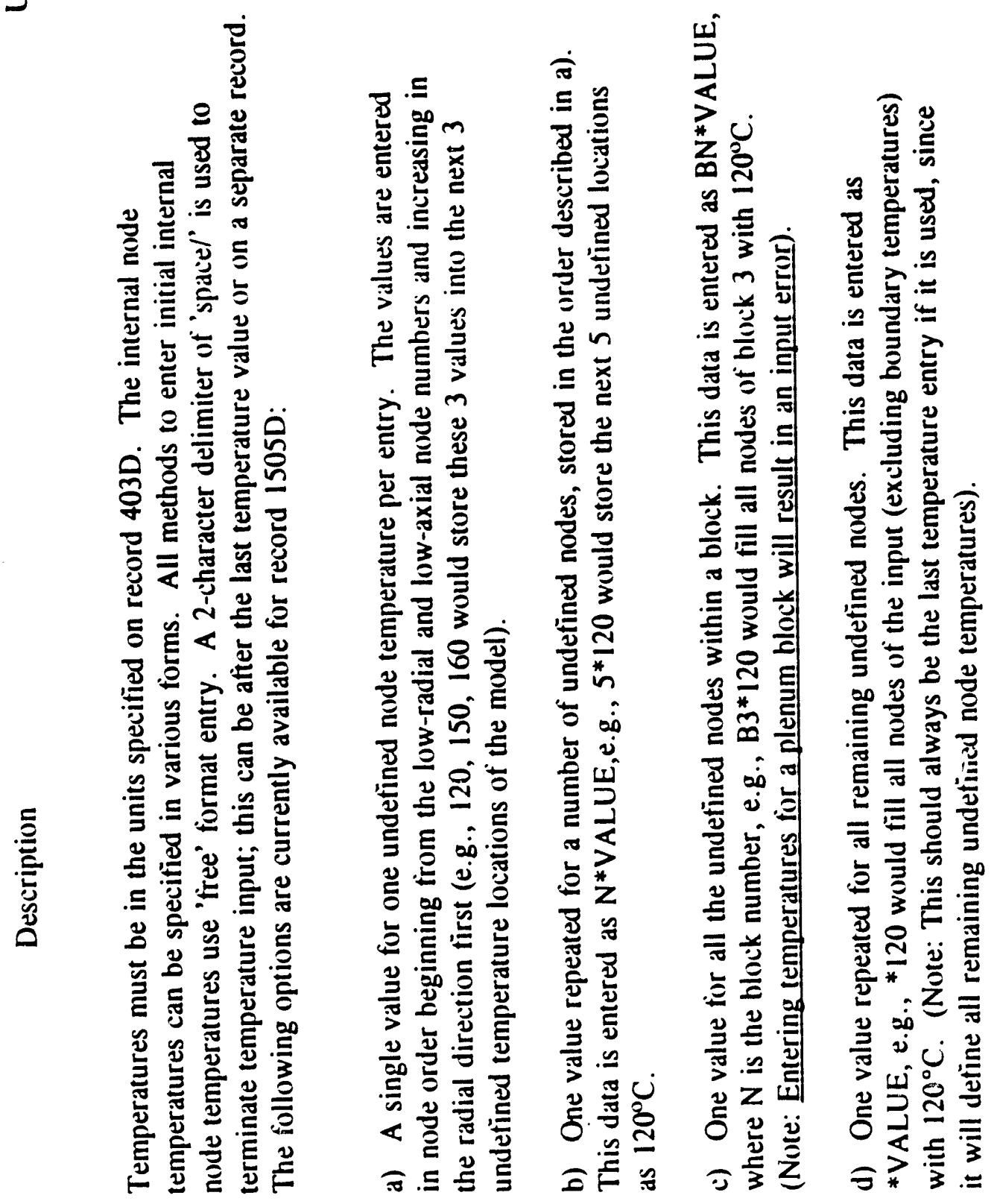

章

خ

ᄅ 
$\stackrel{2}{5}$

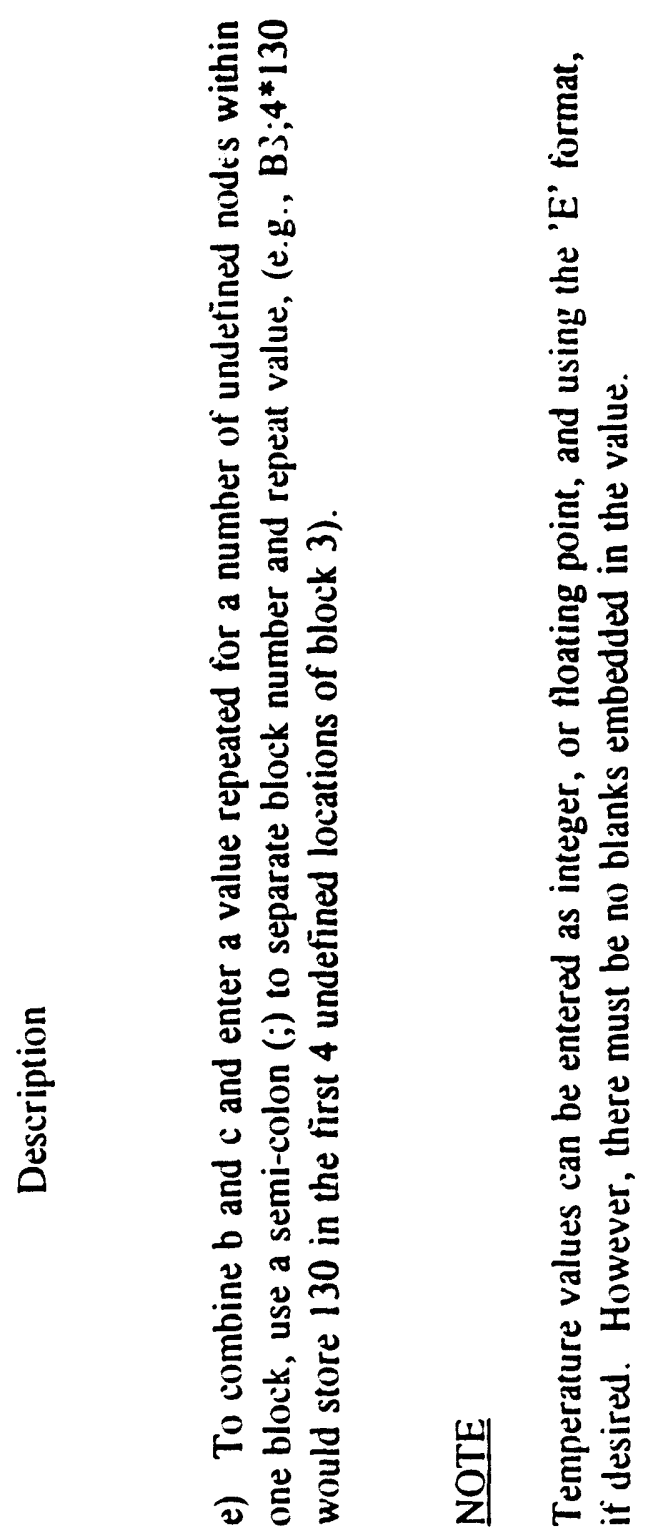

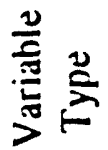

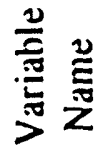

כِّ 


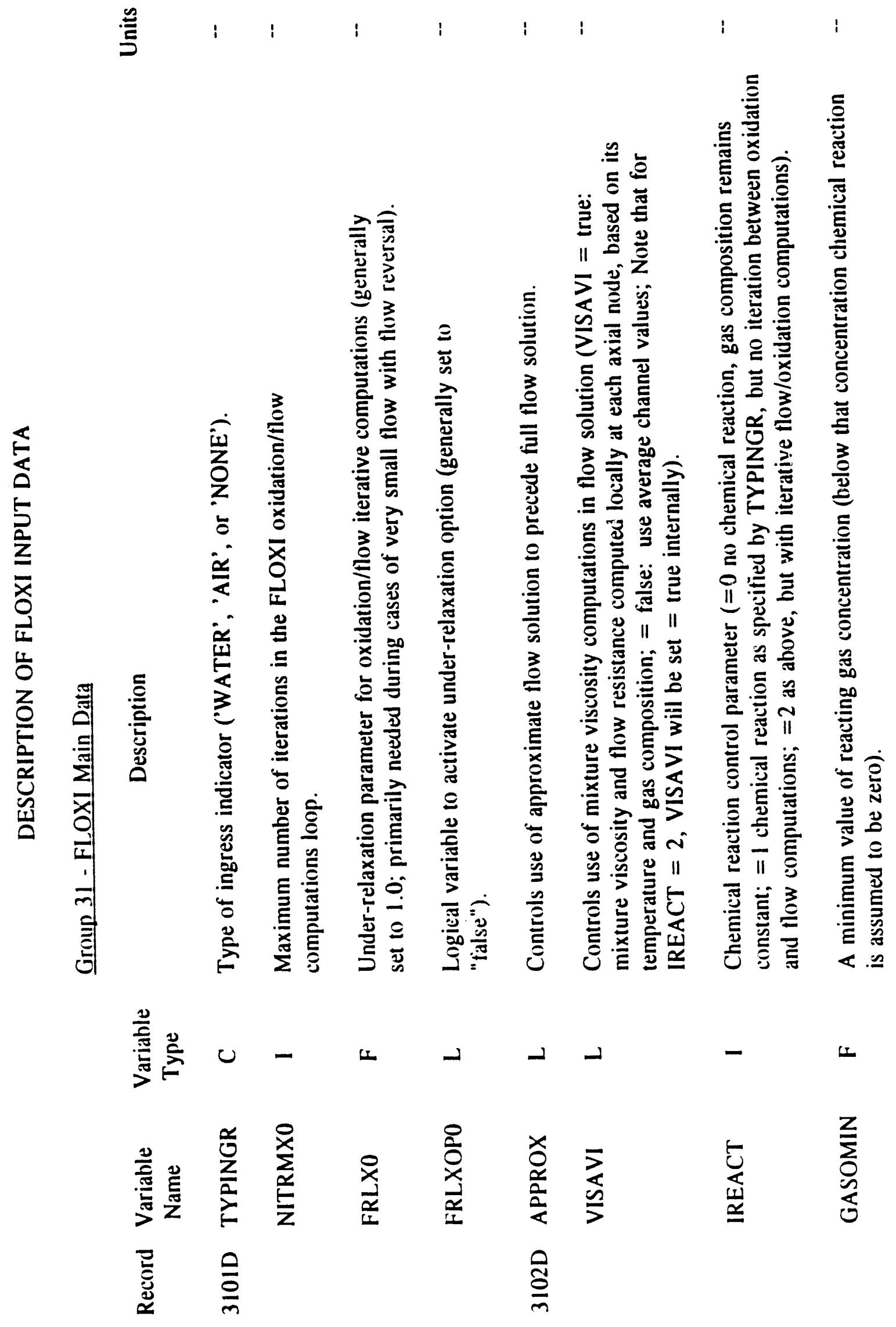



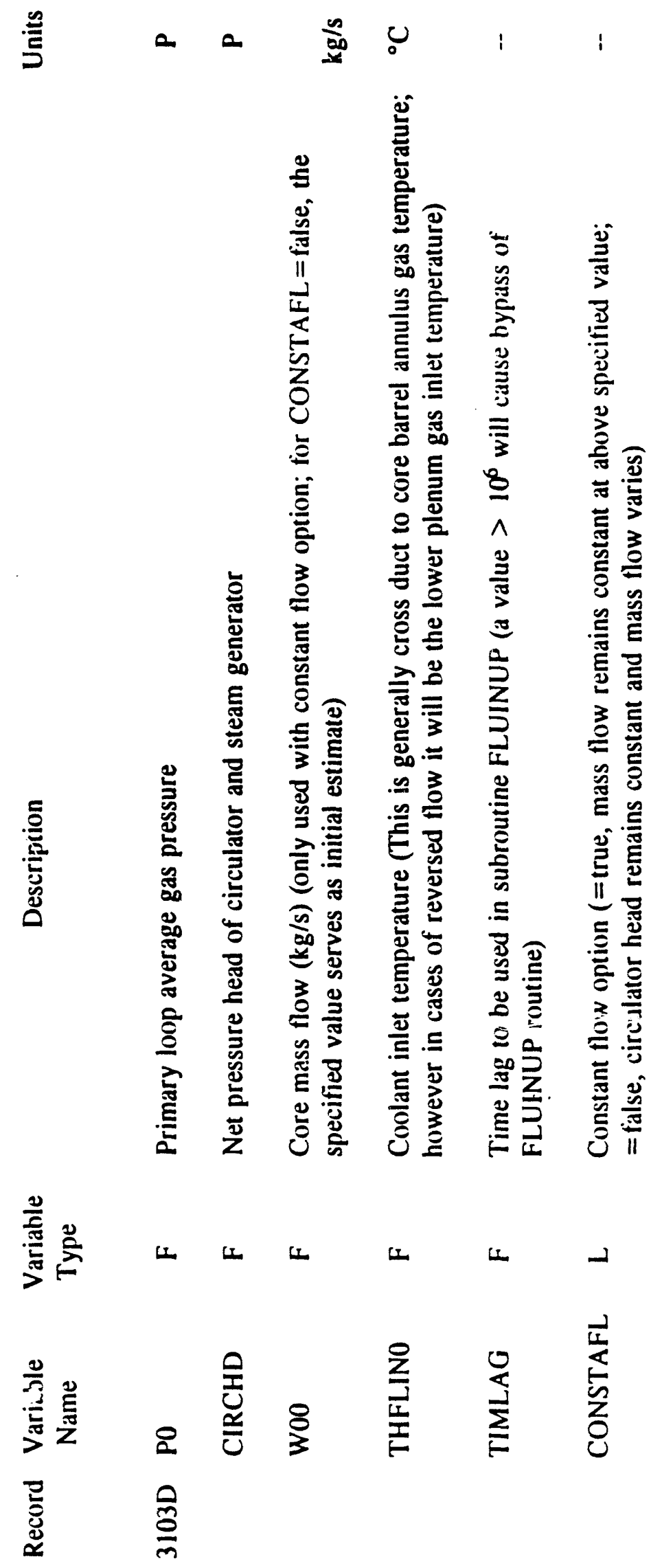
$\stackrel{2}{2}$

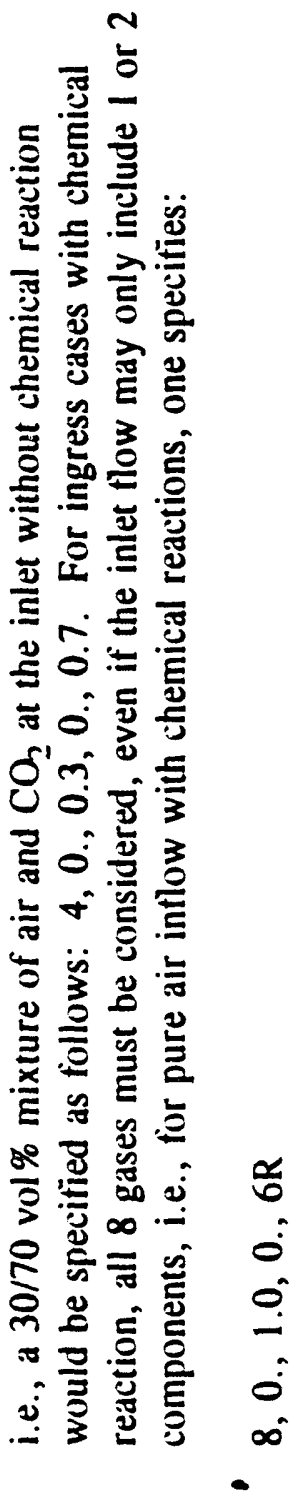

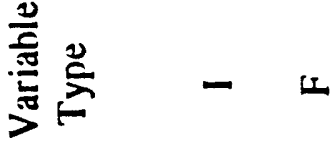

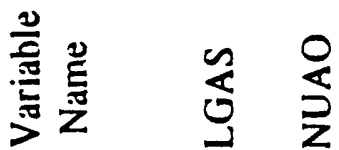

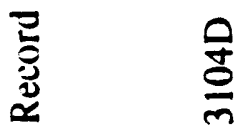




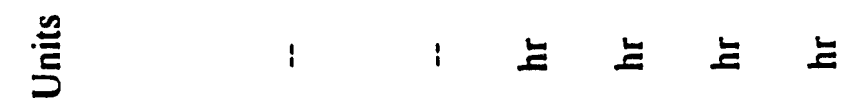
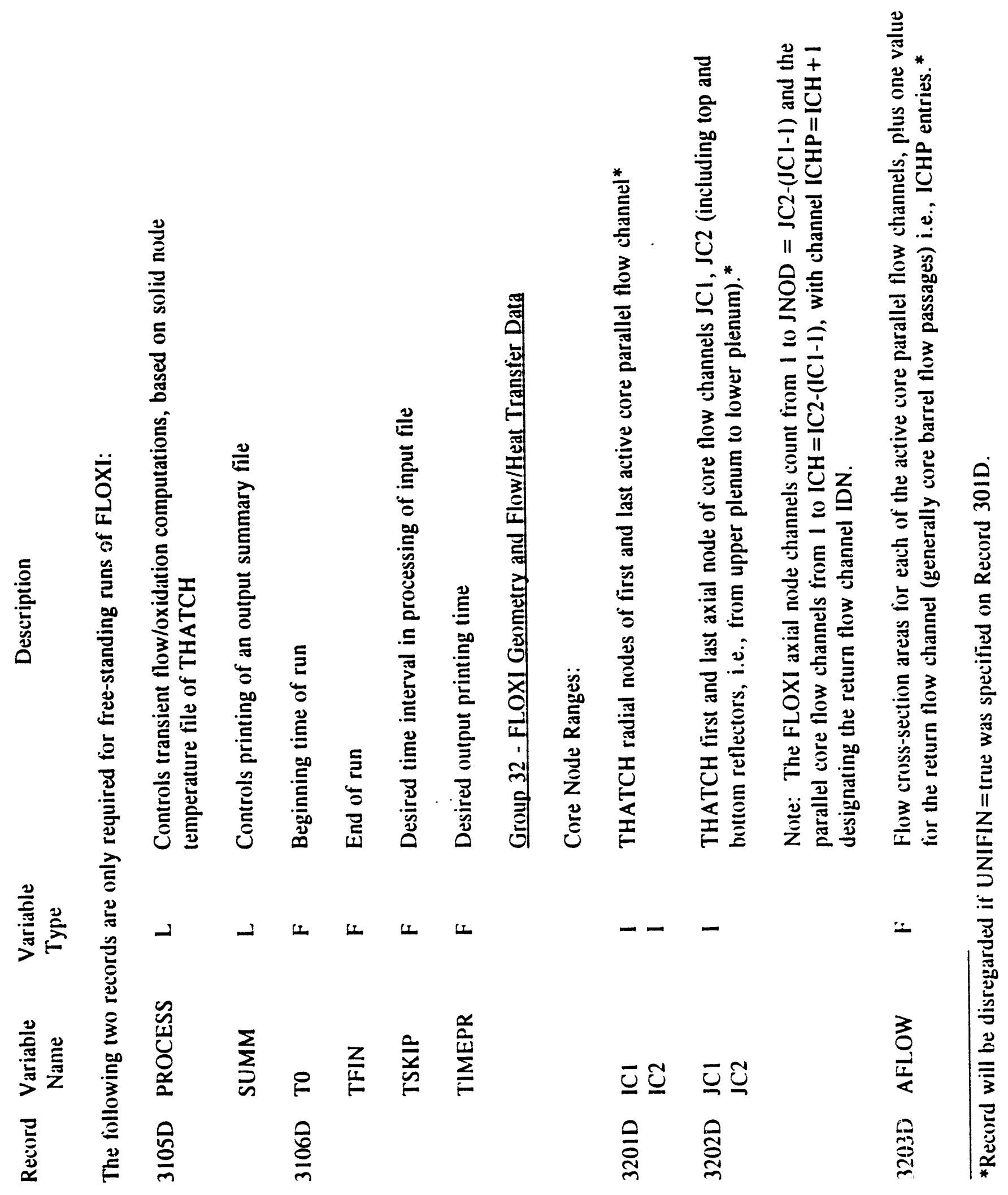


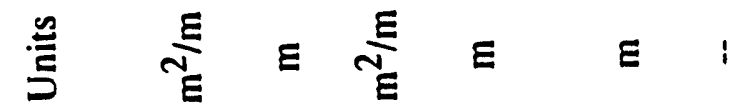

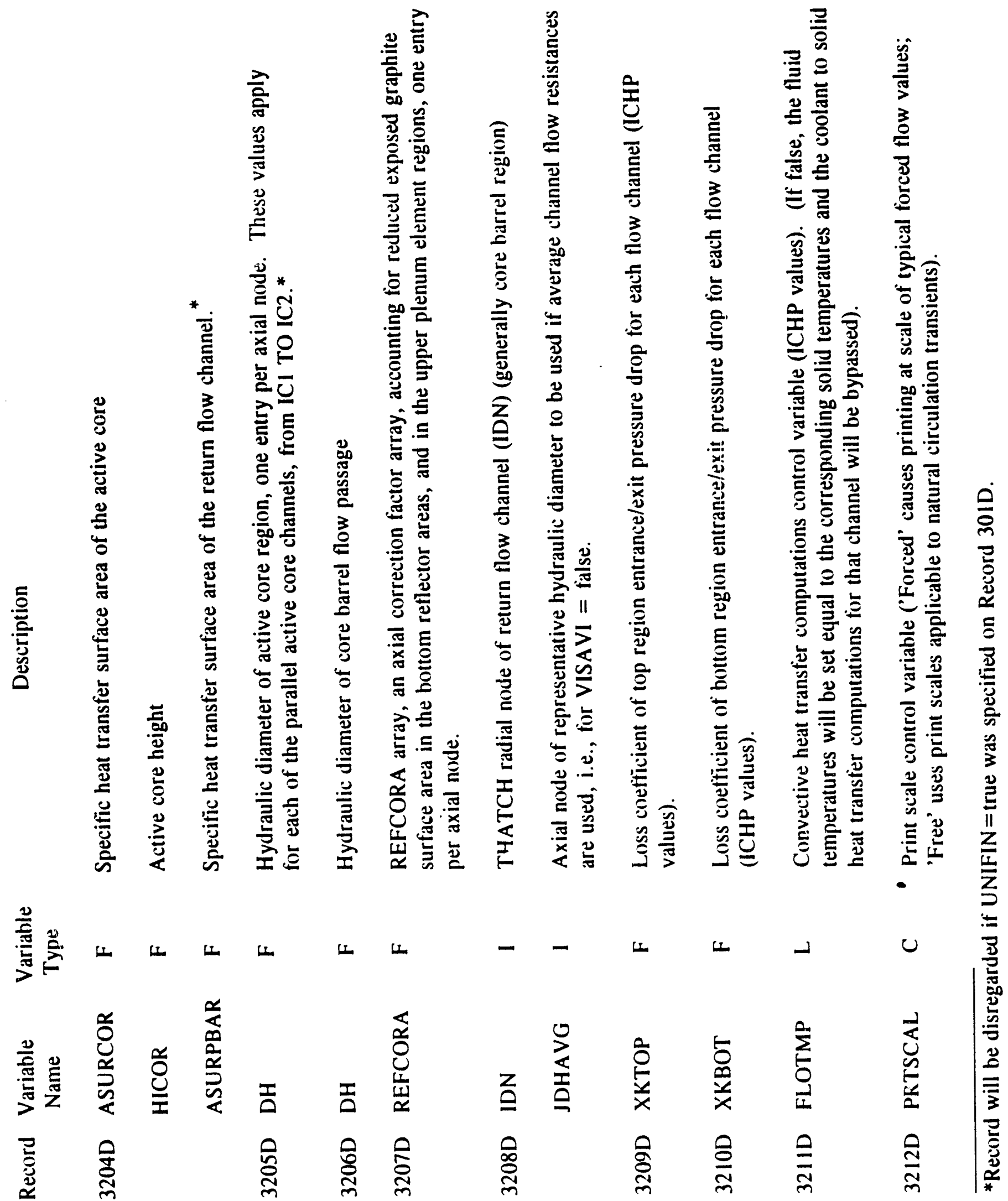


亭

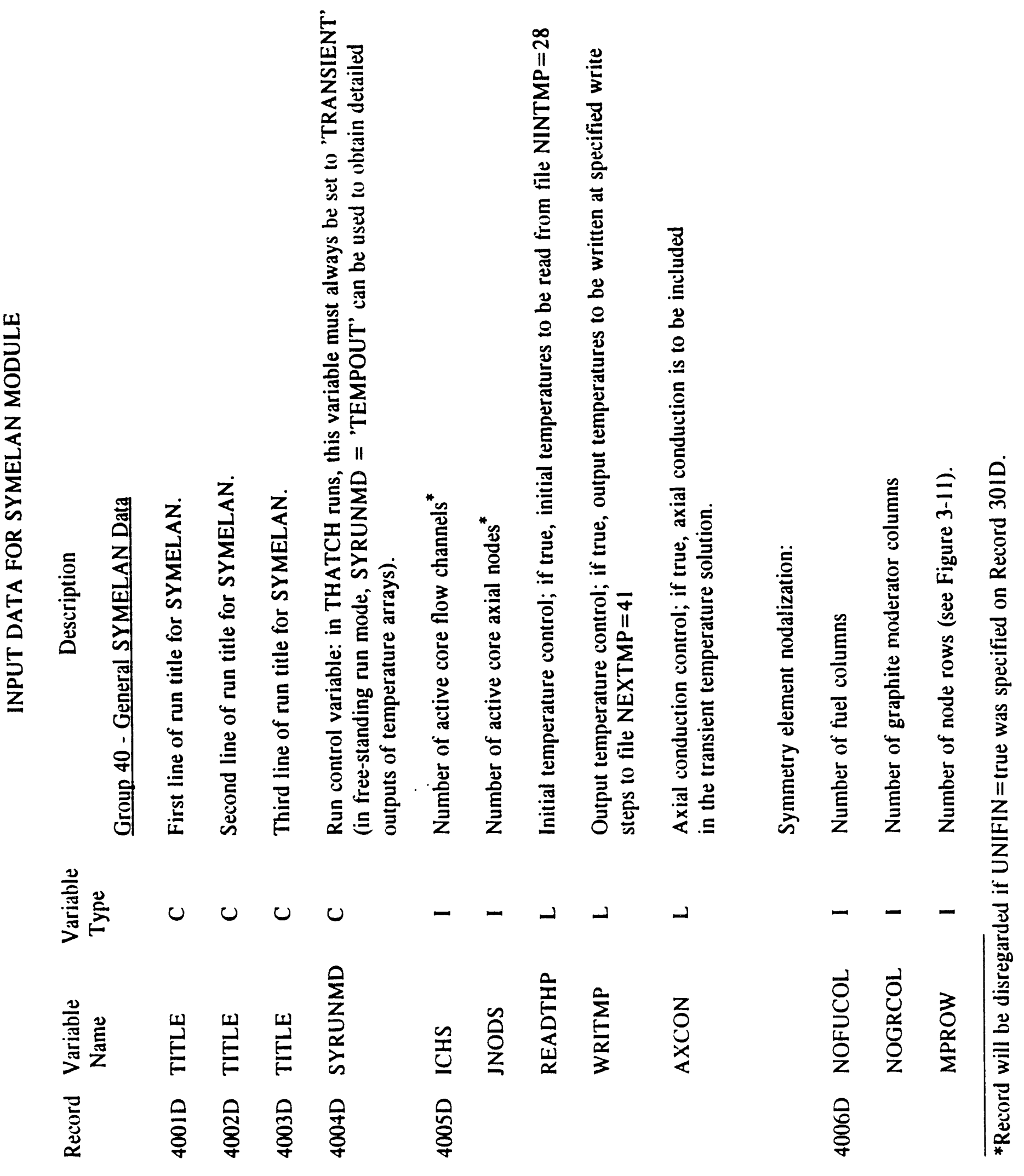




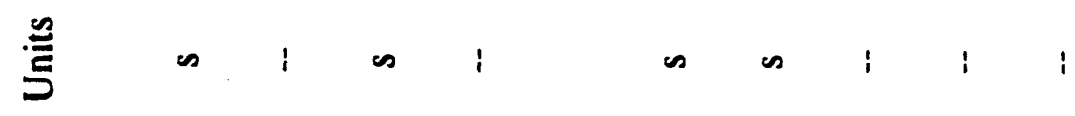

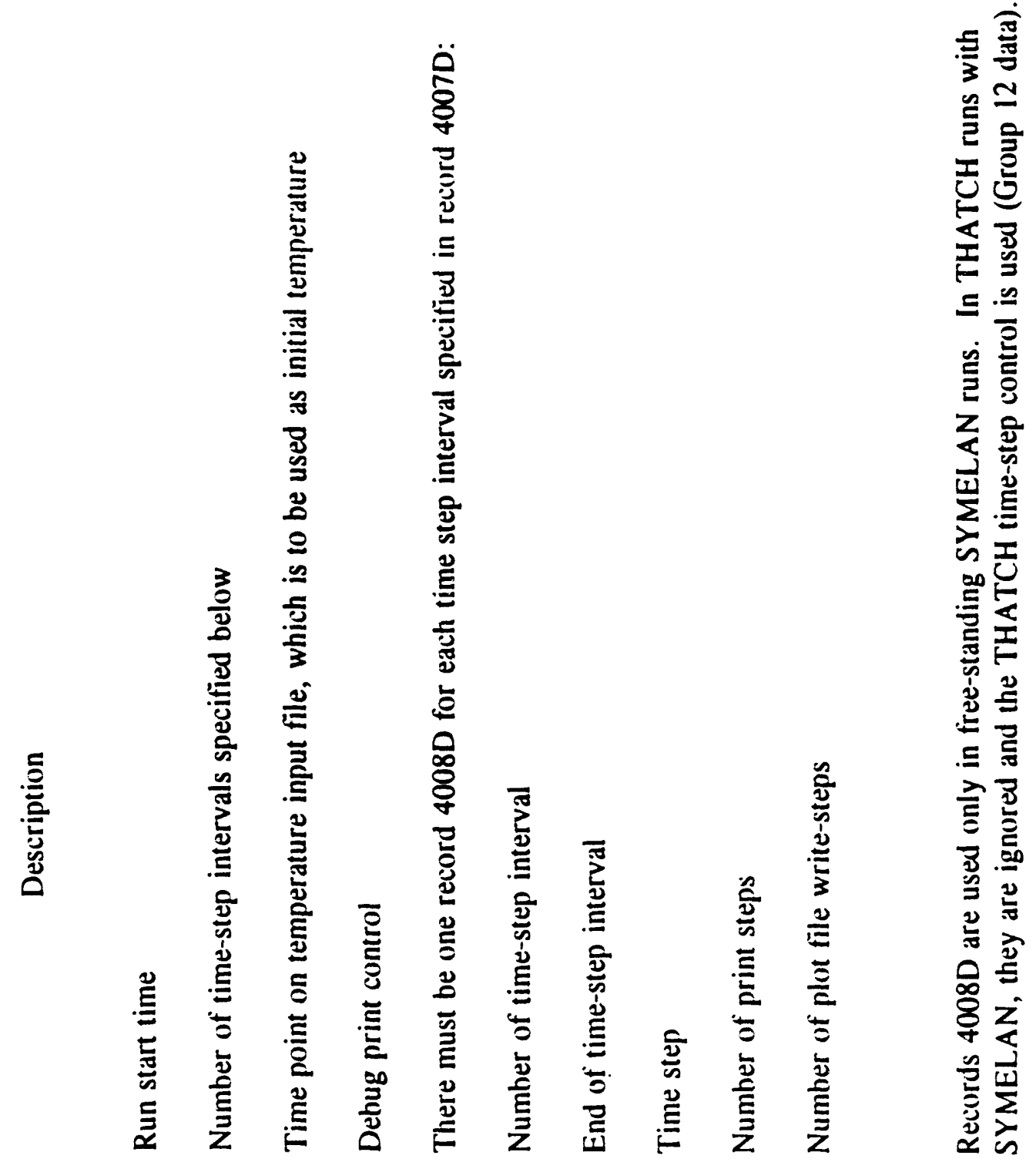

产

紊竞

总

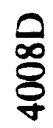


总

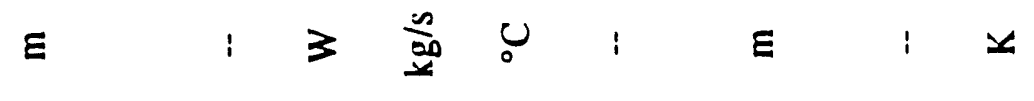
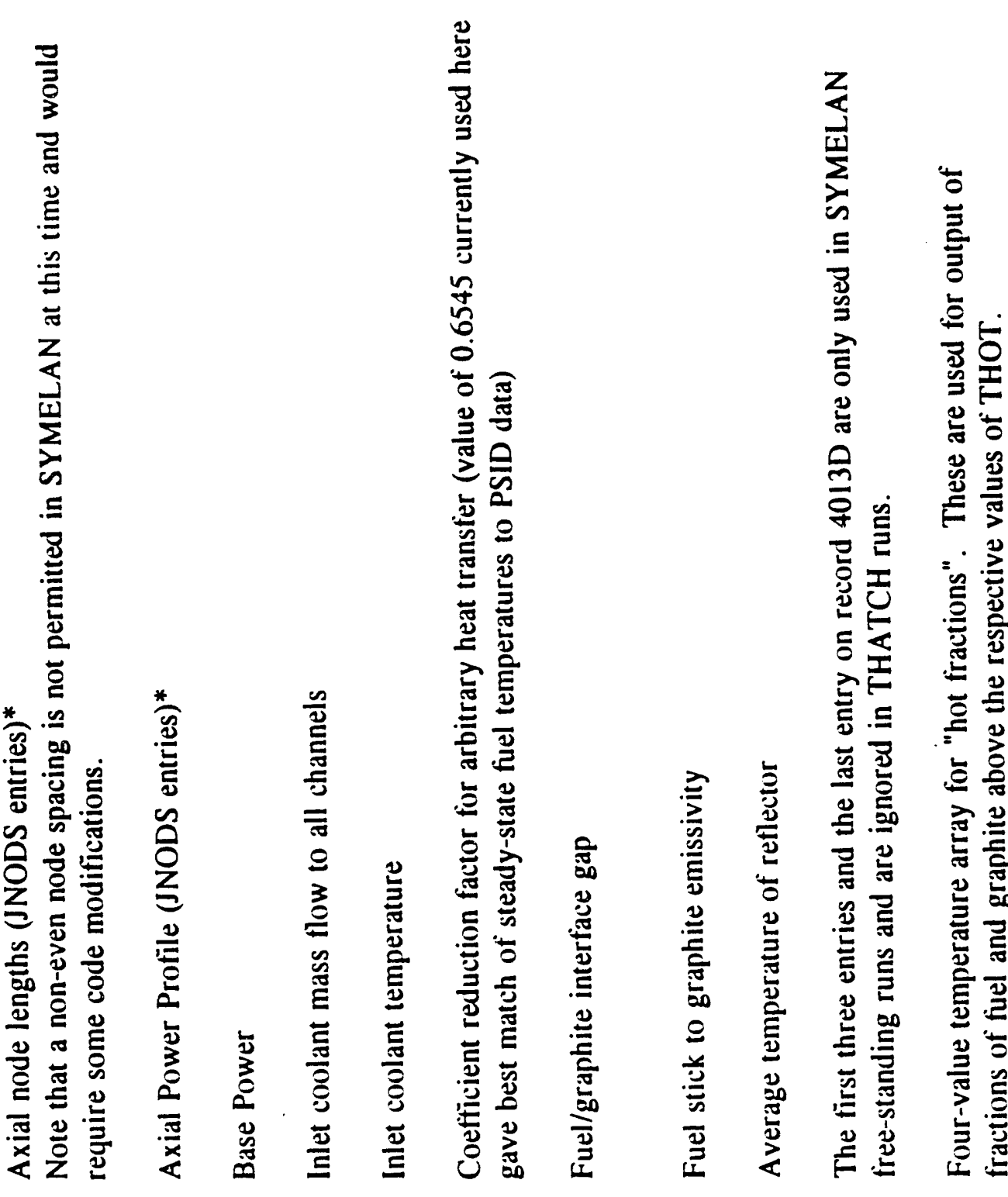

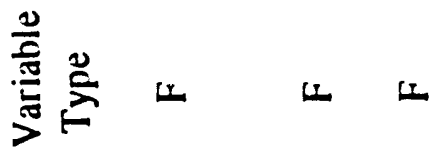

IIIIIIII II I I I

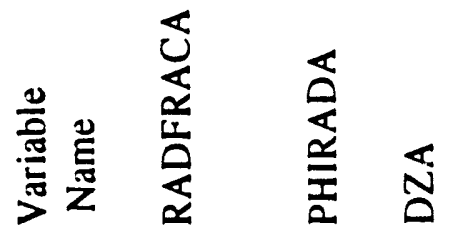

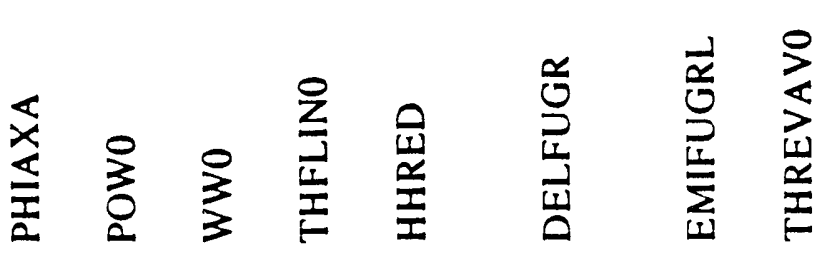

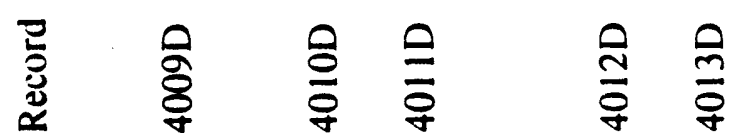




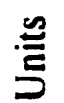
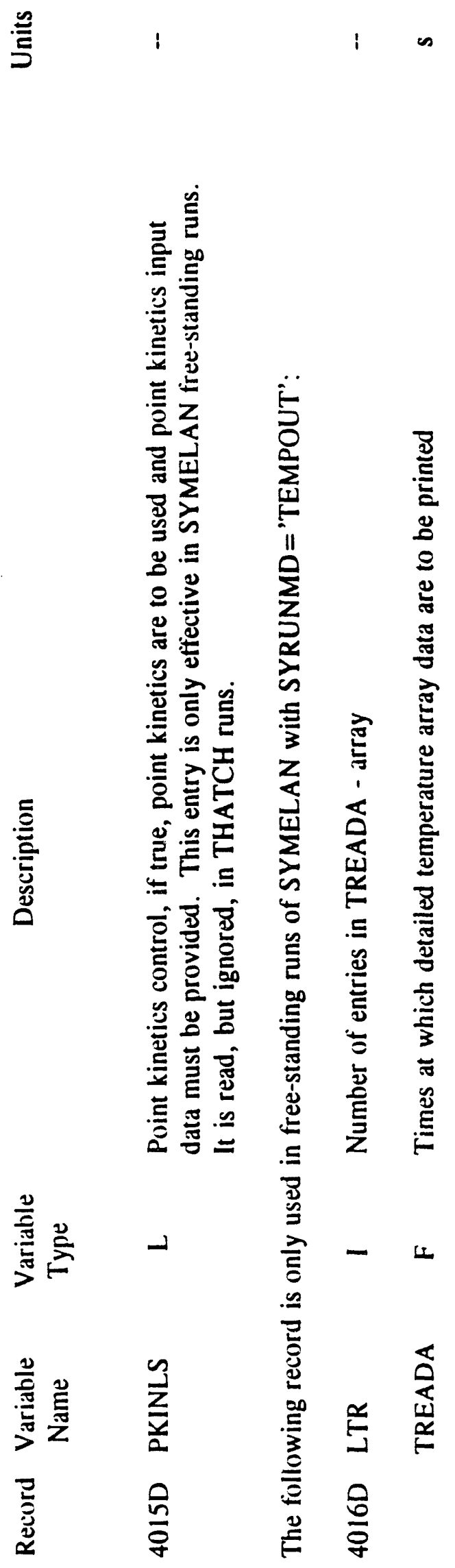
$\stackrel{\varrho}{\Xi}$
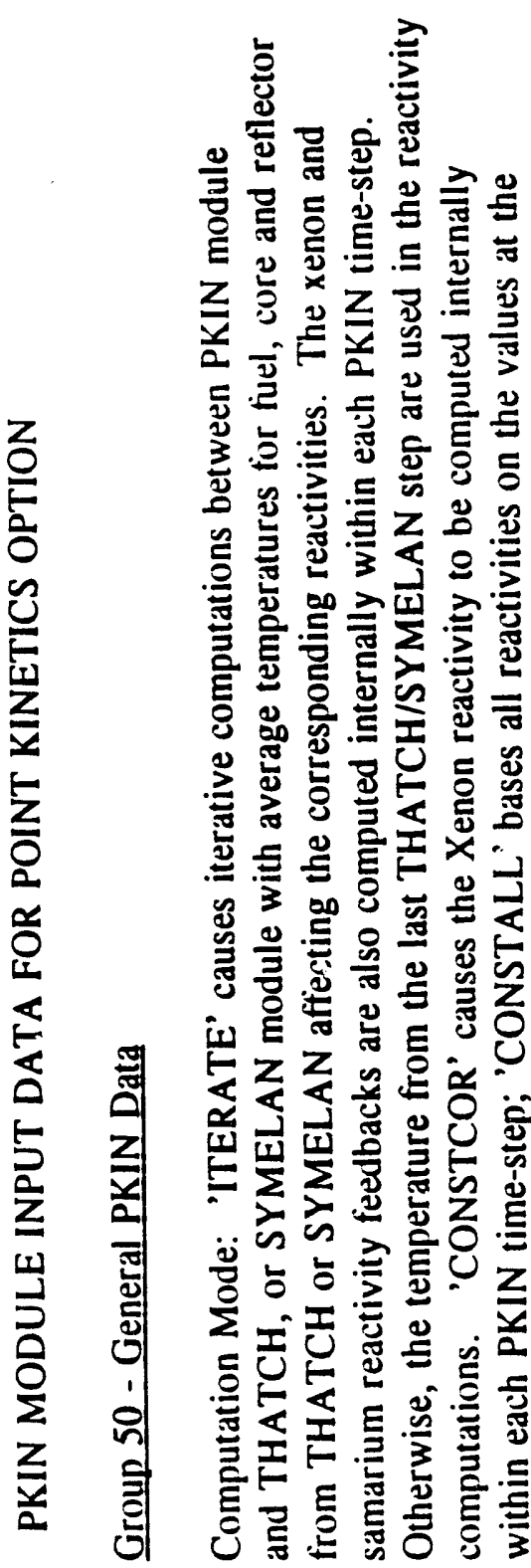

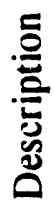

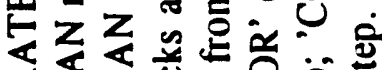

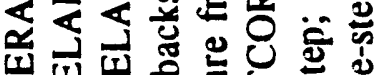

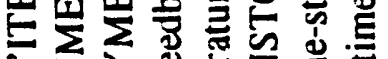

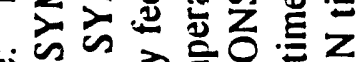

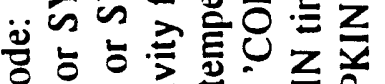

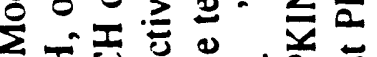

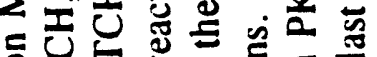

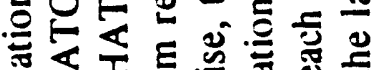

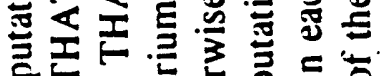

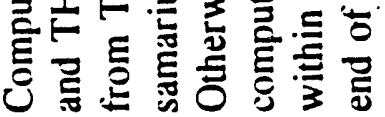

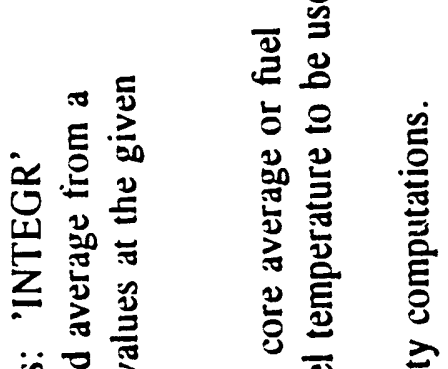

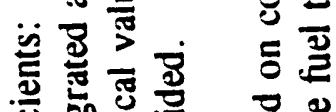

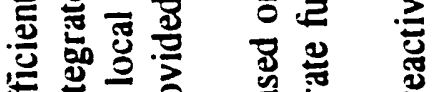

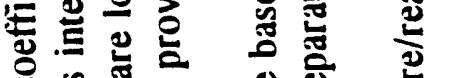

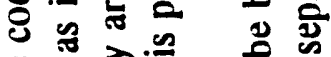

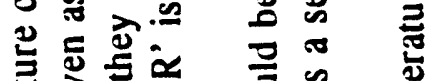

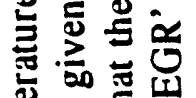

这管焉

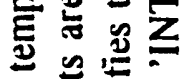

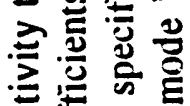

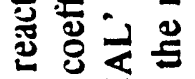

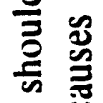

点吉

总 离

롤 包

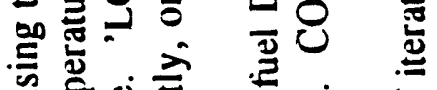

约 ํㅡㄹ

总总总

异

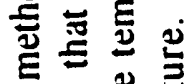

นิ

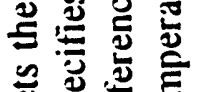

武 $\|$ 焉

ᄂ)

응

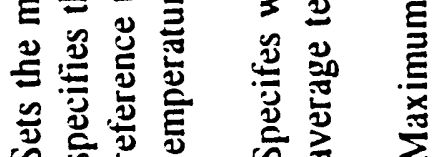

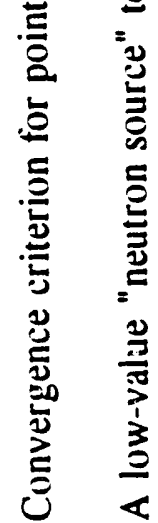

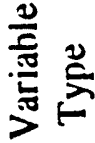

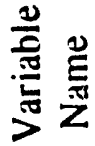

$\sum_{0}^{0}$

$\sum_{\substack{0 \\ \frac{0}{x}}}^{\frac{1}{0}}$

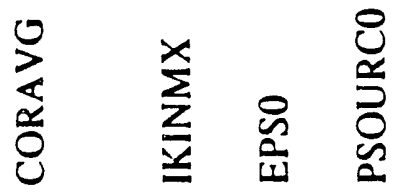

己.

吾 
善 $\quad \geq \quad \infty$

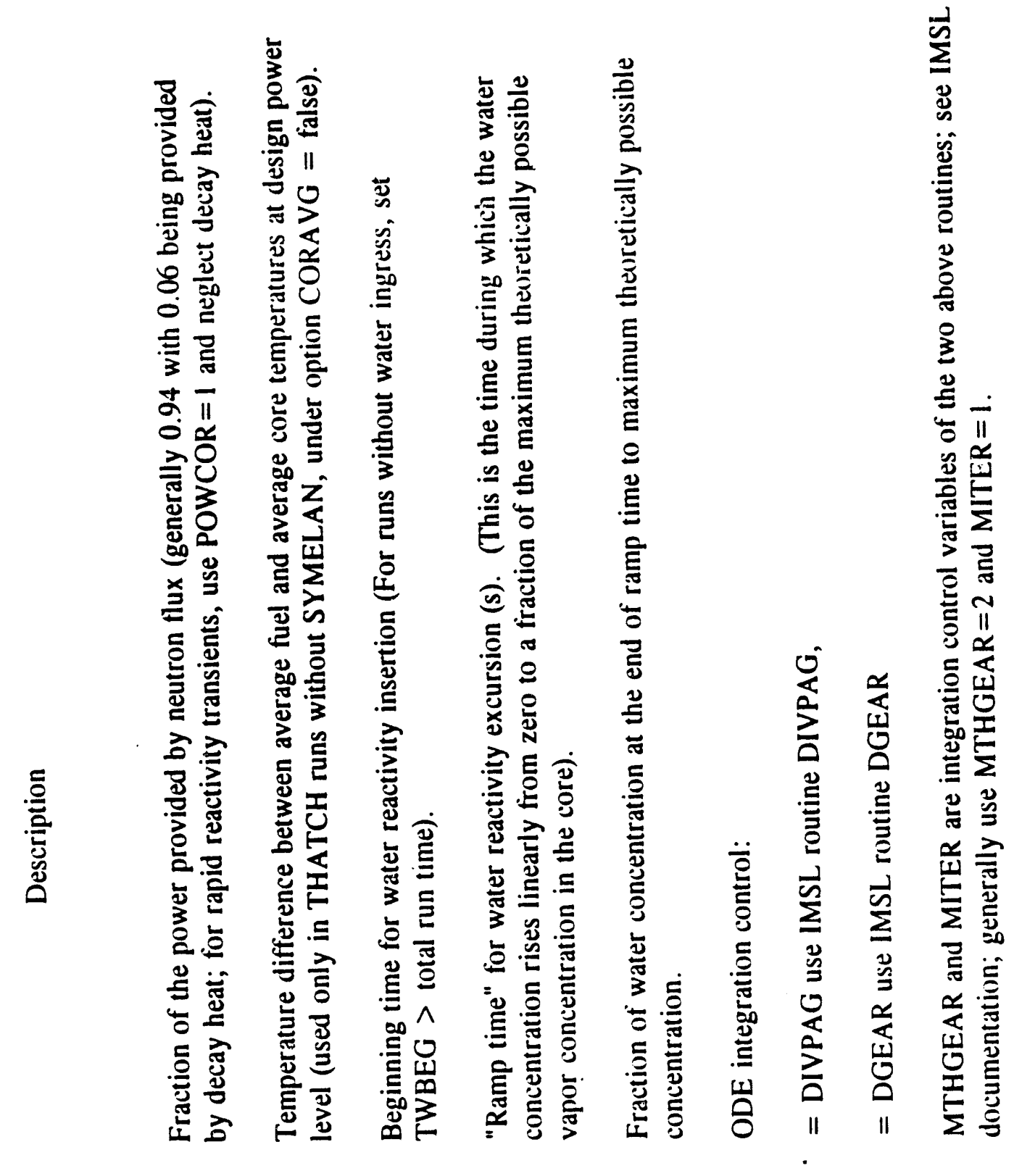

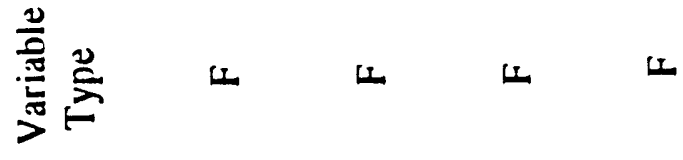

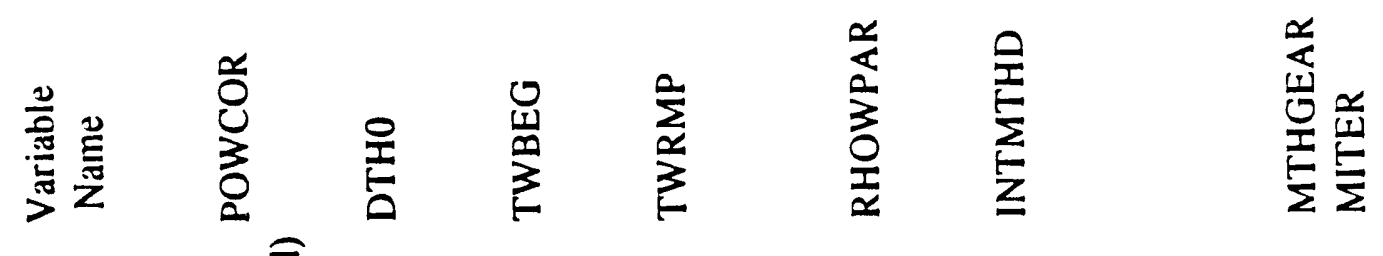

粰

裏高旁 


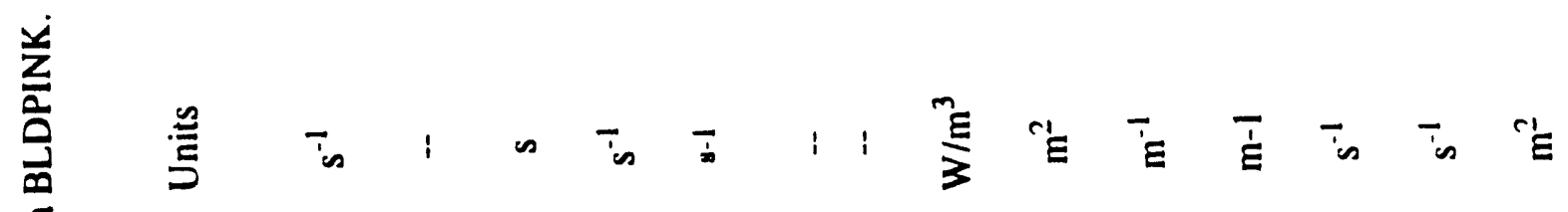

ญี

苟

$\frac{0}{3}$

$\frac{z}{a}$

$\stackrel{5}{5}$

.

异

를

莕

氖

읋

范

总

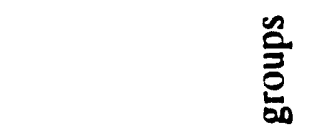

ว

营

로ํ

동

冚

$\overline{\mathrm{F}}$

总泀

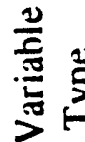

气ั้

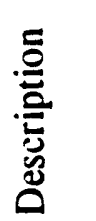
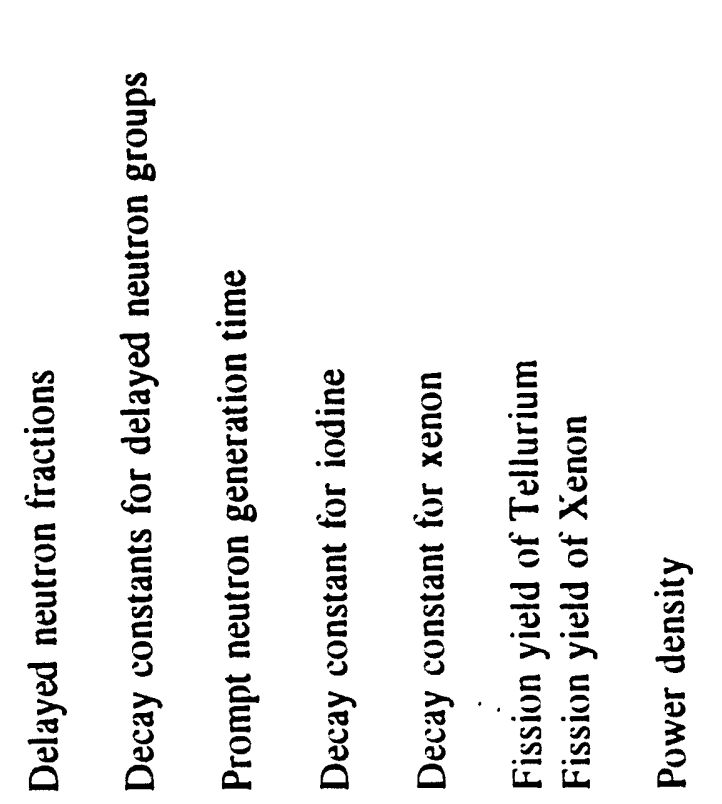

.

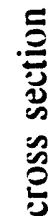

总

战

忧苞

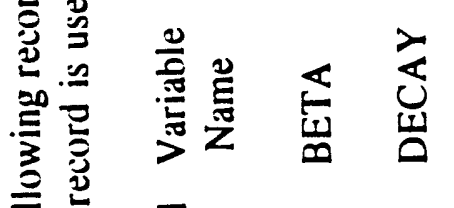

站齐

善善善

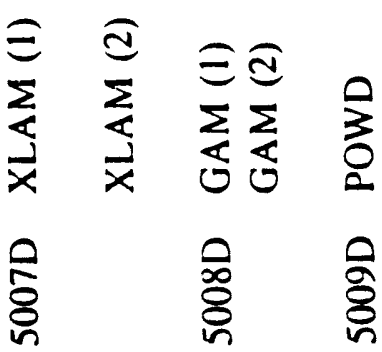

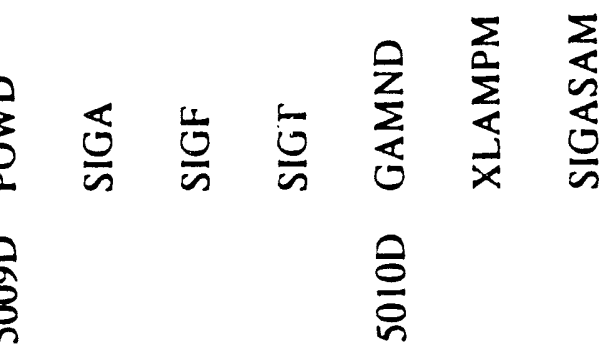


善 ! ! : ' !

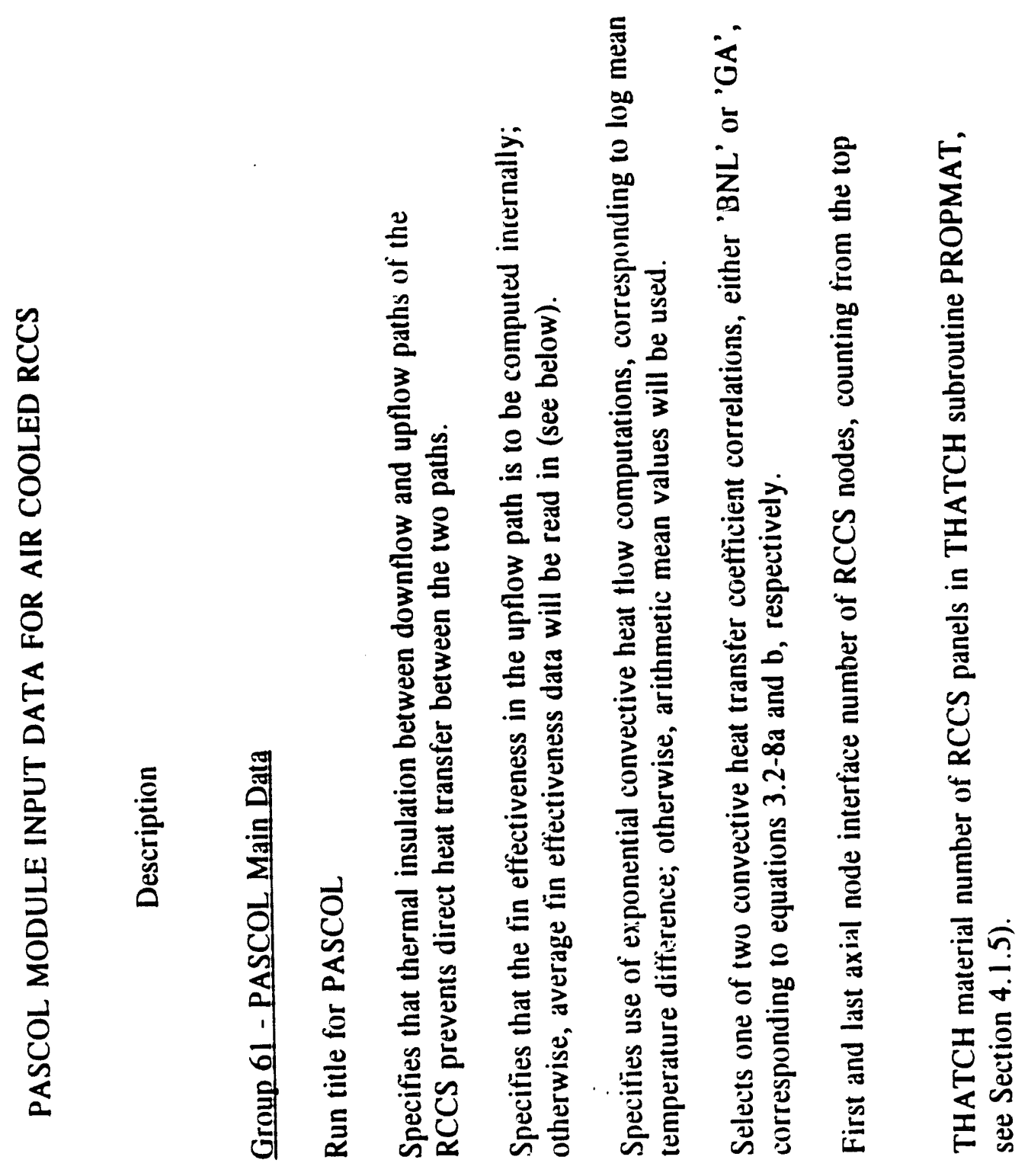

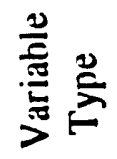
吾
岁站

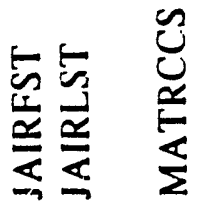

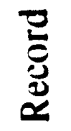
$\frac{0}{0} \frac{0}{0}$
$\frac{2}{0}$ 


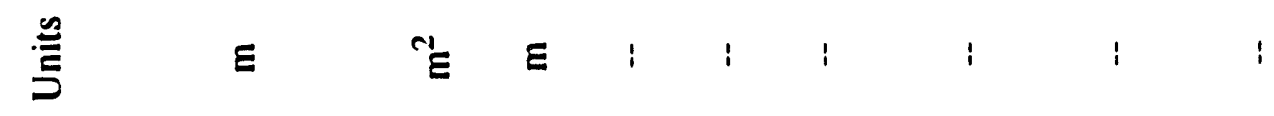
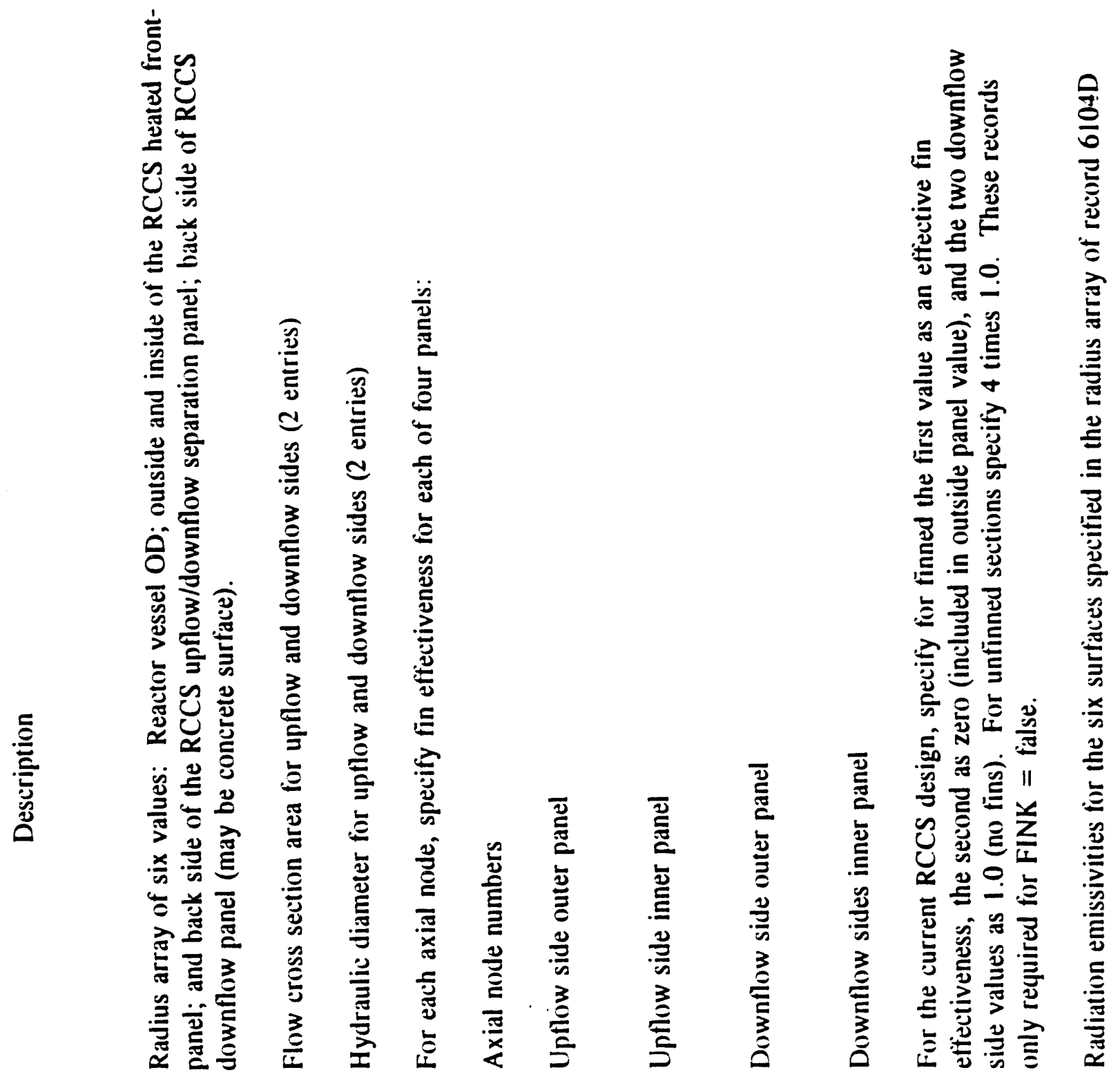

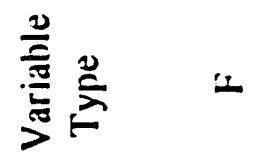

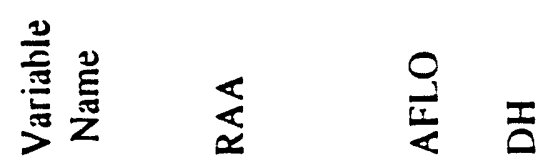

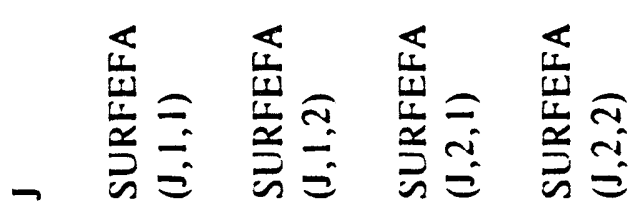

竞言 言

嗳

产 
产

E

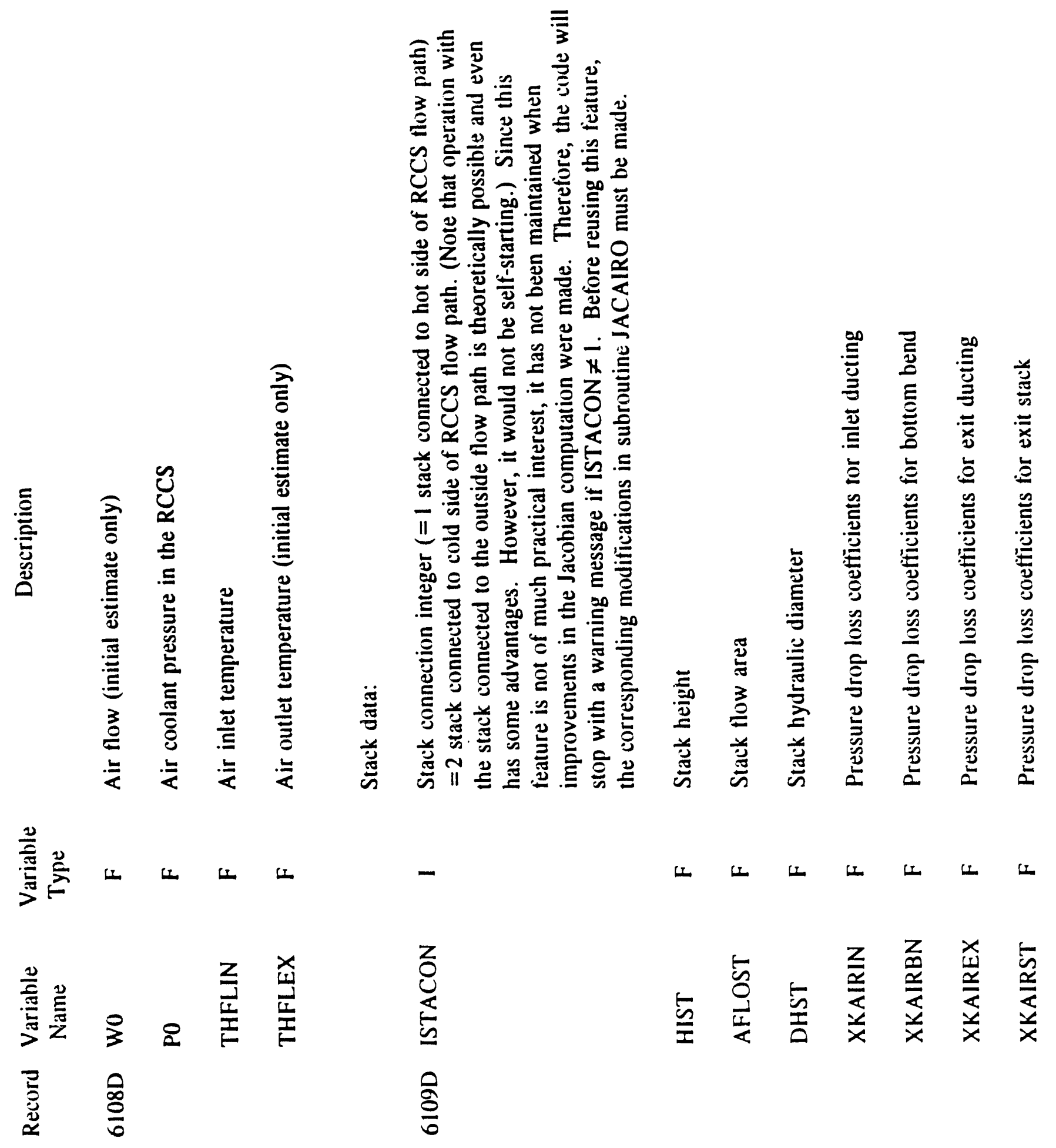


旁
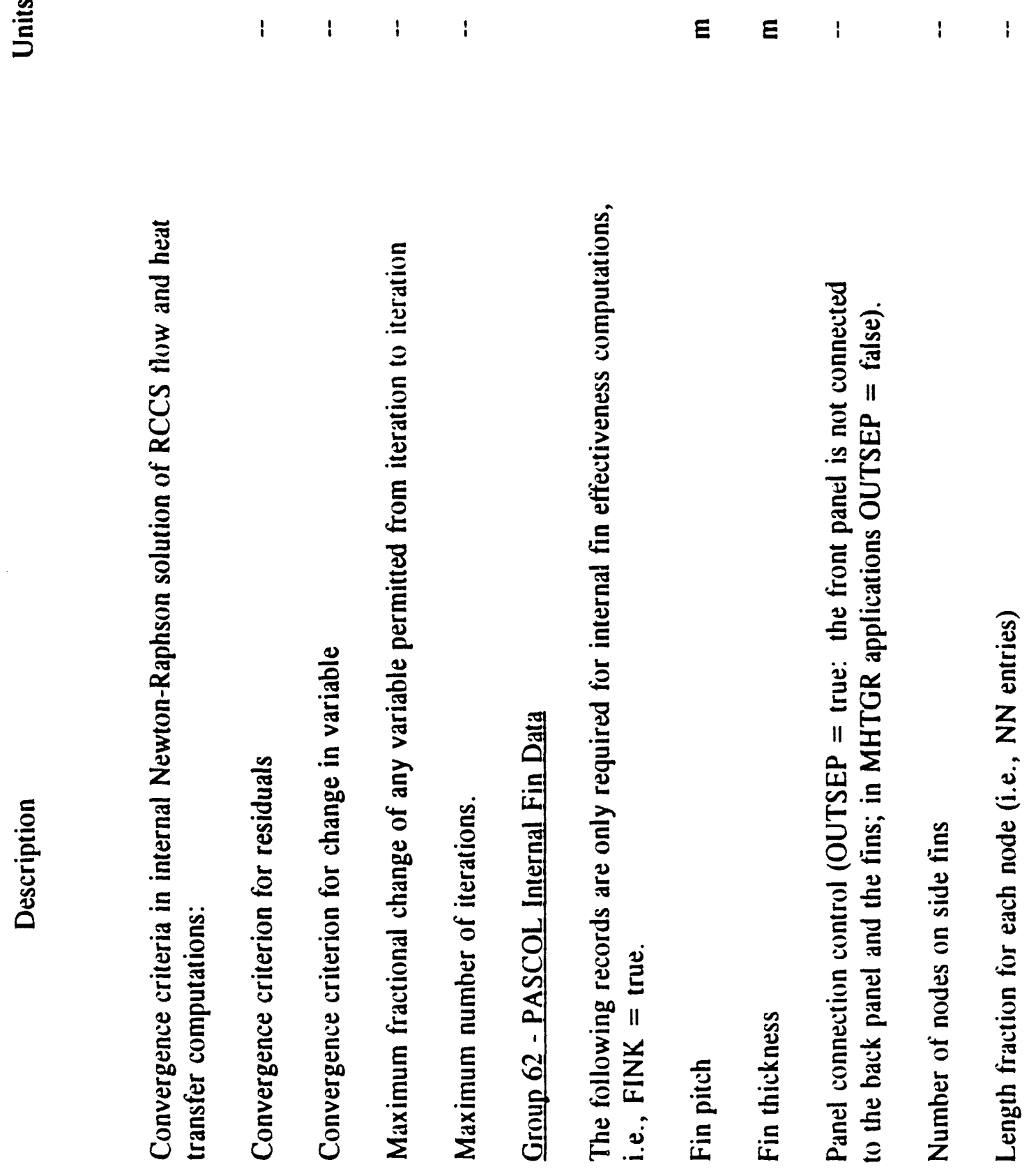

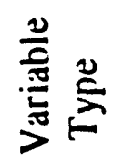

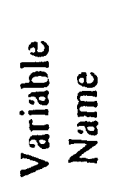

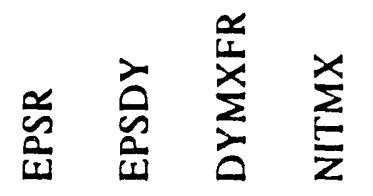

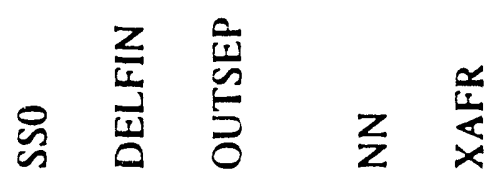
言
言

咅 


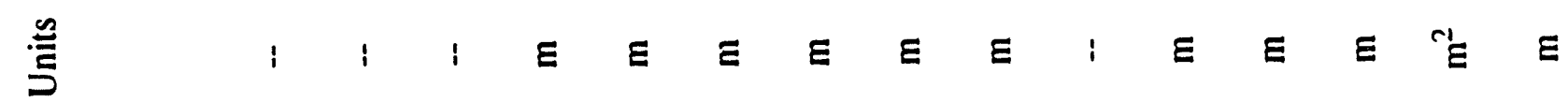
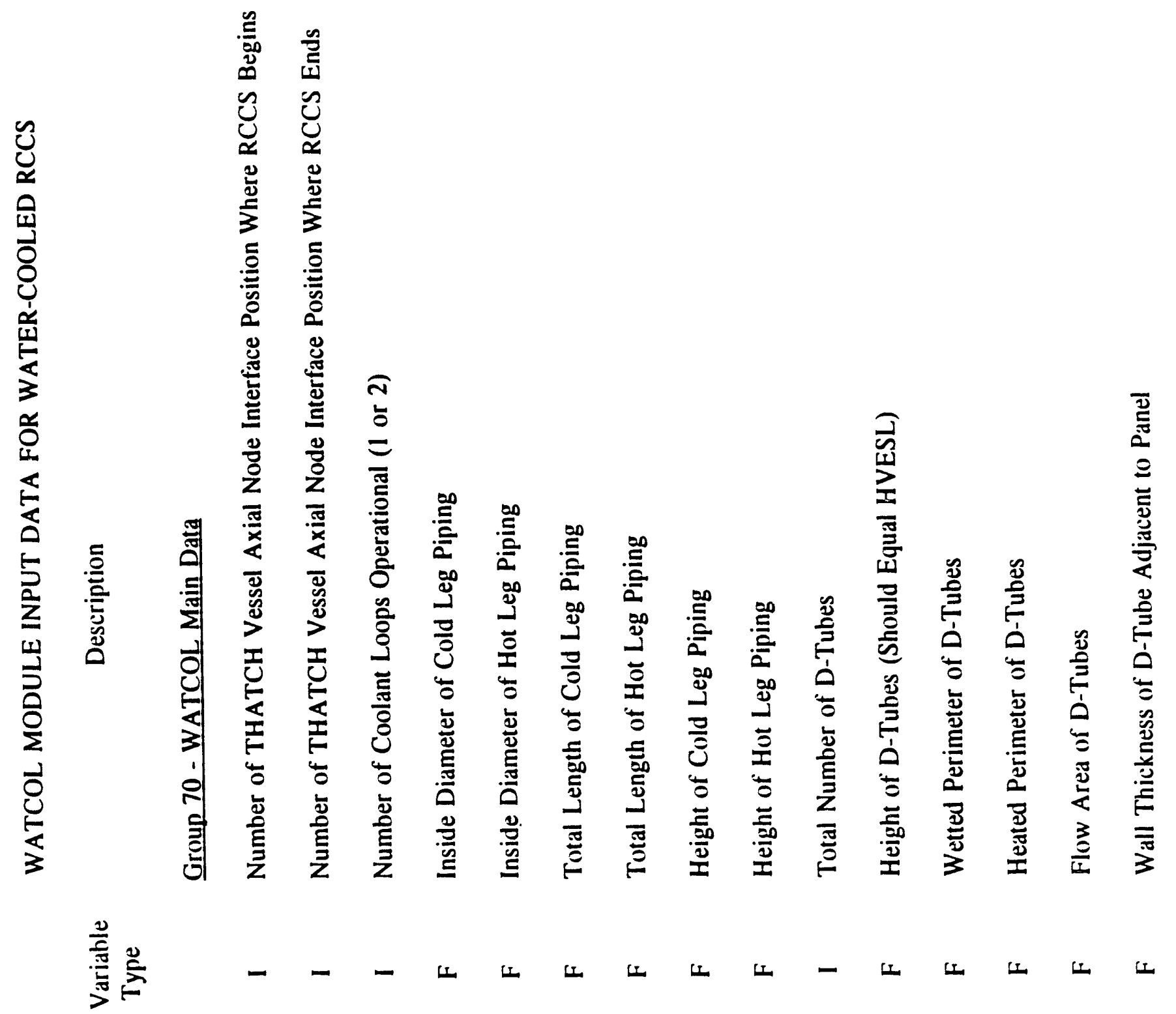

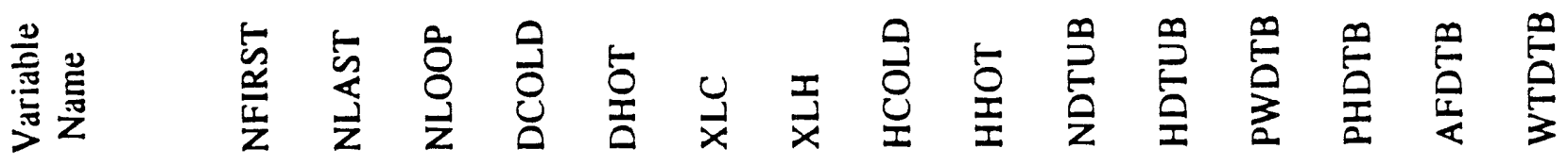
矛 


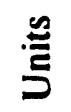

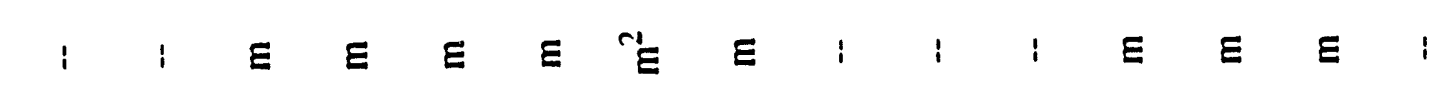

亳

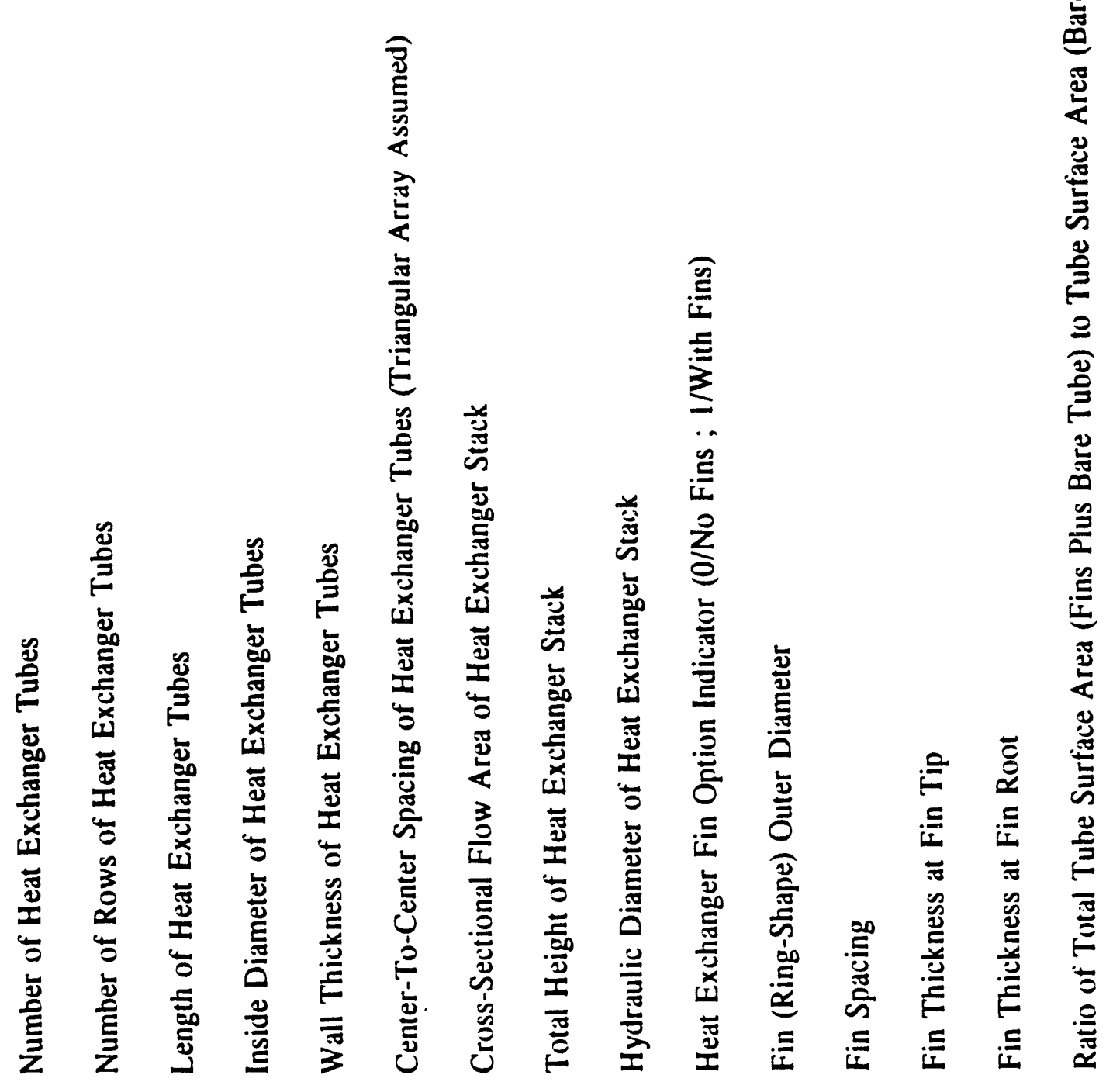

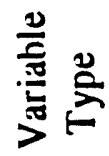

章产

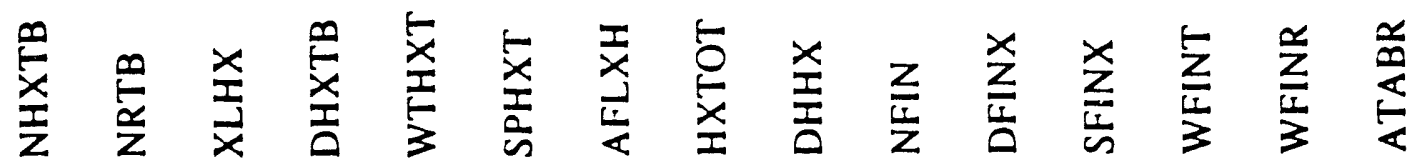

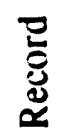

善

率 


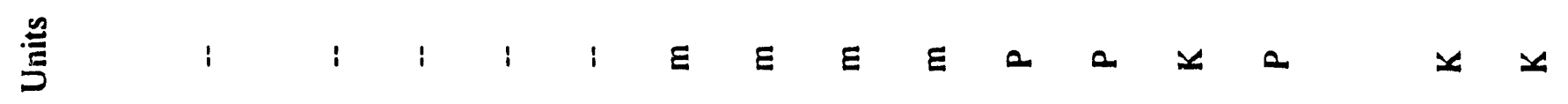

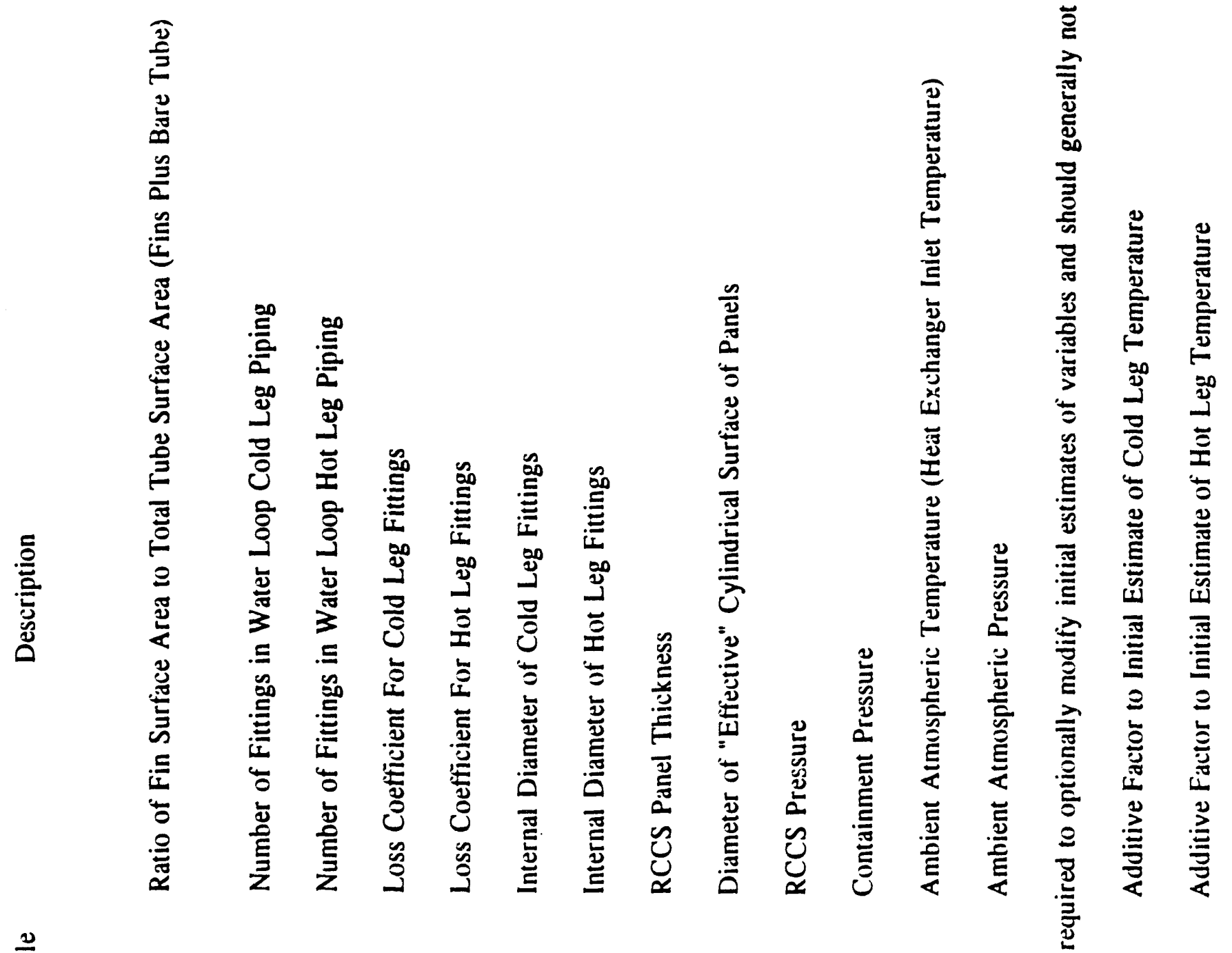

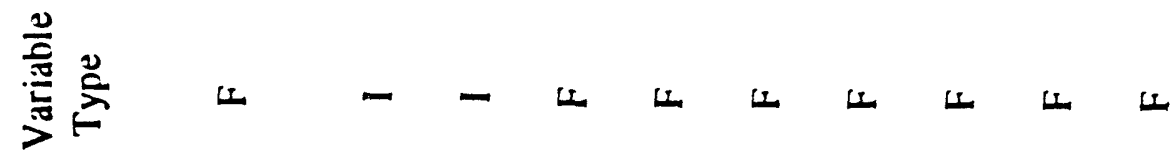

吾 焉旁

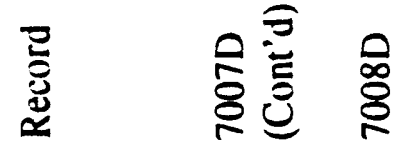

高言 言 突 


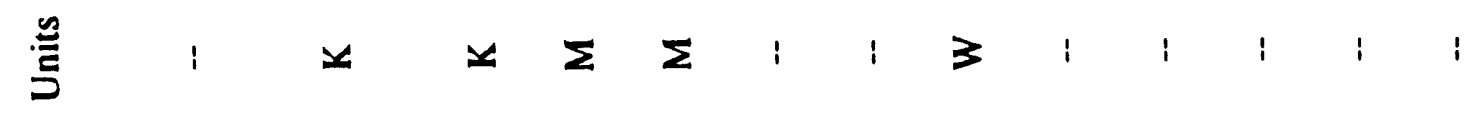

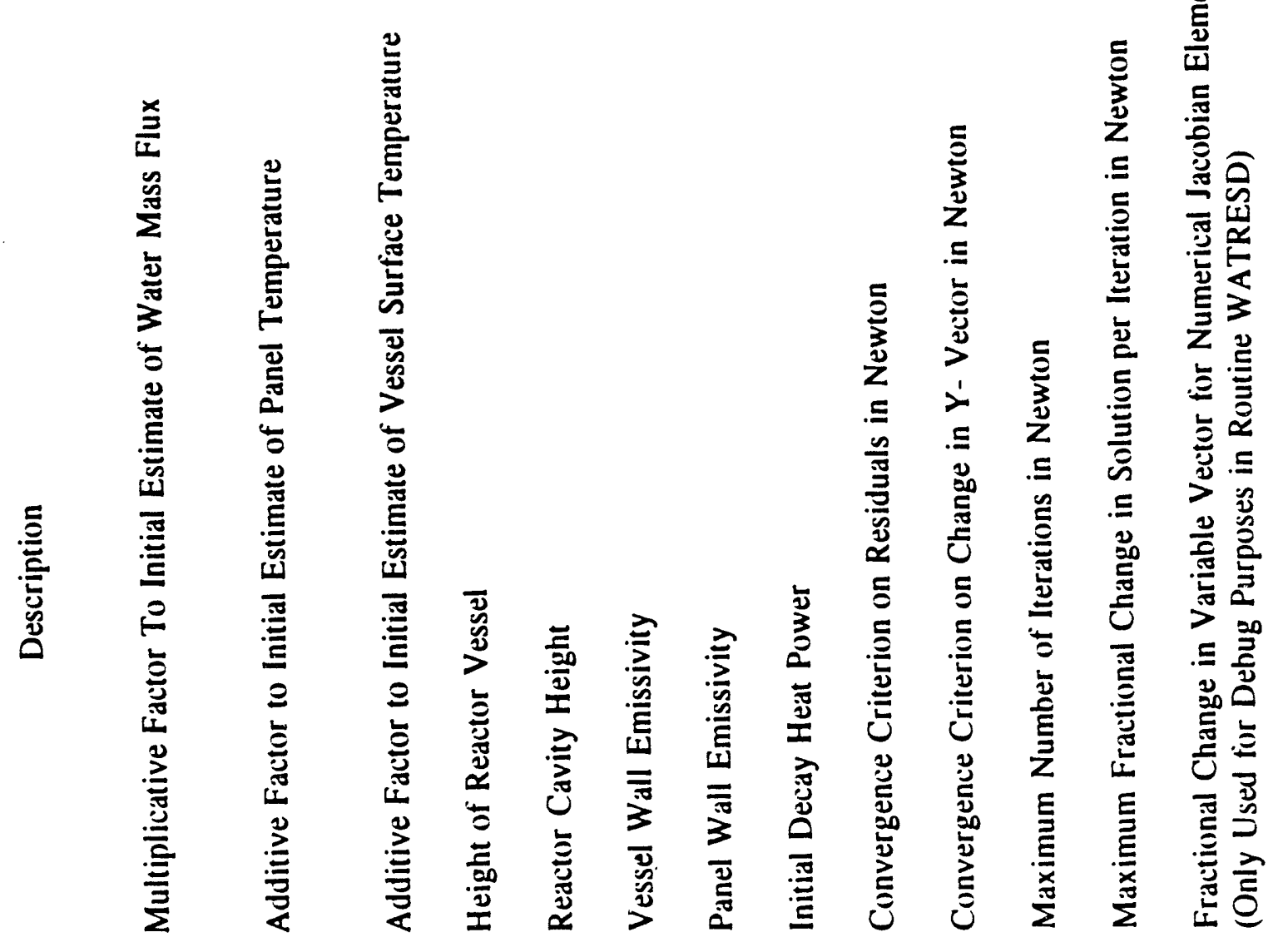

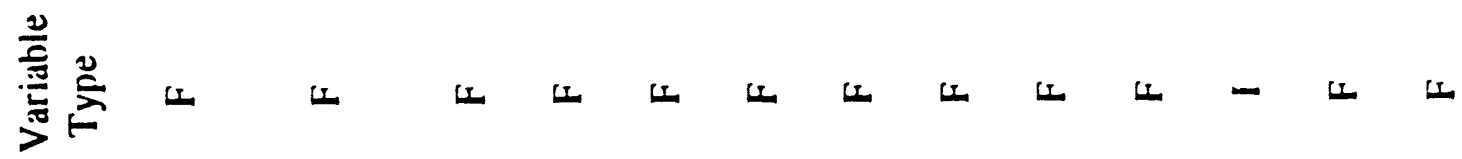

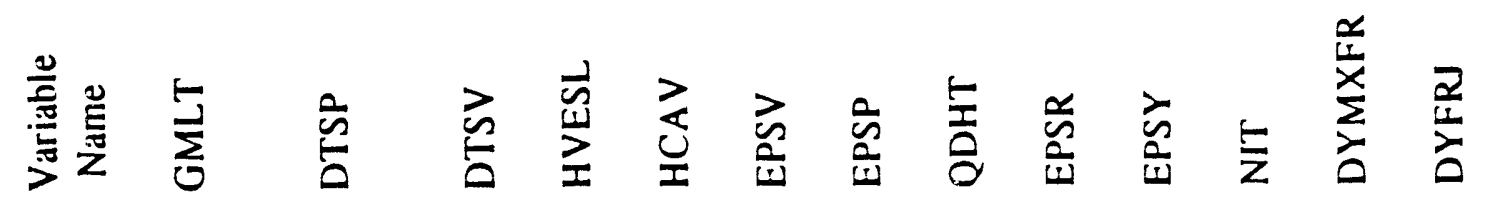

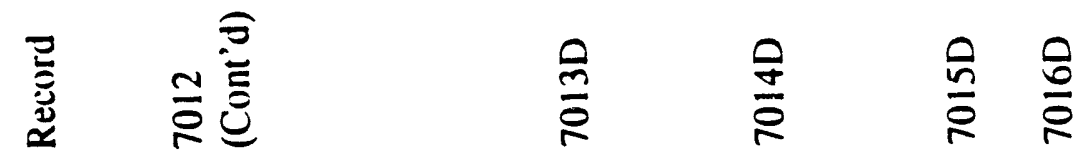




\subsection{Input to THRADLIB Pre-processor for Radiation Coefficients Matrices}

The input data to the THRADLIB pre-processor includes the NAMELIST INPUT, with variables as listed in Table 5-2, followed by the following 3 records:

Record Number Entries

1

2

3

\section{UNITS, RADCHEC}

NOEVAL, NOWRITA array (NOEVAL entries)

THA array (NN entries)

Record 2 is only read if RADCHEC $=$ true.

where

Record 3 is only read if RADCHEC = false.

$\underline{\text { Variable }}$

Type

Description

UNITS

\section{Character *6}

Unit of THA array, must be either 'CELSIUS' or 'KELVIN'

RADCHEC

\section{Logical}

NOEVAL

$$
\text { integer } \leq 6
$$

NOWRITA

integer array

THA

floating point array

NN

If $\mathrm{RADCHEC}=$ true then temperature and heat flow arrays from THATCH debug output will be read from a File 37 debug checks

Number of debug temperature and heat flow records to be read from File 37

Identifier for records on File 37 to be read

Arbitrary surface temperatures (generally in $\mathrm{K}$ ) to be used in debug test

Number of plenum nodes (computed in THRAD; user must provide that many temperatures) 
Table 5-2 Input Data in NAMELIST INPUT for THRADLIB

\begin{tabular}{|c|c|c|c|c|c|}
\hline Variable Description & Symbol & Variable Name & Positions & Units & $\begin{array}{l}\text { Default } \\
\text { Value }\end{array}$ \\
\hline $\begin{array}{l}\text { Character Variable Defining } \\
\text { Geometry } \\
\text { 'CYCLIN' or 'ANCYL' } \\
\text { only are allowed }\end{array}$ & - & GEOM & 1 & - & - \\
\hline Number of Radial Nodes & $n_{r}$ & NRA & 1 & - & - \\
\hline $\begin{array}{l}\text { Number of Axial Nodes } \\
\text { for GEOM = 'CYLIN': } \\
\text { Number of Axial Nodes on } \\
\text { Outer Cylinder for } \\
\text { GEOM = 'ANCYL' }\end{array}$ & $n_{\mathrm{z}}$ & NZAO & 1 & - & - \\
\hline $\begin{array}{l}\text { Number of Axial Nodes on } \\
\text { Inner Cylinder for } \\
\text { GEOM = 'ANCYL' }\end{array}$ & $\mathrm{n}_{\mathrm{zi}}$ & NZAI & 1 & - & - \\
\hline Top Surface Emissivity & $\epsilon_{T}$ & EMMITOP & 1 & - & 0.8 \\
\hline Bottom Surface Emissivity & $\epsilon_{B}$ & EMMIBOT & 1 & - & 0.8 \\
\hline $\begin{array}{l}\text { Outer Cylinder Surface } \\
\text { Emissivity }\end{array}$ & $\epsilon_{3}$ & EMMISDO & 1 & - & 0.8 \\
\hline $\begin{array}{l}\text { Inner Cylinder Surface } \\
\text { Emissivity (used for } \\
\text { GEOM = 'ANCYL' only }\end{array}$ & $\epsilon_{3 i}$ & EMMISDI & 1 & - & 0.8 \\
\hline $\begin{array}{l}\text { Radius Array } \\
\text { (Interface Points) }\end{array}$ & $r$ & RA & $0: 25$ & any & - \\
\hline $\begin{array}{l}\text { Axial Node Interface } \\
\text { Points of Outer Cylinder }\end{array}$ & z & ZAO & $0: 50$ & any & - \\
\hline $\begin{array}{l}\text { Axial Node Interface } \\
\text { Points of Inner Cylinder } \\
\text { (Used for GEOM = 'ANCYL' } \\
\text { only) }\end{array}$ & $z_{I}$ & ZAI & $0: 50$ & any & - \\
\hline $\begin{array}{l}\text { Conversion Constant to Convert } \\
\text { RA, ZAO and ZAI arrays to SI } \\
\text { Unit [m] }\end{array}$ & & CONVTOM & 1 & {$\left[\frac{m}{a n y}\right]$} & 1. \\
\hline $\begin{array}{l}\text { Printed Output Control } \\
\text { Higher Numbers Trigger more } \\
\text { Intermediate Matrix Printing }\end{array}$ & - & IPRINT & 1 & - & 1 \\
\hline $\begin{array}{l}\text { IWRITE >0 will cause Writing } \\
\text { of Output Coefficient array onto } \\
\text { File } 8\end{array}$ & - & IWRITE & 1 & - & 0 \\
\hline
\end{tabular}




\subsection{Input to THPROCS Post Processing and Plot File Writing}

The input data to the THPROCS post processor consists of the following two records:

Record Number

(not part of input line)

1

2

where

Variable

Type

NOTPSIN Integer $(\leq 9)$

PROCESS LOGICAL

PLOT LOGICAL

COMB

LOGICAL

ILEVOUT Integer

TTMPWRT FP

TSTART

TEND

TSTEP

RCCSMOD Character

PKINL

LOGICAL

FLOXI

LOGICAL

NPTS
Entries

NOTPSIN, PROCESS, PLOT, COMB, ILEVOUT, TTMPWRT

TSTART, TEND, TSTEP, AIRCOL, PTKINS, FLOXI, NPTS

\section{Description}

Number of THATCH output files to be processed

$=$ true : Post-processing to be done

= true: Plotting to be done via call to FILEPLT (currently only available at BNL via PLOTLIB and DISSPLAY)

= true: The output files of this post-processing run are sequential, and the plot files to be produced is a single file with plot records extracted from all NOTPSIN files of this run being added sequentially. (This option requires that the start and end times of record 2 are also sequential).

= false: The output tile of this post-processing run are all treated as individual files, each producing its own plot file.

Print level index to control output printing ( 1 to 4 )

Time [hr] at which solid temperature array is to be written to output File 96 in format of a THATCH initial temperature file.

Start time for post-processing [hr]

End time for post-processing [hr]

Minimum time step between records to be processed [hr]

'AIR' or 'WATER' for Air-Cooled or Water-Cooled RCCS options respectively, 'NONE' if neither is used.

$=$ true, if Point Kinetics Option were used

$=$ true, if FLOXI Module were used

Approximate number of time records desired on plot file produced in this run.

There must be one record 2 for each of the THATCH output files to be processed, i.e., NOTPSIN records number 2 . 


\section{FILE USAGE OF THATCH AND SUB-MODULES}

Table 6-1 lists the input files required for a THATCH run; the output files created are listed in Table 6-2.

Intermediate scratch files used in THATCH runs are 13 (for the temporary storage of input data stripped of comments) and 71,72 , and 73 as intermediate binary files.

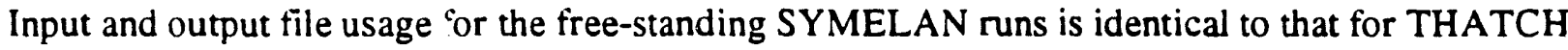
runs, except that Input Files 02, 15, $21 \mathrm{ff}$, and 27 are not required and Output Files 07 and 14 will not be produced. Usage of the intermediate scratch file is identical to that for THATCH. 
Table 6-1

Input Files for THATCH Runs

\begin{tabular}{|c|c|c|c|c|}
\hline $\begin{array}{l}\text { File } \\
\text { No. }\end{array}$ & File Description & $\begin{array}{l}\text { File } \\
\text { Type }\end{array}$ & File Record Format & Remarks \\
\hline 02 & $\begin{array}{l}\text { Spline coefficients } \\
\text { for Gas Properties }\end{array}$ & Binary & $\begin{array}{l}\text { Section } 4.2 .6 \\
\text { Subroutine REDCOF2 }\end{array}$ & $\begin{array}{l}\text { only required if } \\
\text { FLOXI=true }\end{array}$ \\
\hline 05 & Case Input Data & Character & see Section 5.1.2 & \\
\hline 15 & $\begin{array}{l}\text { THATCH Output File } \\
\text { (Output file of } \\
\text { preceding run to } \\
\text { serve as restart } \\
\text { file here }\end{array}$ & Binary & $\begin{array}{l}\text { see Table 4-3 } \\
\text { and Table 4-4 } \\
\text { Section } 4.1 .6\end{array}$ & $\begin{array}{l}\text { only required for } \\
\text { restart (TO }>0 \\
\text { on Input Record } \\
\text { 1201D) }\end{array}$ \\
\hline 27 & $\begin{array}{l}\text { Initial Temperature } \\
\text { File (generally } \\
\text { steady state file) }\end{array}$ & Binary & $\begin{array}{l}1 \text { record: ILP,JLP } \\
1 \text { record: }((T H(I, J), \\
I=1, I L P,), J=1 \\
\text { JLP) }\end{array}$ & $\begin{array}{l}\text { for INPMODE = } \\
\text { 'READRUN' on input } \\
\text { record } 1501 \mathrm{D} \\
\text { (common } \\
\text { run mode) }\end{array}$ \\
\hline $21 \mathrm{ff}$ & $\begin{array}{l}\text { Plenum Radiation } \\
\text { coefficient arrays }\end{array}$ & Binary & $\begin{array}{l}1 \text { record: } N N \\
1 \text { record: }((F(N, M) \\
M=1, N N), N=1, N N)\end{array}$ & $\begin{array}{l}\text { one file for each } \\
\text { plenum with } 2-d \\
\text { radiation. In } \\
\text { sequence of plena } \\
\text { numbers from Input } \\
\text { Record } 603 \mathrm{D}\end{array}$ \\
\hline 28 & $\begin{array}{l}\text { SYMELAN Initial } \\
\text { Temperature File } \\
\text { (generally steady } \\
\text { state file) }\end{array}$ & Binary & see Table 4-13 & $\begin{array}{l}\text { for } \\
\text { THATCH/SYMELAN } \\
\text { runs or SYMELAN } \\
\text { runs of READTMP = } \\
\text { true on Input } \\
\text { Record } 4005 D\end{array}$ \\
\hline
\end{tabular}


Table 6-2

Output Files for THATCH Runs

\begin{tabular}{|c|c|c|c|c|}
\hline $\begin{array}{l}\text { File } \\
\text { No. }\end{array}$ & File Description & $\begin{array}{l}\text { File } \\
\text { Type }\end{array}$ & File Record Format & Remarks \\
\hline 06 & $\begin{array}{l}\text { Printed THATCH } \\
\text { output file }\end{array}$ & Character & & \\
\hline 07 & $\begin{array}{l}\text { Printed catalog of } \\
\text { THATCH output file } \\
\text { records }\end{array}$ & Character & & \\
\hline 09 & $\begin{array}{l}\text { Printed catalog of } \\
\text { SYMELAN plot file } \\
\text { records }\end{array}$ & Character & & $\begin{array}{l}\text { for } \\
\text { THATCH/SYMELAN } \\
\text { or free standing } \\
\text { SYMELAN runs only. }\end{array}$ \\
\hline 14 & $\begin{array}{l}\text { THATCH output file } \\
\text { for post processing } \\
\text { and/or restart }\end{array}$ & Binary & $\begin{array}{l}\text { see Table } 4-3 \text { and } \\
\text { Table } 4-4 \text { in } \\
\text { Section } 4.1 .6 .\end{array}$ & \\
\hline 33 & $\begin{array}{l}\text { Fine time step } \\
\text { output of point } \\
\text { kinetics vector }\end{array}$ & Binary & $\begin{array}{l}\text { see Table } 4-8 \\
\text { Section } 4.2 .4\end{array}$ & $\begin{array}{l}\text { only if } \\
\text { PKIN = true }\end{array}$ \\
\hline 41 & $\begin{array}{l}\text { SYMELAN output } \\
\text { temperature file }\end{array}$ & Binary & $\begin{array}{l}\text { see Table 4-1 } \\
\text { Section } 4.2-5\end{array}$ & $\begin{array}{l}\text { only if } \\
\text { SYMELAN = true }\end{array}$ \\
\hline 42 & SYMELAN plot file & Binary & $\begin{array}{l}\text { see Table } 4-10 \\
\text { Section } 4.2 .5\end{array}$ & $\begin{array}{l}\text { only if } \\
\text { SYMELL = true }\end{array}$ \\
\hline
\end{tabular}




\subsection{PRE-PROCESSING REQUIREMENTS AND POST-PROCESSING OPTIONS}

\subsection{Pre-processing Requirements}

6-1):

For THATCH runs, the following input files must be produced before THATCH runs (see Table

Initial Temperature File. This generally is a steady state file, created by a THATCH steady-state run (FLOXI = true and STEADY = true on Input Record 201D, and 'OTHER' for INPMODE on initial temperature input record 1501D). The output file from the run is processed then by the post-processor THPROCS, with TTMPWRT of input data record 1 set to the time records on the THATCH output file for which an initial temperature tile is to be written. The format of the initial temperature file is given in Table 6-1. The file will be written hy THPROCS as File 96.

Spline Coefficients for Gas Properties. This file is only required for runs with FLOXI=true. The generating program tor the spline coefficients, which was used for the current spline coefficient file, is presently located at BNL, but could be made available on request.

SYMELAN Initial Temperature Files. These files are generated by free-standing SYMELAN runs, specifying no point kinetics option and constant steady state power. This file is required only for combined THATCH/SYMELAN runs.

The Plenum Radiation Coefficient Files. These files must be obtained for each plenum by executing the THRAD program of THRADLIB with input variable IWRITE $=1$ which then will produce a coefficient file as File 8.

\subsection{Post-Processing Options}

The THATCH post-processor THPROCS serves for post-processing. Its subroutines are described in Section 4.2.10. They provide printed output and permit the writing of several output files, listed in Table 4-16. Up to nine THATCH output files can be submitted to the post-processor at one time. The input data requirements of the post processor are described in Section 5.3.

The input and output file requirements for THPROCS are listed in Tables 7-1 and 7.2.; in addition, THPROCS will use internally the following scratch files:

File $\quad$ File

$07 \quad$ IRDIN $\quad$ Temporary input data storage stripped of

Comment records ( $\mathrm{Cbb}$ in columns 1 to 3 )

67

ITPFLX1 Floxi intermediate plot file

68

ITPFLX2 Floxi intermediate plot tile

91

NTPSCR 1

THATCH intermediate plot file 
Intermediate storage for data to be printed later in the same run

93

NTPSCR3

THATCH intermediate plot file and point

Kinetics intermediate plot file

94

NTPSCR4

Currently not used 
Table $7 \cdot 1$

Input Files for THPROCS THATCH Post Processing Runs

\begin{tabular}{l|l|c|l|l||}
\hline \hline $\begin{array}{c}\text { File } \\
\text { No. }\end{array}$ & \multicolumn{1}{|c|}{\begin{tabular}{c}
\multicolumn{1}{c|}{$\begin{array}{c}\text { File } \\
\text { Description }\end{array}$} \\
05
\end{tabular}} & $\begin{array}{c}\text { File } \\
\text { Type }\end{array}$ & \multicolumn{1}{|c|}{$\begin{array}{c}\text { File Record } \\
\text { Format }\end{array}$} & Remarks \\
\hline $11-19$ & $\begin{array}{l}\text { THATCH Output } \\
\text { Files }\end{array}$ & Character & See Section 5.3 & \\
\hline $51-59$ & $\begin{array}{l}\text { THATCH fine time } \\
\text { Step output file } \\
\text { of point kinetics } \\
\text { vectors }\end{array}$ & $\begin{array}{l}\text { See Tables 4-3 } \\
\text { and 4-4 in } \\
\text { Section 4.1.6 }\end{array}$ & $\begin{array}{l}\text { See Table 4-8 } \\
\text { Section 4.2-4 }\end{array}$ & \\
\hline \hline
\end{tabular}

Table 7-2

Output Files for THPROCS THATCH Post Processing Runs

\begin{tabular}{|c|c|c|c|c|}
\hline $\begin{array}{l}\text { File } \\
\text { No. }\end{array}$ & $\begin{array}{c}\text { File } \\
\text { Description }\end{array}$ & $\begin{array}{l}\text { File } \\
\text { Type } \\
\end{array}$ & $\begin{array}{l}\text { File Record } \\
\text { Format } \\
\end{array}$ & Remarks \\
\hline 06 & Printed Output File & Character & Table 4-13 & \\
\hline $21-29$ & Plot Files & Binary & Table 4-13 & \\
\hline $31-39$ & $\begin{array}{l}\text { Condensed Version of Plot } \\
\text { Files }\end{array}$ & Binary & Table 4-15 & \\
\hline$+1-49$ & $\begin{array}{l}\text { Solid Temperature History } \\
\text { File for Free-Standing } \\
\text { FLOXI Runs }\end{array}$ & Binary & Table 4-13 & \\
\hline 61 & $\begin{array}{l}\text { Combined Sequential Plot } \\
\text { File of Files } 31 \text { to } 39\end{array}$ & Binary & Table 4-13 & \\
\hline $71-79$ & $\begin{array}{l}\text { Plot File of Point Kinetics } \\
\text { Data at Fine Time Step }\end{array}$ & Binary & Table 4-X & \\
\hline 96 & $\begin{array}{l}\text { THATCH Initial Temperature } \\
\text { File }\end{array}$ & Binary & $\begin{array}{l}\text { See File } 27 \\
\text { of Table } 6-1\end{array}$ & \\
\hline
\end{tabular}




\section{THATCH MODEL OF THE COMMERCIAL MHTGR}

This section describes the model of the commercial MHTGR that is incorporated in THATCH. The MHTGR model includes descriptions of input data, and also models which are used in certain subroutines.

\subsection{Standard Nodalization and Block Description of the Commercial MHTGR}

The standard THATCH nodalization for the commercial MHTGR was developed early in 1986 for depressurized core heat-up transients. Several active core nodalizations were tried, and the current scheme of six radial active core nodes. two for each radial power zone, with 20 axial core nodes, two per fuel element was found to be economical and accurate (finer nodalizations gave essentially identical results). The remainder of the core nodalization used equivalent node sizes. Since vessel temperatures were of major concern. two radial nodes were used in the steel vessel. The complete nodalization of 21 radial and 44 axial nodes is contained in the input decks.

A scheme of the commercial MHTGR in terms of node blocks is given in Figure 8-1. (It is also available with the printed output of any THATCH run.) Table 8-1 shows the various blocks, their node interface ranges, and material indices.

The material property specifications, including effective thermal conductivity, are given in Section 8.2. The gap characteristics are specified with the boundary data of the input deck. In-core gaps between fuel elements are assumed to be $1 \mathrm{~mm}$ thick in radial direction and $0.5 \mathrm{~mm}$ in axial direction with effective emissivities $\epsilon_{1,2}=0.82$ (corresponding to an assumed value of $\epsilon \approx 0.9$ for graphite). For steel-to-steel, the effective emissivity is generally assumed as $\epsilon_{1,2}=0.68$ (corresponding to an assumed steel emissivity of $\epsilon \approx 0.8$ ).

For radiation from the core-barrel outside to the vessel inside-surface, the flow passages attached to the core-barrel effectively act like a partial radiation shield. The overall emissivity for this configuration, assuming component emissivities of 0.8 was computed to be $\epsilon_{1,2}=0.485$, as included in the input decks (Radial Boundary 4).

Boundary maps are produced with each THATCH printed output file.

\subsection{PROPMAT Subroutine for Solid and Gap Material Properties}

The PROPMAT subroutine for the commercial MHTGR provides the required solid and gap material properties. In general, for the solid rodes, the thermal conductivity and thermal capacitance are required, while for gaps, effective thermal conductivities must be provided.

The materials specified for solid blocks are summarized in Table 8-2. Many of these specifications are self-explanatory, but where necessary, further details are given. 


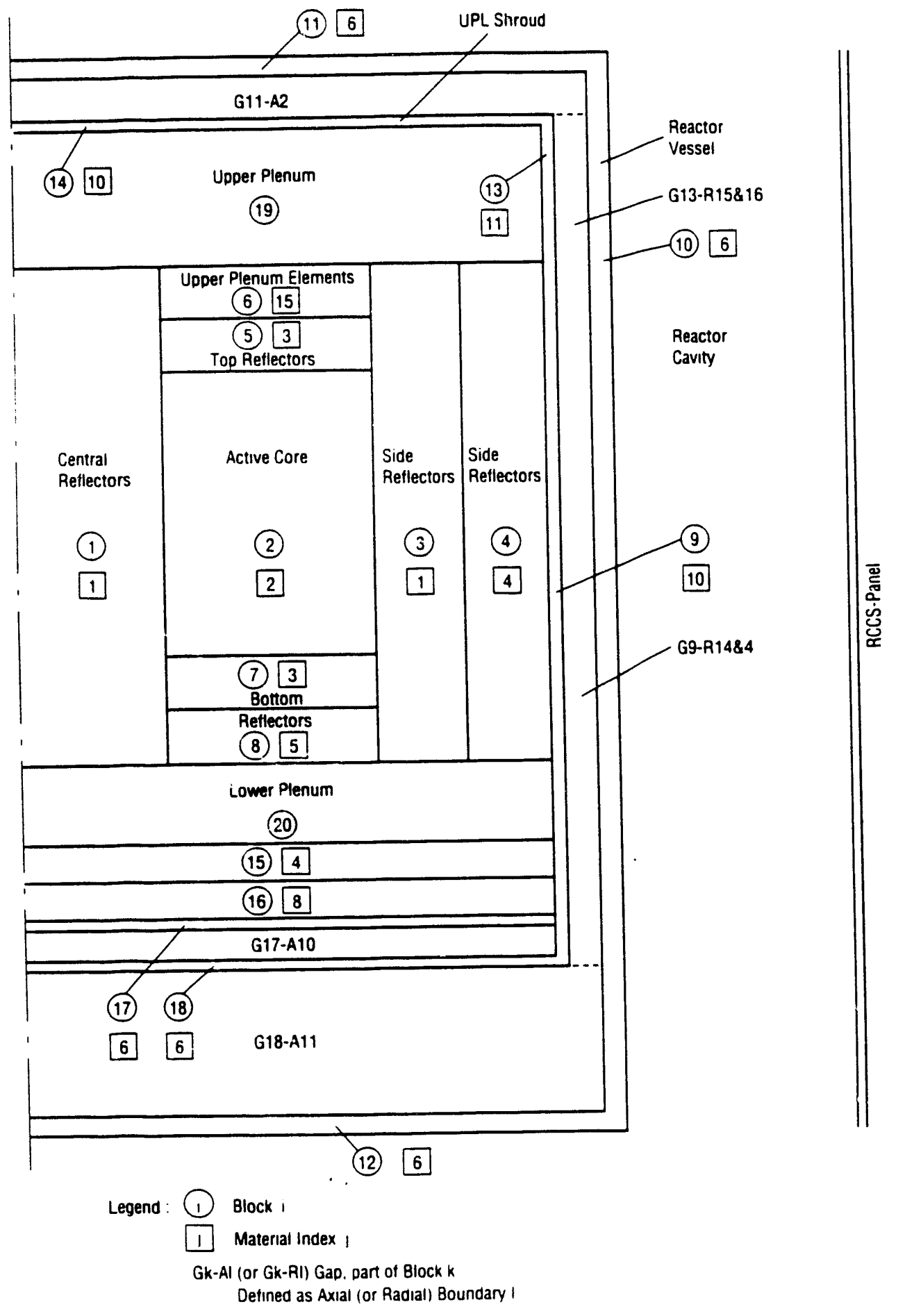

Figure 8-1 Schematic of THATCH Block Nodalization of MHTGR 
TABLE 8-1

Node Block Description of the Commercial MHTGR

\begin{tabular}{|c|c|c|c|c|c|c|}
\hline \multirow{2}{*}{$\begin{array}{l}\text { BLOCK } \\
\text { NUMBER } \\
1 \\
\end{array}$} & \multirow{2}{*}{$\begin{array}{l}\text { BLOCK NAME } \\
\text { Central Reflector }\end{array}$} & \multicolumn{2}{|c|}{$\begin{array}{l}\text { RADIAL } \\
\text { INTERFACE } \\
\text { LO HI }\end{array}$} & \multicolumn{2}{|c|}{$\begin{array}{l}\text { AXIAL } \\
\text { INTERFACE } \\
\text { LO HI } \\
\end{array}$} & \multirow{2}{*}{$\begin{array}{l}\text { MATERIAL } \\
\text { INDEX* } \\
1 \\
\end{array}$} \\
\hline & & 0 & 6 & 8 & 36 & \\
\hline 2 & Active Core & 6 & 12 & 12 & 32 & 2 \\
\hline 3 & Inner Side Reflectors & 12 & 15 & 8 & 36 & 1 \\
\hline 4 & Permanent Side Reflectors & 15 & 18 & 8 & 36 & 4 \\
\hline 5 & Top Reflector & 6 & 12 & 9 & 12 & 3 \\
\hline 6 & Upper Plenum Elements & 6 & 12 & 8 & 9 & 15 \\
\hline 7 & Upper Bottom Reflectors & 6 & 12 & 32 & 34 & 3 \\
\hline 8 & Lower Bottom Reflectors & 6 & 12 & 34 & 36 & 5 \\
\hline 9 & Core Barrel & 18 & 19 & 8 & 43 & 10 \\
\hline 10 & Vessel (side) & 19 & 21 & 1 & 45 & 6 \\
\hline 11 & Vessel (top) & 0 & 19 & 1 & 3 & 6 \\
\hline 12 & Vessel (bottom) & 0 & 19 & 43 & 45 & 6 \\
\hline 13 & Shroud (side) & 18 & 19 & 3 & 8 & 11 \\
\hline 14 & Shroud (top) & 0 & 18 & 3 & 5 & 10 \\
\hline 15 & Upper Core Floor & 0 & 18 & 38 & 40 & 4 \\
\hline 16 & Lower Core Floor & 0 & 18 & 40 & 41 & 8 \\
\hline 17 & Floor Support 1 & 0 & 18 & 41 & 42 & 6 \\
\hline 18 & Floor Support 2 & 0 & 18 & 42 & 43 & 6 \\
\hline 19 & Upper Plenum & 0 & 18 & 5 & 8 & 101 \\
\hline 20 & Lower Plenum & 0 & 18 & 36 & 38 & 102 \\
\hline
\end{tabular}

*Number $>100$ designate plena, with plenum number $=$ material index -100 . 
TABLE 8-2

Solid Node Properties of the

THATCH Commercial MHTGR Model

\begin{tabular}{|c|c|c|}
\hline $\begin{array}{l}\text { Material } \\
\text { Index } \\
\end{array}$ & Material & Application \\
\hline 1 & H451 Graphite & $\begin{array}{l}\text { Central Reflector } \\
\text { Inner Side Reflector }\end{array}$ \\
\hline 2 & $\begin{array}{l}\text { Fuel Element Composite } \\
\text { H451/Fuel/Coolant Holes }\end{array}$ & Active Core \\
\hline 3 & $\begin{array}{l}\text { Reflector Element Composite } \\
\text { H451/Coolant Holes }\end{array}$ & Top and Bottom Reflectors \\
\hline 4 & Stackpole 2020 Graphite & $\begin{array}{l}\text { Permanent Side Reflectors, } \\
\text { Lower Plenum Core Floor }\end{array}$ \\
\hline 5 & $\begin{array}{l}\text { Reflector Element Composite } \\
\text { Stackpole 2020/Coolant Holes }\end{array}$ & Bottom Reflectors \\
\hline 6 & Medium Carbon Steel & $\begin{array}{l}\text { Reactor Vessel and Metallic } \\
\text { Core Support Structure }\end{array}$ \\
\hline 7 & Alloy 800 and Kaowool Composite & Upper Plenum Shroud (top) \\
\hline 8 & Alumina Insulation & Core Floor \\
\hline 9 & Carbon Steel & RCCS Panels \\
\hline 10 & Alloy 800 & $\begin{array}{l}\text { Core Barrel and Upper Plenum } \\
\text { Shroud }\end{array}$ \\
\hline 11 & Alloy 800 and Kaowool Composite & Upper Plenum Shroud (side) \\
\hline 12 & Concrete & * \\
\hline 13 & Clay Soil & * \\
\hline 14 & Non-Operating RCCS Structure & * \\
\hline 15 & $\begin{array}{l}\text { Composite of Alloy } 800 \text { and } \\
\text { Boronated Graphite }\end{array}$ & Upper Plenum Elements \\
\hline 16 & Kaowool & $\begin{array}{l}\text { Core Barrel Gap (top flange } \\
\text { area) }\end{array}$ \\
\hline 17 & Alloy 800 and Kanwool Composite & Upper Plenum Shroud (side) \\
\hline
\end{tabular}

*Currently not used; have been used for analysis of core heat-up without functioning RCCS. 
For the active core-fuel elements and reflectors, the volume fractions of graphite (.584), fuel (.183), and coolant holes (.233) are stated in a data statement; however, previous effective values, as given by the vendor, are still used.

The top region of the upper plenum shroud is modeled as $26 \mathrm{~mm}$ of Alloy 800 (Material 10) plus a quasi-steady layer of $32 \mathrm{~mm}$ Kaowool and $5 \mathrm{~mm}$ cover plates of Alloy 800 (Material 7). The side of the upper plenum shroud also is a composite of Alloy 800 and Kaowool. The radial node thickness for this node $(\mathrm{i}=19)$ is $375 \mathrm{~mm}$. In the core barrel region, $295 \mathrm{~mm}$ of this is the core barrel gap and $80 \mathrm{~mm}$ the effective thickness of the core barrel. A different gap width for this shroud region could have been specified, reducing the solid node thickness to $25 \mathrm{~mm}$; this would be preferable, in general. For historic reasons, a different specification is still in effect here, and will be described, to point out an additional option to the models. The node thickness was retained as $80 \mathrm{~mm}$, but $\mathrm{k}$ and $\rho \mathrm{c}$ of Material 11 were prorated correspondingly to account for the actual node thickness of $25 \mathrm{~mm}$; this requires different proration for radia and axial thermal conductivities.

The side insulation of the upper plenum shroud is a composite layer of $19 \mathrm{~mm} \mathrm{Kaowool}$ and 5 $\mathrm{mm}$ cover plates (Material 17). The insulation is continued downward at the outside of the core barrel to protect the vessel flange region by a $25 \mathrm{~mm}$ layer of Kaowool (Material 16), specified as a layer in Radial Boundary 14.

Table 8-3 summarizes the gap conductivities of the current PROPMAT subroutine, including approximations for the natural convection heat transfer correlations. Since radiation generally dominates the total gap heat transfer; the importance of these correlations is relatively small. However, these models should be improved, particularly if more detail of the upper head region is of interest. 
TABLE 8-3

Gap Effective Thermal Conductivities

of the THATCH Commercial MHTGR Model

\begin{tabular}{|c|c|}
\hline $\begin{array}{l}\text { Material } \\
\text { Index }\end{array}$ & Description \\
\hline 1 & Zero thermal resistance \\
\hline 2 & Stagnant helium \\
\hline 3 & Adiabatic gap \\
\hline 4 & $\begin{array}{l}\text { Reactor cavity natural circulation } \\
\text { (Correlation of Equ } 3.2-4 \text { using cavity dimensions } \\
\mathrm{H}=20.4 \mathrm{~m}, \delta=0.91 \mathrm{~m} \text {, and } \mathrm{p}_{\mathrm{o}}=10^{5} \mathrm{P} \text {.) }\end{array}$ \\
\hline 5 & Air-cooled RCCS thermal insulation* \\
\hline 6 & $\begin{array}{l}\text { Core barrel gap conductance } \\
\text { (Correlation of Equ } 3.2-4 \text { using core barrel dimensions } \\
\mathrm{H}=12.87 \mathrm{~m}, \delta=0.295 \mathrm{~m} \text {, helium as gas and } \mathrm{p}_{\mathrm{o}}=\text { input } \\
\text { value of POUPL which is } 10^{5} \mathrm{P} \text { for depressurized runs and } \\
\text { input via FLOXI for pressurized runs.) }\end{array}$ \\
\hline 7 & $\begin{array}{l}\text { Effective conductance from plenum shroud to vessel } \\
\text { (For depressurized case use approx } 1 / 2 \text { of } h \text { in upper plenum, } \\
\text { i.e. Nu } \approx 100 \text {; For pressurized case use same correlation } \\
\text { as in upper plenum (Equ } 8.5-6 \text { ) with } H=.477 \mathrm{~m} \text {.) }\end{array}$ \\
\hline
\end{tabular}

${ }^{*}$ Currently not used; have been used for analysis of core heat-up without functioning RCCS. 


\subsection{Irradiation Damage and Annealing Model for Thermal Conductivity of Graphite}

The MATLIB submodule of the code includes correlations for the thermal conductivity of H45I graphite with and without irradiation damage. In general, the correlations without irradiation damage will be used. However, the user can specify that irradiation damage is to be considered, and over which range of nodes. In that case a "damage parameter" must be supplied for each node $(i, j)$ where

$$
0<D_{i, j}+\overline{<} 1.0
$$

and the effective thermal conductivity is linearly prorated between damaged and undamaged graphite as

$$
k_{e f f}-k_{i r r a d}\left(\theta_{i, j}\right) D_{i, j}+k_{\text {unirrad }}\left(\theta_{i, j}\right)\left(1-D_{i, j}\right)
$$

During core heatup sequences, the graphite generally will undergo annealing and will recover the thermal conductivity of undamaged graphite. If the user wants to model this process, a temperature range over which annealing is assumed to occur must be specified. As annealing is considered to occur ticit compared to the very slow core heatup scenarios, the damage parameter of any node will be reduced as the node temperature rises such that

$$
\mathrm{D}_{\mathrm{i}, \mathrm{j}}=\min \left(\mathrm{D}_{\mathrm{i}, \mathrm{j}}, \frac{\theta_{2}-\theta_{\mathrm{i}, \mathrm{j}}}{\theta_{2}-\theta_{1}}\right) \quad \begin{aligned}
& \theta<\theta_{2} \\
& \theta \geq \theta_{2}
\end{aligned}
$$

where $\theta_{1}$ to $\theta_{2}$ is the temperature range for annealing. The use of the minimum function ensures that annealing only begins when the appropriate temperature is reached, and will not reverse as cooldown begins. Strictly speaking, this model should be revised if the core becomes critical again later in any scenario, with irradiation being resumed. So far, such scenarios have not been identified or analyzed.

\subsection{Modelling of Solid Heating Rate in THATCH}

The THATCH model for transient solid temperatures of Section 3-1 requires prescription of the volumetric interval heat-generation rate $\mathrm{q}^{\prime \prime \prime}$ at all nodes.

This heating can be due to decay heat, fission power (if PKINL=true), or due to convective heat transfer from the coolant (if FLOXI $=$ true and IREACT $>0$ ).

The radial and axial power distribution is either prescribed with the input data, or taken from PSID data which are given in BLOCK DATA COREDTS of THTCHLIB.

The local power fractions at any node is the product of radial and axial power fractions, i.e.,

$$
\phi_{i, j}-\phi_{R i} \phi_{A j}
$$

where $\phi_{i, j}$ is the local power fraction of node $i . j$ and $\phi_{R i}$ and $\phi_{A j}$ are the radial and axial power tractions. 
The local power fraction at any node is defined as

$$
\phi_{i, j}=\frac{q_{i, j}^{\prime \prime \prime}}{q^{-\prime \prime \prime}}
$$

and is assumed to remain constant with time. (But, note that different power profiles can be prescribed for decay heat and for fission power). Here $\mathrm{q}^{\prime \prime \prime}$ is the local volumetric heating rate and $\mathrm{q}^{\prime \prime \prime}$ is the average volumetric heating rate of the core

$$
\tilde{q}^{\prime \prime \prime}-\frac{1}{\sum V_{i, j}} \sum q_{i, j}^{\prime \prime \prime} v_{i, j}
$$

As total fission power and decay heat vary with time, the average volumetric heating rate can be expressed as

$$
\tilde{q}^{\prime \prime \prime}-\tilde{q}_{0}^{\prime \prime \prime} \times \frac{P(t)}{P_{o}}
$$

where $\mathrm{P}$ is total core power and the subscript o designates initial steady state values.

From (8.4-2) and (8.4-4), the local volumetric heating rate at time $t$ is

$$
q_{i, j}^{\prime \prime \prime}=\phi_{i, j} q_{o}^{\prime \prime \prime} \frac{P(t)}{P_{o}}
$$

This expression is computed for each node at each time step, separately for decay heat and fission power, and finally, the nodal convective heat flows computed in FLOXI as well as chemical reaction heats are added. Thus

$$
\begin{array}{r}
q_{i, j}^{\prime \prime \prime}-\left\{\phi_{D, i, j}\left[\frac{P(t)}{P_{0}}\right]_{D}+\phi_{F, i j}\left[\frac{P(t)}{P_{0}}\right]_{F}\right\} q_{0}^{\prime \prime \prime} \\
+\frac{Q_{F L O X ~ i, j}}{V_{i, j}}
\end{array}
$$

where subscripts $D$ and $F$ denote decay heat and fission power, and $V$ is the node volume and

$$
Q_{\text {FLOX } i, j}-Q_{\text {conv } i, j}+Q_{\text {react } i, j}
$$


$\mathrm{Q}_{\text {CONV } \mathrm{i}, \mathrm{j}}$ is computed from equation (3.4-22) and the reaction heat is

$$
Q_{\text {react } i, j}=-\Gamma_{\text {ox }}^{\prime \prime \prime} H_{o x}
$$

where $\Gamma_{\mathrm{ox}}^{\prime \prime \prime}$ is the volumetric generation rate of the oxidant $\left(\mathrm{O}_{2}\right.$ in the case of air ingress and $\mathrm{H}_{2} \mathrm{O}$ in the case of water ingress) as computed in Section 3.4 .3 and $H_{o x}$ is the energy release

$$
\begin{aligned}
& \mathrm{H}_{\mathrm{O}_{2}}=242 \frac{\mathrm{MJ}}{\mathrm{kmol} \mathrm{O}} \\
& \mathrm{H}_{\mathrm{H}_{2} \mathrm{O}}=-118.8 \frac{\mathrm{MJ}}{\mathrm{kmol} \mathrm{H} \mathrm{O}}
\end{aligned}
$$

(Note that $\Gamma_{\mathrm{ox}}$ is negative)

The FLOXI terms are computed in FLOXI and passed to THATCH routine HEATGEN where the afterheat and fission power terms are computed.

The fission power $\mathrm{P} / \mathrm{P}_{\mathrm{o}}$ is computed by the point kinetics model, and the afterheat fraction is evaluated in routine AFTFR of GENLIB, using the afterheat profile specified by input data records 1108 to $1111 \mathrm{D}$.

\subsection{Natural Convection Model For The Unper Plenum}

During core heatup scenarios, natural convection currents can be significant, since the core is essentially a cavity, heated from below. Natural convection is not of much interest in depressurized core heatup scenarios, where the gas density is low and the convective heat transfer is small compared to radiation and conduction in solids.

However, in pressurized core heatup scenarios this effect can be essential, in particular, because it increases the buoyancy-driven flow circulation through the active core. In such scenarios, gas rises in the coolant channels of the hotter regions of the core, flows through the upper plenum and returns mainly through the coolant channels of colder regions of the core, with some bypass flow through the flow channels of the core-barrel. During the tlow through the upper plenum, which has its own turbulent natural circulation loop at Grashof numbers above $10^{11}$, the gas will be cooled as it flows along the colder plenum shroud, resulting in a temperature drop between the gas streams entering and leaving the upper plenum. An accurate assessment of this effect would require a multi-dimensional model of turbulent convection in the upper plenum with simultaneous gas intlow and outtlow at the bottom of the plenum. Such detail cannot be included here and a simplified model is given. Once the results from detailed evaluations of a separate upper-plenum multi-dimensional code are available, this simplified model can be improved by adjusting its correlations to those results.

The current model assumes convective heat transfer from each plenum node $\ell$ as 


$$
\mathrm{Q}_{\text {conv } \ell}-\mathrm{A}_{\ell} \mathrm{h}_{\text {cav }}\left(\theta_{\mathrm{s} \ell}-\theta_{\text {gav }}\right)
$$

where

$Q_{\text {conv } \ell}$ is the heat removed from the solid surface by convection. $A_{\ell}$ is the node surface area, $h_{\text {cav }}$ a cavity heat transfer coefficient, $\theta_{s}$ the node surface temperature, and $\theta_{\text {gav }}$ an average gas temperature for the upper plenum.

In terms of the general plenum-heat-transfer model of Section 3.1, Equation 3.1-17 which presents a balance of conduction and radiation now would become

$$
Q_{\text {cond }}-Q_{\text {rad }}+Q_{\text {conv }}
$$

or

$$
\begin{gathered}
\mathrm{Q}_{\ell}-\frac{2 \mathrm{~A}_{\ell}}{\Delta \mathrm{Z}_{\mathrm{n}}} \mathrm{k}\left(\theta_{\mathrm{i}, \mathrm{j} \text { avg }}\right)\left(\theta_{\mathrm{i}, \mathrm{j}}-\theta_{\mathrm{s} \ell}\right) \\
-\mathrm{A}_{\ell} \sum_{\mathrm{m}-1}^{\mathrm{L}} \Lambda_{\mathrm{Im}} \sigma \theta_{\mathrm{sm}}^{4}+\mathrm{A}_{\ell} \mathrm{h}_{\mathrm{cav}}\left(\theta_{\mathrm{s} \ell}-\theta_{\mathrm{gav}}\right)
\end{gathered}
$$

A quasi-steady energy balance on the plenum gas flow yields

$$
W c_{p}\left(\theta_{\text {gex }}-\theta_{\text {gin }}\right)-\sum_{\ell=1}^{L} A_{\ell} h_{c a v}\left(\theta_{s \ell}-\theta_{\text {gav }}\right)
$$

and the effective average plenum gas temperature was assumed to be

$$
\theta_{\text {gav }}-1 / 2\left(\theta_{\text {gex }}+\theta_{\text {gin }}\right)
$$

For the convective heat transfer coefficient, h, the code provides an internal option between two correlations (set in calls to UPLCONV):

Either

$$
\mathrm{Nu}-\frac{\mathrm{hH}}{\mathrm{k}}-0.098 \mathrm{Ra}^{0.33}
$$

or 


$$
\mathrm{Nu}=\frac{\mathrm{hH}}{\mathrm{k}}-0.316 \mathrm{Ra}^{0.25}-1.3
$$

where $\mathrm{H}$ is the effective plenum height and $\mathrm{k}$ the gas thermal conductivity.

The first correlation is based on data presented by Catton [1978]. The second correlation was extracted by Moszynski [1982] from Catton [1978], after consulting some of the references in Catton's paper.

To solve the upper plenum heat transfer, including convection, the method of Section 3.1-3 (Equation 3.1-20) must here he amended by adding the convective term, which results in

$$
\sum_{\mathrm{m}=1}^{\mathrm{L}}\left[\delta_{\mathrm{lm}}\left(\alpha_{\ell}+\mathrm{h}_{\mathrm{cav}}\right) \theta_{\mathrm{s} \ell}+\Lambda_{\ell \mathrm{m}} \sigma \theta_{\mathrm{sm}}^{4}\right]-\mathrm{h}_{\mathrm{cav}} \theta_{\mathrm{gav}}-\alpha_{\ell} \theta_{\mathrm{i}, \mathrm{j}} \quad \ell-1, \mathrm{~L}
$$

To this system of $\mathrm{L}$ equations, one equation for $\theta_{\text {gav }}$ must be added, which is obtained from equations $(8.5-3$ and 4$)$ by eliminating $\theta_{\text {gex }}$

$$
\left[2 \mathrm{Wc}_{\mathrm{p}}+\mathrm{h}_{\mathrm{cav}} \sum \mathrm{A}_{\ell}\right] \theta_{\mathrm{gav}}-2 \mathrm{Wc}_{\mathrm{p}} \theta_{\mathrm{gin}}+\mathrm{h}_{\mathrm{cav}} \sum \mathrm{A}_{\ell} \theta_{\mathrm{s} \ell}
$$

This system of $\mathrm{L}+1$ equations, is solved using an analytical Jacobean by the Newton Raphson method. (The code is internally hard-wired to optionally compute the system of $L+2$ equations, including $h_{c a v}$ as additional variable and including equation (8.5-6) for $h_{\text {cav }}$ in the set of equations. The option is set in RADTN via logical variable HUPLINT. From a few test cases, it is not clear which method is preferable. It is currently set to true, i.e., $h_{\text {cav }}$ is included, and a set of $L+2$ equations is solved).

To compute the effective Grashof number for the upper plenum, the temperature difference from bottom to top is computed in UPLCONV by computing area-weighted average temperatures for the top and bottom boundaries of the plenum, and disregarding the side temperatures.

The model is currently coded for helium as a gas only. Because upper plenum convection is only essential in pressurized scenarios, and potential $\mathrm{H}_{2} \mathrm{O}$ concentrations during water ingress scenarios are small, this restriction has not proved to be serious. However, it should be kept in mind, and may have to be removed at a later date. 


\section{SAMPLE CASES AND INSTALLATION REQUIREMENTS}

Four sample cases ware selected, which exercise different options of the code. They are described in Table 9-1 and th sir input decks are reproduced in Appendix F. For each case, a base run, a restart run (except Case 2), and a post-procsising run were executed on the IBM 3090 at BNL and on the CRAY at INEL, to assure complete correspondence of the code's versions. For holders of controlled copies of this document, the printed output files will be enclosed in fiche form with this document.

The source deck of the code is written in FORTRAN 77, including several enhancements which are available on the IBM 3090, as well as on the CRAY computer. The total source disk consists of a dummy main program with $2 \leftrightarrow 9$ subroutines (partitions).

Code installation requires loading of the source disk and code input file from the supplied magnetic tape. After compilation the code can be executed, using the abuve sample cases, following the rules of the installation - specific operating system.

At BNL, the IBM Engineering and scientific Subroutine Library is used to solve sets of simultaneous equations. At the INEL Cray, the IMSL library is used for that purpose. At both installations, the IMSL library is used for the ordinary differenial equation integration of the point kinetics option.

Code compile times are not of any significance. Code execution times vary widely, depending on the options chosen. Table 9-1 includes the IBM 3090 execution times for the four test cases; execution times on the INEL CRAY ranged between $45 \%$ and $85 \%$ of those on the IBM 3090 . 


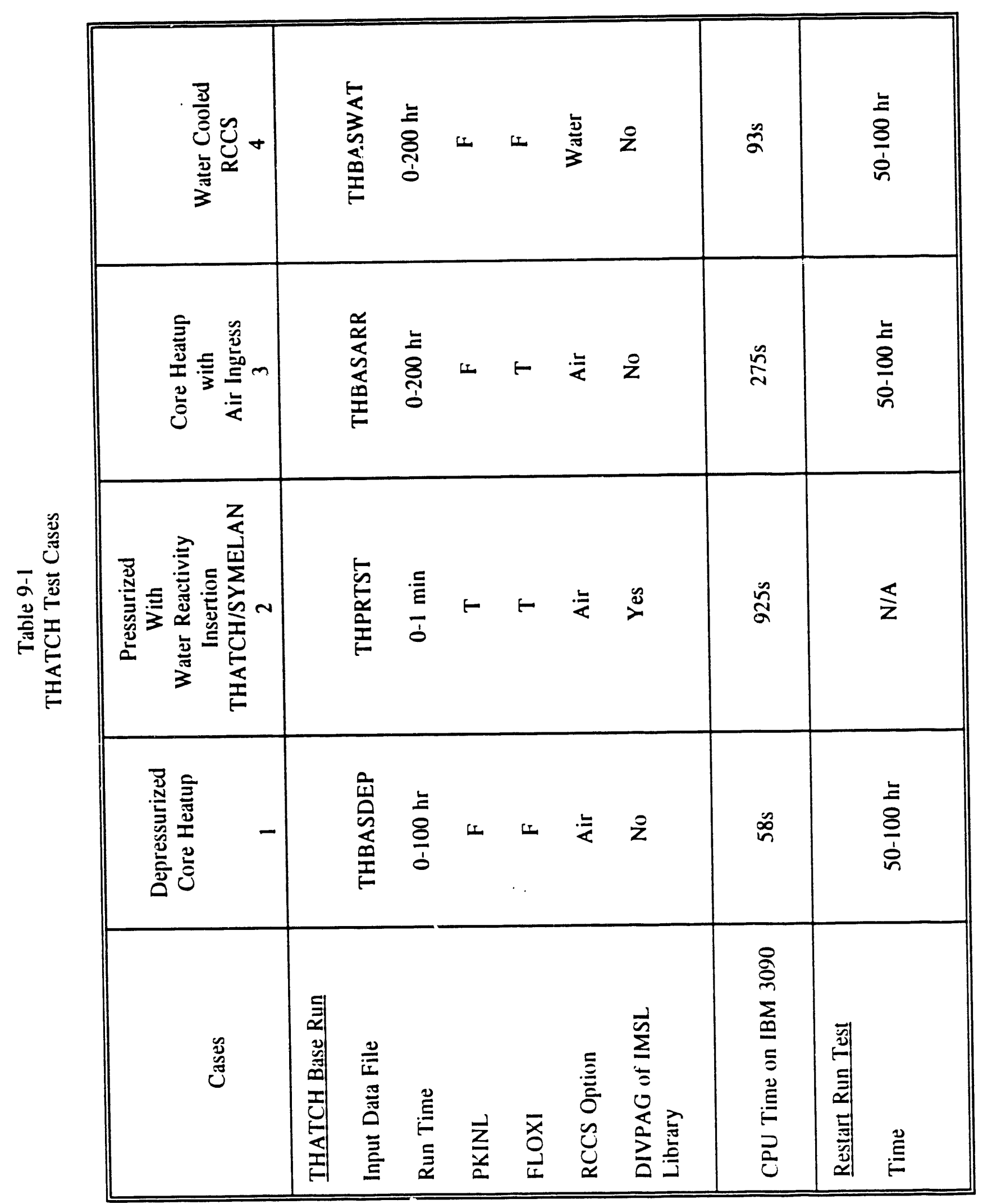




\section{CAPABILITIES AND LIMITATIONS OF THE THATCH CODE}

\subsection{Code Capabilities}

The basic THATCH code can be used to model solid conduction in any network of solid nodes that can be represented in a two-dimensional cylindrical coordinate system of $r-z$ coordinates. Gaps with conduction and radiation and cylindrical plena with two-dimensional radiation also are allowed.

Its major application has been to model thermal-hydraulic transients in the ractor vessel of gas cooled reactor. Since a user-specified nodalization is allowed, the THATCH code can be used to model local effects, employing a very tine nodalization in the areas of primary interest.

In its current model of the commercial MHTGR, described in Section 8, peak fuel and vessel temperatures during pressurized and/or depressurized core-heatup transients with loss of all forced cooling were of primary interest: therefore, the nodalization concentrated on accurate presentation of the center region of the core and center to upper beltline of the vessel.

With its modules for gas flow, convective heat transfer, chemical reactions, and point kinetics the code has been applied to such scenarios as

- $\quad$ pressurized and depressurized core heatup scenarios (also referred to as core cooldown events)

- natural gas circulation in the primary loop during pressurized core-heatup events

- graphite oxidation during air-or water-ingress events, including the evaluation of maximum possible entry of air into the core

- long-term no-scram transients, with recriticality after two days

- rapid reactivity insertions, for instance, due to water ingress (via SYMELAN module)

- parametric evaluations of sensitivity to changes in design data and material properties

- core-heatup scenarios with loss of RCCS performance, including a model of reactor cavity heatup

The code could readily be applied to other events like

- $\quad$ start up and shutdown transients, where vessel temperatures would be of major concern

- modelling of core and vessel temperature transients during load change events

- $\quad$ slow depressurization transients (with prescribed depressurization rate and gas flow)

\subsection{Code Limitations}

The THATCH code presents a model of heat transfer and fll:d tow in the reactor vessel. 
Generally, inputs are the coolant inlet temperature and either the coolant flow rate, or the effective positive pressure head (circulator operating), or a pressure loss head for the flow path outside the reactor (representing steam generator or SCS).

A free-standing quasi-steady model for a steam generator has sometimes been used to estimate changes in the temperatures of the return gas flow, based on changing gas and inlet temperatures, to simulate the corresponding changes in inlet gas flow via THATCH subroutine FLUINUP (Section 4.2.3). This steam generator model could be coupled with FLUINUP, but it has not been done to date.

As peak fuel and vessel temperatures were of primary interest in previous analyses, the cylindrical section of the reactor vessel has been modelled in more detail than the semi-spherical top head and bottom regions. (Some hard-wired special extensions for upper-head cooling have been used; however, they are not part of the current code). The top and bottom regions were modelled as cylindrical, with coolant volume and heat transter surfaces being kept as closely as possible to the design data. When increased accuracy is required in the upper head and the bottom regions, the current models should be refined accordingly.

Upper-and-lower-plenum heat-transfer currently assumes radiation in free spaces. The effect of control rods and fuel support columns on heat transfer has been neglected; this should be revised as these regions gain in relative importance.

The current code does not model changes in system pressure, but uses a user prescribed upper plenum pressure, even though the routines to compute system pressure are available.

The system pressure can be shown to be a function of the sum

$$
\sum \frac{v_{i}}{\theta i}
$$

of gas volumes divided by gas temperatures in all volumes of the primary loop. As the volume data of Table 4-14 show, the bottom plenum, core-barrel side-gap region, and upper plenum are the dominant terms for the reactor vessel. About half the primary loop gas volume is in the steam generator. Any meaningful predictions of pressure would require good models for gas temperatures, including the heat transfer from gas to sidewall, for these regions, many of which are stagnant gas volumes. The main interest of pressure transients is to determine relief-valve lift off, with relief valve pressure being approximately $10 \%$ above steady state pressure. Relatively accurate predictions of pressure would be required to assess whether and when the set point of the relief valve is reached. For this reason, system pressure computations are not made at this time, although they could be implemented. The subroutines for this computation are available.

The point kinetics equations currently are always integrated as full ODEs, with six delayed neutron groups. If computer time is a concern, then, for many transients where the reactivity changes amount to only a few cents, the jump approximation could be implemented to reduce the computer run time. To date, computer time has not been a problem, and the approximation has not been applied. 


\subsection{Error Indications}

Most error messages produced by the code are self explanatory; those that may require experience to interpret will be listed here. Since the developers of the code may not realize what may cause difficulties for new users, we would appreciate feedback, and will expand this section, or others, as required.

At start time, the following message is often produced:

****** WARNING, IN PROPMAT, GR BELOW 1.E7, GR $=1.0$ DELTHGR $=0.0 * *$

This is called in Propmat for Gap Material 107 (Upper plenum shroud to reactor vessel). This is usually due to the initial temperature differences between the insulation of the upper plenum shroud and the reactor vessel's surface being zero on first call and first iteration in BNDCONI. Disregard the message if it only occurs at start time.

The general solution method for THATCH solid temperatures is implicit and, therefore, in principle numerically stable. However, since thermal properties and gap resistances are explicitly updated at each time step, instabilities can arise occasionally when coarse time steps are chosen.

The first message indicating such instability is usually due to non-convergence in the BNDCONI iteration loop for interface resistances. It reads:

***** NON-CONVERGENCE IN BNDCONI ROUTINE

THA $(0: 4)=1.60515 E+031.59636 E+039.21009 E+025.94368 E+025.74552 E+02$

DELSUMR $=1.79725 E-05$

$\mathrm{COF}=2.38685 \mathrm{E}+04$

RADIAL BOUNDARY NO. $=16$

NODE, I,J = 19, 6

TIME $=104.00$ HOURS

Another message indicating instability is non-convergence in plena radiation and convection computations. It generally reads:

From NEWTON:

NON-CONVERGENCE IN NEWRAF-LOOP

JBIGR, BIGR $=\quad 18 \quad 2911884970.86540413$

JBIGDY,BIGDY $=\quad 1846281.7269896565040$ 
FROM RADTN:

TIME $=104.00$ HOURS $* * * * *$ ERROR RETURN FROM NEWTON, PLENUM NUMBER $=1$

$$
\text { IER }=3 \mathrm{NIT}=20 \quad \mathrm{BIGR}=2.9118853+09
$$

Generally, only later in the onset of stability some temperatures will get out of bound in property routine calls, producing a message like:

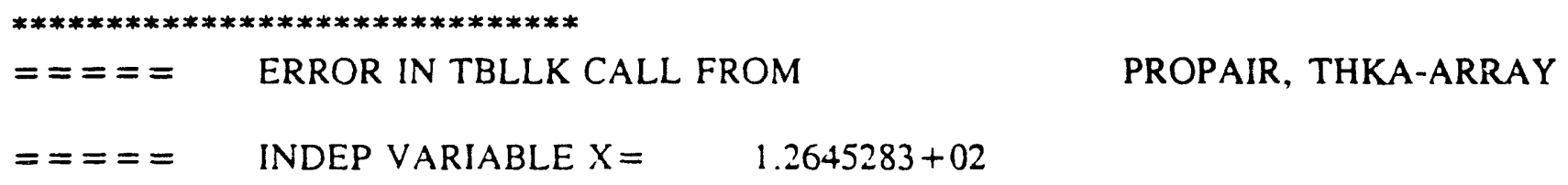

In all these cases, the remedy is a decreased time step. Often this is only required for a limited time. Run times have never posed a problem, therefore no further preventive action, like automatic timestep reductions, have ever been used or planned.

In some cases, the maximum number of function evaluations in DIVPAG have been exceeded. In general, this indicates some error in the modelling, like incorrect or inconsistent input data. The number of permissible function calls is entered in SOLTRAN (part of GENLIB) from Block Data ODEDAT. To increase these parameters may be a temporary solution, but is unlikely to be a permanent remedy. (See also, IMSL documentation of DIVPAG).

\subsection{Code Service}

The THATCH code and its submodules are maintained at Brookhaven National Laboratory, Department of Nuclear Energy, Reactor Analysis Division. Code modifications can be made by one person only

\section{Robert J. Kennett (516) 282-2104 \\ FTS 666-2104}

and only with the concurrence of

Peter G. Kroeger $\quad$ (516) 282-2610

FTS $666-2610$

These people should be the contacts for any problems, questions, or suggestions that may arise. R. J. Kennett is the prime contact for code structure and related subjects: P. G. Kroeger is the prime contact for questions regarding modelling and code capabilities.

The code is maintained, using HISTORIAN PLUS of Opcode Inc. on the IBM 3090 at Brookhaven National Laboratory. This system is similar to the UPDATE system used at other installations.

This document is openly available, as a Brookhaven National Laboratory report. However, in addition to the general copies, there will be a limited number of controlled copies. Holders of such 
copies will be automatically provided with updates as changes are made.

To exercise this code, the user must have general engineering knowledge of nuclear reactor thermal fluid flow and neutronics, as well as some experience in numerical methods. Also, some familiarity with codes and computer programming is required, either in one person or in a team of engineers and programmers. 


\section{REFERENCES}

BECHTEL NATIONAL, Inc. et al., "HTGR Concept Description Report, Reference Modular High Temperature Gas Cooled Reactor Plant," Report DOE-HTGR-86-118, October 1986.

BIRD, R. B., STEWARD, W. E., and LIGHTFOOT, E. N., "Transport Phenomena," John Wiley \& Sons, 1960.

CARNAHAN, B., LUTHER, H. and WILKES, J., "Applied Numerical Methods," John Wiley \& Sons, Inc., 1969.

CATTON, I., "Natural Convection in Enclosures," Proceedings Sixth International Heat Transfer Conference, Vol. 6. p. 13. 1978.

CHENG, H. S., "PKINS - A Point Kinetics Program", Brookhaven National Laboratory, April 26, 1976.

COLE, R, KELLY, D. P., and ELliS, M. A., CORCON-Mod2: A Computer Program for Analysis of Molten-Core Concrete Interactions, NUREG/CR-3920, p. 74, 1984

COX, C. W., "GENRD: A Free-Format Card Input Processor," Los Alamos Scientific Laboratory, LA4793, January 1972

GNIELINSKI, V., ZUKAUSKAS, A. and SKRINSKA, A., "Banks of Plain and Finned Tubes," in Section 2.5.3, Heat Exchanger Design Handbook, Hemisphere Publishing Co., New York pp. 2.5.3-12, 1983.

HOLMAN, J. P., "Heat Transfer," McGraw Hill Book Company, 1981.

HOLMAN, J. P., "Heat Transfer," McGraw Hill, New York, 7th Edition, p. 665, 1990.

ISAACSON, E. and KELLER, H. B., "Analysis of Numerical Methods," John Wiley \& Sons, Inc., 1966.

KAMINSKY, D. A., Ed., Heat Transfer Data Books, Genium Publishing Corp., 1990.

KATCHER, W. and MOORMANN, R., "Graphite Corrosion under Severe HTGR Accident Conditions," IAEA Specialists Meeting on Graphite Component Structural Design, JAERI, Tokai-Muro, Japan, September 1986.

KNIEF, R. A., "Nuclear Energy Technology," Hemisphere Publishing Corp., 1981

KROEGER, P. G., "Safety Evaluation of MHTGR Licensing Basis Accident Scenarios," Brookhaven National Laboratory, NUREG/CR-5261, April 1989.

MC ADAMS, W. H., "Heat Transmission," McGraw - Hill Book Co., New York, p. 272, 1954. 


\section{REFERENCES (CONT'D)}

MOORMANN, R., "Untersuchungen zu Stoerfallen mit massivem Wassereinbruch am Beispiel des Kugelhaufenreaktors PNP-500", Kernforschungsanlage Juelich GmbH , Juel-Spez - 333, 1985.

MOORMANN, R., "Effect of Delays in Afterheat Removal on Consequences of Massive Air Ingress Accidents in High-Temperature Gas Cooled Reactors", Journal of Nuclear Science and Technology, Vol. 21, pp. 824-835, 1984.

MOORMANN, R., KATSCHER, W., HINSSEN, H., and PETERSEN, K., "Verhalten des HTRKugelhaufens bei massivem Lufteinbruch," Atomkernenergie - Kerntechnik, Volume 35, pp. 270 - 274, 1980.

MOORMAN, R., and PETERSEN, R., REACT/THERMIX; Ein Computercode zur Berechnung der Stoerfallbedingten Graphitkorrosion in Kugelhaufenreaktoren," Kernforschungsanlage Juelich GmbH, Juel-1782, April 1982.

MOSZYNSKI, J. R., Memorandum to Files on Natural Convection in Upper Plena, Brookhaven National Laboratory, MF-93, July 23, 1982.

OWCZAREK, J. A., Fundamentals of Gas Dynamics," International Textbook Company, 1964.

PEROOMIAN, M. B., BARSELL, A. W., and SAEGER, J. C., "OXIDE-3: A Computer Code for Analysis of HTGR Steam or Air Ingress Accidents," General Atomic Company, GA-A 112493 (GA-LTR7), January 1974.

REID, R. C., PRAUSNITZ, J. M., and SHERWOOD, T. K., The Properties of Gases and Liquids, McGraw-Hill, New York, 3rd Edition, p. 454, 1977.

ROSSBERG, M, WICKE, E., "Transportvorgaenge und Oberflachenreaktionen bei der Verbrennung Graphitischen Kohlenstoffs", Chemie - Ingenieur Technik, Vol. 23, pp. 181-189, 1956.

SCHMIDT, E., Properties of Water and Steam in SI-Units, Springer-Verliag, Munich, 1979.

SPARROW, E. M. and CESS, R. D., "Radiation Heat Transfer," Brooks/Cole Publishing Company, Inc., 1966.

VARGAFTIK, N. B., "Tables on the Thermophysical Properties of Liquids and Gasses: In Normal and Disassociated States," John Wiley and Sons, Inc., 1975, Second Edition.

ZUKAUSKAS, A. A. and ULINSKAS, R., "Banks of Plain and Finned Tubes," in Section 2.2.4, Heat Exchanger Design Handhook, Hemisphere Publishing Co., New York, p. 2.2.4-6, 1983a.

ZUKAUSKAS, A. A. and ULINSKAS, R., "Banks of Plain and Finned Tubes," in Section 2.2.4, $\underline{\text { Heat }}$ Exchanger Design Handbook, Hemisphere Publishing Co., New York, pp. 2.2.4-13, 1983 b.

ZUKAUSKAS, A. A., "Heat Transfer from Tubes in Cross Flow," Advances in Heat Transfer, Volume 8, pp. 93-160, 1972. 


\section{APPENDIX A}

\section{RADIATION EXCHANGE BETWEEN GRAY SURFACES IN A CLOSED \\ CAVITY WITH A TRANSPARENT GAS.}

It is assumed that the closed cavity can be represented as a set of $L$ isothermal nodes of a prescribed geometry for which the view factors $F_{\mathfrak{l} m}$ are available (see Appendix $B$ ).

For a surface which has the incident radiant energy $\mathrm{H}$ arriving, the net heat loss from the surface can be expressed as

$$
\frac{Q_{\ell}}{A_{l}}-\epsilon_{\ell} \sigma \theta^{4}-\epsilon_{\ell} H_{\ell}
$$

where the first term is the radiation emanating from the surface, and the second term is the fraction of the incident energy being absorbed by the surface, as shown schematically in Figure A-1.

Defining the "radiosity" B as the energy streaming away from a surface we have

$$
B_{\ell}-\epsilon_{\mathfrak{q}} \sigma \theta_{\ell}^{4}+(1-\epsilon) H_{\ell}
$$

Eliminating $\mathrm{H}_{\ell}$ between Equations $(A-1)$ and (A-2), we obtain for the heat loss from the surface $\ell$

$$
\frac{Q_{\ell}}{A_{\ell}}-\frac{\epsilon_{\ell}}{1-\epsilon_{\ell}} \sigma\left(\theta_{\ell}^{4}-B_{\ell}\right)
$$

The radiation incident on surface $\ell, \mathrm{H}_{\ell}$, can be summed over all nodes

$$
A_{\ell} H_{\ell}-\sum_{m-1}^{L} A_{m n} B_{m} F_{m \ell}
$$

where in $F_{m \ell}$ the first index denotes the surfaces from which the radiation emanates and the second subscript denotes the receiving surface.

By using the reciprocity condition

$$
A_{\ell} F_{l i n}-A_{m} F_{m \ell}
$$

this can be rewritten as

$$
H_{\mathfrak{l}}-\sum_{m-1}^{L} B_{m} F_{\ell, n}
$$

Substituting (A-6) into (A-2), we obtain for the radiosity as a function of surface temperatures 


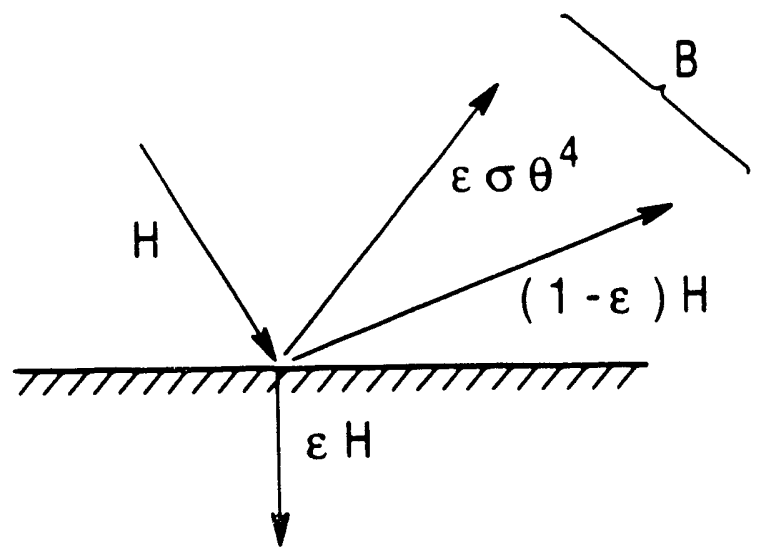

Figure A-1. Schematic of Incident, Absorbed, Emitted, and Retlected Energy and a Gray Surface Element.

A-2 


$$
B_{\ell}-\epsilon_{\ell} \sigma \theta_{\ell}^{4}+\left(1-\epsilon_{\ell}\right) \sum_{m-1}^{L} B_{m} F_{\ell m}
$$

or as a set of simultaneous equations

$$
\mathrm{X} B-\Omega
$$

where the $\mathrm{X}$ matrix has the members

$$
X_{l n}-\frac{1}{\epsilon_{i}}\left[\hat{o}_{l \mathrm{~m}}-\left(1-\epsilon_{\ell}\right) \mathrm{F}_{\ell \mathrm{m}}\right]
$$

with $\delta_{\ell \mathrm{m}}$ being the Kronecker delta, B being the radiosity vector, and $\Omega$ being the black body emittance vector

$$
\omega_{l}-\sigma \theta_{l}^{4}
$$

Inverting the $X$ matrix and defining

$$
\Psi-X^{-1}
$$

we obtain

$$
B_{\ell}-\sum_{m-1}^{L} \Psi_{\ell m} \sigma \theta_{m}^{4}
$$

Substituting (A-12) into (A-3) we obtain

$$
\frac{\mathrm{Q}_{\ell}}{\mathrm{A}_{\ell}}-\sum_{\mathrm{m}-1}^{\mathrm{L}} \Lambda_{\mathrm{m} \ell} \sigma \theta^{4}{ }_{\mathrm{j}}
$$

where

$$
A_{\mathfrak{l} m}-\frac{\epsilon_{\ell}}{1-\epsilon_{i}}\left(\hat{o}_{i j}-\Psi_{i j}\right)
$$

The procedure used in the THATCH 2-d radiation evaluations is to compute the X-matrix of Equation (A-9) from given view tactors and emissivities, to invert the X-matrix and use the resulting $\Psi$ matrix to compute the $\Lambda$ matrix, which then is used in the code to compute heat loss from a surface using Equation (A-13). 


\section{APPENDIX B}

\section{RADIATION VIEW FACTORS FOR PRESCRIBED GEOMETRIES}

In developing radiation view factors between parts of cavities, the following relationships, which are derived from energy conservation principles, are extensively applied [Sparrow and Cess, 1967].

For two surfaces $A_{i}$ and $A_{j}$ the following reprocity relationship applies

$$
A_{i} F_{A_{i}}-A_{j}-A_{j} F_{A_{j}}-A_{i}
$$

In an enclosure with $N$ surfaces. since the view factor $F_{A_{i}-A_{j}}$ is the fraction of the energy leaving $\mathrm{A}_{\mathrm{i}}$ that arrives at $\mathrm{A}_{\mathrm{j}}$

$$
\sum_{j=1}^{N} F_{A_{i}}-A_{j}-1
$$

where $F_{A_{i}}-A_{j}$ is the view factor from area $A_{i}$ to area $A_{j}$.

If one subdivides $A_{j}$ into $A_{j}^{\prime}$ and $A_{j}^{\prime \prime}$, then

$$
A_{i} F_{A_{i}-A_{j}}-A_{i}\left(F_{A_{i}}-A_{j}^{\prime}+F_{A_{i}-A_{j}^{\prime \prime}}\right)
$$

or with Equation (B-1)

$$
\left(A_{j}^{\prime}+A_{j}^{\prime \prime}\right) F_{A_{j}}-A_{i}-A_{j}^{\prime} F_{A_{j}^{\prime}-A_{i}}+A_{j}^{\prime \prime} F_{A_{j}^{\prime \prime}-A_{1}}
$$

In the THATCH code we have to consider cylindrical cavities, described in $r-z$ geometry, symmetric with respect to the $z$-axis. These cavities can be regular cylindrical ones with flat top and bottom sections and an outer side wall or annular cavities which have an inner and an outer side wall.

In this report, surfaces on the top and bottom will be referred to either as "disks" or as "annular disks", depending on whether they include the centel of the top or bottom section. Surfaces on the cylinder walls will be referred to as "outer cylinder rings" or "inner cylinder rings", depending on whether they are parts of the outer or inner wall.

Outer cylinder rings have a view factor onto themselves. All other surfaces to be considered here do not.

\section{B-1 Radiation View Factors in a Cylindrical Cavity}

Following Figure B-1, for two disks of radius $r_{1}$ and $r_{2}$, one from the top surface of the cavity and one from the bottom surface, with cavity height $h$, we have 


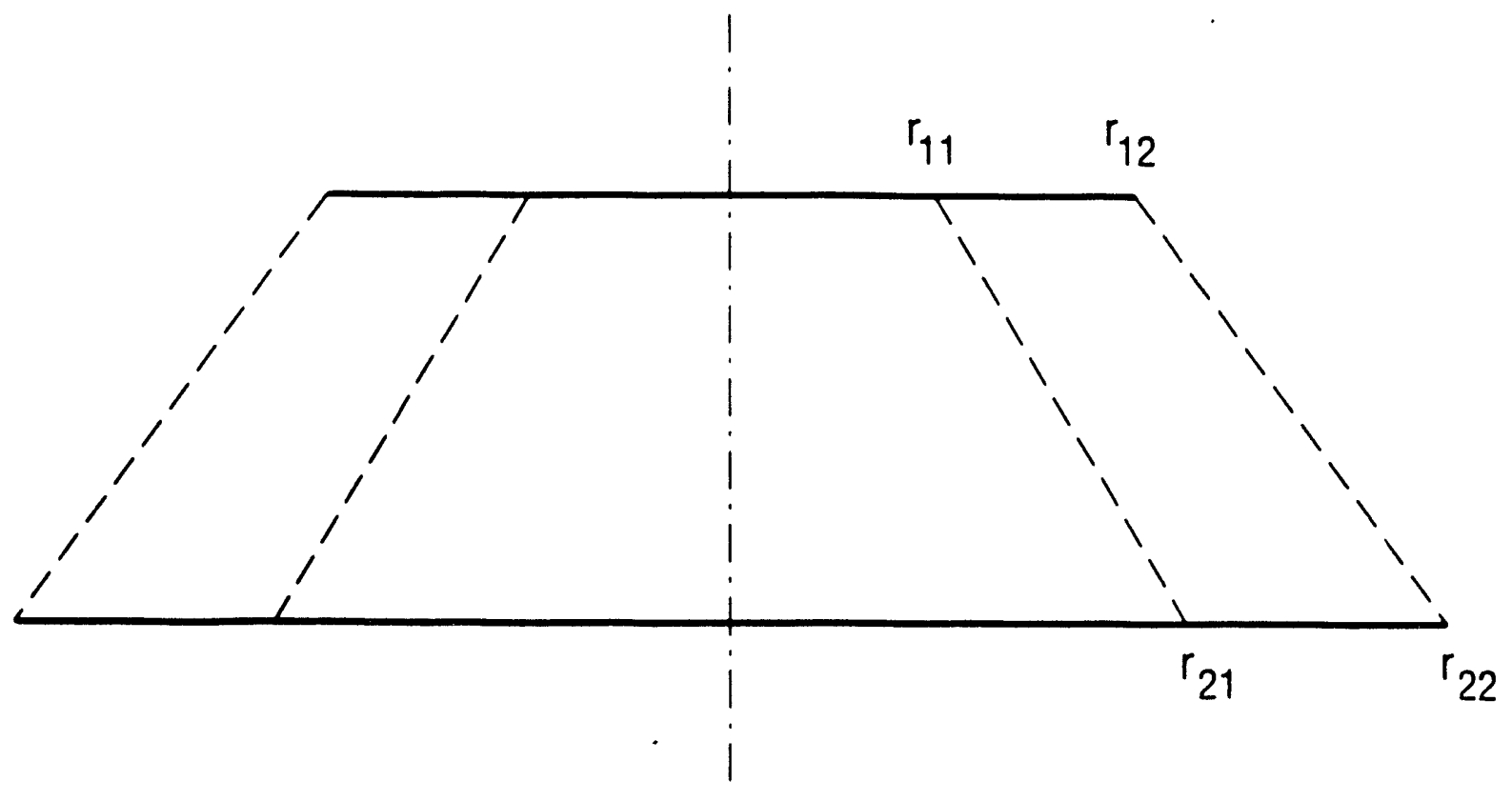

Figure B-1. Schematic of Geometry for Disk and Annular Disk

View Factors 


$$
F_{A_{1}}-A_{2}-\phi\left(r_{1}, r_{2}, h\right)-1 / 2\left(Z-\sqrt{Z^{2}-4 X^{2} Y^{2}}\right)
$$

where

$$
X-\frac{r_{2}}{h} ; Y-\frac{h}{r_{1}} ; Z-1+\left(1+X^{2}\right) Y^{2}
$$

From this disk-to-disk formula, we can apply Equations (B-1) to (B-4) to obtain view factors between any disks or annul i on the opposing top and bottom surfaces. Following the schematic of Figure B.1, $D_{i}$ denotes a disk of radius $r_{i}$, and $A_{i}$ an annular disk extending from $r_{i-1}$ to $r_{i}$.

Then. for the view factor from annular disk $A_{12}$ to disk $D_{21}$ :

$$
\begin{aligned}
\left(r_{12}^{2}-r_{11}{ }^{2}\right) F_{A_{12}}-D_{21} & -r_{12}^{2} \phi\left(r_{12}, r_{21}, h\right) \\
& -r_{11}{ }^{2} \phi\left(r_{11}, r_{21}, h\right)
\end{aligned}
$$

and for the view factor from annular disk $A_{12}$ to annular disk $A_{22}$

$$
\begin{aligned}
\left(r_{12}^{2}-r_{11}^{2}\right) F_{A_{12}}-A_{22} & -r_{12}^{2}\left[\phi\left(r_{12}, r_{22}, h\right)-\phi\left(r_{12}, r_{21}, h\right)\right] \\
& -r_{11}^{2}\left[\phi\left(r_{11}, r_{22}, h\right)-\phi\left(r_{11}, r_{21}, h\right)\right]
\end{aligned}
$$

For a cylindrical cavity of radius $r$ and height $h$, the view factor from the top disk (1) or bottom disk (2) to the side wall (3) is

$$
F_{1,3}-1-F_{1,2}-1-\phi(r, r, h)
$$

With

$$
F_{3,1}-\frac{A_{1}}{A_{3}} F_{1,3}
$$

and

$$
2 \mathrm{~F}_{3,1}+\mathrm{F}_{3,3}-1
$$

we obtain for the view factor of the side wall or any outer cylinder ring onto itself 


$$
F_{i, i}-1-\frac{r_{i}}{h_{i}}\left[1-\phi\left(r_{i}, r_{i}, h_{i}\right)\right]
$$

By further applying Equations (B-1) to (B-4), the correxponding relationships for view factors between outer cylinder rings of different elevation and between outer cylinder rings and disks or annular disks on the top and bottom surface follow from simple algebra.

The view factors, as currently implemented in THATCH, require the same radial division into annular disks for the top and bottom surfaces of the cavity. However, the above equations are general and this restriction can readily be removed.

\section{B-2 Radiation View Factors in an Annular Cylindrical Cavity}

The radiation view factors between the surfaces of an annular cylindrical cavity, following the surface designations of Figure B-2, are as follows [Sparrow and Cess, 1967].

Outer cylinder to inner cylinder:

$$
F_{A_{1}-A_{2}}-\frac{1}{X}-\frac{1}{\pi X}\left\{\begin{aligned}
\cos ^{-1} & \frac{B}{A}-\frac{1}{2 Y}\left[\sqrt{(A+2)^{2}-(2 X)^{2}} \cos ^{-1} \frac{B}{X A}\right. \\
+ & \left.\left.B \sin ^{-1} \frac{1}{X} \frac{\pi A}{2}\right]\right\}
\end{aligned}\right.
$$

Outer cylinder onto itself

$$
\begin{aligned}
F_{A_{1}-A_{1}}-1 & -\frac{1}{X}+\frac{2}{\pi X} \tan ^{-1}\left(2 \frac{\sqrt{X^{2}-1}}{Y}\right] \\
& -\frac{Y}{2} \pi X\left\{\frac{\sqrt{4 X^{2}+Y^{2}}}{Y} \sin ^{-1}\left[\frac{4\left(X^{2}-1\right)+\frac{Y^{2}}{X^{2}}\left(X^{2}-2\right)}{Y^{2}+4\left(X^{2}-1\right)}\right]\right. \\
& \left.-\sin ^{-1}\left(\frac{X^{2}-2}{X^{2}}\right)+\frac{\pi}{2}\left[\frac{\sqrt{4 X^{2}+Y^{2}}}{Y}-1\right)\right\}
\end{aligned}
$$

where 


$$
\begin{gathered}
\mathrm{X}-\frac{\mathrm{r}_{2}}{\mathrm{r}_{1}} ; \mathrm{Y}-\frac{\mathrm{h}}{\mathrm{r}_{1}} \\
\mathrm{~A}-\mathrm{Y}^{2}+\mathrm{X}^{2}-1 \text { and } \mathrm{B}-\mathrm{Y}^{2}-\mathrm{X}^{2}+1
\end{gathered}
$$

From the above equations with Equation (B-2) follows

$$
F_{A_{1}}-A_{3}-1 / 2\left(1-F_{A_{1}}-A_{1}-F_{A_{1}}-A_{2}\right)
$$

With these relationships, one can employ Equations (B-1) to (B-4) to develop, in straightforward algebra, all view factors between elements of these surfaces. The annular cylinder subroutines permit any nodalization of either side surface and do not require equal node spacing on the inside and outside surfaces. The routines also include limiting expressions for the trigonometric functions at small values or values close to multiples of $\pi$. 


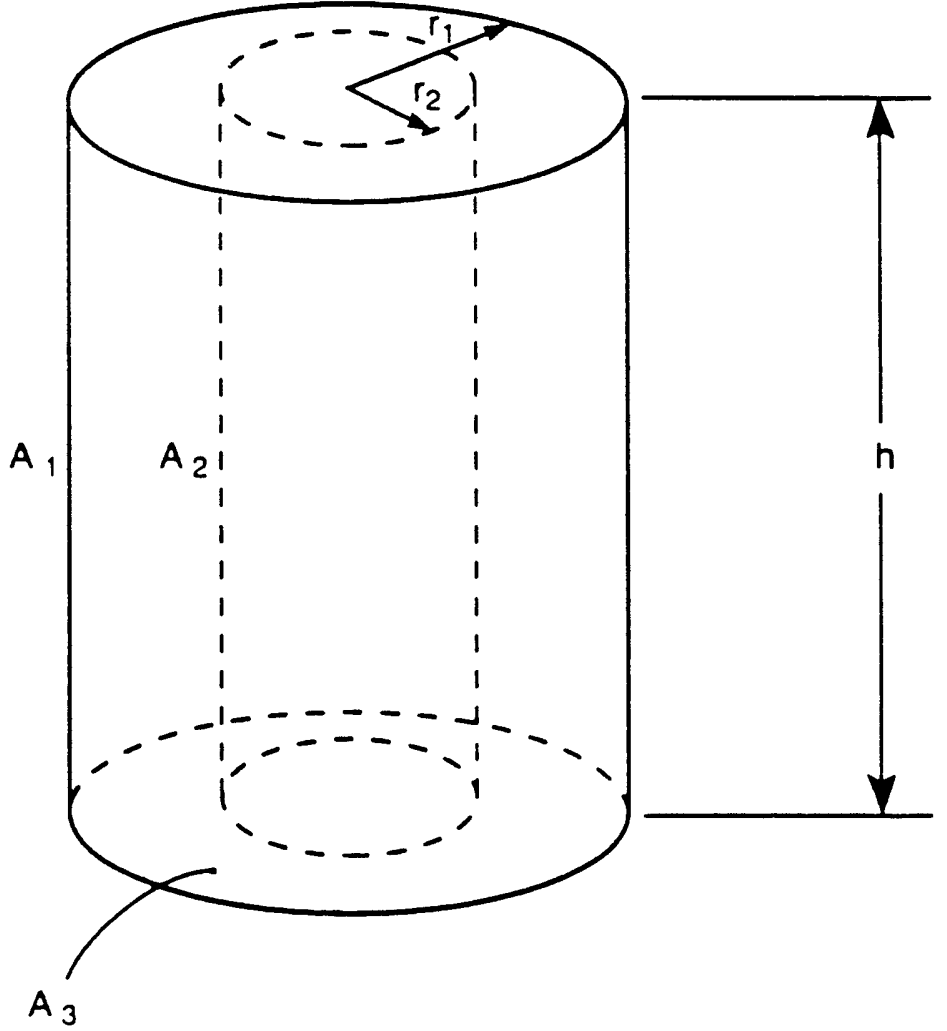

Figure B-2. Schematic of Annular Cylindrical Cavity 


\section{APPENDIX C}

\section{INTERNAL EVALUATION OF HEAT TRANSFER IN FINNED RCCS UPFLOW PANELS}

In the internally finned uptlow channels of the air-cooled RCCS heat transferred from the reactor cavity flows from the front panel of the RCCS by conduction and radiation to the fins and to the rear panel of the RCCS. All sides then transfer heat by convection to the upflowing coolant.

The PASCOL module permits the user to model the effect of the fins at each axial node either by prescribing a fin effectiveness. as outlined in Section 3.2, or by solving internally for simultaneous conduction and radiation in a nodalized fin structure, as shown schematically in Figure C-1.

To evaluate conduction and radiation at each axial node, the geometry of the coolant passage is considered to be rectangular, and radiation among the panel and fin nodes at each axial elevation is assumed to be in the radial and azimuthal direction only, i.e., neglecting any axial radiation. For fin spacing of about $10 \mathrm{~cm}$ and $15 \mathrm{~cm}$ fin lengths, at an RCCs panel radius of about $3.4 \mathrm{~m}$, the curvature effects are indeed small. The effect of axial radiation was found to be very small in the reactor cavity (Kroeger, 1989) and would be even less at the temperature levels within the RCCS channels.

Figure C-2 is a scheme of a nodalized cell of the upflow channel. It consists of one half of each of two opposite fins and the corresponding sections of the front and back panels. Since the THATCH code assumes no variations of temperature in azimuthal direction, the two opposite fin halves have identical temperature distributions, and the solution can be restricted to computing the heat flows between nodes 1 to $i_{\text {op. }}$. However, to compute the radiative heat flow it proved to be logically simpler to sum over all cell nodes.

A quasi-steady energy balance for node $\mathrm{i}$ can be expressed as

$$
Q_{\text {cond in }}-Q_{\text {rad }}+Q_{\text {conv }}+Q_{\text {cond ex }} \text {, }
$$

i.e., the conductive heat intlow equals the radiative plus convective plus conductive heat outtlow. For node 1 the equation is essentially the same, except that $Q_{\text {cond in }}$ is replaced by the heat flow from the reactor vessel

$$
Q_{\text {ves }}-A_{\mathrm{HT} \text { ex }} U_{\text {cav }}\left(\theta_{\text {ves }}-\theta_{1}\right)
$$

where $A_{H T}$ is the exterior heat transfer surface of the RCCS panel, $U_{\text {cav }}$ the overall reactor cavity heat transfer coefticient, $\theta_{\text {ves }}$ the outer vessel node temperature, and $\theta_{1}$ the RCCS front panel temperature (i.e., the temperature of node 1 ).

The various terms of equation $\mathrm{C}-1$ can be expressed as follows:

For the conductive heat flow between nodes $i$ and $i+1$ 


\section{Thermal Insulation}

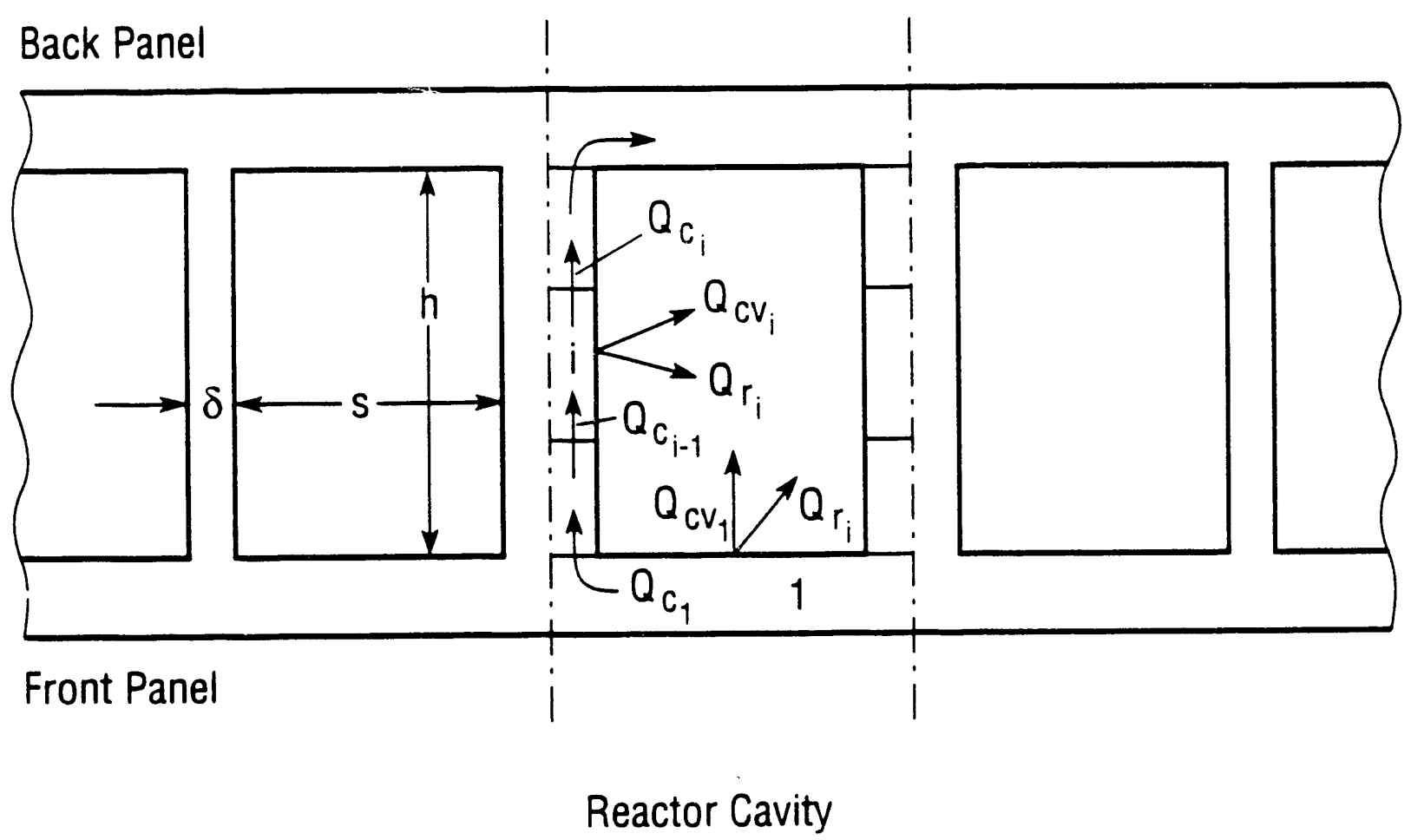

Figure C-1: Schematic of Nodalized Internal Fin Structure of Air-Cooled RCCS

C-2 


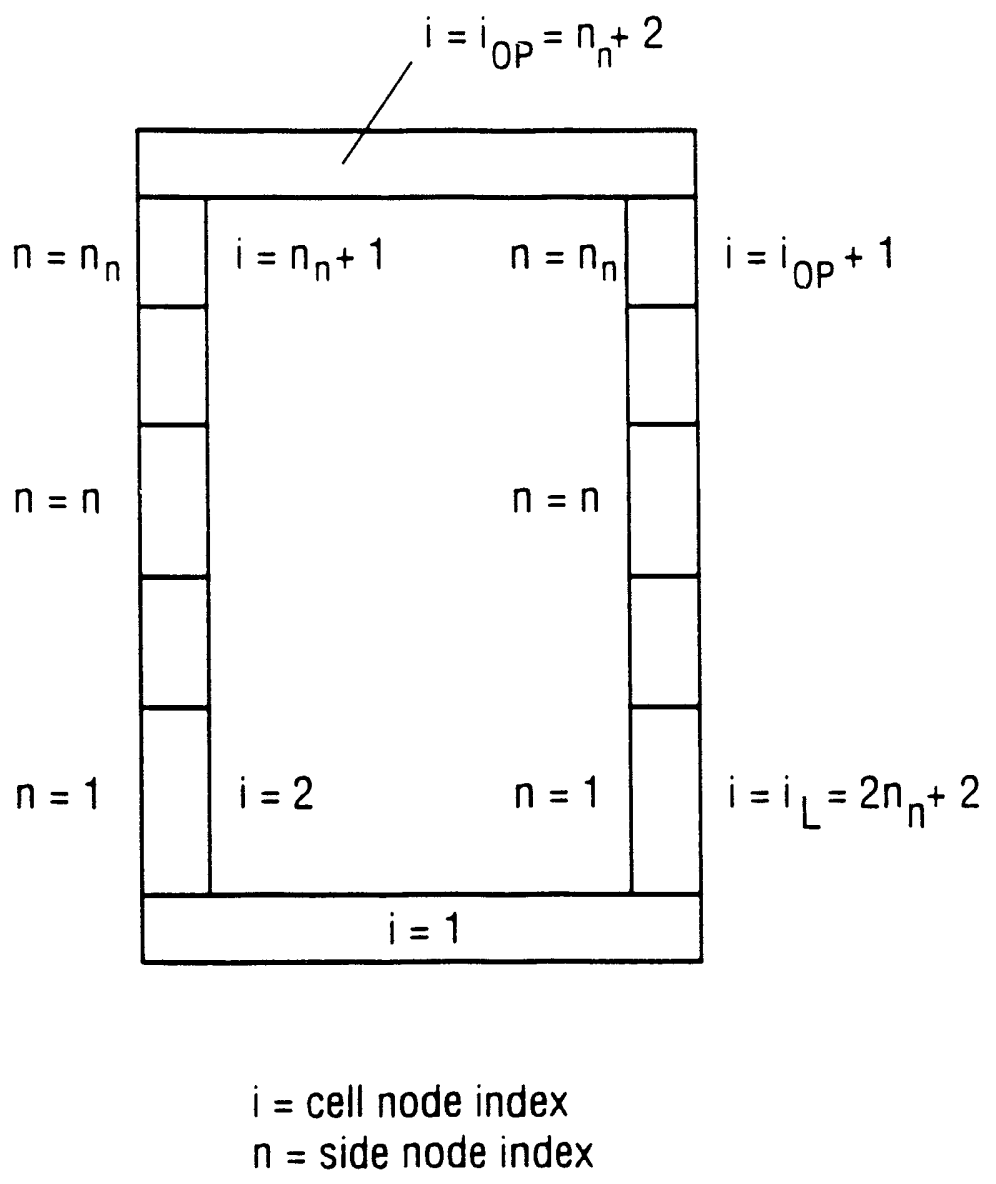

C-2: Schematic of Node Indices for RCCS Uptlow Channel

C-3 


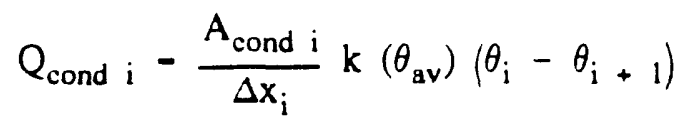

where $\mathrm{A}_{\text {cond } \mathrm{i}}$ is the conductive heat flow cross section area, $\Delta \mathrm{x}_{\mathrm{i}}$ is the distance between the centers of nodes $i$ and $i+1, k\left(\theta_{a v}\right)$ is the thermal conductivity at the average temperature between the two nodes, and $\theta$ is the node temperatures. Note that $A_{\text {cond } i}$ is generally based on one half of the fin thickness, corresponding to the heat flows between side nodes. However, the $Q_{\text {cond ex }}$ term for node 1 and the $Q_{\text {cond in }}$ term for node $i_{\text {op }}$ must be multiplied by two, since two side nodes of one half the fin thickness are connected to each of these two nodes.

The radiative heat flow leaving node $\mathrm{i}$ is

$$
Q_{\text {rad } i}-A_{H T} \sigma \sum_{j-1}^{i_{L}} \lambda_{i, j} \theta_{j}^{4}
$$

where the radiation coefficients $\lambda_{i, j}$ are computed as described in Appendix A. based on the view factors given below. $A_{H T}$ is the node convection and radiation heat transfer area and $\sigma$ the Stefan - Boltzmann constant.

For the convective heat flow leaving node $\mathrm{i}$ we have

$$
Q_{\text {conv } i}-A_{H T} h\left(\theta_{i}-\theta_{n}\right)
$$

where $h$ is the convective heat transfer coefficient (evaluated from Equation 3.2-8), and $\theta_{\mathrm{f}}$ is the coolant temperature.

With Equations (C-2) to (C-5) substituted into (C-1), equation (C-1) represents a set of $\mathrm{i}_{\text {op }}$ equations in the $\mathrm{i}_{\mathrm{op}}$ unknown solid-node temperatures. The PASCOL module solves this set of equations using a Newton-Raphson procedure. For convenience, the equations were scaled by the node heat transfer area $A_{\mathrm{HT}}$. Since the radiation heat exchange couples all node temperatures in each equation, the Jacobian matrix is dense, and the full system of equations must be solved at each iteration.

The radiation view factors between the nodes of a flow channel at a given axial elevation can readily be obtained from Hottel's String Method (Sparrow and Cess, 1966) which actually applies only to long channels, but can be used here since axial radiation is neglected.

Following Figure C-3, the view factors between any two surfaces elongated in the z-direction can be obtained as

$$
F_{1,2}-\frac{1}{251}\left[l_{11-22}+l_{12-21}-l_{11-21}-l_{12-22}\right]
$$

where $s_{1}$ is the length of $A_{1}$ in the $x-y$ plane, and $\ell_{i, j}$ is the length of a straight line between points $i$ and j. 


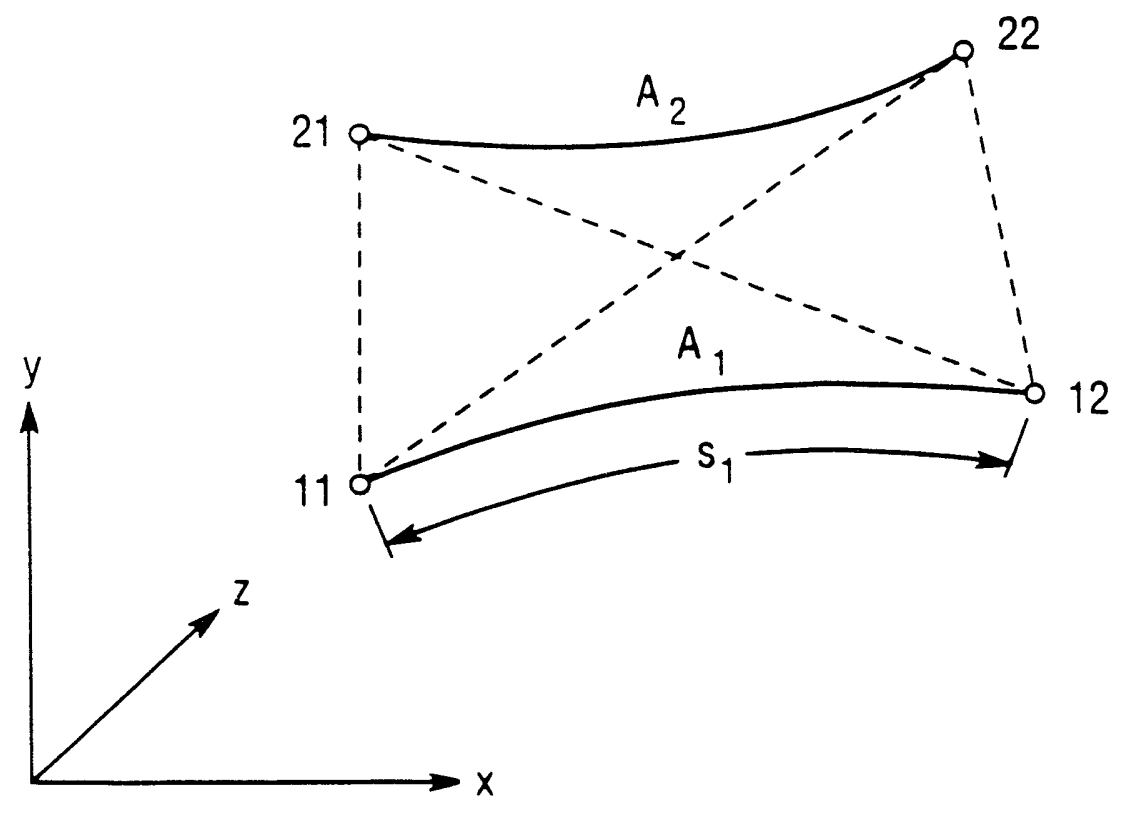

Figure C-3: Schematic for View Factors

Between Surfaces Parallel in the

Z-Direction

C-5 
In a rectangular geometry none of the nodes has a view factor with respect to itself, and the view factors between all nodes are readily computed from specified node end-point coordinates in the $x-y$ plane substituting into equation $\mathrm{C}-6$ the expressions

$$
s_{i}-\sqrt{\left(x_{i 1}-x_{i 2}\right)^{2}+\left(y_{i 1}-y_{i 2}\right)^{2}}
$$

and

$$
\ell_{m-n}-\sqrt{\left(x_{m}-x_{n}\right)^{2}+\left(y_{m}-y_{n}\right)^{2}}
$$




\section{APPENDIX D}

\section{PROPERTIES OF GASES AND GAS MIXTURES}

The THATCH code can handle mixtures of eight component gases.

The gases and their indices in all gas property arrays are

$\begin{array}{cll}\frac{\text { Index }}{1} & & \frac{\text { Gas }}{\text { helium }} \\ 2 & & \text { air } \\ 3 & & \mathrm{CO} \\ 4 & & \mathrm{CO}_{2} \\ 5 & \mathrm{H}_{2} \mathrm{O} \\ 6 & \mathrm{H}_{2} \\ 7 & \mathrm{O}_{2} \\ 8 & \mathrm{~N}_{2}\end{array}$

The properties computed are the four thermal properties, specific heat at constant volume and pressure, internal energy and enthalpy as well as the thermodynamic properties of dynamic viscosity, and for some gases, thermal conductivity and the Prandtl number.

Densities for all gases are computed from the ideal gas state equation

$$
\mathrm{pV}-\mathrm{N} \Re \theta
$$

or

$$
\mathrm{pv}-\mathrm{R} \theta
$$

where $\mathrm{p}$ is the pressure, $\mathrm{V}$ the absolute volume, $\mathrm{N}$ the number of kilomoles, $\Re$ the universal gas constant, $\theta$ the gas temperature, $v$ the specific volume, and $R=\Re / M$ the gas specific gas constant, with

$M$ being the mole weight of the gas species or of a gas mixture.

The gas data to be used are coded in the GASPRLIB section of BIG.

It should be noted that the data presented here for $\mathrm{H}_{2} \mathrm{O}$ are those of an ideal gas, i.e., they apply for states sufficiently far from the saturation line, which means at sufficiently low pressure and high temperature.

\section{D.1 Thermal Gas Properties}

The evaluation of thermal gas properties is based on data for molar internal energy and energy of formation given in tabular form by Owczarek [1964] for all gases of interest, except helium. For helium, ideal gas values can readily be used at all pressures and temperatures of interest, and the corresponding expressions are given below. The given data extends from $300 \mathrm{~K}$ to $4250 \mathrm{~K}$. For 
convenience (to avoid "out of range" error messages), a data point at $273 \mathrm{~K}$ was added, by a linear extrapolation; it was not a measured data point.

From the internal energies, $E$, the enthalpies, $H$, and specific heats, $C_{v}$ and $C_{p}$, are readily computed as

$$
\begin{gathered}
\mathrm{H}-\mathrm{E}+\Re \theta \quad\left[\frac{\mathrm{J}}{\mathrm{kmol}}\right] \\
C_{v}-\frac{\Delta E}{\Delta \theta}\left[\frac{\mathrm{J}}{\mathrm{kmol} \mathrm{K}}\right] \\
C_{p}-C_{v}+\Re\left[\frac{\mathrm{J}}{\mathrm{kmol} \mathrm{K}}\right]
\end{gathered}
$$

where $\Re$ is the universal gas constant and $\Delta E$ and $\Delta \theta$ are the differences in internal energy and temperature. For helium we used

$$
\begin{aligned}
& C_{v}-\frac{3}{2} \Re \quad\left[\frac{\mathrm{J}}{\mathrm{kmol} \mathrm{K}}\right] \\
& C_{p}-\frac{5}{2} \Re \quad\left[\frac{\mathrm{J}}{\mathrm{kmol} \mathrm{K}}\right]
\end{aligned}
$$

and

$$
\begin{aligned}
& E-C_{v} \theta \quad\left[\frac{J}{\mathrm{kmol}}\right] \\
& H-C_{p} \theta \quad\left[\frac{J}{\mathrm{kmol}}\right]
\end{aligned}
$$
data as

As all properties are in molar units, the properties for gas mixtures are obtained from the table

$$
y_{m}-\sum \nu_{l} y_{l}
$$

where $y$ stands for either of the four above properties $\left(E, H, C_{p}\right.$, or $\left.C_{v}\right) . \nu_{\ell}$ is the volume fraction of gas species $\ell$, and subscripts $\mathrm{m}$ and $\ell$ signify "mixture" or "gas species $\ell$ ", respectively.

The actual computations and data tabulations are carried out in the GASPRLIB portion of BIG. The individual routines are described in Section 4 of this report. We note that the gas properties for gas 
mixtures or for a single gas can be either obtained by linear interpolation in the internal energy tables, or by using a spline fit which was computed from the table data assuming a zero second derivative at both end points of the table. The spline fit is generally preferable since linear interpolation of the specific heats from Equation (D-4) leads to discontinuities at the mesh points of the table data, which could cause convergence problems in some instances.

\section{D.2 Gas Viscosities}

The code uses gas viscosity data for the tirst six gases. For the helium viscosity, the following correlation is employed

$$
\mu-C\left(\frac{\theta}{\theta_{0}}\right)^{n}\left[\frac{N s}{m^{2}}\right]
$$

where $\theta$ is the gas temperature in $\mathrm{K}$, and $\theta_{0}$ a reterence temperature. Currently

$$
n=0.674
$$

and

$$
\begin{aligned}
& \theta_{0}=589 \mathrm{~K} \\
& C-3.1137 \times 10^{-5}\left[\frac{\mathrm{Ns}}{\mathrm{m}^{2}}\right]
\end{aligned}
$$

and for coding convenience

$$
\frac{C}{\theta_{0}^{n}}-.4229 \times 10^{-6}
$$

The viscosities for the other gases are mainly given in Table form and are generally computed by linear interpolation. The data are summarized in Tables D-1 to D-4. The actual code data may have more digits than the table entries, but these digits were essentially "generated" by conversion of original data in the literature to SI units using automated procedures to produce these data arrays.

The data source for gas viscosities [Vargattik, 1975] indicated that CO viscosities are essentially equal to those of $\mathrm{CO}_{2}$; therefore, the latter data were used.

For water vapor at temperatures up to $1200 \mathrm{~K}$ the following ASME stream table correlation is used, with table data form table D-3 being used above $1200 \mathrm{~K}$.

$$
\mu-(80.4+0.407 \times \theta[C]) \times 10^{-7}\left[\frac{N_{s}}{m^{2}}\right]
$$


Table D-1

Thermodynamic Properties of Air

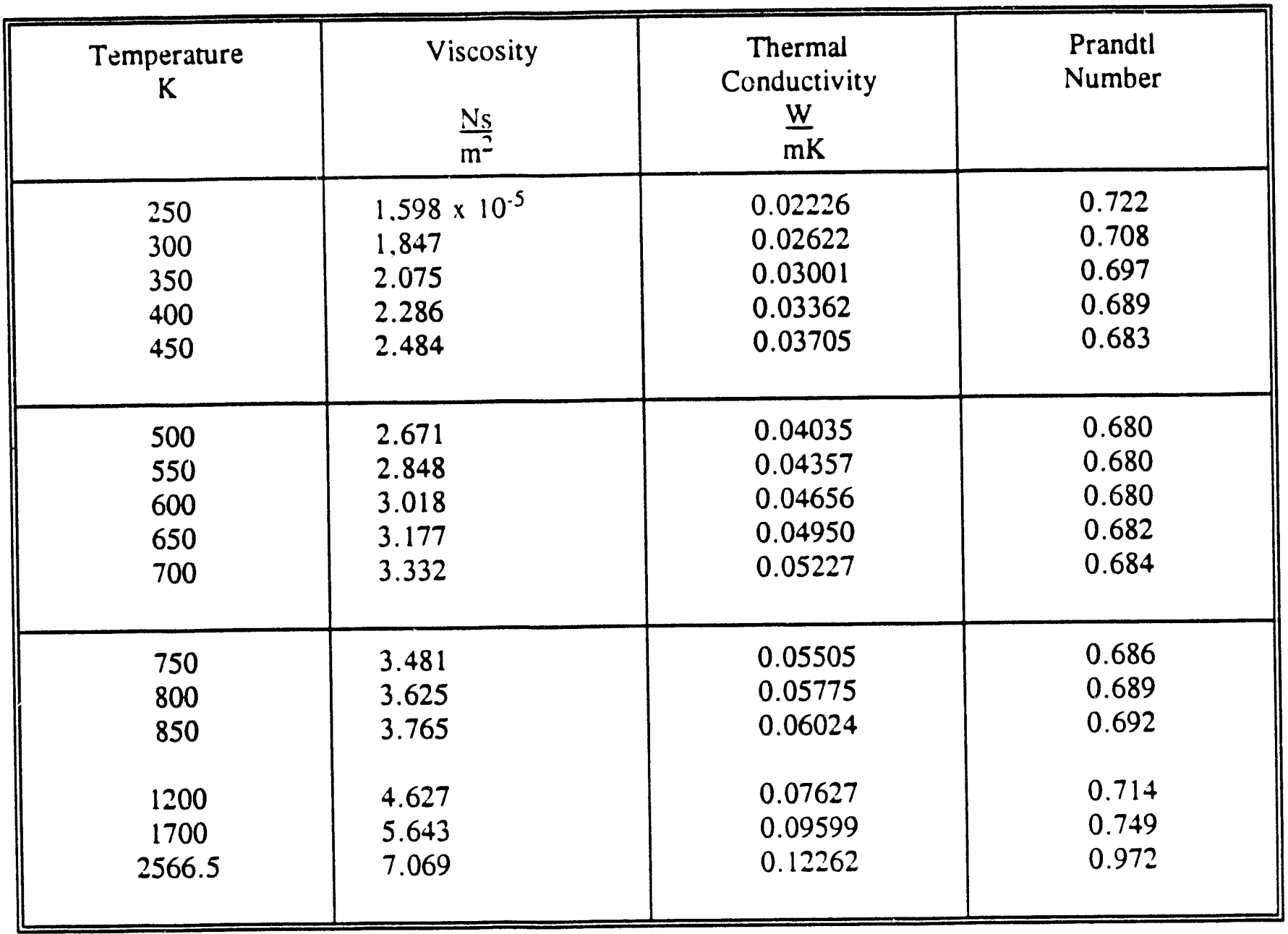


Table D-2

Dynamic Viscosities of $\mathrm{CO}_{2}$

\begin{tabular}{|c|c|}
\hline $\begin{array}{c}\text { Temperature } \\
\mathrm{K}\end{array}$ & Viscosity \\
& $\frac{\mathrm{Ns}}{\mathrm{m}^{2}}$ \\
\hline 300 & $15.1 \times 10^{-6}$ \\
500 & 23.7 \\
700 & 30.7 \\
& \\
\hline 900 & 36.8 \\
1100 & 42.1 \\
1300 & 47.0 \\
& \\
\hline 1500 & 51.5 \\
1700 & 55.6 \\
1900 & 59.5 \\
\hline 2100 & \\
\hline 2300 & 63.2 \\
2500 & 66.6 \\
\hline
\end{tabular}

D-5 
Table D-3

Dynamic Viscosities of $\mathrm{H}_{2} \mathrm{O}^{*}$

\begin{tabular}{||c|c|}
\hline & Viscosity \\
Kemperature & $\frac{\mathrm{Ns}}{\mathrm{m}^{2}}$ \\
& $45.76 \times 10^{-6}$ \\
\hline 1200 & 51.2 \\
1400 & 56.3 \\
1600 & \\
\hline 1800 & 61.2 \\
2000 & 65.9 \\
2200 & 70.3 \\
& \\
\hline 2400 & 74.2 \\
2600 & 77.5 \\
2800 & 79.8 \\
\hline 3000 & \\
\hline 3200 & 81.0 \\
3400 & 81.4 \\
3600 & 81.6 \\
\hline
\end{tabular}

* below $1200 \mathrm{~K}$ use equation $\mathrm{D}-12$ 
Table D-4

Dynamic Viscosity of $\mathrm{H}_{2}{ }^{*}$

\begin{tabular}{|c|l|}
\hline \multicolumn{1}{|c|}{\begin{tabular}{c|} 
Temperature \\
$\mathrm{K}$
\end{tabular}} & Viscosity \\
& $\frac{\mathrm{Ns}}{\mathrm{m}^{2}}$ \\
\hline 250 & $8.0 \times 10^{-6}$ \\
500 & 12.64 \\
750 & 16.6 \\
1000 & 20.1 \\
\hline
\end{tabular}

* beyond $1000 \mathrm{~K}$, linear extrapolation is used 
The gas mixture viscosities are computed from the generally accepted formula of Wilke [1950]

$$
\mu_{m}=\sum_{i=1}^{L} \frac{\mu_{i}}{1+\sum_{j=1}^{L} \frac{\nu_{j}}{\nu_{i}} \phi_{i, j}\left(1-\delta_{i, j}\right)}
$$

with

$$
\phi_{i, j}-\frac{\left[1+\left(\left.\frac{\mu_{i}}{\mu_{j}}\right|^{0.5}\left[\frac{\mathrm{M}_{j}}{\mathrm{M}_{i}}\right)^{0.25}\right]^{2}\right.}{\sqrt{8\left(1+\frac{\mathrm{M}_{\mathrm{i}}}{\mathrm{M}_{\mathrm{j}}}\right)}}
$$

where $\mu$ is the viscosity, $\nu$ is the volume fraction, $M$ is the molar mass, $\delta_{i, j}$ is the Kronecker delta, and the subscript $m$ denotes mixture. $L$ is the number of gases.

In current practice, for viscosities of gas mixtures, THATCH uses three gases in the case of air ingress, namely helium, air, and $\mathrm{CO}$ with the $\mathrm{N}_{2}$ content being added to air. For water ingress, six gases are used, adding $\mathrm{CO}_{2}, \mathrm{H}_{2} \mathrm{O}$, and $\mathrm{H}_{2}$ to the above three gases and, again, considering the $\mathrm{N}_{2}$ concentration with the air.

\section{D.3 Gas Thermal Conductivities}

The present code provides thermal conductivities only for helium and air. For air-ingress scenarios, where $\mathrm{CO}$ and $\mathrm{N}_{2}$ occupy most of the core, the resulting error is not expected to be severe since the thermal conductivity of $\mathrm{N}_{2}$ and $\mathrm{CO}$ is close to that of air. In the water-ingress scenarios investigated so far, the concentrations of $\mathrm{H}_{2} \mathrm{O}$ and $\mathrm{H}_{2}$ remained very small and the use of $\mathrm{He}$ conductivities alone was again justified. However, values for $\mathrm{H}_{2} \mathrm{O}, \mathrm{H}_{2}$ and $\mathrm{CO}$ should be added as soon as possible. Also, a better equation for conductivities of gas mixtures should be provided.

The thermal conductivity of helium is computed here from the helium viscosity. Noting that the Prandtl number remains virtually constant, over the temperature range of interest $\operatorname{Pr}=0.72$, and because the specific heat of helium is constant, we have

$$
k-\left(\frac{C_{p}}{M P r}\right) \mu\left[\frac{W}{m K}\right]
$$

where $\mathrm{k}$ is thermal conductivity, $\mathrm{C}_{\mathrm{p}}$ the molar specitic heat, $M$ the mole mass, and $\mu$ the viscosity.

The thermal conductivity of air is prescribed in tabular form, and was included in table D-1.

For helium-air mixtures, a very crude mixture correlation is currently used, namely:

\section{D-8}




$$
k_{m}-\sum_{\ell-1}^{2} \nu_{i} k_{i}
$$

where $\nu_{i}$ represents the volume fractions.

This relationship should be replaced for more detailed future applications. As stated above, for the air ingress cases handled so far (pure air entering the system), the ensuing error should have been small. 
APPENDIX E

TABULAR SUMMARY OF THATCH SUBROUTINES AND COMMON DECKS

E-1 


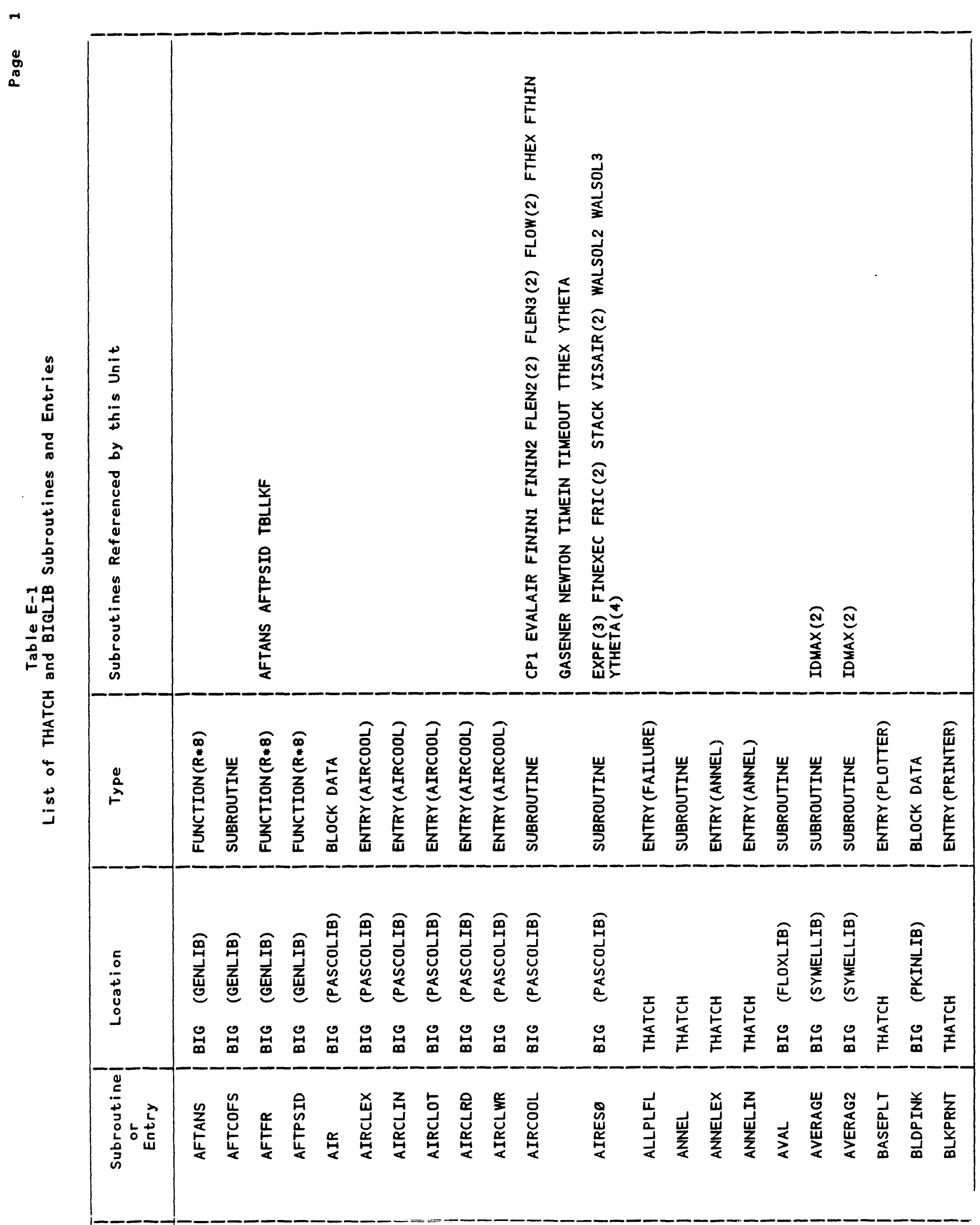




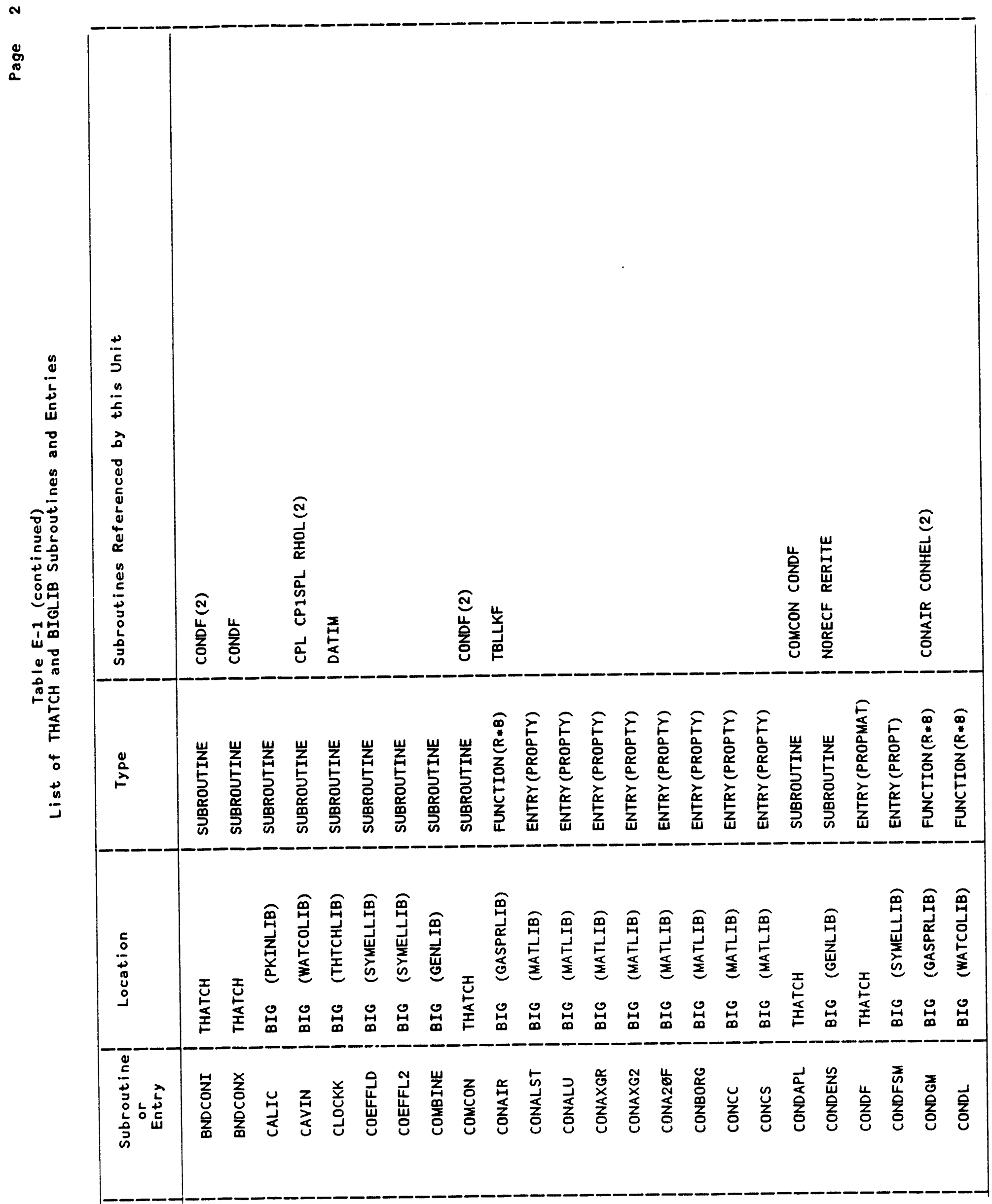




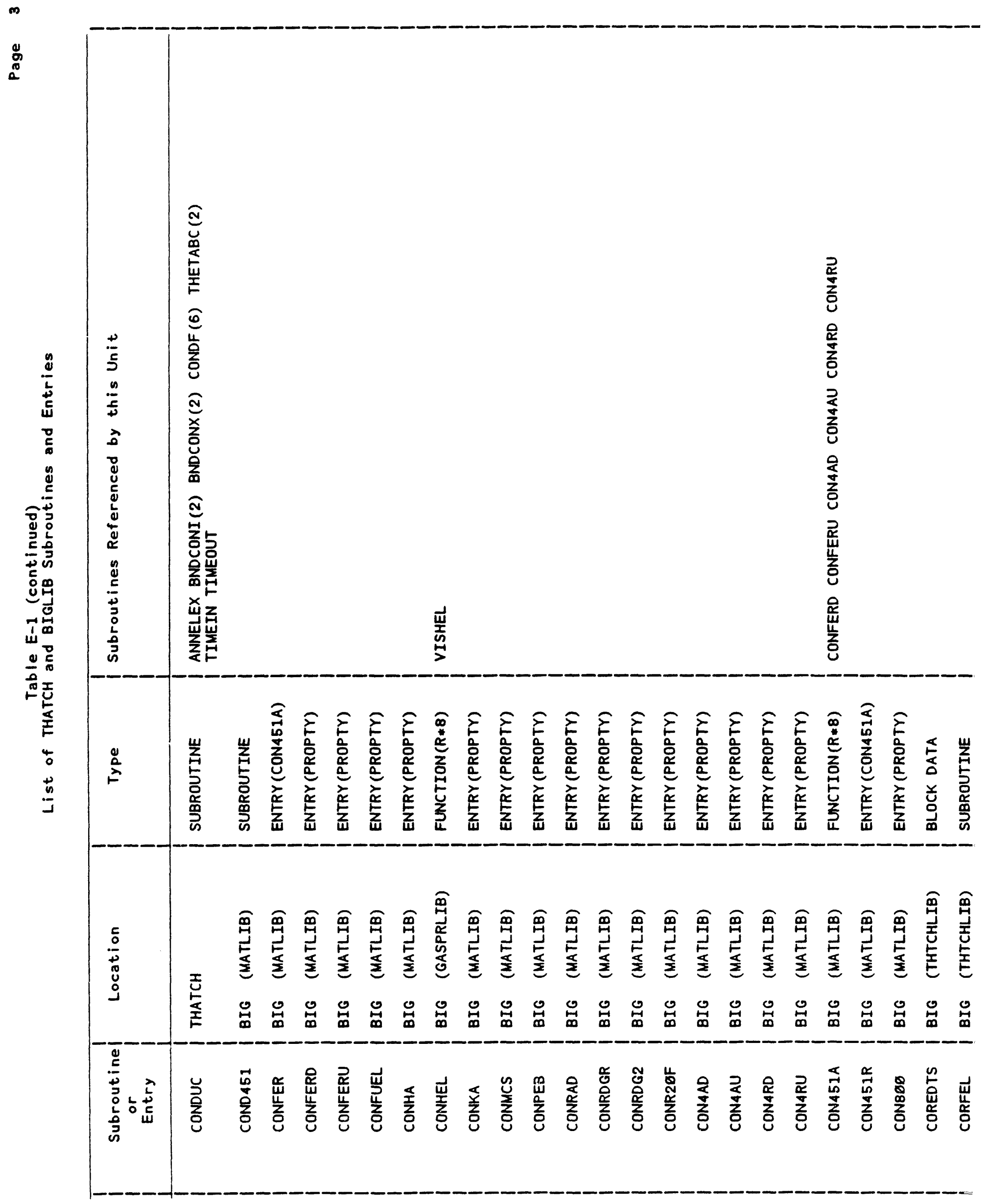




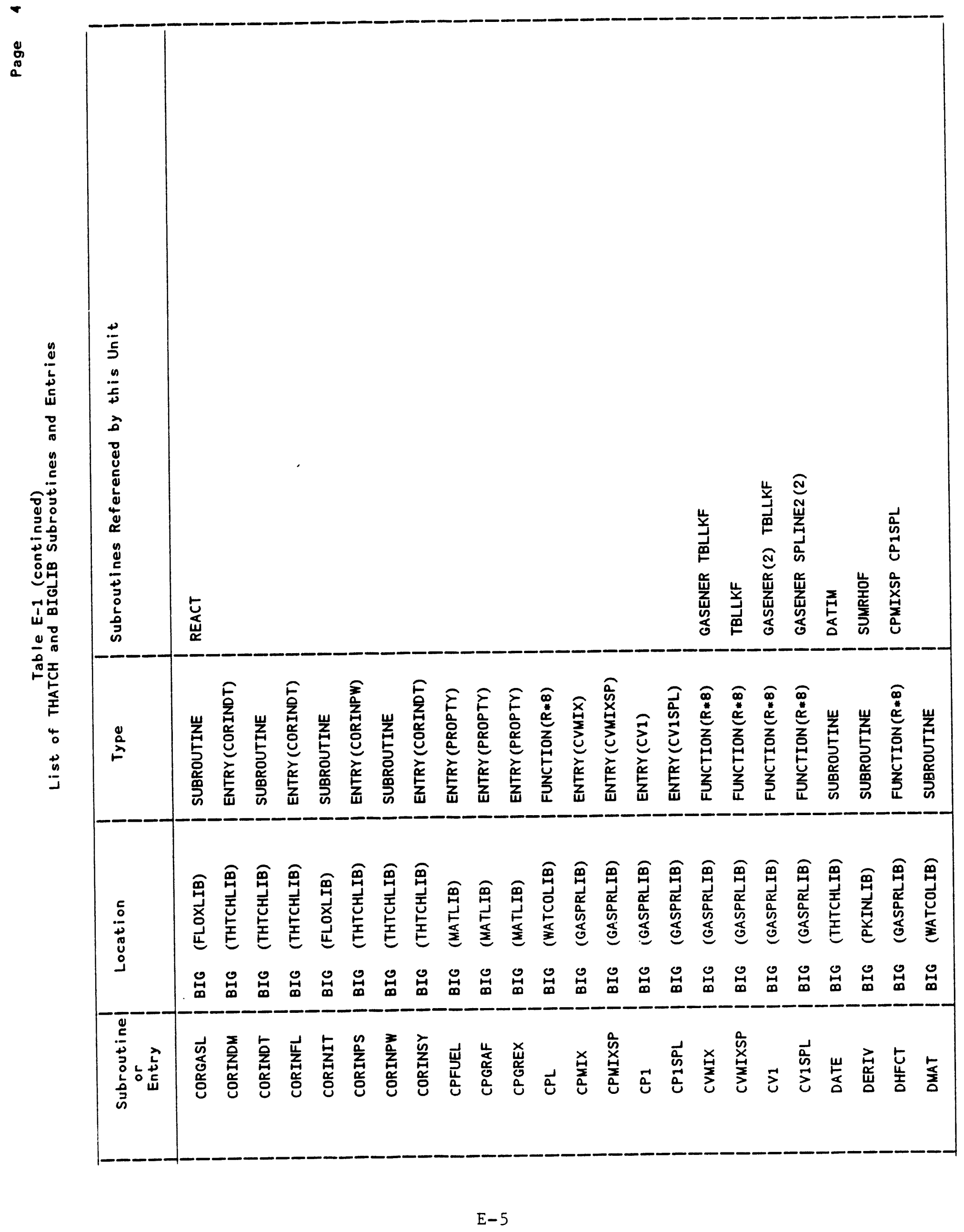


$\omega$

$\underset{0}{0}$

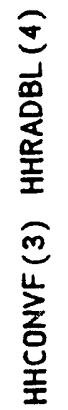

$\stackrel{u}{\stackrel{u}{J}}$

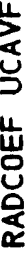

矛

ล્త

志

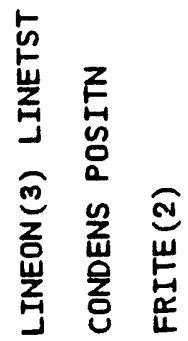

즐

$\widehat{\mathcal{N}}$

总嘉

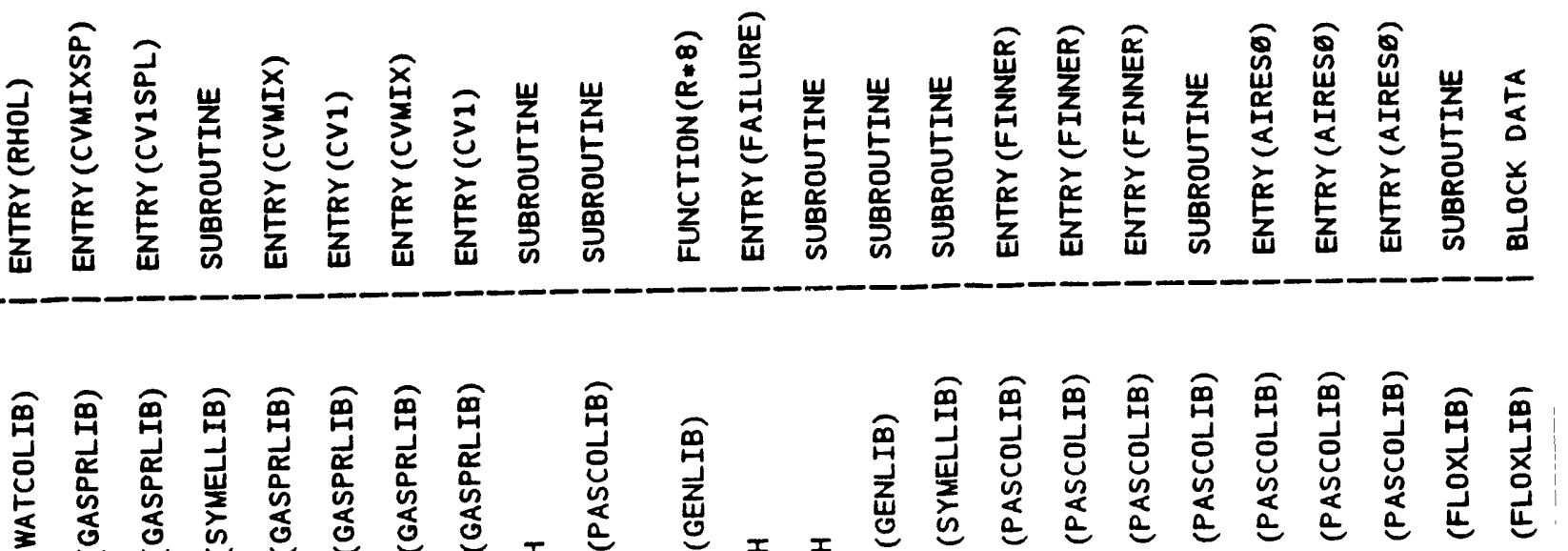

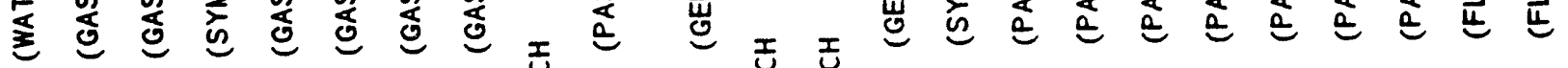

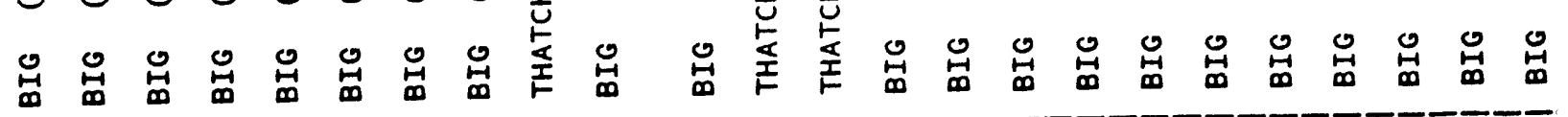

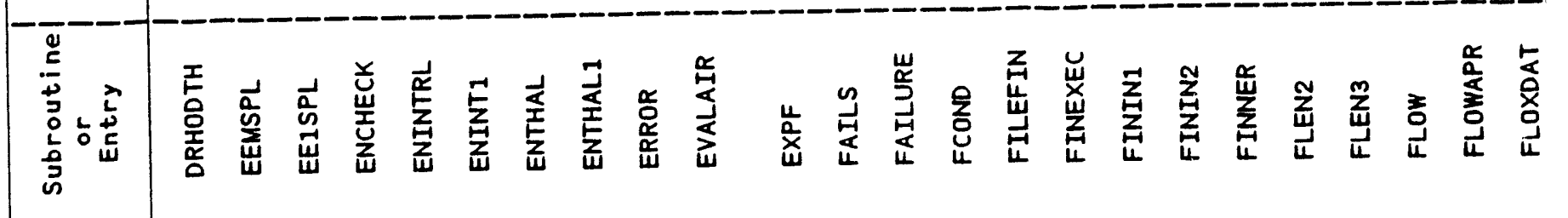


$\vdots$

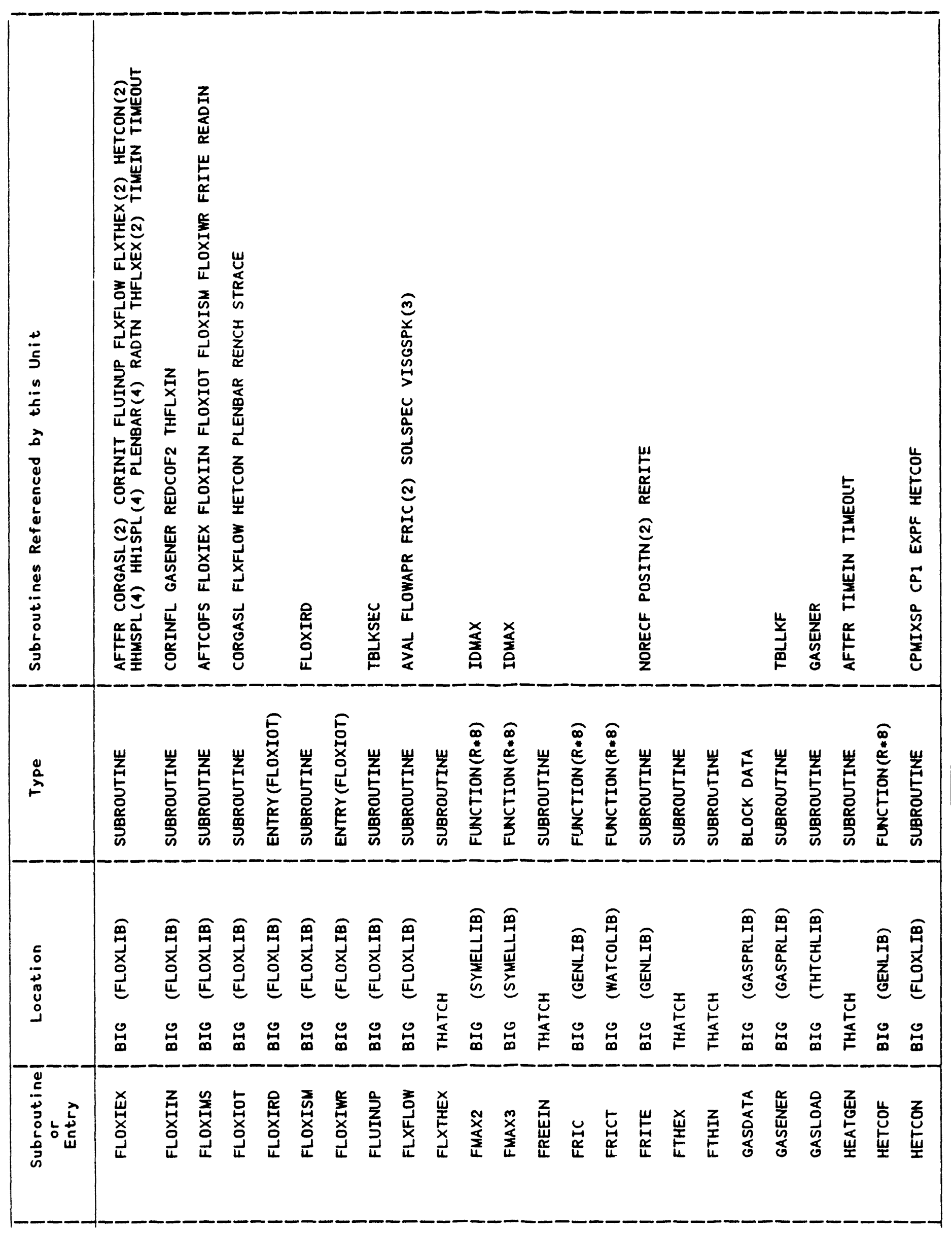




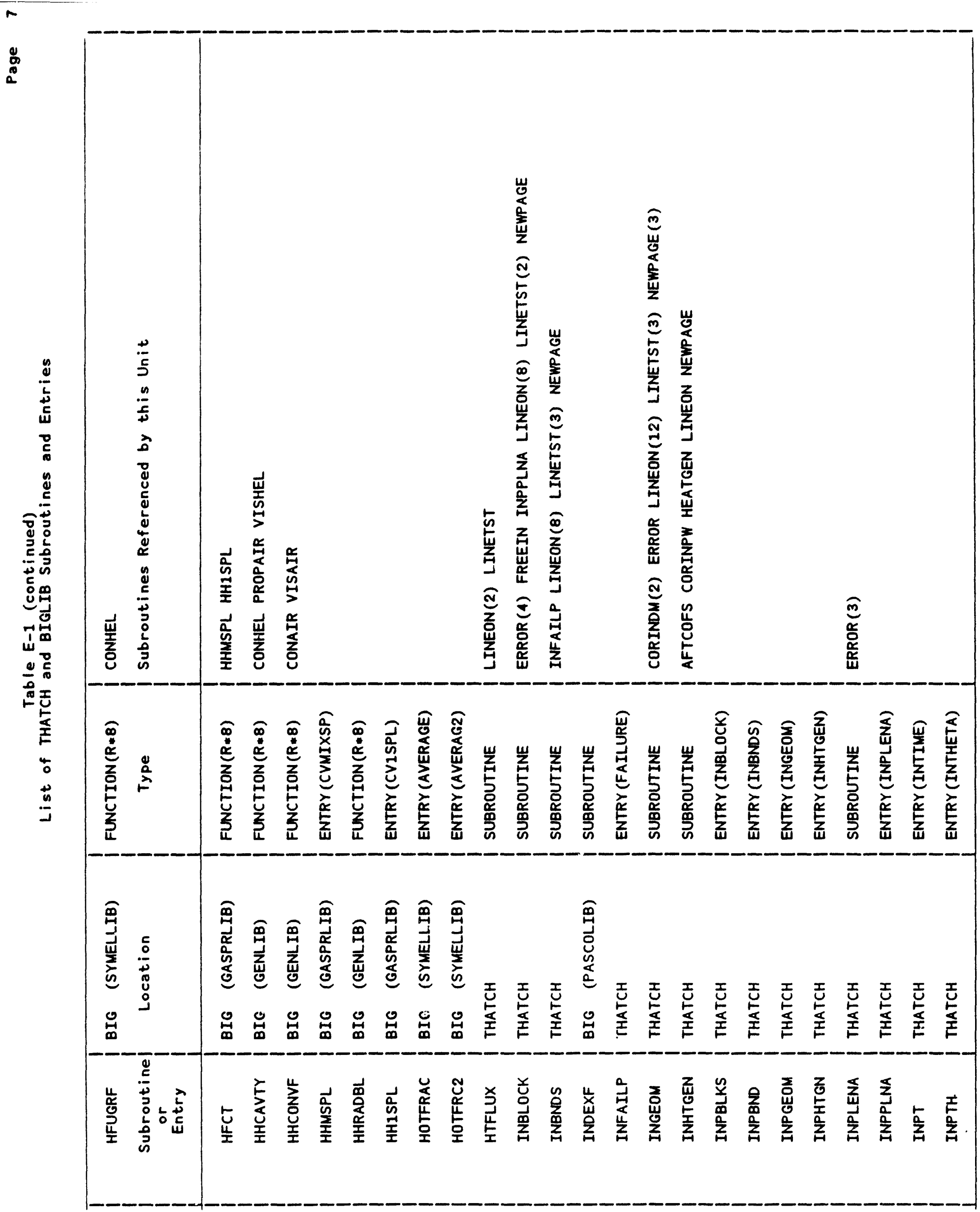




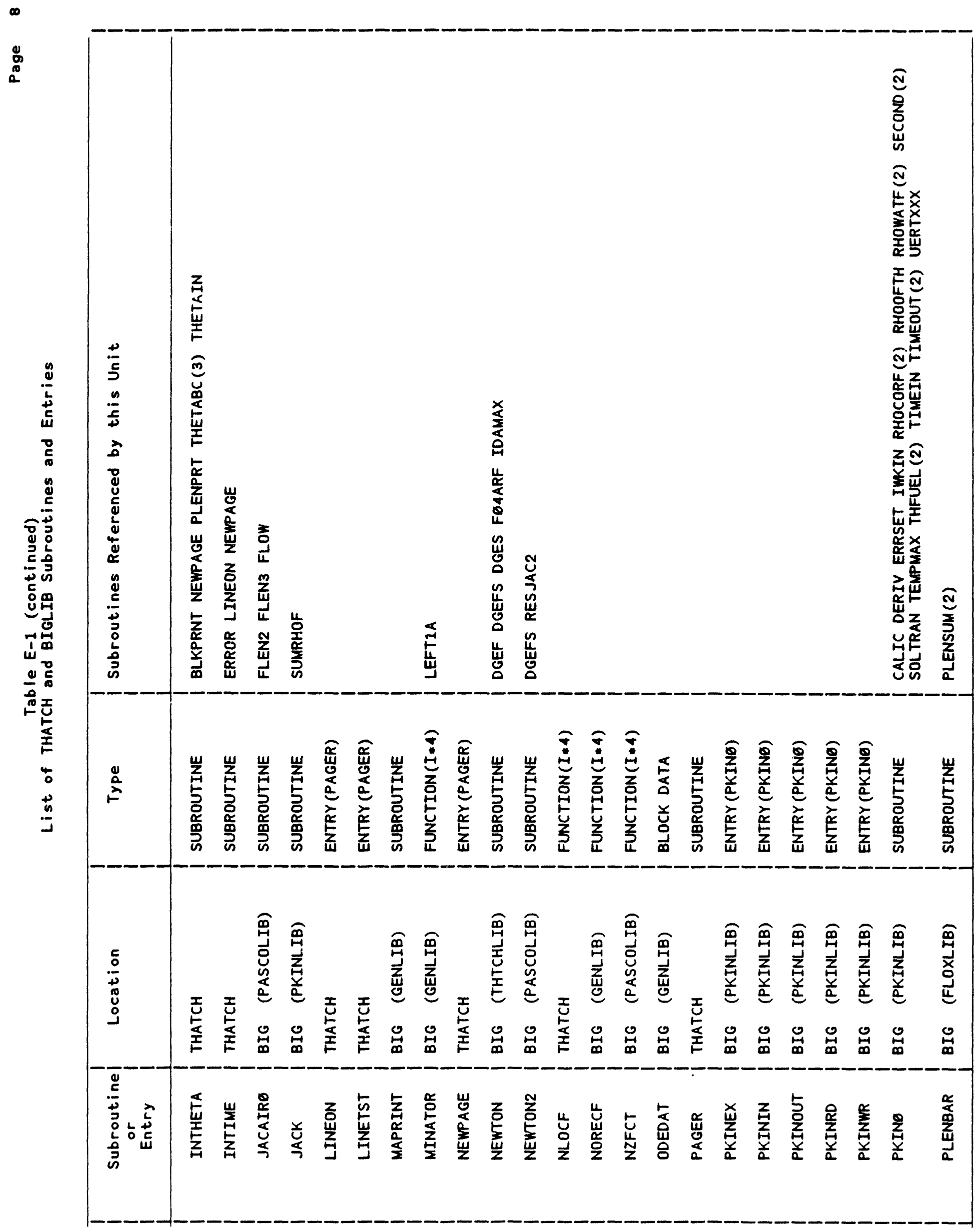




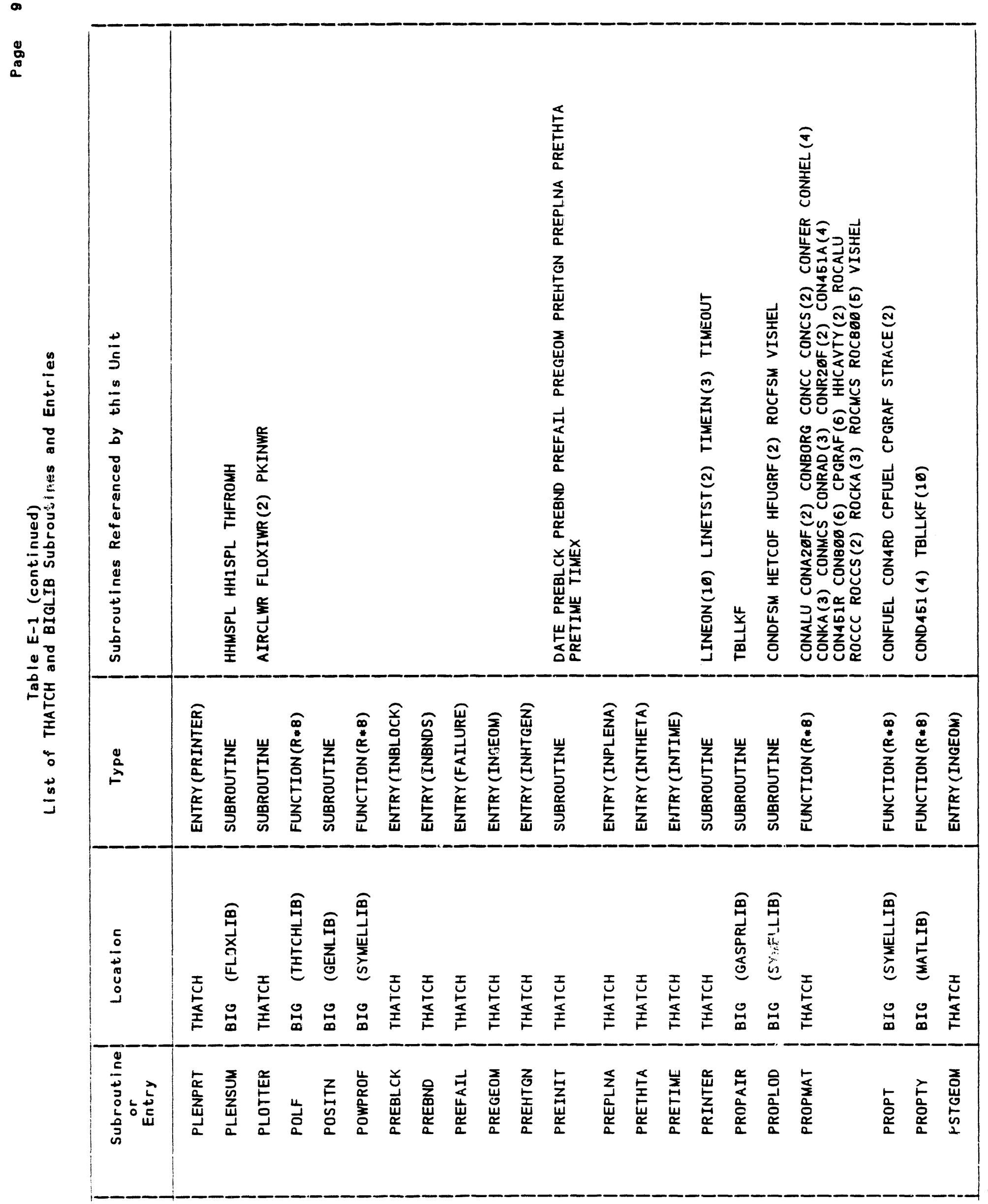




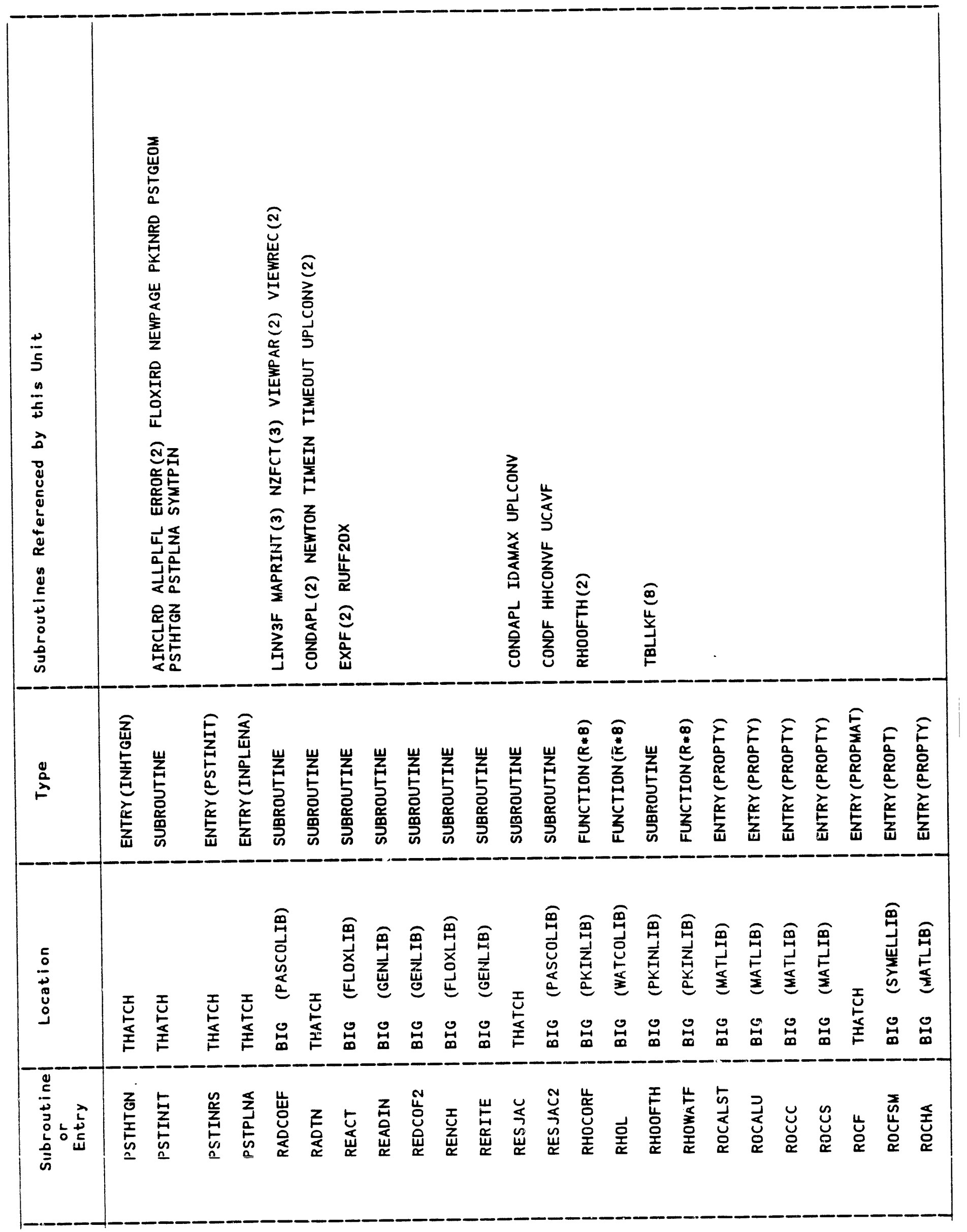




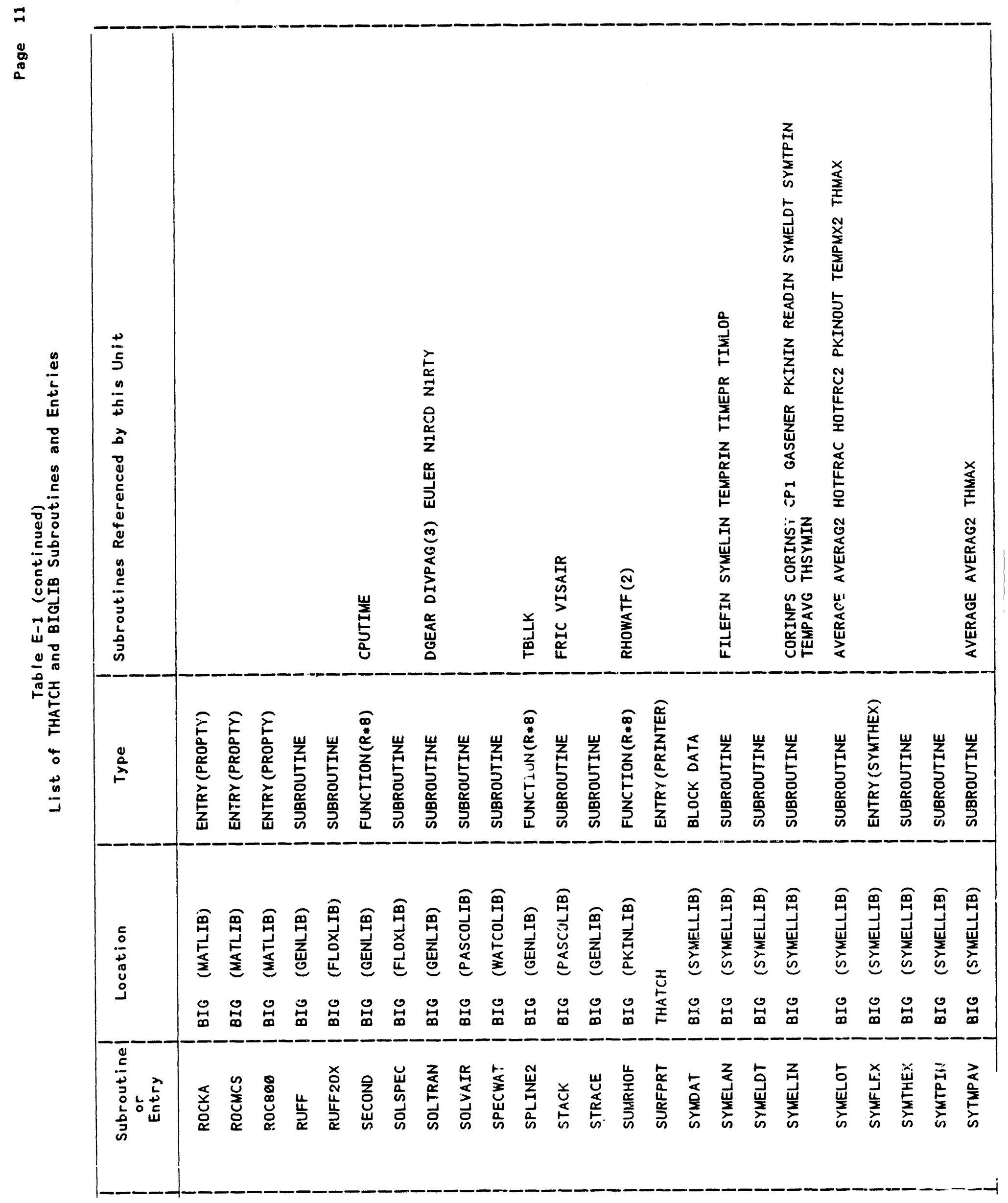




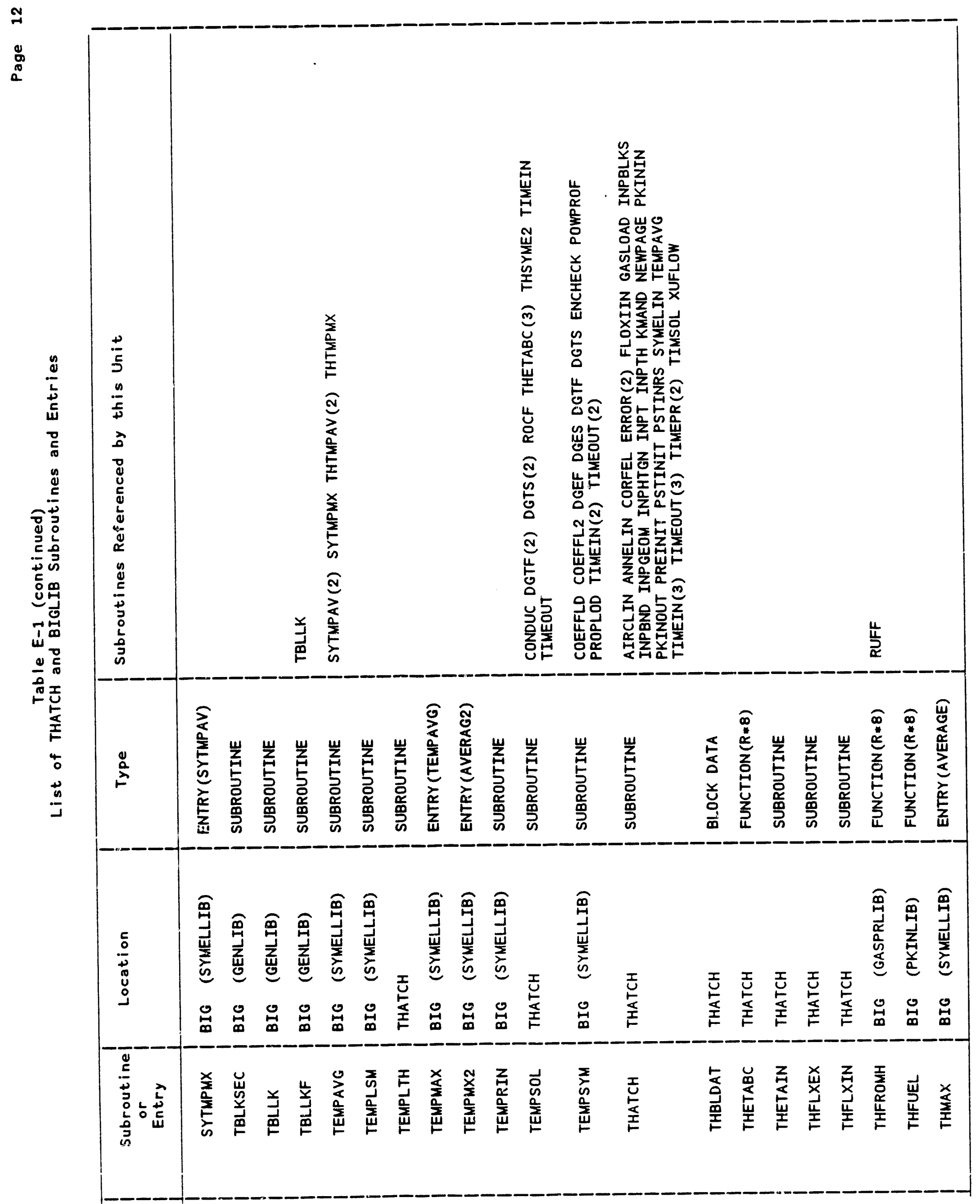




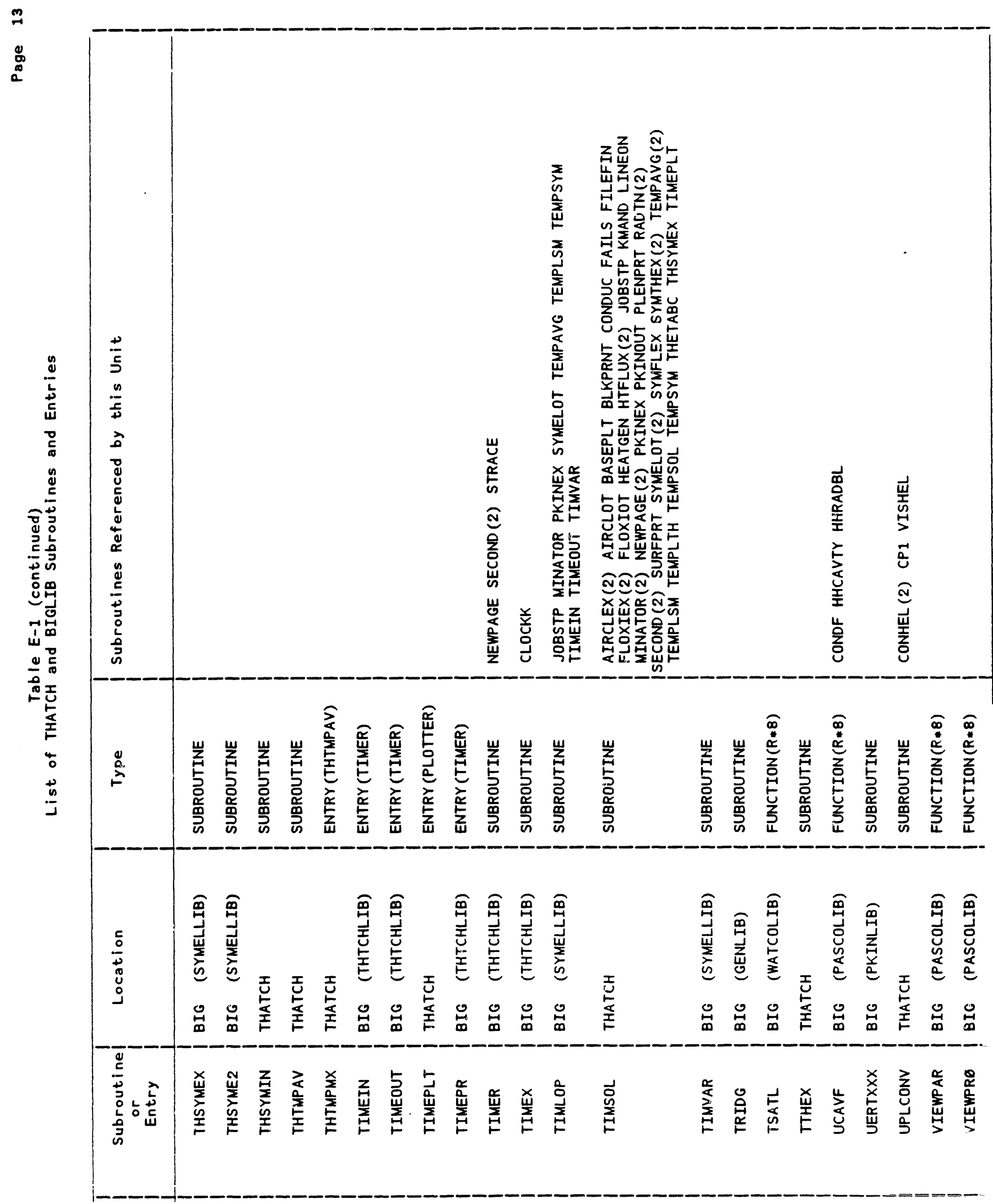




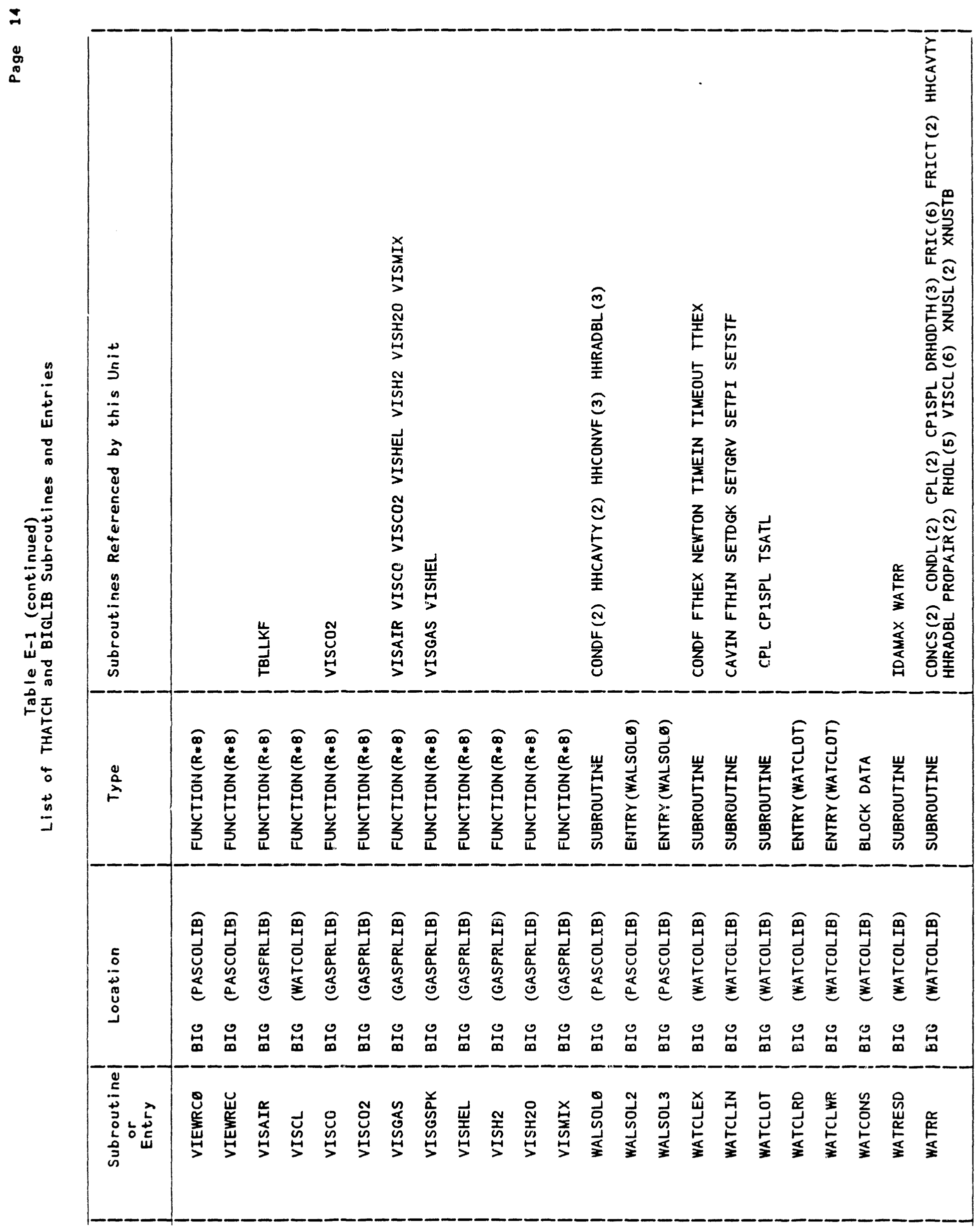




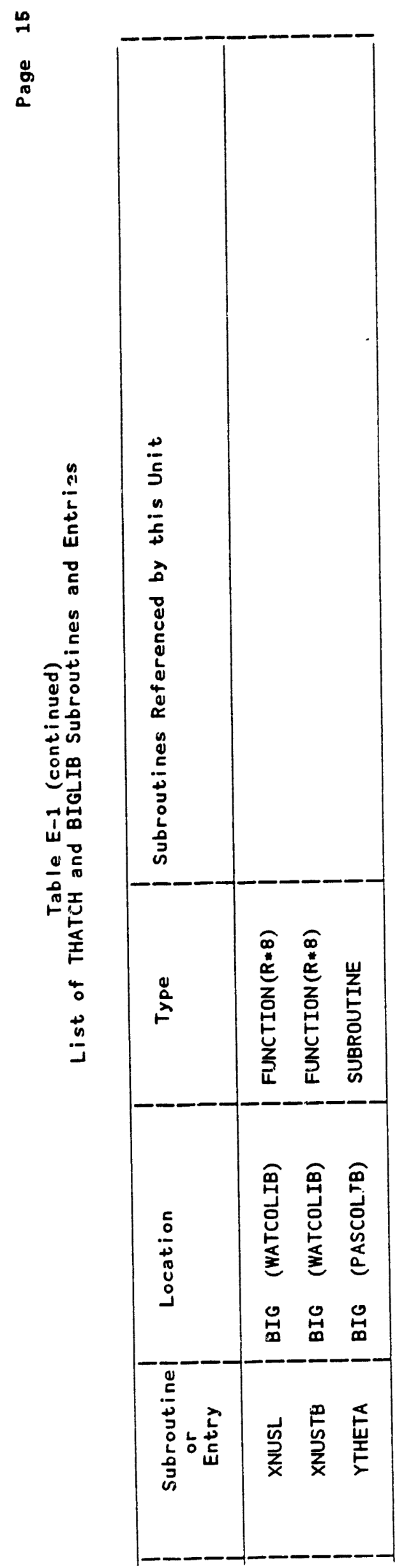

E-16 
$\stackrel{2}{2}$

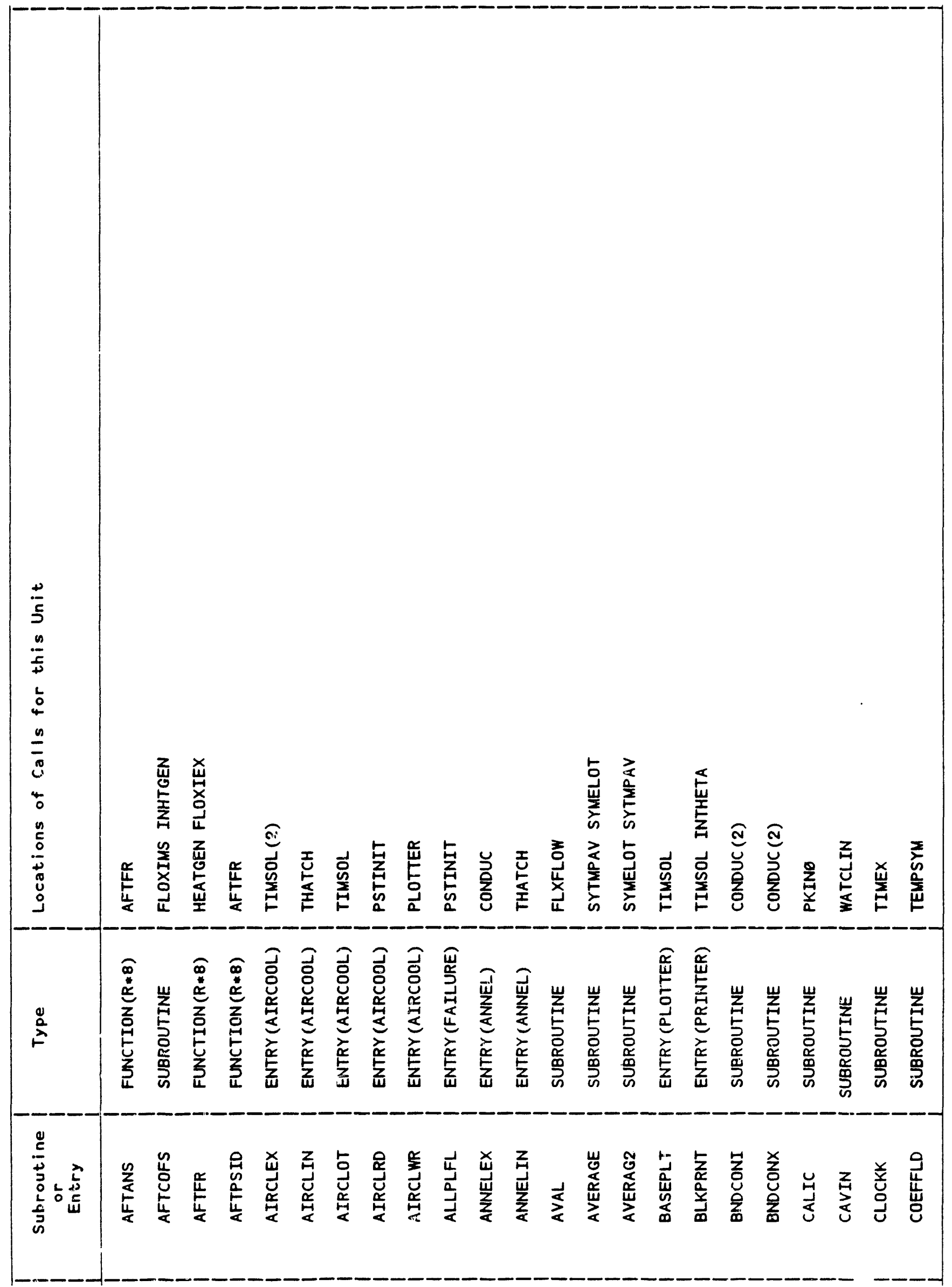




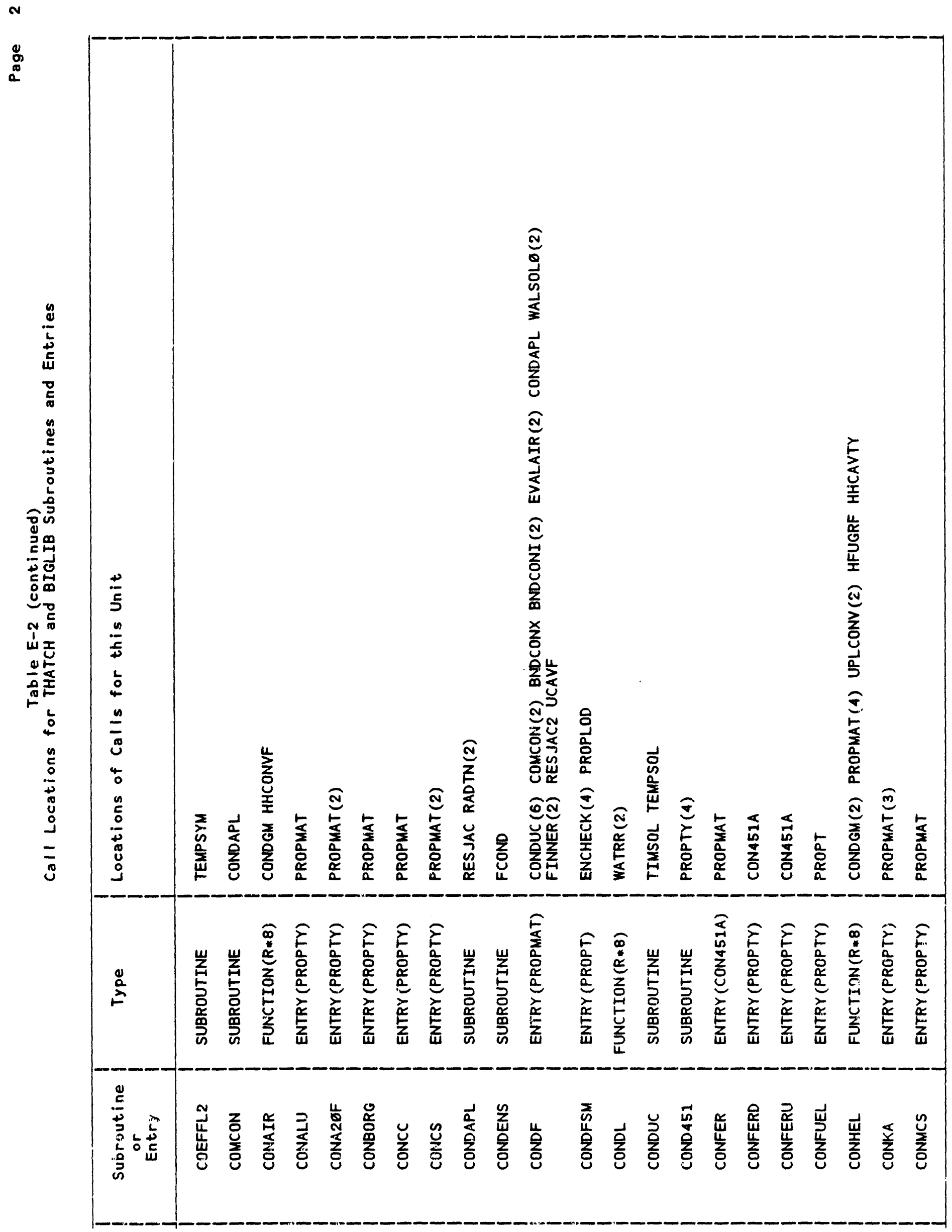




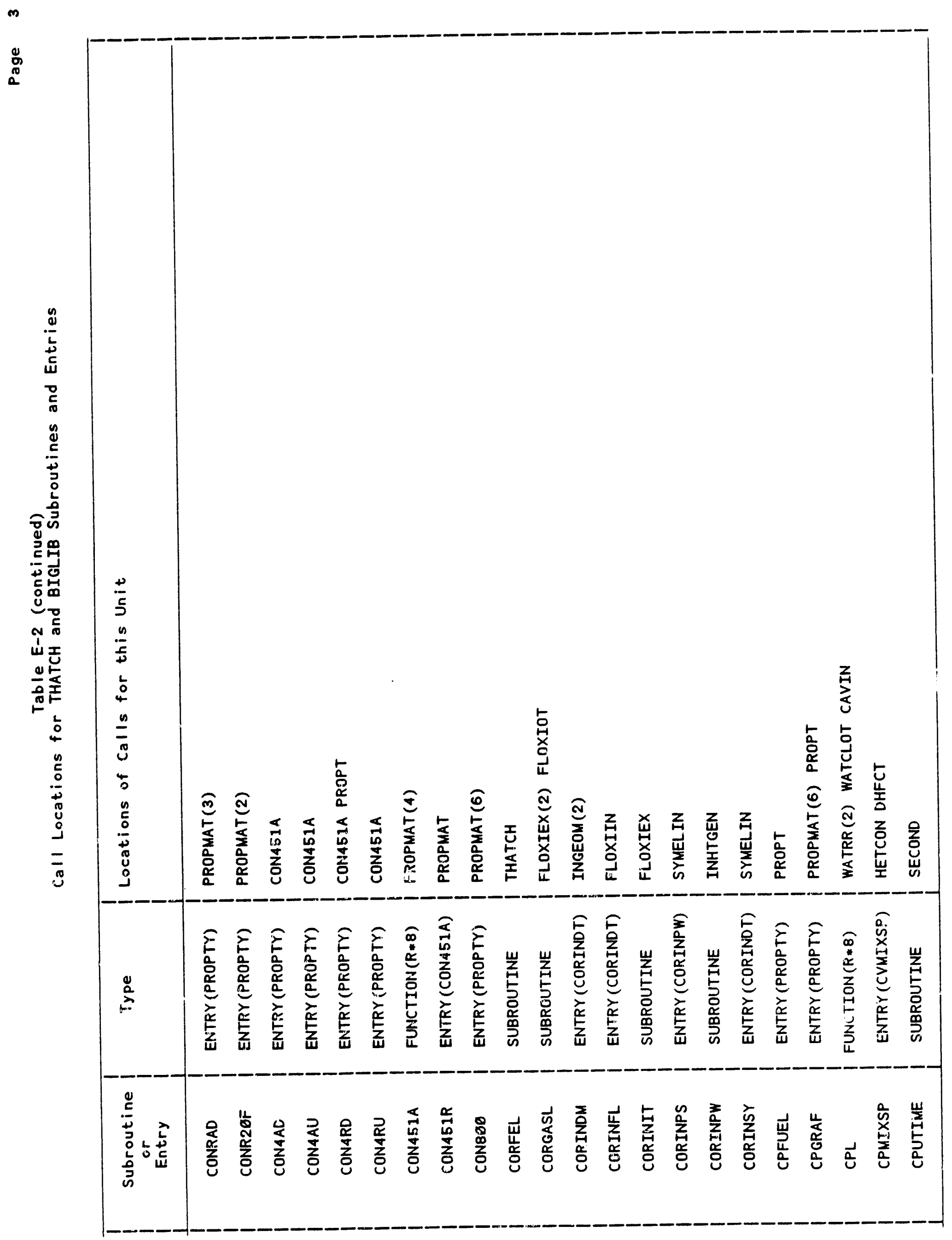




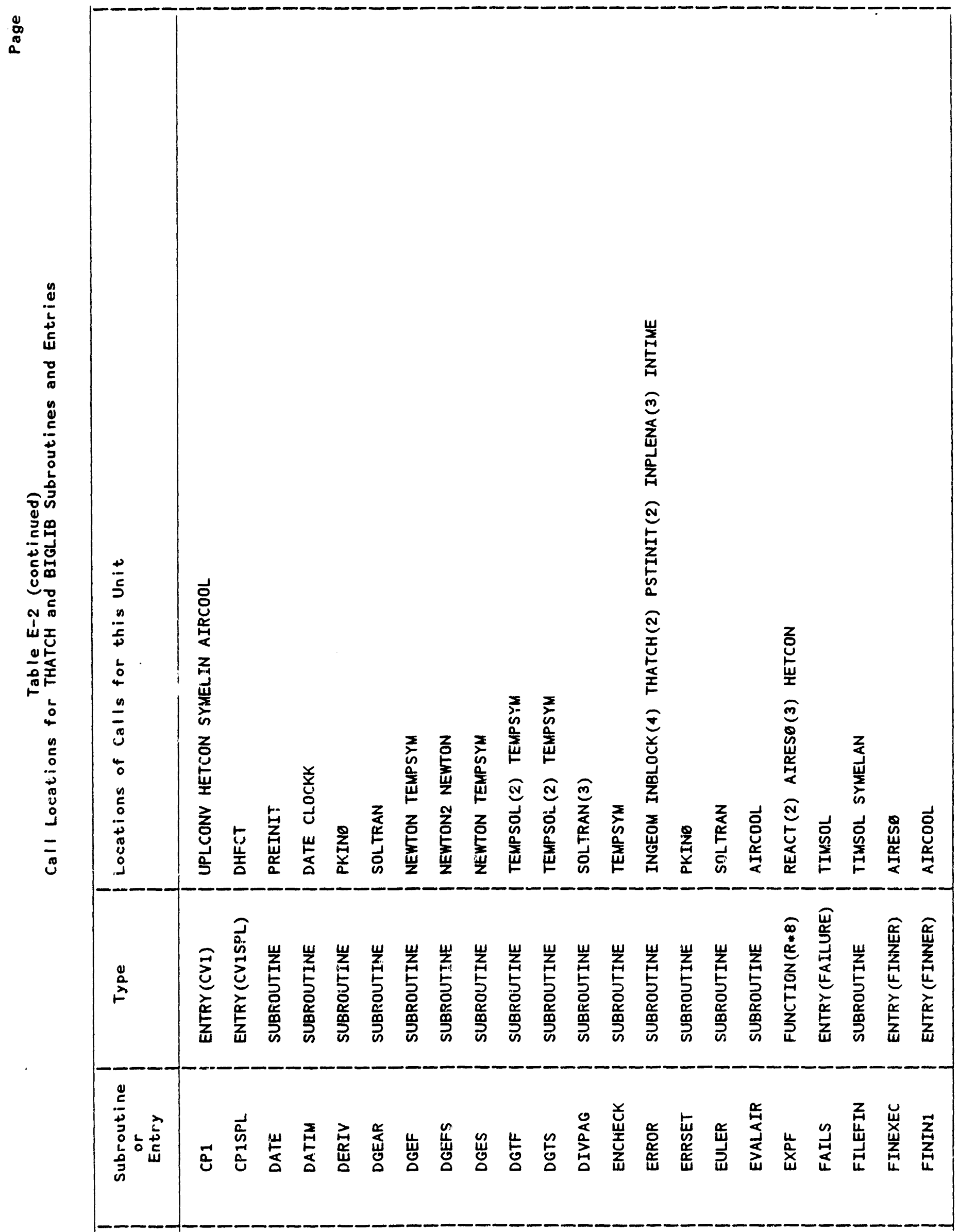




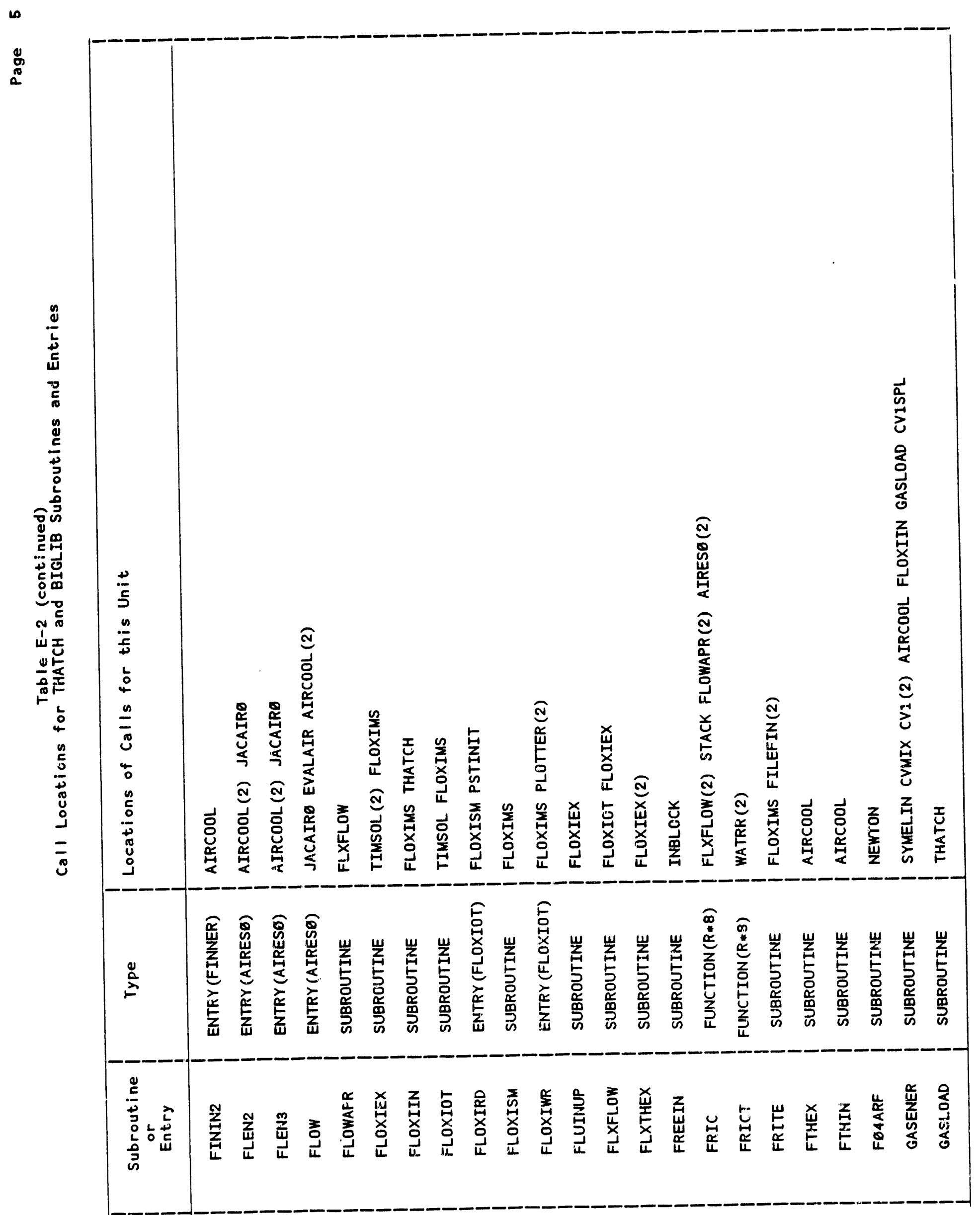




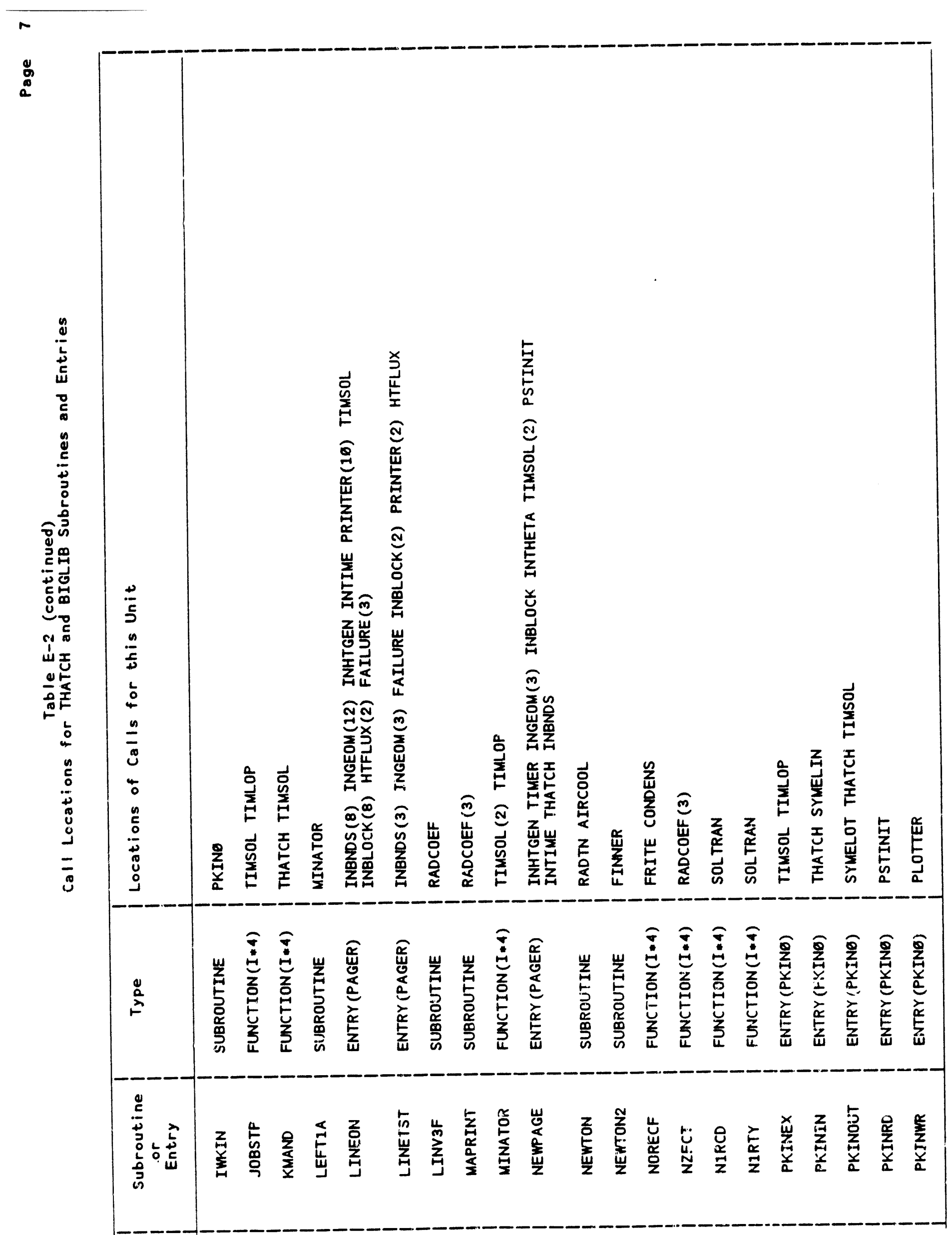




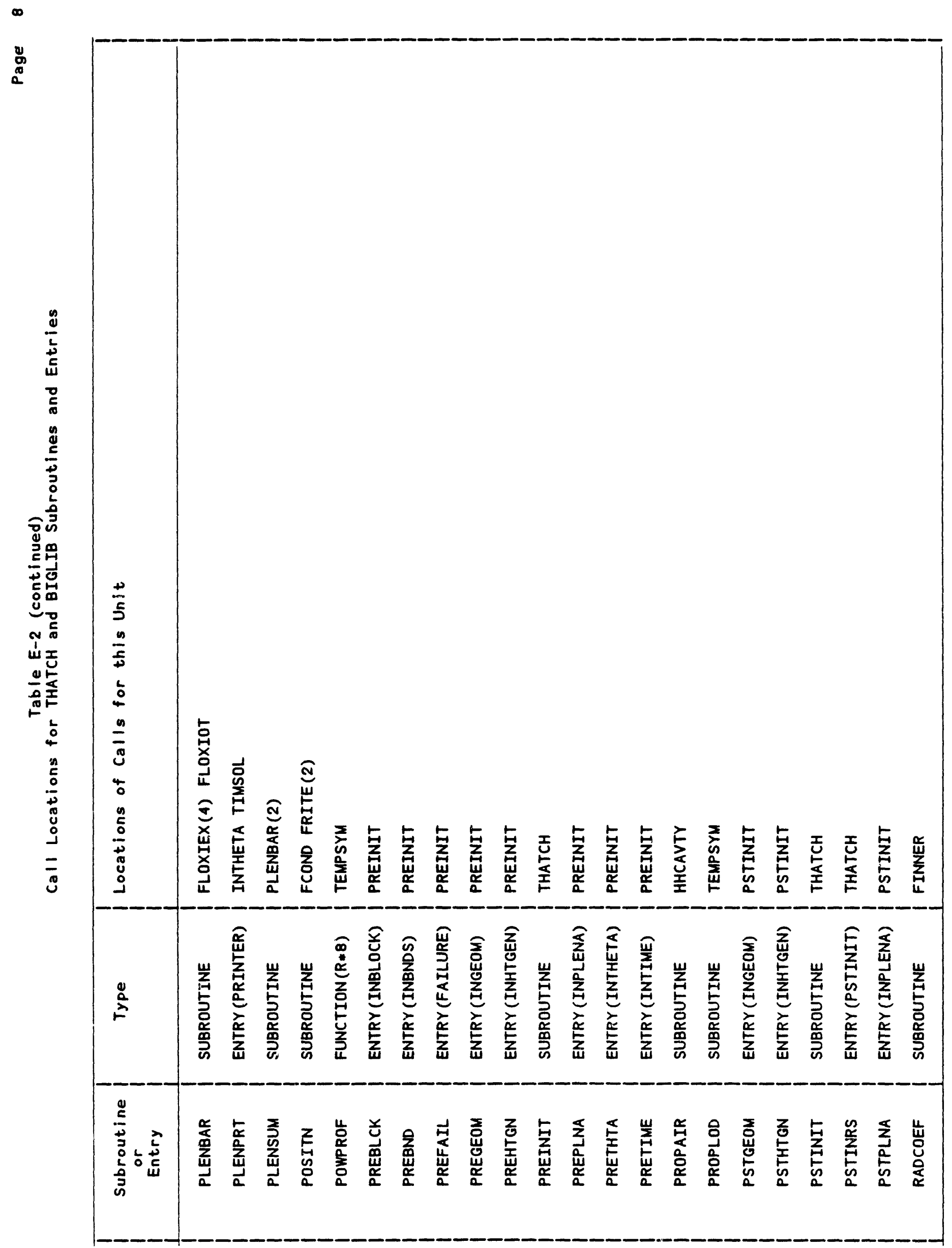




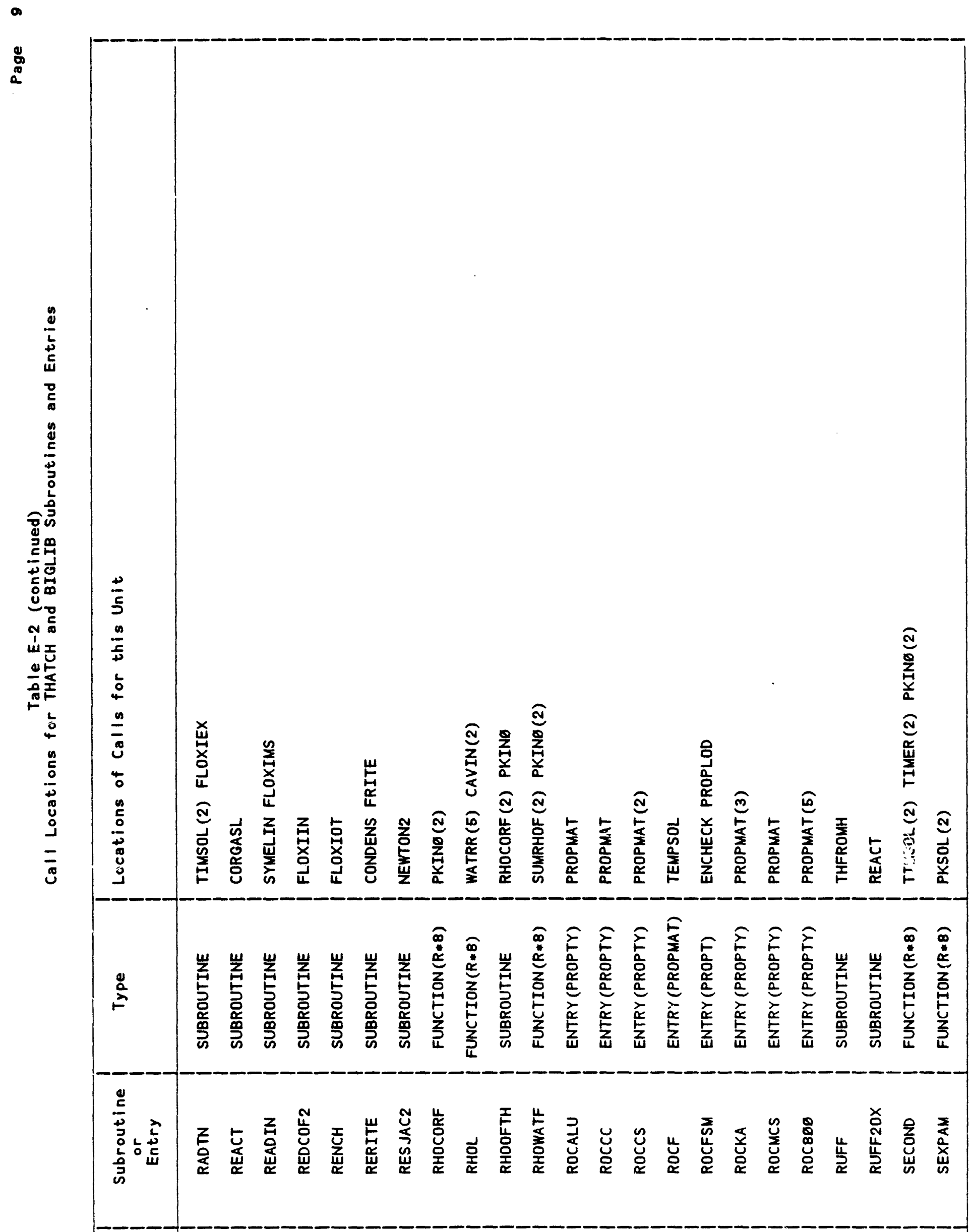




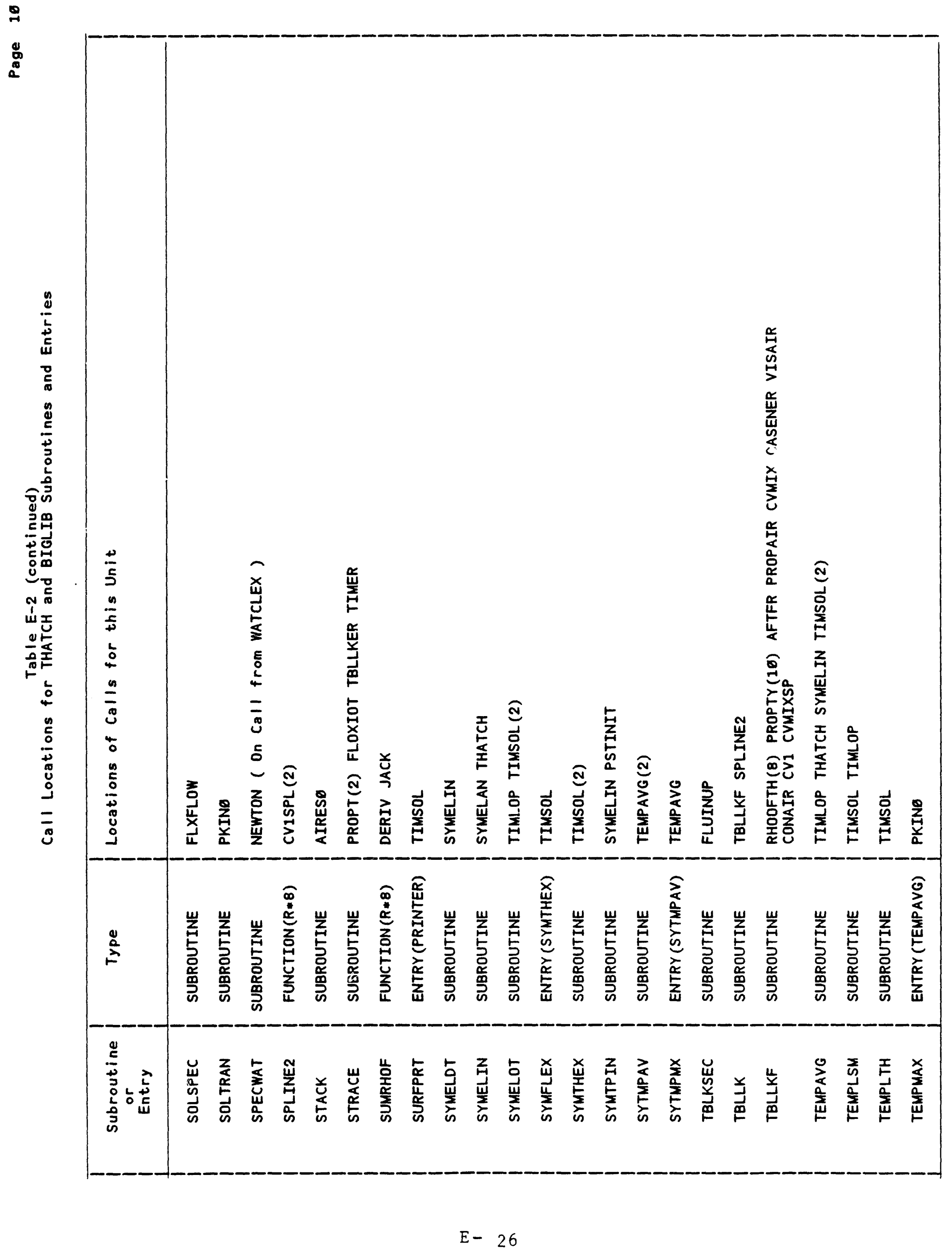




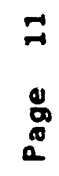

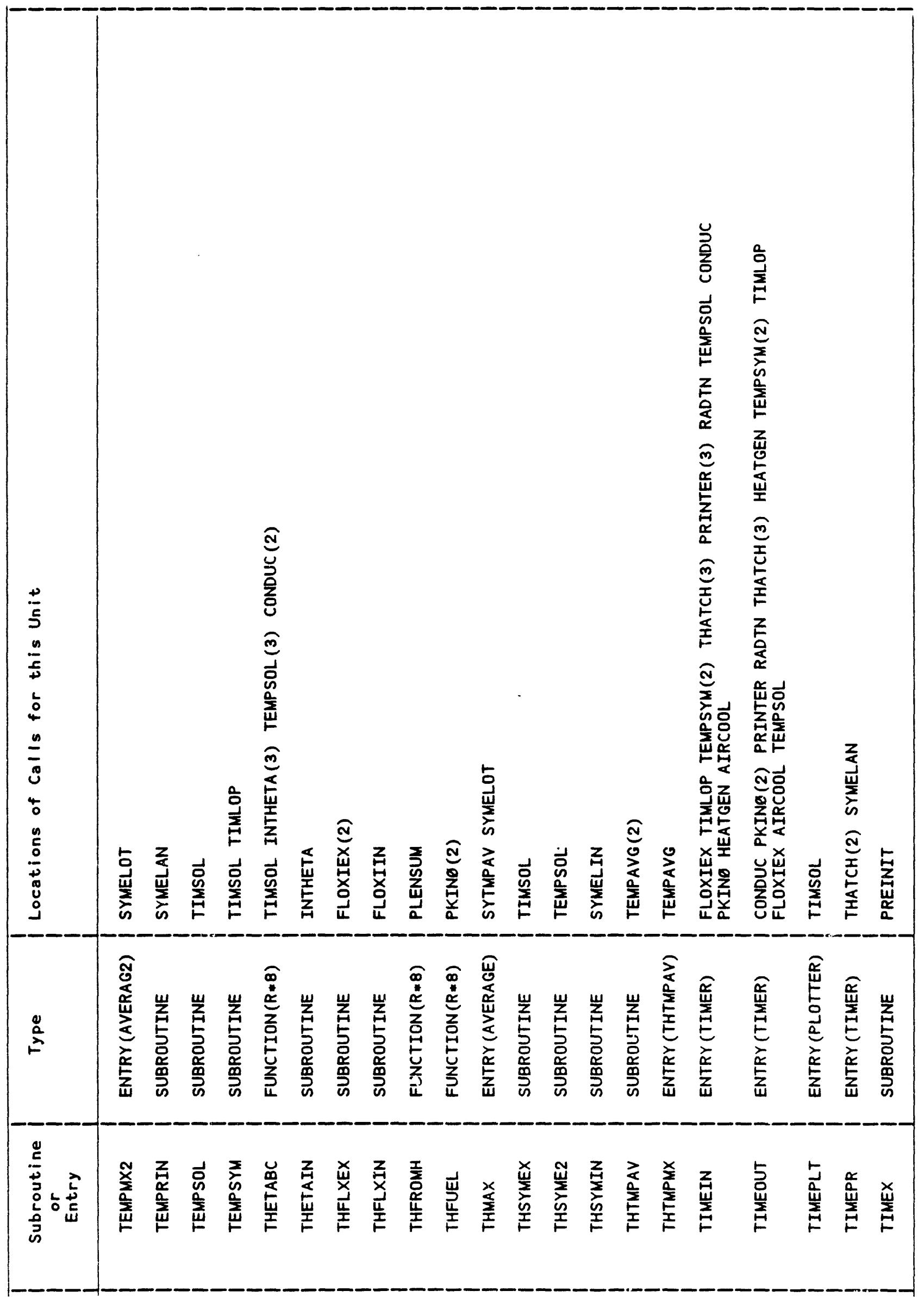




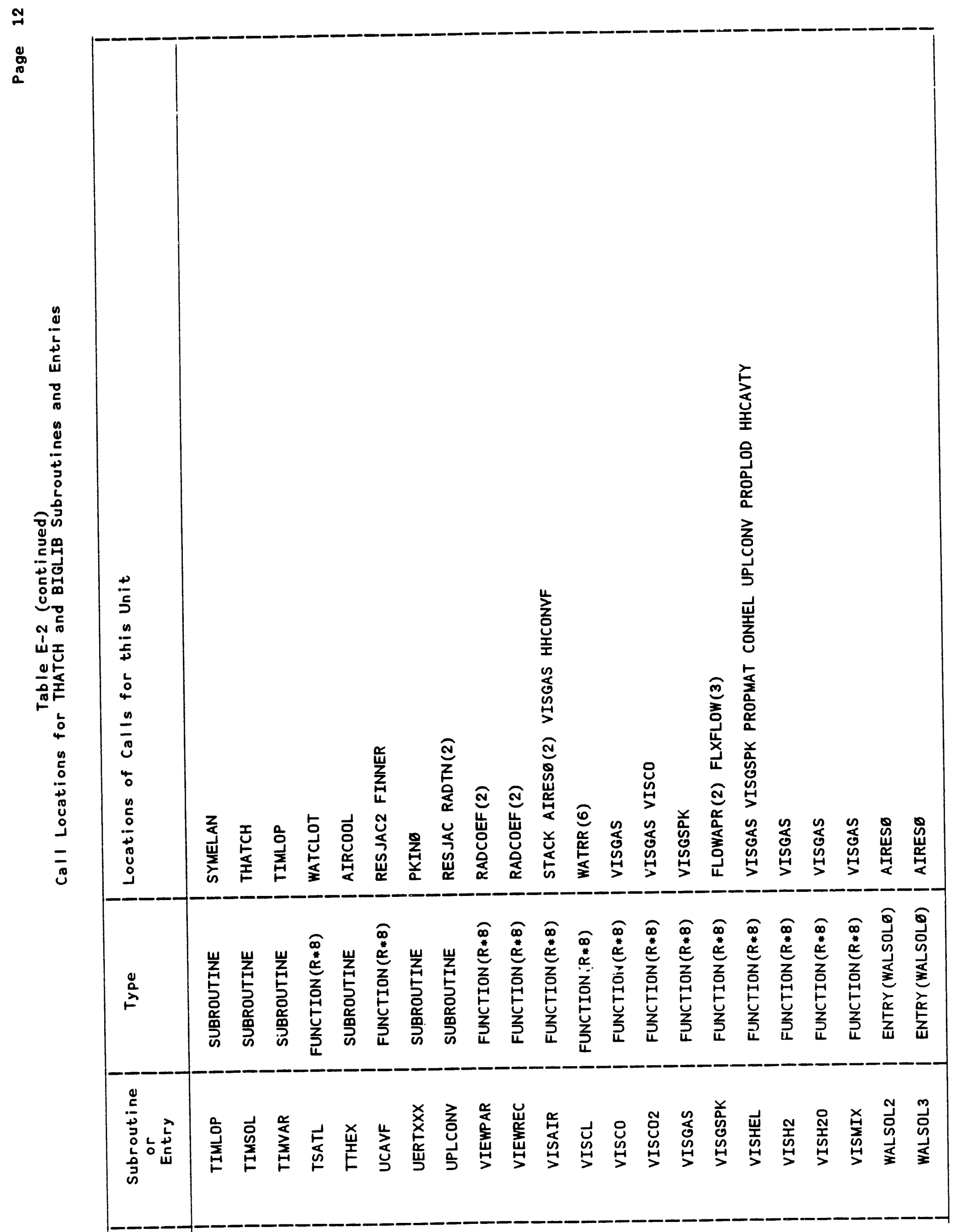




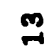

๕ั

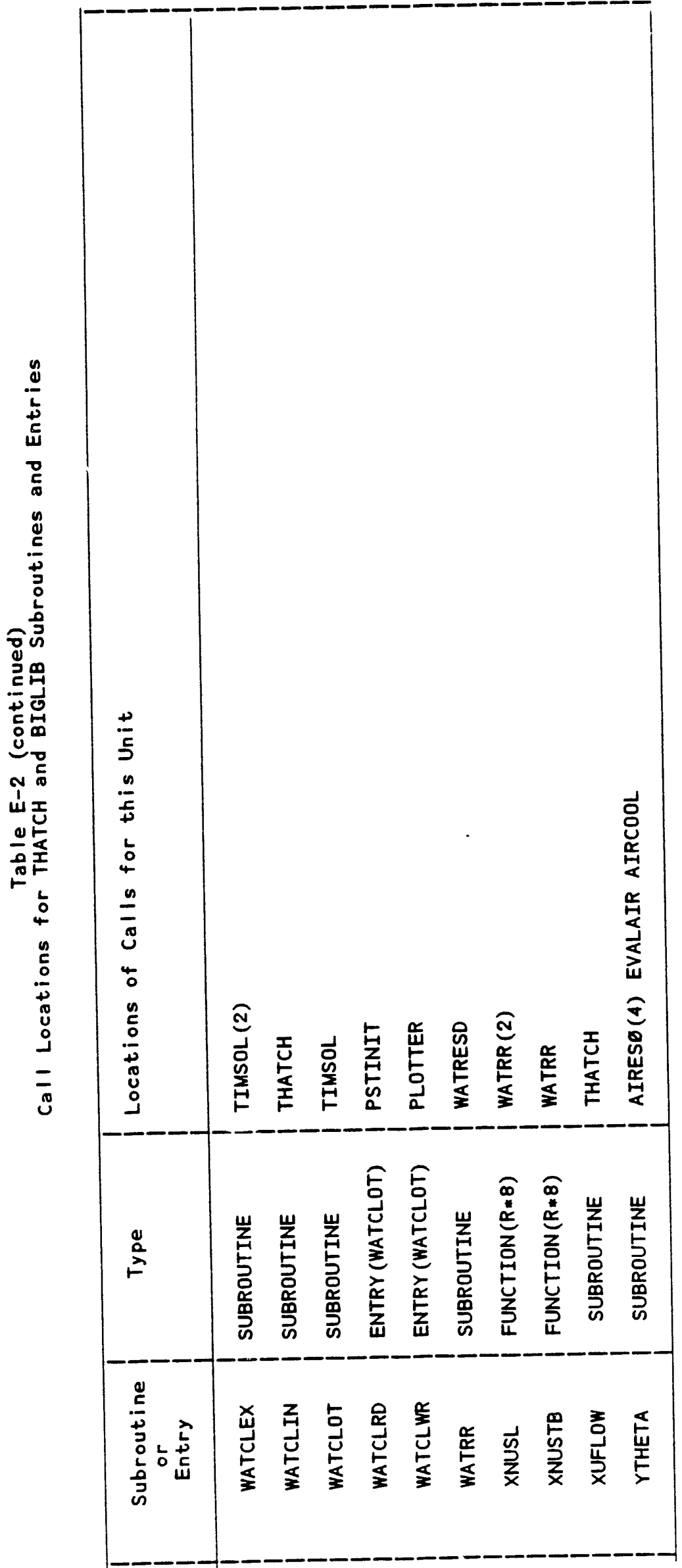

E-29 


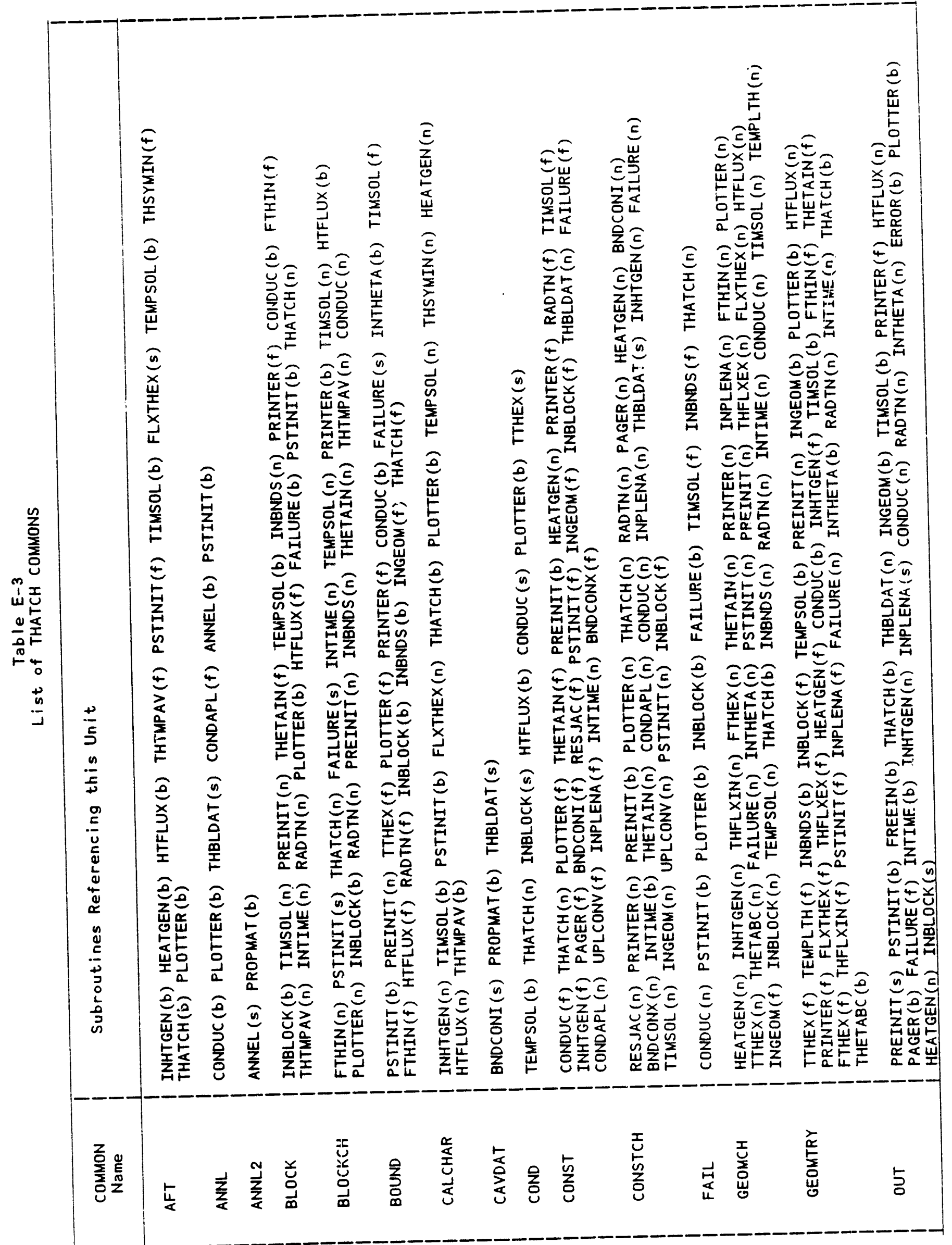




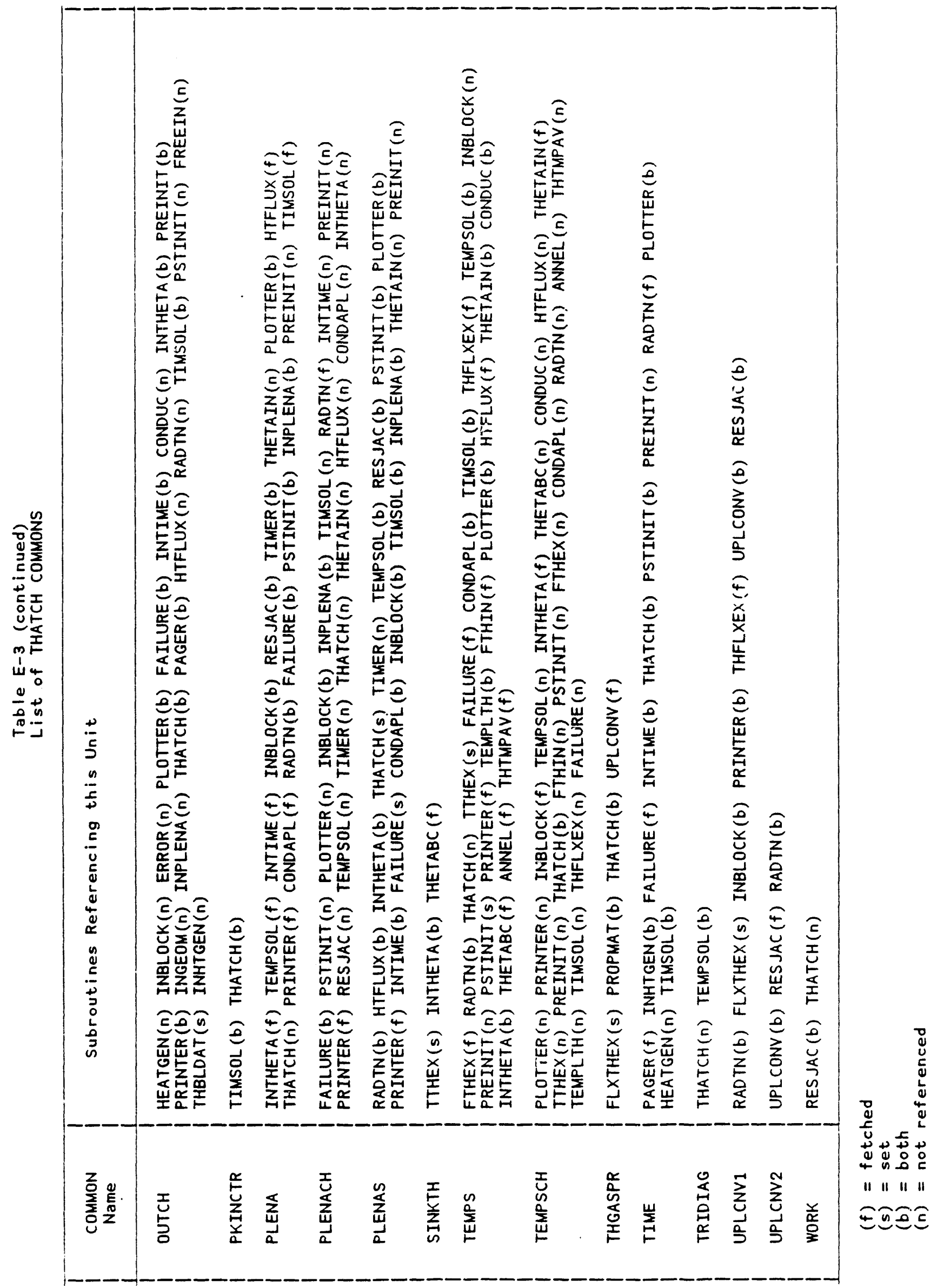




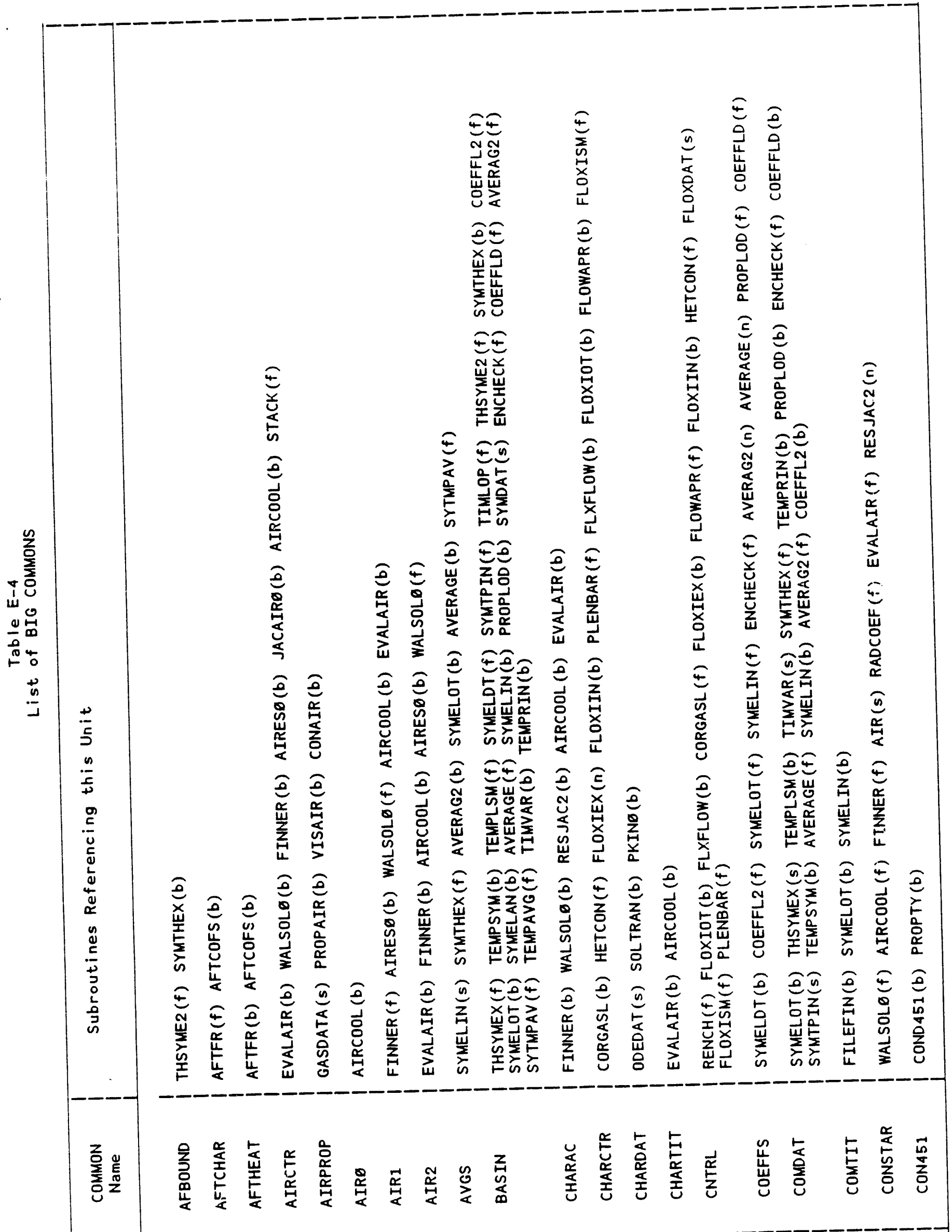


$\infty$
0
0
0
0

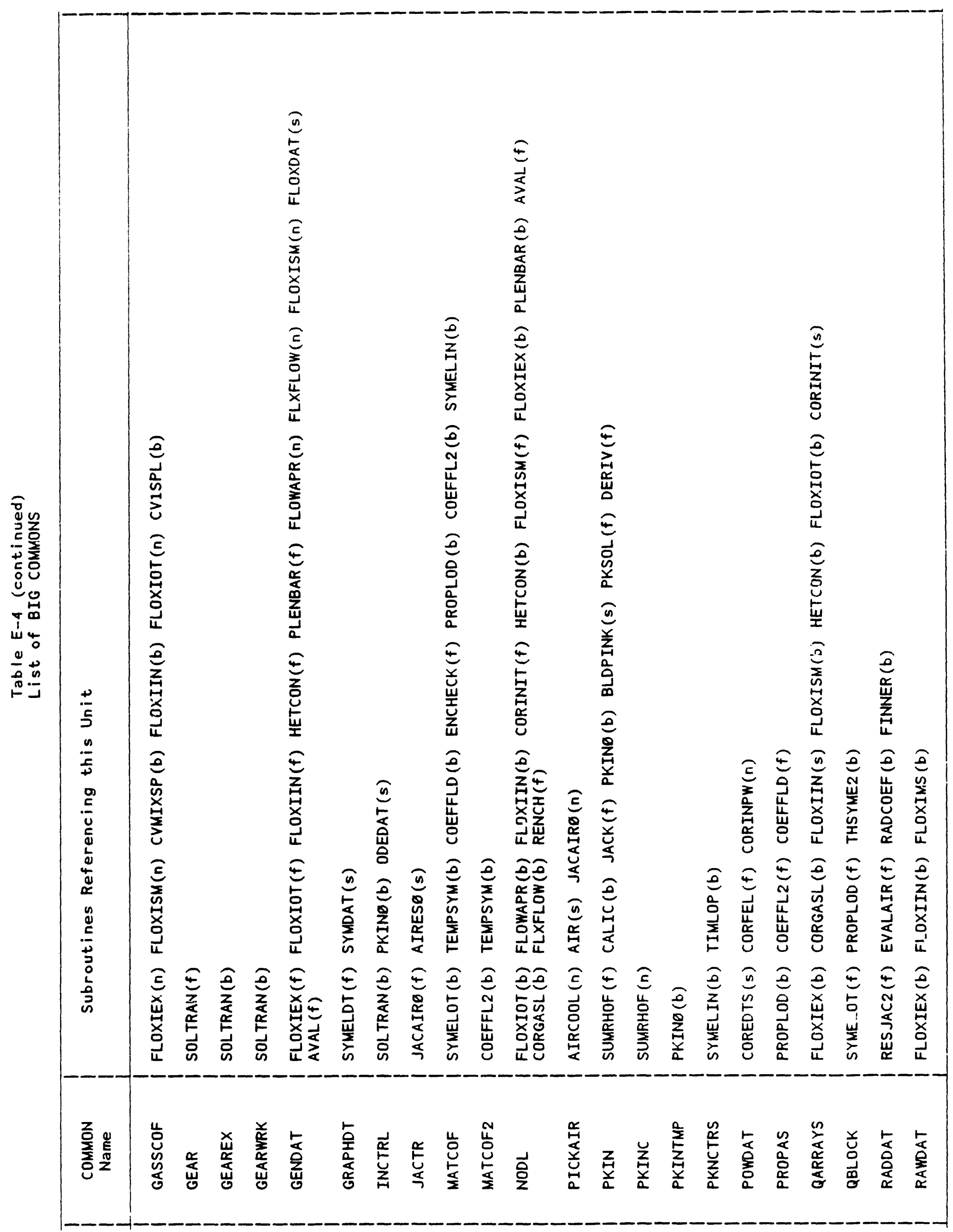




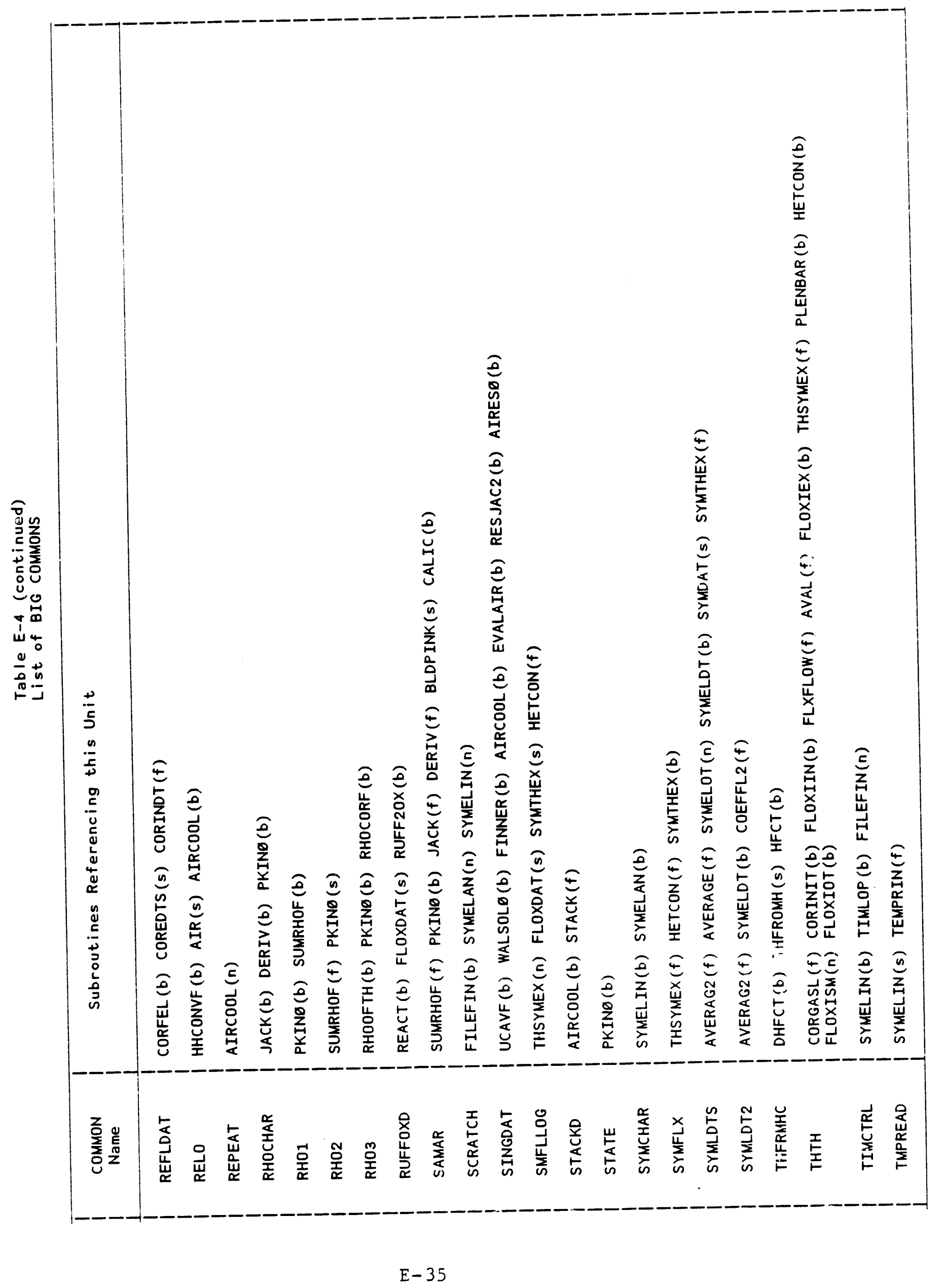




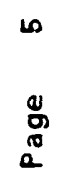

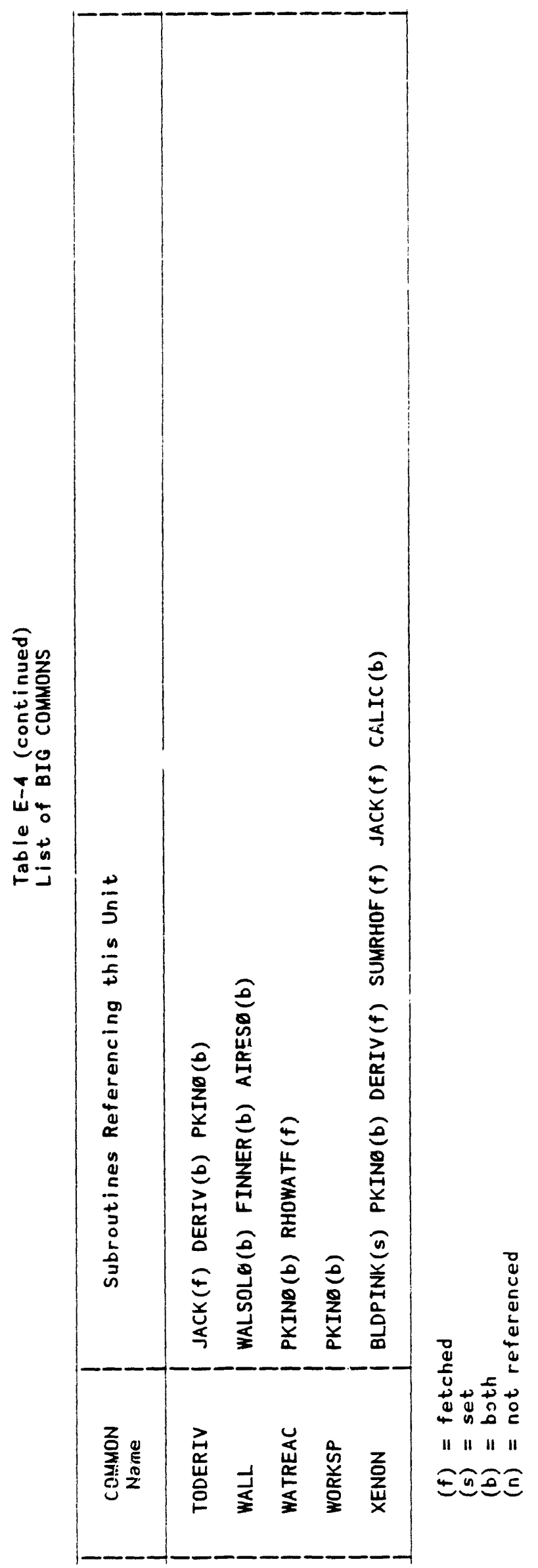




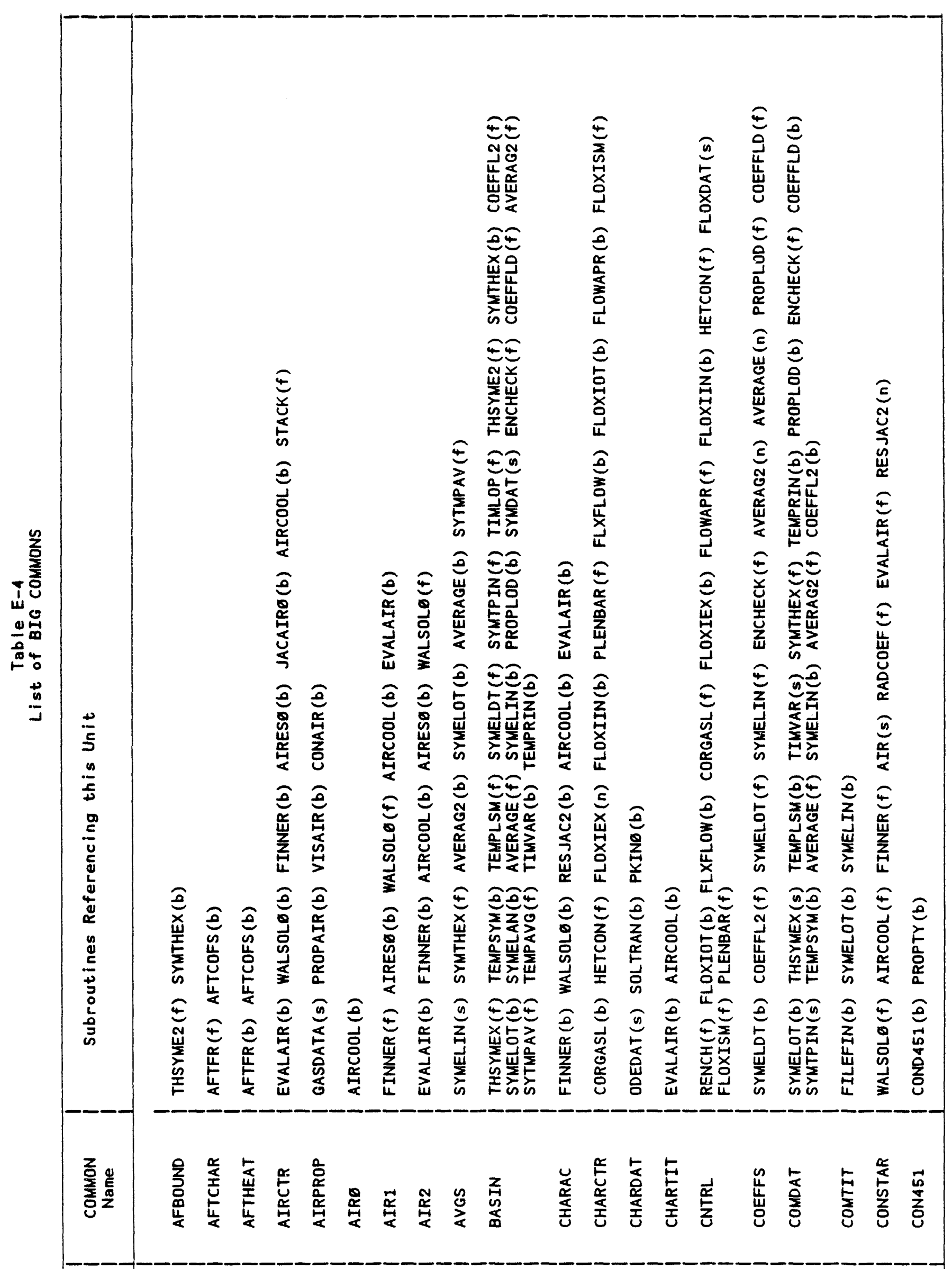


m

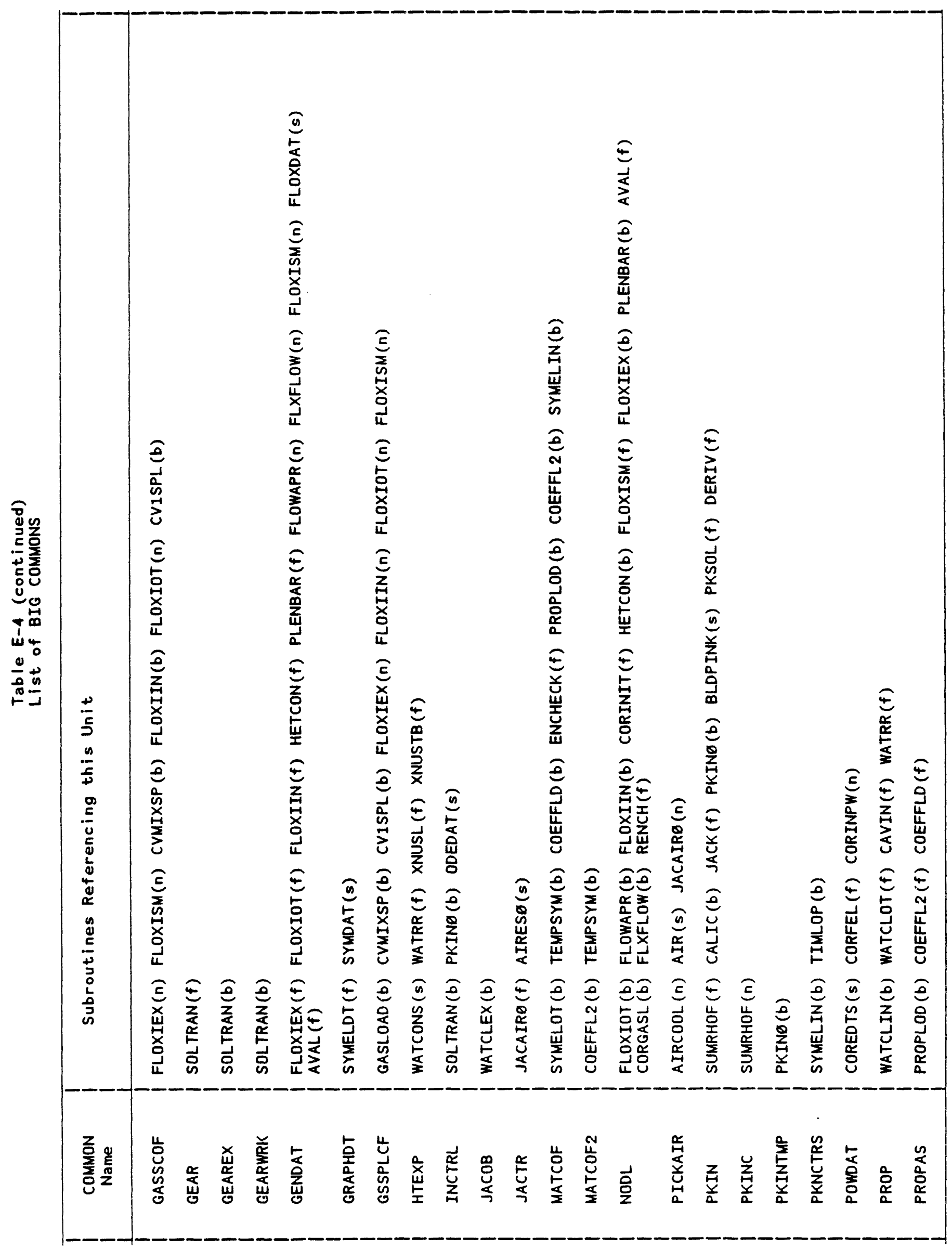




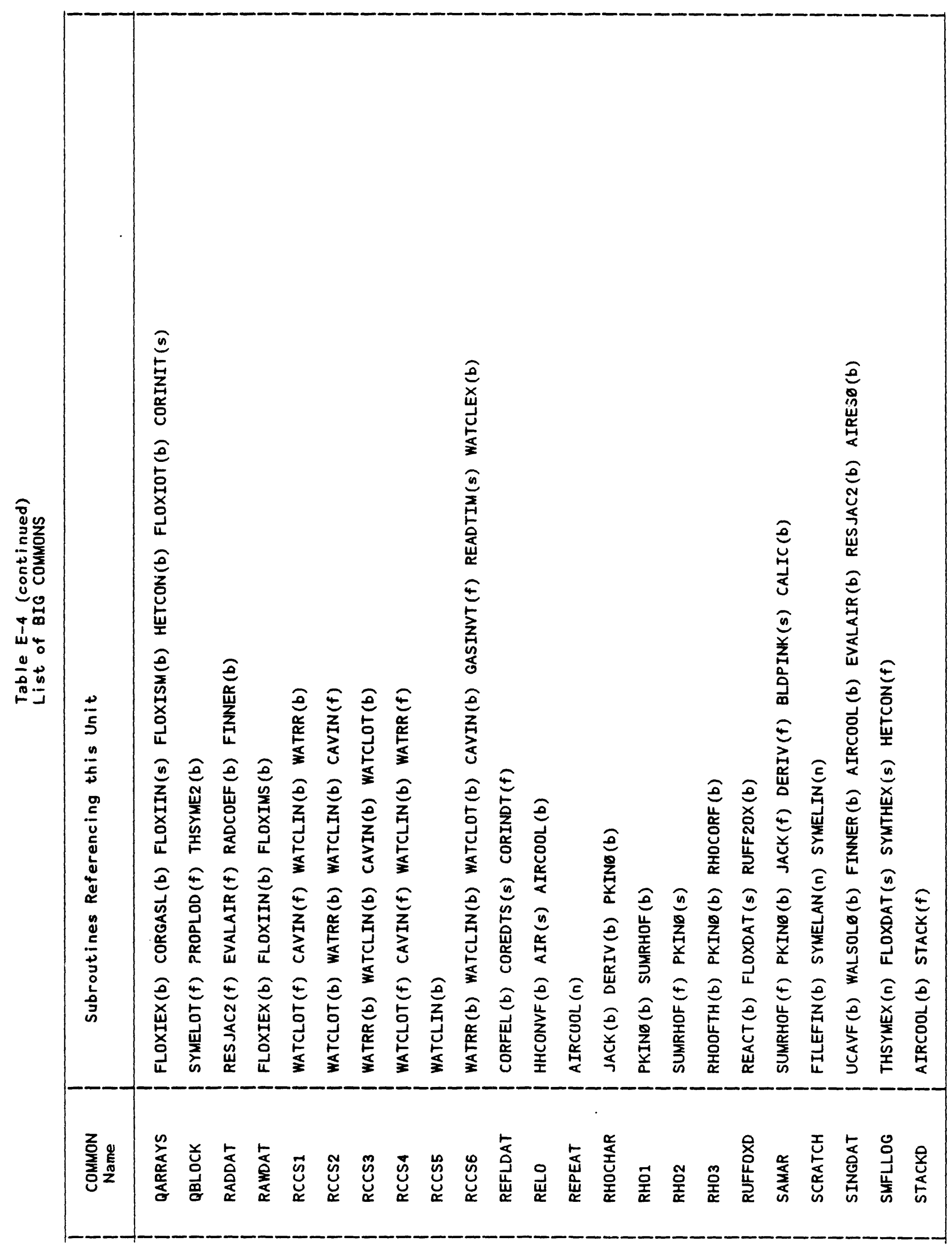


$\because$

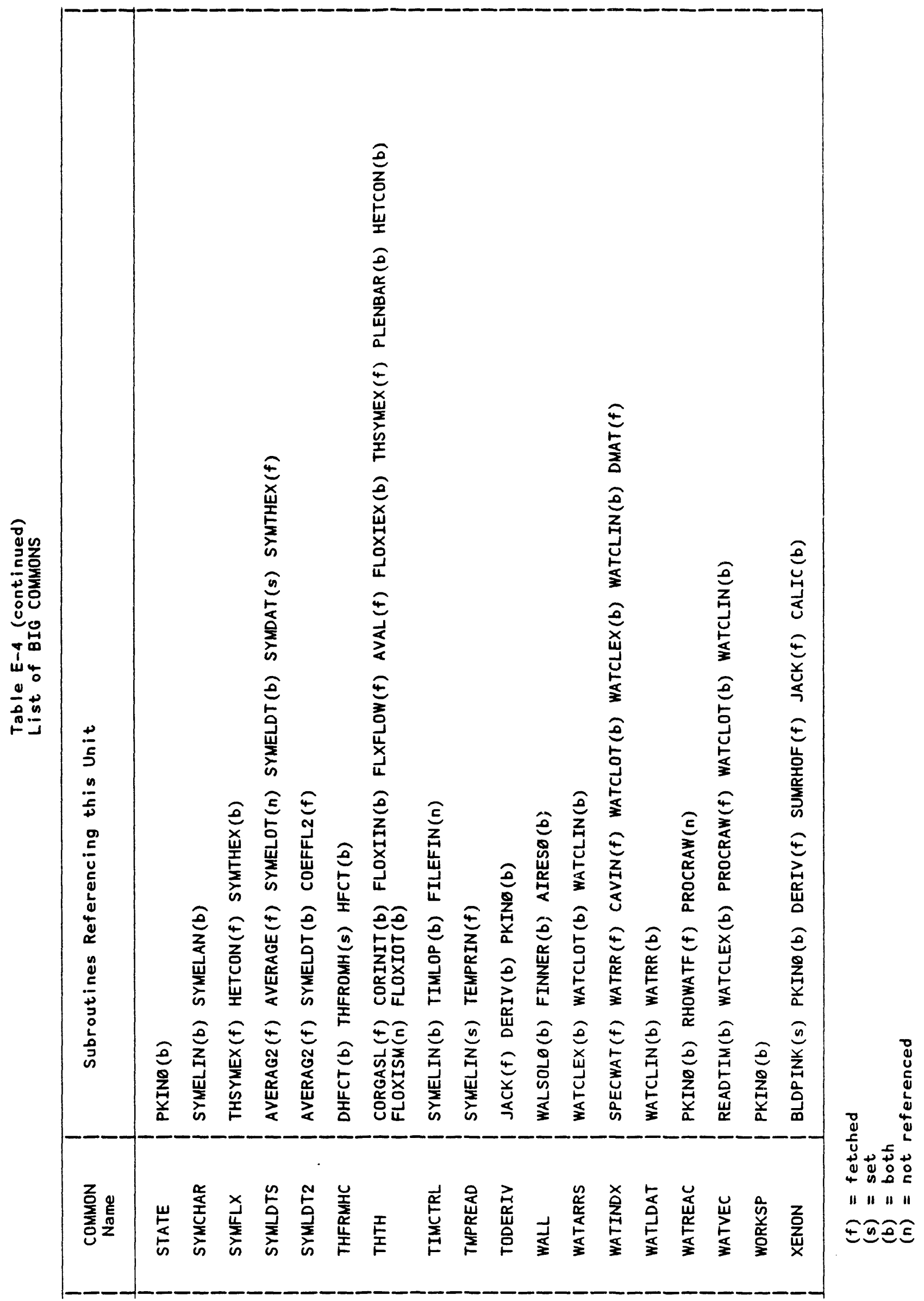


APPENDIX F

INPUT DECKS FOR SAMPLE CASES OF SECTION 9

$\mathrm{F}-1$ 
Input Deck for THATCH Sample Case No 1

THBASDEP

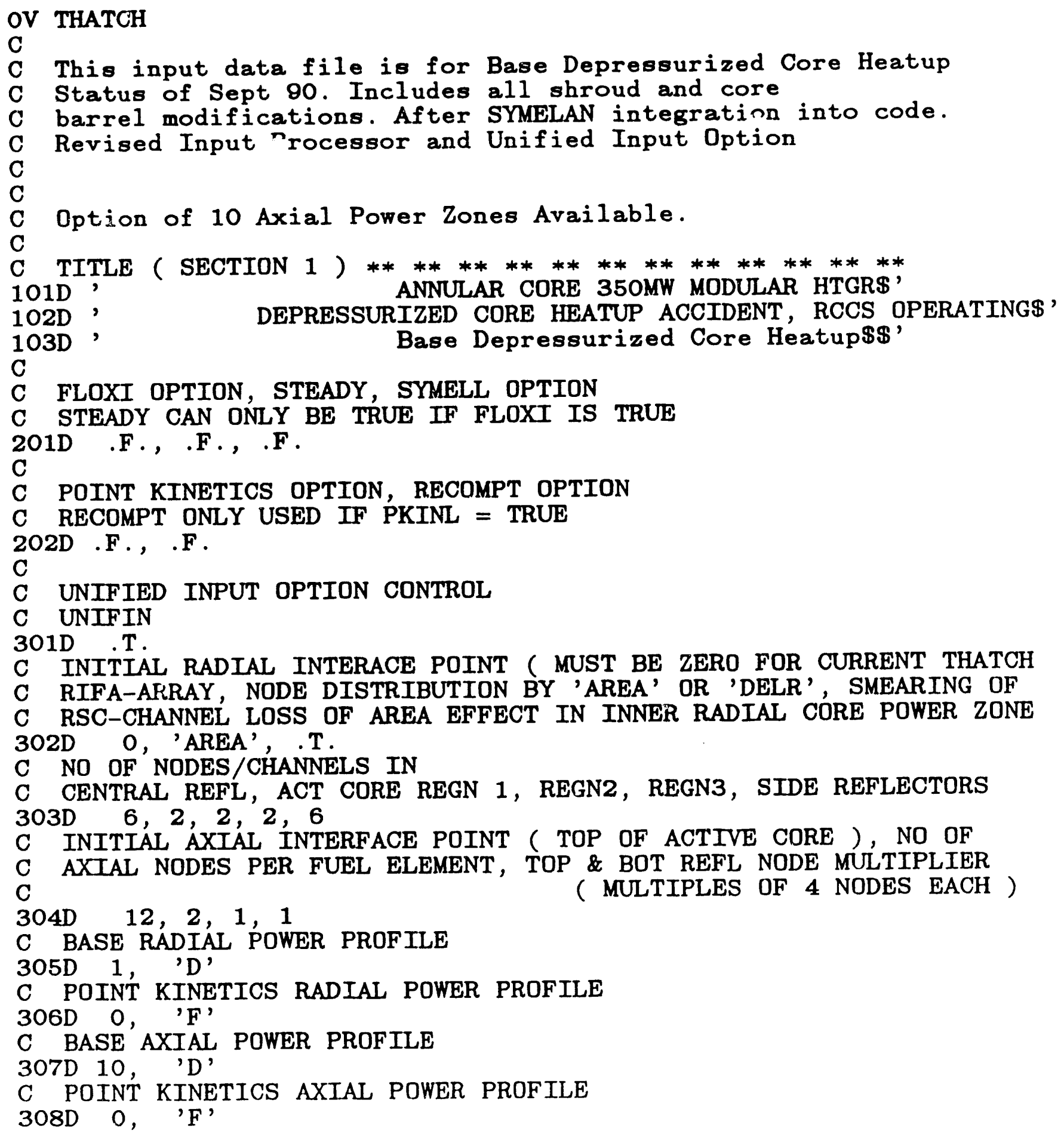




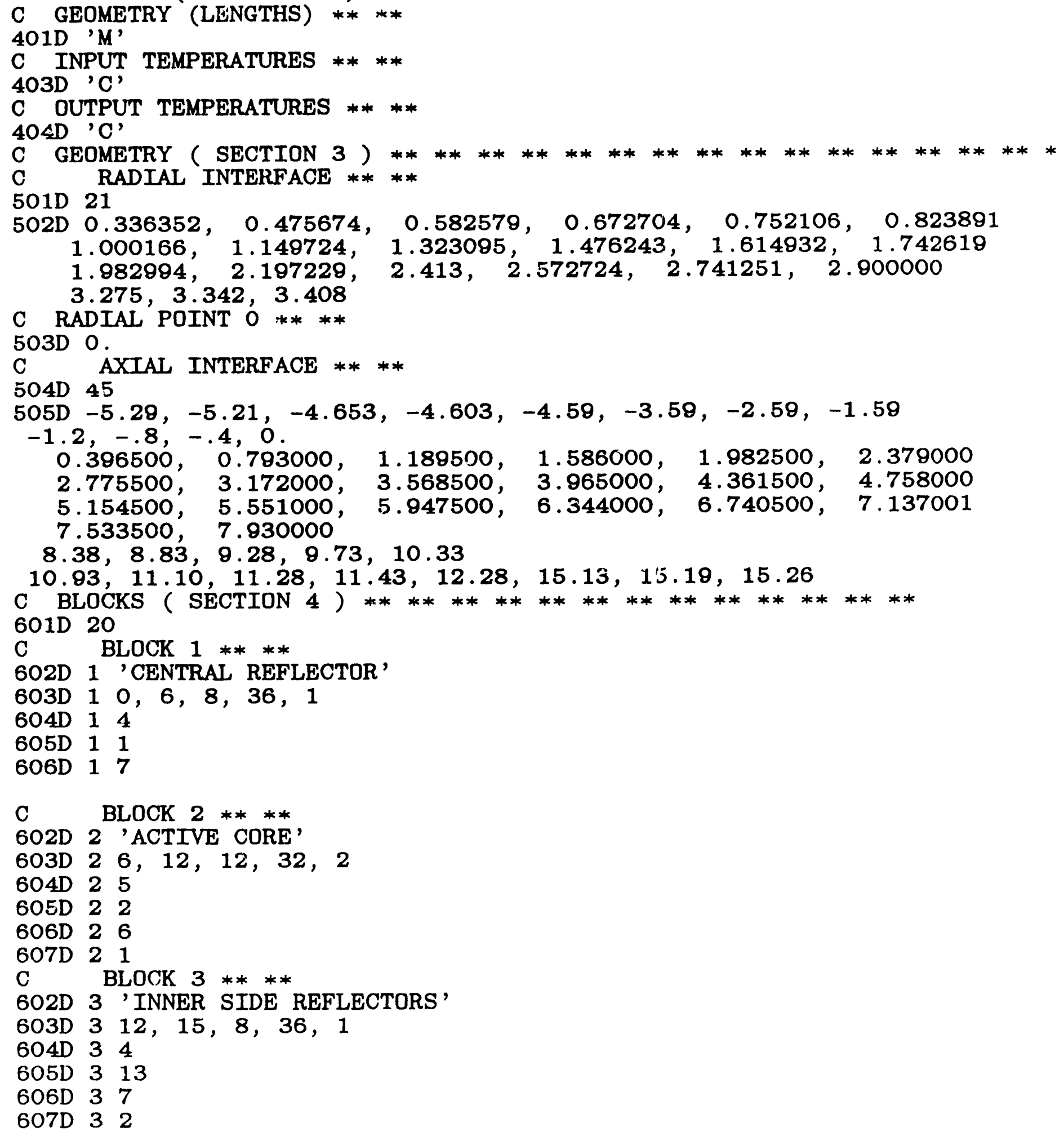


C BLOCK $4 * * * *$

602D 4 'PERMANENT SIDE REFLECTORS'

$603 D 415,18,8,36,4$

604D 44

605D 43

606D 47

607D $4 \quad 13$

C BLOCK $5 * * * *$

602D 5 'TOP REFLECTOR'

603D $56,12,9,12,3$

604D 513

605D 52

606D 55

607D 51

$\begin{array}{lll}\text { C } & \text { BLOCK } 6 & * * * * \\ 602 D & 6 & \text { 'UPPER PLENUM ELEMENTS' }\end{array}$

603D $66,12,8,9,15$

604D 64

605D 62

606D $6 \quad 13$

607D 61

C $62 \mathrm{D} 7$ BLOCK 7 *****

$603 \bar{D} 76,12,32,34,3$

604D 76

605D 72

606D 716

607D 71

C BLOCK 8 ****

602D 8 'LOWER BOTTOM REFLECTORS'

$603 \mathrm{D} 86,12,34,36,5$

604D 816

605D 82

606D 87

607D 81

C BLOCK $9 * * * *$

602D 9 'CORE BARREL'

$603 \mathrm{D} 918,19,8,43,10$

604D 914

/6O5D $9114,9,10,4,11,43$

$605 \mathrm{D} 9,14,8,10,4,11,43$

905D $14,16, .025$

606D $9 \quad 15$

607D $93,9,36,11,37,38,12,39,43$

C **** BLOCK 10***********

602D 10 'VESSEL (SIDE)'

$603 \mathrm{D} 1019,21,1,45,6$

604D 101

605D $107,2,43,8,44,45$

606D $10 \quad 12$

607D $105,2,3,6,44,45$ 


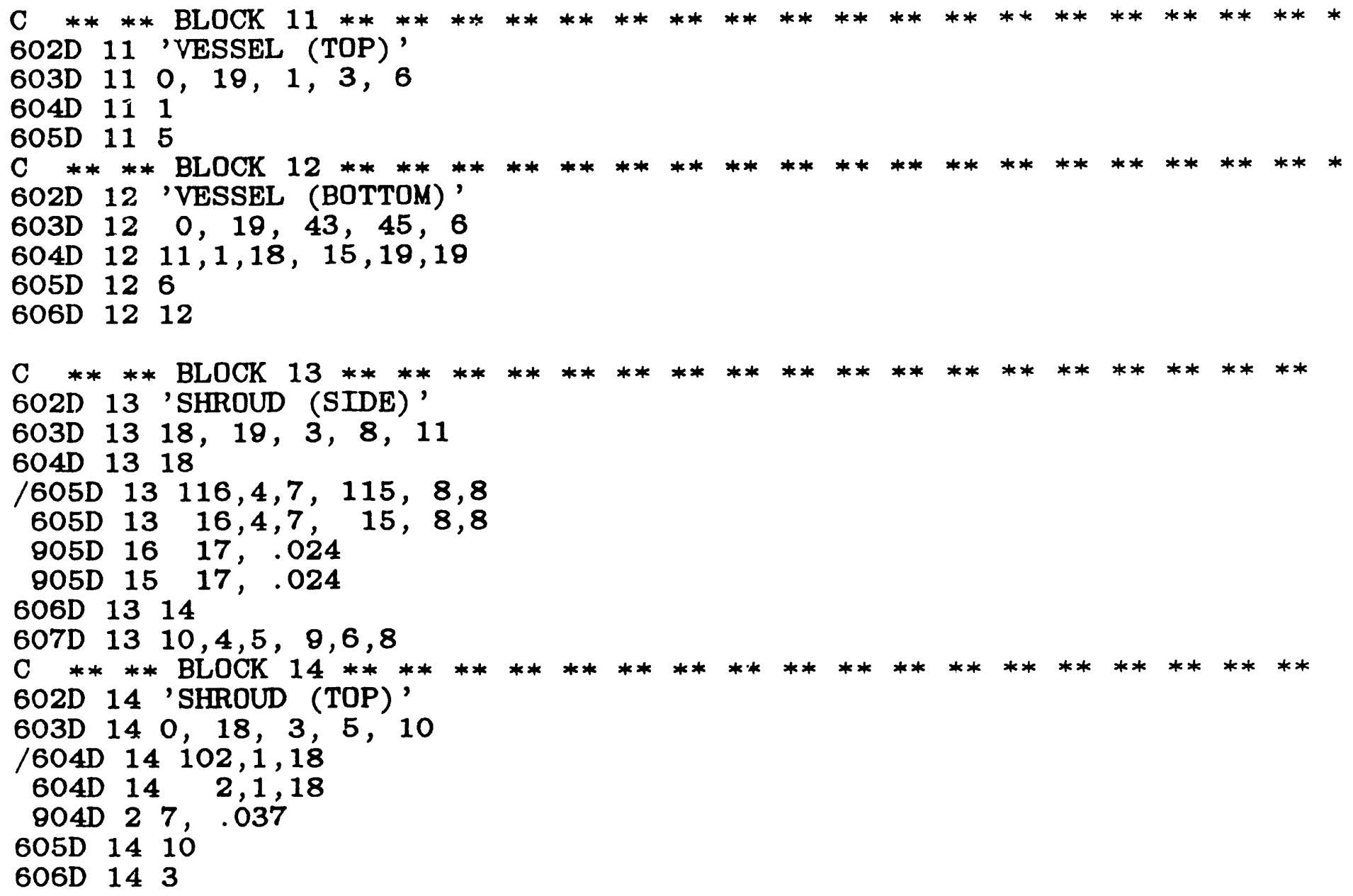




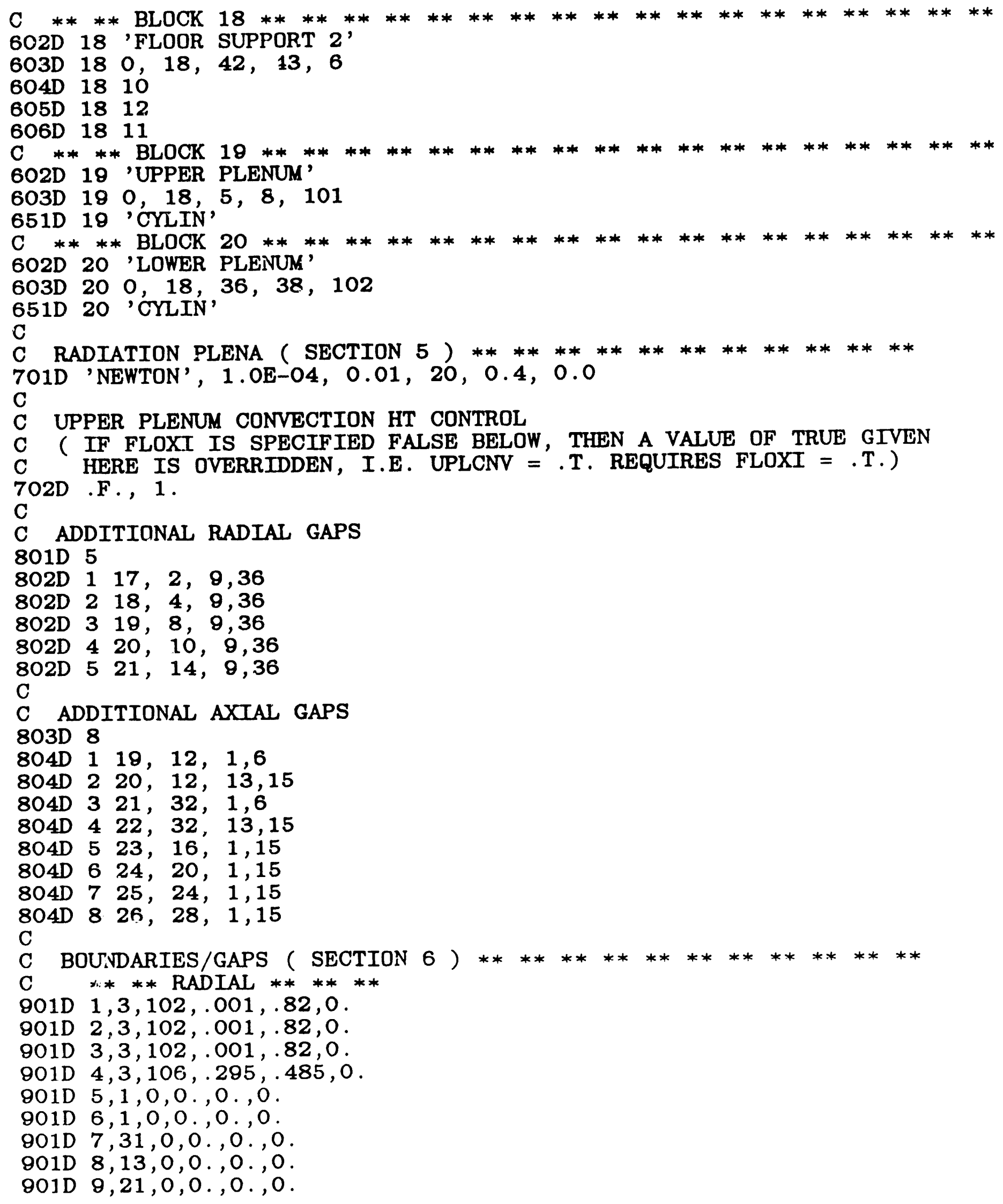




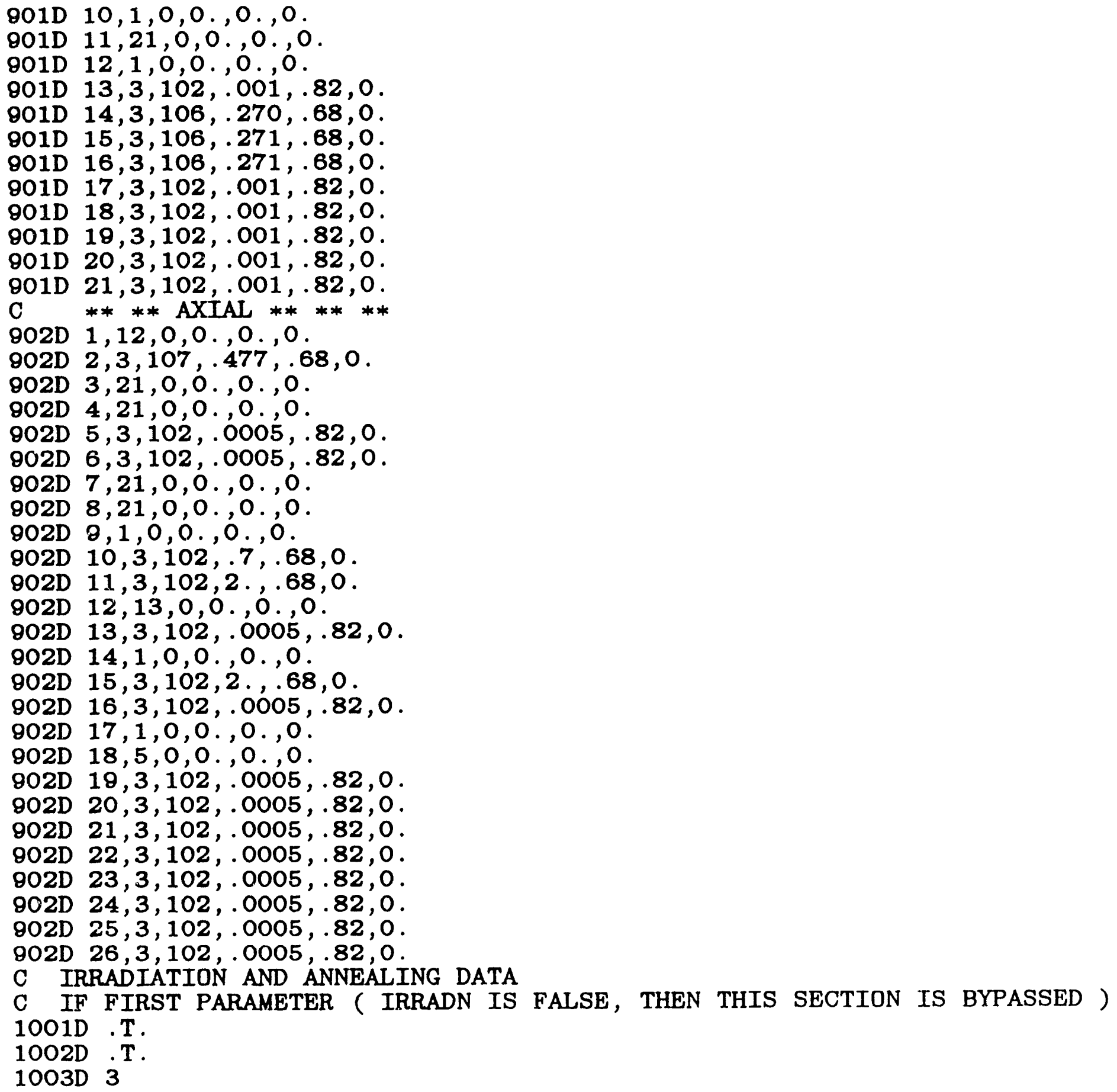




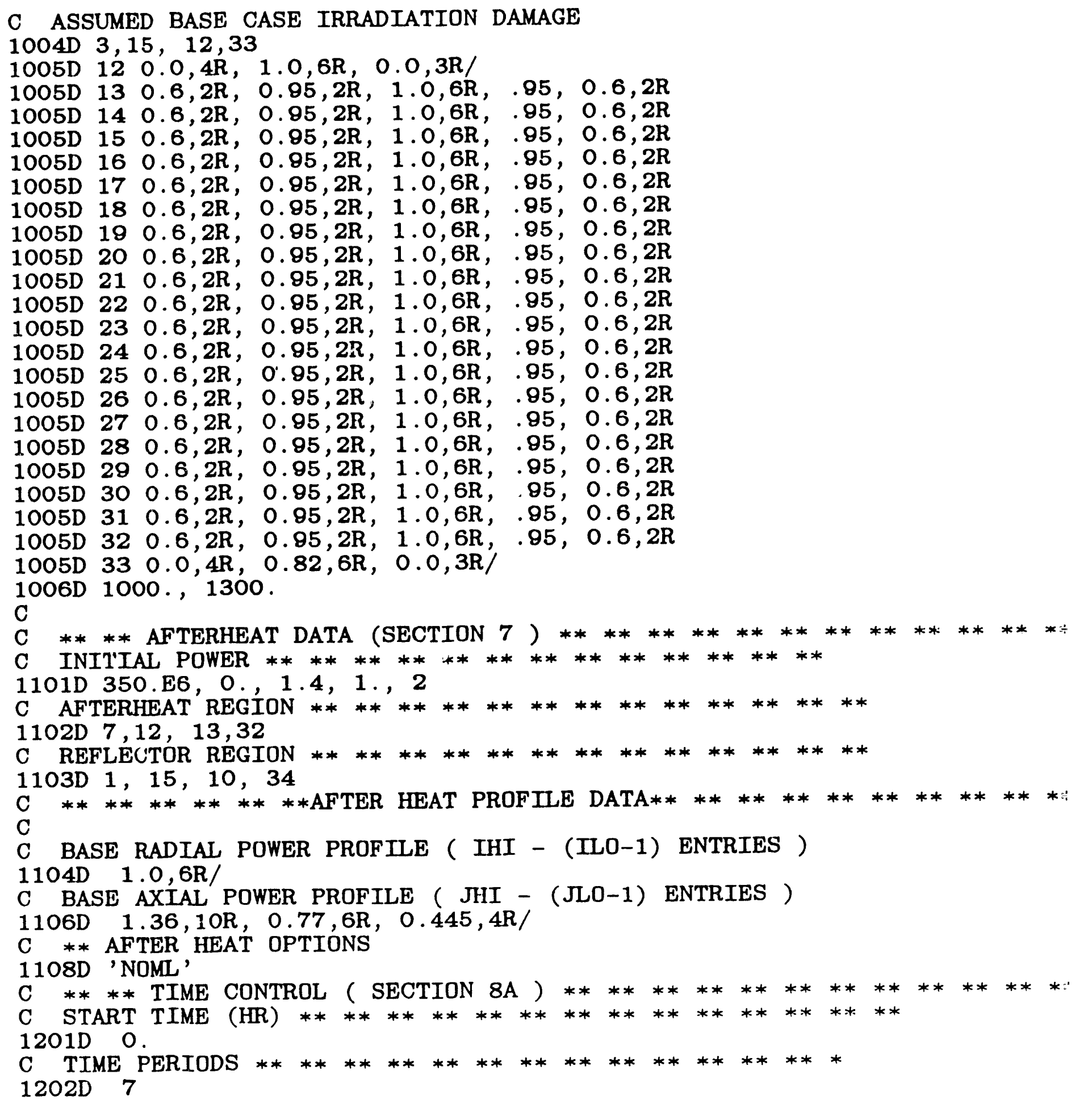


C NORMAL TIME STEPS FOR DEPRESSURIZED TRANSIENTS

$1203 \mathrm{D} 0,240,15,240,240$.

$1,10 ., 1 ., 10,6$.

$1,60,5,50,25$.

$2,5 ., 25,4 ., 1$.

$2,20 ., .5,15 ., 1$.

$2,100,1,20,1$.

$2,200 ., 5,20,1$.

C OUTPUT FORMATS ( SECTION 8B ) *************************** $1301 \mathrm{D} 4,0,8,1$

C BLOCK SURFACES OUTPUT ( SECTION 8C ) ******************* $1401 \mathrm{D} 2$

$1402 \mathrm{D} 10,2,10,4$

C DEBUG (SECTION 8D) *******************************

C DEBUG CONTROL VARIABLE

1403D 0

C DEBUG SET DATA ( BYPASS AT THIS TIME, SETTING NO OF SETS = 0

10

C **** INITIAL TEMPERATIRES ( SECTION 9 ) ****************** 1501D 'READRUN '

C 'OTHER'

$1502 \mathrm{D} 100.0,21 \mathrm{R}$

1503D $100.0,35 \mathrm{R}, 80.0,9 \mathrm{R}$

$1504 \mathrm{D} 100.0,21 \mathrm{R}$

C BLOCK TEMPERATURES

1505D B1;24*258.

$\mathrm{B} 1 ; 6 * 269$.

$B 1 ; 6 * 290$.

$\mathrm{B} 1 ; 6 * 312$.

$\mathrm{B} 1 ; 6 * 333$.

$\mathrm{B} 1 ; 6 * 355$.

$\mathrm{B1} ; 6 * 376$.

$\mathrm{B} 1 ; 6 * 397$.

$\mathrm{B1} ; 6 * 419$.

$\mathrm{B} 1 ; 6 * 440$.

$\mathrm{B} 1 ; 6 * 462$.

$\mathrm{B} 1 ; 6 * 485$.

$\mathrm{B} 1 ; 6 * 505$.

$\mathrm{B1} ; 6 * 526$.

$\mathrm{B1} ; 6 * 548$.

$\mathrm{B} 1 ; 6 * 569$.

$\mathrm{B} 1 ; 6 * 590$.

$\mathrm{B} 1 ; 6 * 612$.

B1; $6 * 633$.

$\mathrm{B} 1 ; 6 * 655$.

$\mathrm{B1} ; 6 * 676$.

B1 ; 24*687.

$\mathrm{B2} ; 6 * 269$.

$\mathrm{B} 2 ; 6 * 290$.

B2; $6 * 312$. 
B2 ; $6 * 333$.

$\mathrm{B2} ; 6 * 355$.

$\mathrm{B2} ; 6 * 376$.

$\mathrm{B2} ; 6 * 397$.

$\mathrm{B} 2 ; 6 * 419$.

$\mathrm{B2} ; 6 * 440$.

$\mathrm{B2} ; 6 * 462$.

$\mathrm{B} 2 ; 6 * \div 83$.

$\mathrm{B} 2 ; 6 * 505$.

$\mathrm{B2} ; 6 * 526$.

$\mathrm{B2} ; 6 * 548$.

$\mathrm{B} 2 ; 6 * 569$.

B2; $6 * 590$.

$\mathrm{B} 2 ; 6 * 612$.

$\mathrm{B} 2 ; 6 * 633$.

$\mathrm{B} 2 ; 6 * 655$.

$\mathrm{B2} ; 6 * 676$.

B3;24*258 , $120 * 400$, , 24*470.

$\mathrm{B} 4 * 258$.

$\mathrm{B} 5 * 687$.

$\mathrm{B} 6 * 258$.

$\mathrm{B} 7 * 219$.

$\mathrm{B} 8 * 219$.

$\mathrm{B} 9 * 219$.

$\mathrm{B} 10 * 258$.

$\mathrm{B} 11 * 258$.

$\mathrm{B} 12 * 500$.

$\mathrm{B} 13 * 400$.

B14*258.

C $1000 * 777$. /

C AIR COOLER INPUT DATA

6101D 'FIN PERFORMANCE COEFFICIENTS ARE INPUT'

6102D .T.,.F.,.F., 'BNL'

$6103 \mathrm{D} 1,43,9$

$6104 \mathrm{D} 3.408,4.316,4.326,4.4784,4.4884,4.85$

$6105 \mathrm{D} 3.95,7.8, .0762, .54$

C SURFACE EFFECTIVENESS DATA

6106D $11.0,4 \mathrm{R}$

6106D $21.0,4 \mathrm{R}$

6106D $31.0,4 \mathrm{R}$

6106D $41.0,4 \mathrm{R}$

$6106 \mathrm{D} 5 \mathrm{1}, 0,4 \mathrm{R}$

$6106 \mathrm{D} 61.0,4 \mathrm{R}$

6106D $71.0,4 \mathrm{R}$

6106D $84.4,0 ., 1 ., 1$.

$6106 \mathrm{D} 94.4,0 ., 1 ., 1$.

$6106 \mathrm{D} 104.4,0 ., 1 ., 1$.

6106D $114.4,0 ., 1 ., 1$.

6106D $124.4,0 ., 1 ., 1$.

6106D $134.4,0 ., 1 ., 1$.

$6106 \mathrm{D} 144.4,0 ., 1 ., 1$. 


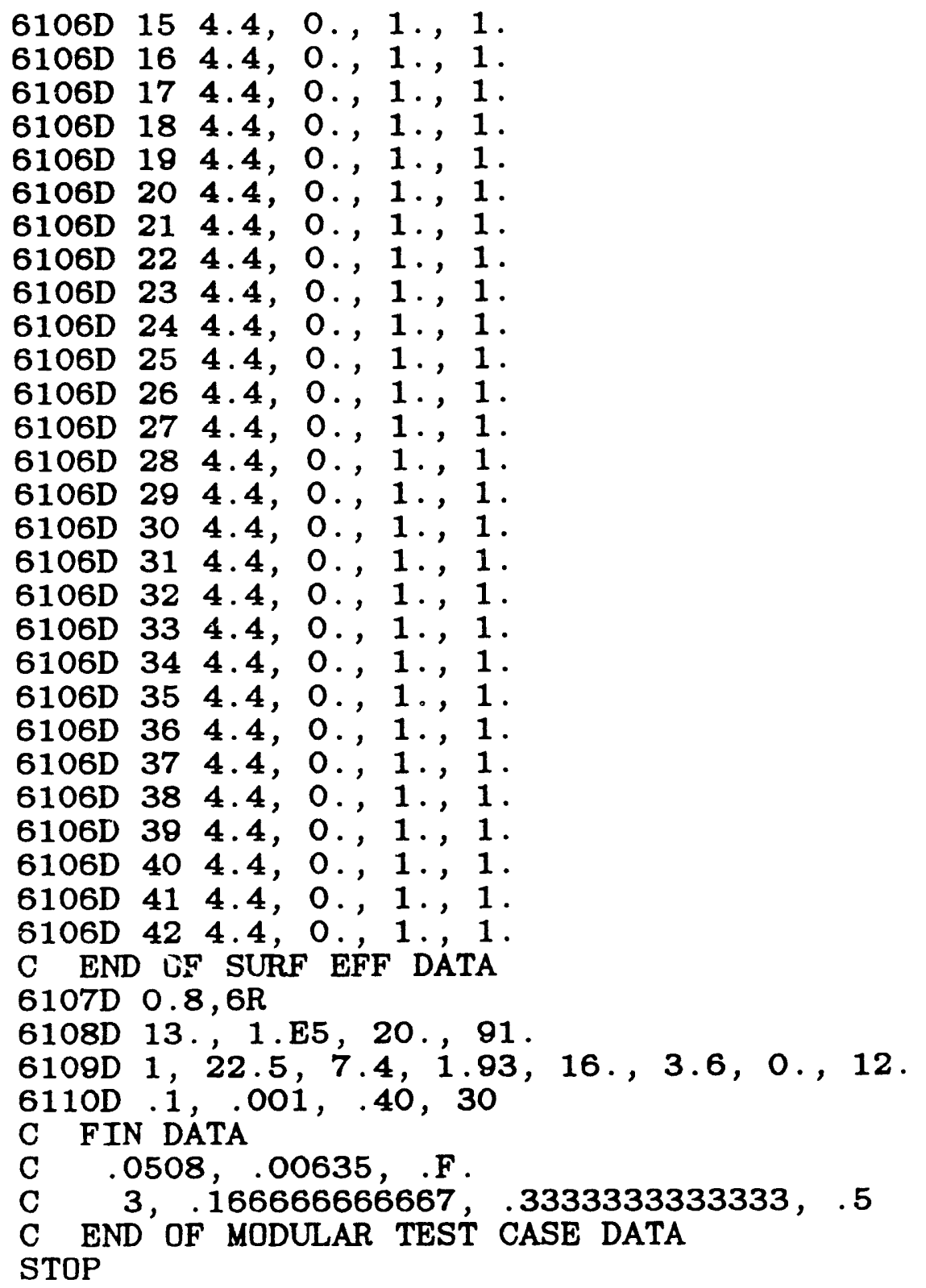


Input Deck for THATCH Sample Case No 2

THPRTST

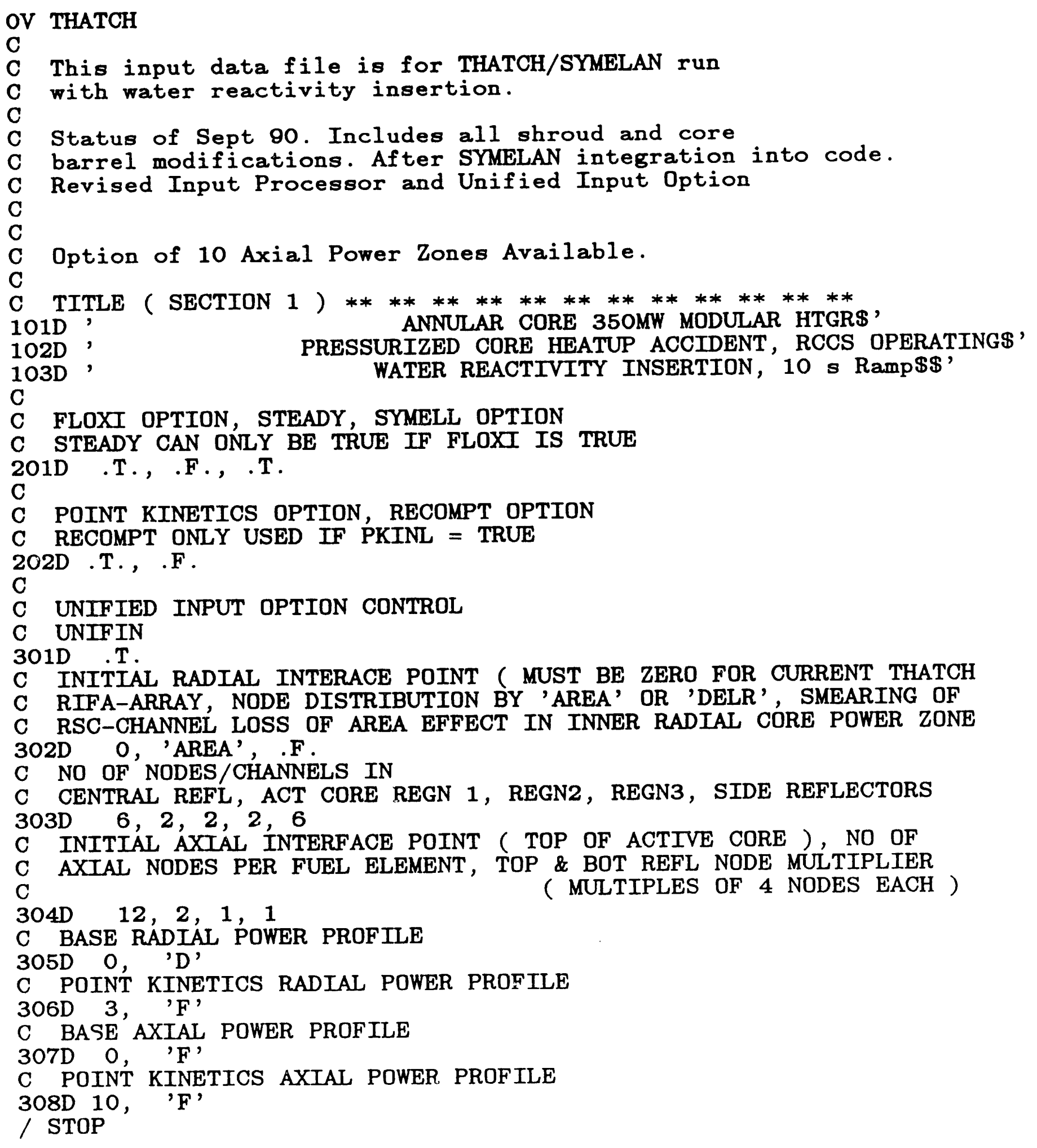



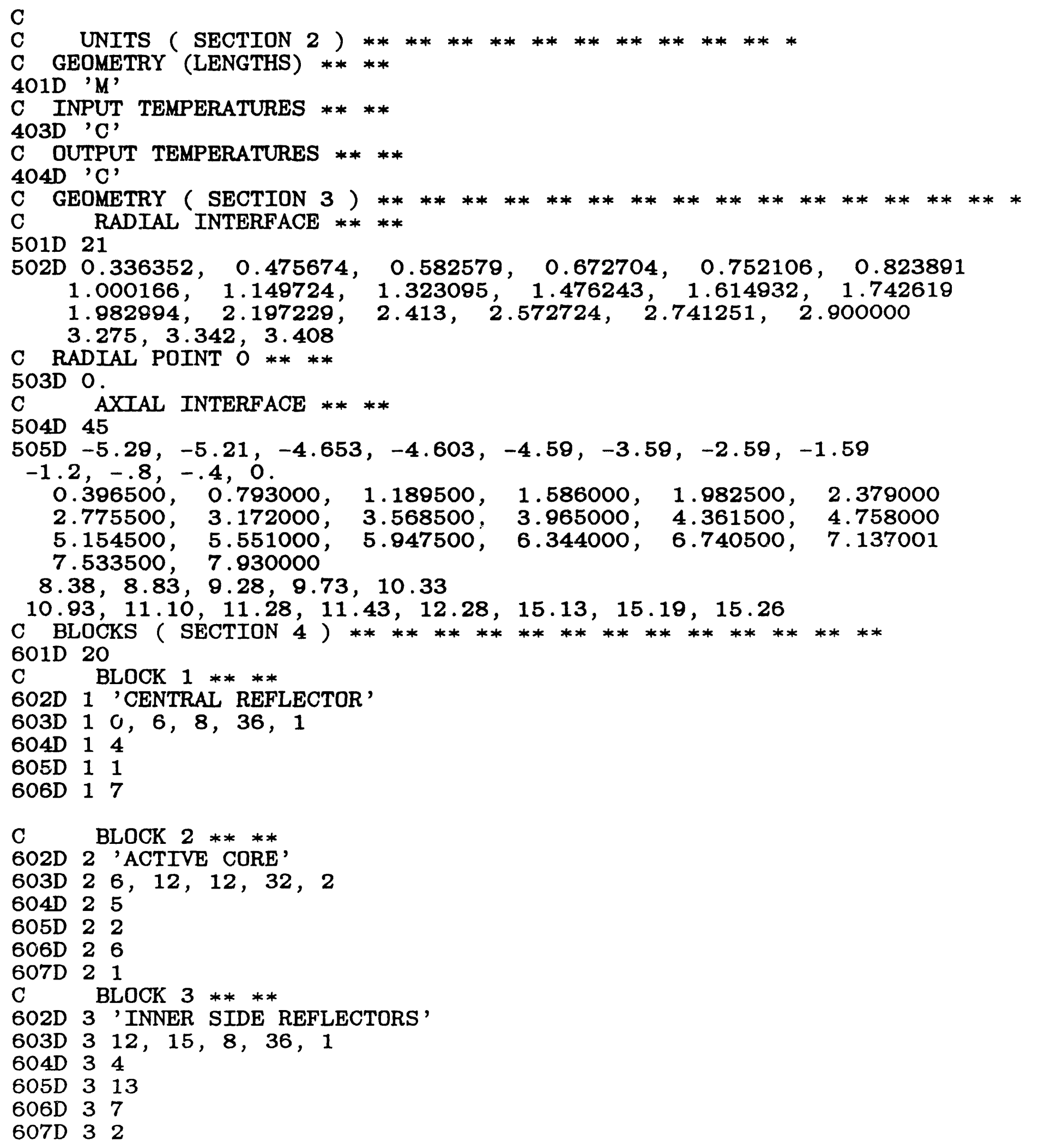


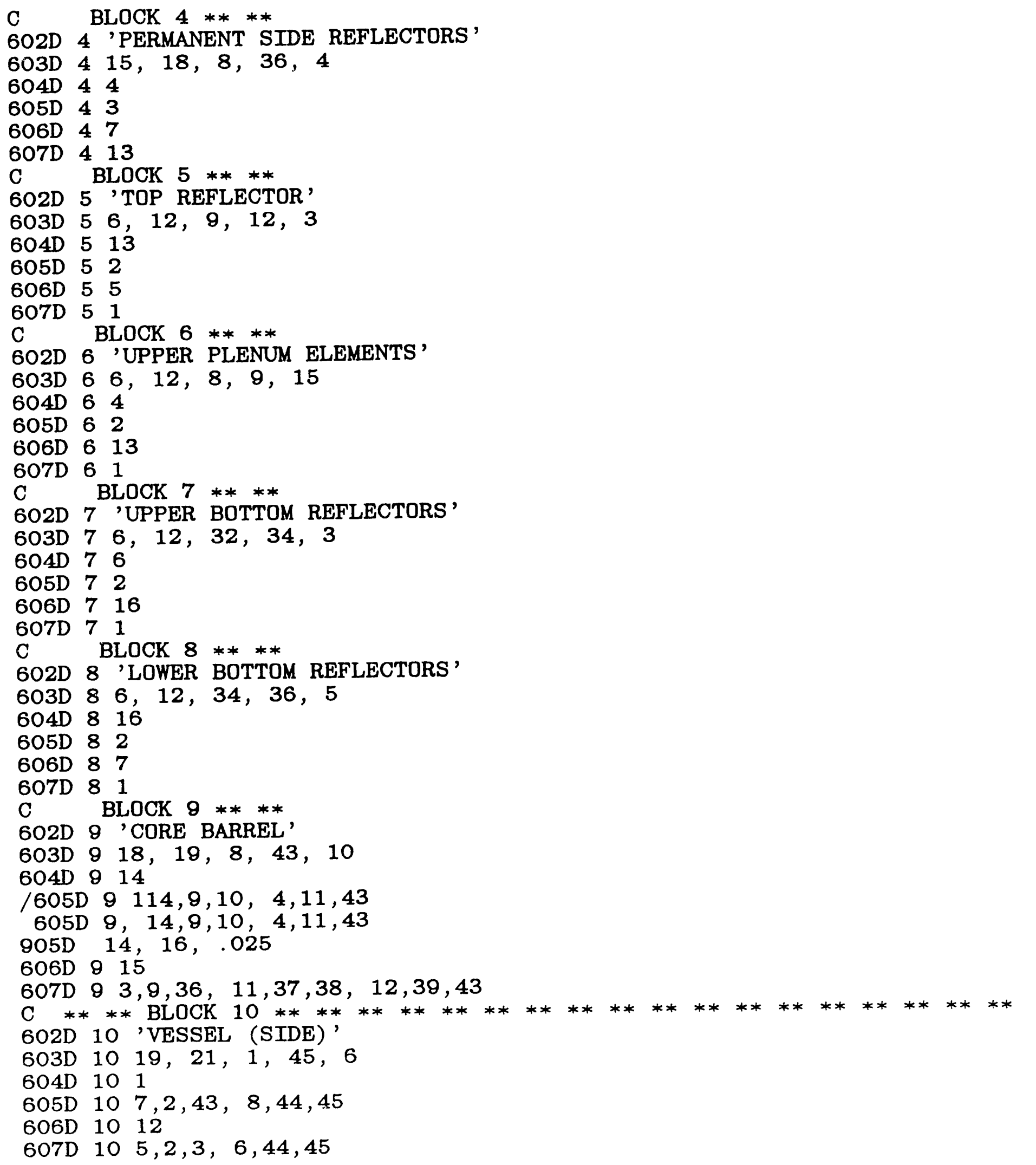




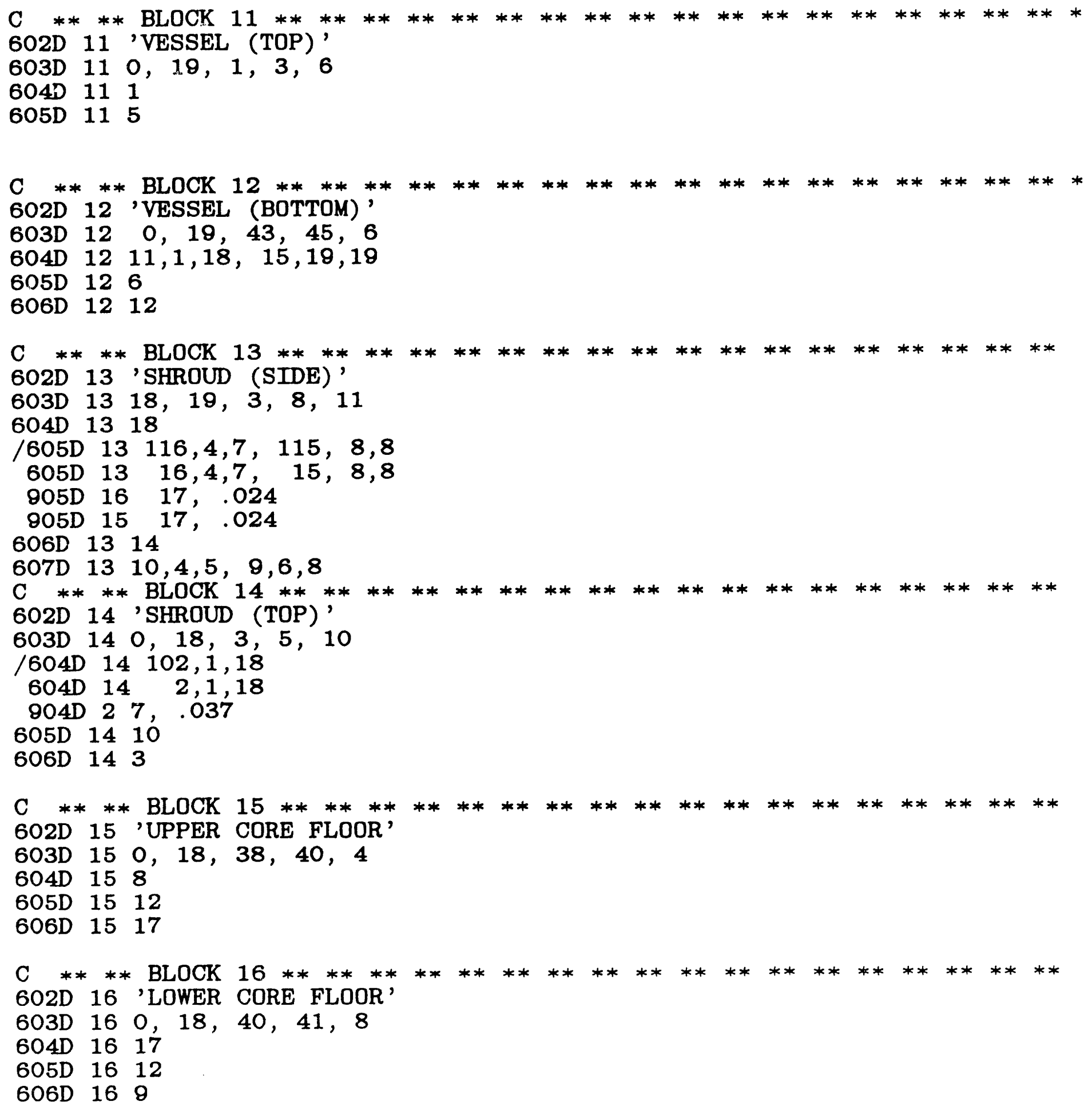




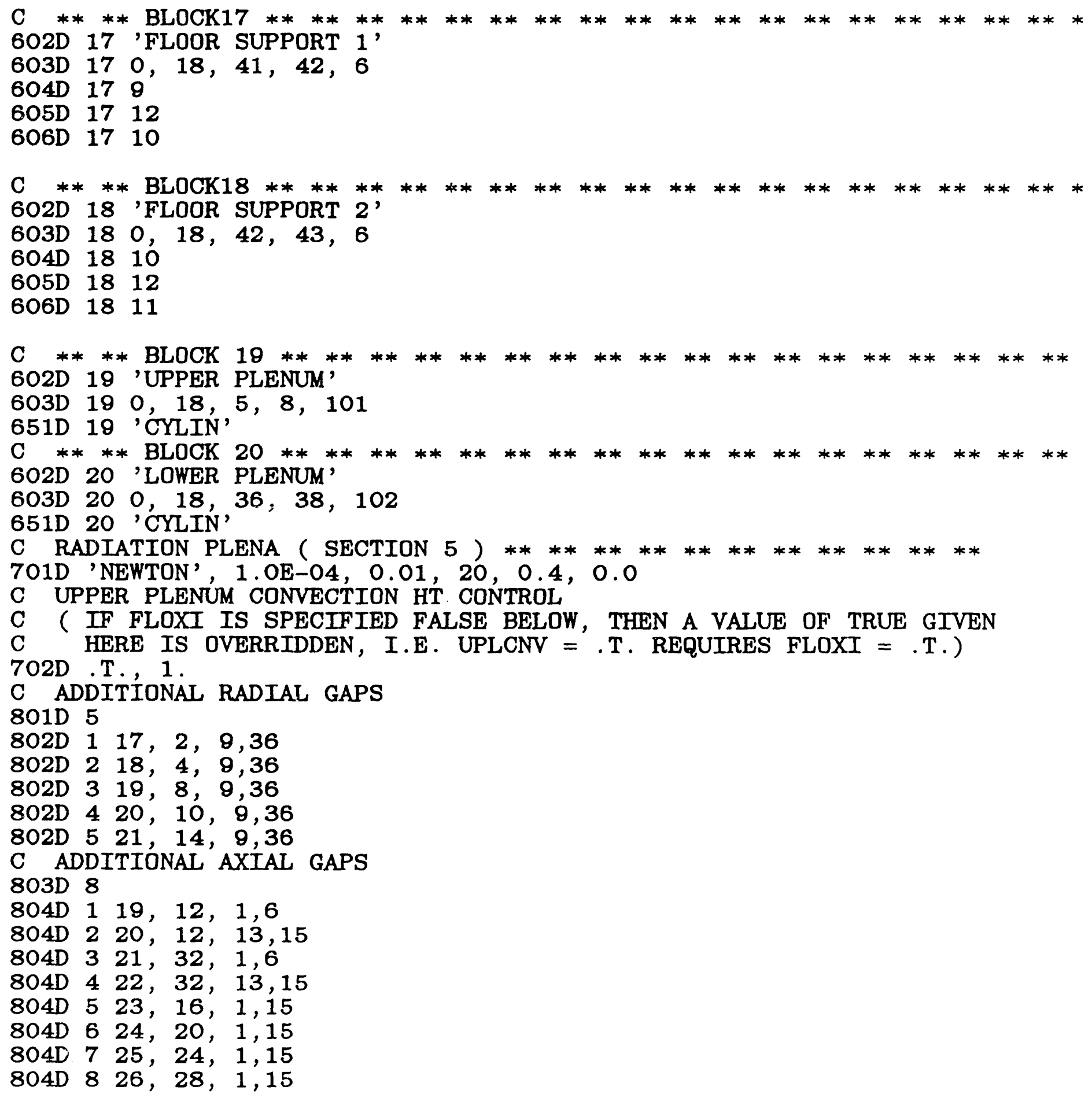


Page 6

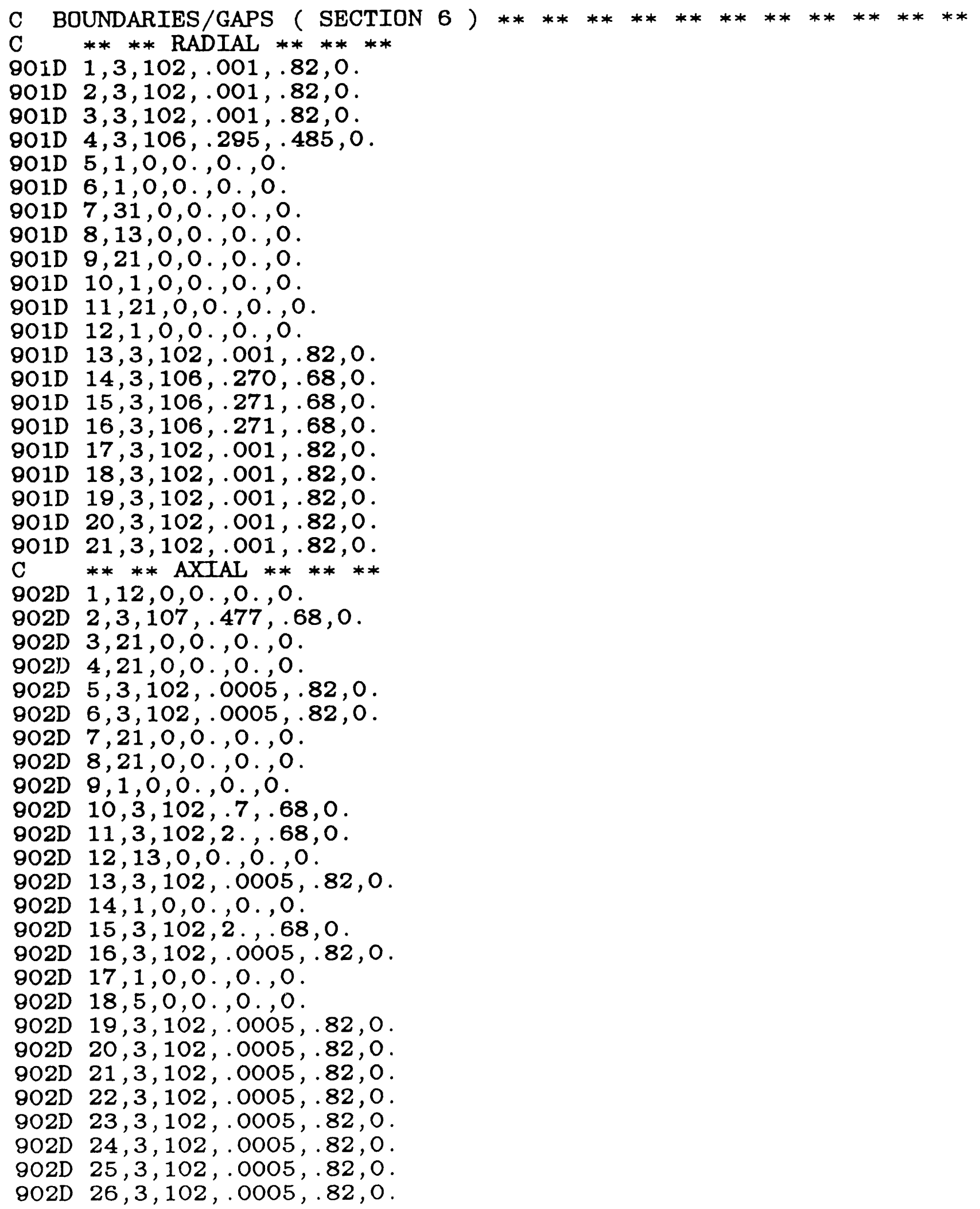


C IRRADIATION AND ANNEALING DATA

C IF FIRST PARAMETER ( IRRADN IS FALSE, THEN THIS SECTION IS BYPASSED) $1001 \mathrm{D} . \mathrm{T}$.

$1002 \mathrm{D} . \mathrm{T}$.

1003 D 3

C ASSUMED BASE CASE IRRADIATION DAMAGE

$1004 \mathrm{D} 3,15,12,33$

$1005 \mathrm{D} 120.0,4 \mathrm{R}, 1.0,6 \mathrm{R}, 0.0,3 \mathrm{R} /$

$1005 \mathrm{D} 130.6,2 \mathrm{R}, 0.95,2 \mathrm{R}, 1.0,6 \mathrm{R}, .85,0.6,2 \mathrm{R}$

$1005 \mathrm{D} 140.6,2 \mathrm{R}, 0.95,2 \mathrm{R}, 1.0,6 \mathrm{R}, .95,0.6,2 \mathrm{R}$

$1005 \mathrm{D} 150.6,2 \mathrm{R}, 0.95,2 \mathrm{R}, 1.0,6 \mathrm{R}, .95,0.6,2 \mathrm{R}$

$1005 \mathrm{D} 160.6,2 \mathrm{R}, 0.95,2 \mathrm{R}, 1.0,6 \mathrm{R}, .95,0.6,2 \mathrm{R}$

$1005 \mathrm{D} 170.6,2 \mathrm{R}, 0.95,2 \mathrm{R}, 1.0,6 \mathrm{R}, .95,0.6,2 \mathrm{R}$

$1005 \mathrm{D} 180.6,2 \mathrm{R}, 0.85,2 \mathrm{R}, 1.0,6 \mathrm{R}, .95,0.6,2 \mathrm{R}$

$1005 \mathrm{D} 190.6,2 \mathrm{R}, 0.95,2 \mathrm{R}, 1.0,6 \mathrm{R}, .95,0.6,2 \mathrm{R}$

$1005 \mathrm{D} 200.6,2 \mathrm{R}, 0.95,2 \mathrm{R}, 1.0,6 \mathrm{R}, .95,0.6,2 \mathrm{R}$

$1005 \mathrm{D} 210.6,2 \mathrm{R}, 0.95,2 \mathrm{R}, 1.0,6 \mathrm{R}, .95,0.6,2 \mathrm{R}$

$1005 \mathrm{D} 220.6,2 \mathrm{R}, 0.95,2 \mathrm{R}, 1.0,6 \mathrm{R}, .85,0.6,2 \mathrm{R}$

$1005 \mathrm{D} 230.6,2 \mathrm{R}, 0.95,2 \mathrm{R}, 1.0,6 \mathrm{R}, .95,0.6,2 \mathrm{R}$

$1005 \mathrm{D} 240.6,2 \mathrm{R}, 0.95,2 \mathrm{R}, 1.0,6 \mathrm{R}, .95,0.6,2 \mathrm{R}$

$1005 \mathrm{D} 250.6,2 \mathrm{R}, 0.85,2 \mathrm{R}, 1.0,6 \mathrm{R}, .95,0.6,2 \mathrm{R}$

$1005 \mathrm{D} 260.6,2 \mathrm{R}, 0.95,2 \mathrm{R}, 1.0,6 \mathrm{R}, .95,0.6,2 \mathrm{R}$

$1005 \mathrm{D} 270.6,2 \mathrm{R}, 0.95,2 \mathrm{R}, 1.0,6 \mathrm{R}, .95,0.6,2 \mathrm{R}$

$1005 \mathrm{D} 280.6,2 \mathrm{R}, 0.95,2 \mathrm{R}, 1.0,6 \mathrm{R}, .95,0.6,2 \mathrm{R}$

$1005 \mathrm{D} 290.6,2 \mathrm{R}, 0.95,2 \mathrm{R}, 1.0,6 \mathrm{R}, .95,0.6,2 \mathrm{R}$

$1005 \mathrm{D} 300.6,2 \mathrm{R}, 0.95,2 \mathrm{R}, 1.0,6 \mathrm{R}, .95,0.6,2 \mathrm{R}$

$1005 \mathrm{D} 310.6,2 \mathrm{R}, 0.95,2 \mathrm{R}, 1.0,6 \mathrm{R}, .85,0.6,2 \mathrm{R}$

$1005 \mathrm{D} 320.6,2 \mathrm{R}, 0.95,2 \mathrm{R}, 1.0,6 \mathrm{R}, .95,0.6,2 \mathrm{R}$

$1005 \mathrm{D} 330.0,4 \mathrm{R}, 0.82,6 \mathrm{R}, 0.0,3 \mathrm{R} /$

$1006 \mathrm{D} 1000 ., 1300$.

C

C **** AFTERHEAT DATA (SECTION 7 )

C INITIAL POWER ** *************************

1101D 350.E6, 0., 1.4, 1., 2

C AFTERHEAT REGION ** ** **********************

$1102 \mathrm{D} 7,12,13,32$

C REFLECTOR REGION ** ************************

$1103 \mathrm{D} 1,15,10,34$

C ************AFTER HEAT PROFILE DATA*****************i***

C BASE RADIAL POWER PROFILE ( IHI - (ILO-1) ENTRIES )

$1104 \mathrm{D} 1.0,6 \mathrm{R} /$

C POINT KINETICS RADIAL POWER PROFILE ( IHI - (ILO-1) ENTRIES )

$1105 \mathrm{D} 0.7121,2 \mathrm{R}, 1.053,2 \mathrm{R}, 1.163,2 \mathrm{R} /$

C BASE AXIAL POWER PROFILE ( JHI - ( $\mathrm{LO}-1$ ) ENTRIES )

$1106 \mathrm{D} 1.36,10 \mathrm{R}, 0.77,6 \mathrm{R}, 0.445,4 \mathrm{R} /$

C POINT KINETICS AXIAL POWER PROFILE ( IHI - (ILO-1) ENTRIES )

$1107 \mathrm{D} 1.36,10 \mathrm{R}, 0.77,6 \mathrm{R}, 0.445,4 \mathrm{R} /$ 


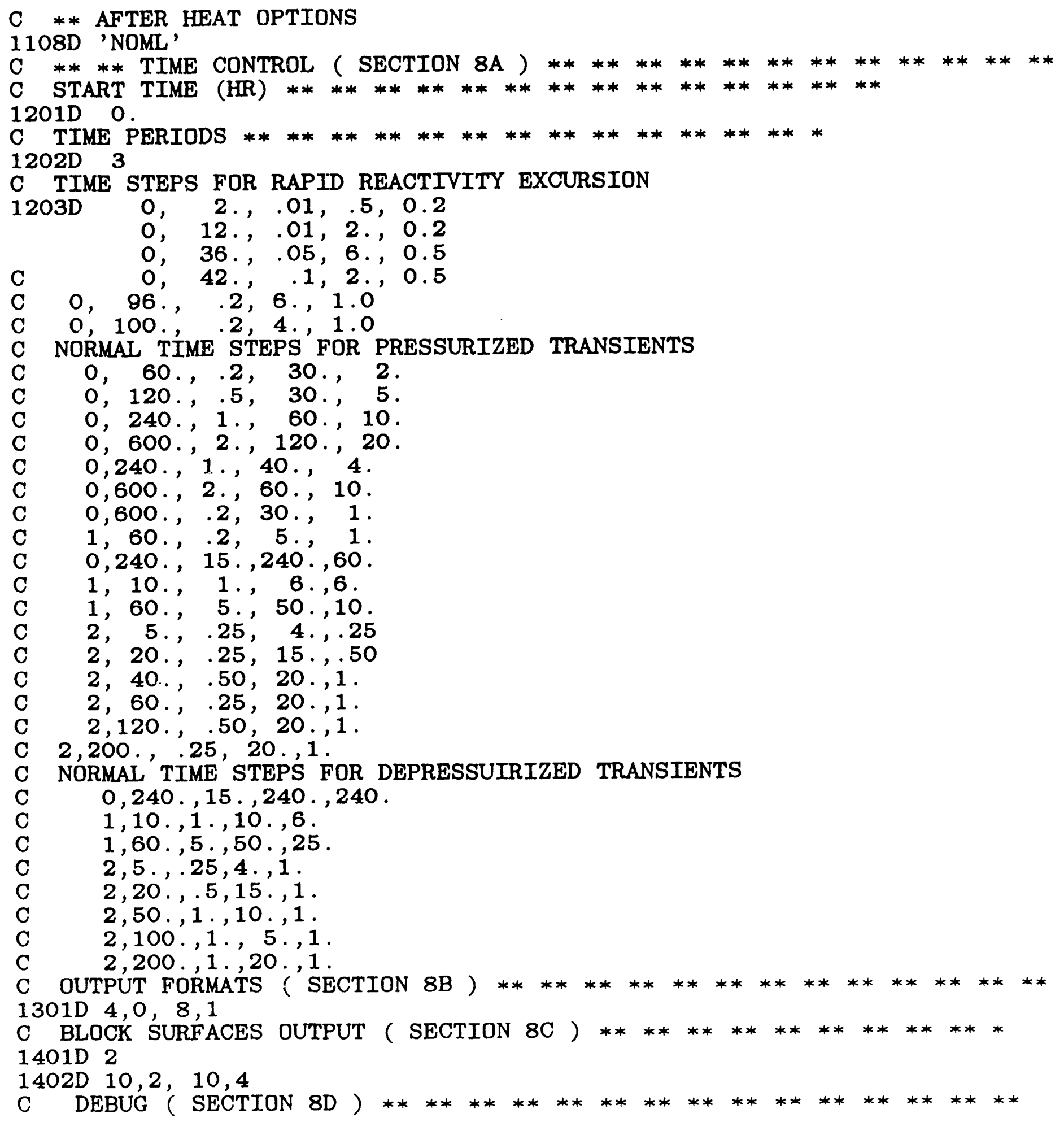


C DEBUG CONTROL VARIABLE

1403D $O$

C DEBUG SET DATA ( BYPASS AT THIS TIME, SETTING NO OF SETS $=0$ / 0 C ** ** INITIAL TEMPERATURES ( SECTION 9 )

1501D 'READRUN'

C 'OTHER'

$\begin{array}{ll}1502 \mathrm{D} & 100.0,21 \mathrm{R} \\ 1503 \mathrm{D} & 100.0,35 \mathrm{R}\end{array}$

$1503 \mathrm{D} 100.0,35 \mathrm{R}, 80.0,9 \mathrm{R}$

1504D 100.0,21R

C BLOCK TEMPERATURES ********************

1505D B1;24*258.

$\mathrm{B} 1 ; 6 * 268$.

$\mathrm{B} 1 ; 6 * 290$.

$\mathrm{B} 1 ; 6 * 312$.

$\mathrm{B} 1 ; 6 * 333$.

B1; $6 * 355$.

$\mathrm{B} 1 ; 6 * 376$.

B1; $6 * 397$.

B1; $6 * 419$.

B1; $6 * 440$.

$B 1 ; 6 * 462$.

B1; $6 * 483$.

B1; $6 * 505$.

B1; $6 * 526$.

B1; $6 * 548$.

B1; $6 * 569$.

B1; $6 * 590$.

B1; $6 * 612$.

B1; $6 * 633$.

B1; $6 * 655$.

B1; $6 * 676$.

B1; $24 * 687$.

B2; $6 * 269$.

B2; $6 * 290$.

B2; $6 * 312$.

B2; $6 * 333$.

B2; $6 * 355$.

B2; $6 * 376$.

B2; $6 * 397$.

B2; $6 * 419$.

B2; $6 * 440$.

B2; $6 * 462$.

B2; $6 * 483$.

B2; $6 * 505$.

B2; $6 * 526$.

B2; $6 * 548$.

B2; $6 * 569$.

B2 ;6*590. 


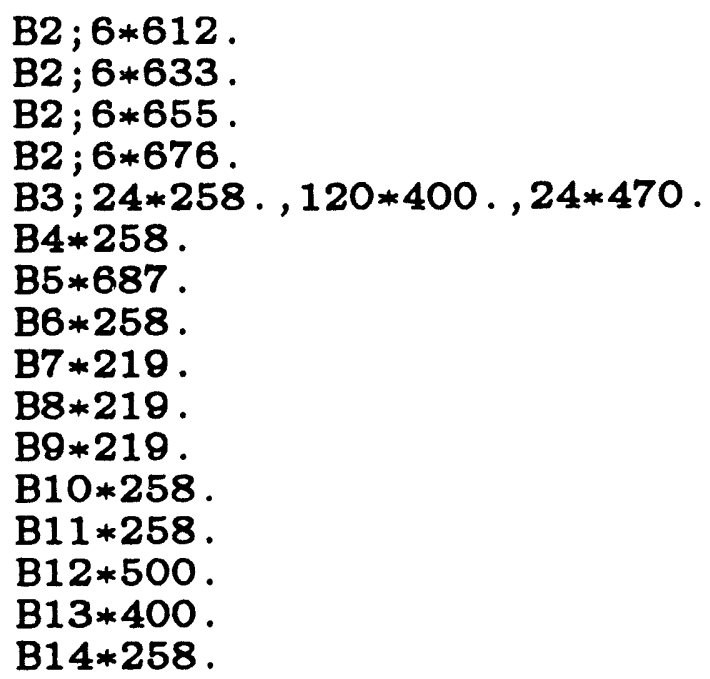




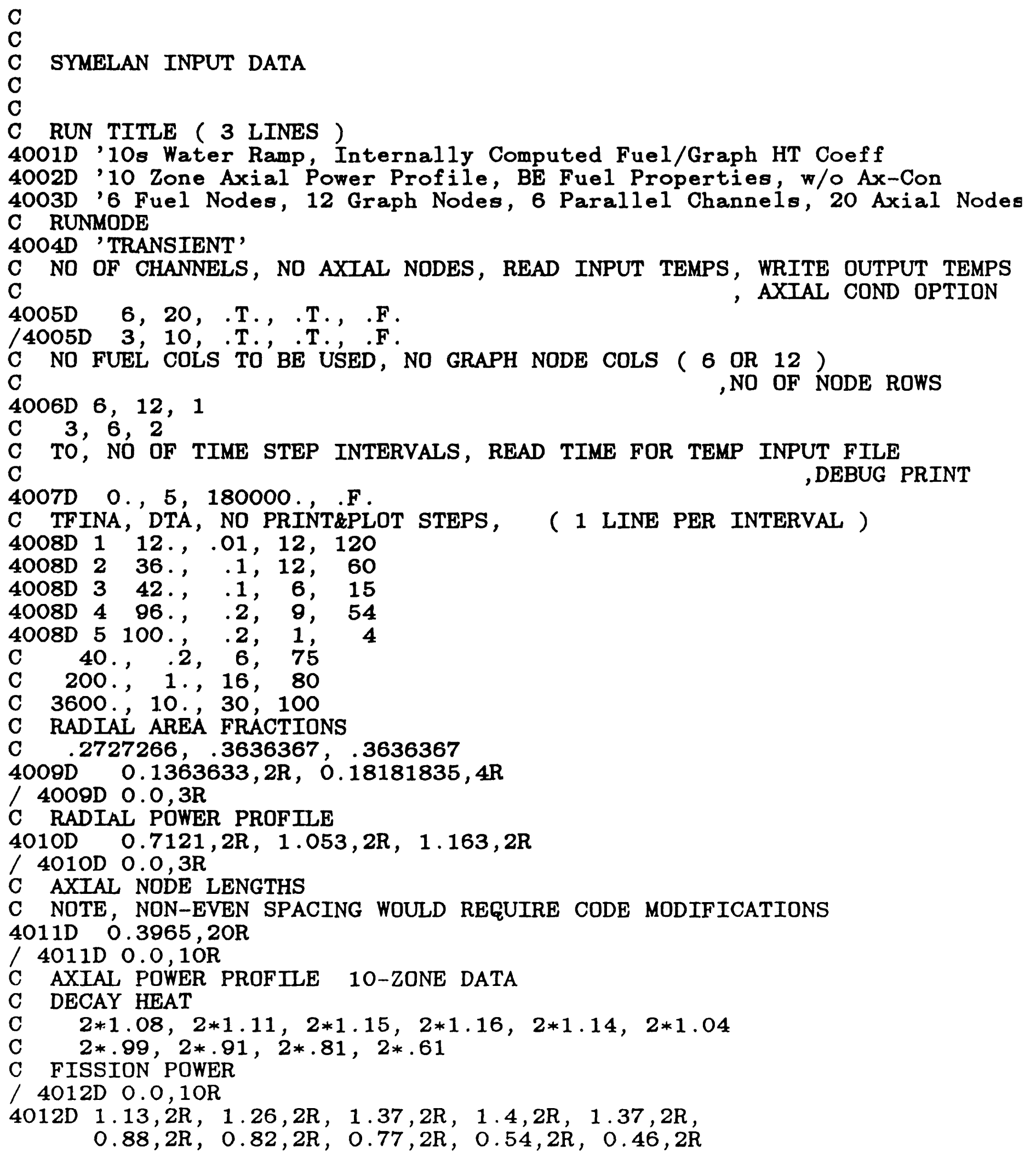


C AXIAL POWER PROFILE 3-ZUIVE DATA

C $\quad 10 * 1.36,6 * .77,4 * .445$

C BASE POWER, INLET MASS FLOW, INLET FLUID TEMP, HT COEFF REDUCTION, C FUEL / GRAPHITE GAP (mm), GAP EMISS, AVG REFLECTOR TEMP (K) ( FOR USE C IN FREE STANDING SYMEL RUNS ONLY)

$4013 \mathrm{D} 350 . \mathrm{E} 6,140 ., 259 ., .6545, .127 \mathrm{E}-3, .81,745.4$

C TEMPERATURE ARRAY FOR HOT FRACTIONS

4014D 1200., 1400., 1600., 1800.

C POINT KINETICS ODTION ( FOR USE WITHIN SYMEL )

4015D . T.

C

C

C

C

POIINT KINETICS INPUT DATA

COMPMOD, RHOMODE, CORAVG, IKINMX, EPSO, PSOURCO, POWCOR, DTHO, VARYIN

FOR SLOW REACTIVITY TRANSIENTS W/O SYMELAN USE POWCOR $=.94$ AND

INCLUDE DECAY HEAT.

FOR RAPID REACTIVITI EXCURSIONS, USE POWCOR $=1.0$, AND NELECT

C DECAY HEAT

5001D 'ITERATE', 'INTEGR', .F . , 20, 1.E-7, 1.E-9, 1.0, 0 .

C ODE INTEFGRATION CONTROL'

C INTMTHO, MTHGEAR, MITER

5003D 'DIVPAG', 2,1

C WATER REACTIVITY INSERTION, BEGIN TIME, RAMP TIME, PARAM COEFFICNT

C NOTE THAT END OF RAMP REACTIVITY IS NOW COMPUTED AND

C INPUT VALUE IS NO LONGER USED

5002D O., 10., 1 .

C OPTIONAL OVERRIDE OF POINT KINETICS DATA IF VARYIN = TRUE

C SVARYINP

C SIGAS. $\perp M=12.32 \mathrm{E}-25$

C \$END

C

C

C AIR COOLER INPUT DATA

6101D 'FIN PERFORMANCE COEFFICIENTS ARE INPUT'

6102D .T., .F., .F., 'BNL'

$6103 \mathrm{D} 1,43,9$

$6104 \mathrm{D} 3.408,4.316,4.326,4.4784,4.4884,4.85$

$6105 \mathrm{D} 3.95,7.8, .0762, .54$

C SURFACE EFFECTIVENESS DATA
6106D $11.0,4 \mathrm{R}$
$6106 \mathrm{D} 221.0,4 \mathrm{R}$
6106D $31.0,4 \mathrm{R}$
$6106 \mathrm{D} 41.0,4 \mathrm{R}$
$6106 \mathrm{D} 5 \mathrm{1}, 0,4 \mathrm{R}$
6106D $61.0,4 \mathrm{R}$
6106D 7 1.0,4R
$6106 \mathrm{D} 84.4,0 ., 1 ., 1$. 


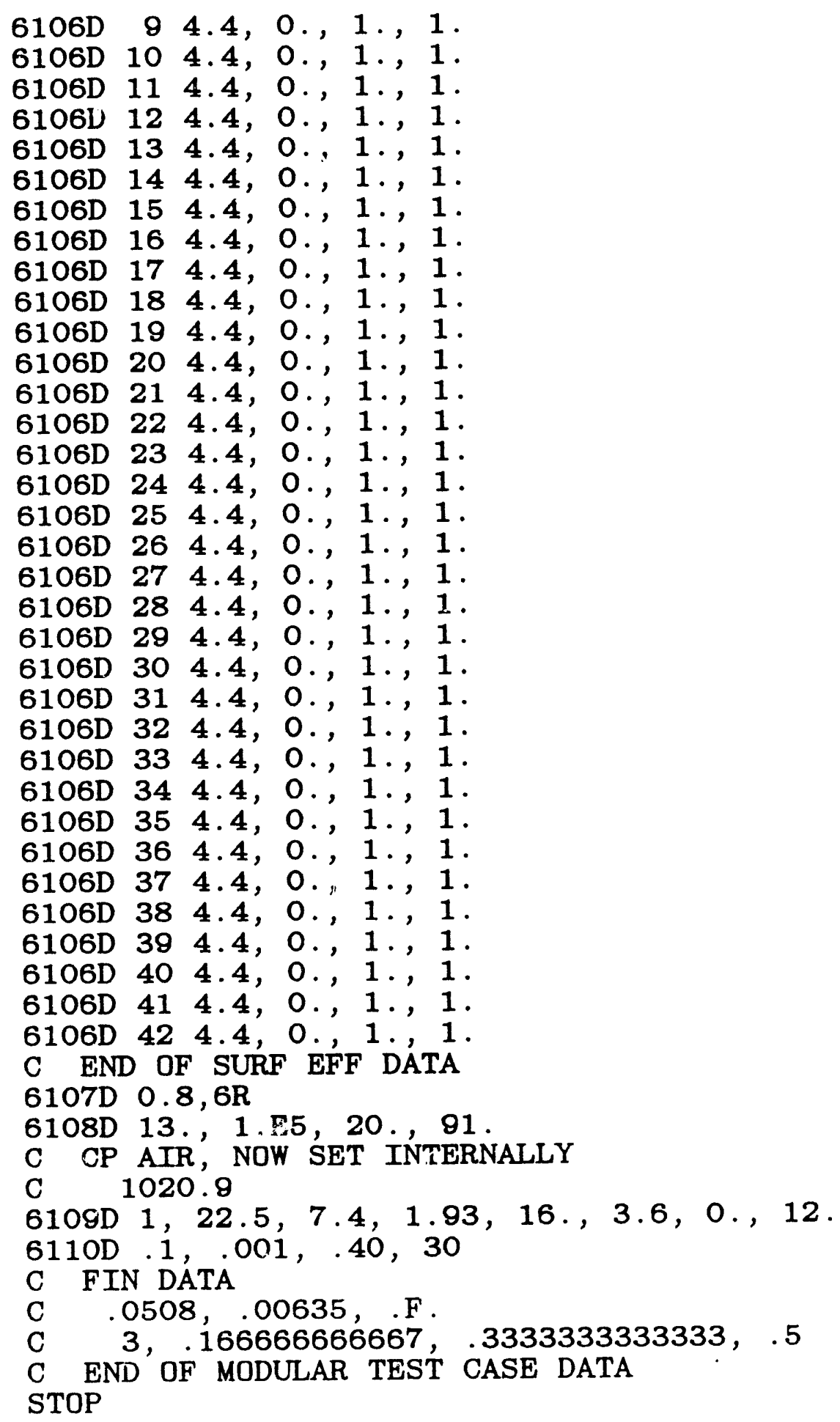


THBASARR

\section{OV THATCH}

C

C

This input data file is for AIR INGRESS SIMULATION

Status of Sept 90 . Includes all shroud and core

barrel modifications. After SYMELAN integration into code.

Revised Input Processor and Unified Input Option

C

C

C

C TITLE ( SECTION 1 ) ***************************

101D' ANNULAR CDRE 350MW MODULAR HTGR\$' 102D ', DEPRESSURIZED CORE HEATUP ACCIDENT, RCCS OPERATING\$
103D

C

C FLOXI OPTION, STEADY, SYMELL OPTION

C STEADY CAN ONLY BE TRUE IF FLOXI IS TRUE

2010 .T., .F., .F.

$\mathrm{C}$

C POINT KINETICS OPTION, RECOMPT OPTION

C RECOMPT ONLY USED IF PKINL = TRUE 2O2D .F., .F.

$\mathrm{C}$

C UNIFIED INPUT OPTION CONTROL

C UNIFIN

301D.T.

C INITIAL RADIAL INTERACE POINT ( MUST BE ZERO FOR CURRENT THATCH

C RIFA-ARRAY, NODE DISTRIBUTION BY 'AREA' OR 'DELR', SMEARING OF

C RSC-CHANNEL LOSS OF AREA EFFECT IN INNER RADIAL CORE POWER ZONE 302D O, 'AREA', .F.

C NO OF NODES/CHANNELS IN

C CENTRAL REFL, ACT CORE REGN 1, REGN2, REGN3, SIDE REFLECTORS 303D $6,2,2,2,6$

C INITIAL AXIAL INTERFACE POINT ( TOP OF ACTIVE CORE), NO OF

C AXIAL NODES PER FUEL ELEMENT, TOP \& BOT REFL NODE MULTIPLIER $\mathrm{C}$

304D $12,2,1,1$

C BASE RADIAL POWER PROFILE

305D 1 , 'D'

C POINT KINETICS RADIAL POWER PROFILE

306D O, 'F'

C BASE AXIAL POWER PROFILE

307D 3, 'D'

C POINT KINETICS AXIAL POWER PROFILE

308D O, 'F' 


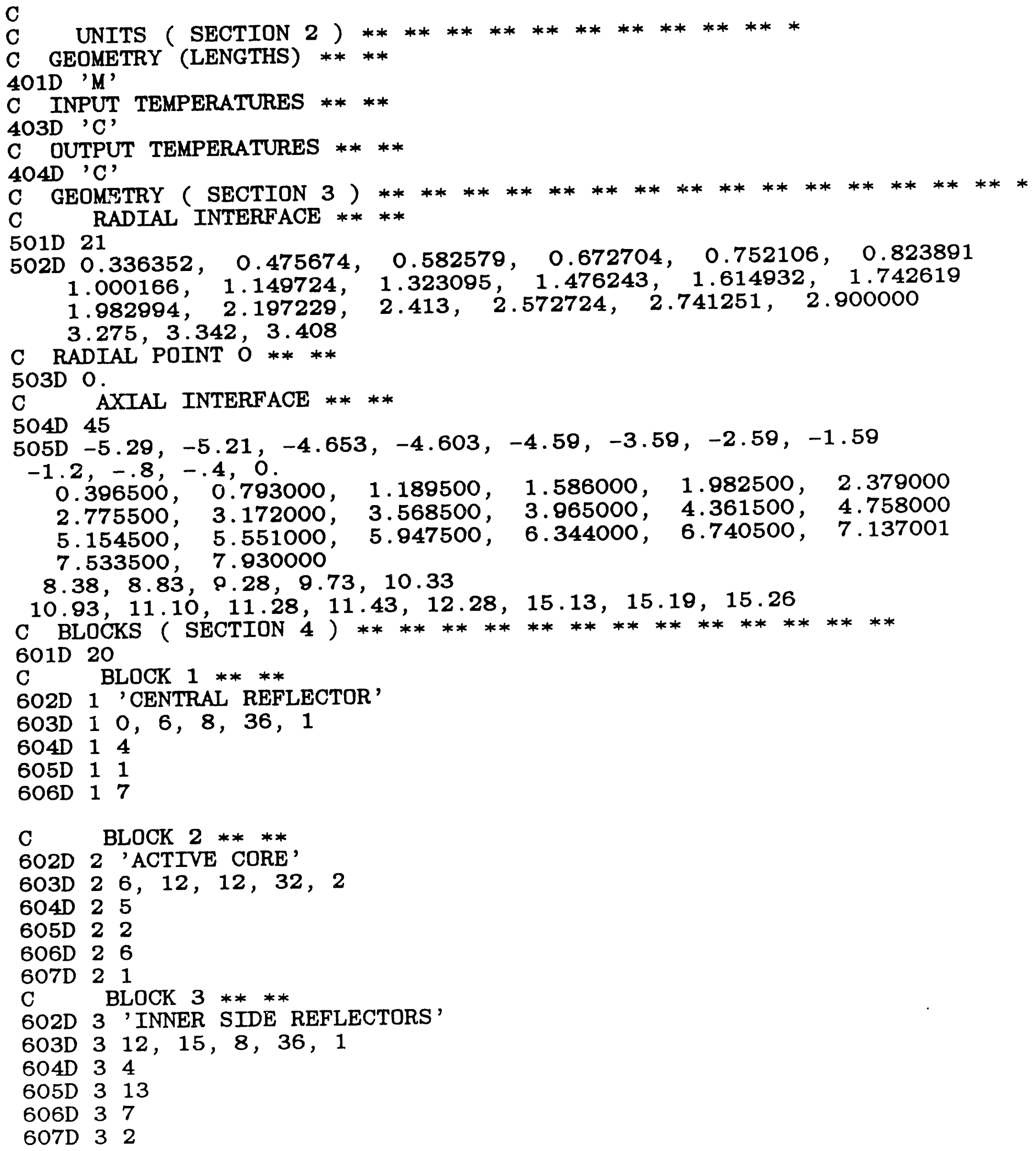




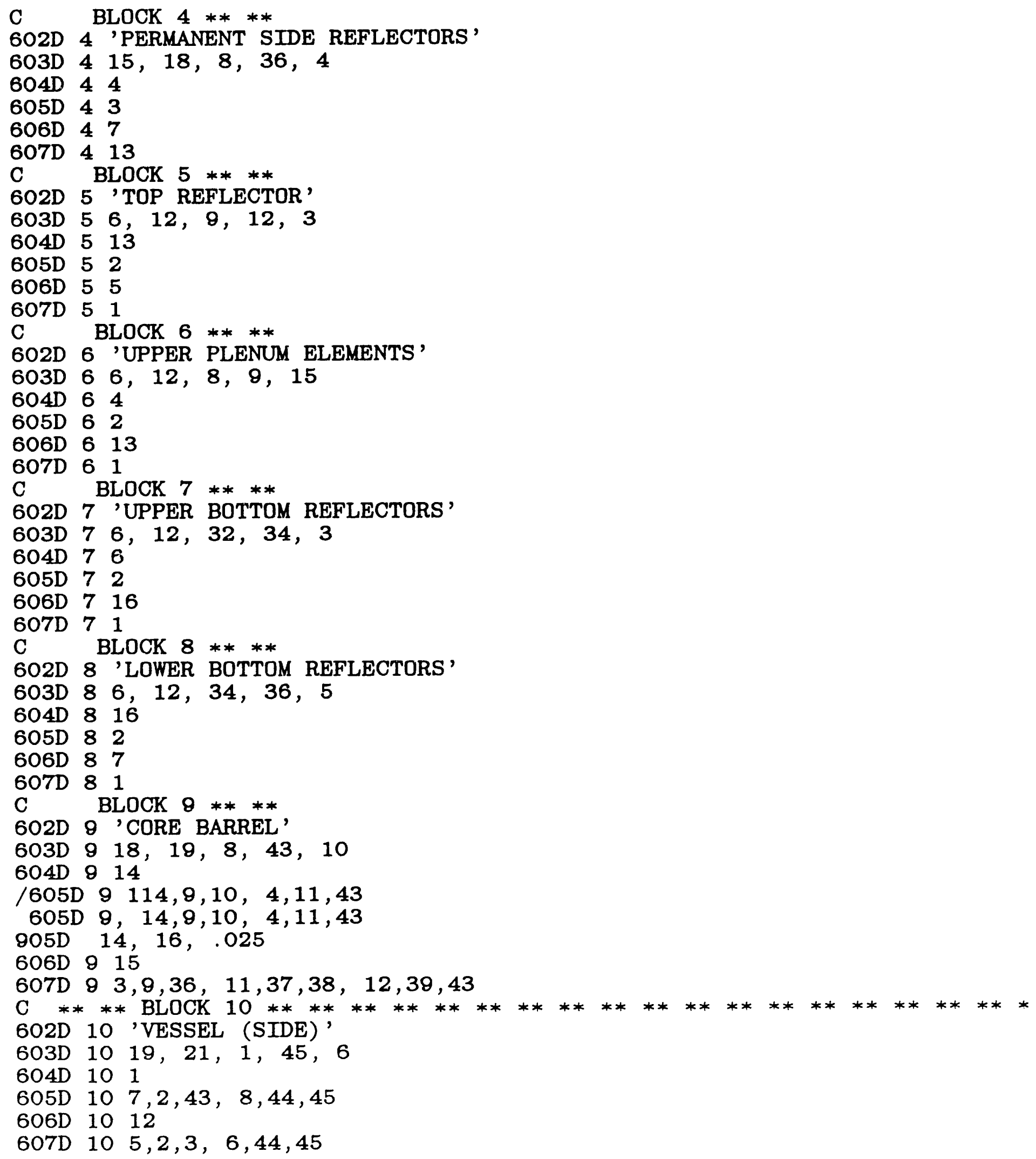


$\mathrm{C} * * * *$ BLOCK $11 * * * * * * * * * * * * * * * * * * * * * * * * * * * * * * * * * * * *$ 602D 11 'VESSEL (TOP)'

603D $110,19,1,3,6$

604D 111

605D 115

$\mathrm{C} * * * * \mathrm{BLOCK} 12 * * * * * * * * * * * * * * * * * * * * * * * * * * * * * * * * * * * *$ 602D 12 'VESSEL (BOTTOM)'

$603 \mathrm{D} 120,19,43,45,6$

$604 \mathrm{D} 1211,1,18,15,19,19$

605D 126

606D 1212

$\mathrm{C} * * * * \mathrm{BLOCK} 13 * * * * * * * * * * * * * * * * * * * * * * * * * * * * * * * * * * * *$

602D 13 'SHROUD (SIDE)'

$603 \mathrm{D} 1318,19,3,8,11$

$604 \mathrm{D} 13 \quad 18$

/605D $13116,4,7,115,8,8$

$605 \mathrm{D} \quad 1316,4,7,15,8,8$

905D $16 \quad 17, .024$

$905 \mathrm{D} \quad 15 \quad 17, .024$

606D $13 \quad 14$

607D $1310,4,5,9,6,8$

C ** ** BLOCK $14 * * * * * * * * * * * * * * * * * * * * * * * * * * * * * * * * * * * *$ 6O2D 14 'SHROUD (TOP)'

$603 \mathrm{D} 140,18,3,5,10$

$/ 604 \mathrm{D} \quad 14102,1,18$

$604 \mathrm{D} \quad 14 \quad 2,1,18$

$904 \mathrm{D} 27, .037$

605D 1410

606D 143

$\mathrm{C} * * * * \mathrm{BLOCK} 15 * * * * * * * * * * * * * * * * * * * * * * * * * * * * * * * * * * * *$ 602D 15 'UPPER CORE FLOOR'

$603 \mathrm{D} 150,18,38,40,4$

$604 D 158$

$605 \mathrm{D} \quad 15 \quad 12$

$606 \mathrm{D} \quad 15 \quad 17$

$\mathrm{C} * * * * \mathrm{BLOCK} 16 * * * * * * * * * * * * * * * * * * * * * * * * * * * * * * * * * * * *$ 6O2D 16 'LOWER CORE FLOOR'

$603 \mathrm{D} 160,18,40,41,8$

$\begin{array}{lll}604 \mathrm{D} & 16 & 17\end{array}$

605D $16 \quad 12$

606D 169 


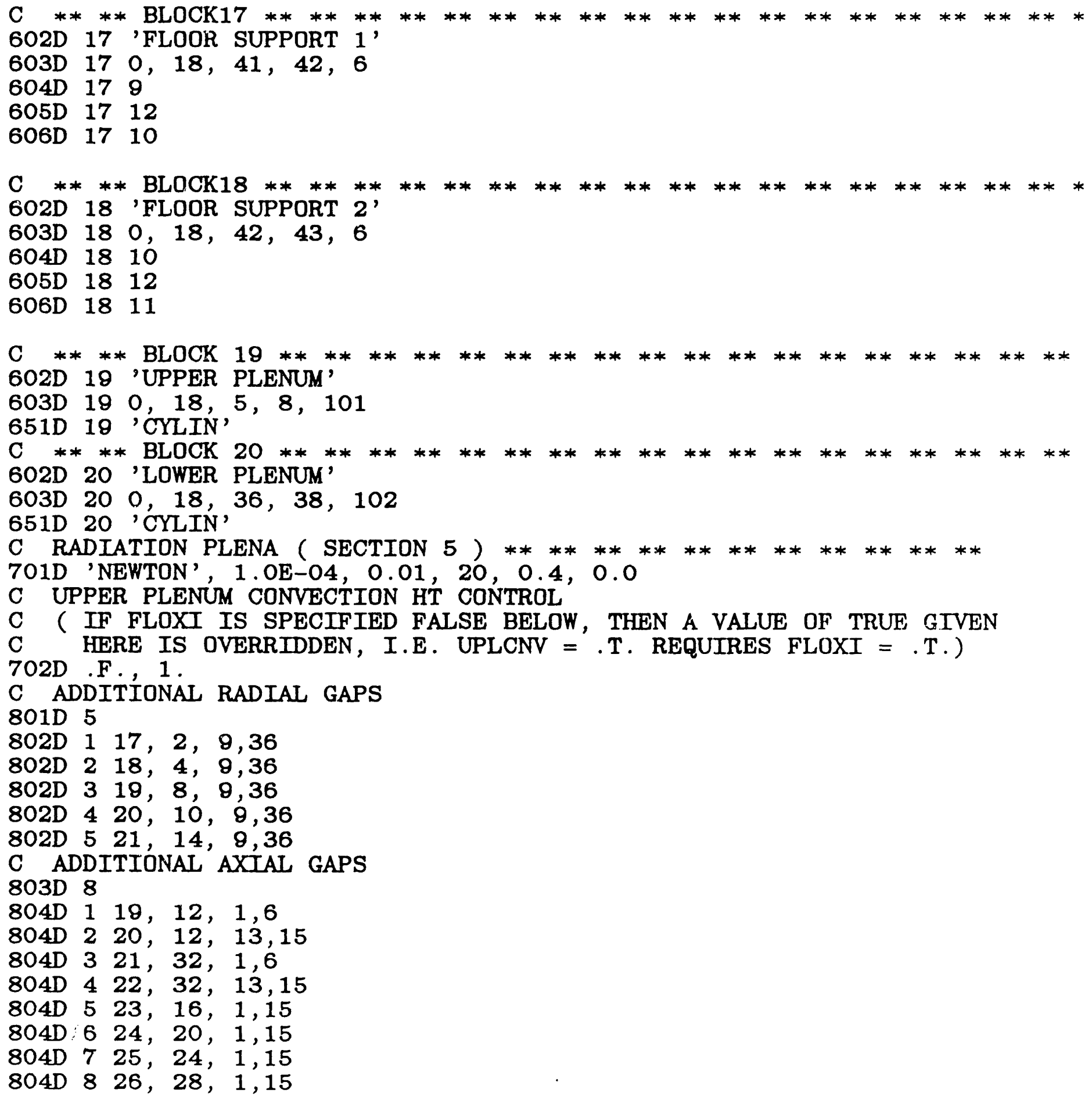




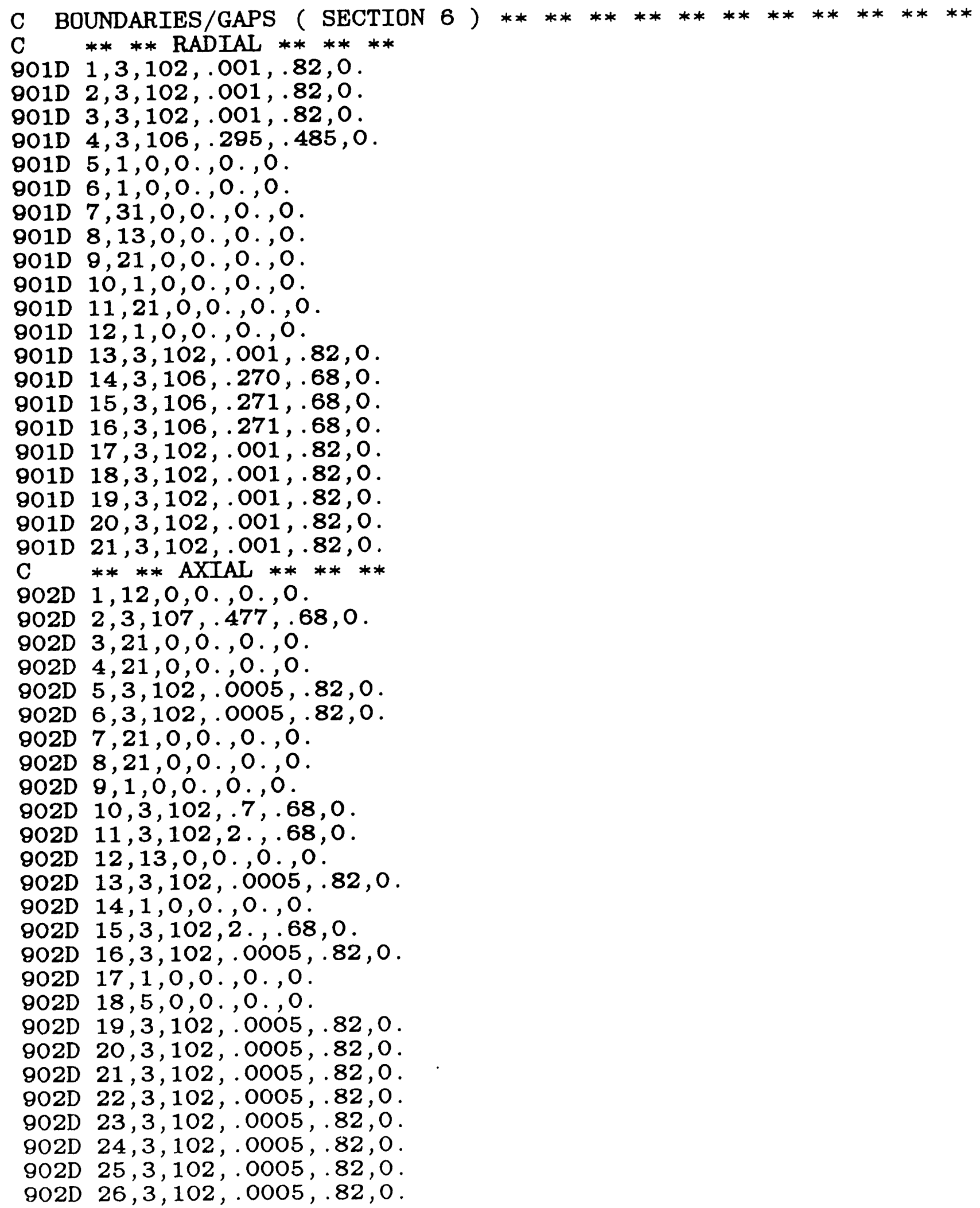


C IRRADIATION AND ANNEALING DATA

C IF FIRST PARAMETER ( IRRADN IS FALSE, THEN THIS SECTION IS BYPASSED) $1001 \mathrm{D} . \mathrm{T}$.

$1002 \mathrm{D} . \mathrm{T}$.

$1003 \mathrm{D} 3$

C ASSUMED BASE CASE IRRADIATION DAMAGE

$1004 \mathrm{D} 3,15,12,33$

$1005 \mathrm{D} 120.0,4 \mathrm{R}, 1.0,6 \mathrm{R}, 0.0,3 \mathrm{R} /$

$1005 \mathrm{D} 130.6,2 \mathrm{R}, 0.95,2 \mathrm{R}, 1.0,6 \mathrm{R}, .95,0.6,2 \mathrm{R}$

$1005 \mathrm{D} 140.6,2 \mathrm{R}, 0.95,2 \mathrm{R}, 1.0,6 \mathrm{R}, .95,0.6,2 \mathrm{R}$

$1005 \mathrm{D} 150.6,2 \mathrm{R}, 0.95,2 \mathrm{R}, 1.0,6 \mathrm{R}, .95,0.6,2 \mathrm{R}$

$1005 \mathrm{D} 160.6,2 \mathrm{R}, 0.95,2 \mathrm{R}, 1.0,6 \mathrm{R}, .85,0.6,2 \mathrm{R}$

$1005 \mathrm{D} 170.6,2 \mathrm{R}, 0.95,2 \mathrm{R}, 1.0,6 \mathrm{R}, .85,0.6,2 \mathrm{R}$

$1005 \mathrm{D} 180.6,2 \mathrm{R}, 0.95,2 \mathrm{R}, 1.0,6 \mathrm{R}, .95,0.6,2 \mathrm{R}$

$1005 \mathrm{D} 190.6,2 \mathrm{R}, 0.95,2 \mathrm{R}, 1.0,6 \mathrm{R}, .95,0.6,2 \mathrm{R}$

$1005 \mathrm{D} 200.6,2 \mathrm{R}, 0.95,2 \mathrm{R}, 1.0,6 \mathrm{R}, .95,0.6,2 \mathrm{R}$

$1005 \mathrm{D} 210.6,2 \mathrm{R}, 0.95,2 \mathrm{R}, 1.0,6 \mathrm{R}, .95,0.6,2 \mathrm{R}$

$1005 \mathrm{D} 220.6,2 \mathrm{R}, 0.95,2 \mathrm{R}, 1.0,6 \mathrm{R}, .95,0.6,2 \mathrm{R}$

$1005 \mathrm{D} 230.6,2 \mathrm{R}, 0.95,2 \mathrm{R}, 1.0,6 \mathrm{R}, .95,0.6,2 \mathrm{R}$

$1005 \mathrm{D} 240.6,2 \mathrm{R}, 0.95,2 \mathrm{R}, 1.0,6 \mathrm{R}, .95,0.6,2 \mathrm{R}$

$1005 \mathrm{D} 250.6,2 \mathrm{R}, 0.95,2 \mathrm{R}, 1.0,6 \mathrm{R}, .95,0.6,2 \mathrm{R}$

$1005 \mathrm{D} 260.6,2 \mathrm{R}, 0.95,2 \mathrm{R}, 1.0,6 \mathrm{R}, .95,0.6,2 \mathrm{R}$

$1005 \mathrm{D} 270.6,2 \mathrm{R}, 0.95,2 \mathrm{R}, 1.0,6 \mathrm{R}, .95,0.6,2 \mathrm{R}$

$1005 \mathrm{D} 280.6,2 \mathrm{R}, 0.95,2 \mathrm{R}, 1.0,6 \mathrm{R}, .95,0.6,2 \mathrm{R}$

$1005 \mathrm{D} 290.6,2 \mathrm{R}, 0.95,2 \mathrm{R}, 1.0,6 \mathrm{R}, .95,0.6,2 \mathrm{R}$

$1005 \mathrm{D} 300.6,2 \mathrm{R}, 0.95,2 \mathrm{R}, 1.0,6 \mathrm{R}, .85,0.6,2 \mathrm{R}$

$1005 \mathrm{D} 310.6,2 \mathrm{R}, 0.95,2 \mathrm{R}, 1.0,6 \mathrm{R}, .95,0.6,2 \mathrm{R}$

$1005 \mathrm{D} 320.6,2 \mathrm{R}, 0.95,2 \mathrm{R}, 1.0,6 \mathrm{R}, .95,0.6,2 \mathrm{R}$

$1005 \mathrm{D} 330.0,4 \mathrm{R}, 0.82,6 \mathrm{R}, 0.0,3 \mathrm{R} /$

$1006 \mathrm{D} 1000 ., 1300$.

C

C **** AFTERHEAT DATA (SECTION 7 ) $* * * * * * * * * * * * * * * * * * * * * * * *$

C INITIAL POWER ** *************************

1101D 350.E6, 0., 1.4, 1., 2

C AFTERHEAT REGION ** **** ********************

$1102 \mathrm{D} 7,12,13,32$

C REFLECTOR REGION ** ** **********************

$1103 \mathrm{D} 1,15,10,34$

C ************AFTER HEAT PROFILE DATA**********************

C BASE RADIAL POWER PROFILE ( IHI - (ILO-1) ENTRIES )

1104D 1.0,6R/

C POINT KINETICS KADIAL POWER PROFILE ( IHI - (ILO-1) ENTRIES )

1105D $0.7121,2 \mathrm{R}, 1.053,2 \mathrm{R}, 1.163,2 \mathrm{R} /$

C BASE AXIAL POWER PROFILE ( JHI - (JLO-1) ENTRIES )

$1106 \mathrm{D} 1.36,10 \mathrm{R}, 0.77,6 \mathrm{R}, 0.445,4 \mathrm{R} /$

C POINT KINETICS AXIAL POWER PROFILE ( IHI - (ILO-1) ENTRIES )

1107D $1.36,10 \mathrm{R}, 0.77,6 \mathrm{R}, 0.445,4 \mathrm{R} /$ 
C ** AFTER HEAT OPTIONS

$1108 \mathrm{D}$ 'NOML'

C ** ** TIME CONTROL ( SECTION 8A ) *************************

C START TIME (HR) ******************************

$1201 \mathrm{D} 0$.

C TIME PERIODS **** ***********************

$1202 \mathrm{D} 7$

C TIME STEPS FOR RAPID REACTIVITY EXCURSION

$1203 \mathrm{D} 0,240,15 ., 120,240$.

$1,10,1,6,6$.

$1,60 ., 5,50 ., 25$.

$2,5, .25,4,1$.

$2,20, .5,15,1$.

$2,100,1,20,1$.

$2,200,1,50,1$.

C $2,400,2,50,2$.

C $2,700,2,20,4$.

C $2,2000,2 ., 100,4$.

C OUTPUT FORMATS ( SECTION 8B ) ************************** 1301D 4, 0, 8,1

C BLOCK SURFACES OUTPUT ( SECTION 8C) $* * * * * * * * * * * * * * * * * * *$ $1401 \mathrm{D} 2$

$1402 \mathrm{D} 10,2,10,4$

C DEBUG ( SECTION 8D) $* * * * * * * * * * * * * * * * * * * * * * * * * * * * * *$

C DEBUG CONTROL VARIABLE

1403D 0

C DEBUG SET DATA ( BYPASS AT THIS TIME, SETTING NO OF SETS = 0 10

C **** INITIAL TEMPERATURES ( SECTION 9 ) ****************** 1501D 'READRUN ,

C 'OTHER'

$1502 \mathrm{D} 100.0,21 \mathrm{R}$

$1503 \mathrm{D} 100.0,35 \mathrm{R}, 80.0,9 \mathrm{R}$

1504D 100.0,21R

C BLOCK TEMPERATURES **** ****************

$1505 \mathrm{D} \quad \mathrm{B} 1 ; 24 * 258$.

$\mathrm{B} 1 ; 6 * 269$.

$\mathrm{B} 1 ; 6 * 290$.

$\mathrm{B1} ; 6 * 312$.

$\mathrm{B} 1 ; 6 * 333$.

$\mathrm{B} 1 ; 6 * 355$.

$\mathrm{B} 1 ; 6 * 376$.

$\mathrm{B} 1 ; 6 * 397$.

$\mathrm{B} 1 ; 6 * 419$.

$\mathrm{B} 1 ; 6 * 440$.

$\mathrm{B} 1 ; 6 * 462$.

$\mathrm{B} 1 ; 6 * 483$.

$\mathrm{B} 1 ; 6 * 505$.

$\mathrm{B} 1 ; 6 * 526$. 


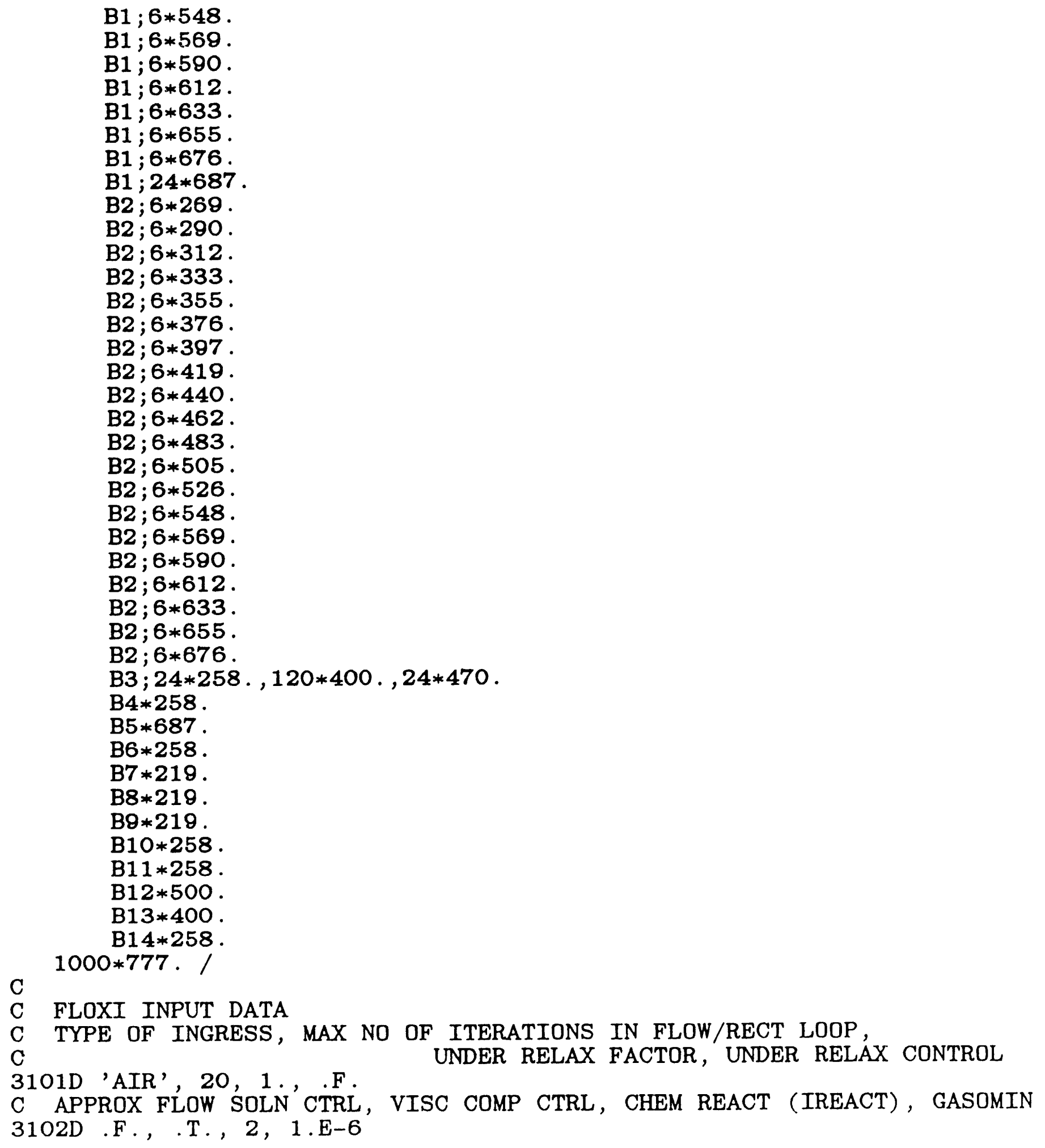




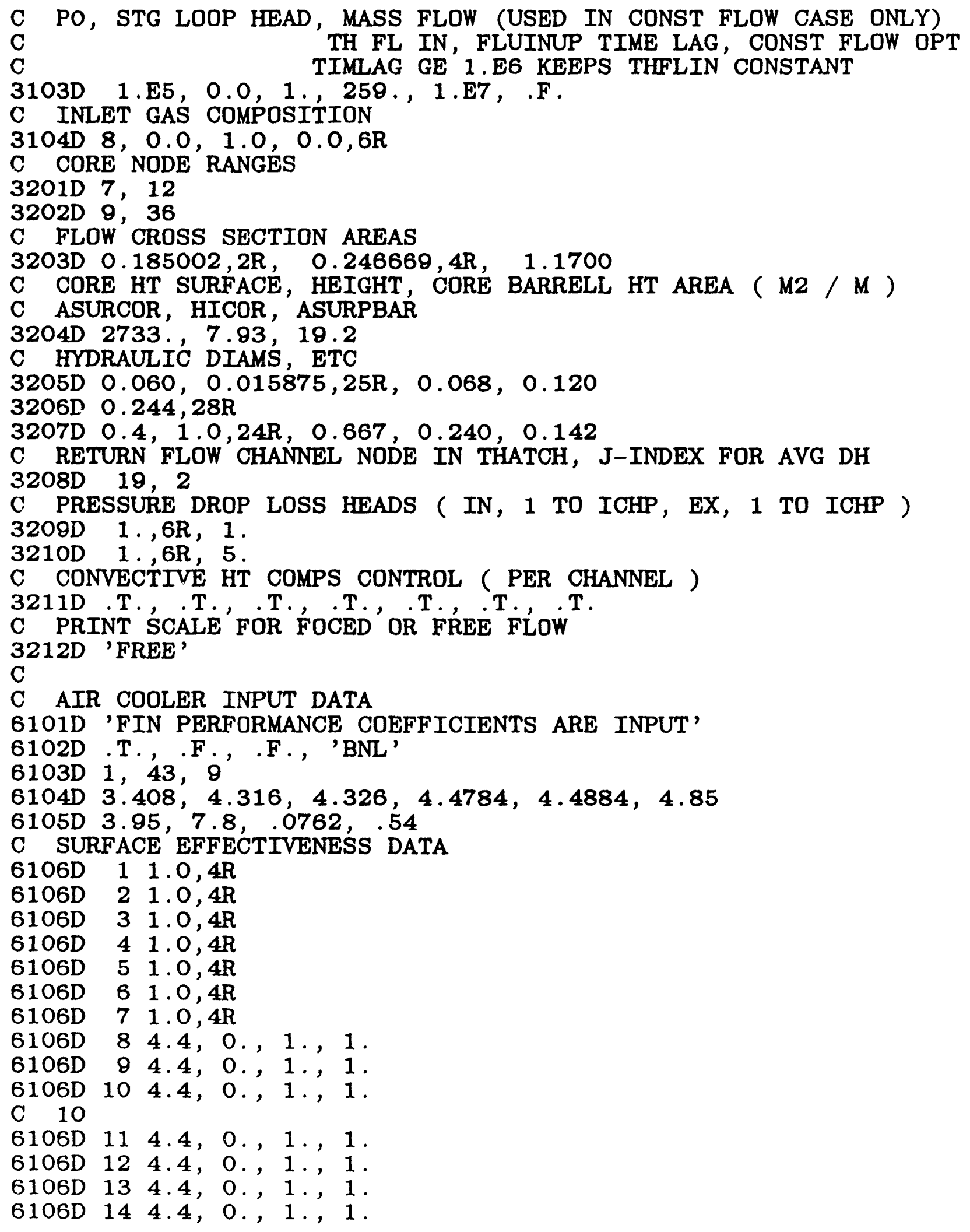




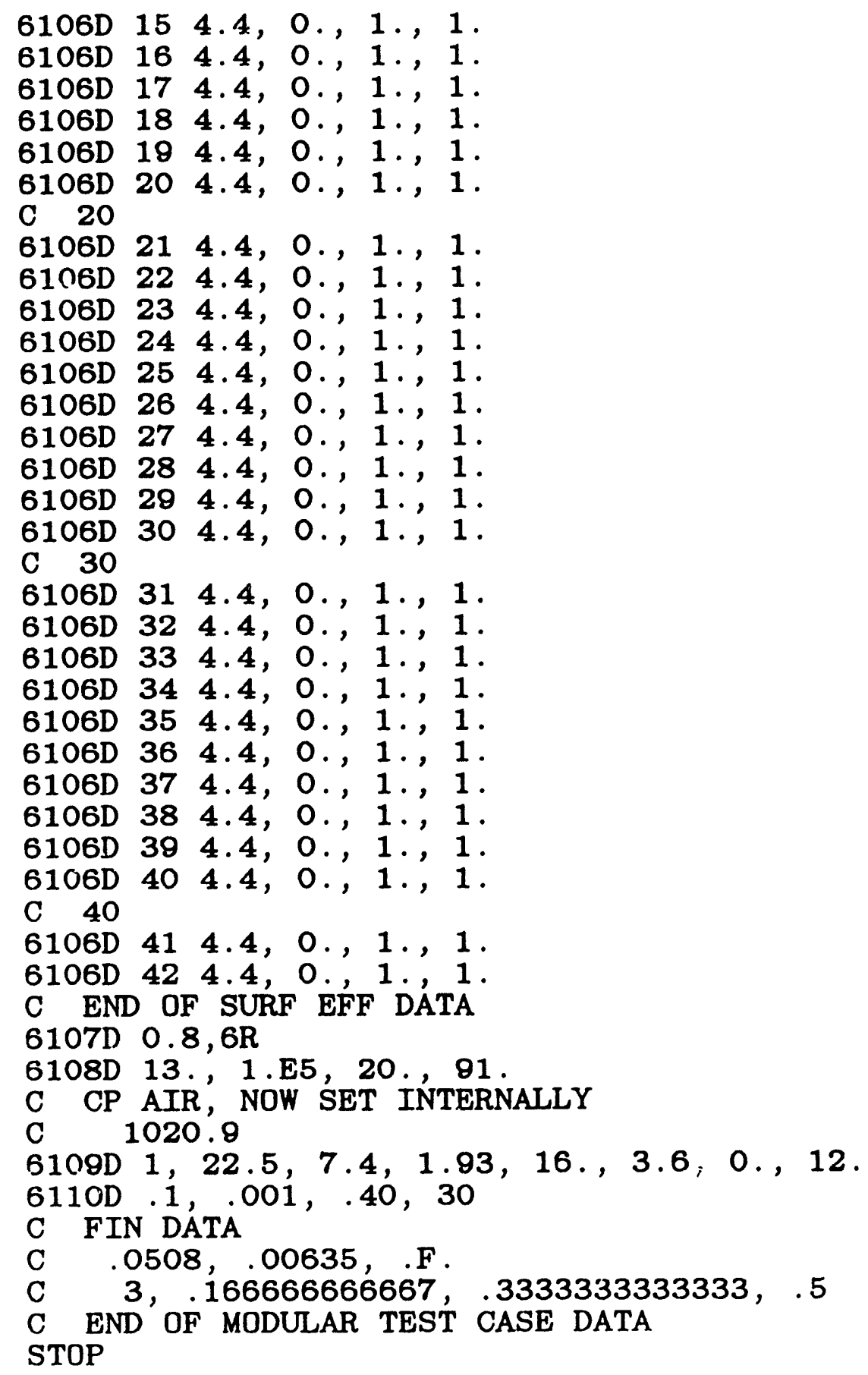


Input Deck for THATCH Sample Case No. 4

BASWAT

This input data file is for Base Depressurized Core Heatup Status of Sept 90 . Includes all shroud and core

barrel modifications. After SYMELAN integration into code.

Revised Input Processor and Unified Input Option

C

C

C

C

C

C

OV THATCH

101D'

102D'

103D'

ANNULAR CORE 35OMW MODULAR HTGR' DEPRESSURIZED CORE HEATUP ACCIDENT, RCCS OPERATING WATER COOLED RCCS'

C

C FLOXI OPTION, STEADY, SYMELL OPTION

C STEADY CAN ONLY BE TRUE IF FLOXI IS TRUE

201D .F., .F., .F.

C

C POINT KINETICS OPTION, RECOMPT OPTION

C RECOMPT ONLY USED IF PKINL = TRUE

202D .F., .F.

C

C UNIFIED INPUT OPTION CONTROL

C UNIFIN

301D . T.

C INITIAL RADIAL INTERACE POINT ( MUST BE ZERO FOR CURRENT THATCH

C RIFA-ARRAY, NODE DISTRIBUTION BY 'AREA' OR 'DELR', SMEARING OF

C RSC-CHANNEL LOSS OF AREA EFFECT IN INNER RADIAL CORE POWER ZONE 302D 0 , 'AREA', .F.

C NO OF NODES/CHANNELS IN

C CENTRAL REFL, ACT CORE REGN 1, REGN2, REGN3, SIDE REFLECTORS

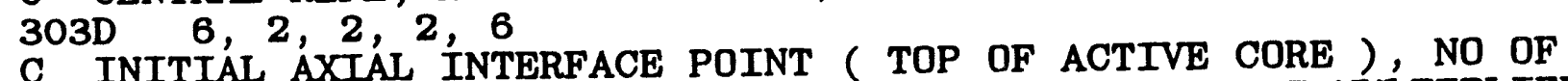
C INITIAL AXIAL INTERFACE POINT ( TOP OF ACTIVE CORE ) C 304D $12,2,1,1$

C BASE RADIAL POWER PROFILE 305D 1, 'D'

C POINT KINETICS RADIAL POWER PROFILE

306D O, 'F'

C BASE AXIAL POWER PROFILE

307D 10, 'D' 
C POINT KINETICS AXIAL POWER PROFIIE

308D 0, 'F'

C

C UNITS ( SECTION 2 ) **********************

C GEOMETRY (LENGTHS) ** **

401D ' $M$ '

C INPUT TEMPERATURES ****

403D ' $C$ '

C OUTPUT TEMPERATURES **** $404 \mathrm{D}$ ' $\mathrm{C}$ '

C GEOMETRY (SECTION 3 ) $* * * * * * * * * * * * * * * * * * * * * * * * * * * * * * *$ C RADIAL INTERFACE ****

501D 21

502D $0.336352,0.475674,0.582579,0.672704,0.752106,0.823891$ $1.000166,1.149724,1.323095,1.476243,1.614932,1.742619$ $1.982994,2.197229,2.413,2.572724,2.741251,2.900000$ $3.275,3.342,3.408$

C RADIAL POINT $0 * * * *$ 503D 0 .

C AXIAL INTERFACE ****

$504 \mathrm{D} 45$

$505 \mathrm{D}-5.29,-5.21,-4.653,-4.603,-4.59,-3.59,-2.59,-1.59$ $-1.2,-.8,-.4,0$.

$0.396500,0.793000$,

$2.775500,3.172000$,

$5.154500,5.551000$,

$7.533500,7.930000$

$1.189500,1.586000,1.982500,2.379000$

$3.568500, \quad 3.965000,4.361500,4.758000$

5.947500,

6.344000,

6.740500,

7.137001

$8.38,8.83,9.28,9.73,10.33$

$10.93,11.10,11.28,11.43,12.28,15.13,15.19,15.26$

C BLOCKS ( SECTION 4 ) ******** ****************** 601D 20

C BLOCK $1 * * * *$

602D 1 'CENTRAL REFLECTOR'

603D $10,6,8,36,1$

604D 14

605D 11

606D 17

C BLOCK $2 * * * *$

602D 2 'ACTIVE CORE'

$603 \mathrm{D} 26,12,12,32,2$

604D 25

605D 22

606D 26

607D 21

C BLOCK $3 * * * *$

6O2D 3 'INNER SIDE REFLECTORS'

$603 \mathrm{D} 312,15,8,36,1$

604D 34

605D 313

606D 37 
607D 32

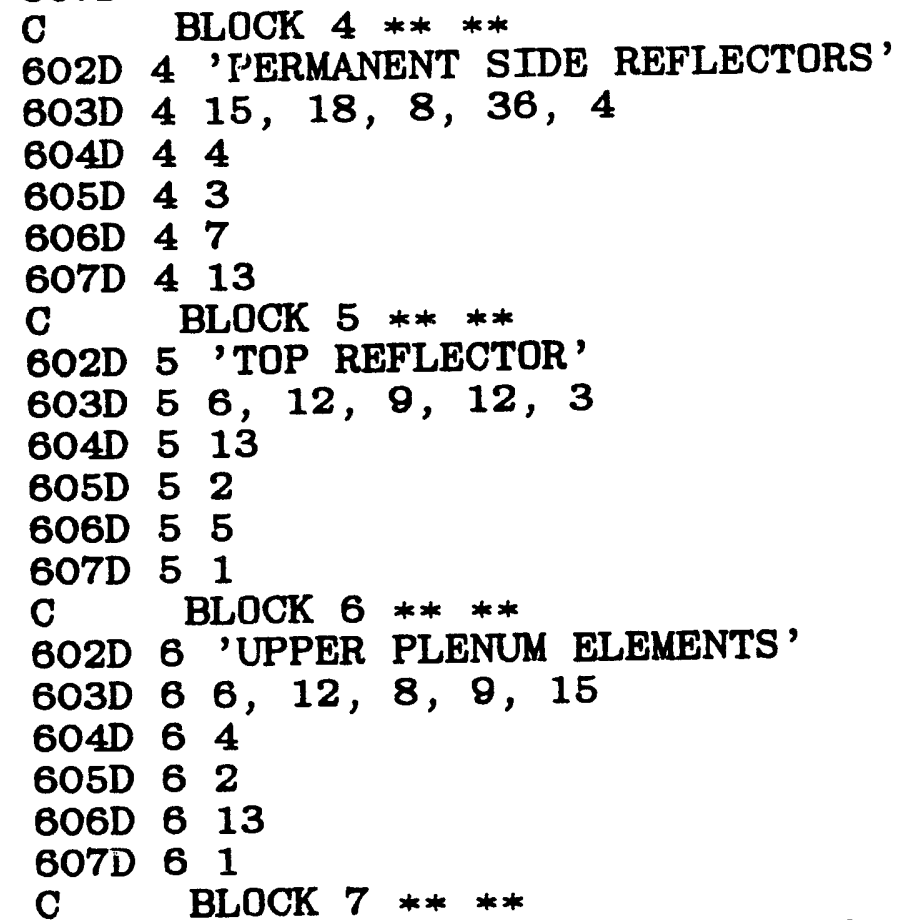

6O2D 7 'UPPER BOTTOM REFLECTORS'

603D $76,12,32,34,3$

604D 76

605D 72

606D 716

607D 71

C BLDCK $8 * * * *$

602D 8 'LOWER BOTTOM REFLECTORS'

$603 \mathrm{D} 86,12,34,36,5$

604D 816

605D 82

606D 87

607D 81

C 6 BLOCK 9 *****

$603 \mathrm{D} 918,19,8,43,10$

604D 914

/6O5D \& $114,9,10,4,11,43$

605D $9,14,9,10,4,11,43$

905D 14, 16,.025

606D $8 \quad 15$

607D $93,9,36,11,37,38,12,39,43$

C $* * * *$ BLOCK $10 * * * * * * * * * * * * * * * * * * * * * * * * * * * * * * * * * * * *$

602D 10 'VESSEL (SIDE)'

$603 \mathrm{D} 1019,21,1,45,6$

604D 101

605D $107,2,43,8,44,45$ 
606D $10 \quad 12$

607D $105,2,3,6,44,45$

C **** BLOCK $11 * * * * * * * * * * * * * * * * * * * * * * * * * * * * * * * * * * * *$ 602D 11 'VESSEL (TOP)'

603D $110,19,1,3,6$

604D 111

605D 115

C **** BLOCK $12 * * * * * * * * * * * * * * * * * * * * * * * * * * * * * * * * * * * *$ 602D 12 'VESSEL (BOTTOM)'

603D $120,19,43,45,6$

$604 \mathrm{D} 1211,1,18,15,18,19$

605D 126

606D 1212

C **** BLOCK $13 * * * * * * * * * * * * * * * * * * * * * * * * * * * * * * * * * * * *$ 602D 13 'SHROUD (SIDE)'

603D $1318,19,3,8,11$

604D 1318

/605D $13116,4,7,115,8,8$

605D $13 \quad 16,4,7,15,8,8$

905D $1617, .024$

905D $1517, .024$

606D $13 \quad 14$

607D $1310,4,5,9,6,8$

C **** BLOCK $14 * * * *$

602D 14 'SHROUD (TOP)'

$603 \mathrm{D} 140,18,3,5,10$

/604D $14102,1,18$

$604 \mathrm{D} 14 \quad 2,1,18$

904D $27, .037$

605D 1410

606D 143

C $* * * *$ BLOCK $15 * * * * * * * * * * * * * * * * * * * * * * * * * * * * * * * * * * * *$ 602D 15 'UPPER CORE FLOOR'

$603 \mathrm{D} 150,18,38,40,4$

604D 158

$\begin{array}{lll}605 \mathrm{D} & 15 & 12\end{array}$

$\begin{array}{lll}606 \mathrm{D} & 15 & 17\end{array}$

$\mathrm{C} * * * * \mathrm{BLOCK} 16 * * * * * * * * * * * * * * * * * * * * * * * * * * * * * * * * * * * * *$ 6O2D 16 'LOWER CORE FLOOR'

603D $160,18,40,41,8$

$604 \mathrm{D} \quad 16 \quad 17$

605D $16 \quad 12$

606D 168

$\mathrm{C} \quad * * * * \mathrm{BLOCK} 17 * * * * * * * * * * * * * * * * * * * * * * * * * * * * * * * * * * * * *$ 602D 17 'FLOOR SUPPORT 1 '

603D $170,18,41,42,6$

$604 D 178$ 


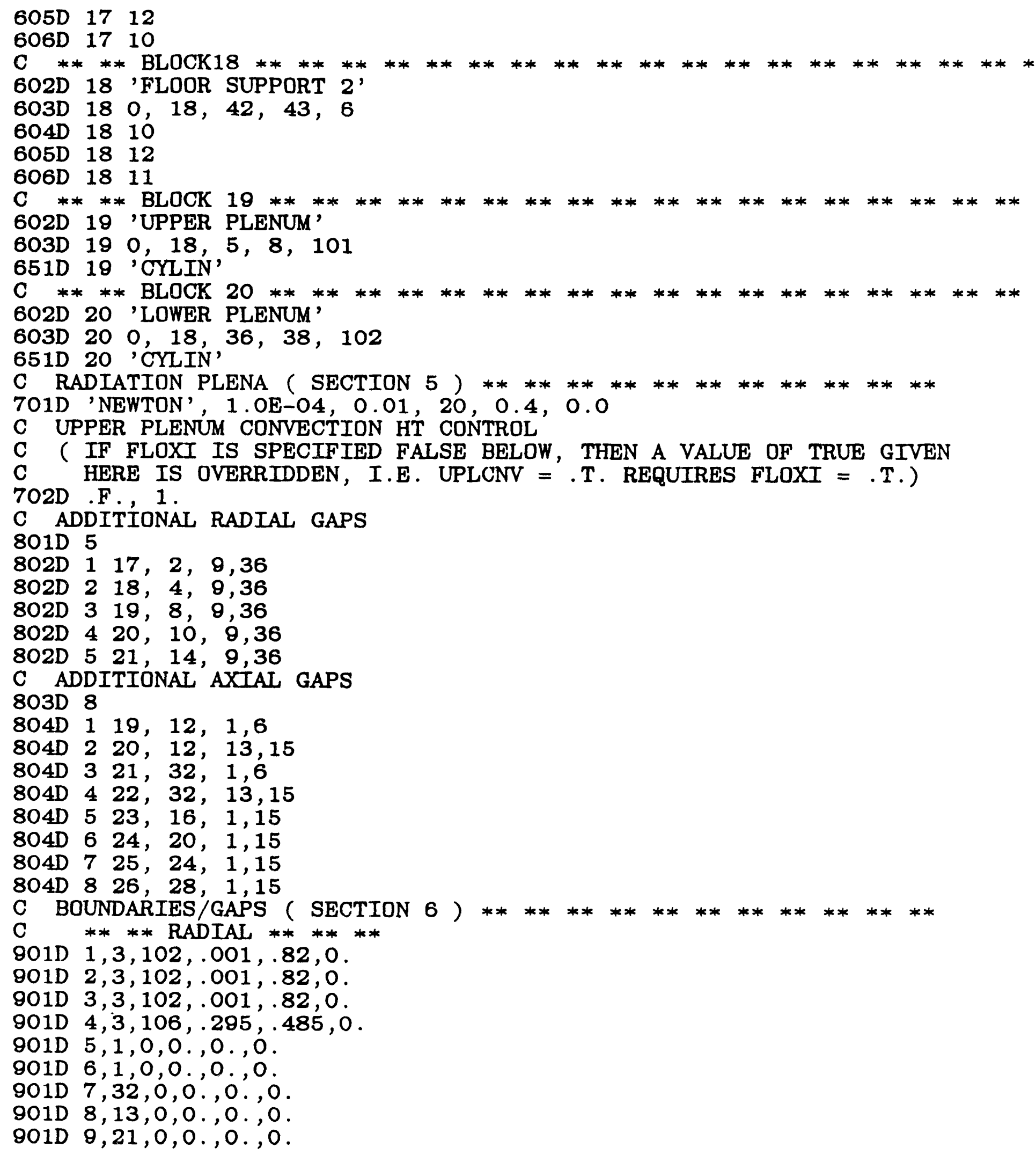


901D $10,1,0,0 \ldots, 0,0$.

901D $11,21,0,0 ., 0 ., 0$.

901D 12,1,0,0.,0., 0 .

901D 13,3,102,.001,.82,0.

901D $14,3,106, .270, .68,0$.

901D $15,3,106, .271, .68,0$.

901D 16, 3, 106,.271,.68,0.

901D $17,3,102, .001, .82,0$.

901D $18,3,102, .001, .82,0$.

901D $18,3,102, .001, .82,0$.

901D $20,3,102, .001, .82,0$.

901D 21, 3, 102,.001,.82,0.

C

** ** AXIAL ** ****

902D $1,12,0,0 ., 0 ., 0$.

902D $2,3,107, .477, .68,0$.

902D 3,21,0,0.,0., 0 .

902D $4,21,0,0 ., 0 ., 0$.

902D $5,3,102, .0005, .82,0$.

902D $6,3,102, .0005, .82,0$.

902D 7,21,0,0.,0.,0.

902D 8,21,0,0.,0.,0.

902D $9,1,0,0 ., 0 \ldots, 0$.

902D $10,3,102, .7, .68,0$.

902D $11,3,102,2, .68,0$.

902D $12,13,0,0 ., 0 ., 0$.

902D $13,3,102, .0005, .82,0$.

902D $14,1,0,0 ., 0 ., 0$.

$902 \mathrm{D} 15,3,102,2 \ldots ., 68,0$.

902D $16,3,102, .0005, .82,0$.

902D $17,1,0,0 ., 0 ., 0$.

902D 18,5,0,0.,0., 0 .

902D $19,3,102, .0005, .82,0$.

902D $20,3,102, .0005, .82,0$.

902D $21,3,102, .0005, .82,0$.

902D $22,3,102, .0005, .82,0$.

902D $23,3,102, .0005, .82,0$.

902D $24,3,102, .0005, .82,0$.

902D 25, 3, 102,.0005,.82,0.

902D 26,3,102,.0005,.82,0.

C IRRADIATION AND ANNEALING DATA

C IF FIRST PARAMETER ( IRRADN IS FALSE, THEN THIS SECTION IS BYPASSED)

1001D.T.

$1002 \mathrm{D} . \mathrm{T}$.

1003D 3

C ASSUMED BASE CASE IRRADIATION DAMAGE

$1004 \mathrm{D} 3,15,12,33$

$1005 \mathrm{D} 120.0,4 \mathrm{R}, 1.0,6 \mathrm{R}, 0.0,3 \mathrm{R} /$

$1005 \mathrm{D} 130.6,2 \mathrm{R}, 0.85,2 \mathrm{R}, 1.0,6 \mathrm{R}, .95,0.6,2 \mathrm{R}$

$1005 \mathrm{D} 140.6,2 \mathrm{R}, 0.95,2 \mathrm{R}, 1.0,6 \mathrm{R}, .85,0.6,2 \mathrm{R}$

$1005 \mathrm{D} 150.6,2 \mathrm{R}, 0.95,2 \mathrm{R}, 1.0,6 \mathrm{R}, .95,0.6,2 \mathrm{R}$ 


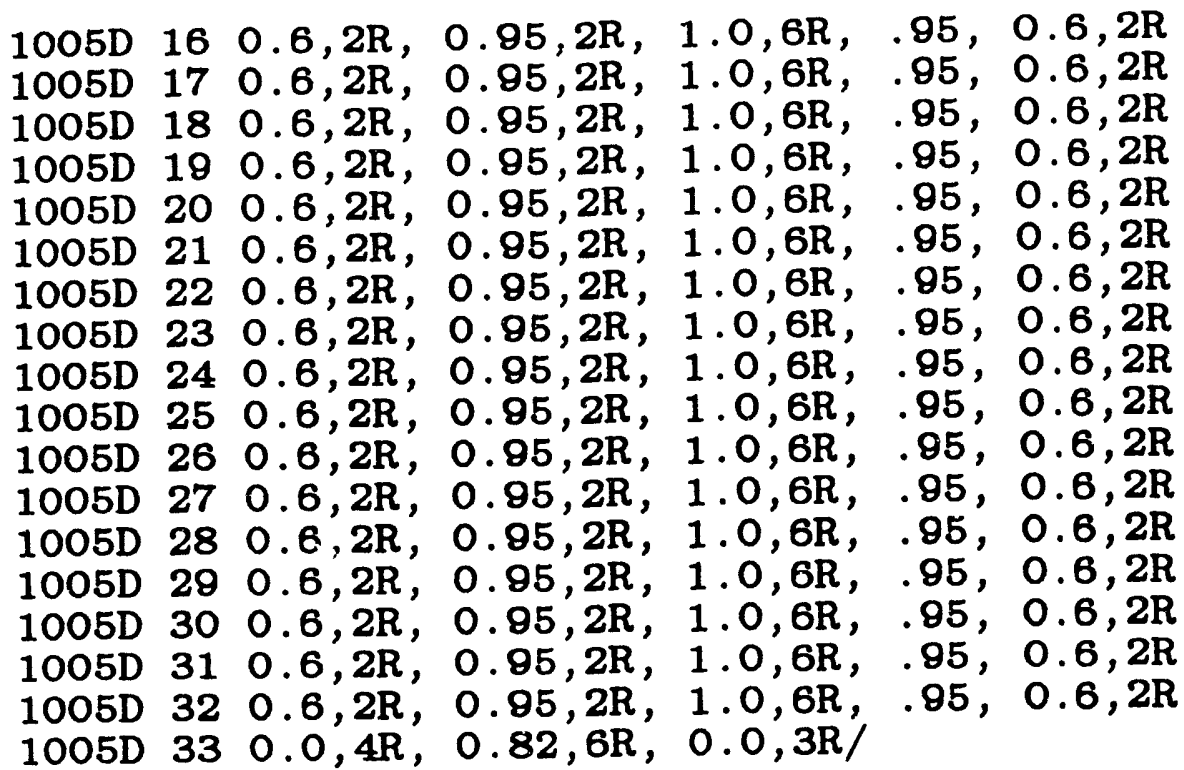

$1006 \mathrm{D} 1000 ., 1300$.

C $\quad * * * *$ AFTERHEAT DATA (SECTION 7) $* * * * * * * * * * * * * * * * * * * * * * * *$

C INITIAL POWER ****************************

1101D 350.E6, 0., 1.4, 1., 2

C AFTERHEAT REGION ** *************************

$1102 \mathrm{D} 7,12,13,32$

C REFLECTOR REGION **************************

$1103 \mathrm{D} 1,15,10,34$

C ************AFTER IEAT PROFILE DATA********************

C BASE RADIAL POWER PROFILE ( IHI - (ILO-1) ENTRIES )

$1104 \mathrm{D} 1.0,6 \mathrm{R} /$

C POINT KINETICS RADIAL POWER PROFILE ( IHI - (ILO-1) ENTRIES )

$1105 \mathrm{D} 0.7121,2 \mathrm{R}, 1.053,2 \mathrm{R}, 1.163,2 \mathrm{R} /$

C BASE AXIAL POWER PROFILE ( JHI - ( $\mathrm{LO}-1)$ ENTRIES )

$1106 \mathrm{D} 1.36,10 \mathrm{R}, 0.77,6 \mathrm{R}, 0.445,4 \mathrm{R} /$

C POINT KINETICS AXIAL POWER PROFILE ( IHI - (ILO-1) ENTRIES )

$1107 \mathrm{D} 1.36,10 \mathrm{R}, 0.77,6 \mathrm{R}, 0.445,4 \mathrm{R} /$

C ** AFTER HEAT OPTIONS

$1108 \mathrm{D}$ 'NOML'

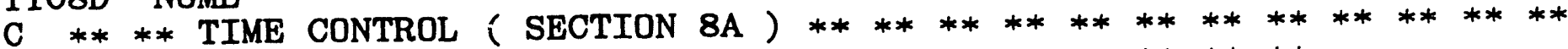

C START TIME (HR) **********************************

$1201 \mathrm{D} 0$.

C TIME PERIODS ** ***************************

1202D 8

C NORMAL TIME STEPS FOR DEPRESSURIZED TRANSIENTS

1203D $0,240 ., 15,, 240 ., 240$.

$1,10 ., 1,10,6$.

$1,60 ., 5 ., 50 ., 25$.

$2,5 ., 25,4 ., 1$.

$2,20 ., .5,15 ., 1$.

$2,100 ., 1 ., 20 ., 1$. 
$2,120,1,20,1$.

$2,200, .5,20 ., 1$.

C OUTPUT FORMATS ( SECTION 8B ) *************************** $1301 \mathrm{D} 4,0,8,1$

C BLOCK SURFACES DUTPUT (SECTION 8C) $* * * * * * * * * * * * * * * * * * *$ 1401D 2

$1402 \mathrm{D} 10,2,10,4$

C DEBUG ( SECTION 8D) $* * * * * * * * * * * * * * * * * * * * * * * * * * * * * *$

C DEBUG CONTROL VARIABLE

$1403 \mathrm{D} 0$

C DEBUG SET DATA ( BYPASS AT THIS TIME, SETTING NO OF SETS $=0$ / 0

C **** INITIAL TEMPERATURES ( SECTION 9 ) ****************** 1501D 'READRUN '

C 'OTHER'

1502D 100.0,21R

1503D $100.0,35 \mathrm{R}, 80.0,9 \mathrm{R}$

1504D 100.0,21R

C BLOCK TEMPERATURES ** ** ****************

$1505 \mathrm{D} \quad \mathrm{B} 1 ; 24 * 258$.

$\mathrm{B} 1 ; 6 * 269$.

$\mathrm{B} 1 ; 6 * 290$.

$\mathrm{B} 1 ; 6 * 312$.

$\mathrm{B} 1 ; 6 * 333$.

$\mathrm{B1} ; 6 * 355$.

$\mathrm{B1} ; 6 * 376$.

$\mathrm{B} 1 ; 6 * 397$.

$\mathrm{B1} ; 6 * 419$.

$\mathrm{B} 1 ; 6 * 440$.

$\mathrm{B} 1 ; 6 * 462$.

$\mathrm{B1} ; 6 * 483$.

$\mathrm{B} 1 ; 6 * 505$.

$\mathrm{B1} ; 6 * 526$.

$\mathrm{B} 1 ; 6 * 548$.

B1; $6 * 569$.

$\mathrm{B1} ; 6 * 590$.

$\mathrm{B1} ; 6 * 612$.

$\mathrm{B1} ; 6 * 633$.

B1; $6 * 655$.

$\mathrm{B1} ; 6 * 676$.

$B 1 ; 24 * 687$.

B2; $6 * 269$.

$\mathrm{B} 2 ; 6 * 290$.

$\mathrm{B2} ; 6 * 312$.

B2; $6 * 333$.

$\mathrm{B2} ; 6 * 355$.

$\mathrm{B2} ; 6 * 376$.

B2 ; $6 * 387$.

B2; $6 * 419$.

B2; $6 * 440$. 


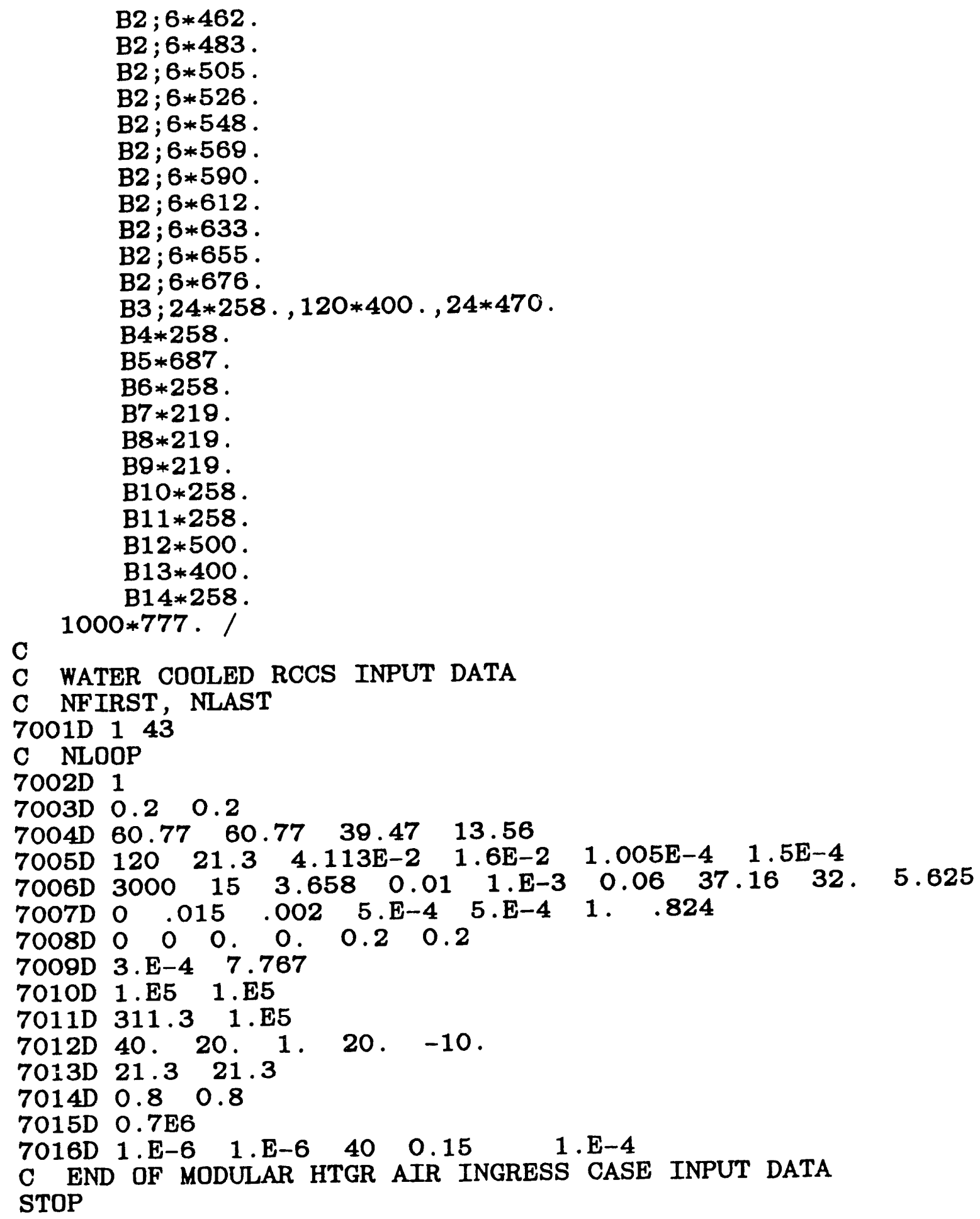


(See inseructions on the reverse)

THATCH: A Computer Code for Modelling Thermal Networks of High-Temperature Gas-Cooled Nuclear Reactors

Brookhaven National Laboratory

Upton, NY 11973

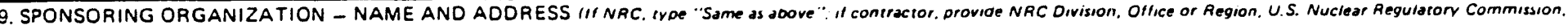
and malling oddress.1

Division of Regulatory Applications

Office of Nuclear Regulatory Research

U.S. Nuclear Regulatory Commission

Washington, DC 20555

10. SUPPLEMENTARY NOTES

11. ABSTRACT (200 words or kess)

This report documents the THATCH code, which models thermal and flow networks of solids and coolant channels in two-dimensional geometries.

The main application of THATCH is for reactor tuermo-hydraulic transients in High-Temperature GasCooled Reactors (HTGRs). The code simulates core heatup transients, heat transfer to general sinks or to specific air or water-cooled reactor cavity cooling systems. Graphite oxidation during air or water ingress can be modelled, including the chemical energy release. A point kinetics model is available for reactivity excursions. For most slow HTGR transients a user-selected nodalization of the core in $r-z$ geometry is used. A separate model of heat transfer in the symmetry element of each fuel element is available for rapid transients.

The report describes the mathematical models and the method of solution. It describes the code structure and its various procedures. Details of the input data and file usage, is given for the code and for the preprocessing and postprocessing options. The THATCH model of the current $350 \mathrm{MW}_{\text {th }}$ reactor is described. Input data for four sample cases are given, with output available in fiche form. Installation requirements, code limitations, and some common error indications are listed.

Heat transfer, mathematical models, Hydraulics, mathematical models, HTGR Type Reactors, Reactor Safety, Reactor Accidents, computerized simulation, Reactor Safety, Computer Codes, T-codes, transients, Geometry, Loss of Coolant, Reactivity, Excursions, fuel. Element Failure, Reactor Kinetics, Mathematical models. HTGR Type Reactors, Heat Iransfer, HTGR Type Reactors, Hydraulics, THATCH Computer Code

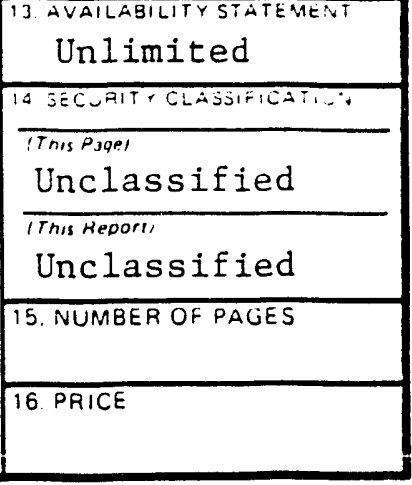



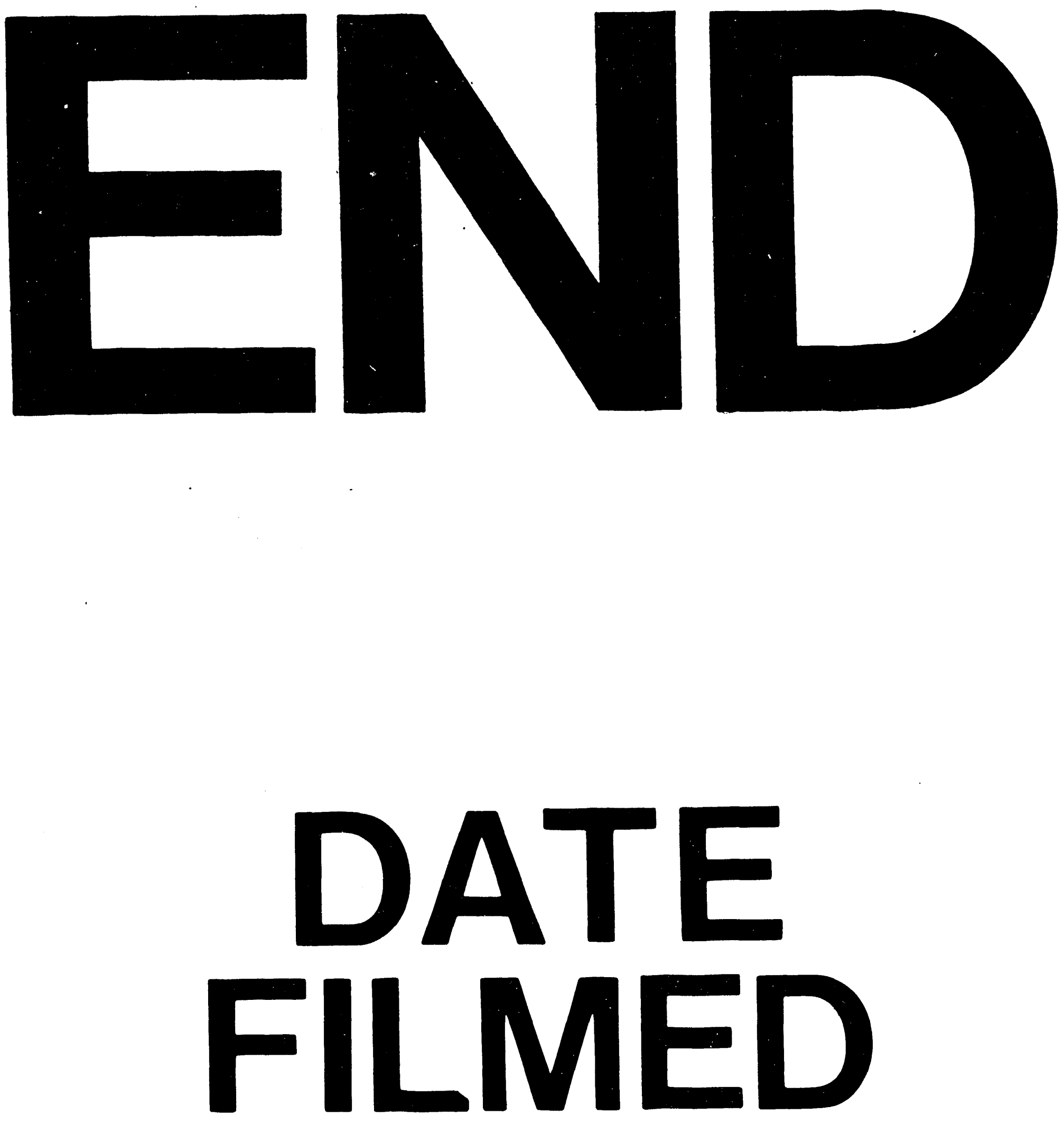
